Katrin Günther

\title{
Historisches Vergleichen:
}

\author{
Vergleichsaufgaben in \\ Lehrwerken des \\ Gesellschaftslehre- und \\ Geschichtsunterrichts \\ der Sekundarstufe II
}



Geschichtsdidaktische Studien

Band 7 


\section{Geschichtsdidaktische Studien}

\section{Band 7}

Herausgegeben von

Markus Bernhardt, Charlotte Bühl-Gramer, Bettina Degner, Marko Demantowsky und Thomas Hellmuth 
Katrin Günther

Historisches Vergleichen:

Vergleichsaufgaben in Lehrwerken

des Gesellschaftslehre- und

Geschichtsunterrichts der Sekundarstufe II

Eine Untersuchung von (Schüler*innen-)Lösungen

zu einer Vergleichsaufgabe am Beispiel eines

Darstellungstextes zur Industriellen Revolution 
Die Arbeit wurde im Jahr 2019 von der Fakultät für Geisteswissenschaften der Universität Duisburg-Essen als Dissertation angenommen.

Bibliografische Information der Deutschen Nationalbibliothek

Die Deutsche Nationalbibliothek verzeichnet diese Publikation in der Deutschen Nationalbibliografie; detaillierte bibliografische Daten sind im Internet über http://dnb.d-nb.de abrufbar.

(C) Copyright Logos Verlag Berlin $\mathrm{GmbH} 2020$

Alle Rechte vorbehalten.

ISBN 978-3-8325-5140-7

ISSN 2363-670X

\section{(c) (1) (8) $\Theta$ \\ BY NC ND}

Logos Verlag Berlin GmbH Georg-Knorr-Str.4, Gebäude 4, 12681 Berlin

Tel.: +49 (0)30 / 42851090

Fax: +49 (0)30/42851092

http://www.logos-verlag.de 


\section{Danksagung}

Diese Arbeit entstand während der Zeit meiner Abordnung als Lehrerin an die Universität DuisburgEssen. Dabei war die Beschäftigung mit dem Thema immer geprägt durch die Verbindung von Schule und Wissenschaft, anfänglich durch die Teilung der Stelle als Spagat zwischen Schule und Hochschule, immer aber durch den engen Praxisbezug, der mir durch die Arbeit im Projekt ProDaZ des Instituts für Deutsch als Zweit- und Fremdsprache ermöglicht wurde.

Ohne die Unterstützung zahlreicher Personen hätte diese Arbeit nicht realisiert werden können. Mein besonderer Dank gilt meiner Doktormutter Frau Prof. Dr. Heike Roll für ihre Unterstützung und Begleitung auf dem langen Weg von den ersten Ideen bis zur Veröffentlichung dieses Dissertationsprojekts.

Ebenso möchte ich Herrn Prof. Dr. Markus Bernhardt für die unterstützende wissenschaftliche Betreuung und Begleitung als Zweitgutachter sehr danken.

Mein herzlicher Dank geht an Tülay Altun, mit der ich über Jahre das Büro und oft meine Gedanken zu dieser Arbeit geteilt habe und die mir immer mit Rat und Tat zur Seite stand.

Den Anstoß für diese Arbeit gab Frau Dr. Claudia Benholz, ihre Motivation und Unterstützung brachte mich insbesondere durch die erste schwierige Zeit des Spagats zwischen Schule und Hochschule. Danken möchte ich außerdem dem Team in ProDaZ und im Institut DaF/DaZ sowie allen, die mit ihren konstruktiven Rückmeldungen bereitstanden, mich unterstützt oder mir einfach durch ihr Angebot, sie bei Schwierigkeiten jederzeit fragen zu können, den Rücken gestärkt haben.

Besonders möchte ich an dieser Stelle Andreas Fischer für die unermüdliche Motivierung und Geduld danken sowie meiner Familie für ihre ausdauernde Unterstützung.

Zum Schluss möchte ich mich bei den Schülerinnen und Schülern bedanken, die durch die Bereitstellung ihrer Texte dazu beigetragen haben, dass diese Arbeit überhaupt entstehen konnte. 


\section{Inhaltsverzeichnis}

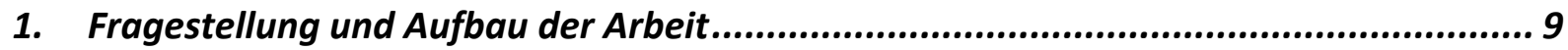

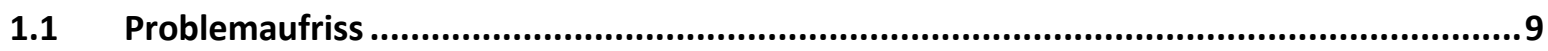

1.2 Begründung für die Auswahl und Herangehensweise .................................................12

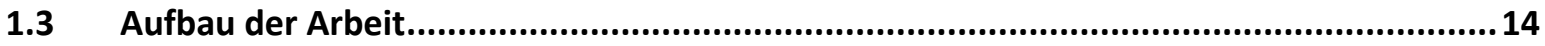

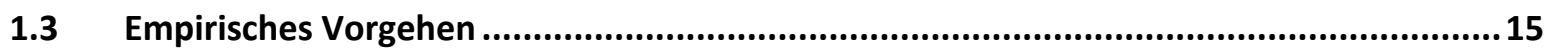

2. Das Vergleichen als Methode des kompetenzorientierten Geschichtsunterrichts...... 17

2.1 Einordnung des Vergleichens als kognitive Operation im Geschichtsunterricht............... 17

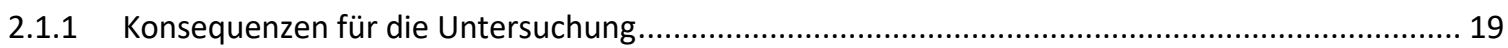

2.1.2 Vergleichen als Methode des kompetenzorientierten Unterrichts - Einpassung in das

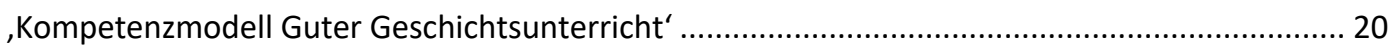

2.2 Aufgaben im kompetenzorientierten Geschichtsunterricht..........................................22

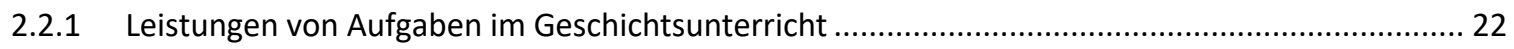

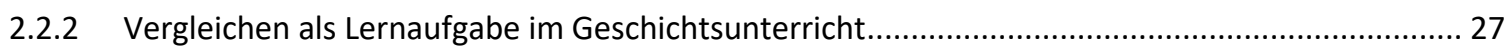

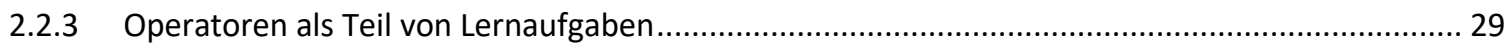

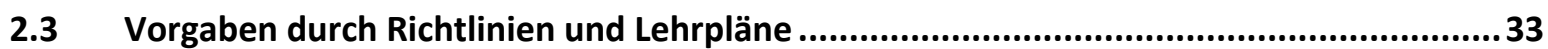

2.4 Wissenschaftspropädeutisches Arbeiten im Geschichtsunterricht .................................35

2.5 Ein funktionales Verständnis von Sprache für den Geschichtsunterricht -

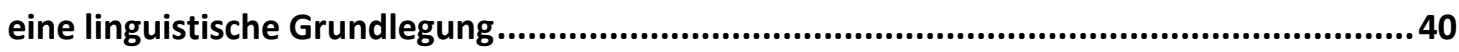

2.5.1 Historische Bewusstseinsbildung über Sprache - eine funktionale Betrachtungsweise ............... 41

2.5.2 Erkenntnisbildung über sprachliches Handeln im Geschichtsunterricht .................................. 47

2.5.3 Die Hinwendung zur Sprache im Geschichtsunterricht: Ein Weg zur Charakterisierung historischer

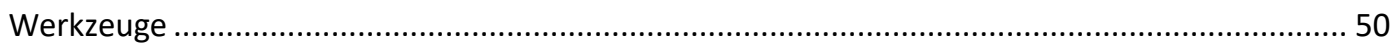

2.5.4 Das Konzept der Alltäglichen Wissenschaftssprache für wissenschaftspropädeutisches Arbeiten im Geschichtsunterricht. 51

2.6 Textsorten- und Textmusterorientierung im Geschichtsunterricht: ein Ansatz zur Entwicklung von Sprech- und Schreibfähigkeit im Geschichtsunterricht.............................55

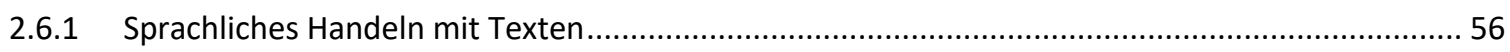

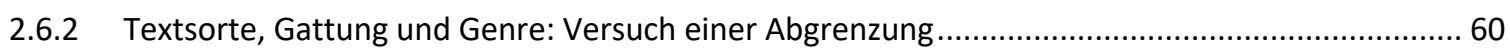

2.7 Schreiben im Geschichtsunterricht: Überlegungen zur Bedeutsamkeit für den

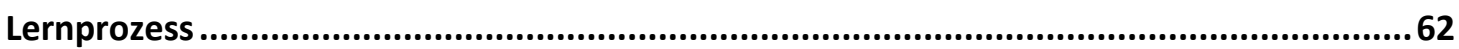

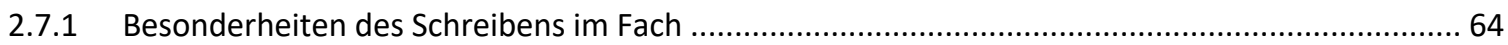

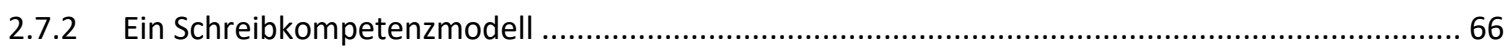

2.7.3 Schreiben im Geschichtsunterricht der Sekundarstufe II: Die Bedeutung von textbezogenem und materialgestütztem Schreiben für die Kompetenzentwicklung ............................................. 70

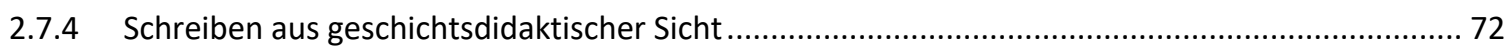

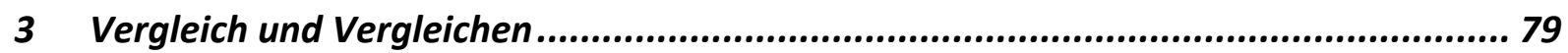

3.1 Alltägliches und fachliches Vergleichen .........................................................................79

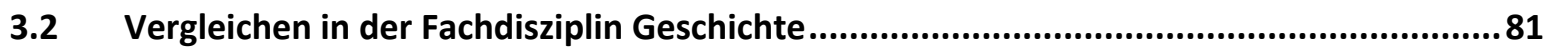

3.2.1 Historisches Vergleichen - ein kurzer Blick in die Geschichte ................................................ 83 
$3.3 \quad$ Vergleichen im Geschichtsunterricht ................................................................89

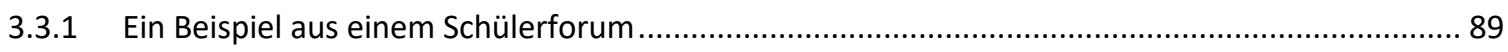

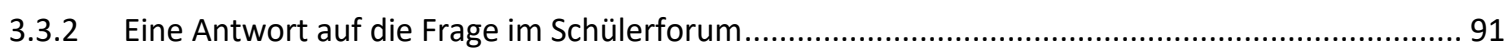

3.3.3 Merkmale des Vergleichs im Geschichtsunterricht ............................................................. 92

3.3.4 Vergleichbares und Unvergleichbares im Geschichtsunterricht? .............................................. 94

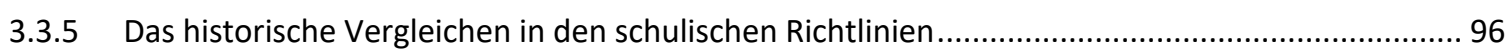

3.3.6 Methodenseiten zum Vergleichen in Geschichtslehrwerken .................................................. 101

3.3.7 Zur Didaktisierung des Vergleichs im Geschichtsunterricht: Ein erster Schluss.......................... 106

Ein sprachlicher Blick auf den Vergleich ............................................................107

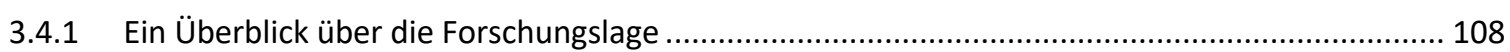

3.4.2 Bedeutung für das Vergleichen im Geschichtsunterricht .................................................. 116

3.5 Sprachliche Teilhandlungen als wesentliche Elemente des Vergleichens im

Geschichtsunterricht ........................................................................................ 118

3.5.1 Gegenüberstellen als Teilhandlung des historischen Vergleichs ............................................ 119

3.5.2 Erklärungen als Elemente des historischen Vergleichs ...................................................... 120

3.5.3 Begründen als Teil des Vergleichens............................................................................. 124

3.5.4 Beurteilen als zentrale sprachliche Teilhandlung des Vergleichs im Geschichtsunterricht .......... 126

3.5.5 Historisches Vergleichen als besondere Form des Beweisens............................................. 127

4 Vergleichsaufgaben in Geschichtslehrwerken: eine Frequenzanalyse....................... 129

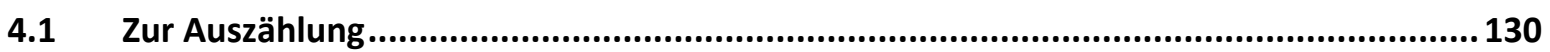

4.1.1 Teil 1: Frequenzanalyse der verwendeten Operatoren - Entscheidungen für die Auszählung ..... 131

4.1.2 Teil 2: Frequenzanalyse der W-Fragen - Entscheidungen für die Auszählung ............................ 132

4.1.3 Allgemeine Hinweise und Entscheidungen ................................................................. 132

$4.2 \quad$ Ergebnisse .................................................................................................. 134

4.2.1 Ergebnisse der vor 2014 erschienenen Lehrwerke .............................................................. 134

4.2.2 Ergebnisse der ab 2014 erschienenen Lehrwerke ................................................................ 151

4.3 Einordnung der Ergebnisse in der Gesamtschau ................................................... 168

5. Analyse von Lernendentexten zum historischen Vergleich: Vorstellung der ausgewählten Vergleichsaufgabe................................................................. 171

5.1 Vorstellung und Einordnung der ausgewählten Vergleichsaufgabe ...........................171

5.2 Der Darstellungstext zur Vergleichsaufgabe .................................................. 173

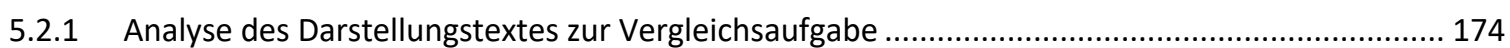

5.3 Einordnung von Text und Aufgabe................................................................. 190

5.4 Die Vergleichsaufgabe als typische Aufgabe ................................................... 196

6 Analyse von Lernendentexten zum historischen Vergleich: Untersuchungsdesign und Methodik ....................................................................................................... 197

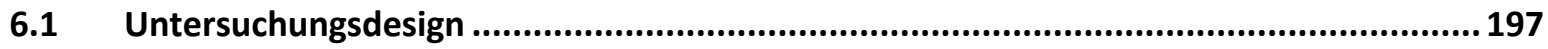

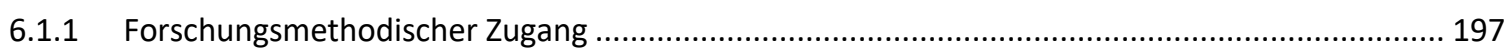

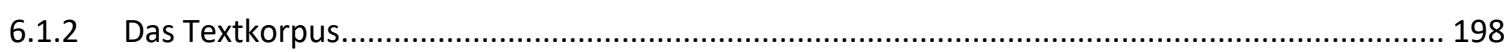

6.2 Darstellung des erarbeiteten Erhebungsinstruments .........................................203

6.2.1 Zum Aufbau des Erhebungsinstruments.............................................................................. 204 


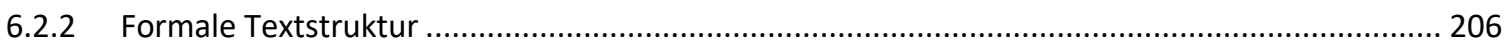

6.2.3 Vergleichsspezifische thematische Inhalte und inhaltlich-thematischer Fokus.......................... 209

6.2.4 Realisierung sprachlicher Teilhandlungen ..................................................................... 211

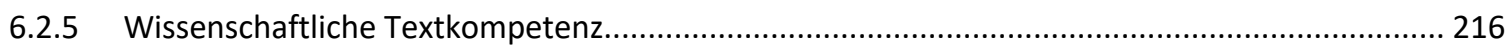

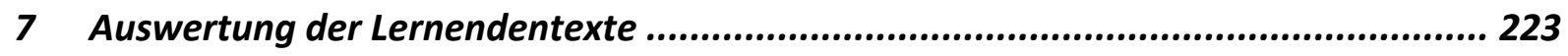

7.1 Interraterreliabilität ............................................................................ 223

7.1.1 Zum Vorgehen bei der Ermittlung der Interraterreliabilität ................................................. 223

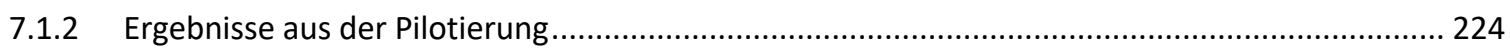

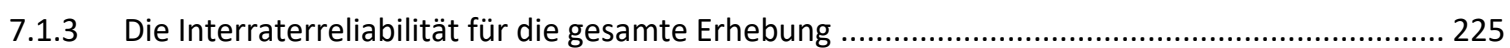

7.2 Die interne Konsistenz ...............................................................................226

7.3 Analyse und Interpretation der Ergebnisse .................................................... 227

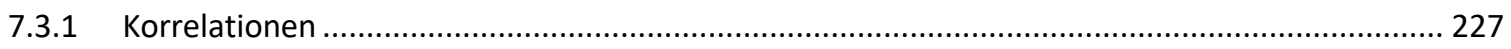

7.3.2 Zum Zusammenhang von Textqualität und der Nutzung expliziter sprachlicher Mittel.............. 230

7.3.3 Durchführung einer explorativen Faktorenanalyse ................................................................ 244

7.3.4 Interpretation der Rotierten Komponentenmatrix................................................................ 245

7.3.5 Zum Einfluss der Textgrundlage auf die Aufgabenlösung ...................................................... 254

8 Qualitative Analyse ausgewählter Lerner*innentexte: Drei Beispiele ..................... 257

8.1 Text 1: Eine Tabelle als Vergleichslösung ............................................................258

8.1.1 Analyse der formalen Textstruktur .................................................................................. 259

8.1.2 Vergleichsspezifische thematische Inhalte und inhaltlich-thematischer Fokus.......................... 260

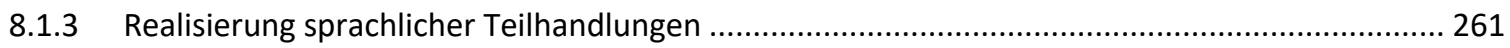

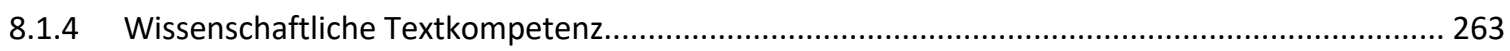

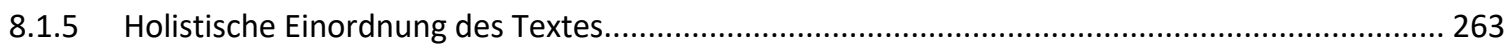

8.2 Text 2: Ein kurzer Vergleich als Fließtext.........................................................264

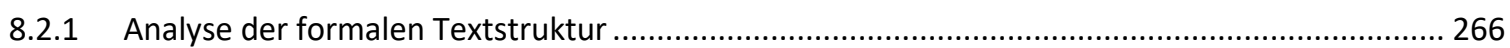

8.2.2 Vergleichsspezifische thematische Inhalte und inhaltlich-thematischer Fokus.......................... 266

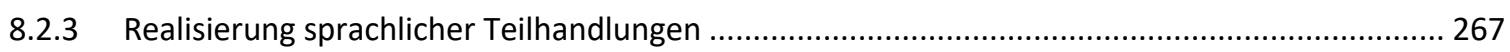

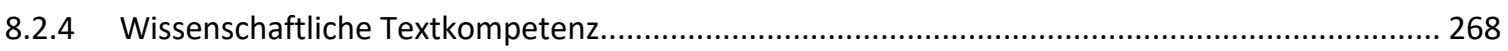

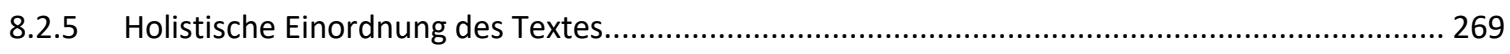

8.3 Text 3: Der Text mit der höchsten Gesamtpunktzahl .........................................271

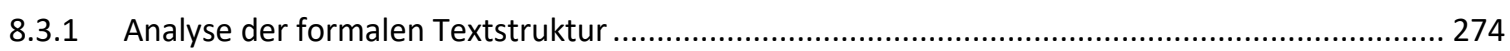

8.3.2 Vergleichsspezifische thematische Inhalte und inhaltlich-thematischer Fokus........................... 274

8.3.3 Realisierung sprachlicher Teilhandlungen ...................................................................... 278

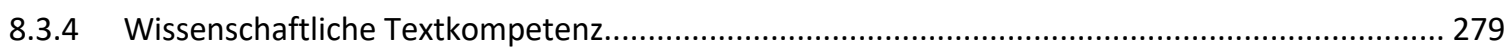

8.3.5 Holistische Einordnung des Textes...................................................................................... 280

8.4. Exkurs: Die Lösung der Aufgabe in der Handreichung für Lehrkräfte ...........................281

8.5 Fazit aus der qualitativen Analyse ........................................................... 283

9 Modell „Vergleichen im Geschichtsunterricht“: Übertragungsmöglichkeiten für die Verwendung im Geschichtsunterricht ..................................................................... 285

9.1 Vorschlag für ein praxistaugliches Kriterienraster für den Vergleich im Geschichtsunterricht: Eine Lehrer*innenhandreichung .......................................285

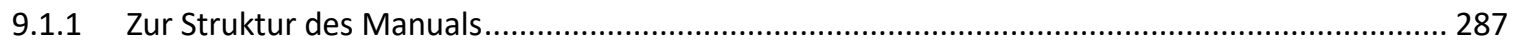


9.1.2 Historisches Vergleichen: Ein Kriterienraster zur Einschätzung einer Vergleichsaufgabe im

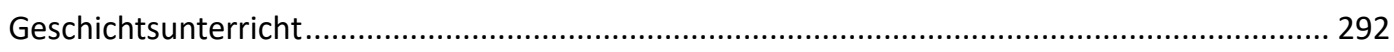

9.1.3 Hinweise zur Auswertung mit dem Vergleichs-Manual ......................................................... 309

9.2 Entwurf für eine Strukturierung von Vergleichstexten für die Schülerhand..................310

9.2.1 Eine Strukturskizze als Hilfe für Schreibnovizen ................................................................... 311

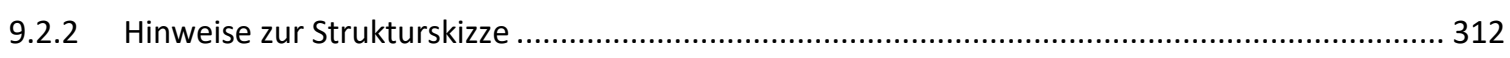

10 Zusammenfassung und weiterführende Überlegungen ............................... 315

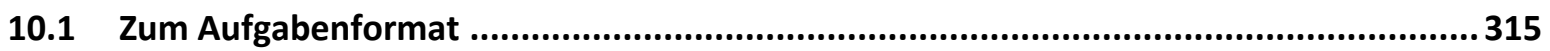

10.2 Zum Stellenwert einer kritischen Lehrwerkanalyse............................................318

10.3 Zum Umgang mit Operatoren im Geschichtsunterricht ........................................319

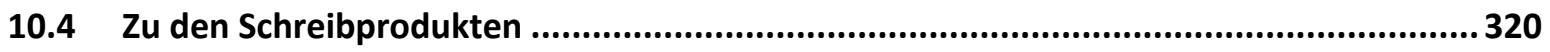

10.5 Zum wissenschaftspropädeutischen Schreiben von Texten in der Sekundarstufe II....... 322

11 Ableitungen: Welche Lehr- und Lernmaterialien werden für den Vergleich im Geschichtsunterricht benötigt, die fachliches und sprachliches Lernen fördern? ... 325

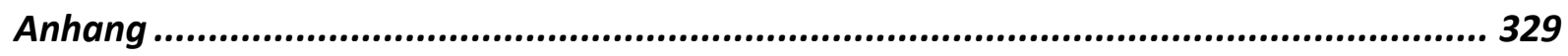

Literaturverzeichnis......................................................................... 396 


\section{Abbildungen}

Abbildung 1: Struktur der Arbeit.

Abbildung 2: Passung des Vergleichens in das Kompetenzmodell 'Guter Geschichtsunterricht' nach Gautschi (2011), (eigene Darst.)

Abbildung 3: Checkliste Kompetenzorientierung (Köster/Bernhardt/Thünemann, 2016) ...................28

Abbildung 4: Sprach- und Wissensmodell nach Ehlich, K.; Rehbein, J. (1986), S. 96. ............................43

Abbildung 5: "Prozessmodell sprachlichen Handelns im Geschichtsunterricht" (Handro, S., 2013, S.

325)

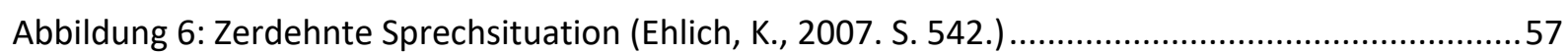

Abbildung 7: Anforderungen durch eine Schreibaufgabe (eigene Darstellung) .................................62

Abbildung 8: Zusammenführung des ,Sprach- und Wissensmodells' nach Ehlich/Rehbein (1986) und

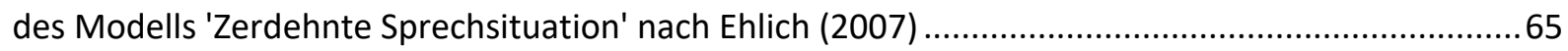

Abbildung 9: "Kompetenzmodell Schreiben" (Becker-Mrotzek, M./Schindler, K., 2007, S. 24.) ..........67

Abbildung 10: Anforderungen durch das Vergleichen im Geschichtsunterricht als Schreibanlass

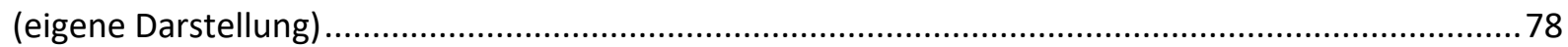

Abbildung 11: Sprachliche Teilhandlungen des Vergleichens im Geschichtsunterricht (eigene

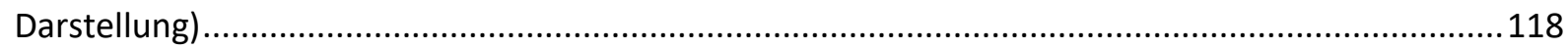

Abbildung 12: Struktur des Vergleichens im Geschichtsunterricht (eigene Darstellung) ...................128

Abbildung 13: Anteile der Aufgaben mit Operator und W-Fragen vor 2014 ....................................135

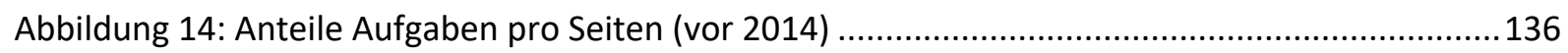

Abbildung 15: Anteil der Aufgaben, die sich auf Darstellungstexte beziehen (vor 2014)..................137

Abbildung 16: Darstellung der am häufigsten verwendeten Operatoren (vor 2014) .........................138

Abbildung 17: Verwendung von Operatoren im Anforderungsbereich I (vor 2014)...........................139

Abbildung 18: Häufigkeit der Verwendung der Operatoren im Anforderungsbereich I in den einzelnen

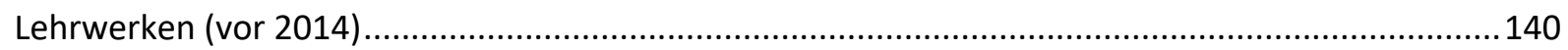

Abbildung 19: Verwendung von Operatoren im Anforderungsbereich II (vor 2014)........................... 141

Abbildung 20: Häufigkeit der Verwendung der Operatoren im Anforderungsbereich II in den

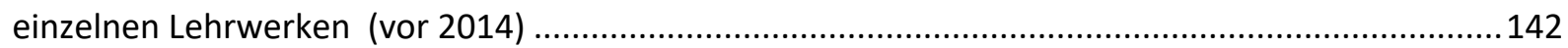

Abbildung 21: Verwendung von Operatoren im Anforderungsbereich II ..........................................144

Abbildung 22: Häufigkeit der Verwendung der Operatoren im Anforderungsbereich III in den

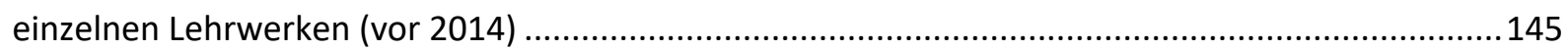

Abbildung 23: Verwendung der Breitbandoperatoren (vor 2014).................................................146

Abbildung 24: Verwendungshäufigkeit im Vergleich von Nicht-EPA-Operatoren und EPA-Operatoren

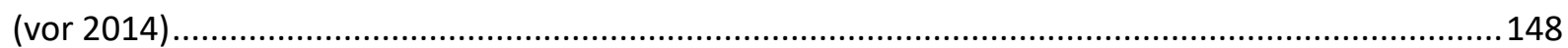

Abbildung 25: Der Operator 'Vergleichen' und seine Teiloperationen: Frequenzen in den Lehrwerken

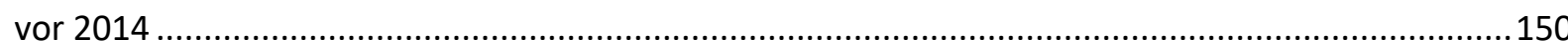

Abbildung 26: Verhältnis von Aufgabenformulierungen durch W-Fragen und Operatoren (ab 2014)

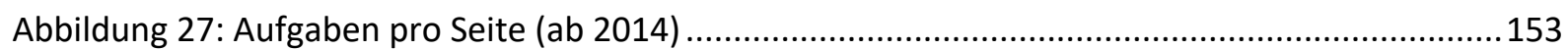

Abbildung 28: Anteil der Aufgaben, die sich auf Darstellungstexte beziehen (ab 2014) ....................154

Abbildung 29: Darstellung der am häufigsten verwendeten Operatoren (ab 2014) .........................155

Abbildung 30: Die am häufigsten verwendeten Operatoren in absoluten Zahlen (ab 2014) .............156

Abbildung 31: Verwendung von Operatoren im Anforderungsbereich I (ab 2014) ...........................157 
Abbildung 32: Häufigkeit der Verwendung von Operatoren im Anforderungsbereich I in den einzelnen

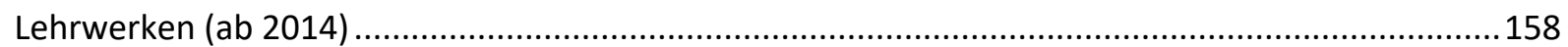

Abbildung 33: Verwendung von Operatoren im Anforderungsbereich II (ab 2014) .......................... 159

Abbildung 34: Häufigkeit der Verwendung von Operatoren im Anforderungsbereich II in den

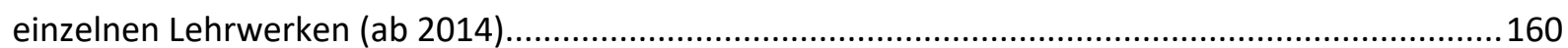

Abbildung 35: Verwendung von Operatoren im Anforderungsbereich III (ab 2014) .......................... 161

Abbildung 36: Häufigkeit der Verwendung der Operatoren im Anforderungsbereich III in den

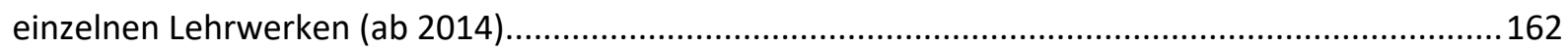

Abbildung 37: Verwendung der Breitbandoperatoren (ab 2014) ..................................................163

Abbildung 38: Verwendungshäufigkeit im Vergleich von Nicht-EPA-Operatoren und EPA-Operatoren

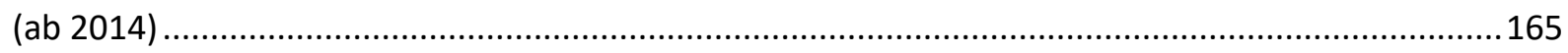

Abbildung 39: Der Operator 'Vergleichen' und seine Teiloperationen: Frequenzen in den Lehrwerken ab 2014 167

Abbildung 40: Kriterien für die Analyse von Vergleichstexten im Geschichtsunterricht (eigene

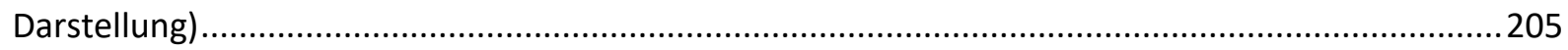

Abbildung 41: Textstrukturskizze 'Vergleich' (eigene Darstellung) ...............................................210

Abbildung 42: Sprachliche Teilhandlungen des Vergleichs (eigene Darstellung) ................................211

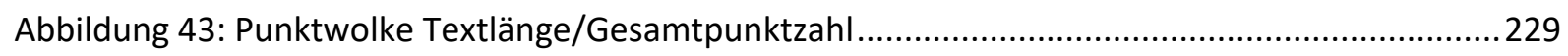

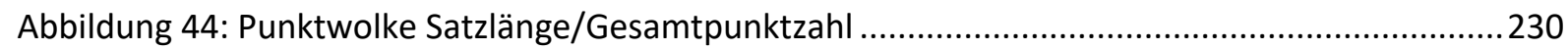

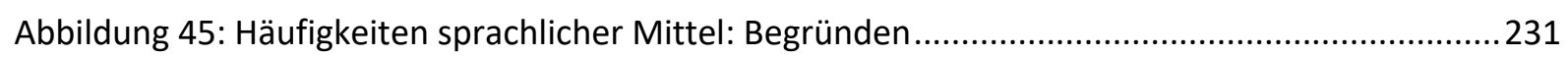

Abbildung 46: Realisierung der sprachlichen Teilhandlung: Begründen ..........................................232

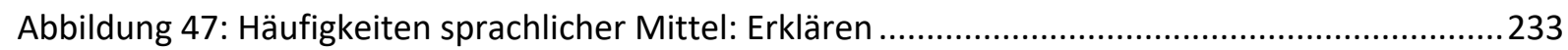

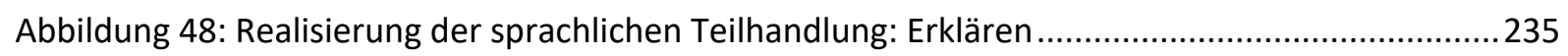

Abbildung 49: Häufigkeiten sprachlicher Mittel von Texten mit guten Erklärstrukturen ....................235

Abbildung 50: Häufigkeiten sprachlicher Mittel: Beurteilen..........................................................237

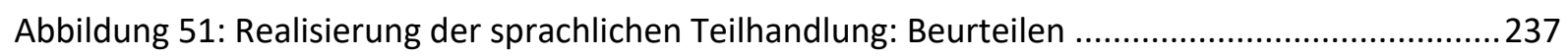

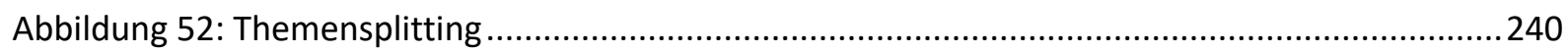

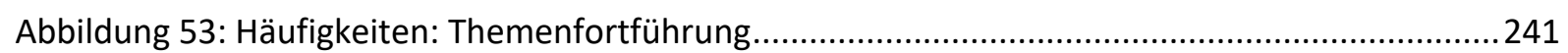

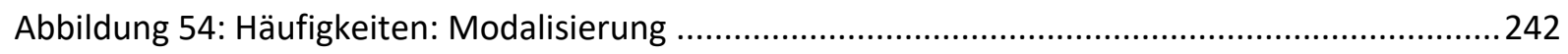

Abbildung 55: Ergebnisse aus den unterschiedlichen Erhebungsteilen...........................................254

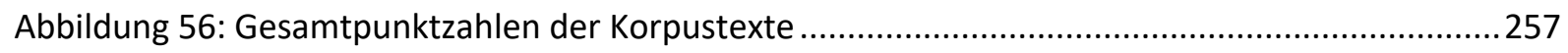

Abbildung 57: Indikatorenmodell „Vergleichen im Geschichtsunterricht“ (eigene Darstellung) .......287

Abbildung 58: Strukturskizze für das Vergleichen im Geschichtsunterricht (eigene Darstellung) .......311 


\section{Tabellen}

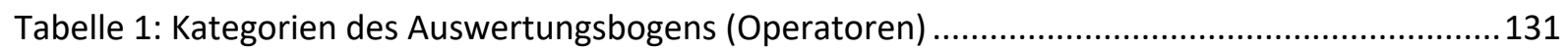

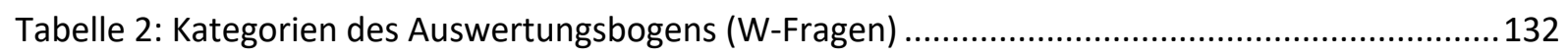

Tabelle 3: Zusammenführung der Prozessmodelle für die Einordung der Vergleichsaufgabe ..........191

Tabelle 4: Anwendung der Checkliste nach Köster/Bernhardt/Thünemann (2016) für die

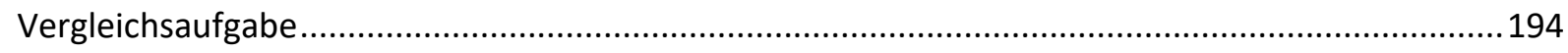

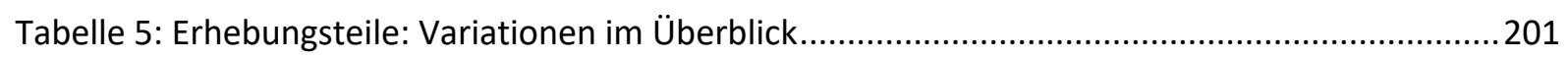

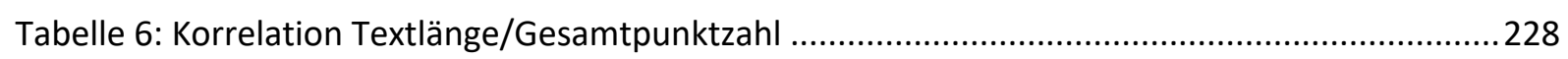

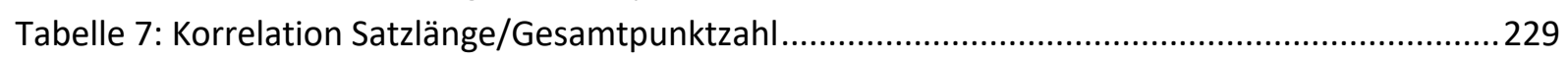

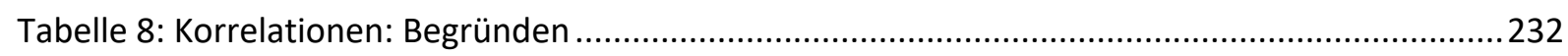

Tabelle 9: Realisierung der Teilhandlung und Nutzung sprachlicher Mittel: Begründen......................233

Tabelle 10: Texte mit den meisten sprachlichen Mitteln: Realisierung der Teilhandlung ...................234

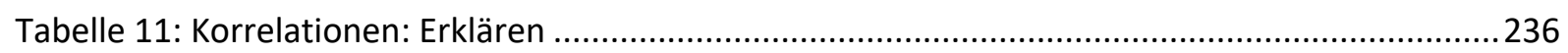

Tabelle 12: Realisierung der Teilhandlung und Nutzung sprachlicher Mittel: Erklären ......................236

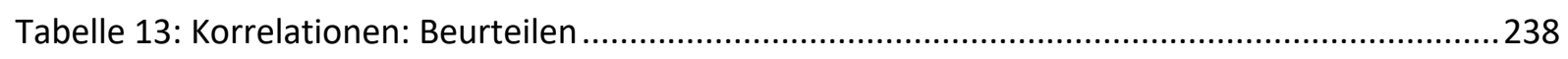

Tabelle 14: Realisierung der Teilhandlung und Nutzung sprachlicher Mittel: Beurteilen....................238

Tabelle 15: Korrelation Realisierung sprachliche Teilhandlungen/Nutzung sprachlicher Mittel

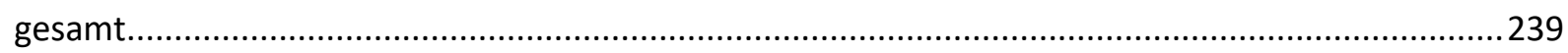

Tabelle 16: Anzahl der Items Themensplitting, Themenfortführung und Modalisierung an der

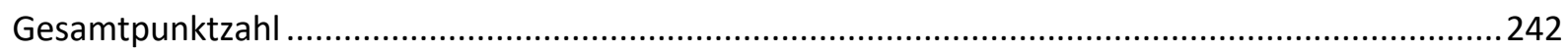

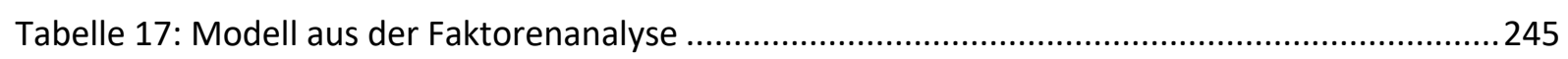

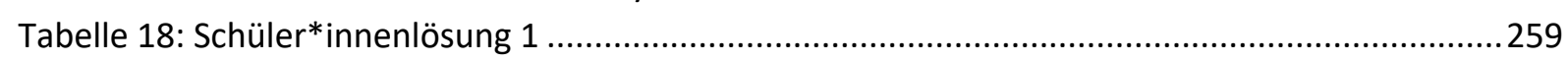

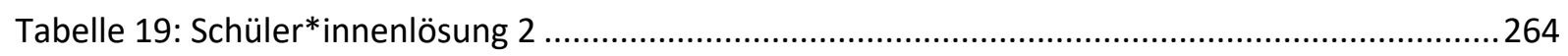

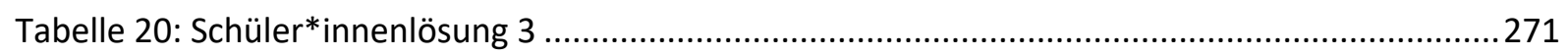

Tabelle 21: Realisierung von Vergleichsstrukturen des Kompetenzniveaus III nach Ausprägung ......285

Tabelle 22: Inhaltsverzeichnis: Kriterienraster für die Lehrerhand.................................................28

Tabelle 23: Kriterienraster für die Hand der Lehrkraft .................................................................292 


\section{Fragestellung und Aufbau der Arbeit}

\subsection{Problemaufriss}

Mit der Kompetenzorientierung des Geschichtsunterrichts stehen in den letzten Jahren Aufgabenstellungen verstärkt im Forschungsinteresse der Geschichtsdidaktik. Ein zentrales Forschungsinteresse richtet sich auf die Frage, was eine fachspezifisch kompetenzorientierte Aufgabe ausmacht. Nach wie vor stehen in der geschichtsdidaktischen Diskussion mehrere Kompetenzmodelle nebeneinander, die zwar alle der Förderung eines reflektierten Geschichtsbewusstseins dienen, sich aber in den Teilkompetenzen unterscheiden. Dennoch ist die grundsätzliche Bedeutung geschichtsspezifischer Kompetenzen für den Geschichtsunterricht unbestritten. Richtlinien und Lehrpläne als maßgebliche Vorgaben für (Geschichts-)Unterricht sind kaum eine Hilfe, wenn es darum geht, geschichtsspezifische Kompetenzen für den Unterricht transparent zu machen. ${ }^{1} \mathrm{Im}$ Gegenteil weisen beispielsweise die Kernlehrpläne Geschichte für die Sekundarstufe I und II der verschiedenen Schulformen in Nordrhein-Westfalen Sach-, Methoden-, Urteils und Handlungskompetenz als für den Geschichtsunterricht wesentliche Teilkompetenzen aus. ${ }^{2}$ Bei diesen Teilkompetenzen handelt es sich weniger um fachspezifische als vielmehr um domänenübergreifende Kompetenzen. Eine Anbindung an eines der aktuell in der Forschung diskutierten Kompetenzmodelle liegt durch die Kernlehrpläne Geschichte NRW nur in Ansätzen vor. Dabei stellen Kompetenzen „kognitive Tools für die Operationen des historischen Denkens oder das Know-how des historischen Lernens ${ }^{\prime 3}$ dar und bedürfen gerade deshalb einer fachdidaktischen Ausformung.

In Bezug auf Aufgaben im Geschichtsunterricht bedeutet dies, dass solche Aufgaben nicht nur Wissen abfragen, sondern beispielweise im Sinne einer historischen Narration eine Um- oder Neustrukturierung von Wissen anregen oder die Urteilskompetenz fördern sollen. Solche Aufgaben regen mentale Prozesse an, die für den Aufbau historischen Bewusstseins von hoher Bedeutung sind. Aufgaben können so ihren Beitrag leisten, das Unterrichtsfach Geschichte im Sinne Pandels zum ,Denkfach' zu machen. ${ }^{4}$ Dabei sollen Aufgaben, die durch handlungsinitiierende Verben formuliert werden, zur Kompe-

\footnotetext{
${ }^{1}$ Vgl. hierzu: Köster, Manuel; Bernhardt, Markus; Thünemann, Holger (2016): Aufgaben im Geschichtsunterricht. Typen, Gütekriterien und Konstruktionsprinzipien. In: Geschichte Lernen 29 (2016), H. 174, S. 2-11. S. 2.

${ }^{2}$ Vgl. z. B.: Ministerium für Schule und Weiterbildung des Landes Nordrhein-Westfalen (Hrsg.) (2007): Kernlehrplan für das Gymnasium - Sekundarstufe I (G8) in Nordrhein-Westfalen. Geschichte. Online einsehbar unter: http://www.schulentwicklung.nrw.de/lehrplaene/upload/lehrplaene_download/gymnasium_g8/gym8_geschichte.pdf (zuletzt eingesehen am 23.02.2017).

oder: Ministerium für Schule und Weiterbildung des Landes Nordrhein-Westfalen (Hrsg.) (2014): Kernlehrplan für das Gymnasium/Gesamtschule - Sekundarstufe II in Nordrhein-Westfalen. Geschichte. Online einsehbar unter: http://www.schulentwicklung.nrw.de/lehrplaene/upload/klp_SII/ge/KLP_GOSt_Geschichte.pdf (zuletzt eingesehen am 23.02.2017).

${ }^{3}$ Köster, Manuel; Bernhardt, Markus; Thünemann, Holger (2016), S. 2.

4 Vgl. Pandel, Hans-Jürgen (2007): Geschichtsunterricht nach PISA. Kompetenzen, Bildungsstandards und Kerncurricula. Schwalbach/Ts.: Wochenschau Verlag.
} 
tenzorientierung beitragen. Richtlinien und Lehrpläne legen nahe, dass diese als Operatoren bezeichneten Verben den Kompetenzerwerb anregen können. ${ }^{5}$ Vielfach wird davon ausgegangen, Operatoren seien klare Handlungsanweisungen, die dahinterstehenden Anforderungen seien also für die Lernenden transparent. Für das Fach Geschichte hat die Kultusministerkonferenz in den Einheitlichen Prüfungsanforderungen in der Abiturprüfung Geschichte ${ }^{6} 32$ Operatoren ausgewählt und mit einer kurzen, auf das Fach Geschichte bezogenen Bestimmung versehen. Manche der Operatoren teilen sich eine Bestimmung, wie bspw. das Analysieren und Untersuchen, anderen, wie dem Vergleichen, ist wiederum eine eigene Bestimmung zugeordnet. Es stellt sich die Frage, welche Teilhandlungen sich hinter den Operatoren verbergen und ob die zugehörigen Bestimmungen diese eindeutiger klären können. Bezieht man zudem in die Überlegungen ein, dass auch für andere Fächer Operatoren in den zugehörigen „Einheitlichen Prüfungsanforderungen“ benannt und fachspezifisch bestimmt werden, so wird deutlich, dass sich Lernende mit einer Vielzahl solcher Operatoren auseinandersetzen und diese entsprechend der Unterrichtsfächer differenzieren müssen. Die Frage um ihre fachspezifische Eingrenzung und damit nach der Eindeutigkeit solcher Handlungsaufforderungen stellt sich also erst recht.

Um eine Aufgabe auf ihre Fachspezifik hin zu hinterfragen, ist es nötig, auch ihre sprachlichen Anforderungen zu berücksichtigen. Sprache stellt die Grundlage für alle Fächer der schulischen Bildung dar oder anders formuliert: Ohne Sprache kann kein Fachunterricht stattfinden. Dies gilt sowohl für sogenannte Sprachfächer wie Deutsch, Englisch etc., aber auch für die Mathematik, das Bewegungsfach Sport, da auch dessen Inhalte sprachlich vermittelt sind, und nicht zuletzt den Geschichtsunterricht. Das Nachdenken über unterrichtliche Gegenstände, zu dem kompetenzorientierter Unterricht anregen soll, wird durch Sprache konstitutiv. Sie ist mehr als ein Instrument, anderen seine Gedanken mitzuteilen. Zwar geht die Psychologie davon aus, dass grundsätzlich Denken ohne Sprache möglich sei, dennoch wird in der Forschungsdebatte ein enger Zusammenhang hergestellt: „Während die vergleichsweise einfachen Formen organischer Tätigkeit [...] ohne Beteiligung der Sprache gesteuert werden können, finden die höheren psychischen Vorgänge auf der Basis sprachlicher Tätigkeit statt"7. Sprache ist bei weitem mehr als ein Ausdrucks- oder Darstellungsinstrument für Wissen, Kenntnisse etc. Hartung stellt im Kontext seiner Fallstudie zu Sprache und konzeptionellem Schreibhandeln im Geschichtsunterricht fest: „Nicht nur unsere Sprache, sondern auch unser Denken verändert sich, wenn es sprachlich wird. Das Denken in neuen Begriffen steigert unser Denkvermögen [...]." ${ }^{8}$

\footnotetext{
${ }^{5}$ Vgl. dazu z. B.: Kultusministerkonferenz (2005): Einheitliche Prüfungsanforderungen der KMK für die Fächer, online einsehbar z. B. für Geschichte: https://www.standardsicherung.schulministerium.nrw.de/abiturgost/fach.php?fach=12 (zuletzt eingesehen am 20.01.18). S. 11.

${ }^{6}$ Kultusministerkonferenz (2005): Einheitliche Prüfungsanforderungen der KMK für die Fächer, online einsehbar z. B. für Geschichte: https://www.standardsicherung.schulministerium.nrw.de/abitur-gost/fach.php?fach=12 (zuletzt eingesehen am 20.01.18). Im Folgenden Zitation in der Kurzform: Kultusministerkonferenz (2005): EPA Geschichte (entspr. für andere Fächer).

${ }^{7}$ Lurija, Alexander R. (1992): Das Gehirn in Aktion. Rowohlt: Reinbek. S. 90. Zitiert nach: Dörner, D. (2006). Sprache und Denken. In J. Funke (Ed.), Denken und Problem-lösen (=Enzyklopädie der Psychologie, Themenbereich C: Theorie und Forschung, Serie II: Kognition, Band 8). Göttingen: Hogrefe. S. 29.

${ }^{8}$ Hartung, Olaf (2013a): Sprache und konzeptionelles Schreibhandeln im Fach Geschichte. Ergebnisse der empirischen Fallstudie "Geschichte-Schreiben-Lernen". In: Michael Becker Mrotzek u.a. (Hrsg.): Sprache im Fach. Sprachlichkeit und fachliches Lernen. Münster u.a.: Waxmann, S. 335-353. S. 336.
} 
Hartung steht damit in der Tradition Humboldts, der Sprache als wirkende Kraft benennt ${ }^{9}$. Humboldt nimmt an:

„Die Sprache, in ihrem wirklichen Wesen aufgefaßt, ist etwas beständig und in jedem Augenblick Vorübergehendes. [...] Sie selbst ist kein Werk (Ergon), sondern eine Tätigkeit (Energeia). Ihre wahre Definition kann daher nur eine genetische sein. Sie ist nämlich die sich ewig wiederholende Arbeit des Geistes, den artikulierten Laut zum Ausdruck des Gedanken fähig zu machen. ${ }^{\text { }} 10$

Humboldt weist der Sprache eine epistemische Funktion zu. Der ausdrückliche Hinweis auf die sich wiederholende Arbeit des Geistes zeigt die Bedeutung von Sprache für die Deutung von Wirklichkeit und damit für Erkenntnis und Sinnbildung. Er verweist außerdem auf die Chance, über die Kenntnis verschiedener Sprachen verschiedene Perspektiven einnehmen zu können. Über beide Aspekte kann ein direkter Bogen zu den Zielen von Geschichtsunterricht und der Forderung nach Multiperspektivität geschlagen werden, die sich über Sprache entwickeln. ${ }^{11}$ Damit steht die Beschäftigung mit Sprache als Werkzeug des Historikers bzw. im Geschichtsunterricht in einer langen Tradition.

In der Aussage Humboldts, Sprache sei eine andauernde Arbeit des Geistes, zeigt sich zudem ein funktionaler Blick auf Sprache, der über eine rein strukturalistische Betrachtung sprachlicher Phänomene hinausgeht. Vielmehr stehen für Humboldt grammatische Formen in direkter Beziehung zum Denken. In die Tradition Humboldts sind funktional-linguistische Ansätze einzuordnen, die Sprache und Denken in zweckhafter Verknüpfung sehen und Sprache als Form des Handelns begreifen. Ein solcher funktional-linguistischer Ansatz liegt dieser Arbeit zugrunde. Das Wissen um sprachliche Handlungen ist gerade für den Geschichtsunterricht von hoher Bedeutung.

Durch die Überlegungen zur Erkenntnisfunktion von Sprache wird nach Ehlich die besondere Rolle der Sprache als Medium der Überlieferung in Geschichtswissenschaft und Geschichtsunterricht deutlich:

„Die Bindung von Geschichte an Sprache ist also unaufgebbar. Dies macht sich auf eine eigenartige Weise im deutschen Wort Geschichte selbst bemerkbar. Es enthält nämlich in seinem Doppelsinn eine Rücknahme der Scheidung von Wort und Sache. Die Geschichte ist einerseits das Geschehen, sie ist andererseits die Erzählung 'Vom Geschehen'“. ${ }^{12}$

Es ist keine neue Erkenntnis, dass Geschichte ohne Sprache nicht denkbar ist. Schon früh weist Lucas darauf hin. ${ }^{13}$ Sprache ermöglicht überhaupt erst das Narrativieren von Geschichte.

\footnotetext{
${ }^{9}$ Vgl. dazu auch: Hartung, Olaf (2010a): Die, sich ewig wiederholende Arbeit' des Geschichtsbewusstseins - Sprache als Medium des historischen Lernens, in: Zeitschrift für Geschichtsdidaktik 9. S. 181-191. S. 183.

${ }^{10}$ Humboldt, Wilhelm v. (1973): Über die Verschiedenheit des menschlichen Sprachbaues und ihren Einfluß auf die geistige Entwicklung des Menschengeschlechts. In: Humboldt, Wilhelm v. (Hrsg.): Schriften zur Sprache Stuttgart: Reclam [Orig. v. 1836]; S.36.

${ }_{11}$ „Durch die Mannigfaltigkeit der Sprachen wächst unmittelbar für uns der Reichtum der Welt und die Mannigfaltigkeit dessen, was wir in ihr erkennen; es erweitert sich zugleich dadurch für uns der Umfang des Menschendaseins, und neue Arten zu denken und empfinden stehen in bestimmten und wirklichen Charakteren vor uns da.", Humboldt, Wilhelm v. (1908): Fragmente der Monographie über die Basken. Hrsg.: Leitzmann, Albert: Gesammelte Schriften Bd. VII. 2. Hälfte. Berlin. S. 602ff. Zit. n.: Hofmannsthal, Hugo von (1927): Wert und Ehre deutscher Sprache. München: Verlag der Bremer Presse. S. 146.

${ }^{12}$ Ehlich, Konrad (2005): dabar und logos. Kursorische Überlegungen zum Verhältnis von Sprache und Geschichte. In: Trabant, Jürgen (Hrsg.): Sprache der Geschichte. München: Oldenbourg. S. 27-39. S. 34.

${ }^{13}$ Vgl. dazu z. B.: Lucas, Friedrich J. (1975): Zur Funktion der Sprache im Geschichtsunterricht. In: Die Funktion der Geschichte in unserer Zeit. Hrsg.: Jäckel, Eberhard; Weymar, Ernst. Stuttgart. S. 326-342.
} 
Die Folge daraus ist, dass auch im Geschichtsunterricht die Bearbeitung einer Aufgabe ohne Sprachbeherrschung nicht möglich ist: „In geschichtsdidaktischer Perspektive stellt die Frage nach dem Verhältnis von Sprache und Fach keine Neuentdeckung dar [...]. Vielmehr gehört es zu den geschichtsdidaktischen Prämissen, historisches Lernen und Denken und Handeln, als historische Orientierung und Sinnbildung über Sprache zu begreifen. ${ }^{14}$ Soll bspw. etwas erklärt, begründet oder verglichen werden, geht dies nur mit Hilfe sprachlicher Formen und Mittel. Die Beantwortung von Aufgaben bezieht den Blick auf ihre sprachlichen Dimensionen unmittelbar ein. Ohne die den Aufgaben innewohnenden sprachlichen Handlungen oder Teilhandlungen zu berücksichtigen, können Aufgaben nicht bearbeitet werden. Dabei zeigt sich im Begriff der sprachlichen Handlung, dass es bspw. nicht nur um semantisches oder grammatisches Sprachinventar geht. Die sprachliche Handlung berücksichtigt vielmehr einen kommunikativen Zweck. ${ }^{15}$ Beispielsweise bei historischen Lernaufgaben sind kommunikativer Zweck und ihre epistemische Funktion eng verknüpft, denn: „Nicht nur unsere Sprache, sondern auch unser Denken verändert sich, wenn es sprachlich wird. ${ }^{\text {16 }}$

Der Frage nach (fachgebundenem) kommunikativem Zweck und epistemischer Funktion von Aufgaben im Geschichtsunterricht soll im Folgenden am Beispiel eines ausgewählten Operators nachgegangen werden: Für das Vergleichen im Geschichtsunterricht soll nachfolgend am Beispiel einer authentischen Schulbuchaufgabe seine Leistung im Hinblick auf historisches Lernen geprüft werden. Ausgangspunkt ist dabei die Frage, welche Fertigkeiten und welche Kompetenzen Lernende zur Lösung einer historischen Vergleichsaufgabe benötigen.

\subsection{Begründung für die Auswahl und Herangehensweise}

Das Vergleichen hat grundsätzlich eine bedeutende Funktion für die Verortung des Individuums in seiner Lebenswelt. Hierzu stellt etwa Eggs fest, dass das Vergleichen die Erschließung der Umwelt ermöglicht. Neues wird integriert, indem es in Beziehung zu Bekanntem gesetzt wird, wodurch Gemeinsamkeiten und Unterschiede aufgedeckt werden können. ${ }^{17}$ Aus der Entwicklung von Kindern ist etwa bekannt, dass sich diese permanent vergleichen, sich auf diese Art und Weise eben in ihrer Umwelt verorten. Auch der erwachsene Mensch vergleicht, denn: „Das Vergleichen ist [...] fundamental für den

Handro, Saskia: Sprache und historisches Lernen. In: Michael Becker-Mrotzek, Karen Schramm, Eike Thürmann, Helmut Johannes Vollmer (Hrsg.): Sprache im Fach. Sprachlichkeit und fachliches Lernen. Münster: Waxmann S. 318-333. Bernhardt, Markus; Wickner, Mareike-Cathrine: Die narrative Kompetenz vom Kopf auf die Füße stellen - Sprachliche Bildung als Konzept der universitären Geschichtslehrerausbildung. In Benholz, Claudia; Frank, Magnus; Gürsoy, Erkan (Hrsg.). (2015). Deutsch als Zweitsprache in allen Fächern. Konzepte für Lehrerbildung und Unterricht. Beiträge zu Sprachbildung und Mehrsprachigkeit aus dem Modellprojekt ProDaZ. Stuttgart: Fillibach bei Klett. S. 281-296.

${ }^{14}$ Handro, Saskia (2013), S. 320.

${ }^{15}$ Dieser Annahme liegt der linguistische Ansatz der Funktionalen Pragmatik zugrunde, der im Folgenden noch auszuführen sein wird.

${ }^{16}$ Hartung, Olaf (2013a), S. 336.

${ }^{17}$ Vgl. Eggs, Frederike (2006b): Vergleichen und Vergleiche - Implikationen der Sprachwissenschaft für die Sprachdidaktik In: Becker, Tabea und Peschel, Corinna (Hrsg.): Gesteuerter und ungesteuerter Grammatikerwerb. Hohengehren 2006, S. 48. 
Erkenntnisprozess und die Begriffsbildung schlechthin. ${ }^{18}$ Die mentale Operation des Vergleichens ist also elementar für das Lernen in der Schule. ${ }^{19}$

Die Geschichtswissenschaft spricht dem Vergleichen die Funktion einer historischen Methode ${ }^{20} \mathrm{zu}$. Schreiber beschreibt das Vergleichen ${ }^{21}$ in ihrer Herausgeberschrift schon im Titel als "Methode zur Förderung historischer Kompetenzen“". ${ }^{22}$ Es nimmt die Funktion einer Erkenntnismethode im Sinne Pandels ${ }^{23}$ ein.

Dieser wichtigen Rolle des Vergleichens für den Geschichtsunterricht scheint der Umgang mit Vergleichsaufgaben im unterrichtlichen Kontext jedoch entgegenzustehen. So ist das Vergleichen in den EPA nur grob bestimmt. Eine Klärung, was das Vergleichen im Geschichtsunterricht überhaupt ausmacht, steht aus. Damit ist auch die Frage verbunden, welche Rolle das Vergleichen in Geschichtslehrwerken spielt und wie bzw. ob das Vergleichen für die Lernenden aufgeschlüsselt wird, damit sie Vergleichsaufgaben bearbeiten können.

Zentrales Element ist daher die Frage, welche sprachlichen wie fachlichen Teilhandlungen Lernende benötigen, um das Vergleichen in ihren Aufgabenlösungen erfolgreich umsetzen zu können. Um dieser Frage nachzugehen, wurde in der vorliegenden Untersuchung exemplarisch eine Aufgabe ausgewählt. Die ausgewählte Aufgabe ist angebunden an den Themenbereich der Industriellen Revolution und beinhaltet einen Darstellungstext ${ }^{24}$ als Materialgrundlage. Er ist im Inhaltsfeld 4 „Die moderne Industriegesellschaft zwischen Fortschritt und Krise" der Richtlinien und (Kern-)Lehrpläne für das Fach Geschichte in der Sekundarstufe II - Gymnasium/Gesamtschule in Nordrhein-Westfalen ${ }^{25}$ verortet. Dadurch wird das für die Sekundarstufe II zentrale Element des wissenschaftspropädeutischen Arbeitens zu berücksichtigen sein. Die Komplexität von Aufgabe und Textgrundlage und die Anbindung an den Oberstufenunterricht führen weiterhin zu der Entscheidung, die Aufgabe schriftlich lösen zu lassen.

\footnotetext{
${ }^{18}$ Eggs (2006b), S. 46.

${ }^{19} \mathrm{Vgl}$. ebd.

${ }^{20}$ Vgl. z. B.: Kaelble, Hartmut: Historischer Vergleich, Version: 1.0, in: Docupedia-Zeitgeschichte, 14.

8.2012, URL: http://docupedia.de/zg/Historischer_Vergleich (zuletzt eingesehen am 01.08.2018).

${ }^{21}$ Im Rahmen dieser Arbeit werden die Begriffe Vergleichen und Vergleich weitgehend synonym gebraucht, wenn nicht eine klare Abgrenzung zwischen der Tätigkeit (Vergleichen) und dem Produkt (Vergleich) gegeben ist.

${ }^{22}$ Schreiber, Waltraud (2005a): Durch Vergleiche lernen - vergleichen lernen. In: Schreiber, Waltraud (Hrsg.): Der Vergleich - Eine Methode zur Förderung historischer Kompetenzen. Ausgewählte Beispiele. Neuried: ars una. S. 31-59.

${ }^{23}$ Vgl. dazu z. B.: Pandel, Hans-Jürgen (2013): Geschichtsdidaktik. Eine Theorie für die Praxis. Schwalbach/Ts.: Wochenschau Verlag.

${ }^{24}$ Auch wenn Pandel feststellt, dass der Begriff ,Darstellungstext' unspezifisch sei, weil Darstellungen auch nichtnarrativ sein können (s. Pandel, Hans-Jürgen 2010: Historisches Erzählen. Narrativität im Geschichtsunterricht. Schwalbach/Ts.: Wochenschau Verlag. S. 10.), wird an ihm festgehalten, weil er für Geschichts-Lehrwerke als gängig angenommen werden kann.

${ }^{25}$ Vgl. Ministerium für Schule und Weiterbildung des Landes Nordrhein-Westfalen (Hrsg.) (2014).
} 
Beforscht werden sollen die aufgeworfenen Fragen durch zwei Untersuchungen. Die erste, eine Frequenzanalyse der Aufgabenstellungen in Geschichts- und Gesellschaftslehrelehrwerken (ein Querschnitt der in NRW zugelassenen Lehrwerke der Sekundarstufe II), kann Hinweise auf relevante Aufgabenstellungen und den Einsatz von Vergleichsaufgaben bieten. Die anschließende Korpusanalyse von 88 schriftlichen Lerner*innenlösungen ${ }^{26}$ auf der Grundlage eines selbsterstellten Kriterienrasters (corpus-based und corpus-driven ${ }^{27}$ ) prüft dann sprachliche wie fachliche Merkmale dieser Vergleichslösungen.

Auf der Basis der Ergebnisse werden abschließend die fachlichen und sprachlich-kognitiven Merkmale, die sich aus der Untersuchung der Korpustexte ergeben, herausgearbeitet und Vorschläge für den Umgang mit Vergleichsaufgaben im Geschichtsunterricht gemacht.

\subsection{Aufbau der Arbeit}

An den unter 1.1 vorgestellten Grundelementen orientiert sich der Aufbau der Arbeit.

Das Vergleichen wird grundlegend strukturiert durch fachliche und sprachlich-kognitive Anforderungen. Doch welche sind das genau? Bei der Untersuchung dieser Anforderungen müssen Elemente wie die Textgrundlage, die Ausgestaltung als Schreibaufgabe oder die Bedeutung wissenschaftspropädeutischen Schreibens in der Oberstufe Berücksichtigung finden, da diese implizit oder explizit in der Aufgabenstellung gefordert werden. Der Blick auf fachliche und sprachlich-kognitive Anforderungen spielt dabei eine Rolle für alle Untersuchungsaspekte zum Vergleichen im Geschichtsunterricht. Denn die fachlichen Anforderungen sind im Unterrichtsfach Geschichte nur dann zu bewältigen, wenn sie auch versprachlicht werden können. Zudem eröffnet dieser Weg die Möglichkeit die epistemische Funktion des Vergleichens im Geschichtsunterricht zu konkretisieren.

Die nachfolgende Abbildung soll das Miteinander von theoretischem und empirischem Teil und damit die Kapitelstruktur dieser Arbeit veranschaulichen:

\footnotetext{
${ }^{26}$ Die Arbeit bedient sich in der Regel des sog. Gender-Stars. Würde der Textfluss gestört, wurde an einzelnen Stellen auf ein Gendering verzichtet, solche Formulierungen sind dennoch geschlechtsneutral und keinesfalls ausgrenzend gemeint.

${ }^{27}$ Diese Herangehensweisen werden in der Korpuslinguistik als Paradigmen eingeordnet (vgl. dazu z. B. Steinhoff, Torsten (2017): Untersuchung von Textkorpora. In: Becker-Mrotzek, Michael; Grabowski, Joachim; Steinhoff, Torsten (Hrsg.): Forschungshandbuch empirische Schreibdidaktik. Münster: Waxmann. S. 353-368. S. 365). Durch die Verbindung beider Ansätze können mögliche Einschränkungen eines Zugriffs über eine isolierte Herangehensweise vermieden werden.
} 


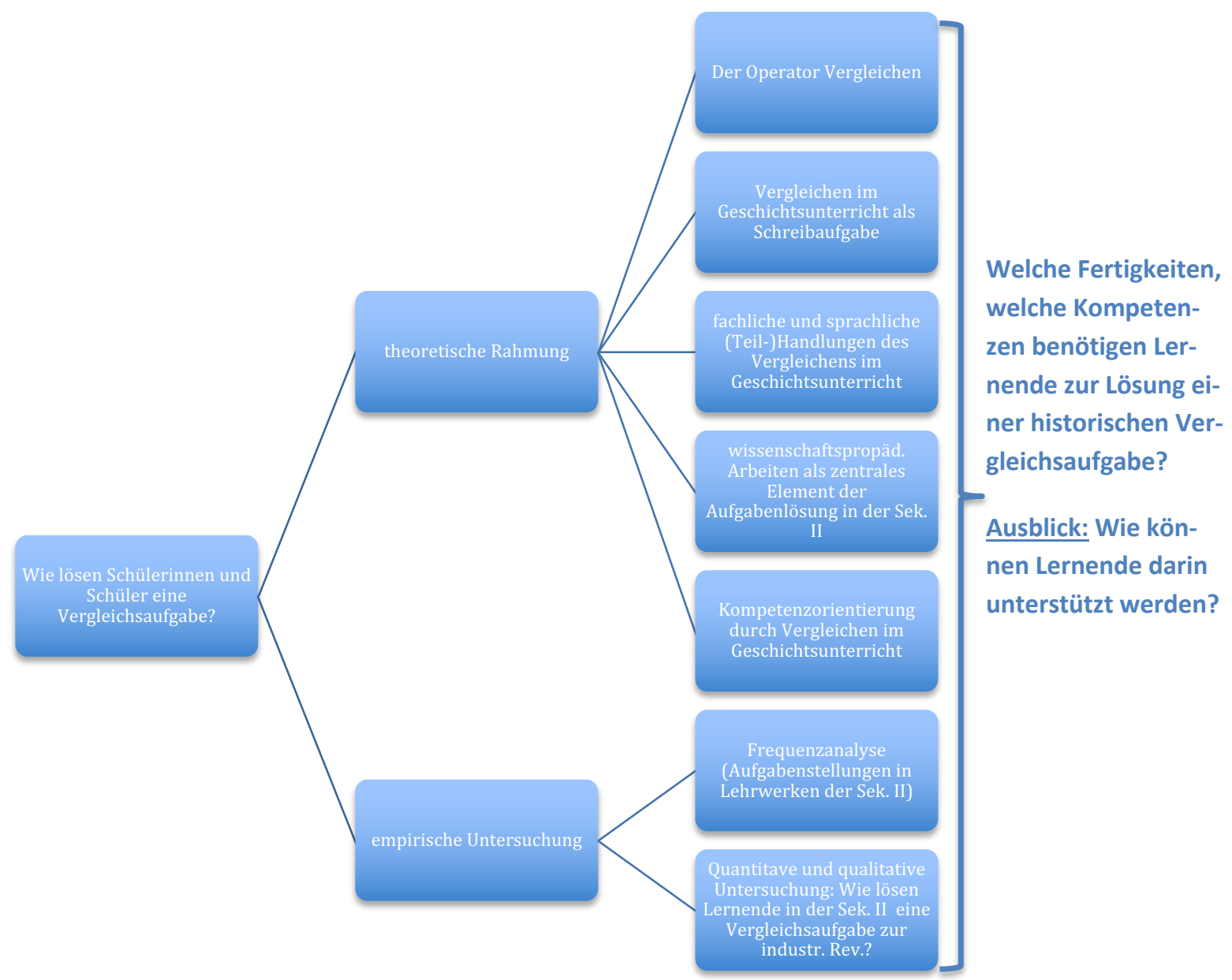

Abbildung 1: Struktur der Arbeit

\subsection{Empirisches Vorgehen}

Eine einfache Frequenzanalyse ${ }^{28}$ soll im ersten Untersuchungsteil Hinweise darauf geben, welche Operatoren in Schulbüchern relevant sind und welcher Anteil im Besonderen dem Vergleichen zukommt (Kap. 4). Untersucht werden dazu die zugelassenen Lehrwerke des Geschichts- und Gesellschaftslehreunterrichts der Sekundarstufe II in Nordrhein-Westfalen. Da es in 2014 durch die Einführung der neuen Kernlehrpläne zu einer Umstellung der Lehrwerke kam, ist die Untersuchung zweigeteilt in zugelassene

\footnotetext{
28 „Die einfachste Art inhaltsanalytischen Arbeitens besteht darin, bestimmte Elemente des Materials auszuzählen und in ihrer Häufigkeit mit dem Auftreten anderer Elemente zu vergleichen." aus: Mayring, Philip (2003): Qualitative Inhaltsanalyse. Grundlagen und Techniken. Weinheim: Beltz. S. 13.
} 
Lehrwerke vor und ab 2014. Darüber kann nachfolgend abgesichert werden, ob zum einen das Vergleichen überhaupt in den Schulbüchern eine wesentliche Rolle spielt, ob es also als bekannt vorauszusetzen ist. Zum anderen wird im zweiten Untersuchungsteil zu analysieren sein, inwieweit eigenständige Operatoren als Teilhandlungen für das Vergleichen eine Rolle spielen und wie sie von den Lernenden realisiert werden (Kap. 7). Über diese vorgeschaltete Frequenzanalyse kann im Hinblick auf diesen zweiten Untersuchungsteil gefragt werden, welche Rolle solche Teiloperationen als eigenständige Operationen in Aufgabenstellungen spielen. Die Frequenzanalyse bestimmt die Häufigkeit bestimmter Wörter oder Wortgruppen, hier der in den EPA vorgegebenen Operatoren. Darüber hinaus werden Operationen, die nicht in den EPA aufgeführt sind, und der Anteil von W-Fragen miterfasst, sodass diese Ergebnisse korreliert werden können.

Eine Absicherung, welche Operatoren im Unterricht tatsächlich eine Rolle spielen, kann allerdings nicht durch eine solche Frequenzanalyse, sondern nur durch die Beobachtung von Geschichtsunterricht geleistet werden. Eine solche Untersuchung stellt ein Desiderat dar. Deshalb bietet das quantitative Auszählen von Geschichts- und Gesellschaftslehre-Lehrwerken immerhin Hinweise, ob es Operatoren gibt, die häufig in Schulbüchern genutzt werden und vermutlich deshalb auch eine höhere Wahrscheinlichkeit besitzen, im Unterricht eingesetzt zu werden.

Da dieser erste Untersuchungsteil zwar einen quantitativen, aber noch keinen qualitativen Aufschluss über die Elemente des Vergleichs gibt, wird unter Bezugnahme auf den theoretischen Teil über den zweiten Teil der Erhebung zu zeigen sein, welche Teilhandlungen dem Vergleichen innewohnen.

Das Kernstück der Untersuchung auf der Basis der ausgewählten Aufgabe (Kap. 5) bildet deshalb ein Textkorpus aus 88 Lernendentexten und 4 Texten von Lehrenden (diese Texte wurden zur Entwicklung des Kriterienrasters herangezogen) in der gymnasialen Oberstufe (Kap. 6 und 7). Dabei handelt es sich um Schüler*innen der Klassen 12 und 13 an nordrhein-westfälischen Berufskollegs. Die Lerner*innen haben eine Vergleichsaufgabe aus einem in NRW zugelassenen Lehrwerk schriftlich gelöst. Ihre Lösungen werden mit Hilfe eines dafür entwickelten Kriterienrasters (Kap. 6.2) in einem Ratingverfahren ausgewertet. Dieses Raster berücksichtigt fachliche und sprachliche Kategorien, die sowohl theoriegeleitet als auch auf der Grundlage des Korpus entstanden sind. So gesehen stellt der als „Theoretische Rahmung" bezeichnete Teil der Arbeit mehr als nur eine Rahmung dar. Er ist vielmehr eine wichtige Grundlage für die Auswertung, die in diesem Teil dargestellten Kategorien finden sich im Raster wieder. Die Ergebnisse der Auswertung des Lerner*innenkorpus werden in einer qualitativen Überprüfung einzelner Korpustexte ausgeführt (Kap. 8) und auf ihre Bedeutung für den Umgang mit Vergleichsaufgaben im Geschichtsunterricht hinterfragt und eingeordnet. Abschließend werden auf der Grundlage dieses Untersuchungsteils Vorschläge für die Praxis des Geschichtsunterrichts gemacht (Kap. 9). 


\title{
2. Das Vergleichen als Methode des kompetenzorientierten Geschichtsunterrichts
}

\author{
2.1 Einordnung des Vergleichens als kognitive Operation im Geschichtsunter- \\ richt
}

Setzt man sich mit Kompetenzen im Geschichtsunterricht auseinander, trifft man auf eine anhaltende geschichtsdidaktische Diskussion um solche Kompetenzen.

Zu den Aufgaben und Zielen des Fachs Geschichte stellt Sauer grundsätzlich fest:

„Geschichtsunterricht legitimiert sich nicht einfach von seinem Gegenstand her. Es geht um dessen aktuelle Relevanz und seine Bedeutung für die Adressaten. Ihnen soll Geschichtsbewusstsein vermittelt und historisch fundierte Gegenwartsorientierung ermöglicht werden. “29

Auch Rüsen beschreibt die Bedeutung des Geschichtsbewusstseins als grundlegende Kategorie. Er spricht vom Geschichtsbewusstsein als der mentalen Tätigkeit historischer Erinnerung, „die die Erfahrung der Vergangenheit deutend so vergegenwärtigt, daß gegenwärtige Lebensverhältnisse verstanden und Zukunftsperspektiven der Lebenspraxis erfahrungskonform entwickelt werden können. Der mentale Modus dieser Erinnerungsleistung ist das Erzählen von Geschichten [...]. " 30 Über die Grundkategorie des Geschichtsbewusstseins zeigt Rüsen die Bedeutung der Narration, also des Erzählens von Geschichten im oben gemeinten Sinne. Er leitet daraus die narrative Kompetenz als „Fundamentalkompetenz"31 ab.

Einhellig plädiert die neuere Didaktik für einen Geschichtsunterricht, der statt zum traditionellen Pauken zum Denken anregt. ${ }^{32}$ Auch im Hinblick auf die Entwicklung von Geschichtsbewusstsein als zentrales Ziel von Geschichtsunterricht scheint Einigkeit in der Debatte um die Frage, was Schüler*innen im Geschichtsunterricht lernen sollen, zu bestehen. Differenzen finden sich jedoch in anzubahnenden Teilkompetenzen.

Was bedeuten diese Grundkategorien Geschichtsbewusstsein und Gegenwartsorientierung für das diskursive Vergleichen im Geschichtsunterricht?

\footnotetext{
${ }^{29}$ Sauer, Michael (2012): Geschichte unterrichten. Eine Einführung in die Didaktik und Methodik. 10. Aufl. Seelze: Friedrich Verlag. S. 19.

${ }^{30}$ Rüsen, Jörn (1992): Das ideale Schulbuch. Überlegungen zum Leitmedium des Geschichtsunterrichts. In: Internationale Schulbuchforschung, 14. S. 237-250. S. 238.

${ }^{31}$ Vgl. Rüsen, Jörn (1992), S. 240.

32 Zum Thema "Geschichte denken“ gibt es eine Vielzahl an Publikationen. Exemplarisch seien deshalb die folgenden Titel genannt:

Bramann, Christoph; Kühberger, Christoph; Bernhard, Roland (Hrsg.) (2018): Historisch Denken lernen mit Schulbüchern. Schwalbach/Ts.: Wochenschau Verlag. (zur Zeit der Erstellung dieser Arbeit noch im Druck, so dass nur auf den Titel verwiesen sei). Kühberger, Christoph (Hrsg.) (2012): Historisches Wissen. Geschichtsdidaktische Erkundung zu Art, Tiefe und Umfang für das historische Lernen. Schwalbach/Ts.: Wochenschau Verlag. Schreiber Waltraud (2005b): Geschichte denken statt pauken. Theoretische Grundlegung für ein praktisches Konzept. Basisbeitrag. In: Mebus, Sylvia/ Schreiber, Waltraud (Hrsg.): Geschichte denken statt pauken. Didaktischmethodische Hinweise zur Förderung historischer Kompetenzen (Siebeneichener Diskurse 3). Meißen. S. 17-23. S. 17.
} 
„Aus der Sicht eines methodenorientierten Geschichtsunterrichts ist das Ziel des Vergleichs das Vergleichen selbst. " 33 Riekenberg grenzt in dieser Aussage den Vergleich vom Vergleichen ab. Kann der Vergleich als Produkt oder als, wie er sagt, „Denkfigur“34 eingeordnet werden, meint das Vergleichen die Tätigkeit, die zum Vergleich als Produkt führt. Riekenberg spricht hier von der „kognitiven Operation" ${ }^{35}$ Im Folgenden wird es vor allem um das Vergleichen als Tätigkeit im Geschichtsunterricht gehen. Die Einordnung als ,kognitive Operation' markiert die Bedeutsamkeit des Vergleichens für einen kompetenzorientierten Unterricht: „Sie ist u.a. für die Begriffsbildung sowie bei der Übertragung von gelerntem ( $\rightarrow$ Transfer) von großer Bedeutung. “"

Riekenberg verweist auf die Funktion des Vergleichs als kognitive Operation; dieser Grundannahme folgend, stellt der Vergleich ein Instrument zur Anbahnung von Geschichtsbewusstsein dar. Insbesondere die Möglichkeit, Gelerntes zu transferieren, es neu zu ordnen oder einzuordnen, kann, wie Sauer sagt, für die Anbahnung einer historisch fundierten Gegenwartsorientierung ${ }^{37}$ genutzt werden.

Aus sprachtheoretischer Sicht ist der Vergleich als kognitiver Begriff einzuordnen, dem, so Hahnemann, aus linguistischer Perspektive kein einheitlicher Formtyp gegenübersteht: „Um einen dieser unterschiedlichen, kognitiv denkbaren Vergleichstypen zu realisieren, stehen einer Sprache potentiell alle syntaktischen [...] und morphologisch-lexikalischen Mittel zur Verfügung. “38 Diese Annahme bedeutet, dass auf der sprachlichen Oberfläche Formen und Mittel des Vergleichs im Geschichtsunterricht charakterisiert werden müssen. Diese sind wiederum untrennbar verbunden mit den fachlichen Zielen. Das Zusammenspiel von sprachlichen und kognitiven Tätigkeiten, um einen geschichtlichen Vergleich zu realisieren, markiert diesen als sprachlich-kognitive Operation, die der unterrichtlichen Kompetenzentwicklung im Sinne der Anbahnung von Geschichtsbewusstsein dient.

Doch ist damit noch nicht geklärt, welche Kompetenzen sich in ihm abbilden. Der Begriff des ,kompetenzorientieren Geschichtsunterrichts' ist, wie einleitend schon dargestellt, viel diskutiert. Will er nicht zur Worthülse werden, muss er hinsichtlich seiner Fachspezifik deutlich umrissen werden. Erst dann können auch die für das Vergleichen relevanten Kompetenzen charakterisiert werden.

Wenn Pandel feststellt, dass man die moderne Geschichtsschreibung als „diskursiv angereicherte Erzählung" bezeichnen könne ${ }^{39}$, muss die Frage nach der Leistung des Vergleichens als diskursive Textsorte für das Narrativieren im Geschichtsunterricht gestellt werden. Dies geht nur im Zusammenhang mit der Klärung der durch diese Operation angebahnten Kompetenzen. Dies führt wiederum zur Anbindung der Untersuchung an ein Kompetenzmodell, das zur Klärung dieser Fragen hilfreich erscheint.

\footnotetext{
${ }^{33}$ Riekenberg, Michael: Der Vergleich. In: Ulrich Mayer, Hans-Jürgen Pandel, Gerhard Schneider (Hrsg.) (2008): Handbuch Methoden im Geschichtsunterricht. Schwalbach/Ts. S. 269-285. S. 269.

${ }^{34}$ Ebd.

35 Ebd.

${ }^{36}$ Ebd.

${ }^{37}$ Vgl. Sauer, Michael (2012), S. 19.

${ }^{38}$ Hahnemann, Suzan (1999): Vergleiche im Vergleich. Zur Syntax und Semantik ausgewählter Vergleichsstrukturen mit >als< und >wie< im Deutschen. Tübingen: Niemeyer (Linguistische Arbeiten; 397). S. 4.

${ }^{39}$ Vgl. Pandel, Hans-Jürgen): Historisches Erzählen. Narrativität im Geschichtsunterricht. Schwalbach/Ts.: Wochenschau Verlag. S. 26.
} 


\subsubsection{Konsequenzen für die Untersuchung}

Aus den Vorüberlegungen und der Verortung des Vergleichs stellt sich das Kompetenzmodell "Guter Geschichtsunterricht ${ }^{\prime 40}$ nach Gautschi als Grundlegung für diese Arbeit als besonders geeignet dar ${ }^{41}$. Auf eine Darstellung der übrigen derzeitig gängigen Kompetenzmodelle soll an dieser Stelle verzichtet werden.

Gautschi schließt seinen Kompetenzbegriff zunächst an den erziehungswissenschaftlichen Kompetenzbegriff Weinerts an und stellt dann in geschichtsdidaktischer Hinsicht Rüsen folgend die Fähigkeit, durch historisches Lernen Sinn über Zeiterfahrung zu bilden, in den Mittelpunkt. ${ }^{42}$ Eine solche Art der Sinnbildung benennt er mit Barricelli als narrative Kompetenz und rückt sie in den Mittelpunkt seines Modells: „Diese narrative Kompetenz auszubilden, ist das zentrale Lernziel des Geschichtsunterrichts. ${ }^{413}$

Die narrative Kompetenz gliedert Gautschi wiederum in vier verschiedene Teilkompetenzen auf, die seiner Annahme nach auf vier ,Problemen' von Individuen beruhen, wenn sie historisch lernen:

"-Wie finde und erkenne ich historische Zeugnisse und Menschen, die mir über Vergangenes berichten können? Wie komme ich zu Fragen und Vermutungen, die mich ins Universum des Historischen führen?

-Wie erschließe ich Quellen und Darstellungen, die über das Universum des Historischen erzählen? Wie komme ich zu einer Sachanalyse, und wie kann ich sie überprüfen?

-In welchem Zusammenhang stehen die einzelnen Sachanalysen zu anderen Sachanalysen, wo sind sie im Universum des Historischen verortet, was sind Ursachen und Wirkungen? Wie komme ich zu einem Sachurteil, und wie kann ich es überprüfen?

-Was ist der Sinn, den ich der Beschäftigung mit dem Universum des Historischen entnehme? [...] $]^{\text {u44 }}$

Die Fundierung dieses Modells auf den genannten Grundproblemen weisen es als eindeutig geschichtsdidaktisches Modell aus. ${ }^{45}$ Das Kompetenzmodell Gautschis ist derzeit das einzige, das auf einer Studie mit einem qualitativen und quantitativen Forschungsdesign aufbaut und deshalb nicht allein normativ begründet ist. Dies unterscheidet es wesentlich von anderen gängigen Modellen. Gleichzeitig ist das Modell in einem Theoriekonzept fundiert und in seiner Ausformung der Kompetenzen als klar geschichtsdidaktisches Modell konzipiert. Die Beschreibungen der Teilkompetenzen wurden durch die

\footnotetext{
${ }^{40}$ Vgl. Gautschi, Peter (2011).

${ }^{41}$ Auch die Bedeutung, die Gautschi den Aufgaben in einem kompetenzorientierten Geschichtsunterricht zuweist, unterstützt die Auswahl des Modells. Vgl. dazu bspw. Gautschi, Peter (2016): Plausibilität der Theorie, Spuren der Empirie, Weisheit der Praxis. Zum Stand der geschichtsdidaktischen Kompetenzdiskussion. In: Geschichte für heute. Zeitschrift für historisch-politische Bildung, Heft 3 (2016), S. 5-19.

Zum Stand der geschichtsdidaktischen Kompetenzdiskussion.

${ }^{42}$ Vgl. Gautschi, Peter (2011), S. 48.

${ }^{43} \mathrm{Ebd}$.

${ }^{44}$ Ebd. S. 50.

${ }^{45}$ Vgl. Mayer, Ulrich (2014), S. 9.
} 
Auswertung des empirischen Materials konkretisiert und sind als Indikatoren nutzbar ${ }^{46}$, es basiert auf dem ebf. geschichtsdidaktisch fundierten Modell Pandels und entwickelt es weiter.

Grundsätzlich lässt sich für das Modell ,Guter Geschichtsunterricht' feststellen:

„Es knüpft sowohl am Modell historischen Lernens von Rüsen (2008) mit den Dimensionen Erfahrung, Deutung, Orientierung als auch an die von Jeismann vorgeschlagene Unterteilung des historischen Erkenntnisprozesses in Sachanalyse, Sachurteil und Werturteil an (Jeismann 1978, 2000) und betont damit die Prozesshaftigkeit des Umgangs mit historischen Inhalten. ${ }^{407}$

In Bezug auf das Vergleichen bedeutet dies, dass über das Modell die Prozesshaftigkeit des historischen Vergleichens und nicht nur das Produkt sichtbar gemacht werden kann. Diese Prozesshaftigkeit impliziert wiederum die Berücksichtigung des Ziels eines historischen Vergleichs (bzw. des Vergleichs im Geschichtsunterricht), welches wiederum über seine Funktionalität hergeleitet werden muss. Dieser Ansatz passt zu dem gewählten funktional-pragmatischen Rahmen der linguistischen Pragmatik ${ }^{48}$, so dass das Kompetenzmodell ,Guter Geschichtsunterricht' zusammen mit dem der Arbeit zugrundeliegenden sprachlichen Ansatz eine plausible Basis für die Untersuchung des Vergleichens im Geschichtsunterricht darstellt.

\subsubsection{Vergleichen als Methode des kompetenzorientierten Unterrichts - Einpassung in} das ,Kompetenzmodell Guter Geschichtsunterricht‘

Wie passt sich der Vergleich in das Kompetenzmodell "Guter Geschichtsunterricht" ein, auf welche der darin formulierten Kompetenzen nimmt er Bezug? Im Folgenden sollen die zuvor dargelegten Überlegungen und Entscheidungen anhand dieser Leitfragen konkretisiert werden.

Die noch zu diskutierenden „Einheitlichen Prüfungsanforderungen in der Abiturprüfung Geschichte" (Beschluss der Kultusministerkonferenz in der Fassung vom 10.02.2005) geben kurze Bestimmungen für die dort aufgeführten Operatoren vor. Für das Vergleichen wird hier formuliert: „auf der Grundlage von Kriterien historische Sachverhalte problembezogen gegenüberzustellen, um Gemeinsamkeiten, Unterschiede, Teil-Identitäten, Ähnlichkeiten, Abweichungen oder Gegensätze zu beurteilen “ ${ }^{49}$ Ohne an dieser Stelle schon auf die hier für das Vergleichen genannten Teiloperationen im Einzelnen einzugehen, weist diese Begriffsbestimmung auf die Bildung eines Sachurteils hin, die eingefordert wird. Dem geht die Analyse der für den Vergleich herangezogenen historischen Gegenstände voraus, die gegenübergestellt werden müssen. Ein Werturteil wird durch diese Bestimmung nicht zur Bedingung gemacht, kann aber wiederum über die Art der Aufgabenstellung gefordert werden. Generell kann davon ausgegangen werden, dass der Vergleich nicht nur mit dem Sachurteil abschließen muss, sondern die Frage nach der Bedeutung des Vergleichs für die Gegenwart ein Werturteil möglich macht.

\footnotetext{
${ }^{46} \mathrm{Vgl}$. ebd. S. 62.

${ }^{47}$ Waldis, Monika; Hodel, Jan; Fink, Nadine (2012): Lernaufgaben im Geschichtsunterricht und ihr Potential zur Förderung historischer Kompetenzen. In: zdg (Zeitschrift für Didaktik der Gesellschaftswissenschaften), Heft 1/2012. S. 142-157. S. $145 f$.

48 in Anlehnung an Ehlich/Rehbein (vgl. bspw. Ehlich, Konrad; Rehbein, Jochen (1979) Sprachliche Handlungsmuster. In: Soeffner, Hans-Georg (Hrsg.): Interpretative Verfahren in den Sozial- und Textwissenschaften. Stuttgart: Metzler, S. 243-274).

${ }^{49}$ Kultusministerkonferenz (2005): EPA Geschichte, S. 8.
} 


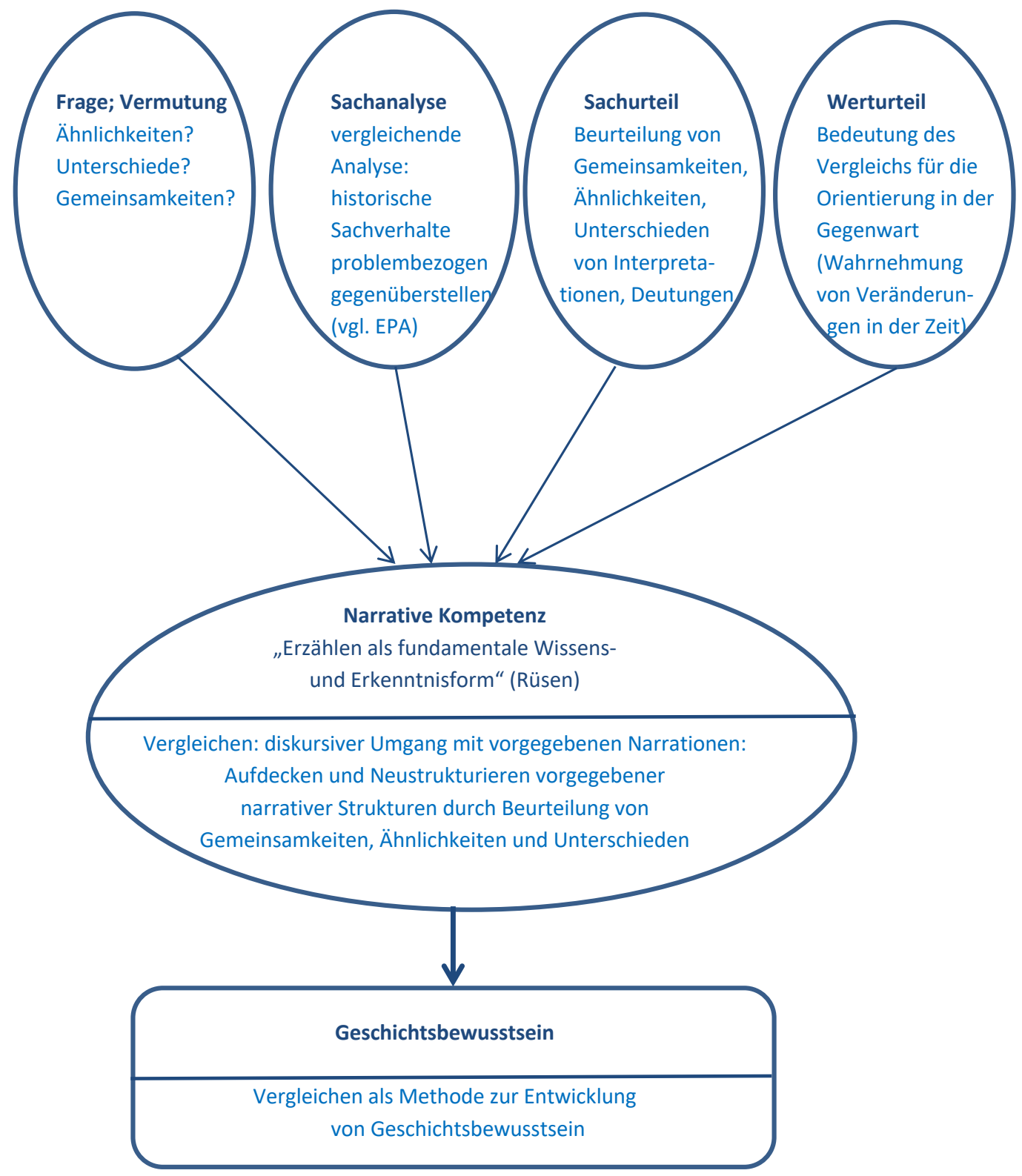

Die Darstellung orientiert sich insbesondere am ,Kompetenzmodell Guter Geschichtsunterricht' (Gautschi, P., 2011) sowie den Vorgaben in den ,Einheitlichen Prüfungsanforderungen in der Abiturprüfung Geschichte' (Beschluss der Kultusministerkonferenz, 2005)

Abbildung 2: Passung des Vergleichens in das Kompetenzmodell 'Guter Geschichtsunterricht' nach Gautschi (2011), (eigene Darst.)

In diesem ersten generalisierten Zugriff auf das Vergleichen kann mit Hilfe des ausgewählten Kompetenzmodells festgestellt werden, dass das Vergleichen grundsätzlich eine kompetenzorientierte Me- 
thode darstellt. Zu prüfen wäre, ob die z. B. in den Lehrwerken formulierten unterschiedlichen Aufgabenstellungen immer auch auf Kompetenzorientierung abzielen oder ob ein bestimmtes Aufgabenumfeld benötigt wird. Für die vorliegende und nachfolgend zu überprüfende Vergleichsaufgabe soll diese Frage gestellt werden, im Hinblick auf weitere Formulierungen von Vergleichsaufgaben muss sie jedoch offenbleiben, wenngleich erste Vermutungen angestellt werden können.

\subsection{Aufgaben im kompetenzorientierten Geschichtsunterricht}

Da geschichtsdidaktische Kompetenzmodelle sich bisher gar nicht oder nur zum Teil auf empirische Daten stützen, wirft Zülsdorf-Kersting die Frage auf: „Mit welchen Kriterien und mit welchem Anspruch auf Methodenreflexion werden beispielsweise Aufgaben im Geschichtsunterricht formuliert? ${ }^{\prime 50}$ Hinter dieser Fragestellung steht die prinzipielle Frage nach Aufgabenformaten für den kompetenzorientierten Geschichtsunterricht. Dieser soll in Bezug auf das Vergleichen nachgegangen werden.

\subsubsection{Leistungen von Aufgaben im Geschichtsunterricht}

Mit der Diskussion um die Kompetenzorientierung ist in den letzten Jahren auch die Debatte um die Aufgabenkultur im Geschichtsunterricht in Gang gekommen. Wenn Geschichtsunterricht historisches Denken vermitteln soll, muss es entsprechende Aufgabenformate geben, die diesem Anspruch gerecht werden. Darauf ist gerade auch in den neueren Veröffentlichungen zu Aufgaben im Geschichtsunterricht immer wieder hingewiesen worden. Heuer bringt mit Verweis auf Wenzel ${ }^{51}$ diesen Anspruch wie folgt auf den Punkt:

„Lange Zeit sollten Schülerinnen und Schüler im Geschichtsunterricht durch einzelne Aufgaben lediglich zur Auseinandersetzung mit einer bestimmten Quelle oder Darstellung angehalten werden. Die einzelnen vom Lehrer oder dem Schulgeschichtsbuch vorgegebenen Aufgaben dienten dann in erster Linie dazu, historischen Wissenserwerb anzuleiten bzw. zu erleichtern [...]. Erst im Zuge der Outputorientierung und der infolge dessen geführten Kompetenzdiskussion wurde auch für den Geschichtsunterricht die Bedeutung von Aufgabenformaten für die Förderung von fachspezifischen Kompetenzen betont. ${ }^{\text {“52 }}$

Wie können Aufgaben das leisten, wie werden sie zu spezifischen "Geschichtsaufgaben“, welche historisches Lernen anregen? Was leisten insbesondere solche Aufgaben hinsichtlich ihrer Kompetenzorientierung, die auf diskursive Operationen abzielen wie bspw. Erklären, Begründen und eben auch das Vergleichen?

Wie können Schüler*innen überhaupt Kompetenzen aufbauen, wenn es, wie Heuer anführt, ein generelles Problem gibt:

\footnotetext{
${ }^{50}$ Zülsdorf-Kersting, Maik (2012), S. 10.

${ }^{51}$ Vgl. Wenzel, Birgit, (2007, hier in der Neuauflage von 2015): Aufgaben im Geschichtsunterricht. In: GüntherArndt, Hilke; Handro, Saskia (Hrsg.): Geschichtsmethodik. Handbuch für die Sekundarstufe I und II. 5. Aufl. Berlin: Cornelsen. S. 84.

52 Heuer, Christian (2012): Zur Aufgabenkultur im Geschichtsunterricht. In: Keller, Stefan; Bender, Ute (Hrsg.): Aufgabenkulturen. Fachliche Lernprozesse herausfordern, begleiten, reflektieren. Seelze-Velber: Kallmeyer. S. 100-112. S. 100.
} 
„Nun stellt sich für den täglichen Geschichtsunterricht [...] ein grundsätzliches Problem: Kompetenzen lassen sich erstens nicht vermitteln und zweitens nicht lernen. Kompetenzen können nur gefördert werden.

Diese Einsicht rückt die Frage nach den Möglichkeiten - den $>$ Werkzeugen< $<$ ins Zentrum der didaktischen Analyse. “53

Um diesen Fragen nachzugehen, sollen zunächst verschiedene Aufgabentypen und -formate unterschieden werden.

Bezüglich der Formate ist allgemein in geschlossene, halbgeschlossene und offene Aufgaben zu differenzieren. Für den Geschichtsunterricht in der Sek. II spielen, dies lässt sich aus der nachfolgenden Lehrwerkanalyse ersehen, insbesondere offene Aufgabenformate eine Rolle: „Als halboffen gelten Kurzantwort-Items und - bei Schreibaufgaben - Lückentexte. Überall da, wo die Lerner*innen kleinere oder größere Texte mündlich oder schriftlich produzieren, handelt es sich um offene Formate. "54 Geschlossene Formate, wie Multiple-Choice-Aufgaben, sind so gut wie nicht zu finden, Lückentexte u. ä. ebenso kaum.

Die Entscheidung für die offenen Formate durch die Schulbuchautoren lässt sich darin begründen, dass sie in der geschichtsdidaktischen Diskussion durchweg als besonders wichtig für das historische Lernen angesehen werden. Sie sollen im Folgenden im Mittelpunkt der Betrachtung stehen.

Weiterhin sind verschiedene Aufgabentypen zu unterscheiden. Ihre Zuordnung hängt vom Ziel ab, das mit den Aufgaben erreicht werden soll. Grundsätzlich ist in Diagnose-, Lern- und Prüfungsaufgaben zu differenzieren. ${ }^{55}$ Sollen Diagnoseaufgaben Auskunft über den Kompetenzstand der Lernenden erteilen, Lernfortschritte dokumentieren und so Hinweise für die weitere Struktur des Lern- und Unterrichtsverlauf geben, fragen Prüfungsaufgaben den Kenntnisstand im Sinne der Leistungsbewertung zu einem bestimmten Zeitpunkt ab. Lernaufgaben hingegen dienen für den Geschichtsunterricht als Hilfsmittel, um historisches Lernen zu ermöglichen und narrative Kompetenzen zu erwerben.

Die Unterscheidung der verschiedenen Aufgabentypen ist entscheidend, wie Köster/Lindauer für die Lern- und Prüfungsaufgaben zeigen:

„Eine Aufgabe kann für die Lernsituation überaus geeignet sein und für die Leistungssituation denkbar ungünstig. Denn in einer Leistungssituation bemüht man sich in erster Linie darum, Erfolge zu erzielen und Misserfolge zu vermeiden. Demgegenüber geht es in Lernsituationen darum, Neues zu lernen, Wissenslücken zu schließen oder unklar Gebliebenes besser zu verstehen. Während es in der Leistungssituation Fehler zu vermeiden gilt, sind sie in der Lernsituation ein Erkenntnismittel. ${ }^{\prime 56}$

Die Autoren zeigen, warum Lernaufgaben so wichtig sind: Sie geben die Chance auf Entwicklung. Was macht nun Lernaufgaben im Geschichtsunterricht aus?

\footnotetext{
${ }^{53}$ Heuer, Christian (2010): Für eine „neue“ Aufgabenkultur - Alternativen für historisches Lehren und Lernen an Hauptschulen. In: Zeitschrift für Geschichtsdidaktik 9. S. 79-97. S. 91.

${ }^{54}$ Köster, Juliane; Lindauer, Thomas (2008): Zum Stand wissenschaftlicher Aufgabenreflexion aus deutschdidaktischer Perspektive. In: Didaktik Deutsch 14, Sonderheft 2. S. 148-158. S. 152.

${ }^{55}$ Vgl. hierzu auch: Köster, Manuel; Bernhardt, Markus; Thünemann, Holger (2016). S. $3 \mathrm{ff}$.

${ }^{56}$ Köster, Juliane; Lindauer, Thomas (2008), S. 152.
} 
In der Einordnung der Bedeutsamkeit von Lernaufgaben für den Geschichtsunterricht scheint in der fachdidaktischen Diskussion zwar insgesamt Einigkeit zu herrschen. Dennoch wird um ihre Ausgestaltung, insbesondere in fachspezifischer Hinsicht gerungen: „Lernaufgaben stellen den bislang wohl am intensivsten diskutierten Aufgabentyp dar “57, stellen Köster/Bernhardt/Thünemann zum jüngeren Stand der Debatte fest.

Gautschi definiert Lernaufgaben als „[...] schriftlich abgefasste inhaltsbezogene Problemstellungen und Arbeitsanleitungen. Sie haben einen fachspezifischen Aspekt („Lerngegenstand“) sowie lernbezogene Hinweise („Prozessstruktur“). Schüler*innen bearbeiten Lernaufgaben selbständig, sie entdecken und lernen dabei etwas Neues und entwickeln ein Produkt (,Nutzung $\left.{ }^{\prime}\right)^{\prime \prime}{ }^{58}$

Gautschi geht von einem allgemeinen Lernaufgabenbegriff aus, der nur durch das Setting geschichtsdidaktische Lernaufgaben beschreibt. Eine genauere Ausdifferenzierung gattungsspezifisch historischer Lernaufgaben ist hier noch nicht eindeutig abgegrenzt.

Grundsätzlich sollen sie, verfolgt man die geschichtsdidaktische Diskussion, den Aufbau von narrativer Kompetenz anregen. Diese in der Geschichtsdidaktik weitgehend akzeptierte Kompetenz ist im Kapitel zuvor bereits angesprochen worden, jedoch diskutieren Barricelli et al. eine Schwierigkeit, die dieser Kompetenz innewohnt: „Nun ist aber Narrativität kein didaktischer Begriff, sondern vielmehr das konstitutive Merkmal aller historischen Aufgaben, seien sie nun vor-wissenschaftlich (rhetorisch), ,außerwissenschaftlich' (alltagsweltlich) oder wissenschaftlich [...]. ${ }^{159}$ Es muss deshalb an dieser Stelle erneut gefragt werden, wie gerade solche Aufgaben unter den Begriff der Narrativität eingeordnet werden können, die (wie eben Aufgaben zum Vergleichen im Geschichtsunterricht) auf diskursive Formate abzielen, denn „Im Geschichtsunterricht kann nicht nur die eine - die narrative - Textsorte fachliche Wertschätzung beanspruchen, sondern diskursive Textsorten gehören ebenfalls unmittelbar zum sprachlichen Werkzeug des Historikers [...]. ${ }^{\text {“60 }}$. Eine solche Differenzierung scheint im Moment im geschichtsdidaktischen Diskurs noch nicht genügend ausdifferenziert.

Damit Geschichte nun aber nicht beliebig wird, leiten Barricelli/Gautschi/Körber aus ihren Überlegungen Konsequenzen für den Unterricht ab: „Wer Geschichte lehren möchte, muss daher den Lernenden im Besonderen ermöglichen, dass sie das spezifische Strukturmerkmal ,Narrativität' und die dazugehörigen methodischen Regularien verstehen. “61 Geschichte zu erzählen bedeutet demnach eben nicht, ,irgendeine' Geschichte zu erzählen, sondern eine, die etwa geschichtsadäquate Methoden zugrunde legt ${ }^{62}$. Auch oder gerade dieser Forderung müssen Lernaufgaben gerecht werden. Aus dieser Überlegung leitet etwa Barricelli das Ziel der Arbeit an und mit Lernaufgaben ab:

\footnotetext{
${ }^{57}$ Köster, Bernhardt, Thünemann (2016), S. 6.

58 Gautschi, Peter (2009); S. $246 f$.

${ }^{59}$ Barricelli, Michele; Gautschi, Peter; Körber, Andreas (2012): Historische Kompetenzen und Kompetenzmodelle. In: Barricelli, Michele; Lücke, Martin (Hrsg.): Handbuch Praxis des Geschichtsunterrichts. Bd. 1. Schwalbach/Ts.: Wochenschau-Verlag. S. 207-235. S. 210f.

${ }^{60}$ Bernhardt, Markus; Conrad; Franziska (2018): Sprachsensibler Geschichtsunterricht. Sprachliche Bildung als Aufgabe des Fachs Geschichte. In: Geschichte lernen 31 (2018). H. 182, S. 2-9. S. 5.

${ }^{61}$ Barricelli, Michele; Gautschi, Peter; Körber, Andreas (2012), S. 211.

${ }^{62} \mathrm{Vgl}$. hierzu etwa auch die Wahrheitskriterien des historischen Denkens bei Rüsen, Jörn (1983): Historische Vernunft. Göttingen: Vandenhoeck und Ruprecht. S. 76ff.
} 
„Das Rückgrat des kompetenzorientierten Unterrichts sind Aufgaben - verstanden als schriftlich abgefasste inhaltsbezogene Arbeitsanleitungen mit Materialien: Die Lernenden sollen sich selbstständig mit Vergangenheit und Geschichte auseinandersetzen und auf diese Weise Möglichkeiten erhalten, um ihre Selbstwirksamkeit zu erfahren. “63

Wie müssen Aufgaben jedoch gestaltet sein, um diesen Anforderungen gerecht zu werden? Wie passen die diskursiven Aufgabenformate in dieses Anforderungprofil? Waldis/Hodel/Fink formulieren drei Anforderungen:

„Im Hinblick auf die Förderung von Kompetenzen besteht zudem ein Bedarf an Lernaufgaben, die Schülerinnen und Schüler dazu anregt, Fragen an die Geschichte zu stellen, Informationen zu suchen und zu erschließen sowie Informationen zueinander in Bezug zu setzen und zu deuten. [...] Zudem sollten solche Aufgaben einen fachlich begründeten Umgang mit Geschichte herausfordern, wie er derzeit in verschiedenen Kompetenzmodellen beschrieben wird. Idealerweise sind sie auf unterschiedlichen Taxonomiestufen angesiedelt. “64

Um insbesondere dem Anspruch der Kompetenzentwicklung zu entsprechen, bindet Heuer Lernaufgaben in ein Lernarrangement ein und entwickelt darüber eine Aufgabenkultur. ${ }^{65}$ Die daraus entwickelten sieben Gütekriterien sind allerdings, so der Vorwurf Thünemanns, nicht genuin geschichtsspezifisch. ${ }^{66}$ So nennt Heuer beispielsweise das Kriterium der Verwendung von Operatoren ${ }^{67}$. Ohne ein konkretes Setting muss man jedoch feststellen, dass Operatoren nicht nur für den Geschichtsunterricht formuliert bzw. bestimmt wurden. So ist Thünemann in seinem Hinweis auf die fehlende Geschichtsspezifik zu folgen ${ }^{68}$, wenngleich die Kriterien Heuers als wesentliche Grundlage auch für Lernaufgaben im Geschichtsunterricht bedeutsam sind.

Thünemann konstatiert in Bezug auf die fachliche Ausformung von Lernaufgaben:

„Obwohl diese Einschätzung [dass Aufgaben eine Schlüsselfunktion im Lernprozess zukommen. Anm. d. Verf.] prinzipiell kaum bestritten wird, ist auffällig, dass eine genuin fachspezifische Profilierung des Aufgabenbegriffs in der Geschichtsdidaktik bislang noch weitgehend fehlt. “69

Aus diesem Desiderat leitet er einen Vorschlag zur „fachspezifischen Modellierung historischer Lernaufgaben "70 ab. Er benennt in seinem Vorschlag zunächst drei Qualitätskriterien historischer Lernaufgaben: „historische Leitfragen, historische Werturteilsbildung und historische Reflexion. “71

Kriterium I definiert Thünemann als „[...] problemorientierte Lernimpulse, sie strukturieren den Lernprozess der Schülerinnen und Schüler, sind also gewissenmaßen ein roter Faden, der für den gesamten

\footnotetext{
${ }^{63}$ Barricelli, Michele; Gautschi, Peter; Körber, Andreas (2012), S.231.

${ }^{64}$ Waldis, Monika; Hodel, Jan; Fink, Nadine (2012), S.143f.

${ }^{65}$ Heuer, Christian (2011): Gütekriterien für kompetenzorientierte Lernaufgaben im Fach Geschichte. In: GWU 62. S. 443-458. S. $445 f$.

${ }^{66}$ Thünemann, Holger (2013): Historische Lernaufgaben. Theoretische Überlegungen, empirische Befunde und forschungspragmatische Perspektiven. In: Zeitschrift für Geschichtsdidaktik 12, S. 141-155. S. 142.

${ }^{67}$ Vgl. Heuer, Christian (2011). S. 449.

${ }^{68}$ Dies gilt auch für die weiteren Kriterien. Aufgaben sollen weiterhin: zum Erzählen anregen, verständlich sein, Offenheit aufweisen, fordern, differenzieren, zur Kooperation anregen. Vgl. hier: Heuer, Christian (2011), ebd.

${ }^{69}$ Thünemann, Holger (2013). S. 142.

${ }^{70} \mathrm{Vgl}$. ebd.

${ }^{71}$ Vgl. ebd. S. 146.
} 
Lernweg eine Orientierungshilfe bedeutet. ${ }^{\text {“72 }}$ Dabei grenzt er historische Leitfragen von der reinen Faktendarstellung deutlich ab.

Kriterium II definiert Thünemann in Anlehnung an Jeismann und setzt voraus, dass dieser Werturteilsbildung ein Sachurteil vorausgegangen sein muss. Er stellt zusammenfassend fest:

„Historische Werturteilsbildung als Ziel und Qualitätskriterium historischer Lernaufgaben auszuweisen, bedeutet somit, dass es in diesen Aufgaben immer auch um die aktuelle Relevanz historischer Phänomene gehen muss. Es reicht also nicht aus, wenn Schülerinnen und Schüler beispielsweise lernen, wann die Französische Revolution begann, wann sie endete oder durch welche Akteure sie getragen wurde. Entscheidend ist, die Ursachen und Wirkungen dieser Revolution sowie ihre aktuelle historische Bedeutung herauszuarbeiten. ${ }^{473}$

Mit Kriterium III weist er auf die Metakompetenz der Selbstreflexion hin:

„Damit ist einerseits gemeint, dass Schülerinnen und Schüler dazu befähigt werden, ihren eigenen Lernweg bzw. ihren eigenen historischen Denkprozess reflexiv-evaluativ nachzuvollziehen. Andererseits sollen sie ihren eigenen Standpunkt reflektieren und verstehen, warum ihre Mitschüler möglicherweise zu ganz anderen Ergebnissen, Werturteilsbildungen und Orientierungsleistungen kommen. ${ }^{174}$

Lernaufgaben ermöglichen folglich den Aufbau eines „reflektierten Geschichtsbewusstseins“ ${ }^{75}$ Der Beitrag des Vergleichens hierzu ist also zu überprüfen.

Oft werden Aufgaben erst durch ihre Situierung zu historischen Aufgaben, Thünemann geht in seiner Darstellung deutlich über die reine Situierung hinaus, indem er fachspezifische Kriterien entwickelt. Die drei genannten Qualitätskriterien fußen auf der Debatte um historische Kompetenzmodelle. Er beruft sich beispielsweise auf Rüsen und Jeismann und hebt die Bedeutung, historisches Denken anzuregen, heraus. Hierauf nehmen seine drei Kriterien direkten Bezug. Er stellt jedoch auch fest, dass Aufgaben, die diesen Kriterien entsprechen, im Unterricht gegenwärtig im Prinzip nicht vorkommen. ${ }^{76}$ Bestätigung findet diese Einschätzung etwa bei Mägdefrau/Michler, die auf empirischer Grundlage konstatieren:

„Dabei fällt auf, dass neuere Studien, die sich der empirischen Erforschung von Arbeitsaufträgen in Schulgeschichtsbüchern widmen, die Ergebnisse älterer Untersuchungen, die zum Teil bereits vor über 20 Jahren durchgeführt wurden, im Großen und Ganzen immer noch bestätigen: Arbeitsaufträge in den Schulbüchern sind in ihren Anforderungen hauptsächlich auf Reproduktion angelegt, allenfalls werden noch Erklärungs- und Transferleistungen eingefordert. Aufgaben, die auf historische Beurteilung und Bewertung abzielen, sind dagegen deutlich unterrepräsentiert. ${ }^{\text {“77 }}$

Es ist also davon auszugehen, dass Aufgaben, die weder Diagnose- noch Prüfungsaufgaben darstellen, sondern vielmehr auf das historische Lernen von Schüler*innen hin angelegt sind (also beispielsweise

\footnotetext{
72 Ebd. S. 147.

73 Ebd. S. 148.

${ }^{74}$ Ebd. S. 149.

75 Ebd.

76 Ebd.

77 Mägdefrau, Jutta; Michler, Andreas (2012): Individualisierende Lernaufgaben im Geschichtsunterricht. Eine empirische Untersuchung zur Rolle von Schulbuchaufgaben und Eigenkonstruktionen der Lehrkräfte. In: Zeitschrift für Geschichtsdidaktik, 208-232. S. 210.
} 
solche in Lehrwerken formulierten Aufgaben), nicht automatisch eine Lernaufgabe im hier beschriebenen Sinne darstellen, weil sie eben nicht oder kaum Kompetenzen anregen, wie Mägdefrau/Michler feststellen.

Die Autoren stellen weiterhin auf der Grundlage ihrer Untersuchung zu individualisierenden Lernaufgaben im Geschichtsunterricht fest: „Arbeitsaufträge müssen gründlicher untersucht werden, da sie das zeigen auch unsere Befunde eindrücklich - den Unterricht in hohem Maße bestimmen. ${ }^{\text {"78 }}$ Köster/Bernhardt/Thünemann stellen zudem klar, dass es mehr bedarf als der reinen Aufgabenformulierung, damit eine Aufgabe zu einer echten Lernaufgabe werden kann:

„Die Qualität einer Lernaufgabe bemisst sich also nicht allein an der Aufgabe selbst (Aufgabentext), sondern auch an der Frage, wie diese Aufgabe im Unterricht bearbeitet wird, welche Materialien zur Verfügung stehen, wie viel Zeit den Lernenden zur Bearbeitung eingeräumt wird und wie die Ergebnisse der Aufgabenbearbeitung reflektiert und diskutiert werden (Aufgabenkontext). ${ }^{\text { }} 79$

Auf der Grundlage dieser Überlegungen und Einschätzungen wird im Folgenden von Aufgaben die Rede sein, auch wenn im Kern die Kategorie der Lernaufgaben gemeint ist. Da nicht sichergestellt werden kann, dass Aufgaben immer diesen Zweck erfüllen, auch wenn sie ihn anvisieren, fällt die Wahl auf den breit angelegten Begriff der Aufgaben. Mit Heuer kann abschließend festgestellt werden: „Was bislang klar geworden sein sollte ist, dass wer von Lernaufgaben spricht, vom Aufgabenkontext, also vom Design des Geschichtsunterrichts selbst und vom Lehrer- und Schülerhandeln nicht schweigen darf. ${ }^{480}$

\subsubsection{Vergleichen als Lernaufgabe im Geschichtsunterricht}

Was bedeuten die Überlegungen nun für den Einsatz von Vergleichsaufgaben im Geschichtsunterricht? Bevor der Blick auf die Leistungen des Operators Vergleichen fällt, muss auch für das Vergleichen festgestellt werden, dass es in den Aufgabenkontext einzubetten ist.

Köster/Bernhardt/Thünemann schlagen eine Checkliste zur Prüfung von Aufgaben im Hinblick auf ihre Kompetenzorientierung vor:

\footnotetext{
${ }^{78}$ Mägdefrau, Jutta; Michler, Andreas (2012). S. 231.

${ }^{79}$ Köster, Manuel; Bernhardt, Markus; Thünemann, Holger (2016), S. 8.

${ }^{80}$ Heuer, Christian (2014): Geschichtsunterricht anders machen. Zur Aufgabenkultur als Möglichkeitsraum. In: Blumscheid, Patrick (Hrsg.): Lernaufgaben - didaktische Forschungsperspektiven. Bad Heilbrunn: Klinkhardt. S. 231-241. S. 232.
} 


\begin{tabular}{|l|l|}
\hline \multicolumn{2}{|l|}{ Checkliste 1: Lernaufgaben } \\
\hline Wird mit der Aufgabe bzw. den Aufgaben ein historisches Problem bearbeitet? & \\
\hline Wird die Problembearbeitung durch eine historische Leitfrage strukturiert? & \\
\hline Werden die Lernenden zur Bildung eigener Sach- und Werturteile aufgefordert? & \\
\hline $\begin{array}{l}\text { Werden die Lernenden angeregt, ihren eigenen Lernprozess oder das Zustandekommen unterschiedlicher Antworten } \\
\text { zu reflektieren? }\end{array}$ & \\
\hline Ist die Aufgabe verständlich formuliert? & \\
\hline Ist die Aufgabe inhaltlich offen und nicht auf eine spezifische Lösung festgelegt? & \\
\hline Liegt das Anforderungsniveau der Aufgabe in der "Zone der nächsten Entwicklung" der Mehrzahl der Lernenden? & \\
\hline Kann die Aufgabe von Lernenden unterschiedlichen Niveaus auf verschiedene Art und Weise betrachtet werden? & \\
\hline Regt die Aufgabe die Lernenden zur Kooperation an? & \\
\hline Finden standardisierte Operatoren Verwendung? & \\
\hline Werden die Lernenden zur Produktion eigener Darstellungen aufgefordert? & \\
\hline
\end{tabular}

Aus: Köster, Manuel; Bernhardt, Markus; Thünemann, Holger (2016): Aufgaben im Geschichtsunterricht. Typen, Gütekriterien und Konstruktionsprinzipien, In: Geschichte lernen, 174. S. 7.

Abbildung 3: Checkliste Kompetenzorientierung (Köster/Bernhardt/Thünemann, 2016)

Wie für andere Aufgabenstellungen, kann diese Checkliste zur Überprüfung von Vergleichsaufgaben eingesetzt werden.

Ein Beispiel: „Vergleichen Sie das Industriepotential der feindlichen Kriegskoalitionen“81

Diese authentische Schulbuchaufgabe ist dem Lehrwerk Horizonte II entnommen. Dieses Lehrwerk von 2006 ist vor der Veröffentlichung der kompetenzorientierten Lehrpläne für das Fach Geschichte in der Sekundarstufe II erschienen. Die Aufgabe ist eingebettet in das Thema „Ausbruch und Verlauf des Krieges“ (1. Weltkrieg) und bezieht sich explizit auf eine Tabelle („Industrieller/technologischer Vergleich der Bündnisse“) ${ }^{82}$ und eine Karte („Europa im Ersten Weltkrieg (1914-1918)“). ${ }^{83}$

Arbeitet man an dieser Aufgabenstellung die Kriterien der Checkliste ab, kann Folgendes festgestellt werden: Mit der Aufgabe wird ein historisches Problem bearbeitet und die Arbeit durch die Leitfrage strukturiert. Da der Operator Vergleichen im Geschichtsunterricht entsprechend der Einheitlichen Prüfungsanforderungen für das Fach Geschichte ${ }^{84}$ die Bildung eines Sachurteils verlangt, sollten Lernende den Vergleich mit einem Sachurteil abschließen. Dies müssen Lernende wissen, weil die Anforderung,

\footnotetext{
${ }^{81}$ Aus: Bahr, Frank (Hrsg.) (2006): Horizonte II. Geschichte für die Oberstufe. Vom Absolutismus bis zum Ersten Weltkrieg. Braunschweig: Westermann. S. 306.

82 Bahr, Frank (Hrsg.) (2006), S. 305.

83 Bahr, Frank (Hrsg.) (2006), S. 306.

${ }^{84}$ Kultusministerkonferenz (2005): Einheitliche Prüfungsanforderungen der KMK. Geschichte. Beschluss der Kultusministerkonferenz vom 01.12.1989 i. d. F. vom 10.02.2005. Online einsehbar unter:

https://www.kmk.org/fileadmin/veroeffentlichungen_beschluesse/1989/1989_12_01-EPA-Geschichte.pdf (zuletzt eingesehen am 01.08.2018).
} 
die Aufgabe mit einem Sachurteil abzuschließen, nicht explizit im Aufgabentext herausgehoben wird. Ebenso gibt es keine Explizierung hinsichtlich eines Werturteils. Die Aufgabenstellung regt nicht zur Reflexion des Lernprozesses an, dies muss durch das Unterrichtssetting geleistet werden. Grundsätzlich ist die Aufgabe verständlich formuliert, wenn, und an dieser Stelle spielt die Sprache eine wesentliche Rolle, insbesondere die Komposita „Industriepotenzial“ und „Kriegskoalitionen“ verstanden werden. Die Aufgabe ist nur bedingt offen, die Auswertung von Tabelle und Karte führt zum Ergebnis, dass die Kriegsgegner (gemeint ist hier die Entente 1914: Frankreich, Russland, Großbritannien) der Mittelmächte Deutsches Reich und Österreich-Ungarn in ihrer Gesamtheit ein höheres Industriepotenzial besitzen als die Mittelmächte. Die Zone der proximalen Entwicklung kann ohne den Blick auf eine konkrete Lerngruppe an dieser Stelle nicht bestimmt werden. Eine Differenzierung unterschiedlicher $\mathrm{Ni}$ veaus bietet die Aufgabe nicht, auch gibt sie keine Hinweise, ob sie kooperativ oder in Einzelarbeit gelöst werden soll. Mit dem Verb „Vergleichen“ wird ein standardisierter Operator verwendet. Es wird in der Aufgabe kein Hinweis gegeben, ob eigene Darstellungen produziert werden sollen. Die Formulierung ist auf den relevanten Fachinhalt reduziert, der bearbeitet werden soll, das Aufgabensetting wird nicht berücksichtigt. Dies ist Aufgabe der Lehrkraft. Insgesamt kann mit Heuer festgestellt werden, dass diese Vergleichsaufgabe eher auf historischen Wissenserwerb hin angelegt ist. Es zeigt sich also, dass eine solche Aufgabe nicht per se eine kompetenzorientierte Lernaufgabe ist. Im Gegenteil können nur wenige Fragen der Checkliste mit „Zutreffend“ beantwortet werden.

Auch bei der im empirischen Teil eingesetzten Vergleichsaufgabe wird gefragt werden müssen, ob es sich um eine Aufgabe handelt, die zum Kompetenzerwerb beiträgt. Da dies aber nur im Zusammenhang mit dem zugehörigen Material, also dem Darstellungstext, geleistet werden kann, wird die Beantwortung erst in Kapitel 5.3 vorgenommen.

\subsubsection{Operatoren als Teil von Lernaufgaben}

Die Beantwortung der Frage nach der Leistung von Lernaufgaben kann nur hinreichend geleistet werden, wenn die Aufgabenformulierung in den Blick genommen wird. Diese erfolgt grundsätzlich in Form einer Frage mit oder ohne Fragepartikel oder über ein handlungsinitiierendes Verb.

Analysiert man ältere und neuere Lehrwerke im Hinblick auf ihre Aufgaben, fällt auf, dass sich die Formulierung der Aufgabenstellung verändert hat. Findet man in den älteren Lehrwerken häufig Formulierungen mit W-Fragen, herrschen in den neueren Lehrwerken Aufgabenstellungen durch sogenannte Operatoren vor. ${ }^{85}$

\footnotetext{
${ }^{85}$ Eigene Auszählung von in NRW zugelassenen Geschichts- oder Gesellschaftslehrelehrwerken der Sekundarstufe II. Die Auszählung wird in Kapitel 4 vorgestellt. Dabei wurden Formulierungen mit Fragepartikeln und/oder Operatoren erhoben. Formulierungen als Frage ohne Fragepartikel wurden nicht gesondert ausgezählt, da sie zwar vorkommen, aber quantitativ nur eine sehr begrenzte Rolle spielen.
} 
Im Zuge der Durchsetzung des Zentralabiturs sind diese Operatoren zunächst durch die Kultusministerkonferenz (KMK) in den Einheitlichen Prüfungsanforderungen in der Abiturprüfung (EPA) ${ }^{86}$ vereinheitlicht und dann durch die Länder übernommen worden. ${ }^{87}$

Der Begriff ,Operator' wird im Allgemeinen wie folgt definiert: „Operatoren sind handlungsinitiierende Verben, die signalisieren, welche Tätigkeiten beim Bearbeiten von Aufgaben erwartet werden. In der Regel sind sie den einzelnen Anforderungsbereichen zugeordnet. ${ }^{\text {"88 }} \mathrm{Ihr}$ Ziel ist Sicherheit in der Aufgabenerwartung für Lehrkräfte und Schüler*innen.

Die von der KMK dem Fach Geschichte zugeordneten 32 Operatoren sind den verschiedenen Anforderungsbereichen zugeordnet und mit einer kurzen Bestimmung versehen. ${ }^{89}$ Sie sind weiterhin als verbindlich für die Konstruktion von Abituraufgaben zu verwenden, wie die Konstruktionsvorgaben für Abiturprüfungsaufgaben im Fach Geschichte (NRW) zeigen (hier: Teilkapitel 3. zur Abfolge und Formulierung der Teilaufgaben):

„• Die Teilaufgaben müssen in einem klar erkennbaren inneren Zusammenhang stehen und somit die Einheit der Aufgabenstellung (vgl. LP S. 109) sichtbar machen.

- Die Teilaufgaben sollen durch eine progressiv gestufte Abfolge Leistungen in allen drei Anforderungsbereichen ermöglichen (vgl. LP S. 104 ff., S. 109 ff.).

- Es sind ausschließlich Operatoren der veröffentlichten Operatorenliste in der Aufgabenstellung zu verwenden. ${ }^{490}$

Wie bereits in Kapitel 2.2 (Einordnung: Kompetenzmodelle und schulische Rahmenbedingungen der gymnasialen Oberstufe als Rahmung des Forschungsvorhabens) gezeigt, werden in den Einheitlichen Prüfungsanforderungen die Anforderungsbereiche I bis III sowie ein übergreifender Anforderungsbereich festgelegt, denen die Operatoren zugeordnet werden: Anforderungsbereich I ist auf die Reproduktion von Sachverhalten und Arbeitstechniken begrenzt, Anforderungsbereich II umfasst Reorganisation und Transfer, Anforderungsbereich III den reflexiven Umgang mit neuen Fragestellungen, er soll zu eigenständigen Folgerungen führen. ${ }^{91}$ Diese Zuordnung lässt auf eine Progression schließen, die man aus der Kompetenzdarstellung so nicht ersehen kann. Die Operatoren bauen nicht in dem Maße aufeinander auf, wie es die Anforderungsbereiche darstellen.

Für das Fach Geschichte wurden durch die KMK die folgenden Operatoren festgelegt:

Zum Anforderungsbereich I gehören nennen, aufzählen, bezeichnen, schildern, skizzieren,

86 Einheitliche Prüfungsanforderungen der KMK für die Fächer, online einsehbar z. B. für Geschichte: https://www.standardsicherung.schulministerium.nrw.de/abitur-gost/fach.php?fach=12 (zuletzt eingesehen am 27.02.18).

${ }^{87}$ Vgl. Bildungsportal des Landes Nordrhein-Westfalen: Zentralabitur in der gymnasialen Oberstufe. Geschichte. Online einsehbar: https://www.standardsicherung.schulministerium.nrw.de/cms/zentralabitur-gost/faecher/fach.php?fach=12 (zuletzt eingesehen am 22.05.20).

${ }^{88}$ Lehrerfortbildungsserver Baden-Württemberg: Operatorenliste. Online einsehbar: http://lehrerfortbildungbw.de/faecher/gwg/fb1/modul1/geo/operator/ (zuletzt eingesehen am 01.08.18).

${ }^{89}$ Kultusministerkonferenz (2005): EPA Geschichte.

${ }^{90}$ Qualitäts- und Unterstützungsagentur - Landesinstitut für Schule (2017) Konstruktionsvorgaben für Abiturprüfungsaufgaben Geschichte (NRW). https://www.standardsicherung.schulministerium.nrw.de/abiturgost/fach.php?fach=12 (zuletzt eingesehen am 03.02.18).

${ }^{91}$ Vgl. ebd. S. 6. 
aufzeigen, beschreiben, zusammenfassen und wiedergeben. Diese Operatoren sollen laut Vorgaben der EPA insbesondere zur Reproduktion bekannter Arbeitstechniken anregen.

Dem Anforderungsbereich II sind die Operatoren analysieren, untersuchen, begründen, nachweisen, charakterisieren, einordnen, erklären, erläutern, herausarbeiten, gegenüberstellen und widerlegen zugeordnet. Dieser Bereich soll zum Transfer bekannter Methoden und Inhalte anregen. Zum Anforderungsbereich III gehören beurteilen, bewerten, Stellung nehmen, entwickeln, sich auseinandersetzen, diskutieren, prüfen, überprüfen und vergleichen. Schließlich werden interpretieren, erörtern und darstellen als sogenannte übergeordnete Operatoren bestimmt, die aus allen drei Anforderungsbereichen Leistungen verlangten. ${ }^{92}$ Den Operatoren ist jeweils eine kurze Bestimmung zugeordnet, manche Operatoren haben auch eine gemeinsame Bestimmung. Leider ist jedoch nicht nachvollziehbar, welche Entscheidungen zur Aufnahme der 32 Operatoren in die Liste geführt haben.

Die nachfolgende Schulbuchanalyse wird, wie eingangs schon darauf hingewiesen, zeigen, dass nur in Neuauflagen älterer Lehrwerke W-Fragen zu finden sind, wohingegen in den neueren Schulbüchern W-Fragen maximal zur Aspektierung eines Operators genutzt werden. In wieder anderen Lehrwerken fällt auf, dass geradezu peinlich genau darauf geachtet wurde, W-Fragen ganz zu vermeiden. Sie tauchen dort auch nicht als Aspektierung einer Aufgabe im Nebensatz auf. Dies hat sicherlich damit zu tun, dass W-Fragen oft auf Inhaltswissen rekurrieren. Sie stellen meist Teilleistungen eines umfangreicheren Operators dar. Ein Beispiel: Fragt man Schüler*innen beispielsweise auf der Basis einer Textgrundlage, welche Argumente der Autor einer Rede nutzt, werden sie vermutlich die entsprechenden Argumente aus dem Redetext heraussuchen. Fordert man dagegen im Sinne einer Aufgabenformulierung mit Operator, die Autorargumentation zu erklären, reicht es nicht aus, die Argumente herauszusuchen; bei dieser Formulierung müssen der Argumentationsweg dargelegt und die einzelnen Argumente eingeordnet werden. Als Formulierung mit W-Fragen müssten mehrere W-Fragen hintereinandergeschaltet werden, um ebendieses Ziel zu erreichen (Welche Argumente nennt der Redner? Wie sind sie hintereinander aufgebaut? Was sagen die Argumente aus? u.a.m.). Sicherlich wäre auch eine Kombination aus Operator und W-Frage möglich (z. B.: Erläutern Sie, wie der Redner seine Argumente aufbaut). Es wäre zum Beispiel zu diskutieren, ob nicht eine Aspektierung durch W-Fragen im Sinne einer Binnendifferenzierung schwächere Schüler bei der Aufgabenlösung unterstützen kann. Im obigen Beispiel würde das Augenmerk der Schüler durch die „Wie“-Frage darauf gelenkt, dass es einen logischen Aufbau gibt, den sie nun herausfinden müssten. Dies kann, je nach Lerngruppe, ein Hinweis für die Aufgabenlösung sein.

Doch auch die Operatoren können Schwierigkeiten bergen, wie Schönemann et al. feststellen:

„Eine genauere Betrachtung der Operatoren und ihrer Verwendung offenbart mindestens drei Defizite. Erstens dürften Schüler in der Regel damit überfordert sein, sich alle Operatoren und die mit ihnen verbundenen Definitionen zutreffend einzuprägen. [...] Zweitens ist es systematisch kaum überzeugend, nicht nur spezifische, sondern auch übergeordnete Operatoren auszuweisen. Wenn bei diesen Operatoren Leistungen in allen Anforderungsbereichen erforderlich sind, dann sollte man sie - im Sinne der Vereinheitlichung - dem AFB III zuordnen. Denn anderenfalls müsste man Operatoren für den AFB II auch als übergeordnete Operatoren für den ersten Anforderungsbereich

\footnotetext{
${ }^{92}$ Vgl. jeweils: Kultusministerkonferenz (2005): EPA Geschichte, S. 7f.
} 
klassifizieren. Und drittens ist ein Mangel an Präzision bei der Verwendung der Operatoren zu kritisieren. ${ }^{\text {"93 }}$

Die Autoren verweisen im ersten Punkt auf die Menge der vorhandenen Operatoren für das Fach Geschichte und stellen die Vielzahl der Operatoren auf den Prüfstand. Dem ist hinzuzufügen, dass die Schüler*innen nicht nur die 32 Operatoren für das Fach Geschichte kennen müssen, sondern dass diese Operatoren mit zum Teil unterschiedlichen Bestimmungen auch in anderen Fächern verwendet werden. Die Lernenden müssen also zudem in der Lage sein, sie fachspezifisch zu unterscheiden und anzuwenden. Vergleicht man die Operatorenlisten „verwandter" Fächer wie etwa Geschichte und Sozialkunde/Politik, fällt z. B. auf, dass der Operator Erörtern in beiden Listen auftaucht. Dabei ist er in Sozialkunde/Politik dem Anforderungsbereich III zugeordnet, in Geschichte gehört er dagegen zu den übergeordneten Operatoren, die Leistungen aus allen drei Bereichen einfordern. Auch die Bestimmungen legen nahe, dass unterschiedliche Teilleistungen von den Lernenden gefordert sind, wenn sie die Aufgabe lösen wollen. So wird in Politik Erörtern wie folgt bestimmt: „Zu einer vorgegebenen Problemstellung eine reflektierte, kontroverse Auseinandersetzung führen und zu einer abschließenden, begründeten Bewertung gelangen. ${ }^{\text {“94 }}$ In Geschichte wird verlangt: „Eine These oder Problemstellung durch eine Kette von Für-und-Wider-bzw. Sowohl-als-Auch-Argumenten auf ihren Wert und ihre Stichhaltigkeit hin abwägend prüfen und auf dieser Grundlage eine eigene Stellungnahme dazu entwickeln. Die Erörterung einer historischen Darstellung setzt deren Analyse voraus. ${ }^{495}$ Es fällt auf, dass die Teilschritte in den EPA Geschichte enger festgelegt sind. Insbesondere wird hier eine vorausgehende Analyse explizit verlangt. Es zeigt sich aber auch, dass die Bestimmung des Operators Erörtern in den EPA Geschichte keinen expliziten historischen Bezug erhält. Lediglich der Hinweis auf eine „historische Darstellung" stellt den Zusammenhang zum Fach her. Hier ist zu fragen, was das Erörtern zu einem spezifisch historischen Operator macht. Im Rückgriff auf die Kritik Schönemanns et al. ist eine klare Spezifizierung des Operators bedeutsam.

Diese Explikation muss im Folgenden auch für den Operator Vergleichen geleistet werden. Die Bestimmung in den Einheitlichen Prüfungsanforderungen in der Abiturprüfung Geschichte stellt eine Geschichtsspezifik lediglich über die Bearbeitung „historischer Sachverhalte ${ }^{\text {“96 }}$ her. Umso bedeutsamer ist es, die in der Bestimmung genannten Teilhandlungen auf ihre Geschichtsspezifik zu überprüfen. Dies geht nur über den Einbezug von Sprache und der Frage, wie diese Teilhandlungen sprachlich realisiert werden können, und wird den Kern dieser Arbeit darstellen.

\footnotetext{
${ }^{93}$ Schönemann, Bernd; Thünemann, Holger; Zülsdorf-Kersting, Maik (2010): Was können Abiturienten? Zugleich ein Beitrag zur Debatte über Kompetenzen und Standards im Fach Geschichte. LIT Verlag, Münster. S. 18.

${ }^{94}$ Kultusministerkonferenz (2005): Einheitliche Prüfungsanforderungen in der Abiturprüfung Sozialkunde/Politik. Beschluss der Kultusministerkonferenz vom 01.12.1989 i. d. F. vom 17.11.2005. Online verfügbar unter: http://www.kmk.org/fileadmin/veroeffentlichungen_beschluesse/1989/1989_12_01-EPA-Sozialk-Politik.pdf. S. 18.

${ }^{95}$ Kultusministerkonferenz (2005): EPA Geschichte. S. 7.

${ }^{96}$ Ebd.
} 
Es ist an dieser Stelle festzustellen, dass Operatoren zur Kompetenzentwicklung beitragen sollen, indem sie die Lernenden zur Selbstständigkeit anregen. Es wird aber auch deutlich, dass damit Schwierigkeiten verbunden sind. Über die Bedeutsamkeit sprachlicher Fähigkeiten zur Aufgabenlösung wird an späterer Stelle im Zusammenhang mit dem Operator Vergleichen noch ausführlicher zu reden sein. Es fällt insgesamt auf, dass Operatorbestimmungen häufig durch den Verweis auf „historische Sachverhalte" oder "historische Problemstellung “97 einen geschichtlichen Zusammenhang erhalten. Dabei handelt es sich wiederum, wie von Thünemann kritisiert (vgl. Kapitel 2.4.1), um das Setting, weniger um eine wirkliche Gattungsspezifik. Diese auszuarbeiten bleibt zunächst ein Desiderat.

\subsection{Vorgaben durch Richtlinien und Lehrpläne}

Mit dem Blick auf Kompetenzen und Aufgaben im Geschichtsunterricht sind wesentliche Felder bereits eröffnet, auf deren Grundlage die Untersuchung zum Vergleichen basiert. Dennoch fehlt noch der Bezug zum institutionellen Rahmen, in dem Aufgaben und hier speziell das Vergleichen im Geschichtsunterricht eingebunden sind.

Da die der Arbeit zugrundeliegende Vergleichsaufgabe einem Lehrwerk der gymnasialen Oberstufe entstammt, ist das Augenmerk auf die Vorgaben der Sekundarstufe II für das Fach Geschichte (bzw. für das Berufskolleg: Gesellschaftslehre mit Geschichte) in Nordrhein-Westfalen gerichtet. Weil die Datenerhebung zudem in NRW durchgeführt wurde und die für die Aufgabe ausgewählte Lehrwerkausgabe für NRW zugelassen ist, sind die Vorgaben dieses Bundeslandes im Weiteren von Bedeutung. Der unterrichtliche Rahmen wird grundsätzlich durch die Richtlinien und Lehrpläne, im Fall von Nordrhein-Westfalen durch den Kernlehrplan für die gymnasiale Oberstufe im Fach Geschichte ${ }^{98}$ bzw. die Einheitlichen Prüfungsanforderungen (EPA) ${ }^{99}$ der Kultusministerkonferenz und die „Vorgaben zu den unterrichtlichen Voraussetzungen für die schriftlichen Prüfungen im Abitur in der gymnasialen Oberstufe ${ }^{1100}$, abgesteckt (sie werden jährlich angepasst und nach dem Jahr der Abiturprüfung benannt). Da es in der Folge um die Untersuchung einer Vergleichsaufgabe geht, sind die unterrichtlichen Voraussetzungen für die Abiturprüfung nur in zweiter Linie bedeutsam, so dass vor allem die schon zuvor genannten EPA, die eine Bestimmung der Operatoren für das Lösen einer Vergleichsaufgabe vorgeben, für die Arbeit herangezogen werden. Der Kernlehrplan für das Fach Geschichte in der gymnasialen Oberstufe findet im Rahmen dieser Arbeit als eine rechtliche Grundlage für den Geschichtsunterricht Beachtung; da die dort angeführten Kompetenzen weitgehend fachunspezifisch sind, wird er für die Untersuchung der Vergleichsaufgabe nur bedingt berücksichtigt.

\footnotetext{
${ }^{97}$ Vgl. ebd.

${ }^{98} \mathrm{vgl}$. Ministerium für Schule und Weiterbildung des Landes Nordrhein-Westfalen (Hrsg.) (2014): Kernlehrplan für die Sekundarstufe II Gymnasium/Gesamtschule in Nordrhein-Westfalen. Geschichte. Online verfügbar unter: http://www.standardsicherung.schulministerium.nrw.de/lehrplaene/upload/klp_SII/ge/KLP_GOSt_Geschichte.pdf (zuletzt eingesehen am 07.10.14).

${ }^{99}$ Kultusministerkonferenz (2005): EPA Geschichte.

${ }^{100} \mathrm{Vgl}$. z. B. Ministerium für Schule und Weiterbildung des Landes Nordrhein-Westfalen (Hrsg.) (2014): Vorgaben zu den unterrichtlichen Voraussetzungen für die schriftlichen Prüfungen im Abitur in der gymnasialen Oberstufe 2015. Online verfügbar unter: https://www.standardsicherung.schulministerium.nrw.de/abitur-gost/getfile.php?file=3213 (zuletzt eingesehen am 07.10.14).
} 
Zu den Rahmenbedingungen ist zudem zu bemerken, dass neben den Bildungsgängen, die nicht fachgebunden zur allgemeinen Hochschulreife führen, außerdem Bildungspläne für die Erlangung der allgemeinen Hochschulreife am Berufskolleg sowie am Abendgymnasium und Kolleg ${ }^{101}$ existieren. Die Besonderheit insbesondere der zum Berufskolleg gehörenden Bildungsgänge ist, dass sie berufliche Kenntnisse oder einen Berufsschulabschluss nach Landesrecht vermitteln. Die Bildungspläne der Fächer sind den jeweiligen Schwerpunkten der beruflichen Kenntnisse, die vermittelt werden, zugeordnet. So existieren z. B. spezielle Pläne für den Fachbereich ,Technik' oder ,Wirtschaft und Verwaltung' u.a.m. Exemplarisch soll der Bildungsplan Geschichte des Fachbereichs Wirtschaft und Verwaltung berücksichtigt werden, da die Erhebung vor allem in Klassen des Schwerpunktes, Wirtschaft und Verwaltung' durchgeführt wurde. ${ }^{102}$

Der Kernlehrplan für die gymnasiale Oberstufe weist den Aufbau eines „reflektierten Geschichtsbewusstseins “103 als wesentliche Kompetenz des Geschichtsunterrichts aus. Dies soll erreicht werden durch den Aufbau der Teilkompetenzen Sach-, Methoden-, Urteils- sowie Handlungskompetenz. Er schließt damit an den Lehrplan Geschichte der Sek. I an. Diese Kompetenzen sollen wiederum im Rahmen verschiedener Inhaltsfelder gefördert werden. Der Lehrplan bestimmt weiter die Teilkompetenzen, die bis zum Ende der Einführungsphase bzw. der Qualifikationsphase erlangt werden sollen. Da im Kernlehrplan explizit das historische Denken als charakteristisch für das Fach gekennzeichnet wird $^{104}$, fügt er sich durch diese Grundkompetenz an die gängigen Kompetenzmodelle an. Die Teilkompetenzen passen sich jedoch nur teilweise in die Modelle ein. Am ehesten kann man den Anschluss an das Modell der FUER-Gruppe vermuten, wenn etwa von Re- und Dekonstruktion die Rede ist ${ }^{105}$. Die zu erreichenden Kompetenzen werden dann durch die Formulierungen mit Hilfe von Operatoren anhand der einzelnen Inhaltsfelder konkretisiert. Auch der Bildungsplan zur Erprobung für das Fach Gesellschaftslehre mit Geschichte für das Berufskolleg geht von den Teilkompetenzen Sach-, Methoden-, Urteils- und Handlungskompetenz aus, deren Ausbildung ebenfalls an Inhaltsfelder gekoppelt ist.

Die ausgewählte Vergleichsaufgabe zur Industrialisierung ist in den Themen- und Inhaltsbereich „Politische und wirtschaftliche Systeme“ des Bildungsplans für das Berufskolleg und das Inhaltsfeld 4 "Die moderne Industriegesellschaft zwischen Fortschritt und Krise" des Kernlehrplans Geschichte für die gymnasiale Oberstufe ${ }^{106}$ einzuordnen.

\footnotetext{
${ }^{101}$ Ministerium für Schule und Weiterbildung des Landes Nordrhein-Westfalen (2015): Kernlehrplan für das Abendgymnasium und Kolleg in Nordrhein-Westfalen Geschichte / Sozialwissenschaft https://www.schulentwicklung.nrw.de/lehrplaene/lehrplan/191/KLP_WbK_GESO.pdf (zuletzt eingesehen am 10.02.2018).

102 Ministerium für Schule und Weiterbildung des Landes Nordrhein-Westfalen (Hrsg.) (2012): Bildungspläne zur Erprobung für die Bildungsgänge, die zu einem Berufsabschluss nach Landesrecht und zur allgemeinen Hochschulreife oder zu beruflichen Kenntnissen und zur allgemeinen Hochschulreife führen, Teil III: Fachlehrplan Gesellschaftslehre mit Geschichte Fachbereich Wirtschaft und Verwaltung. Grundkurs. Online verfügbar unter: Abendgymnasium (zuletzt eingesehen am 01.08.18).

${ }^{103} \mathrm{Vgl}$. Ministerium für Schule und Weiterbildung des Landes Nordrhein-Westfalen (Hrsg.) (2014): Kernlehrplan für die Sekundarstufe II Gymnasium/Gesamtschule in Nordrhein-Westfalen. Geschichte. S. 14.

${ }^{104} \mathrm{Vgl}$. ebd. S. 10.

${ }^{105} \mathrm{Ebd}$.

${ }^{106}$ Vgl. Kernlehrplan für die Sekundarstufe II Gymnasium/Gesamtschule in Nordrhein-Westfalen. Geschichte. S. 28.
} 
Es fällt auf, dass im Bildungsplan des Berufskollegs keine Differenzierung von Themen und Inhalten vorgenommen wird, die Begriffe quasi synonym verwendet werden.

Die Ausbildung der Kompetenzen in den genannten Bildungsplänen ist insofern fachunspezifisch, da diese Aufteilung für die gesellschaftswissenschaftlichen Fächer insgesamt gilt, die Kompetenzen also nicht aus den Anforderungen an das Fach Geschichte abgeleitet sind (vgl. dazu Kap. 2.1). Die Zuordnung der Kompetenzen zu den Themen und Inhalten ist zudem nicht themenspezifisch, im Lehrplan ist von „übergeordneten Kompetenzerwartungen “107 die Rede, so dass kein direkter Bezug von Aufgabe und Thema zu den Kompetenzen hergestellt werden kann.

So geben die Oberstufenlehrpläne zwar die Rahmung des Forschungsvorhabens vor, werden aber für die historische Analyse der Aufgabenlösungen zum Vergleichen nur als Orientierungsrahmen behandelt. Zudem erscheint die Handlungskompetenz für die Auswertung der als Schreibaufgabe gelösten Vergleichsaufgabe unbrauchbar, da zwar ein historisches Werturteil (vgl. Gautschi) erkennbar sein kann, jedoch das als Handlungskompetenz formulierte Ziel, „[...]Prozesse und Ergebnisse historischen Denkens lebensweltlich wirksam werden zu lassen “108, in einem solchen Setting nicht bestimmbar ist. Zunächst soll damit jedoch das Feld abgesteckt sein, in das die Vergleichsaufgabe einzuordnen ist.

\subsection{Wissenschaftspropädeutisches Arbeiten im Geschichtsunterricht}

Die ausgewählte Vergleichs-Aufgabe ist einem Lehrwerk der Sekundarstufe Il entnommen, sie ist damit an den Geschichtsunterricht in der Oberstufe angebunden. Es ist also zu fragen, was die spezifischen Ziele des Geschichtsunterrichts der Sek. II sind, die auch bei der Lösung der Vergleichsaufgabe zu berücksichtigen sind, und was den Geschichtsunterricht der Sekundarstufe II von dem der Sekundarstufe I unterscheidet.

Pandel stellt dazu fest: „Es gibt zwar allgemeine Beschreibungen für diese Schulstufe, aber nicht für den Geschichtsunterricht, der auf ihr erteilt wird. Das besondere Ziel für den Geschichtsunterricht in der Sek. II ist bisher noch nicht eindeutig definiert worden. “109

Im Hinblick auf die Frage nach Unterscheidungsmerkmalen zwischen dem Geschichtsunterricht der Sekundarstufe I und II macht Pandel mit dem Hinweis auf fehlende spezifische Ziele ein wesentliches Problemfeld auf, das spätestens bei didaktischen Entscheidungen etwa im Hinblick auf die Unterrichtsplanung wichtig wird. Bedeutsam wird diese Feststellung auch für die Operation des Vergleichens: Gibt es einen Unterschied zwischen dem Vergleichen in der Sekundarstufe I und II und falls ja, worin manifestiert er sich?

Zwar weisen Richtlinien und Lehrpläne auf die Funktion wissenschaftspropädeutischen Arbeitens für die Sekundarstufe II hin, doch ist auch hier zu fragen, ob es als Kriterium nicht auch schon für den

\footnotetext{
107 Ebd. S. 19.

108 Ebd. S. 15.

109 Pandel, Hans-Jürgen (2012): Geschichtsunterricht in der Sekundarstufe II. In: Barricelli, Michele; Lücke, Martin (Hrsg.): Handbuch Praxis des Geschichtsunterrichts. Bd. 1. Schwalbach/Ts.: Wochenschau Verlag. S. 176-188. S. 176.
} 
Unterricht in der Sekundarstufe I beachtenswert ist und deshalb nur scheinbar eine Abgrenzung darstellt. Für das Vergleichen bedeutet dies herauszufinden, welche Formen und Arbeitsweisen im Geschichtsunterricht notwendig und als wissenschaftsorientiert bzw. wissenschaftspropädeutisch auszumachen sind. Diese Fragen können nur über die Merkmale von Wissenschaftspropädeutik für den Geschichtsunterricht geklärt werden.

Dazu muss in einem ersten Schritt überprüft werden, wie das Kriterium des wissenschaftspropädeutischen Arbeitens institutionell verankert ist.

Erste Auskunft gibt die sog. KMK-Vereinbarung („Vereinbarung zur Gestaltung der gymnasialen Oberstufe in der Sekundarstufe II, Beschluss der Kultusministerkonferenz vom 07.07.1972 i. d. F. vom 08.12.2016“110). Unter der Überschrift „Zielsetzung“ wird die Vermittlung wissenschaftspropädeutischer Bildung formuliert:

„Der Unterricht in der gymnasialen Oberstufe vermittelt eine vertiefte Allgemeinbildung, allgemeine Studierfähigkeit sowie wissenschaftspropädeutische Bildung. Von besonderer Bedeutung sind dabei vertiefte Kenntnisse, Fähigkeiten und Fertigkeiten in den basalen Fächern Deutsch, Fremdsprache und Mathematik. ${ }^{1111}$

Das Ziel einer wissenschaftspropädeutischen Bildung ist damit deutschlandweit vereinbart. Allerdings wird der Begriff für den Geschichtsunterricht inhaltlich nicht gefüllt oder abgegrenzt.

An einer zweiten Stelle der Vereinbarung taucht der Verweis unter dem Titel „Gestaltung und Gliederung" auf. Hier heißt es:

„Der Fachunterricht wird auf unterschiedlichen Anspruchsebenen nach den Bildungsstandards für die Allgemeine Hochschulreife oder den „Einheitlichen Prüfungsanforderungen in der Abiturprüfung" (EPA) erteilt; die Zuordnung obliegt den Ländern. Dabei repräsentiert Unterricht mit grundlegendem Anforderungsniveau das Lernniveau der gymnasialen Oberstufe unter dem Aspekt einer wissenschaftspropädeutischen Bildung. Unterricht mit erhöhtem Anforderungsniveau repräsentiert das Lernniveau der gymnasialen Oberstufe unter dem Aspekt einer wissenschaftspropädeutischen Bildung, die exemplarisch vertieft wird. ${ }^{\text {112 }}$

Zwar wird in verschiedene Anforderungsniveaus differenziert, eine klare Füllung des Begriffs fehlt aber auch hier. Der Begriff der wissenschaftspropädeutischen Bildung taucht im ersten Zitat der KMK-Vereinbarung im Zusammenhang mit den Kategorien Allgemeinbildung und allgemeiner Studierfähigkeit auf. Es wird jedoch nicht deutlich, ob damit die Vorbereitung auf das Studium der Geschichte oder der

\footnotetext{
110 online einsehbar unter: http://www.kmk.org/fileadmin/Dateien/veroeffentlichungen_beschluesse/1972/1972_07_07-Vereinbarung-Gestaltung-Sek2.pdf (zuletzt eingesehen am 07.02.2017).

${ }^{111}$ Kultusministerkonferenz (2016): Vereinbarung zur Gestaltung der gymnasialen Oberstufe in der Sekundarstufe II, Beschluss der Kultusministerkonferenz vom 07.07.1972 i. d. F. vom 08.12.2016. S. 5. Online einsehbar unter: http://www.kmk.org/fileadmin/Dateien/veroeffentlichungen_beschluesse/1972/1972_07_07-Vereinbarung-Gestaltung-Sek2.pdf (zuletzt eingesehen am 07.02.2017).

112 Kultusministerkonferenz (2016), S. 6.
} 
Begriff eher im Hinblick auf selbständiges Arbeiten gemeint ist. Diese Abgrenzung, das zeigt die fachwissenschaftliche Debatte, ist aber bedeutsam und grundlegend für das Arbeiten im Fach Geschichte. ${ }^{113}$ Pandel stellt dazu fest:

„Das Ziel einer Wissenschaftspropädeutik ist nicht, eine Einführung in die Geschichtswissenschaft zu geben. Sie soll nicht helfen, Wissenschaft zu betreiben, sondern verstehend mit ihr umzugehen. Es geht nicht darum, professionelle Historikerinnen und Historiker auszubilden, sondern um das historische Denken und das lebenspraktische Umgehen mit Produkten der Geschichtswissenschaft. “114

Diese Aussage ist konstitutiv für alle weiteren Überlegungen. In der Debatte wird verschiedentlich darauf hingewiesen, dass nur ein geringer Teil der die Sekundarstufe II besuchenden Schüler*innen ein Geschichtsstudium aufnehmen wird. ${ }^{115}$ Auch aus dieser Perspektive erscheint die Einschränkung Pandels sachlogisch. Allerdings fehlt der Feststellung Pandels eine Konkretisierung im Hinblick auf den Geschichtsunterricht. Es muss also bspw. hinsichtlich des Vergleichs gefragt werden, wie und durch Berücksichtigung welcher wissenschaftspropädeutischen Mittel der Vergleich im Geschichtsunterricht historisches Denken anregen kann. Solche wissenschaftspropädeutischen Mittel (sprachlich wie fachlich) müssten erst einmal bestimmt werden. Dieses gilt ebenso für andere Elemente des Geschichtsunterrichts.

Häufig findet man in Abhandlungen zum Geschichtsunterricht in der Sekundarstufe II einen auf Studierfähigkeit eingegrenzten Begriff von Wissenschaftspropädeutik ${ }^{116}$ :

„Der Geschichtsunterricht der gymnasialen Oberstufe hat einen wissenschaftspropädeutischen Auftrag. Er ist ausgerichtet an der Bildung zur Studierfähigkeit allgemein und der des Faches Geschichte im Besonderen. Zur Studierfähigkeit allgemein gehört unter anderem die Fähigkeit der selbstständigen Erarbeitung eines Themas in Form einer schriftlichen Hausarbeit. “117

\footnotetext{
${ }^{113}$ In diesem Kapitel wird mehrfach Bezug auf verschiedene Handbucheinträge genommen. Dies hat damit zu tun, dass längere Abhandlungen zum Thema Geschichtsunterricht in der Sekundarstufe II und Wissenschaftspropädeutik viel weniger pointiert die Schwierigkeiten einer klaren Zielsetzung in den Blick nehmen. Dies wird im Folgenden noch zu zeigen sein.

${ }^{114}$ Pandel, Hans-Jürgen (2012), S. 181f.

${ }^{115} \mathrm{Vgl}$. z. B. Rüsen Jörn (1997): Wissenschaftspropädeutik im Geschichtsunterricht. In: Bergmann, Klaus; Fröhlich, Klaus; Kuhn, Annette; Rüsen, Jörn; Schneider, Gerhard (Hrsg.): Handbuch der Geschichtsdidaktik. 5. überarb. Aufl. Seelze-Velber: Kallmeyer'sche Verlagsbuchhandlung. S. 340-342. S.343.

${ }^{116}$ Auch der Blick in Richtlinien und Lehrpläne unterstützt diesen Begriff von Wissenschaftspropädeutik. Nennt der Rahmenlehrplan noch sehr allgemeine Zielsetzungen wie „Die Schülerinnen und Schüler erhalten die Möglichkeit, Defizite auszugleichen und Stärken weiterzuentwickeln. Sie vertiefen bzw. erwerben fachbezogen und fachübergreifend Grundlagen für wissenschaftspropädeutisches Arbeiten und bewältigen zunehmend komplexe Aufgabenstellungen selbstständig" (Rahmenlehrplan Geschichte Sek. II, S. V), findet sich in den Kernlehrplänen der Hinweis in Bezug auf die Arbeit im Grundkurs:

"Grundkurse im Fach Geschichte repräsentieren das Lernniveau der gymnasialen Oberstufe im Hinblick auf eine fundierte Allgemeinbildung und eine grundlegende wissenschaftspropädeutische Ausbildung. Sie sollen in grundlegende Fragestellungen, Sachverhalte, Problemkomplexe und Strukturen einführen und wesentliche Arbeitsmethoden des Faches vermitteln" (Ministerium für Schule und Weiterbildung des Landes Nordrhein-Westfalen (Hrsg.) (2014): Kernlehrplan für die Sekundarstufe II Gymnasium/Gesamtschule in Nordrhein-Westfalen. Geschichte. S. 13).

${ }^{117}$ Mieles, Bernhard (1999): Wissenschaftspropädeutisches Arbeiten in der Sekundarstufe II. In: Geschichte lernen, 68/1999. S. 50-53. S. 50.
} 
Der Hinweis Mieles' auf die schriftliche Hausarbeit als wichtige Erarbeitungsform erscheint typisch. In der Fachliteratur findet sich häufiger der Bezug auf einschlägige Erarbeitungsformen wie Hausarbeit, Referat etc. Mieles expliziert beispielweise die Arbeitsformen Referat, Protokoll, Klausur, Gruppenarbeit sowie die Arbeit mit Fachliteratur als typische Arbeitstechniken in der Sek. II. Dabei vereint er hier Sozial- und Produktformen sowie die Arbeit mit einem Medium. Allerdings können diese unterschiedlichen Formen genauso relevant für die Sek. I sein. Es handelt sich nicht um rein auf die Sekundarstufe Il zugeschnittene Formen.

Rüsen weist darauf hin, dass es im Geschichtsunterricht darum gehe, insbesondere historisches Denken auszubilden. Er beschreibt hier also kompetenzorientierten Geschichtsunterricht. Geschichtsunterricht, der die Ausbildung historischen Denkens zum Ziel habe, sei grundsätzlich wissenschaftspropädeutisch, vor allem da die Vermittlung methodischer und diskursiver Fähigkeiten elementar sei. ${ }^{118}$ Daher kann wissenschaftspropädeutisches Arbeiten kein Kriterium der Sekundarstufe II allein sein. Auch das Vergleichen wird in der Sekundarstufe I als Methode im Geschichtsunterricht nicht auf einer alltagsweltlichen Basis verbleiben, sobald ein geschichtsspezifisches Konzept in den Blick kommt, muss es zumindest in Ansätzen wissenschaftspropädeutisch sein.

Rüsen führt diese Überlegungen zusammen, wenn er weiter feststellt:

„Die besondere Nähe des Geschichtsunterrichts zum wissenschaftspropädeutischen Lernen hängt mit dem Konstruktcharakter des Fachs zusammen, der nur einen indirekten Zugang zu seinem Gegenstand zulässt und diesen im Unterricht in aller Regel erst herstellen muss. Weil alle Geschichte vermittelte Geschichte ist und ein naiver Zugang auf die Geschichte nicht möglich ist, sind Fragen der Erkenntnisgewinnung sowie Methodenschulung integrative Bestandteile des Geschichtsunterrichts. ${ }^{.119}$

Wenn man eine solche Differenzierung von wissenschaftspropädeutischem Arbeiten und nicht wissenschaftspropädeutischem Arbeiten trifft, wie man sie in den zitierten Rahmenvorgaben sowie Richtlinien und Lehrplänen vorfindet, so muss klar sein, was damit gemeint ist. Die Formulierungen in diesen schulischen Vorgaben haben mit dem Ausdruck des „wissenschaftspropädeutischen Arbeitens" eher eine stärkere Ausdifferenzierung wissenschaftlicher Methodik und damit der Versprachlichung von Vorgehen und Ergebnissen im Blick als eine Einführung fachmethodischer Herangehensweisen erst in der gymnasialen Oberstufe. ${ }^{120}$ Zudem ist der Begriff damit noch nicht fachspezifisch ausdifferenziert. Einen solchen Zugang formuliert Pandel:

\footnotetext{
${ }^{118}$ Rüsen, Jörn (1997), S. 340.

${ }^{119}$ Ebd.

${ }^{120}$ Z. B.: „Was unter ,Wissenschaftspropädeutik' zu verstehen ist, ist ebensowenig eindeutig wie der Begriff ,Allgemeinbildung'. Gegenüber dem weitgespannten Anspruch, auf verschiedenen Gebieten wenigstens in Umrissen Bescheid zu wissen, führt das Ziel der Studienvorbereitung schnell zu einer Spezialisierung auf eine ganz bestimmte Disziplin. Dieses widerspruchsvolle Verhältnis zwischen Bildung und Wissenschaft verliert an Spannung, wenn man weniger die konkrete Studienvorbereitung im Blick hat, als vielmehr einen Einblick in die Welterschließung qua Wissenschaft ermöglichen will. Eine solche Wissenschaftsorientierung, die in der Sekundarstufe II durchaus in eine Wissenschaftspropädeutik münden kann, ermöglicht es, im Bildungsbegriff die fachwissenschaftlichen Ansprüche mit dem Lebensweltbezug der Schüler zu vermitteln.", aus: Baumgärtner, Ulrich (2002): Wissenschaftspropädeutik oder historische Bildung? Der Geschichtsunterricht am Gymnasium. In: Schönemann, Bernd; Voit, Hartmut (Hrsg.): Von der Einschulung bis zum Abitur. Prinzipien und Praxis des historischen Lernens in den Schulstufen. Idstein: Schulz-Kirchner Verlag. S. 230-242. S. $238 f$.
} 
„Wissenschaftspropädeutik besitzt eine pragmatische und eine reflexive Ebene. Auf der pragmatischen Ebene werden ausgesuchte Operationen historischen Arbeitens ausprobiert (z. B. Quellen interpretieren und aus Quellen eine Geschichtsdarstellung schreiben). Zur reflexiven Ebene gehört die Kenntnis eines Kategoriensystems, um sich mit ihm über Ergebnisse von Wissenschaft zu verständigen, sie einzuordnen, sie zu kritisieren, zu befragen, ihre Geltungsansprüche zu akzeptieren bzw. zurückzuweisen. Ein kleines Stück Geschichtstheorie gehört ebenfalls dazu. ${ }^{\text {} 121}$

Bezogen auf die zuvor genannten Formen wie Hausarbeit, Referat etc. bedeutet dies, dass ganz unterschiedliche Operationen als wissenschaftspropädeutisch bezeichnet werden können. Lediglich in Bezug auf die Selbstständigkeit kann für Hausarbeit und Referat festgestellt werden, dass es sich um Formen handelt, die den Lernenden ein recht hohes Maß an eigenständigem Arbeiten abverlangen. Es sind aber nicht die einzigen Formen, ebenso die Erstellung eines Vergleichs kann eine hochanspruchsvolle und selbstständige Arbeitsform darstellen.

Pandel benennt vier Elemente einer Wissenschaftspropädeutik: Geschichtskultur, Erkenntnisverfahren, Geschichtstheorie und Historiografiegeschichte. ${ }^{122}$

Bezieht man diese Überlegungen auf das Vergleichen im Geschichtsunterricht, scheint das Element „Erkenntnisverfahren“ insbesondere von Bedeutung zu sein, es wird im Weiteren auch als „historische Methode" benannt. ${ }^{123}$ Der Vergleich stellt ein Erkenntnisverfahren dar, da die Elemente, die miteinander verglichen werden, auf Gemeinsamkeiten, Ähnlichkeiten und Unterschiede hinterfragt werden und ein Sachurteil am Ende steht. Zudem ist ein Vergleich nur erkenntnisfördernd, wenn er unter Berücksichtigung historischer Arbeitsweisen realisiert und auch reflektiert wird. Das wiederum bedeutet, nimmt man historisches Lernen ernst, zumindest ein Minimum an Kenntnissen über die Theorie des historischen Vergleichs, so dass die Lernenden „[...] im Laufe ihrer Schulzeit ein methodisches Instrumentarium erwerben, mit dem man ,Geschichte' lernt ${ }^{\prime 124}$, das sie also letztlich in die Lage versetzt, fachgerecht mithilfe solcher diskursiven Erkenntnisverfahren fachgerecht zu narrativieren ${ }^{125}$. Insbesondere Rüsen und Pandel bestimmen sehr pointiert die Rahmenbedingungen für den Begriff der Wissenschaftspropädeutik. ${ }^{126}$

\footnotetext{
121 Pandel, Hans-Jürgen (2012), S. 182.

122 Vgl. Pandel, Hans-Jürgen (2012), S. 183.

123 „Die historische Methode bezieht sich auf drei miteinander verknüpfte Teile: Arbeit mit Quellen, dem Schreiben von Geschichte und die Adaption von Theorien, die die beiden anderen Bereiche verbinden.", aus: Pandel, Hans-Jürgen (2012), S. 184.

124 Müller, Bernhard (2013): Wissenschaftspropädeutisches Arbeiten. in: Ulrich Mayer/Hans-Jürgen Pandel/Gerhard Schneider (Hrsg.), Handbuch Methoden im Geschichtsunterricht. Klaus Bergmann zum Gedächtnis, Schwalbach/Ts. 4. Aufl. S. 308-324. S. 309.

125 Die Bedeutung diskursiver Elemente stellt Rüsen im Hinblick auf die Glaubwürdigkeit von historischen Geschichten bereits Anfang der 80er Jahre dar: „Geschichten begegnen einem solchen Zweifel [gemeint ist der Zweifel an ihrer Glaubwürdigkeit, Anm. d. Verf.] dadurch, daß sie ihre Glaubwürdigkeit begründen. Sie geben Gründe dafür, warum man ihnen trauen und sich mit ihnen in der Zeit orientieren kann." (Rüsen, Jörn, 1983: Historische Vernunft. Göttingen: Vandenhoeck und Ruprecht. S. 78.)

${ }^{126}$ Auch Wunderer bestimmt bspw. das Ziel wissenschaftspropädeutischen Arbeitens in Anlehnung an Rüsen und stellt deutlich klar, dass es nicht darum gehe „fachwissenschaftliche Professionalität zum Lernziel zu erheben. Ziel des Geschichtsunterrichts ist es vielmehr, die Schülerinnen und Schüler in die Grundlagen der Geschichtswissenschaft einzuführen, indem er ihnen auf der Basis ihrer eigenen Erfahrungen (,Lebenswelt') eine Vorstel-
} 
Die Probleme des Begriffs Wissenschaftspropädeutik und seiner Füllung liegen auf dem Tisch. Gleichwohl ist durch diese Überlegungen immer noch nicht geklärt, was denn nun eigentlich im Geschichtsunterricht der Sekundarstufe II genau gelernt werden soll, so dass es im weitesten Sinne als Vorbereitung auf wissenschaftliches Arbeiten gelten kann.

Mit Baumgärtner kann als Desiderat festgehalten werden:

„Historische Bildung ist also nur in Orientierung an und Auseinandersetzung mit der Geschichtswissenschaft möglich, aber erst im Rückbezug auf die eigene Lebenswelt garantiert. Insofern kommt der Geschichtsdidaktik die Aufgabe zu, ein im Geschichtsunterricht erreichbares Konzept wissenschaftsorientierter historischer Bildung zu entwickeln, das die Vermittlung zwischen der Alltagspraxis der Schüler und der theoretischen Alltagsdistanz der Wissenschaften zu gewährleisten und weniger künftige Historiker als informierte Laien im Sinn hätte. ${ }^{1127}$

Lösungsansätze für ein solches Konzept bietet Baumgärtner nicht an, es stellt vielmehr eine Forderung an die Geschichtsdidaktik dar.

Ausgehend von der Operation des Vergleichens im Geschichtsunterricht wird im Rahmen dieser Arbeit gefragt, welche Formen und Arbeitsweisen im Geschichtsunterricht notwendig und als wissenschaftsorientiert bzw. wissenschaftspropädeutisch auszumachen sind. Die für den Vergleich benötigten (vor-)wissenschaftlichen Formen sollen im folgenden Kapitel im Zusammenhang operationalisiert und so ein Beitrag zur Entwicklung des von Baumgärtner geforderten Konzepts geleistet werden. Dazu muss jedoch zunächst der sprachliche Ansatz, den diese Arbeit verfolgt, offengelegt werden, an den ein Konzept zum Umgang mit wissenschaftspropädeutischem Arbeiten geknüpft wird.

\subsection{Ein funktionales Verständnis von Sprache für den Geschichtsunterricht - eine linguistische Grundlegung}

Im vorhergehenden Kapitel konnte festgestellt werden, dass Ziel von Unterricht sein muss, Formen alltäglicher Wissenschaftssprache zu explizieren und Lernende zu unterstützen, (sprachliche) Werkzeuge in Bezug auf das jeweilige Fach weiterzuentwickeln. Dies gilt umso mehr für den Unterricht in der Sekundarstufe II. Die folgenden Überlegungen zur Funktion von Sprache im Geschichtsunterricht knüpfen an diese Grundüberlegung an. Über einige Anmerkungen zum Verständnis von Sprache im Geschichtsunterricht allgemein soll in diesem Kapitel über eine funktionale Herangehensweise gezeigt werden, inwiefern die Hinwendung zur Sprache die Kompetenzorientierung im Geschichtsunterricht unterstützen kann und welche Rolle dabei die Explizierung geschichtsspezifischer Operationen und Textsorten bzw. Textmuster im Geschichtsunterricht spielen kann.

\footnotetext{
lung von historischer Arbeitsweise, der Leistung und der Grenzen wissenschaftlicher historischer Erkenntnis vermittelt." Wunderer, Hartmann (2000): Geschichtsunterricht in der Sekundarstufe II. Schwalbach/Ts.: Wochenschau Verlag. S. 19.

127 Baumgärtner, Ulrich (2002), S. 239.
} 


\subsubsection{Historische Bewusstseinsbildung über Sprache - eine funktionale Betrachtungs-}

weise

Die Auseinandersetzung mit dem Thema Sprache im Geschichtsunterricht in einem Geschichtsblog zeigt, dass die Bedeutung von Sprache allgemein, konzeptionell schriftlich wie mündlich, in der neueren geschichtsdidaktischen Forschung zunehmend an Beachtung findet. Auf die im Geschichtsblog „Public History Weekly“ durch Handro aufgeworfene Frage „Sprachlos im Geschichtsunterricht?"128 antwortet Hartung im Hinblick auf spezifische sprachliche Ressourcen im Geschichtsunterricht: „Besonders vordringlich erscheint mir zu untersuchen, welche sprachlichen Mittel Schüler*innen wie auch Lehrkräfte in verschiedenen Kommunikationssituationen zum Versprachlichen geschichtlicher Sachverhalte tatsächlich verwenden. “129 Der Blogbeitrag und der zugehörige Kommentar zeigen die Aktualität der Suche nach fachspezifischen sprachlichen Strukturen. Dabei gehen beide Autoren deutlich darauf ein, dass diese sprachlichen Ressourcen in engem Zusammenhang mit der Funktionalität der durch die sprachlichen Mittel evozierten Bedeutungen im Hinblick auf den historischen Untersuchungsgegenstand stehen.

Auch wenn der Ansatz nicht völlig neu ist, sondern bereits in älteren Veröffentlichungen Bezug darauf genommen wird ${ }^{130}$, so sind jüngst vermehrt Publikationen entstanden, die den Zusammenhang von historischem Bewusstsein und Sprache explizieren. So stellen etwa Handro/Schönemann fest:

„Die Reflexion des Verhältnisses von ,Geschichte und Sprache' ist zentral für geschichtsdidaktisches Denken. Ob bei der Rekonstruktion vergangener Wirklichkeit, beim historischen Verstehen, bei der Darstellung von Geschichte oder beim Diskurs über Geschichte - stets ist man auf Sprache angewiesen. “"131

Generell scheint im Forschungsdiskurs Einigkeit über die prinzipielle Bedeutung von Sprache zu bestehen, gerade im Hinblick auf die in der Geschichtsdidaktik als grundlegend angesehene narrative Kompetenz. Soll Geschichte ,erzählt' werden, braucht es dafür spezielle sprachliche Mittel.

In diese Überlegung bringen Handro/Schönemann einen weiteren Aspekt ein:

„Nimmt man den lernpsychologisch fundierten Zusammenhang zwischen Denken und Sprache und die konstitutive Bedeutung von Sprache im Prozess des Wissenserwerbs ernst, dann erweist sich "Sprache" als ein Schlüsselproblem des Geschichtsunterrichts. Historisches Denken und historisches Lernen sind durch Sprache determiniert. Lernprozesse können immer nur im Wechselspiel

${ }^{128}$ Vgl. Handro, Saskia (2014): Sprachlos im Geschichtsunterricht. Blogbeitrag vom 9.1.14. In: Public History Weekly. The International Blogjournal. Online-Ressource: https://public-history-weekly.degruyter.com/2-20141/sprachlos-im-geschichtsunterricht/ (zuletzt eingesehen am 07.03.17).

${ }^{129}$ Vgl. Hartung, Olaf (2014): Die Fragen, die Saskia Handro in ihrem Beitrag zum Forschungsfeld Sprache und Geschichtslernen stellt, sind für die Gestaltung historischer Lernprozesse essentiell [Blog-Kommentar], veröffentlicht am 27.01.2014 um 14:25 auf Public History Weekly. The International Blogjournal. Online-Ressource: https://public-history-weekly.degruyter.com/2-2014-1/sprachlos-im-geschichtsunterricht/ (zuletzt eingesehen am 07.03.17).

${ }^{130}$ Vgl. dazu z. B. Lucas, Friedrich J. (1975): Zur Funktion der Sprache im Geschichtsunterricht. In: Die Funktion der Geschichte in unserer Zeit. Hrsg.: Jäckel, Eberhard; Weymar, Ernst. Stuttgart. S. 326-342 oder Günther-Arndt, Hilke (2010): Hinwendung zur Sprache in der Geschichtsdidaktik - Alte Fragen und neue Antworten. In: Saskia Handro/Bernd Schönemann (Hrsg.): Geschichte und Sprache (Zeitgeschichte - Zeitverständnis 21). Berlin: LiT-Verlag, S. 17-46. S. 18.

${ }^{131}$ Handro, Saskia; Schönemann, Bernd (2010): Geschichte und Sprache - Eine Einführung. In: Dies. (Hrsg.): Geschichte und Sprache (Zeitgeschichte - Zeitverständnis 21). Berlin: LiT-Verlag, S. 3-15. S. 3. 
zwischen mündlichen/schriftlichen Sprachprodukten und den sprachgebundenen Vorwissens-

strukturen der Rezipienten gedacht werden. "132

Die Autoren stellen also eine sich über Sprache bestimmende Wechselbeziehung zwischen historischem Denken und Lernen und Sprache fest, sie führen jedoch nicht aus, wie sich diese ausgestaltet. Bernhardt/Wickner charakterisieren dieses Wechselspiel am Beispiel der Metapher vom Mauerfall: „Das Fallen ist vielmehr eine metaphorische Fassung eines Vorgangs, der damit gleich eine historische Deutung erhält, indem die Relevanz des Tages durch die Metapher aufgewertet wird [...]. ${ }^{\text {“133 Dieses }}$ Beispiel zeigt, dass das Erzählen von Geschichte unterschiedliche sprachliche Strukturen benötigt, die über eine ,sachlich-neutrale' Sprache hinausgehen. Deshalb ist es nicht sinnvoll, auf der Ebene der Nennung solcher Strukturen zu verbleiben, vielmehr müssen sie auf ihre Funktionalität hin hinterfragt werden. ${ }^{134}$

Im Folgenden wird der Versuch unternommen, diese Wechselbeziehung von historischem Denken, Lernen und Sprache über das Sprach- und Wissensmodell nach Ehlich/Rehbein zu explizieren. ${ }^{135}$ Ehlich/Rehbein gehen in Anlehnung an Searle davon aus, dass ein Bestandteil von Sprechakten ihr propositionaler Gehalt ist. Damit ist der Inhalt von (längeren oder kürzeren, schriftlichen oder mündlichen) Äußerungen gemeint.

Über das Vergleichen im Geschichtsunterricht werden beispielsweise bestimmte Inhalte vermittelt, wie im untersuchten Beispiel etwa der zeitliche Beginn der Industrialisierung in England und Deutschland und anderes mehr. Dabei hat Kommunikation in der Regel keinen Selbstzweck, sondern es soll ein Inhalt an einen Zuhörer oder Leser vermittelt werden. Erst dann wird Kommunikation wirkungsvoll. In dem Moment, in dem ein Zuhörer oder Leser den Inhalt versteht, kann durch die Kommunikation das Wissen des Hörers verändert werden. Nennt ein Sprecher im Vergleich des Beginns der Industriellen Revolution in England mit Deutschland zum Beispiel den Startzeitpunkt der Industrialisierung und der Hörer kannte ihn bis dahin nicht, kann er diesen in sein Wissen integrieren. Die ausgewählten Inhalte sind dabei immer abhängig vom Zweck der sprachlichen Operation. Für den Vergleich des Beginns der Industriellen Revolution in England und Deutschland bedeutet dies, dass nur solche Elemente berücksichtigt werden, die es ermöglichen, England und Deutschland in Beziehung zu setzen und dadurch abschließend zu einer Bewertung zu kommen. Dieser Zweck (oder: die Absicht des Sprechers) wird als illokutiver Akt bezeichnet. Die mögliche Absicht eines Sprechers, einem Hörer den Beginn der Industriellen Revolution in England und Deutschland zu vermitteln, kann jedoch nur dann gelingen, wenn der Hörer über genügend Wissenselemente verfügt, um die Information einordnen zu können. Kennt

\footnotetext{
132 Handro, Saskia/Schönemann, Bernd (2010), S. 9.

133 Bernhardt, Markus; Wickner, Mareike-Cathrine (2015), S. $281 \mathrm{f}$.

${ }^{134}$ Einen ähnlichen Ansatz wählt auch Schrader, die es sich zur Aufgabe macht, „,...] anhand einer funktionallinguistischen Analyse von Darstellungstexten deutscher Schulgeschichtsbücher narrative Strukturen zu eruieren, um damit Ansätze aufzuzeigen, wie eine gezielte Förderung der narrativen Kompetenz in einem sprachsensiblen Geschichtsunterricht aussehen könnte." (Schrader, Viola, 2013: Geschichte als narrative Konstruktion. Eine funktional-linguistische Analyse von Darstellungstexten in Geschichtsschulbüchern. Berlin: Lit Verlag. S. 11.). Schrader wählt einen Zugang über Hallidays „Functional Grammar“ und untersucht Darstellungstexte über die Dimensionen Field, Tenor und Mode. Allerdings schließt sie daran statt eines sprachbildenden einen nur auf Sprachförderung angelegten Ansatz für ihre Förderung eines sprachsensiblen Geschichtsunterrichts an.

135 Ehlich, Konrad; Rehbein, Jochen (1986): Muster und Institution. Untersuchungen zur schulischen Kommunikation. Tübingen: Narr. S. $95 \mathrm{ff}$.
} 
er etwa das Konzept der Industriellen Revolution nicht oder weiß mit England und/oder Deutschland nichts anzufangen, wird die Mitteilung nicht zur gewünschten Wissensveränderung beim Hörer führen. Dem kann der Sprecher entgegenwirken, indem er genügend Informationen mitliefert, um die fehlenden Wissensstrukturen beim Hörer auszugleichen. Aus Gründen der Veranschaulichung wurde mit diesem Beispiel zunächst eine sehr basale Form von Wissen gewählt, die durch den Rückbezug auf einen Zeitpunkt eine Form des deklarativen Wissens repräsentiert. Doch auch für das Transferieren von anderen Wissensformen, wie dem prozeduralen und metakognitiven Wissen hat dieses Modell durch seine sehr grundlegende Herangehensweise seine Berechtigung ${ }^{136}$. Denn ihm liegt die Vorstellung zugrunde, dass über sprachliche Praktiken Bedeutungen geteilt werden können bzw. Sprache es ermöglicht, einen gemeinsamen Handlungsraum aufzubauen. ${ }^{137}$

Um die Zusammenhänge zwischen Proposition und Illokution in Bezug auf die Veränderung des Hörerbzw. Leserwissens abbildbar zu machen, arbeiten Ehlich/Rehbein mit dem nachfolgenden Modell:

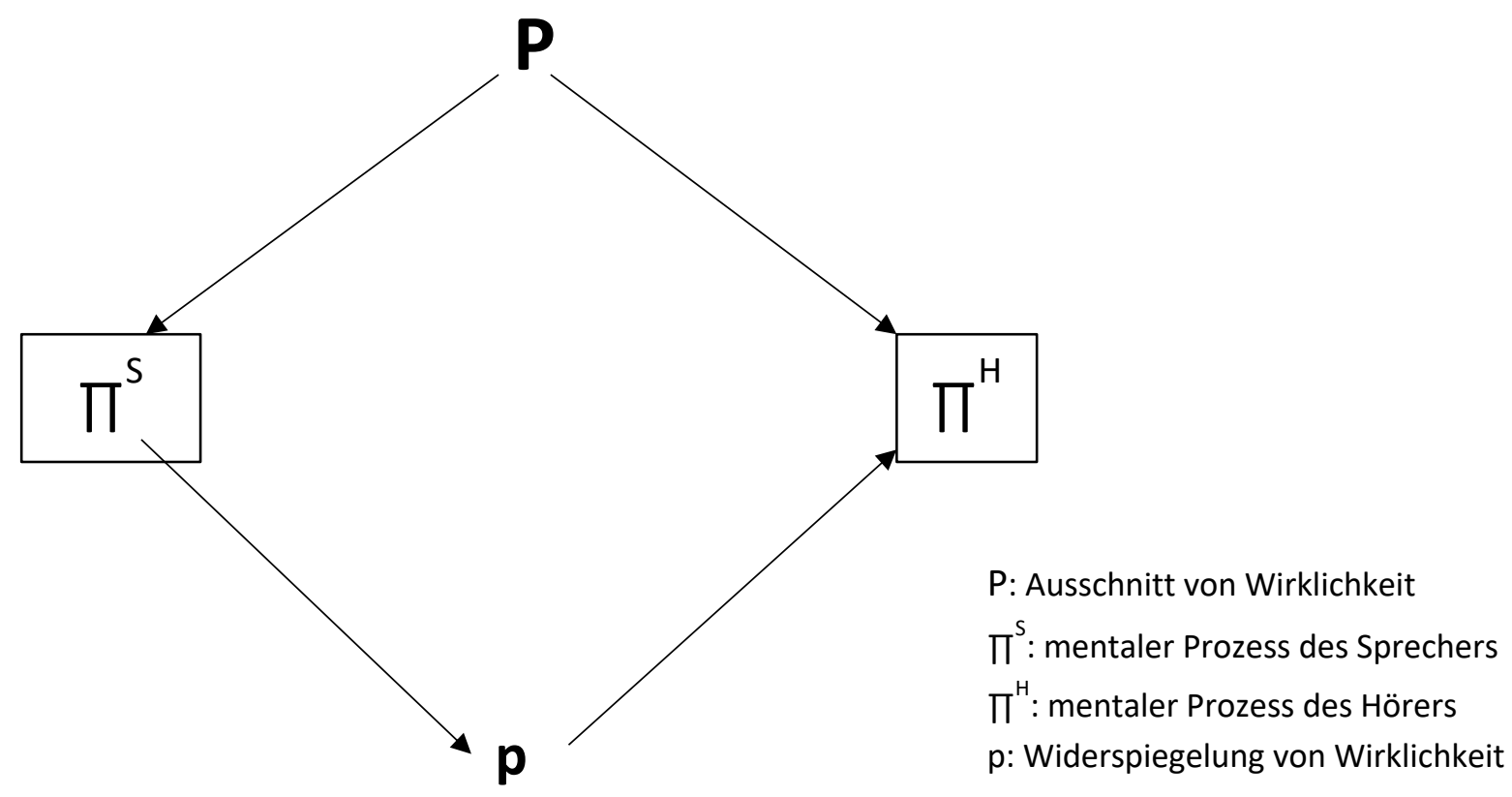

Abbildung 4: Sprach- und Wissensmodell nach Ehlich, K.; Rehbein, J. (1986), S. 96.

\footnotetext{
${ }^{136}$ Günther-Arndt differenziert die Wissenstypen in metakognitives, strategisches, domänenspezifisches, deklaratives und prozedurales Wissen aus. Das Transferieren des Startzeitpunkts der Industriellen Revolution wäre nach Günther-Arndt als Chunking einzuordnen. Vgl. dazu Günther-Arndt, Hilke (2014): Historisches Lernen und Wissenserwerb. In: Günther-Arndt, Hilke; Zülsdorf-Kersting, Meik (Hrsg.): Geschichtsdidaktik. Praxishandbuch für die Sekundarstufe I und II. 6. Aufl. Berlin: Cornelsen. S. 24-49.S.40f.

${ }^{137}$ Ehlich/Rehbein gehen vom Begriff des Handlungsraums aus. Dieser findet sich wieder in der Handlungstheorie von Bourdieu (vgl. Bourdieu, Pierre (1982): Die feinen Unterschiede. Kritik der gesellschaftlichen Urteilskraft. 1. Aufl. Frankfurt a. M.: Suhrkamp Verlag). Bourdieu geht von den gesellschaftlichen Akteuren aus, die sich entsprechend der Dimensionen ihres gesellschaftlichen Kapitals im sozialen Raum verteilen. Hieraus entwickelt er seinen Habitusbegriff, dem unterschiedliche gesellschaftliche Praktiken zugrunde liegen. Diese werden wiederum über kommunikative Strukturen verbalisiert. Schule stellt einen solchen institutionellen Handlungsraum dar, der bestimmte sprachliche Handlungen einfordert, die vermittelt werden müssen und nicht vorausgesetzt werden können.
} 
Auf der linken Seite des Modells bilden sie mit der Bezeichnung " $\Pi$ " den mentalen Prozess des Sprechers (gekennzeichnet durch " $\Pi^{S^{\prime \prime}}$ ) bei der Vermittlung, Auswahl, Strukturierung der Elemente, die er in Sprache transformiert, ab. Will nun der Sprecher eine sprachliche Handlung erfolgreich ausführen, muss er Kenntnis über das Wissen des Hörers haben, um das zu vermittelnde Wissen strukturieren und übermitteln zu können. Dies geschieht über die Sprache. Ausgehend von der Annahme, dass Sprache Wirklichkeit widerspiegelt, bezieht sich der inhaltliche Gehalt (also die Proposition) auf einen ,Ausschnitt der Wirklichkeit'. Diesen Ausschnitt von außersprachlicher Wirklichkeit bezeichnen die Autoren mit „P", die sprachliche Widerspiegelung dieser Wirklichkeit in der Proposition als „p“. Ist die Übermittlung des Wissens erfolgreich, verändert sie mittels eines psychischen Prozesses beim Hörer $\left({ }, \Pi^{\mathrm{H}^{\prime \prime}}\right)$ dessen Wissen. ${ }^{138}$

Der Begriff „Wirklichkeit“ ist in der Geschichtswissenschaft nicht unumstritten, Goertz stellt dazu fest: „Historische $>$ W. $<$ [gemeint ist die Wirklichkeit. Anm. d. Verf.] kann keinen Zustand unabhängig von seinem Erkanntwerden in der Gegenwart meinen, sondern allenfalls eine Beziehung, die in der Veränderlichkeit der Gegenwart zur Vergangenheit gesucht wird und die Zukunft zu eröffnen verspricht. “139 Er grenzt mit dieser Aussage einen „naiven Realismus“ von einem „reflektierten Realismus“ ab ${ }^{140}$. Auch Ehlich/Rehbein gehen mit ihrem Wirklichkeitsbegriff nicht von einem „naiven Realismus", sondern von einer kollektiven und damit gesellschaftlich konstruierten Wirklichkeit ${ }^{141}$ aus. Da die Überlegungen Goertz' auch die Schwierigkeiten der Geschichtswissenschaft, den Begriff "Wirklichkeit“ zu fassen, zeigen, muss der geschichtswissenschaftliche Begriff von Wirklichkeit im Hinblick auf seine Wirksamkeit für das ausgewählte Modell hinterfragt werden. Dafür kann das von Lorenz vorgeschlagene Konzept des „internen Realismus“ ${ }^{\prime 142}$ nutzbar gemacht werden: Lorenz überträgt den Begriff des „internen Realismus", der von Putnam ${ }^{143}$ für die Naturwissenschaften geprägt wurde, auf die Geschichtswissenschaft ${ }^{144}$. Dazu bedient er sich der Grundannahmen, „erstens daß die Wirklichkeit unabhängig von unserem Wissen von ihr existiert, und zweitens daß unsere wissenschaftlichen Aussagen - unsere Theorien eingeschlossen - sich auf diese Wirklichkeit beziehen. ${ }^{145}$ Diese Beschreibung von Wirklichkeit ist übertragbar auf „P“ des Modells von Ehlich/Rehbein. Interessant ist insbesondere die Fortführung dieses Blickes auf Wirklichkeit: „Der Ausgangspunkt des, internen Realismus' ist die Erkenntnis, daß all unser Wissen von der Wirklichkeit durch die Sprache vermittelt ist; Wirklichkeit ist für uns also immer

\footnotetext{
138 Vgl. ebd.

139 Goertz, Hans-Jürgen (2002): Wirklichkeit. In: Jordan, Stefan (Hrsg.): Lexikon Geschichtswissenschaft. Hundert Grundbegriffe. Stuttgart: Reclam. S. 328-330. S. 330.

140 Vgl. ebd. S. 328.

${ }^{141}$ Vgl. dazu: Rehbein, Jochen (1977): Komplexes Handeln. Elemente zur Handlungstheorie der Sprache. Stuttgart: Metzlersche Verlagsbuchhandlung. S. 35.

142 Lorenz, Chris (2004): Historisches Wissen und historische Wirklichkeit: Für einen „internen Realismus“. In: Schröter, Jens; Eddelbüttel, Antje: Konstruktion von Wirklichkeit. Beiträge aus geschichtstheoretischer, philosophischer und theologischer Perspektive (Reprint 2013). Berlin; New York: De Gruyter. S. 65-106.

${ }^{143}$ Vgl. z. B.: Putnam, Hillary (1982): Reason, Truth and History, Cambridge: Cambridge University.

Press.

$-(1982 a)$

144 Ebd.

145 Ebd. S: 79.
} 
Wirklichkeit innerhalb des Rahmens einer bestimmten Beschreibung. "146 Und weiter: „Die Beschreibungen verkörpern Standpunkte oder Perspektiven [Hervorh. i. Orig., Anm. d. Verf.], von denen aus die Wirklichkeit betrachtet wird. Die Perspektiven als solche gehören zum Beschreibungsrahmen und nicht zur Wirklichkeit selbst. ${ }^{1147}$, wodurch sich das Sprechen über Wirklichkeit immer auf den ausgewählten Bezugsrahmen richtet. Diese Beschreibung von Wirklichkeit ist übertragbar auf "p" des Modells von Ehlich/Rehbein. Lorenz stellt daraus folgernd fest, dass der Sprache eine „performative Funktion“148 zukomme, und leitet hierüber die Bedeutung eines linguistisch-pragmatischen Zugangs für seinen Wirklichkeitsbegriff ab:

„In der Geschichte sind die Historiker die Sprecher, ihre Texte Ansammlungen von Sprechakten und ihr Publikum die Hörer. Die Hauptfunktionen von Sprechakten sind es, Kontakte und Beziehungen zu stiften, Informationen zu liefern, Emotionen auszudrücken, zu bewerten, sich zu engagieren und eine ästhetische Rolle zu spielen. ${ }^{.149}$

Es geht also, verkürzt gesagt, um die ,Widerspiegelung von Wirklichkeit' nach Ehlich/Rehbein, die in Form einer Wiedergabe von Fakten, Beschreibungen, aber eben auch von argumentativen Strukturen vermittelt sein kann. Damit über historische Texte wiederum die von Lorenz angesprochenen Beziehungen gestiftet, Informationen erfolgreich geliefert oder bewertet werden können, ist die Kenntnis der mentalen Prozesse bedeutsam (im Modell durch $\pi$ gekennzeichnet): Daraus folgt, dass nur, wenn der Sprecher oder Schreiber das Wissen des Hörers oder Lesers berücksichtigt, Kommunikation erfolgreich sein und das gewünschte Wissen übertragen werden kann.

Lorenz argumentiert, dass mit Hilfe des, internen Realismus' das bisher vernachlässigte Verständnis für die normative Funktion der Sprache des Historikers geklärt werden kann: „Die Vernachlässigung liegt in Objektivismus und Relativismus begründet, da beide voraussetzen, daß die normative Funktion der Sprache die Repräsentationsfunktion als Folge der angeblich, unüberbrückbaren Kluft' zwischen Tatsachen- und Werturteil ausschließt. ${ }^{\text {“150 }}$ Die Schließung dieser Kluft kann über das Sprach- und Wis-

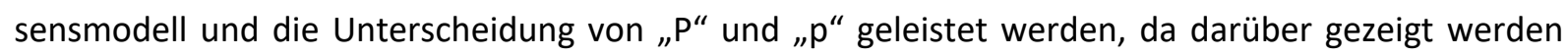
kann, dass eine Aussage unterschiedliche Funktionen innerhalb eines Diskurses haben kann. Wenn Lorenz die strikte Trennung von Tatsachen und Werten in Frage stellt, bieten ihm die funktionale Pragmatik und die Grundlegung dieser im Sprach- und Wissensmodell die Basis, die Aufhebung einer solchen Trennung in der Geschichtswissenschaft zu fundieren ${ }^{\prime 151}$. Denn dieser Ansatz geht von der Zweckgebundenheit einer sprachlichen Handlung aus und kann so nicht nur zeigen, dass eine Aussage mehrere Funktionen besitzen kann ${ }^{152}$, sondern darüber hinaus diese Funktionen offenlegen.

Auch und gerade für den Geschichtsunterricht ist dieses Modell bedeutsam, um Funktionen historischer Aussagen (und damit sprachlicher Handlungen) im Unterricht sichtbar zu machen und Lernenden

\footnotetext{
146 Ebd. S. 85.

${ }^{147}$ Ebd.

148 Ebd. S. 98.

149 Ebd.

150 Ebd. S. 99.

${ }^{151}$ Vgl. ebd. S. 100.

152 Ebd.
} 
darüber ein Werkzeug an die Hand zu geben, zu eigenen Narrationen zu gelangen. Denn das Nachdenken über den Zweck einer sprachlichen Handlung führt letztlich dazu, bestehende oder eigene historische Narrationsansätze prüfen zu können und mögliche Erklärungen oder Erkläransätze für ein historisches Phänomen einzuordnen und diese dann für eine eigene Narration zu nutzen oder auch zu verwerfen.

Für das Vergleichen als sprachliche Handlung im Geschichtsunterricht lassen sich hieraus folgende Überlegungen ableiten:

Kennt der Sprecher den Zweck eines geschichtlichen Vergleichs nicht, ist ihm bspw. nicht klar, dass der Vergleich mind. zwei Elemente in Beziehung setzt und abschließend beurteilt, muss der Vergleich misslingen oder bleibt etwa im Gegenüberstellen stecken. Denn der Zweck eines Vergleichs im Geschichtsunterricht geht über das Gegenüberstellen hinaus und verlangt eine Beurteilung, der Vergleich wäre dann zumindest nicht abgeschlossen. Gleichzeitig muss dem Modell entsprechend das Hörer- bzw. Leserwissen berücksichtigt werden, damit das Vergleichen gelingen, also vom Hörer oder Leser antizipiert werden kann; auch dies kann nur über die Kenntnis der Zweckgebundenheit sprachlicher Handlungen funktionieren. Geschichtsunterricht muss deshalb wesentliche Operationen (wie z. B. das Beschreiben, Erklären, Begründen, Vergleichen u.a.m.) zweckgebunden sprachlich wie fachlich explizieren, damit fachliche Kommunikation und dadurch der Aufbau historischen Bewusstseins als Kernziel des Geschichtsunterrichts ermöglicht wird. Dabei kann ein textmusterorientierter Weg helfen, der nachfolgend dargestellt wird.

Das Modell verdeutlicht weiterhin, dass Unterricht sprachliche Strukturen auf der Seite der Lernenden aufbauen muss, diese also nicht aus sich selbst heraus als vorhanden angenommen werden können. Diese Einsicht wiederum bedeutet zunächst die Klärung solcher sprachlichen Strukturen, die für historisches Lernen bedeutsam sind, für und durch die Lehrenden. Dies ist bisher nur in Ansätzen geschehen. Auch die Einheitlichen Prüfungsanforderungen (EPA) klären diese Strukturen nicht.

Das Modell nach Ehlich/Rehbein basiert auf mündlicher Kommunikation, nimmt also die Kopräsenz von Sprecher und Hörer an. In der Untersuchung der Lösungen zur Vergleichsaufgabe handelt es sich jedoch um Schreibprodukte, Schreiber und Leser sind nicht kopräsent und der Text muss Zeit und Raum überbrücken. ${ }^{153}$ Das bedeutet, dass der Schreiber noch viel stärker über das mögliche Wissen des Lesers nachdenken muss, will er erfolgreich Wissen übertragen: Welches Wissen muss vermittelt werden? Welchen Zweck hat der Text? Wie kann das Wissen strukturiert werden? In welcher Form muss es aufbereitet werden? Welche sprachlichen Mittel sind dazu notwendig? u.a.m. Diesen Fragen will die Arbeit für das Vergleichen nachgehen und zum Abbau des Desiderats für das Vergleichen im Geschichtsunterricht beitragen.

\footnotetext{
${ }^{153}$ Vgl. dazu Ehlich, Konrad (1984): Zum Textbegriff. In: Rothkegel, Annely; Sandig, Barbara (Hrsg.) Text - Textsorten - Semantik. Hamburg: Buske, S. 9-25.
} 


\subsubsection{Erkenntnisbildung über sprachliches Handeln im Geschichtsunterricht}

Das Sprach- und Wissensmodell nach Ehlich/Rehbein lässt sich in hohem Maße an das Modell Handros zur Sprache im Geschichtsunterricht anschließen. Handro geht statt von einer Zuordnung über den Begriff der Narration als Ziel des Geschichtsunterrichts, wie bspw. Günther-Arndt sie vornimmt ${ }^{154}$, im Sinne einer stärkeren Prozessorientierung direkt von der Bedeutung der Sprache für den Erkenntnisund Lernprozess aus und unterscheidet im Hinblick auf Struktur, Stellung und Funktion innerhalb der genannten Prozesse vier Sprachen im Geschichtsunterricht. Sie unterscheidet die Ebene der Quellen, der historischen Darstellung, der Schülersprache und der fachspezifischen Schulsprache. ${ }^{155}$ Die Ebene der historischen Darstellung definiert sie als: „Repräsentation gegenwärtiger Sprachhandlungen und damit verbundener Sinnbildungen“156. Bernhardt/Conrad unterstreichen die Chancen, die dieses Modell für unterschiedliche Textsorten bietet: „Das überzeugende an diesem Modell besteht darin, dass abseits der komplexen Anforderungen der ,narrativen Kompetenz' auf einer mittleren Ebene Operationalisierungen von Sprachhandlungen im Geschichtsunterricht formuliert worden sind, die in entsprechende Textverfahren umgesetzt werden können, um zu fachlichen Sprachhandlungen zu gelangen. [...] Die narrative Darstellung erscheint hier zu Recht nur als eine von mehreren Möglichkeiten. “157

Die Differenzierung in die genannten vier Ebenen kann veranschaulicht werden, wenn man sie auf die im Rahmen dieser Arbeit untersuchte Vergleichsaufgabe bezieht. Die vorliegende Vergleichsaufgabe basiert auf einem Darstellungstext, der innerhalb des Ebenen-Schemas auf der Ebene der historischen Darstellung verortet werden kann. Die Voraussetzung für das Gelingen dieser Schreibaufgabe ist das Verstehen des Darstellungstextes zur Industrialisierung. ${ }^{158}$ Das Lösen und damit die Versprachlichung der Vergleichsaufgabe ist innerhalb dieses Modells insbesondere der Ebene der fachspezifischen Schulsprache zuzuordnen, „[...] die unterschiedliche erkenntnis- und diskursorganisierende Elemente umfasst und in Schulbüchern, in Aufgabenstellungen, in der Lehrersprache sowie in den selten explizierten Anforderungen der Unterrichtskommunikation zum Ausdruck kommt. "159 Sicherlich spielt im Zusammenhang der Vergleichsaufgabe auch die Schülersprache eine Rolle, eine erfolgreiche Aufgabenlösung nur auf dieser Basis ist allerdings gerade in der Sekundarstufe II keine ausreichende Basis.

\footnotetext{
154 Vgl. Günther-Arndt, Hilke (2010): Hinwendung zur Sprache in der Geschichtsdidaktik - Alte Fragen und neue Antworten. In: Saskia, Handro; Schönemann, Bernd (Hrsg.): Geschichte und Sprache (Zeitgeschichte - Zeitverständnis 21). Berlin: LiT-Verlag, S. 17-46.

155 Handro, Saskia (2013): Sprache und historisches Lernen. Dimensionen eines Schlüsselproblems des Geschichtsunterrichts. In: Becker Mrotzek, Michael u.a. (Hrsg.): Sprache im Fach. Sprachlichkeit und fachliches Lernen. Münster u.a.: Waxmann, S. 317-333. S. 320.

${ }^{156}$ Ebd. S. 321.

157 Bernhardt, Markus; Conrad, Franziska (2018): Sprachsensibler Geschichtsunterricht. Sprachliche Bildung als Aufgabe des Fachs Geschichte. In: Geschichte lernen 31 (2018). H. 182, S. 2-9. S. 5.

${ }^{158}$ Die Anforderungen, die der Text an seine Leser stellt, werden in einem der folgenden Kapitel noch ausführlicher dargestellt.

159 Handro, Saskia (2013), S. 321.
} 
Handro führt diese vier Sprachen des Geschichtsunterrichts mit den derzeit gängigen Modellen zur Verbindung von historischem Lernen und Sprache zusammen. Daraus entwickelt sie ein „Prozessmodell sprachlichen Handelns im Geschichtsunterricht “. ${ }^{160}$ Für die Einordnung der Untersuchungsgegenstände Darstellungstext und Schülerlösungen zur Vergleichsaufgabe bietet es eine gute Orientierung:

\begin{tabular}{|c|c|c|c|c|c|c|c|c|c|c|}
\hline & $\begin{array}{r}\text { Vers } \\
\text { als geger }\end{array}$ & $\begin{array}{l}\text { (He } \\
\text { ebund }\end{array}$ & $\begin{array}{l}\text { utik) } \\
\text { tungsa }\end{array}$ & Str & gien histor & ien De & & $\begin{array}{r}\mathbf{E r} \\
\text { als Anwen }\end{array}$ & $\begin{array}{l}\text { ung ( } \\
\text { fachspe } \\
\text { pte und }\end{array}$ & $\begin{array}{l}\text { tik) } \\
\text { er Theorien, } \\
\text { fe }\end{array}$ \\
\hline & & & & sche M & lode & & & & & \\
\hline & Erker & $\begin{array}{l}\text { Leuris } \\
\text { tiation }\end{array}$ & erche & $\begin{array}{r}\text { Qu } \\
\text { Erkennt } \\
\text { method }\end{array}$ & $\begin{array}{l}\text { nkritik } \\
\text { roduktionu. } \\
\text { le Reflexion }\end{array}$ & \begin{tabular}{|r} 
Inte \\
Erkenn \\
rung u
\end{tabular} & $\begin{array}{l}\text { etation } \\
\text { sstrukturie } \\
\text { nnbildung }\end{array}$ & Erkenntn & $\begin{array}{l}\text { arstellu } \\
\text { sentation } \\
\text { reflexiol }\end{array}$ & stiftung/- \\
\hline 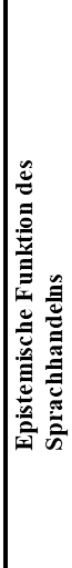 & 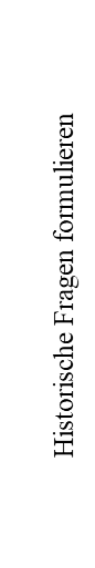 & 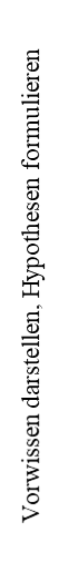 & 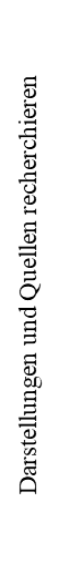 & 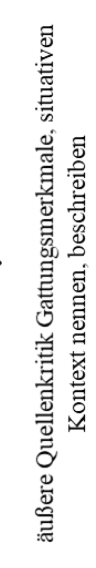 & 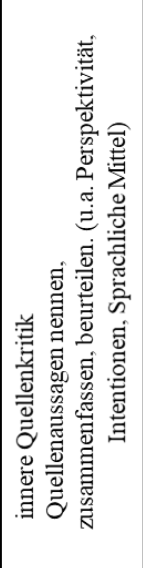 & 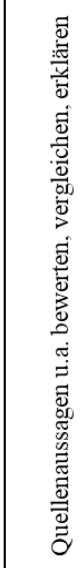 & 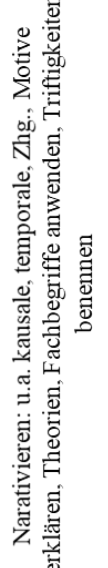 & 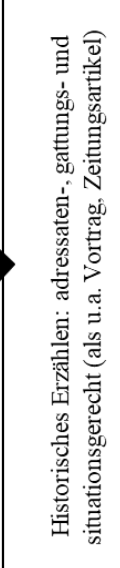 & 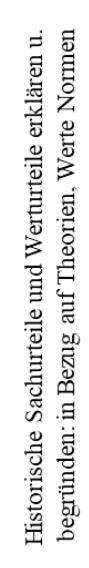 & 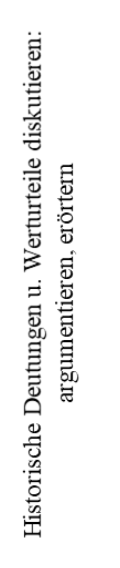 \\
\hline & & & & nntnis & & & & & nik & \\
\hline
\end{tabular}

Abbildung 5: "Prozessmodell sprachlichen Handelns im Geschichtsunterricht" (Handro, S., 2013, S. 325)

Das Modell spannt die beiden Pole Verstehen und Erklärung als Strategien historischen Denkens auf. Dabei sind auch die Teilkompetenzen des Modells „Guter Geschichtsunterricht" nach Gautschi erkennbar. Handro nennt in ihrem Modell wesentliche Wissensstrukturen, die Lernende im Geschichtsunterricht benötigen, um entweder über Heuristik und Quellenkritik zu einer Interpretation als Erkenntnisbildung zu gelangen oder eine vorgegebene Erkenntnis (Darstellung) zu hinterfragen. Sie spricht von einem Erkenntnis- bzw. von einem Kommunikationsprozess und zeigt dadurch an, dass historisches Denken nicht an sich schon da ist, sondern sich über den historischen Umgang mit fachspezifischen Textsorten wie Quellen, Darstellungen etc., letztlich also über Sprache, erst entwickelt. In ihrem Modell nennt sie dafür wesentliche Operationen, wie das Fragen formulieren, das Nennen und Beschreiben, Beurteilen, Bewerten, Erklären, Vergleichen u.a.m. Zum Teil verbergen sich hinter diesen Operationen komplexe Muster, die immer über Sprache vermittelt oder über sie generiert werden. Die Spannbreite ist groß: Ist das Formulieren von Fragen eine sprachliche Handlung, die sich auf kurze (nicht minder komplexe) sprachliche Äußerungen bezieht, handelt es sich beim Erklären und Vergleichen etwa um längere Zusammenhänge oder sogar, je nach Aufgabenstellung, um eigenständige Textsor-

160 Ebd, S. 325. 
ten. Handro nimmt in ihrem Modell wesentliche Anforderungen an den Geschichtsunterricht auf (historische Fragen formulieren, Produktion historischer Erkenntnis über analytisches Vorgehen und Interpretation bis hin zur Sinnbildung oder der Reflexion vorgegebener Sinnbildung), die Leistung dieses Modells ist dabei die Verknüpfung dieser Anforderungen mit epistemischen Formen von Sprache im Geschichtsunterricht, die sie in Bezug auf wesentliche Arbeitsweisen des Geschichtsunterrichts konkretisiert und auf ihren Erkenntnisgewinn hin verortet. Das Modell von Ehlich/Rehbein findet sich insofern in ihrem „Prozessmodell sprachlichen Handelns im Geschichtsunterricht" wieder, als dass Handro nicht von sprachlichen Kleinstformen ausgeht, sondern Handlungsmuster ${ }^{161}$ zuordnet. Übermittlung von Wissen kann nur erfolgreich sein, wenn der Zweck explizit wird. Das bedeutet eine Generierung fachlicher und sprachlicher Strukturen beim Sprecher (oder Schreiber) und Transformierung dieser Strukturen hinsichtlich des beabsichtigten Zwecks. Dabei werden schon beim Sprecher (oder Schreiber) und nicht nur beim Hörer (oder Leser) erkenntnisbildende Prozesse wirksam (im Modell nach Ehlich/Rehbein abgebildet durch „ $\left.\Pi^{\prime \prime}\right)$. Handro stellt diesen Zusammenhang durch die Zuweisung von Heuristik, Quellenkritik und Interpretation als Erkenntnisprozess dar.

Bezieht man das Modell Handros nun auf die ausgewählte Vergleichsaufgabe, kann man zunächst feststellen, dass auch zur Lösung der Vergleichsaufgabe die Lernenden auf der Grundlage des Darstellungstextest und der Fragestellung eine Hypothese formulieren müssen, da sie für einen vollständigen Vergleich im Geschichtsunterricht am Ende fundiert (oder besser: begründet) beurteilen sollen, inwiefern zwei Gegenstände vergleichbar sind. Die historische Methode wäre weniger die einer Quellenkritik, da es sich bei dem zugrundliegenden Text um einen Darstellungstext handelt. Dennoch müsste der Text mit der historischen Methode des Vergleichs erarbeitet werden. Der Begriff des Vergleichens taucht im genannten Prozessmodell unter "Interpretation: Erkenntnisstrukturierung und Sinnbildung auf", stellt aber eigentlich eine eigene Methode dar, wie nachfolgend zu zeigen ist.

Am Ende des Vergleichs steht ein Sachurteil und damit eine Interpretation des historischen Gegenstandes, der in einer Darstellung erklärt und begründet werden muss. Handro ermöglicht also durch ein solches Prozessmodell eine Phasierung des Vergleichsprozesses und damit der fachlichen und sprachlichen Handlungen. Ist also beispielsweise klar, dass am Ende eines Vergleichs ein begründetes Sachurteil steht, können im Unterricht Strukturen des Begründens und der Urteilsbildung thematisiert werden. Durch ein solches Vorgehen lassen sich sprachliche Teilhandlungen herausfiltern, die Lernende auch für andere Operationen einsetzen können. Sind Zweck und Struktur beispielsweise des Begründens bekannt, können Lernende sie auch für andere Aufgaben nutzbar machen, für die Begründungsstrukturen bedeutsam sind.

Ziel dieser Arbeit ist, das Modell für den Vergleich zu profilieren.

${ }^{161}$ Gerade aus diesem Grund ist die nachfolgende Differenzierung von Textsorte und Handlungsmuster bedeutsam. 
2.5.3 Die Hinwendung zur Sprache im Geschichtsunterricht: Ein Weg zur Charakterisierung historischer Werkzeuge

Anhand Handros Prozessmodell sprachlichen Handelns im Geschichtsunterricht konnte die Bedeutung eines funktionalen sprachlichen Ansatzes gezeigt werden, der von den Bedarfen des Fachs ausgeht und die fachlichen Ziele berücksichtigt. Dazu ist die Hinwendung zu geschichtlichen Werkzeugen, Operationen und Textprodukten bedeutsam. Ein wesentliches Textprodukt ist die historische Narration. Um diese Grundform historischen Denkens zu untersuchen, beschreitet Günther-Arndt einen textsortenorientierten Ansatz. Auch sie ordnet wie Handro Sprache hinsichtlich ihrer Funktionalität für die Geschichte ein. Der Weg über die Musterhaftigkeit von Operationen wie über die Textsorte und damit über Prozess und Produkt kann eine gute Unterstützung zur Klärung für den Geschichtsunterricht wesentlicher Operationen bieten.

Günther-Arndt skizziert den Verlauf der geschichtsdidaktischen Diskussion um das Verhältnis von Geschichte und Sprache und bezieht Stellung zur Diskussion, ob die Hinwendung zur Sprache einen, linguistic turn' bedeute. ${ }^{162}$ Der Begriff des ,linguistic turn' wird im geschichtsdidaktischen Diskurs immer wieder diskutiert und es scheint die Sorge durch, sich in einzelsprachlichen Aspekten zu verlieren, weshalb er an dieser Stelle kurz diskutiert werden muss:

Trabant füllt diesen Begriff, indem er inn als "Sprachliche Wende“ oder „Hinwendung zur Sprache“ beschreibt ${ }^{163}$, denn, so Trabant:

„Er hat nichts (oder kaum etwas) mit Linguistik zu tun, sondern bezeichnet eine Hinwendung der Geschichtswissenschaft zur ,Sprache der Geschichte'. Er ist wegen der Ambiguität von ,Geschichte' sogar eine doppelte Hinwendung: einerseits zur Sprachlichkeit der Historiographie und andererseits zur Sprachlichkeit ihrer Gegenstände. “164

Es ist sicher richtig, dass die Linguistik, wenn Sie für Geschichte und den Geschichtsunterricht nutzbar gemacht werden soll, eine besondere Rolle in Bezug auf das Fach spielen muss. Es geht eben nicht darum Wörter, Texte und andere Strukturen um ihrer selbst willen linguistisch zu charakterisieren. Dennoch kann die Linguistik einige Bedeutung für den Geschichtsunterricht haben, wenn sie hilft Strukturen zu explizieren, Lernende zu unterstützen, historisch zu erzählen oder vorliegende Narrationen kritisch zu hinterfragen etc., also Geschichte als Konstruktion wahrzunehmen. So soll die Linguistik nicht die Geschichte überlagern, vielmehr kann sie im Forschungszusammenhang, aber gerade auch für die Geschichtsdidaktik ein Werkzeug zur Auseinandersetzung mit historischen Gegenständen sein. Gerade die von Trabant festgestellte doppelte Hinwendung zur Geschichte macht diesen Zusammenhang deutlich: Geschichtsschreibung bedarf der Sprache. Dies ist keine neue Erkenntnis. Lucas weist im Zusammenhang mit dem Geschichtsunterricht bereits in den 60er Jahren darauf hin, dass Sprache bestimmte Funktionen übernimmt. Sie könne verschleiern, verharmlosen, Einstellungen suggerieren,

\footnotetext{
162 Günther-Arndt, Hilke (2010), S.24f.

${ }^{163}$ Vgl. Trabant, Jürgen (2005): Zur Einführung: Vom linguistic turn der Geschichte zum historical turn der Linguistik. In: Trabant, Jürgen (Hrsg.): Sprache der Geschichte (Schriften des Historischen Kollegs 62). München: Oldenbourg Verlag. S. VII-XXII. S. VII.

${ }^{164}$ Ebd. S. IX.
} 
sie könne jedoch nach Lucas niemals wertneutral sein. ${ }^{165}$ Dies könne jedoch nur erkennen, wer sich mit Sprache auseinandersetze. Folgt man Lucas, ist Sprache sowohl produktiv als auch rezeptiv ein Werkzeug des Historikers.

Handro spitzt diese Überlegungen in Bezug auf den Zusammenhang von Sprache und Denken für den Unterricht zu, wenn sie formuliert:

„In der Lesart der funktionalen Linguistik eröffnet erst das Wissen um die Funktion sprachlicher Mittel Schülerinnen und Schülern sprachliche Wahl- und damit auch alternative Denkmöglichkeiten, die im Unterricht durch aktives und domänenspezifisches Sprachhandeln gefördert werden müssen. ${ }^{1166}$

Handro weist darauf hin, dass ein impliziter Wissensbestand bei den Lernenden nicht ausreicht, sondern Unterricht für die Explizitmachung sprachlicher Mittel Sorge tragen muss. Solche sprachlichen Mittel wiederum strukturieren sich über historische Erkenntnismittel, wie etwa den Vergleich. Die hinter solchen Erkenntnismitteln (oder Werkzeugen) stehende Musterhaftigkeit, die sich über den Zweck generiert, muss für Lernende sichtbar werden. Deshalb gehört auch das Wissen um die Sprache im Fach zu den unterrichtlichen bzw. fachlichen Grundprinzipien.

2.5.4 Das Konzept der Alltäglichen Wissenschaftssprache für wissenschaftspropädeutisches Arbeiten im Geschichtsunterricht

Wissenschaftspropädeutik, also die Vorbereitung auf wissenschaftliches Arbeiten, bedient sich zumindest im Ansatz der Methoden und Herangehensweisen der entsprechenden fachwissenschaftlichen Disziplin. Für Geschichte als Fach, das durch seinen Konstruktionscharakter der Sprache unmittelbar bedarf, bedeutet dies, dass Strukturen einer (zumindest vor-)wissenschaftlichen Sprache der Geschichte mitgedacht und im Unterricht mitgelehrt werden müssen. Im Kontext der Wissenschaftspropädeutik bedeutet dies ein Nachdenken über wissenschaftliche Strukturen von Sprache überhaupt und für die Geschichte im Besonderen.

Dabei erfolgt der erste Zugriff auf Wissenschaftssprache häufig über die Lexik als Merkmal für Fachsprache. Steinhoff stellt im Hinblick auf die Funktion von Fachsprache fest, in der traditionellen Fachsprachenforschung werde Wissenschaftssprache „[...] oft als eine „Extremform" von Fachsprache eingeschätzt: Sie sei extrem gegenstandsgebunden, eindeutig, ökonomisch und anonym. ${ }^{167}$ Dabei sind

\footnotetext{
165 Vgl. Lucas, Friedrich (1975). S. 327.

166 Handro, Saskia (2014): Sprachlos im Geschichtsunterricht. Blogbeitrag vom 9.1.14. In: Public History Weekly. The International Blogjournal. Online-Ressource: https://public-history-weekly.degruyter.com/2-2014-1/sprachlos-im-geschichtsunterricht/ (zuletzt eingesehen am 09.03.17).

${ }^{167}$ Steinhoff, Torsten (2007): Wissenschaftliche Textkompetenz. Sprachgebrauch und Schreibentwicklung in wissenschaftlichen Texten von Studenten und Experten. Tübingen: Max Niemeyer Verlag. S. 10.
} 
diese traditionellen Einschätzungen von Fachsprache kritisch zu hinterfragen. Zumindest für die Fachsprache ${ }^{168}$ der Historiker und des Geschichtsunterrichts ist bspw. festzustellen, dass sie nicht durchgängig eindeutig ist. Im Gegenteil stellt Lucas bereits in den 1970er Jahren klar, dass die Sprache der Geschichte und auch des Geschichtsunterrichts verschleiern, verharmlosen kann. ${ }^{169}$ Steinhoff stellt der traditionellen Einschätzung von Fachsprache, zurückgehend auf den Ansatz der funktionalen Pragmatik, einen pragmatischen Zugang gegenüber, indem er nach der kommunikativen Funktion von Wissenschaftssprache fragt.

Wissenschaftssprache steht im Zusammenhang eines wissenschaftlichen Textes oder wissenschaftlichen Diskurses. ${ }^{170}$ Gerade die Überbrückung von Zeit und Raum im Sinne einer ,zerdehnten Sprechsituation ${ }^{171}$ scheint ein tragendes Element von Wissenschaft und damit von Wissenschaftssprache zu sein ${ }^{172}$, da nur so Forschungsergebnisse Verbreitung finden können. Grundsätzlich kann ein Text auch mündlich sein, Ehlich folgend, ist für ihn aber die Ruptur kennzeichnend. Der Text dient der Überbrückung eines fehlenden gemeinsamen Wahrnehmungsraums. ${ }^{173}$

Schule bereitet auf wissenschaftliches Schreiben durch Einführung schulischer Textsorten vor. Das Schreiben wird an späterer Stelle noch zu thematisieren sein. Aber auch das Lesen (vor-)wissenschaftlicher Texte spielt eine wesentliche Rolle. Dies gilt auch oder gerade für den Geschichtsunterricht, in dem Lernende mit Quellen und Darstellungstexten konfrontiert werden.

Was unterscheidet zum Beispiel einen Darstellungstext, der wesentliche Aspekte zu einem ausgewählten historischen Thema fokussiert und darstellt, von einem wissenschaftlichen Artikel eines Historikers? Schließlich kann auch der Darstellungstext von einem Historiker verfasst worden sein und bei beiden Textsorten steht die Weitergabe von Wissen im Mittelpunkt. ${ }^{174}$

Darauf gibt Graefen eine Antwort: „Ob ein Text ein wissenschaftlicher ist, ergibt sich zunächst einmal nicht aus sprachlichen Merkmalen. Es ist eine Frage der Adressierung und des Zwecks, den ein Text

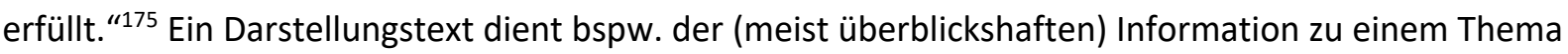

\footnotetext{
${ }^{168}$ Der im geschichtswissenschaftlichen Diskurs von manchen Historikern vertretene Auffassung, die Geschichtswissenschaft besitze keine formalisierte Fachsprache (vgl. dazu z. B. Rüth, Axel (2005): Erzählte Geschichte: narrative Strukturen in der französischen Annales-Geschichtsschreibung. Berlin, New York: de Gruyter. S. 22.), muss widersprochen werden. Denn alltagssprachliche Begriffe wie bspw. „Bürger“ werden durch die Einordnung in einen historischen Kontext gerade zu einem Element von Fachsprache, da ihnen dadurch ein festes Konzept zugeordnet wird. Vgl. dazu bspw. Bernhardt, Markus; Wickner, Mareike-Cathrine (2015). S. 288.

${ }^{169} \mathrm{Vgl}$. Lucas, Friedrich J. (1975), S. 327.

170 In Anbindung an die Funktionale Pragmatik meint Diskurs die Kopräsenz der Aktanten im gemeinsamen Handlungsraum, also eine mündliche Gesprächssituation (vgl. dazu Ehlich, Konrad (2007): Sprache und sprachliches Handeln. Bd. 1. Pragmatik und Sprachtheorie. Berlin, New York: de Gruyter. S.18f).

${ }^{171}$ Vgl. Ehlich, Konrad (2007), S. 542. Eine Darstellung dieses Ansatzes folgt in Kapitel 2.6.1.

${ }^{172}$ Vgl. dazu z. B. auch: Pohl, Torsten (2010): Das epistemische Relief wissenschaftlicher Texte - systematisch und ontogenetisch. In: Pohl, Thorsten; Steinhoff, Torsten (Hrsg.): Textformen als Lernformen. Duisburg: Gilles \& Francke. S. 105.

${ }^{173} \mathrm{Vgl}$. ebd.

${ }^{174}$ Vgl. dazu Ehlich, Konrad (1993): Deutsch als fremde Wissenschaftssprache. In: Wierlacher, A. et al. (Hrsg.) Jahrbuch Deutsch als Fremdsprache 19, 13-42. S. 24.

${ }^{175}$ Gabriele Graefen, München (2002): Probleme mit der Alltäglichen Wissenschaftssprache in Hausarbeiten ausländischer StudentInnen. In: Rehbein, Jochen; Riedel, Sabine (Hrsg.): Lernen in der zweiten Sprache. Online-Zugriff: www.wissenschaftssprache.de/Texte/GG_Probleme_mit_AWS_2002.pdf (zuletzt eingesehen am 09.02.17).
} 
und ist auf die Lerner*innengruppe hin meist inhaltlich und sprachlich angepasst. Die dort gegebenen Informationen müssen in der Regel nicht argumentiert oder sogar verteidigt werden, wie in einem wissenschaftlichen Text, der einem Fachpublikum neue Einsichten eines oder mehrerer Forscher vermitteln möchte. Ein solcher an ein Fachpublikum gerichteter Text verwendet somit auch andere sprachliche Strukturen als ein Darstellungstext in einem Geschichtslehrwerk.

Diese Beispiele zeigen, dass es nicht in erster Linie die Lexik sein kann, die einen Text zu einem wissenschaftlichen macht. Gleichwohl benötigt der Autor sprachliche Mittel, die die Schwierigkeit überbrücken, dass der Leser nicht kopräsent ist. Er benötigt durchaus dazu lexikalische Mittel, die seinen Fachinhalt vermitteln, aber mehr noch sprachliche Strukturen, die den wissenschaftlichen Artikel argumentativ absichern, Erklärungen liefern und ggf. Begründungen vorwegnehmen. So ist die dafür benötigte hohe Komplexität und Abstraktionsebene ein Aspekt, der wissenschaftliche Sprache ausmacht:

„Der Zusammenhang zwischen Wissenschaftstext, wissenschaftlichem Diskurs, Wissenschaftssprache und den Grundlagen des wissenschaftlichen Handelns als einer spezifischen Form gesellschaftlicher Praxis ist intensiver und deutlicher miteinander vermittelt, als üblicherweise angenommen wird. ${ }^{1176}$

Ehlich zeigt, dass zur Wissenschaftssprache mehr gehört als etwa die Kenntnis von lexikalisch fachsprachlichen Strukturen. Vielmehr weist er ausgehend vom Gedanken der „Ordinary language philosophy “177 die alltägliche Sprache als konstitutives Element von Wissenschaftssprache aus. Über einen Verweis auf die Sprache Schillers und Hegels führt er diesen Zusammenhang aus:

„Schiller hat mit seinen Behandlungen historischer Themen in einer Sprache, die von seiner sonstigen Sprache nicht so sehr entfernt war, die Möglichkeit nachgewiesen, daß man Wissenschaft betreiben kann, indem man eine entwickelte alltägliche Sprache dafür verwendet. Der Philosoph Hegel wiederum zeichnet sich dadurch aus, daß er in seiner Sprache für die Zwecke der Philosophie in extremem Maß die alltägliche Sprache, das Deutsche, zur Grundlage genommen hat.." ${ }^{178}$

Ehlich untersucht alltagssprachliche Elemente in wissenschaftlichen Texten, er zeigt dazu Konstruktionen, die sowohl im alltagssprachlichen wie im wissenschaftlichen Kontext Verwendung finden, wie etwa die Ausdrücke ,darstellen', ,ausgehend von' oder die Nominalphrase ,nähere Umgebung. ${ }^{179}$ Schon diese drei Beispiele zeigen, dass alltägliche Wissenschaftssprache nicht auf bestimmte grammatische Phänomene beschränkt werden kann. Solche Ausdrücke und sprachliche Formen können aber wiederum im Hinblick auf den Zweck des Textes über ihre Funktionalität erfasst werden.

Auch in der Unterrichtssprache finden sich fachsprachliche und alltagssprachliche Elemente, diese auszuformen im Hinblick auf die alltägliche Wissenschaftssprache des spezifischen Faches muss dabei Aufgabe des Fachunterrichts sein. Ohne das Wissen um solche fachspezifischen Formen und die Explizierung dieser im Unterricht ist es für Lernende, die solche Kenntnisse nicht aufbauen konnten, schwer, die dahinterstehenden Konzepte zu erkennen. Ehlich weist in diesem Zusammenhang auf die Bedeutung hin, solche Formen für den Unterricht aufzubereiten. Dabei geht er davon aus, dass für sogenannte, native speakers' keine grundsätzliche Hürde im Erwerb solcher Strukturen zu sehen ist, er setzt

\footnotetext{
${ }^{176}$ Ehlich, Konrad (1993), S. 31.

177 Vgl. Ehlich, Konrad (1999): Alltägliche Wissenschaftssprache. In: Info DaF 1/1999. S. 3-24. S. 6.

${ }^{178}$ Ehlich, Konrad (1999), S. 4.

${ }^{179}$ Vgl. ebd. S. 9.
} 
damit die alltagssprachlichen Formen für diese Lerner*innengruppe als in der Regel bekannt voraus, weist aber auf die besonderen Herausforderungen für mehrsprachige Lerner*innen ${ }^{180}$ hin. Dabei stellt er selber fest, dass er hier ein idealisiertes Bild zeichnet. Die Explikation fachsprachlicher Strukturen ist für alle Lernenden bedeutsam, da, wie die Unterrichtswirklichkeit in vielen Schulen zeigt, nicht davon ausgegangen werden kann, dass solche Strukturen bei sog. ,native speakers' vorausgesetzt werden können. Auch die PISA-Studie der OECD stützt diesen Ansatz, da sie einen Zusammenhang zwischen sozioökonomischen Status und Schülerleistung ausmacht. ${ }^{181}$

Mit Ehlich muss insgesamt festgehalten werden:

"Ohne die Basis der Alltagssprache ist im allgemeinen eine wissenschaftliche Verständigung nicht möglich. Wenn an dieser Stelle ein Lernproblem vorliegt, werden noch so spezifizierte fachterminologische Kenntnisse wirkungslos bleiben, weil die Zusammenhänge zwischen dem, was in den Fachtermini ausgedrückt ist, nicht deutlich werden können; diese Zusammenhänge werden aber gerade in der wissenschaftlichen Alltagssprache artikuliert. ${ }^{\text {"182 }}$

Ziel von Unterricht muss es also sein, Formen alltäglicher Wissenschaftssprache und damit wiederum wissenschaftspropädeutischen Arbeitens zu explizieren und damit die (sprachlichen) Werkzeuge der Lerner*innengruppe in Bezug auf das jeweilige Fach weiterzuentwickeln und diese auszubauen.

Für das Forschungsvorhaben stellt sich nun die Frage, welche Formen alltäglicher Wissenschaftssprache durch das historische Vergleichen konstruiert werden. Auch wenn es sich dabei um eine ,vorwissenschaftliche Form handelt', ist das Vergleichen im Unterricht diskursiv, weil es entsprechend den EPA in einem (begründeten) Urteil mündet. ${ }^{183}$ Es stellt damit einen Grundtyp (vor-)wissenschaftlicher Texte dar. ${ }^{184}$

Bevor das historische Vergleichen auf seine Bestandteile, sprachlich wie fachlich, überprüft werden soll, folgen weiterführende Überlegungen zum Zusammenhang von Sprache und Geschichte, an die die Frage geknüpft sein wird, was das Schreiben im Geschichtsunterricht ausmacht. Hier wird die Anbindung an die Schreibforschung gesucht. Dieser Schritt legitimiert sich einerseits daraus, dass die hier untersuchte komplexe und an einen längeren Text gebundene Vergleichsaufgabe von den Lernenden schriftlich gelöst werden sollte, und zum anderen durch die Verknüpfung mit den hier dargestellten Überlegungen zum (vor-)wissenschaftlichen Text.

\footnotetext{
${ }^{180}$ Vgl. Ehlich, Konrad (1999), S. 11.

${ }^{181}$ Vgl. hierzu die Ergebnisse der PISA-Studie von 2015, zusammengefasst z. B. in: OECD (2016): PISA 2015. Ergebnisse im Fokus. S. 4. Online verfügbar unter: https://www.oecd.org/berlin/themen/pisa-studie/PISA_2015_Zusammenfassung.pdf (zuletzt eingesehen am 04.03.2017).

182 Ehlich, Konrad (1999), S. 10.

${ }^{183} \mathrm{Vgl}$. Einheitliche Prüfungsanforderungen in der Abiturprüfung Geschichte (2005).

${ }^{184}$ Ehlich beschreibt zwei Grundstrukturen wissenschaftlicher Texte: Assertion und Eristik: „Wenn wissenschaftliche Texte also in diesem Sinne Kombinationen von einerseits Sachverhaltswiedergaben in der Familie der assertiven illokutiven Typen, andererseits ein Niederschlag von jeweils wissenschaftshistorisch und wissenschaftstheoretisch spezifisch geprägten und ausgeprägten Streitkulturen sind, dann, denke ich, erweisen sich diese Texte als außerordentlich komplexe Einheiten, Einheiten, die praktisch das schwierige Verhältnis von Diskurs und Text in der textuellen Oberfläche abbilden [...]."aus: Ehlich, Konrad (1993), S. 29. Pohl geht bei wissenschaftlichen Texten von drei zentralen Dimensionen aus: der Gegenstands-, Diskurs- und Argumentationsdimension: „Erst wenn jene drei Dimensionen aufeinandertreffen bzw. in Perspektive des Schreibenden: in einem Text aufeinander bezogen und miteinander vermittelt werden, haben wir es mit wissenschaftlichem Schreiben im engeren Sinne zu tun." aus: Pohl, Thorsten (2010), S. 101.
} 


\title{
2.6 Textsorten- und Textmusterorientierung im Geschichtsunterricht: ein
} Ansatz zur Entwicklung von Sprech- und Schreibfähigkeit im Geschichtsunterricht

Der Ort, an dem sprachliches Handeln im Geschichtsunterricht verankert sein müsste, ist der Lehrplan. Tatsächlich wird Sprache dort jedoch kaum berücksichtigt. Dies gilt sowohl für den Kernlehrplan Geschichte für die Sekundarstufe II in NRW an Gymnasien und Gesamtschulen ${ }^{185}$ als auch für den Lehrplan Politik/Gesellschaftslehre bzw. Gesellschaftslehre mit Geschichte am Berufskolleg. ${ }^{186}$

In Bezug auf die Methodenkompetenz taucht ein Hinweis im Zusammenhang mit sprachlicher Korrektheit auf. Dort heißt es: „Zur Methodenkompetenz gehört auch die Fähigkeit, historische Sachverhalte eigenständig, adressatengerecht und (fach-)sprachlich korrekt darzustellen und zu präsentieren.“187 Sprache wird hier auf ein Ausdrucksmittel verkürzt, ein Erkenntnismittel stellt es in diesem Zusammenhang nicht dar. Auch wenn Sprache nicht explizit genannt wird, erscheint der Zusammenhang, in dem dieses Zitat eingebettet ist, von Bedeutung:

\begin{abstract}
„Methodenkompetenz im Fach Geschichte bedeutet, dass die Schülerinnen und Schüler über diejenigen Verfahrensweisen verfügen, die notwendig sind, um Fragen aus der Gegenwart an die Vergangenheit zu stellen, Informationen zu beschaffen, historische Verläufe und Strukturen zu analysieren, Zusammenhänge zu erklären und darzustellen sowie mit konkurrierenden Deutungen umzugehen. Fundament dieser Arbeit ist die Beschäftigung mit Quellen und Darstellungen. Schwerpunkte der Methodenkompetenz sind vor diesem Hintergrund der fachgerechte Umgang mit der Interpretation von Quellen verschiedener Gattungen und der Analyse von und der kritischen Auseinandersetzung mit verschiedenen Formen historischer Darstellung sowie die Entwicklung eigenständiger historischer Argumentationen. ${ }^{1188}$
\end{abstract}

Diese Beschreibung von Methodenkompetenz wird durch das zuerst genannte Zitat abgeschlossen. Dabei ist gerade der längere erste Teil für die Betrachtung sprachlichen Handelns weitreichender als der abschließende direkte Bezug auf Sprache. Der hier dargestellte Begriff von Methodenkompetenz ist allerdings nicht ausdifferenziert. Wenn ganz allgemein von Verfahrensweisen die Rede ist, bleibt unklar, ob Methode als Erkenntnismethode, Unterrichtsmethode etc. verwendet wird. Der Begriff erschließt sich im Zitat nur über geschichtliche Operationen, wie das Analysieren, Erklären, Darstellen u.a.m. Dabei stellen die Ergebnisse solcher Operationen für den Geschichtsunterricht gleichzeitig wichtige Produkte dar. Insbesondere Interpretation und Analyse sowie die Argumentation werden im Lehrplan als Schwerpunkte der Methodenkompetenz benannt und z. B. in Abituraufgaben von den Lernen-

\footnotetext{
${ }^{185}$ Ministerium für Schule und Weiterbildung des Landes Nordrhein-Westfalen (Hrsg.) (2014): Kernlehrplan für die Sekundarstufe II Gymnasium/Gesamtschule in Nordrhein-Westfalen. Geschichte. Online verfügbar unter: http://www.standardsicherung.schulministerium.nrw.de/lehrplaene/upload/klp_SII/ge/KLP_GOSt_Geschichte.pdf (zuletzt eingesehen am 08.06.17).

${ }^{186}$ Ministerium für Schule und Weiterbildung des Landes Nordrhein-Westfalen (Hrsg.) (2007): Lehrplan für das Berufskolleg in Nordrhein-Westfalen. Politik/Gesellschaftslehre bzw. Gesellschaftslehre mit Geschichte. Bildungsgänge der Fachoberschule (Anlage C9 bis C11 und D29). Online verfügbar unter: https://www.berufsbildung.nrw.de/cms/upload/_lehrplaene/d/politikgesellschaftslehre-fos_40012.pdf (zuletzt eingesehen am 08.06.17).

${ }^{187}$ Ministerium für Schule und Weiterbildung des Landes Nordrhein-Westfalen (Hrsg.) (2014), S.16.

188 Ebd.
} 
den gefordert. Es sind also immer zugleich Prozess und Produkt gemeint. Hinter der Produktorientierung verbirgt sich wiederum der Textsortenbegriff. Beese/Roll rekurrieren für die Einordnung des fachlichen Textsortenbegriff auf den Ansatz des generischen Lernens ${ }^{189}$ und kennzeichnen inn durch die Bezeichnung als „didaktischen Hebel“ ${ }^{“ 190}$ als hochrelevant für den Unterricht:

„Textsorten, - wie Textaufgaben in der Mathematik, Versuchsprotokolle in Physik, Steckbriefe in Biologie, Schaubildbeschreibungen in den Gesellschaftswissenschaften, Quellen in Geschichte, sind Ausdruck fachlicher Systematik und spezifischer Denk- und Erkenntnisformen und sie begründen ein begrenztes Repertoire an bildungs- und fachsprachlichen Mitteln. ${ }^{\text {} 191}$

Zu den Textsorten im Geschichtsunterricht können an dieser Stelle auch die Darstellungen ergänzt werden. Bedeutsam an dieser Einordnung des Textsortenbegriffs ist insbesondere die Bestimmung von Textsorten als Denk- und Erkenntnisform. Auch Süssmann unterstreicht aus fachlich-historischer Sicht die Hinwendung zu einer Textsortenorientierung für die Geschichte über eine Differenzierung des Erzählbegriffs und typischer Textsorten des historischen Erzählens. ${ }^{192}$ Prozess und abschließendes Produkt gehören damit untrennbar zusammen. Gerade deshalb muss der Methodenbegriff im Kernlehrplan auch in Bezug auf die Funktion von Sprache ausdifferenziert werden und bestärkt die Hinwendung zu Musterorientierung und Textsorte. Der Hinweis im Lehrplan auf eine eigenständige, adressatengerechte und (fach-)sprachlich korrekte Darstellung bzw. Präsentation greift zu kurz.

Im Folgenden soll ein muster- und textsortenorientierter Ansatz in Bezug auf seine Bedeutung für das Vergleichen überprüft werden.

\subsubsection{Sprachliches Handeln mit Texten}

Auch wenn ein Text sowohl schriftlich als auch mündlich ausgestaltet sein kann, bezieht er sich im schulischen Kontext sehr häufig auf schriftliche Texte. Für den Rahmen dieser Arbeit wird nachfolgend mit einem schriftlichen Textbegriff weitergearbeitet, weil die ausgewählte Vergleichsaufgabe schriftlich gelöst werden sollte.

${ }^{189}$ Vgl. dazu z. B. Hallet, Wolfgang: Hallet, Wolfgang (2013): Generisches Lernen im Fach. In: Becker-Mrotzek, Michael et al. (Hrsg.) (2013): Sprache im Fach. Münster/New York: Waxmann, 59-76.

190 Vgl. Beese, Melanie; Roll, Heike (2015). Textsorten im Fach - zur Förderung von Literalität im Sachfach in Schule und Lehrerbildung. In Benholz, Claudia; Frank, Magnus; Gürsoy, Erkan (Hrsg.). (2015). Deutsch als Zweitsprache in allen Fächern. Konzepte für Lehrerbildung und Unterricht. Beiträge zu Sprachbildung und Mehrsprachigkeit aus dem Modellprojekt ProDaZ. Stuttgart: Fillibach bei Klett. S. 51-72. S. 51.

191 Ebd. S. $51 \mathrm{f}$.

${ }^{192}$ Süssmann, Johannes (2000): Für eine Textsortenlehre der Geschichtsliteratur. In: ders.: Geschichtsschreibung oder Roman? Zur Konstitutionslogik von Geschichtserzählungen zwischen Schiller und Ranke (1780-1824). Stuttgart. S. 11-32.

Dabei stellt Süssmann fest, „„[...] daß das Erzählen weniger das starre Gitter ist, als das es White betrachtet, denn ein flexibles, höchst differenziert einsetzbares Instrument, das ganz verschiedenen Darstellungsinteressen dienen kann [...]." (ebd. S. 31). Hieran knüpft der Textmusterbegriff an, dabei kann das Textmuster verkürzt dargestellt als Teil einer Textsorte gefasst werden. Eine ausführlichere Betrachtung der verschiedenen Begriffe folgt in Kapitel 2.6. 
Im Gegensatz zu mündlichen Texten sind für schriftliche Texte besondere Bedingungen zu berücksichtigen, die über das Modell Ehlichs, der in Abgrenzung zum mündlichen Diskurs den (schriftlichen) Text als „zerdehnte Sprechsituation“193 einordnet, gezeigt werden können: Durch den Begriff der zerdehnten Sprechsituation beschreibt Ehlich die besondere Situation der Kommunikation über einen schriftlichen Text: „Wir haben es nämlich sozusagen mit zwei halben, mit zwei unvollständigen Sprechsituationen zu tun - an der Stelle einer einheitlichen, über die sinnliche Wahrnehmbarkeit zugänglichen. “194 Ehlich spricht in diesem Zusammenhang vom einsamen Sprecher, dem Schreiber, und vom einsamen Hörer, dem Leser ${ }^{195}$, die sich in einer indirekten Kommunikationssituation befinden.

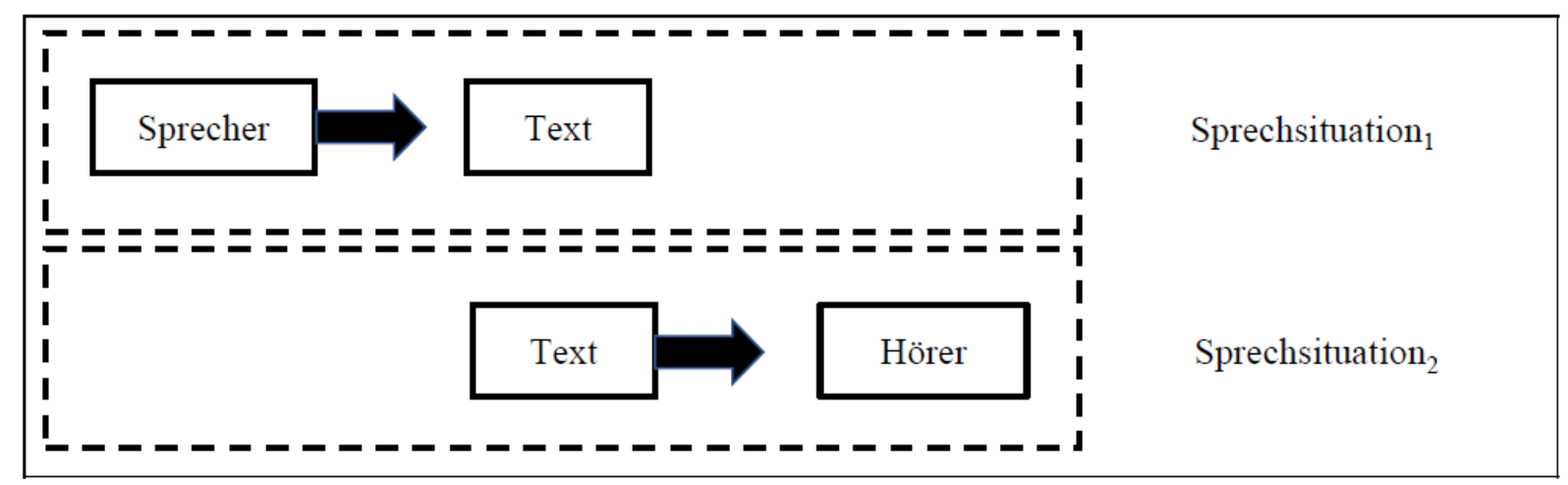

Abbildung 6: Zerdehnte Sprechsituation (Ehlich, K., 2007. S. 542.)

Für den Schreibenden bedeutet dieser Zusammenhang, dass er aufgrund der fehlenden direkten Reaktion des Lesers nicht kontrollieren kann, wie dieser das Geschriebene versteht. Er muss deshalb vorwegdenken, welche Wissenslücken zu füllen sind oder an welchen Textstellen ein Einverständnismangel auftreten könnte. Über den Rückgriff auf bestehende (oder sich entwickelnde) Handlungsmuster, über die möglichst Schreiber und Leser Kenntnis haben, kann diese indirekte Kommunikationssituation überbrückt werden.

Der Zugriff über einen muster- und textsortenorientierten Ansatz legt die Betrachtung von solchen Handlungsmustern zugrunde. Dieser kann sich auf Textmuster oder Textsorten beziehen.

Der Begriff des Handlungsmusters wird in dem linguistischen Ansatz der Funktionalen Pragmatik (vgl. z. B. Ehlich/Rehbein, 1979) bestimmt. Bei Handlungsmustern handelt es sich um gesellschaftliche standardisierte Formen des Handelns, „[...] die im konkreten Handeln aktualisiert und realisiert werden. Die Handelnden verwirklichen in ihrem Handeln ihre Zwecke. " 196

Bachmann/Becker-Mrotzek konkretisieren den Begriff des Musters im Hinblick auf den Zusammenhang von sprachlichen Mitteln und kommunikativer Absicht:

\footnotetext{
193 Ehlich, Konrad (2007), S. 542.

194 Ehlich, Konrad (2007), S. 541.

195 Vgl. ebd.

${ }^{196}$ Ehlich, Konrad; Rehbein, Jochen (1979) Sprachliche Handlungsmuster. In: Soeffner, Hans-Georg (Hrsg.): Interpretative Verfahren in den Sozial- und Textwissenschaften. Stuttgart: Metzler, 243-274. S. 250.
} 
„Die Muster beinhalten für unterschiedliche Handlungszwecke je spezifische Verhältnisse von prototypischen Inhalten, sprachlichen Mitteln und Strukturen, die den Sprachteilhabern zur Realisierung ihrer individuellen Ziele zur Verfügung stehen. Die sprachlichen Mittel und Strukturen stehen dabei nicht in einem beliebigen Verhältnis, sondern leiten sich systematisch aus den (kommunikativen) Zwecken her." ${ }^{197}$

Solche Handlungsmuster bestehen aus Teilhandlungen, über die der Zweck des Handlungsmusters realisiert werden kann. Für das Vergleichen im Geschichtsunterricht konnte schon mit Hilfe der Bestimmung durch die EPA festgestellt werden, dass es mindestens das Gegenüberstellen und das Beurteilen beinhaltet. Diese Teilhandlungen werden in der Folge noch genauer ausdifferenziert. Ebenso ist das Vergleichen zweckgebunden. Über den Zweck geben die EPA ebenfalls Auskunft, nämlich über die Herstellung von Ähnlichkeiten, Gegensätzen und Unterschieden ein Sachurteil zu bilden.

Da das historische Vergleichen als geschichtswissenschaftliches Werkzeug eingeordnet wird, stellt es eine Handlung dar, die dazu führt, neues Wissen zu generieren. ${ }^{198}$ Dies geschieht über die Realisierung der Teilhandlungen, die den Handelnden als Musterwissen zur Verfügung stehen. Diese Teilhandlungen sind zwar als Muster ausgeprägt, aber dennoch durchaus variabel einsetzbar, um letztlich das Muster des historischen Vergleichs erfolgreich zu generieren. So müssen beim historischen Vergleichen bspw. die verglichenen Elemente je nach Kontext mehr oder weniger stark erklärt werden, damit der Hörer oder Leser den Vergleich nachvollziehen kann. Das Erklären kann damit als weitere Teilhandlung eine stärkere oder schwächere Rolle spielen, es ist also variabel einsetzbar. Dabei kann das Handlungsmuster historisches Vergleichen (wie im Beispiel das Erklären) auch zu einer Teilhandlung innerhalb einer dem Vergleichen übergeordneten Operation werden. Das Muster würde sich also innerhalb eines übergeordneten Musters realisieren. Dies müssen Lernende wissen. Wenn ihnen deutlich ist, welche Zwecke verschiedene Handlungsmuster verfolgen, können Sie dieses Wissen zur Lösung einer Aufgabe im Geschichtsunterricht nutzbar machen. Ein solches Vorgehen würde echte Kompetenzorientierung bedeuten.

Handlungsmuster lassen sich wiederum als Strukturelemente in Textsorten finden: Sie strukturieren diese.

Der Begriff der Textsorte ist grundlegend für Schule und Unterricht. Für diese Arbeit ist der Textsortenbegriff als Ausgangspunkt weiterer Überlegungen insofern bedeutsam, weil die Textsorte nach Brinker über ihre Textfunktion bestimmt werden kann: Brinker weist den Textsorten eine „fundamentale Bedeutung für die kommunikative Praxis“ ${ }^{\prime 199}$ zu und stellt als zentrales Analyse- und Unterscheidungskriterium die jeweilige kommunikative Funktion in den Mittelpunkt der Betrachtung: „Die Textfunktion fungiert gewissermaßen als Basiskriterium sowohl für die Beschreibung der Textqualität als

\footnotetext{
${ }^{197}$ Bachmann, Thomas; Becker-Mrotzek, Michael (2017): Schreibkompetenz und Textproduktion modellieren. In: Becker-Mrotzek, Michael; Grabowski, Joachim; Steinhoff, Torsten (Hrsg.): Forschungshandbuch empirische Schreibdidaktik. Münster: Waxmann. S.25-53. S. 29.

${ }^{198} \mathrm{vgl}$. dazu Rehbein, Jochen in Anlehnung an Ehlich: Rehbein, Jochen (1977): Komplexes Handeln. Elemente zur Handlungstheorie der Sprache. Stuttgart: Metzler. S. 111.

${ }^{199}$ Brinker, Klaus (2010): Linguistische Textanalyse. Eine Einführung in Grundbegriffe und Methoden. 7. Aufl. Berlin: Erich Schmidt Verlag. S. 135.
} 
auch für die Differenzierung von Textsorten. ${ }^{200}$ Der Ansatz über die Funktion fügt sich damit in das gewählten Sprach- und Wissensmodell nach Ehlich/Rehbein ein.

Während in der Textlinguistik der Begriff der Textsorte gängig ist, wird er in der Funktionalen Pragmatik kritisch gesehen und durch den Begriff Textart ersetzt. Graefen hält den Begriff Textart für besser geeignet, ,[[...] denn ,Art' ist ein allgemeiner Ausdruck zur Bezeichnung der Klassenzugehörigkeit, wobei es auf die Eigenschaft der klassifizierten Sache selbst ankommt. “201 Der Textsortenbegriff ist aber (im Gegensatz zur Textart) für Schule und Unterricht und damit auch für den Geschichtsunterricht gängig, so dass er aufgrund seiner leichteren Anschlussfähigkeit hier weiter Verwendung findet. Steinhoff/Pohl sprechen in Anlehnung an den semiotisch-funktionalen Ansatz Feilkes ${ }^{202}$ von einer Textform ${ }^{203}$ und heben dabei den Erwerbsprozess von Lernenden heraus. Da es in dieser Arbeit zunächst um die Ausformung des Vergleichs im Geschichtsunterricht und die Klärung des dahinterstehenden Musters geht, wird die Klärung der für die Didaktisierung wichtigen Erwerbsprozesse ausgeklammert.

Fix bestimmt schulisches Textsortenwissen im Hinblick auf die oft sehr starke Normung wie folgt: „Textsortenwissen könnte man zunächst als deklaratives Wissen (»knowing that«) klassifizieren, weil es sich um einen Bestandteil des Welt- oder Sachwissens handelt. " ${ }^{204}$ Für den Geschichtsunterricht könnte hinzugefügt werden, dass sich in den Textsorten auch eine fachliche Systematik wiederfindet, die sich aus dem Zweck (und damit aus den hinter den Textsorten stehenden Handlungsmustern) ableitet und die bestimmte Textsorten ausmacht.

Fachspezifisches Textsortenwissen kann wiederum als eine Art Strategiewissen den Lernenden vermittelt werden, sofern die Systematik bereits ausgearbeitet wurde. Daran knüpfen sich im Weiteren die sprachlichen Werkzeuge, wie bspw. Textverknüpfungsmittel, Verweisformen u.a.m. Verstehen Lernende bspw. die Funktion sprachlicher Mittel zur Gegenüberstellung (...als..., ...wie... etc.), können sie diese Mittel im Sinne eines Strategiewissens für den Vergleich einsetzen. Ähnliches gilt für kausale Strukturen (und entsprechende Satzverknüpfungsmittel), die z. B. für Begründungen notwendig sind. Insofern stellt die Textsortenorientierung eine strukturierte Unterstützungsmöglichkeit für den Fachunterricht dar.

Fix stellt allerdings auch fest, dass hinter den normierten Textsorten Muster als Werkzeuge oder Grundbausteine kommunikativen Handelns stehen. ${ }^{205}$ Am Beispiel von Erzählung und Bericht führt er diese Differenzierung aus:

\footnotetext{
${ }^{200}$ Brinker, Klaus (1983): Textfunktionen. Ansätze zu ihrer Beschreibung. In: Zeitschrift für germanistische Linguistik. Bd. 11 H. 2, S. 127-148. S. 128. Online verfügbar unter: https://www.degruyter.com/downloadpdf/j/zfgl.1983.11.issue-2/zfgl.1983.11.2.127/zfgl.1983.11.2.127.pdf. Zuletzt eingesehen am 13.03.2018, ${ }^{201}$ Graefen, Gabriele (1997), S. 41.

202 Vgl. z. B. Feilke, Helmuth (2011): Was sind Textroutinen? In: Feilke, Helmuth, Lehnen, Katrin (Hrsg.): Schreibund Textroutinen. Frankfurt a. M. Lang. S. 1-31.

203 vgl. Pohl, Thorsten; Steinhoff, Torsten (2010): Textformen als Lernformen. In: Pohl, Thorsten; Steinhoff, Torsten (Hrsg.): Textformen als Lernformen. Duisburg: Gilles \& Francke. S. 5-26.

${ }^{204}$ Fix, Martin (2008), S. 92.

${ }^{205}$ Vgl. ebd.
} 
„Hinter den Aufsatzarten »die Erzählung» und »der Bericht» stehen somit Muster des Erzählens und Berichtens [...]. Das Wissen darüber ist als abstraktes Rahmenschema gespeichert und enthält prototypische Elemente über z. B. das erzählende, beschreibende und erörternde Muster [...]. “206 Ähnliches konnte bereits für den Vergleich festgestellt werden: Als Werkzeug des Geschichtsunterrichts steht hinter ihm ein Muster (in Anlehnung an Ehlich/Rehbein wird im Rahmen dieser Arbeit damit das Handlungsmusters gemeint bzw. der Begriff des Handlungsmusters genutzt). ${ }^{207}$ Dieses Muster kann sowohl in einer Textsorte ,Historischer Vergleich' ausgestaltet als auch als Muster in anderen Textsorten (z. B. im Rahmen einer Quellenanalyse) wiederzufinden sein.

Mit Riekenberg wird für den Vergleich im Geschichtsunterricht nachfolgend festzustellen sein, dass es keine feste Form des Vergleichs im Geschichtsunterricht gibt. Gerade deshalb lohnt es sich, den Zugriff über die Textsorte und den Begriff des Handlungsmusters als zusammengehörig zu denken, so ist das Handlungsmuster Teil der Textsorte.

Diese Überlegung verdeutlicht die Notwendigkeit eines Musterwissens zur Lösung einer Vergleichsaufgabe. Wird durch die Aufgabenstellung der Prozess („Vergleiche/Vergleichen Sie“) angeregt, für den ein Musterwissen benötigt wird, stellt der am Ende stehende Vergleich ein Textprodukt dar.

Nach Handro lassen sich über einen solchen Ansatz nicht nur die Muster hinter sprachlichen Handlungen ableiten, sondern es können darüber auch fachspezifische Herangehensweisen fundiert werden:

„Aus der sprachlichen Verfasstheit des Untersuchungsgegenstands lassen sich fachspezifische Mediengattungen und Textgenres ableiten; mit ihr können Sprachhandlungen, die den historischen Erkenntnisprozess begleiten, systematisiert werden und über die Sprachlichkeit der historischen Überlieferung können methodische und kommunikative Praktiken des Geschichtsunterrichts begründet werden [...].“208

Diese Herangehensweise über die Hinwendung zur Sprache macht das Fach Geschichte im Sinne der Kompetenzorientierung im besten Sinne zu einem Denkfach.

\subsubsection{Textsorte, Gattung und Genre: Versuch einer Abgrenzung}

War bisher von Textsorte und Handlungsmuster die Rede, bringt Handro für die Fachdisziplin Geschichte zwei weitere Begriffe ins Spiel: Gattung und Genre. Sie bezieht die Gattung auf das Medium, das Genre auf den Text, klärt diese Begriffe allerdings nicht. Genre und Gattung werden im Folgenden als der Textsorte übergeordnete Kategorie verstanden. Den Begriffen liegt das Verständnis zugrunde, Genres in Bezug auf ihren Inhalt aufzufassen, die Gattung (Handro spricht von Mediengattung) dagegen in Bezug auf das Ausdrucksmedium in seinem kommunikativen Zusammenhang. Demzufolge sind Quellen und Darstellungen als unterschiedliche Genres zu verstehen, die wiederum in verschiedene Gattungen einteilbar sind (für das Genre Quelle könnte bspw. die literarische Quelle und für das Genre Darstellungstext die Gattung Darstellungstext im Schulbuch folgen). Folglich ist das Genre im Sinne

\footnotetext{
206 Ebd.

207 Vgl. z. B. Ehlich, Konrad; Rehbein, Jochen (1979) Sprachliche Handlungsmuster. In: Soeffner, Hans-Georg (Hrsg.): Interpretative Verfahren in den Sozial- und Textwissenschaften. Stuttgart: Metzler, 243-274. 208 Handro, Saskia (2014), S. 321.
} 
einer Systematisierung als Oberkategorie zu verstehen, dem die Gattung folgt. Hierunter schließt sich die Textsorte an.

"Genres oder auch kommunikative Gattungen bzw. Diskursfunktionen sind diejenigen Einheiten mit sprachlich-symbolischen und textuellen Eigenschaften, in denen das Geschichtswissen erzeugt und manifest wird. [...] Sie sind anerkannte sprachliche Handlungsmuster für die Bearbeitung spezifischer, wiederkehrender kommunikativer Zwecke. “209

Hartung verbindet die Begriffe Genre und Gattung aus geschichtsdidaktischer Sicht mit dem der Textsorte übergeordneten Handlungsmuster. Sicherlich ist diese Zuschreibung diskutierbar, ermöglicht aber gerade Lernenden, über eine solche Kategorisierung den Zweck einer Textsorte zu erschließen:

„Für die Herstellung und für das Verstehen von Texten sind wir tatsächlich auf Muster angewiesen. Anders nämlich, als wenn es um die Rechtschreibung oder den Satzbau geht, um nur zwei Beispiele zu nennen, können wir beim Textproduzieren und -rezipieren mit Regeln wenig anfangen. Wir haben die sehr komplexen Text- und Stilvorgaben nicht als abzuarbeitende Regelinventare, die notwendigerweise unüberschaubar wären, verinnerlicht, sondern als Muster. Muster sind im Sinne der kognitiven Psychologie Möglichkeitsfelder, in denen es sowohl einige überindividuelle Handlungsorientierungen gibt als auch Ermessensspielräume. “210

Fix unterstreicht die Kenntnis von bestimmten wiederkehrenden und damit regulativen Strukturen, die es zu kennen gilt, soll ein bestimmter Zweck in einem Text erreicht werden. Gleichzeitig grenzt sie dieses Musterwissen von einer festen Formvorgabe für die eigene Textproduktion ab. Diese Forderung ist gerade für unterrichtliche Formen der Textproduktion hochbedeutsam: Es geht nicht darum, Lernenden feste Textstrukturen vorzugeben, die sie reproduzieren sollen, vielmehr muss im Sinne einer Reflexion auf der Metaebene Wissen über den Zweck angeregt werden, damit nachfolgend Strukturen reflektiert werden können, die das Erreichen der Schreibabsicht unterstützen, also das Musterwissen. ${ }^{211}$ Dieser bei Fix fachübergreifende Ansatz trifft auch für die Geschichte zu:

"Genres sind trotz ihres hohen Grades der Konventionalisierung keine für alle Zeiten feststehenden Gebilde oder fixe diskursive Muster, die - einmal erlernt - bloß noch angewendet werden müssen. [...] Historiker/innen, Geschichtslehrkräfte und Schüler/innen greifen in unterschiedlichen diskursiv-interaktionalen Situationen auf verschiedene ihnen bekannte Formen zurück. Diese konfigurieren sie entsprechend ihren jeweiligen kommunikativen Bedürfnissen zu einer neuen Konkretisierung, womit sie auf lange Sicht zur Erweiterung des kulturell verfügbaren Genre-Repertoires beitragen. ${ }^{\prime 212}$

\footnotetext{
209 Hartung, Olaf (2016): Generische Lernaufgaben im Geschichtsunterricht. In: Handro, Saskia; Schönemann, Bernd (Hrsg.): Aus der Geschichte lernen? Weiße Flecken der Kompetenzdebatte. Münster, Hamburg, Berlin, London: LIT. S. 187-198. S.189f.

${ }^{210} \mathrm{Fix}$, Ulla (2008): Texte und Textsorten - sprachliche, kommunikative und kulturelle Phänomene. Berlin: Frank \& Timme. S. 65.

${ }^{211}$ Die im schulischen Kontext häufig geführte Diskussion um die Erörterung kann diesen Zusammenhang erhellen: Bei der Erörterung knüpfen sich Fragen von Lernenden häufig an den, richtigen Aufbau': Soll zunächst die Argumentation des zugrundeliegenden Verfassertextes wiedergegeben werden oder darf der Text sofort kommentiert werden? Diese Frage stellt sich nur bei einer zu engen Textmustervorgabe. Thematisiert man hingegen den Zweck und nützliche Musterstrukturen, wird die Frage obsolet.

${ }^{212}$ Hartung, Olaf (2016), S. 192.
} 
Hartung zeigt die Anschlussfähigkeit des Begriffs Handlungsmuster für die Konzepte Genre und Gattung in Bezug auf die Fachdisziplin bzw. das Unterrichtsfach Geschichte. Das Handlungsmuster ist wiederum ausformbar über die Textsorte.

Für die zu untersuchende Vergleichsaufgabe liegt dabei ein komplexes Wissen über Textsorten zugrunde, weil es sowohl auf Textverstehen des zur Aufgabe gehörenden Darstellungstext im Schulbuch (im Sinne einer Textsorte „Erklärung“) als auch das Produzieren des in der Aufgabe angelegten Vergleichs ausgerichtet ist. Dies verlangt von den Lernenden Musterwissen zum Lesen und zum Schreiben im Hinblick auf das Fach Geschichte.

Zwar ist der Begriff Text nicht an Schriftlichkeit gebunden, dennoch legt er diese im unterrichtlichen Kontext nahe, erst recht, wenn es sich um komplexe Aufgaben handelt, die einer Textproduktion zugrunde liegen. Ausgehend von der Annahme, dass das wissenschaftspropädeutische Arbeiten im Geschichtsunterricht der Sekundarstufe II komplexe Textproduktion und damit Schreiben bedingt, soll nachfolgend das Schreiben im Geschichtsunterricht fokussiert werden.

\subsection{Schreiben im Geschichtsunterricht: Überlegungen zur Bedeutsamkeit für den Lernprozess}

Der in Kapitel 2.6 dargelegte Textsortenbegriff lässt sich übertragen auf bzw. konkretisieren anhand der ausgewählten Vergleichsaufgabe. An diese Überlegungen kann die Annahme angeschlossen werden, dass Aufgaben in Geschichtslehrwerken sehr häufig Texte (damit sind kontinuierliche wie diskontinuierliche Texte gemeint) zugrunde liegen. Die nachfolgende Grafik soll das Anforderungsprofil einer Schreibaufgabe umreißen:

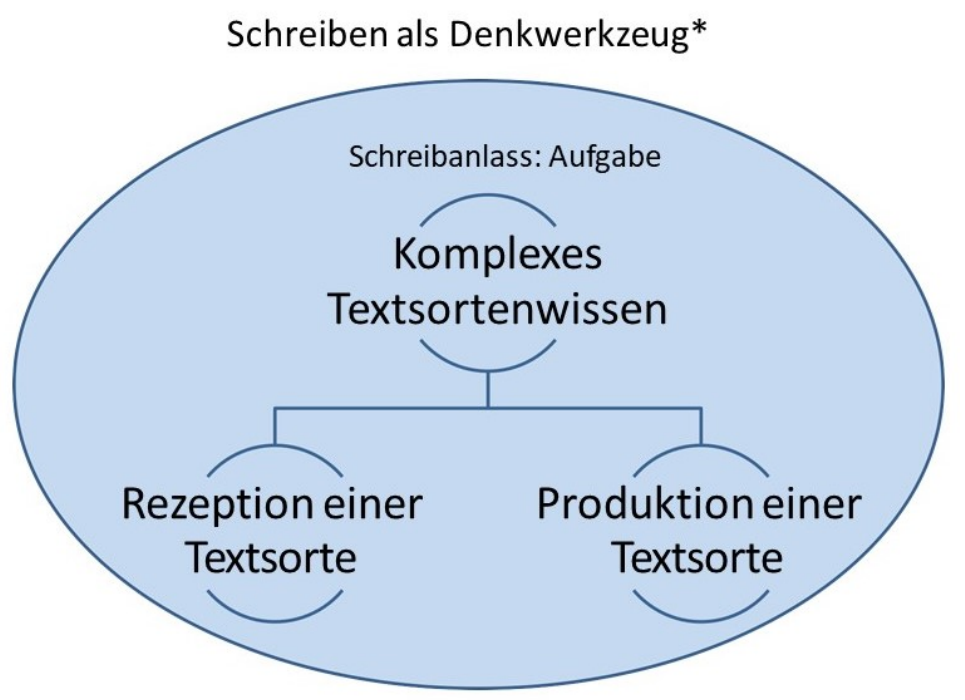

(*vgl. Thürmann, 2012)

Abbildung 7: Anforderungen durch eine Schreibaufgabe (eigene Darstellung) 
Die Grafik zeigt, dass die zu rezipierende Textsorte von der zu produzierenden zu trennen ist. Für das Leseverstehen ist es dabei bedeutsam, die Textsorte, auf deren Grundlage eine Aufgabe gelöst werden soll, zu kennen. Die zu produzierende kann sich aufgrund des Operators wiederum völlig von der rezipierten Textsorte unterscheiden. Die Komplexität von Aufgaben bestimmt wiederum die Produktionsart (konzeptionell und/oder medial schriftlich). Ausgehend von der Annahme, dass Aufgaben in der Sekundarstufe II gegenüber solchen der Sekundarstufe I an Komplexität zunehmen, lässt sich folgern, dass der Anteil der konzeptionell und medial schriftlich zu lösenden Aufgaben zunehmen müsste. Für das Fach Geschichte muss allerdings festgestellt werden, dass nur in den wenigsten Aufgaben vermerkt ist, ob diese mündlich oder schriftlich zu lösen sind. Schreiben im Fachunterricht scheint also stark durch die Schwerpunktsetzungen der Lehrkräfte bestimmt zu sein. Dabei muss spätestens in Klausuren geschrieben werden. Zur Beantwortung von Klausuraufgaben wird von den Lernenden Textsortenwissen gefordert. Wird es im Unterricht nicht oder kaum thematisiert, bedeutet dies, dass es als bekannt vorausgesetzt wird (z. B. die Textsorte Quellenanalyse im Geschichtsunterricht). Abgesehen von komplexen Schreibanlässen stellt Schmölzer-Eibinger fest, dass es beim Schreiben im Fachunterricht sehr häufig darum geht, Informationen festzuhalten, epistemisches Schreiben dagegen kaum vorkommt ${ }^{213}$ : „Dort, wo dies dennoch geschieht, gehen die SchülerInnen meist sowohl mit Sprache als auch mit Inhalten genauer, reflektierter und kritischer um - eine intensive Spracharbeit und ein besseres inhaltliches Verstehen sind die Folge. ${ }^{\text {"214 }}$

Sie beantwortet damit die Frage, warum denn überhaupt im Fachunterricht geschrieben werden soll, wenn es sich doch um eine sehr zeitintensive Tätigkeit handelt, die zudem bei den Lernenden in der Regel sehr unbeliebt ist.

Die Bedeutung des Schreibens im Hinblick auf die Schule und die verschiedenen Fächer wird in der Schreibdidaktik immer wieder thematisiert. Zudem finden sich verschiedene Untersuchungen zum wissenschaftlichen Schreiben von Studierenden, z. T. auch mit Blick auf den Übergang von Schule und Studium. ${ }^{215}$ Im wissenschaftlichen Diskurs zur Schreibdidaktik scheint dabei Einigkeit über die Bedeutung des Schreibens zu bestehen.

\footnotetext{
${ }^{213}$ Schmölzer-Eibinger, Sabine: Sprache als Medium des Lernens im Fach. In: Michael Becker Mrotzek u.a. (Hrsg.): Sprache im Fach. Sprachlichkeit und fachliches Lernen. Münster u.a.: Waxmann, S. 25-40. S. 32.

${ }^{214}$ Ebd.

${ }^{215} \mathrm{Vgl}$. z. B. Steinhoff, Thorsten (2007): Wissenschaftliche Textkompetenz. Sprachgebrauch und Schreibentwicklung in wissenschaftlichen Texten von Studenten und Experten. Tübingen: Niemeyer.

oder Scholten-Akoun, Dirk; Kuhnen, Angela; Mashkovskaya, Anna (2012): Sprachkompetenzen Studierender. Design und erste Ergebnisse einer empirischen Studie. In: Feilke, Helmuth; Köster, Juliane; Steinmetz, Michael (Hrsg.) (2012): Textkompetenzen in der Sekundarstufe II. Stuttgart: Fillibach bei Klett, S. 207-228. u.a.m.
} 


\subsubsection{Besonderheiten des Schreibens im Fach}

In Anlehnung an Ehlichs Modell der „zerdehnten Sprechsituation ${ }^{216 “ ~ k o n n t e ~ z u v o r ~ d a s ~ S c h r e i b e n ~ a l s ~}$ Denkwerkzeug konzeptualisiert werden.

Im Hinblick auf den Schreibprozess machen Pohl/Steinhoff zudem die folgenden Faktoren als bedeutsame Faktoren für das Lernen durch Schreiben aus: wegen ihres Planungspotenzials die Langsamkeit, wegen des Überarbeitungspotenzials die Vorläufigkeit und wegen des Reflexionspotenzials die Objektivation (damit meinen sie die Tatsache, dass es sich beim Schreiben um nicht-flüchtige Zeichen handelt, die eine vertiefte inhaltliche Auseinandersetzung beim Schreiben ermöglicht). ${ }^{217}$

Nimmt man stärker das Schreibprodukt in den Blick, lässt sich insbesondere über den Zusammenhang von Rezeption zu Produktion die Bedeutung des Schreibens als Denkwerkzeug für den Geschichtsunterricht darstellen, da über die Aufgabenstellung eine Überführung von Inhalten einer bestimmten für das Fach relevanten Textsorte in eine andere angeregt wird ${ }^{218}$. Diese Umformung bedeutet eine Neustrukturierung von Inhalten und Hinzufügungen weiterer als relevant gesehener Aspekte im Hinblick auf das Schreibziel (also den Zweck der Textsorte). Dies funktioniert wiederum über die Realisierung passender Handlungsmuster und zugehöriger sprachlicher Mittel und Strukturen. ${ }^{219}$

Für das in Kapitel 2.5.1 dargestellte Sprach- und Wissensmode ${ }^{220}$ sind für das Schreiben weitere Komponenten hinzuzufügen. Um diese zu kennzeichnen, kann das Modell mit dem Modell „Zerdehnte Sprechsituation “221 zusammengeführt und ergänzt werden:

\footnotetext{
${ }^{216}$ Vgl. Ehlich, Konrad (1984). (Im Rahmen dieser Arbeit wiedergegeben auf S. 58)

${ }^{217}$ Vgl. Pohl, Thorsten; Steinhoff, Thorsten (2010), S. 9f.

${ }^{218}$ Im Rahmen dieser Arbeit steht das Schreiben im Mittelpunkt der Betrachtung. Gleichwohl gehört zu dieser Betrachtung eine Analyse fachspezifischer Faktoren, die auf das Entschlüsseln von Texten ausgerichtet ist, im Rahmen dieser Arbeit aber nicht geleistet werden können. Zu den verschiedenen Aspekten, die zum Lesen von Texten im Geschichtsunterricht gehören, vgl. z. B.: Köster, Manuel (2013): Historisches Textverstehen. Rezeption und Identifikation in der multiethnischen Gesellschaft, Berlin: LIT Verlag.

${ }^{219}$ Vgl. Bachmann, Thomas; Becker-Mrotzek, Michael (2017), S. 30.

${ }^{220}$ Ehlich, Konrad; Rehbein, Jochen (1986). S. 96.

${ }^{221}$ Ehlich, Konrad (2007), S. 542.
} 


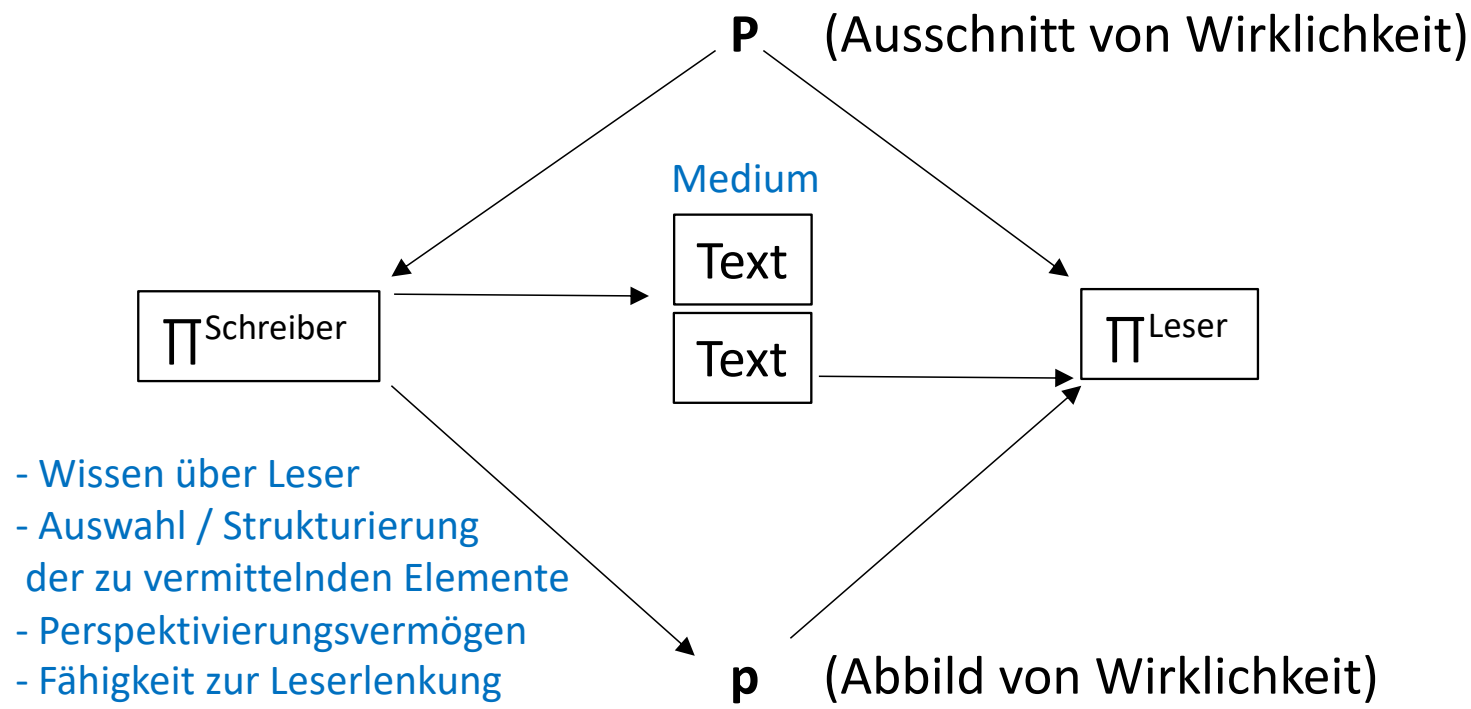

Abbildung 8: Zusammenführung des, Sprach- und Wissensmodells' nach Ehlich/Rehbein (1986) und des Modells 'Zerdehnte Sprechsituation' nach Ehlich (2007)

Hinsichtlich des mentalen Prozesses des Schreibers $\left(\right.$ „ $\left.\Pi^{S^{\prime \prime}}\right)$ bei der Vermittlung, Auswahl und Strukturierung der Elemente, ist festzustellen, dass der Schreiber Aspekte berücksichtigen muss, die in der direkten Kommunikation eine nur geringe Rolle spielen, wie beispielsweise das Perspektivierungsvermögen und die Fähigkeit zur Leserlenkung, weil in der schriftlichen Kommunikation eine direkte Rückmeldung fehlt. Er muss sich in einen angenommenen Leser hineinversetzen, ohne konkret dessen Wissen zu kennen (also dessen mentale Prozesse bei der Rezeption vorwegdenken). Der Schreiber muss vorausblickend fehlende Wissenselemente transferieren, wie etwa den fehlenden Kontext. Dazu benötigt er Elemente der Fachsprache, Textsortenkenntnis und Musterwissen, Wissen über Mittel der Leserlenkung, Textverknüpfungsmittel u.a.m. Umso wichtiger erscheint es, das Schreibziel, also den Zweck (oder die kommunikative Absicht des Schreibanlasses), im Blick zu haben, um so das oder die hinter der Textsorte stehende(n) sprachliche(n) Handlungsmuster erkennen zu können: Geht es um den Transfer von Wissen, wie beim Erklären? Müssen beim Erklären Wissenselemente hinzugefügt oder ausgeführt werden, weil diese wohlmöglich im Leserwissen fehlen? Geht es darum eine Position zu untermauern, die gegebenenfalls angezweifelt werden könnte, wie beim Begründen? Aufgrund der fehlenden Kopräsenz von Schreiber und Leser erscheint es noch wichtiger zu sein, sprachliche Handlungsmuster, Textsorten und Schreibziele zu klären bzw. in Zusammenhang zu bringen. Diese zunächst fachübergreifenden allgemeinen Überlegungen hinsichtlich des Muster- und Textsortenwissens und damit des überfachlichen Schreibens gelten im Besonderen für das Schreiben im Fach, wie am Beispiel des Vergleichens zu zeigen ist. Eine allgemeine Textsortenkenntnis wird ergänzt durch die Prinzipien der dahinterstehenden Fachwissenschaft. Ist Lernenden dieser Zusammenhang klar, kann in der unterrichtlichen Didaktisierung darüber leichter ein Schreibplan generiert werden, aus dem sich wiederum benötigte sprachliche Mittel ableiten lassen. Gerade für Lernende mit Unterstützungsbedarf kann 
eine solche Herangehensweise eine Hilfe bei der Aufgabenlösung sein. Deshalb werden im Folgenden solche Elemente, die das Vergleichen im Geschichtsunterricht strukturieren, herausgearbeitet.

\subsubsection{Ein Schreibkompetenzmodell}

Um den Blick im Sinne des wissenschaftspropädeutischen Schreibens auf das epistemische Schreiben zu fokussieren und es von anderen Kompetenzen abzugrenzen, kann das Kompetenzmodell nach Bereiter dienlich sein: Bereiter beschreibt fünf Kompetenzstufen des Schreibens, die höchste Ausformung und damit die Stufe 5 ist für ihn das epistemische oder erkenntnisbildende Schreiben. ${ }^{222}$ Dies ist die Ebene, auf der Schreibaufgaben der Progression entsprechend im Unterricht der Sekundarstufe II angesiedelt sein müssten. Dies bedeutet nicht, dass epistemisches Schreiben keine Relevanz im Unterricht der Sekundarstufe I hat, da aber gerade in der Sekundarstufe II über Schreiben im Sinne wissenschaftspropädeutischen Arbeitens Wissen generiert werden soll, müssten Schreibaufgaben auf epistemisches Schreiben ausgerichtet sein. In Abstufung nennt Bereiter weiterhin das kritische Schreiben, bei dem der eigenen Text einer selbstkritischen Prüfung unterzogen wird, das leserbezogene Schreiben, das die Fähigkeit voraussetzt, sich in den Leser hineinzuversetzen, das normorientierte Schreiben, das die Berücksichtigung von Schreibnormen auf der Textoberfläche meint, und das assoziative Schreiben als Grundstufe des Kompetenzmodells. ${ }^{223}$ Die Abstufungen zeigen, welcher Anspruch an Lernende gestellt wird, wenn von innen epistemisches Schreiben gefordert wird. Unterrichtspraktisch ist aber mit Schmölzer-Eibinger ${ }^{224}$ zu fragen, auf welcher Kompetenzstufe sich das Schreiben in der Schule und im Geschichtsunterricht im Besonderen in der Regel befindet. Auch hinsichtlich des Operators Vergleichen wäre dies zu untersuchen, da angenommen werden kann, dass Aufgabenformulierung und zugrunde liegender Text einen nicht unerheblichen Einfluss auf das angestrebte Kompetenzniveau haben.

Schreiben ist nicht nur Lerngegenstand, sondern gerade beim epistemischen Schreiben vielmehr Lernmedium. ${ }^{225}$ Steinhoff weist die Wissensbereiche aus, die (epistemisches) Schreiben nicht nur erfordert, sondern auch aktiviert. Schreiben regt, so Steinhoff, die Entwicklung von Weltwissen, sprachlichem Wissen und metakognitivem Wissen an. ${ }^{226}$

Um die Wissensbereiche weiter auszudifferenzieren, kann das ,Kompetenzmodell Schreiben' von Becker-Mrotzek/Schindler ${ }^{227}$ Anhaltspunkte bieten. Es schlüsselt die Anforderungen des Schreibens in Bezug auf unterschiedliche Wissenstypen als Kompetenzdimensionen auf. Auch wenn dies kein Kom-

\footnotetext{
${ }^{222}$ Bereiter, Carl (1980): Development in Writing. In: Gregg, Lee W.; Steinberg; Erwin R. (Hrsg.): Cognitive Processes in Writing. Hillsdale: Erlbaum, S. 73-93.

223 Vgl. ebd.

${ }^{224}$ Schmölzer-Eibinger, Sabine (2013), S. 32.

${ }^{225}$ vgl. Steinhoff, Thorsten (2014): Lernen durch Schreiben. In: Feilke, Helmuth/Pohl, Thorsten (Hrsg.): Schriftlicher Sprachgebrauch - Texte verfassen. Baltmannsweiler: Schneider. S. 316-330. S. 332.

${ }^{226}$ vgl. Steinhoff, Thorsten (2014), S. 332f.

${ }^{227}$ Becker-Mrotzek, Michael; Schindler, Kirsten (2007): Schreibkompetenz modellieren. In: Becker-Mrotzek, Michael; Schindler, Kirsten (Hrsg.): Texte schreiben. Duisburg: Gilles \& Francke. S. 7-26. S. 24.
} 
petenzmodell für das Schreiben im Geschichtsunterricht darstellt, sondern es sich um ein überfachliches Modell handelt, kann es eine Unterstützung für die Annäherung an die schulische Schreibaufgabe ,historischer Vergleich' darstellen:

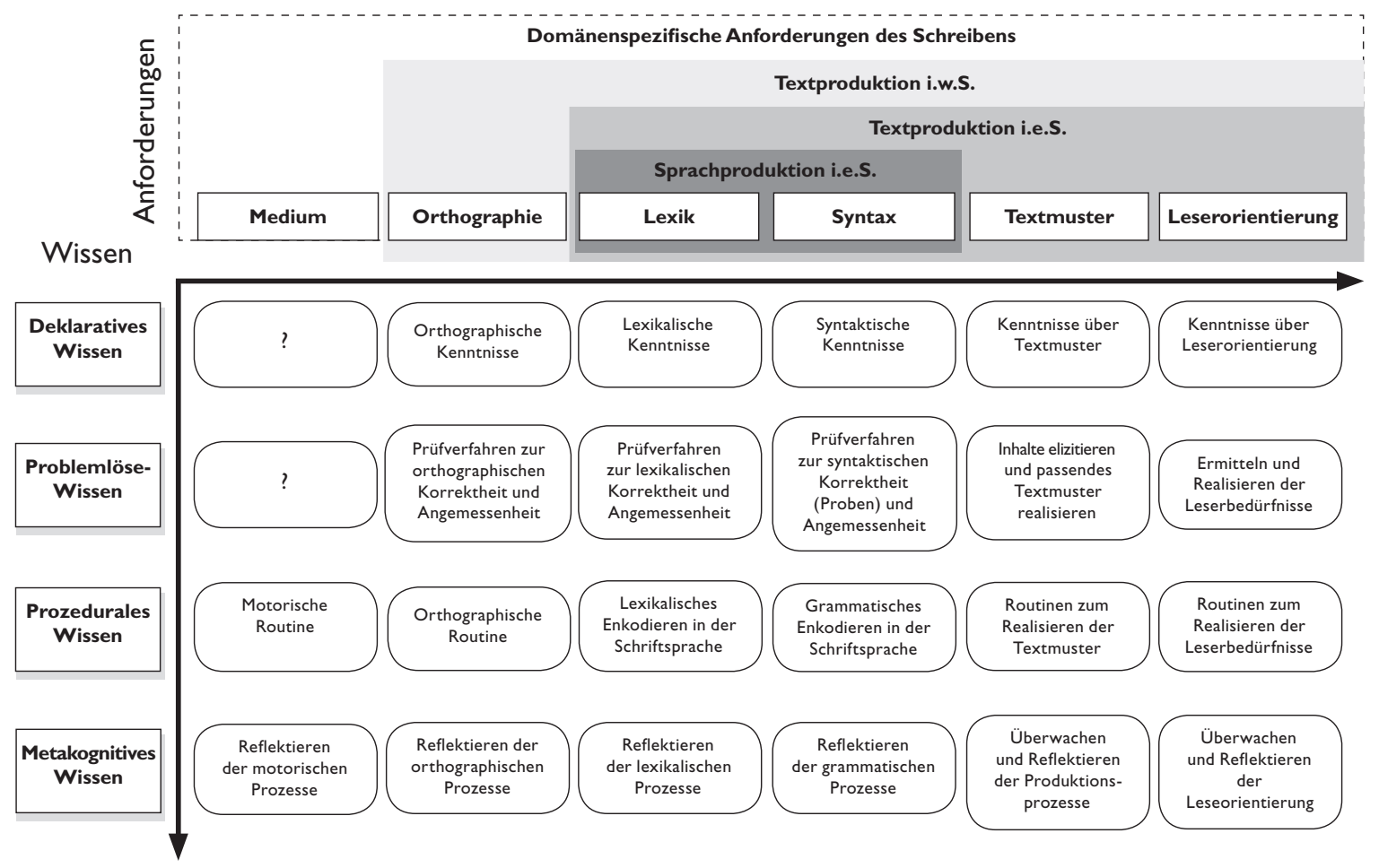

Abbildung 9: "Kompetenzmodell Schreiben" (Becker-Mrotzek, M./Schindler, K., 2007, S. 24.)

Bei der Betrachtung der für die Textproduktion im engeren Sinne dargestellten Anforderungen zeigt das Modell die Bedeutsamkeit von Textmusterwissen und Leserorientierung. Beide Kategorien sind hoch bedeutsam für wissenschaftliches Schreiben und damit auch für die wissenschaftspropädeutische Textproduktion. Dabei zeigt die Begriffswahl ,Textmusterwissen', dass es den Autoren nicht um eine Zuweisung zu den speziellen Anforderungen von Textsorten geht, sondern um Textmuster als übergeordnete und flexible Elemente.

Für die Kategorie „Textmuster" konstruieren die Autoren verschiedene Wissensebenen. Hier stellt das deklarative Wissen die grundlegende Kompetenzstufe dar. Diese ist erfüllt, wenn Lernende Kenntnisse über das Textmuster haben. An dieser Stelle kann Unterricht anknüpfen und die nötigen Wissenselemente bereitstellen, die wiederum auf der nächsten Wissensstufe (problemlösendes Wissen) für das Zusammenbringen von Inhalten und Textmuster bspw. im Hinblick auf eine zu realisierende Textsorte benötigt werden. Auswahl und Strukturierung von Mustern und Inhalten ist ohne Wissen über den Zweck des zu produzierenden Textes nicht sinnvoll. Zuvor wurde der Zusammenhang von Zweck und Auswahl der strukturellen und sprachlichen Mittel dargestellt. Im Modell taucht dieser unter der Kategorie „Prozedurales Wissen“ auf. Dabei ist von Routinen zur Textmusterrealisierung die Rede. Auch 
an dieser Stelle kann Unterricht ansetzen, indem Lernende textstrukturelle bzw. textmusterrealisierende Mittel kennenlernen, aus denen sie für ihre Textproduktion wählen können. ${ }^{228}$ Die Möglichkeit der Auswahl, die über Musterwissen, aber auch sprachliches Wissen, das auf der Metaebene reflektiert wird, angeleitet werden kann, versetzt Lernende in die Lage, selbständig über ihren Text zu entscheiden. Die Realisierung des Zweckes einer Textsorte meint also weniger die Reproduktion eines festen Textschemas als vielmehr die eigenständige Zweckrealisierung mit Hilfe von Musterwissen, das Varianzen zulässt. Gerade durch die Variation mithilfe von Text- und/oder sprachlichen Handlungsmustern wird (vor-)wissenschaftliche Textproduktion erst möglich. Im Modell wird diese Ebene in der Kategorie „Metakognitives Wissen“ durch die Zuschreibung des Überwachens und Reflektierens der Textproduktion ausgedrückt. Nachfolgend wird der hier verwendete Begriff des Textmusters im Anschluss an Kapitel $2.6 \mathrm{im}$ Sinne eines sprachlichen Handlungsmusters verwendet.

Ebenso lässt sich die Kategorie „Leserorientierung“ im Modell abarbeiten, sie folgt dem gerade dargestellten Schema für das Textmuster. Auch hier werden zunächst Kenntnisse über die Leserorientierung vorausgesetzt, die dann auf die Leserschaft angewandt werden, um diese bei der Textproduktion zu berücksichtigen. Dabei helfen wiederum Routinen, die auf der Metaebene reflektiert werden. Dabei darf jedoch gerade im Hinblick auf die Fähigkeit der Leserlenkung nicht unterschätzt werden, welch hohe Anforderung diese an Schreibnovizen bedeutet. Es entwickelt sich dem Modell Bereiters entsprechend erst als dritte Kompetenzstufe. Dabei beinhaltet das schulische Schreiben, wie schon zuvor angesprochen, die besondere Hürde, dass ein meist unwissender Leser angenommen, die Schreibaufgabe aber in der Regel für die Lehrkraft, die einen Wissensvorsprung hat, bearbeitet wird. Dieser Zusammenhang von Schreibentwicklung in Fähigkeit zur Leserlenkung macht die besondere Relevanz authentischer Schreibaufgaben im schulischen Kontext sichtbar.

Das Modell zeigt, dass für das epistemische Schreiben sämtliche Wissensebenen aktiviert und alle domänenspezifischen Anforderungen realisiert werden müssen. Welchen Nutzen besitzt es für das Schreiben im Fach Geschichte und für das Schreiben eines historischen Vergleichs?

Wendet man das Modell auf das Vergleichen im Geschichtsunterricht an, entstehen auf der Ebene des deklarativen Wissens erste Hürden: Die Bestimmung der EPA strukturiert zwar die wesentlichen Wesensmerkmale des historischen Vergleichs, ein klares Handlungsmuster ${ }^{229}$ muss aber erst generiert

\footnotetext{
${ }^{228}$ Der Begriff der Routine wird durch Ehlich/Rehbein als wiederkehrende sprachliche Handlungsmuster verstanden.

Nach Feilke muss der Begriff der Routine im Zusammenhang mit der Kategorie Textmuster als Textroutine verstanden werden: „Im Unterschied zu Schreibroutinen sind Textroutinen also bezogen auf die kommunikativfunktionale Strukturierung von Texten und die entsprechenden sprachlichen Ordnungsleistungen." (Feilke, Helmuth (2012): Was sind Textroutinen? In: Feilke, Helmuth; Lehnen, Katrin (Hrsg.): Schreib- und Textroutinen. Theorie, Erwerb und didaktisch-mediale Modellierung. Frankfurt a. M.: Peter Lang Verlag. S. 1- 31. S. 10.

Eine klare Abgrenzung nehmen die Autoren des Kompetenzmodells Schreiben nicht vor, da der Begriff der Routine aber im Zusammenhang mit dem Textmuster auftaucht, soll der Strukturaspekt an dieser Stelle besonders herausgehoben werden. Er widerspricht dem sprachlichen Handlungsmuster nicht, da Ehlich/Rehbein Strukturen solcher Handlungsmuster herausarbeiten.

${ }^{229}$ In Anlehnung an Ehlich/Rehbein wird nachfolgend der Begriff des Handlungsmusters statt des Textmusters benutzt (vgl. Kap. 2.6).
} 
werden. Dieses für die vorliegende Schreibaufgabe zu tun, ist das Ziel dieser Arbeit. Denn um Verfahren für die Didaktisierung von Handlungsmustern zu entwickeln oder um „Werkzeuge des Schreibens“ bereitstellen zu können, muss zunächst geklärt sein, durch welche Handlungsmuster bzw. Teilhandlungen, sprachlich wie fachlich, das Vergleichen gekennzeichnet ist. Es wird zu zeigen sein, dass solche (Teil-)Handlungsmuster nicht allein auf der Basis der EPA erarbeitet werden können. Liegen grundlegende Strukturelemente auf dem Tisch, können diese auf die zu lösende Aufgabe hin abgestimmt und angepasst werden. Erst daraus ergeben sich Routinen zum Handlungsmuster. Über die Analyse von Vergleichsaufgabe und Aufgabenlösungen können solche Routinen ${ }^{230}$ gezeigt werden. Dennoch zeigt die EPA-Bestimmung, wie bedeutsam es ist, Aufgaben in den Fachkontext einzubetten. Lernende, die nicht wissen, dass für den historischen Vergleich eine Beurteilung gefordert ist, könnten so auf der Ebene des Gegenüberstellens verbleiben, das fehlende Element kann jedoch nicht als fehlend erkannt werden.

Der Aspekt der Leserorientierung gestaltet sich für die ausgewählte Vergleichsaufgabe im schulischen Kontext als schwierig. Wie oben gezeigt, ist die ausgewählte Vergleichsaufgabe keinem authentischen Schreibkontext zugeordnet, eine Leserschaft also nicht klar umrissen, wenngleich sich die Einbettung in einen authentischen Kontext für das Vergleichen im Geschichtsunterricht grundsätzlich realisieren ließe. Im Zusammenhang mit der vorliegenden Untersuchung schreiben die Lernenden für die Lehrenden bzw. in diesem Fall für jemanden in der Universität.

Aufgrund der noch anzuschließenden Lehrwerkanalyse lässt sich vermuten, dass nur ein geringer Teil von Lernaufgaben und insbesondere von Vergleichsaufgaben in einen solchen authentischen Kontext eingebettet ist.

Die Beispiele machen die Grenzen eines solchen überfachlichen ,Kompetenzmodell Schreibens' deutlich. Dennoch kann dieses Modell sehr hilfreich sein, weil auf dieser Grundlage gezeigt werden kann, welche Bedarfe der Geschichtsunterricht an das Schreiben stellt. Es kann als Folie für die fachliche Ausdifferenzierung dienen.

${ }^{230}$ Der Begriff der Routine wird nachfolgend an Ehlich/Rehbein im Sinne wiederkehrender sprachlicher Handlungsmuster angeschlossen. 
2.7.3 Schreiben im Geschichtsunterricht der Sekundarstufe II: Die Bedeutung von textbezogenem und materialgestütztem Schreiben für die Kompetenzentwicklung

Im Rückgriff auf die Bedeutung von Textkompetenzen formulieren Schindler/Siebert-Ott den Zusammenhang von Textkompetenz und Fach. Zwar betrachten sie in diesem Zusammenhang das studentische Schreiben, allerdings kann die Aussage auch als gültig für das Schreiben in den Unterrichtsfächern angenommen werden:

„Für die Entwicklung von Textkompetenzen in diesem Kontext spielt nicht nur die Domäne (Wissenschaft), sondern auch die Disziplin (das studierte Fach, die studierten Fächer) eine besondere Rolle. So ist bei der Nutzung fremder Texte etwa nicht nur die Fähigkeit, zu zitieren, zu entwickeln, sondern auch die Bereitschaft, diese Fächer jederzeit entsprechend den in dieser Domäne gültigen Regeln zu nutzen. Das Fachstudium macht Studierende darüber hinaus zu Mitgliedern einer Diskursgemeinschaft mit besonderen Diskurspraktiken und besonderen Diskurstraditionen. ${ }^{2331}$

Auch wenn es beim schulischen Schreiben noch nicht um die Zugehörigkeit zu einer Diskursgemeinschaft geht, müssen gerade beim Schreiben in der Sekundarstufe II das universitäre Schreiben vorbereitet und zumindest historische Werkzeuge im weiteren Sinne vermittelt werden. ${ }^{232}$ Zieht man die Überlegungen von Feilke/Köster/Steinmetz hinzu, die in Abgrenzung zur Sekundarstufe I Kompetenzen für das Schreiben in der Sekundarstufe II benennen, findet man die Fähigkeit zum Verstehen, zur Reflexion und Kritik fremder Texte sowie produktiven Weiterverarbeitung des Gelesenen in eigenen Texten, die die Autoren herausstellen ${ }^{233}$ :

„Pointiert formuliert sollten damit nach unserer Auffassung zum einen metatextuelle - das heißt auf das Verstehen und die Analyse von Texten bezogene - und zum anderen intertextuelle - das heißt auf die Darstellung und produktive Weiterverarbeitung gelesener Texte bezogene Kompetenzen im Mittelpunkt stehen. ${ }^{2234}$

Die Aussagen der Autoren schließen sich an die eingangs vorgestellte Darstellung an, in der von einem komplexen Textsortenwissen ausgegangen wird.

Textproduktion in der Sekundarstufe II darf nicht auf einer reproduktiven Ebene verbleiben, sondern reflektiert die üblicherweise zugrundeliegende Textgrundlage (damit sind sämtliche Textsorten, kontinuierlich wie diskontinuierlich, mitgedacht), indem sie diese weiterverarbeitet. Wenn Hartung bspw. feststellt, dass reproduktive Schreibformen im Unterricht dominant sind ${ }^{235}$, zeigt dies die Bedeutung, solche Strukturen im Unterricht aufzunehmen und zu thematisieren. Hartung widmet sich der besonderen Bedeutung des Schreibens im Geschichtsunterricht ${ }^{236}$ im Hinblick auf Schüler*innen am Ende

\footnotetext{
${ }^{231}$ Schindler, Kirsten; Siebert-Ott, Gesa (2012): Textkompetenzen im Übergang Oberstufe - Universität. In: Feilke, Helmuth; Köster, Juliane; Steinmetz, Michael (Hrsg.): Textkompetenzen in der Sekundarstufe II. Stuttgart: Fillibach bei Klett. S.151-175. S. 151.

${ }^{232}$ Damit ist nicht gemeint, im Geschichtsunterricht der Oberstufe Historiker auszubilden. Vgl. dazu die vorhergehenden Kapitel 2.4 und 2.5.4.

${ }^{233}$ Vgl. Feilke, Helmuth; Köster, Juliane; Steinmetz, Michael (2012): Zur Einführung - Textkompetenzen in der Sekundarstufe II. In: Feilke, Helmuth; Köster, Juliane; Steinmetz, Michael (Hrsg.): Textkompetenzen in der Sekundarstufe II. Stuttgart: Fillibach bei Klett. S. 7-18. S. 8.

${ }^{234}$ Ebd. S. 8 f.

${ }^{235} \mathrm{Vgl}$. Hartung, Olaf (20123b), S. 50.

${ }^{236}$ Hartung, Olaf (20123b).
} 
der Sekundarstufe I und II, der ebenfalls eine geschichtsdidaktische und funktional-pragmatische Betrachtungsweise verbindet. Auf seine Studie sei an dieser Stelle besonders hingewiesen, im Weiteren wird mehrfach auf sie verwiesen.

Im Gegensatz zu reproduktiven Schreibformen stellt das historische Vergleichen eine (reflexive) Weiterverarbeitung dar. Historisches Vergleichen mit der über das Gegenüberstellen hinausgehenden Anforderung des Beurteilens ist damit eine typische Textsorte der Sekundarstufe II. ${ }^{237}$

Der Bearbeitung der eigentlichen Schreibaufgabe geht im Geschichtsunterricht (wie auch in anderen Unterrichtsfächern) häufig das Lesen und Verstehen einer Textgrundlage voraus. Dabei kann es sich um kontinuierliche oder um diskontinuierliche Texte handeln. Hier sind in Anlehnung an die Bildungsstandards für die Allgemeine Hochschulreife im Fach Deutsch der Kultusministerkonferenz von 2012 zwei Grundtypen des Schreibens zu unterscheiden: das textbezogene und das materialgestützte Schreiben. ${ }^{238}$ Beim textbezogenen Schreiben muss der zugrunde liegende Text (oder auch die Texte) genau wiedergegeben oder analysiert werden. Das materialgestützte Schreiben umfasst dagegen die Berücksichtigung verschiedener Texte, die im Schreibprozess aufeinander bezogen werden und z. T. nur selektiv gelesen werden ${ }^{239}$. Die Qualität des materialbezogenen Schreibens leiten Abraham/Baurmann/Feilke über die Fähigkeit der Lernenden ab, „[...] eigene strukturierende Gesichtspunkte für die Integration der heterogenen Materialien zu entwickeln und für das Schreiben zu nutzen. ${ }^{\text {“240 }}$ Es handelt sich damit um eine kompetenzorientierte Aufgabe.

Bei der ausgewählten Vergleichsaufgabe handelt es sich, dieser Definition folgend, um eine klassische textbezogene Schreibaufgabe, deren Bearbeitung eine genaue Analyse und Gegenüberstellung der Elemente des Darstellungstextes bzw. in der veränderten Erhebung zwei Darstellungstexte vorausgehen. Wenngleich insbesondere die veränderte Erhebung mit den zwei gegenüberzustellenden Texten näher an ein materialbezogenes Schreiben herankommt als der authentische Text im ersten Erhebungsteil, der, wie noch zu zeigen ist, schon Vergleichselemente beinhaltet, muss die Textgrundlage genau ausgewertet und abgearbeitet werden, was dem Ansatz des materialgestützten Schreibens entgegensteht. Abraham/Baurmann/Feilke führen die materialgestützte Form des Schreibens aus, indem sie feststellen:

„Das neue Aufgabenformat verlangt sowohl bei literarisch als auch nichtliterarisch orientierten Aufgaben eine Auswertung bereitgestellter Materialien unter einem thematischen Gesichtspunkt und auf der Basis eines selbst zu entwickelnden Textplans bei vorgegebener Textsorte. Damit ist eine spezifische Textsorte gefordert, die weit über die bloße Wiedergabe deklarativen Wissens zu einem Thema hinausreicht. “241

\footnotetext{
${ }^{237}$ Das bedeutet nicht, dass das Vergleichen für den Unterricht der Sekundarstufe I zu komplex sei, im Gegenteil müsste es dort schon angebahnt werden, was bedeutet, dass innewohnende Teilhandlungen geklärt und geübt werden müssen.

${ }^{238}$ Ständige Konferenz der Kultusminister (2012): Bildungsstandards im Fach Deutsch für die Allgemeine Hochschulreife. S31. Online einsehbar unter: http://www.kmk.org/fileadmin/Dateien/veroeffentlichungen_beschluesse/2012/2012_10_18-Bildungsstandards-Deutsch-Abi.pdf (zuletzt eingesehen am 05.04.17).

${ }^{239} \mathrm{Vgl}$. Abraham, Ulf; Baurmann, Jürgen; Feilke, Helmuth (2015): Materialgestütztes Schreiben. In: Praxis Deutsch 251. S. 4-11. S. 5.

${ }^{240}$ Ebd.

${ }^{241}$ Ebd. S. 6.
} 
Zwar passt der von den Autoren formulierte Anspruch durchaus auf die vorliegende Vergleichsaufgabe, da die Textgrundlage unter einem thematischen Schwerpunkt (dem Beginn der Industriellen Revolution in England und Deutschland) ausgewertet werden und ein Textplan für die Textsorte „Historisches Vergleichen" entwickelt werden muss. Dazu bedarf es aber eines entsprechenden Rahmens hinsichtlich der Aufgabenformulierung. Die Aufgabenformulierung, verführt'zur Übernahme von Textteilen statt zum eigenständigen Vergleichen. Es wird weiterhin zu zeigen sein, dass sich die Lernenden bei der Aufgabenlösung stark auf das (in den Texten vorgegebene) deklarative Wissen stützen.

Der dem materialgestützten Schreiben zugrundeliegende überfachliche Ansatz des, reading to write' passt sich hinsichtlich seines Konstruktionscharakters in den geschichtsdidaktischen Forschungsdiskurs ein, so dass eine Entwicklung materialgestützten Schreibens nicht nur lohnenswert erscheint, sondern dieser Ansatz für die Forschungen zum Schreiben im Geschichtsunterricht im aktuellen Diskurs aufgenommen wird.

\subsubsection{Schreiben aus geschichtsdidaktischer Sicht}

Für den Geschichtsunterricht scheint Einigkeit im geschichtsdidaktischen Diskurs über die Bedeutung des Schreibens für das historische Lernen zu bestehen. Hartung stellt beispielsweise in diesem Zusammenhang fest:

„Konkret geht es um das selbstständige Schreibhandeln der Schülerinnen und Schüler im sog. Sachfach Geschichte, das mehr sein kann, als ,nur' ein Werkzeug zur Wiedergabe vorgegebenen Wissens oder zur externen Wissensspeicherung zum Zwecke der Gedächtnisentlastung. Vielmehr kann das Verfassen eigener Texte zur Geschichte unter bestimmten Voraussetzungen zum Prozess des historischen Lernens selbst werden. ${ }^{\text {"242 }}$

Dies bedeutet, dass den Lernenden eine aktive Rolle im Lernprozess zukommt. Indem sie schreiben, entwickeln sie ihre Narration, ihr Bild von Geschichte und historischen Zusammenhängen. Daher erscheint es zu kurz gegriffen, wenn Hartung zunächst feststellt: „Die eigene ,innere Sprache" unterscheidet sich von der äußeren, weshalb Sprechen und besonders Schreiben immer auch einen Transformationsprozess beinhaltet, bei dem die innere Sprache in eine angemessene äußere Form ,gegossen" werden muss. ${ }^{\prime 243}$

Sicherlich beinhaltet Schreiben einen Transformationsprozess, doch geschieht beim Schreiben mehr, denn der Transformationsprozess greift weiter:

\footnotetext{
${ }^{242}$ Hartung, Olaf (2010b): Historisches Lernen und (Schreib-)Kultur. Zur Bedeutung einer „Kulturtechnik" für das Geschichtslernen. In: Hartung, Olaf u.a. (Hrsg.): Lernen und Kultur. Kulturwissenschaftliche Perspektiven in den Bildungswissenschaften (Schule und Gesellschaft 46). Wiesbaden: Verlag für Sozialwissenschaften, S. 67-79. S. 67.

${ }^{243}$ Hartung, Olaf (2010b), S. 67.
} 
Die meisten Schreibmodelle legen das Planen, Formulieren und Überarbeiten als typische Teilprozesse für das Schreiben zugrunde. ${ }^{244}$ Dabei handelt es sich nicht um voneinander abgegrenzte zeitlich nacheinander abfolgende Teilprozesse, sondern eher um einen kreisförmigen Prozess ${ }^{245}$, in dem die einzelnen Teilhandlungen ineinander greifen. Wenn der Schreibende an jedem Punkt seiner Textproduktion eingreifen kann, Überlegungen verwirft, umformuliert, neu strukturiert etc., zeigt dies, dass Schreiben mehr bedeutet als nur die innere in eine äußere Sprache zu transformieren. Man kann also von einer epistemischen Funktion des Schreibens ausgehen. Dies gilt gerade für das Schreiben im Geschichtsunterricht, weshalb es mehr betrifft als das Dauerhaftmachen der eigenen Gedanken und Aussagen, Hartung stellt dazu selber fest: „[...] denn die Schülerinnen und Schüler sind Teil des großen textuellen ,Bedeutungsgewebes' der Geschichtskultur, und zwar nicht nur passiv als Adressaten, sondern auch aktiv als Produzenten von kultureller Bedeutung. ${ }^{\text {"246 }}$

Für das Unterrichtsfach Geschichte weisen Hartung/Memminger auf die Kompetenzen hin, die das Schreiben im Geschichtsunterricht von der Mündlichkeit unterscheiden:

„Im Vergleich zur Mündlichkeit unterstützt das schreibende Lernen bestimmte kognitive Fähigkeiten für die Verarbeitung von Wissensinhalten, wie z. B. distanzierte Betrachtung und Multiperspektivität, Abstraktion, Klassifikation und kategoriales Denken sowie komplexe Schlussfolgerungen und Sinnbildungen [...]. “247

Die zweifellos bedeutsamen fachübergreifenden Fähigkeiten, die das Schreiben anregt, verknüpfen die Autoren mit der geschichtsspezifischen Fähigkeit der Multiperspektivität. Dabei sind auch die fachübergreifenden Fähigkeiten in einer geschichtsspezifischen Ausgestaltung zu denken. Beispielsweise meint Sinnbildung auf der Grundlage eines historischen Textes eine geschichtsspezifische Sinnbildung und im Sinne Barricellis narratives Wissen: „Historisches Wissen ist immer narratives Wissen. Indem Historiker Quellen beschreiben, analysieren, interpretieren, fallbezogen, vergleichend oder seriell, erzählen sie Geschichte“248. Dies bedeutet, dass auch diskursive Formen wie der Vergleich zum narrativen Wissen im Geschichtsunterricht gehören.

Dieses narrative Wissen wird wiederum über die historische Methode hergeleitet, zur Bedeutung der Schriftlichkeit für diese Methode stellen Hartung/Memminger fest: „Das Erkenntnisverfahren der Historischen Methode als komplexe Abfolge der Arbeitsschritte Historische Frage - Heuristik - Kritik Interpretation und Historische Antwort ist ohne das Distanzmedium der Schrift kaum sinnvoll anzuwenden $[\ldots]^{\prime \prime 249}$

\footnotetext{
${ }^{244} \mathrm{Vgl}$. z. B. Hayes, John R.; Flower, Linda S.: Identifying the Organization of Writing Processes. In: Gregg, Lee W.; Steinberg, Erwin R.: Cognitive processes in writing. Hillsdale: Lawrence Erlbaum Associates, 1980, S.3-30.

${ }^{245}$ Kast nutzt bspw. den Begriff des kreisförmigen Prozesses, weil es kein Nacheinander der einzelnen Teilprozesse gibt, sondern diese immer wieder kreisförmig ineinandergreifen. (vgl. Kast, Bernd (2003): Fertigkeit Schreiben. Das Fernstudienangebot Deutsch als Fremdsprache. Fernstudieneinheit 12. München: Langenscheidt-Verlag. S. 23).

${ }^{246}$ Hartung, Olaf (2010), S. 67.

${ }^{247}$ Hartung, Olaf; Memminger, Josef (2017): Schreibend Geschichte lernen. Mehr als ein „mündliches Fach“ - die Vielfalt des Schreibens im Geschichtsunterricht entdecken. In: Geschichte lernen, 176. S. 2-11. S. 2.

${ }^{248}$ Barricelli, Michele (2017): Narrativität. In: Barricelli, Michele; Lücke, Martin (Hrsg.): Handbuch Praxis des Geschichtsunterrichts. Bd. 1. 2. Aufl. Schwalbach/Ts.: Wochenschau Verlag. S. 255-280. S. 258.

${ }^{249}$ Hartung, Olaf; Memminger, Josef (2017), S. 4f.
} 
Die Autoren knüpfen an den Ansatz des materialgestützten Schreibens an, wenn sie die Bedeutsamkeit des Schreibens gerade im Hinblick auf das Narrativieren und das Verfassen einer Narration herausheben: „Für den komplexen Vorgang des Auswählens von Aussagen zu historischen Ereignissen, deren Sequenzierung, sprachlogische Verknüpfung und Narrativierung durch „emplotment“ [...] eignet sich

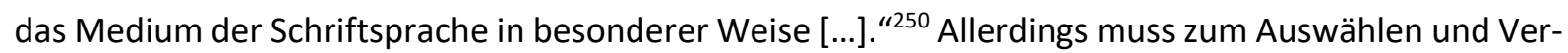
knüpfen ein entsprechendes Aufgabenumfeld geschaffen werden, das eben dazu anregt. Wenn man allerdings bedenkt, „[...] dass bei rund der Hälfte der Arbeitsaufträge die Bearbeitungszeit unter fünf Minuten liegt ${ }^{\prime 251}$, ist ein materialgestütztes Schreiben im Geschichtsunterricht nicht denkbar. Hodel/Waldis ergänzen diese Ergebnisse durch den Hinweis, dass bei einem weiteren Viertel der Arbeitsaufträge die Bearbeitungszeit bei 5 bis 10 Minuten liegt. ${ }^{252}$

Hartung/Memminger weisen dem Schreiben im Geschichtsunterricht eine weitere Rolle zu, die das geschichtsdidaktische Schreiben besonders heraushebt. Sie stellen fest, dass das Schreiben als Distanzmedium besonders gut zum Ansatz des historischen Lernens im Geschichtsunterricht passt:

„Das Distanzmedium der Schriftsprache kann Schülerinnen und Schüler dabei unterstützen, vergangene Epochen und Kulturen nicht nur nach den historischen Denkweisen und Wertesystemen zu beurteilen, indem sie damit eine größere Distanz zu ihrem eigenen Denken und Fühlen herzustellen vermögen. Als Distanzmedium eignet sich Schreiben auch besonders gut als Mittel der (Meta-)Reflexion. “253

Dieser Fokus macht das Schreiben zu einem geschichtsspezifischen Werkzeug, über das Annäherung an Geschichte gelingen kann. In dieser Hinsicht ist es umso wichtiger, Lernende an dieses Werkzeug ${ }^{254}$ heranzuführen, Schreiben also im Geschichtsunterricht zu explizieren. Dabei ist Kritik von Seiten der Lehrkräfte, es sei zu wenig Zeit für das Schreiben da, ernst zu nehmen, so dass Richtlinien und Lehrpläne auf Dauer hierauf reagieren müssen. Schreiben ist ein zeitintensiver Prozess, der gut angeleitet werden muss, eine Auslagerung in die Hausaufgaben der Lernenden kann deshalb keine Lösung sein. Empirische Untersuchungen zum Schreiben im Geschichtsunterricht zeigen, dass Lernende grundsätzlich in der Lage sind, geschichtsdidaktische Schreibaufträge, die eine Narration verlangen, zu erfüllen, aber Unterstützung etwa hinsichtlich der sprachlichen Werkzeuge benötigen. Zwar ist das Schreiben im Unterrichtsfach Geschichte noch nicht sehr umfänglich untersucht, doch weisen die bereits existierenden Untersuchungen in diese Richtung. In diesem Zusammenhang ist zunächst die Untersuchung Barricellis „Schüler erzählen Geschichte“ zu nennen ${ }^{255}$, dessen zentrales Anliegen die Untersuchung narrativer Kompetenz von Lernenden ist, sein Medium der Analyse von Schülernarrationen sind die entsprechenden Schreibprodukte. Barricelli sucht in diesen Schreibprodukten nach beobachtbaren

\footnotetext{
250 Ebd. S. 4.

${ }^{251}$ Hodel, Jan; Waldis, Monika (2007): Sichtstrukturen im Geschichtsunterricht - die Ergebnisse der Videoanalyse. In: Gautschi, Peter; Moser, Daniel; Reusser, Kurt; Wiher, Pit (Hrsg.): Geschichtsunterricht heute. Eine empirische Analyse ausgewählter Aspekte. Bern: h.e.p. Verlag. S. 91-142. S. 136.

252 Ebd.

${ }^{253}$ Hartung, Olaf; Memminger, Josef (2017), S. 4.

${ }^{254}$ Hartung benennt sogar den Text als Werkzeug: „Geschichtstexte sind nicht nur Repräsentationsmedien für schon fertige Gedanken, sondern auch und gerade Werkzeuge zur historischen Sinnbildung." (Hartung, Olaf (2013a)). Er versteht den Text also nicht als Produkt, sondern als Prozess.

255 Barricelli, Michele (2005): Schüler erzählen Geschichte. Narrative Kompetenz im Geschichtsunterricht. Schwalbach/Ts. Wochenschau-Verlag.
} 
„Regelhaftigkeiten im Bemühen der Schüler um Bedeutung“. ${ }^{256}$ Dazu nimmt er Formulierungen der Lernenden in den Blick und untersucht sie auf Regelhaftigkeiten im Sinne fachlicher Aspekte. Barricelli stellt fest, dass Lernende der von inm untersuchten 9. Jahrgangsstufe in der Lage sind, Geschichte zu erzählen und unverbundene Einzelaussagen, Inhalte etc. in einen neuen Zusammenhang bringen und diese zudem funktional ausgestalten können. ${ }^{257}$ Er konstatiert aber auch: „Wer (historisch-) narrative Kompetenz als elementare Fach-Fähigkeit ausbilden (und abfragen und bewerten) möchte, muss sie ausführlich einführen, demonstrieren, reflektieren, einüben, sprich: lehren. ${ }^{258}$ Dabei denkt Barricelli zwar auch an die „Bauformen der Narrativität ${ }^{\text {“259 }}$, weniger aber an die sprachlichen Instrumente, die eine ebenso hohe Bedeutung tragen.

Memminger beschäftigt sich in seiner Untersuchung mit kreativem Schreiben im Geschichtsunterricht. ${ }^{260}$ Er wertet kreative Schreibprodukte (z. B. Reportage) von Lernenden der Jahrgangsstufe 7 und der gymnasialen Oberstufe aus, analysiert dabei einzelne sprachliche Mittel, bringt sie jedoch nicht in einen größeren systematischen Zusammenhang, der einen stärker linguistisch konturierten Ansatz erforderlich macht. So bleibt die Einordnung der Ergebnisse z. T. auf der Ebene der Feststellung, dass z. B. die Sprache der Reportage keine Probleme bereite oder der Text im Wesentlichen flott formuliert sei. $^{261}$

Sowohl Barricelli als auch Memminger legen für ihre Untersuchung die Erstellung einer Narration zu Grunde, andere für den Geschichtsunterricht relevante Textsorten betrachten sie nicht, insbesondere fehlt die Berücksichtigung diskursiver Textsorten. Diese erscheinen aber gerade bei der Arbeit mit Aufgaben in Geschichtslehrwerken als besonders relevant, wie die nachfolgende Frequenzanalyse von Aufgaben in Geschichtslehrwerken zeigen wird.

Hodel dagegen berücksichtigt nicht ausschließlich narrative Textelemente ${ }^{262}$. Er zeigt allerdings in seiner Untersuchung zum Recherchieren und Verfassen von Referaten, dass Lernende in diesen Referaten vorhandene Narrative reproduzieren (er bezeichnet diese Form als Nacherzählung), im Internet bereitgestellte Inhalte kopieren; insgesamt stellt er aber fest, dass auch in dieser Textsorte durch das Verknüpfen im Netz recherchierter Informationen Fragmente zu einer Form der Narration verknüpft werden. ${ }^{263}$ Interessant an den Ergebnissen Hodels ist für diese Arbeit insbesondere die Feststellung, dass Lernende in hohem Ausmaß Inhalte aus den recherchierten Dokumenten kopieren. Er differenziert

\footnotetext{
${ }^{256}$ Barricelli, Michele (2002): Narrative Kompetenz als Ziel des Geschichtsunterrichts - Eine empirische Studie zu Erfahrungen von Schülern, Geschichte zu erzählen, in: Handro, Saskia/Schönemann, Bernd (Hrsg.): Methoden geschichtsdidaktischer Forschung (Zeitgeschichte - Zeitverständnis 10), Münster, S. 73-84. S. 77.

${ }^{257}$ Vgl. ebd. S. 273.

258 Ebd. S. 283.

259 Ebd.

${ }^{260}$ Memminger, Josef (2007): Schüler schreiben Geschichte. Kreatives Schreiben zwischen Fiktionalität und Faktizität. Schwalbach/Ts.: Wochenschau-Verlag.

${ }^{261} \mathrm{Vgl}$. ebd. S. 127.

${ }^{262}$ Zwar kann in Referaten eine Narration entstehen; da das Referat in der Regel unterschiedliche Teilhandlungen beinhalten kann, sind aber genauso diskursive Elemente wahrscheinlich.

${ }^{263}$ Vgl. Hodel, Jan (2013): Verkürzen und Verknüpfen. Geschichte als Netz narrativer Fragmente: Wie Jugendliche digitale Netzmedien für die Erstellung von Referaten im Geschichtsunterricht verwenden. Bern: hep Verlag. S. $327 f$.
} 
dabei in komplett übernommene Textstellen und bearbeitete Fassungen. ${ }^{264}$ Hodel hinterfragt seine Ergebnisse im Hinblick auf die Überlegung, ob es um vorsätzliche Täuschung oder undifferenzierte Angabe der zugrunde liegenden Quellen geht. ${ }^{265}$ Für die Vergleichsaufgabe spielt diese letzte Überlegung eine eher untergeordnete Rolle. Allerdings werden die Ergebnisse zeigen, dass in den Schülerschreibprodukten zur Vergleichsaufgabe viele Übernahmen aus dem zugrundeliegenden Darstellungstext zu finden sind, weniger direkte Übernahmen von Textpassagen als vielmehr mehr oder weniger gelungene Paraphrasierungen oder Übernahmen von Begriffen, die im Text genutzt werden, häufig jedoch, auch das wird die Auswertung zeigen, ohne diese zu kennzeichnen. Für den Geschichtsunterricht muss hier überlegt werden, wie mit dem Wissen um fehlende Kennzeichnung umgegangen werden kann und wie Lernende dazu angeleitet werden können, im Sinne wissenschaftlichen oder wissenschaftspropädeutischen Arbeitens Übernahmen korrekt zu kennzeichnen. Voraussetzung dafür ist die Schaffung eines Bewusstseins für die Übernahme vorhandener Strukturen.

Hartung verbindet in seiner Studie „Geschichte Schreiben Lernen“ geschichts-, schreib- und textlinguistische Ansätze. ${ }^{266}$ Er hebt nicht nur die grundsätzliche Bedeutung des Schreibens im Geschichtsunterricht sehr deutlich hervor, sondern thematisiert auch die Werkzeuge, die Lernende dazu benötigen, wenn er feststellt:

„Zum erfolgreichen Schreiben von und über Geschichte bedürfen die Schüler/innen sowohl dem jeweiligen Textgegenstand und Schreibziel angemessene Schreibstrategien als auch Kenntnisse über die dafür in Frage kommenden Vertextungsmuster. Insofern sollte konzeptionelles Schreiben über historische Sachverhalte sowohl Medium als auch Gegenstand des Lernens im Geschichtsunterricht sein. ${ }^{267}$

Hartung will auf der Grundlage von Falluntersuchungen u.a. Antworten auf die Fragen geben, wie Lernende konzeptionelle Schreibaufgaben zu historischen Fragestellungen lösen, welchen Einfluss Faktoren wie individuelle Schreibhandlungen, verfügbare Schreibstrategien, Aufgabenstellungen und Textsorte haben, ob sie beim Schreiben von Geschichtetexten epistemische Effekte erzielen können bzw. wie sich diese beschreiben lassen und welche Erfahrungen die Lernenden mit konzeptionellen Schreibaufgaben im Geschichtsunterricht machen. ${ }^{268}$ Die Untersuchung ist in der Sek. I und II angesiedelt. Er lässt die Textsorten Rede, Erörterung und Zeitschriftenessay produzieren. Hartung stützt sich auf den Ansatz der Funktionalpragmatik und untersucht die Schülertexte etwa auf ihre sprachlichen Handlungen. Er stellt einen engen Zusammenhang zwischen Textsorte und sprachlichen Handlungen fest, kommt aber auch zu dem Schluss, dass selbst in der Textsorte Erörterung in Schülertexten Bewertungen nur wenig vorkommen. ${ }^{269}$ Dieses Ergebnis ist auch für die Vergleichsaufgabe interessant, da das historische Vergleichen durch das Urteilen im Sinne eines Sachurteils auch das Bewerten einfordert. Die Texte des Vergleichskorpus werden eine ähnliche Tendenz zeigen. Diese Tendenz konnte Hartung

\footnotetext{
264 Ebd. S. 242.

265 Ebd. S. $243 f$.

${ }^{266}$ Hartung, Olaf (2013b): Geschichte Schreiben Lernen. Empirische Erkundungen zum konzeptionellen Schreibhandeln im Geschichtsunterricht. Berlin: LIT Verlag. S. 9.

267 Ebd. S. 20.

268 Ebd. S. 143.

${ }^{269}$ Vgl. ebd. S. 298.
} 
bereits 2010 in Bezug auf den Essay feststellen und einordnen: „Die textsortendifferenzierte Betrachtung offenbart einen auffällig hohen Anteil an Paraphrasen bei den Erörterungen (15,6 \%), was auf mögliche Schwierigkeiten der Schüler/innen hindeutet, mit ihren eigenen Formulierungen historisch zu argumentieren. “270 Für die Untersuchung der Schreibprodukte zur Vergleichsaufgabe spielen die produktiven Fähigkeiten, die das selbständige Formulieren einfordert, eine Rolle, die zu solchen Paraphrasierungen führen, weiterhin ist aber die Frage mitzudenken, welchen Einfluss die rezeptiven Fähigkeiten der Lernenden hinsichtlich des zugrunde liegende Textes/der zugrunde liegenden Texte spielen. So ist es denkbar, dass die Übernahme oder Umformulierung von Textteilen fehlendem Textverständnis geschuldet ist, hierauf lassen zumindest grammatische Schwierigkeiten oder die Herstellung eines ,schiefen' semantischen Zusammenhangs bei manchen Reformulierungen schließen.

Ausgehend von der Feststellung, dass für das Schreiben von Geschichte die Operationen des Erklärens, Begründens, Belegens und Bewertens konstitutiv sind, bemängeln Mierwald/Brauch für das dem wissenschaftlich historischen Schreiben vorausgehende Schreiben im Geschichtsunterricht: „[...] jedoch fehlen immer noch konkrete Aussagen zur narrativen Verfasstheit und fachspezifischen Konzeption diskursiv-argumentativer Sprachhandlungen im Geschichtsunterricht.“271 Das Argumentieren bzw. Teilhandlungen des Argumentierens finden sich als Element in unterschiedlichen Operatoren wieder. Die Füllung der Operation Argumentieren ist damit wesentlich für das Lösen von Aufgaben im Geschichtsunterricht. Auf der Grundlage ihrer Arbeitsdefinition von historischem Argumentieren untersuchen die Autoren insbesondere Schwierigkeiten der Lernenden bei dieser sehr umfassenden Operation und ziehen Schlüsse für die Förderung der Argumentationsfähigkeit Lernender daraus. Anhand eines Schüler*innenbeispiels skizzieren sie das Schreiben einer Argumentation mit Hilfe einer Instruktionsmethode. Dabei fällt auf, dass die Auswertung stark den Aufbau einer Argumentation bzw. eines Arguments in den Blick nimmt, die dazu notwendigen sprachlichen Mittel, wie etwa Satzverknüpfungsmittel u.Ä., jedoch nicht untersucht. Eine linguistische Fundierung, wie bspw. Hartung sie zugrunde legt, ist hier nicht erkennbar.

Schon in Kapitel 2 (vgl. z. B. 2.2.3) zu Kompetenzen im Geschichtsunterricht spielten Überlegungen aus der Untersuchung Schönemanns et al. ${ }^{272}$ hinsichtlich der Rahmenbedingungen des Zentralabiturs und der Vorgaben der EPA eine Rolle. Auch die eigentliche Untersuchung muss an dieser Stelle mitgedacht werden. Auf der Grundlage einer Erhebung von Leistungskursklausuren eines Abiturjahrgangs an unterschiedlichen Schulen analysieren die Autoren anhand eines Kriterienrasters auf der Grundlage normativer Vorgaben für den Geschichtsunterricht bzw. die Abiturprüfung, „[...] welche Lernleistungen Abiturienten am Ende des schulischen historischen Lernprozesses der Sekundarstufe II zu erbringen imstande sind und wie sich diese Leistungen systematisch, aber zugleich auch material- und praxisnah

\footnotetext{
${ }^{270}$ Hartung; Olaf (2010c): Geschichte - Schreiben - Lernen. Eine empirische Studie. In: Handro, Saskia; Schönemann, Bernd (Hrsg.): Sprache und Geschichte (Zeitgeschichte - Zeitverständnis, Bd. 21). Berlin: LIT-Verlag. S. 6177. S. 70.

${ }^{271}$ Mierwald, Marcel, Brauch, Nicola (2015): Historisches Argumentieren als Ausdruck historischen Denkens. Theoretische Fundierung und empirische Annäherungen. Zeitschrift für Geschichtsdidaktik 14/2015. S. 104-120. S. 107.

${ }^{272}$ Schönemann, Bernd; Thünemann, Holger; Zülsdorf-Kersting, Maik (2010): Was können Abiturienten? Zugleich ein Beitrag zur Debatte über Kompetenzen und Standards im Fach Geschichte. LIT Verlag, Münster.
} 
beschreiben und beurteilen lassen. ${ }^{1273}$ Außerdem fragen die Autoren danach, ob sich die Schülerleistungen über die normativen Vorgaben überhaupt angemessen abbilden lassen. ${ }^{274}$ Anders als die zuvor dargestellten Studien stehen hier nicht einzelne Textsorten im Mittelpunkt der Betrachtung, sondern Textprodukte werden im Zusammenhang von Aufgabenstellungen, Materialgrundlage und erwarteten Lösungen geprüft und hinsichtlich der Schülerschreibprodukte untersucht. Der Zugang ist damit ein doppelter. Dabei konstatieren sie u.a. sehr unterschiedliche Formen der Sachurteilsfindung ${ }^{275}$ in den Schülertexten, ein Ergebnis, das auch im Hinblick auf das Vergleichen im Geschichtsunterricht von Bedeutung sein dürfte.

Auch wenn insbesondere Schönemann et al. Aufgabenstrukturen auf der Grundlage von Schülertexten kritisch hinterfragen, sind Anforderungen durch Aufgabenstellungen und Operatoren, die Lernende zum Schreiben im Geschichtsunterricht anregen sollen, kaum untersucht.

Legt man abschließend das zu Beginn des Kapitels allgemein formulierte Anforderungsprofil an Schreibaufgaben auf einer Textgrundlage zugrunde, kann man bereits an dieser Stelle für die zu untersuchende Vergleichsaufgabe im Geschichtsunterricht nachfolgendes Profil beschreiben:

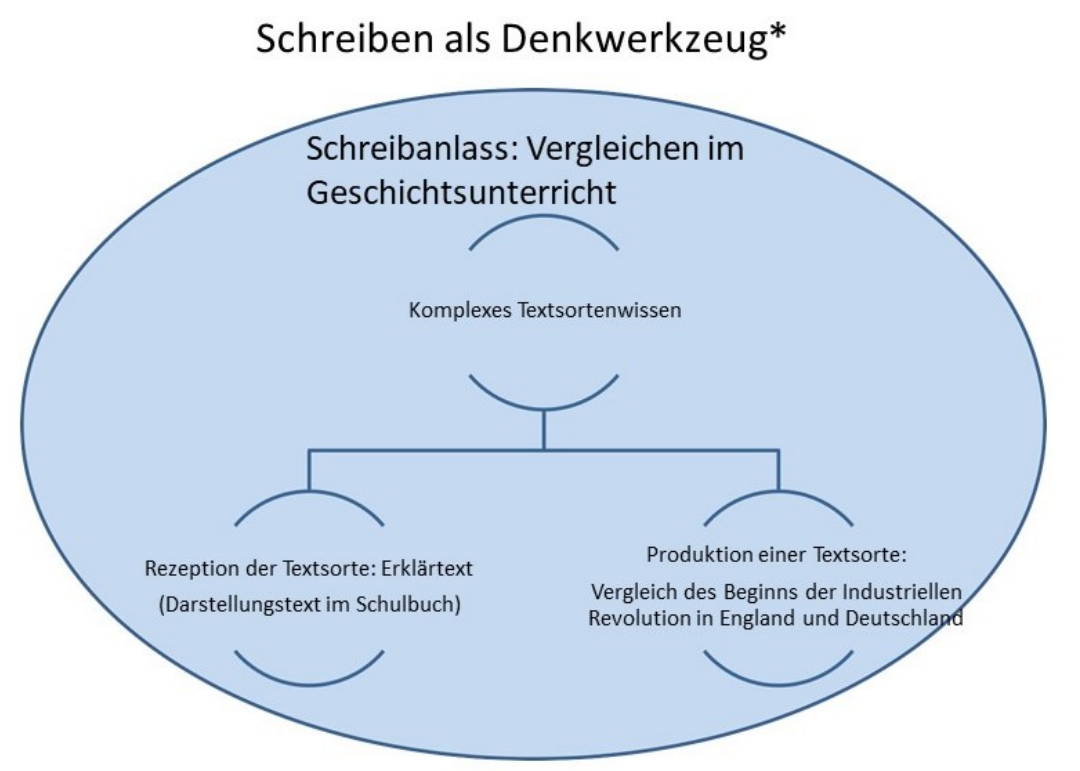

(*vgl. Thürmann 2012. S. 53)

Abbildung 10: Anforderungen durch das Vergleichen im Geschichtsunterricht als Schreibanlass (eigene Darstellung)

Inwieweit die ausgewählte Vergleichsaufgabe dem Anspruch, ein Denkwerkzeug zu sein, tatsächlich gerecht wird, kann jedoch erst die Auswertung der Texte der Lernenden zeigen.

\footnotetext{
273 Ebd. S. 3.

274 Ebd.

275 Ebd. S. 68.
} 


\section{Vergleich und Vergleichen}

In Kapitel 2 hat das Vergleichen bereits mehrfach eine Rolle gespielt, so dass die Leistungen und Anforderungen von Vergleichsaufgaben im Geschichtsunterricht nun systematisiert aufgearbeitet werden sollen.

Von einem allgemeinen Blick auf das Vergleichen ausgehend, soll im Folgenden zunächst kurz auf die Leistungen des Vergleichs in Alltag und Schule geschaut werden, bevor dann der Vergleich in Geschichtswissenschaften und Geschichtsunterricht fokussiert wird. Im Weiteren wird der historische Blick auf den Vergleich durch den linguistischen Blick ergänzt und beide Herangehensweisen zusammengeführt.

In diesem Kapitel im Besonderen, aber auch im Rahmen dieser Arbeit wird sowohl vom Vergleich als auch vom Vergleichen die Rede sein. Sicherlich stellt der Begriff Vergleich eher den Produktcharakter, das Vergleichen den Prozess in den Vordergrund. Da aber an manchen Stellen der Darstellung die Differenzierung verschwimmt, werden die Begriffe im Folgenden weitestgehend synonym verwendet ${ }^{276}$.

\subsection{Alltägliches und fachliches Vergleichen}

Der Mensch nutzt schon im Kleinkindalter das Vergleichen zum Erschließen seiner Umwelt ${ }^{277}$, Vergleichen ist elementar für die eigene Verortung in der Umwelt. Für diese Form des Vergleichs kommt ein alltägliches Vergleichskonzept zum Tragen. Da es sich beim Vergleichen im Geschichtsunterricht um ein fachliches Vergleichen handelt, lohnt sich der Blick auf die Unterscheidung zwischen alltäglichem und fachlichem Vergleichen.

Was macht das Vergleichen im Fach aus? In diesem Zusammenhang kann mit Petersen gefolgert werden, wenn sie für das Argumentieren als sprachliche Handlung feststellt: „Der Erwerb von Argumentationskompetenz ist für ein Individuum nicht nur eine höchst relevante, sondern auch eine komplexe Aufgabe.[...] Der Sachverhalt muss durch die Umstrukturierung von Wissen erst hergestellt werden, Argumente müssen entsprechend ihrer Relevanz ausgewählt, gewichtet und angeordnet werden. “278 Ähnliches gilt für das fachliche Vergleichen, es ist hoch relevant für die Generierung neuer Wissensstrukturen, da für den Vergleich wie beim Argumentieren Wissen umstrukturiert werden muss. Gleichzeitig müssen diese Umstrukturierung und die sich daraus ergebenen Folgerungen argumentativ un-

\footnotetext{
${ }^{276} \mathrm{Vgl}$. dazu auch Anm. 21.

277 Vgl. Eggs, Frederike (2006b): Vergleichen und Vergleiche - Implikationen der Sprachwissenschaft für die Sprachdidaktik In: Becker, Tabea und Peschel, Corinna (Hrsg.): Gesteuerter und ungesteuerter Grammatikerwerb. Hohengehren. S. 48.

278 Petersen, Inger (2013): Entwicklung schriftlicher Argumentationskompetenz bei ein- und mehrsprachigen Oberstufenschüler/-innen und Studierenden. In: Brandl, Heike; Arslan, Emre; Langelahn, Elke; Riemer, Claudia (Hrsg.): Mehrsprachig in Wissenschaft und Gesellschaft. Mehrsprachigkeit, Bildungsbeteiligung und Potenziale von Studierenden mit Migrationshintergrund. Bielefeld, 69-79. Online verfügbar unter http://biecoll.ub.unibiele-feld.de/volltexte/2013/5284/pdf/08_petersen_entwicklung\%20schriftlicher\%20argumentationskompetenz korrigiert.pdf (zuletzt eingesehen am 01.08.2018). S. 70.
} 
terstützt werden, um als diskursives Element Eingang in einen fachlichen oder sogar fachwissenschaftlichen Diskurs zu finden. Das fachliche Vergleichen kann deshalb als Form des Argumentierens angenommen werden. Im Gegensatz zum alltäglichen Vergleich kann der fachliche Vergleich in Anlehnung an das Konzept Koch/Oesterreichers ${ }^{279}$ als konzeptionell schriftlich eingeordnet werden, da er eine komplexe Struktur darstellt, die fachlich differenziert und elaboriert einem Leser oder Hörer vermittelt werden muss. Im Gegensatz dazu ist das alltägliche Vergleichen in der Regel konzeptionell mündlich geprägt, solche Vergleiche sind weniger komplex und bedürfen kaum fachsprachlicher Ausformung. Dies zeigt sich bspw. in kurzen Vergleichsstrukturen, wie „Er/Sie hat mehr/weniger davon als ich.", die im Gegensatz zu fachlichen Verweisen über deiktische Verweise (z. B. Zeigegesten) funktionieren.

Was leistet solch ein fachlicher Vergleich für den schulischen Lernprozess? Aus einem pädagogischen Ansatz heraus gibt Spanhel darauf eine erste Antwort: Bei einem „[...] Vergleich, der aus einem oder mehreren Aussagesätzen besteht, werden zwei Sachverhalte (Personen oder Dinge) nebeneinandergestellt, um so deren Eigenart dem Schüler deutlich und besser verständlich machen zu können.“280 Spanhel weist hier insbesondere auf das Ziel des Vergleichens hin, nämlich Verstehen von (Unterrichts-)Inhalten zu erleichtern. Er weist damit dem Vergleich einen durchaus hohen pädagogischen Anspruch zu. Allerdings differenziert er in diesem Zusammenhang die wesentlichen Elemente des Vergleichs noch nicht aus: Zwar nimmt er Bezug auf die beiden Sachverhalte, die verglichen werden, bezieht aber in diesem Zusammenhang nicht das Tertium Comparationis als gemeinsames Drittes ein, über das die beiden Sachverhalte überhaupt nur verglichen werden können. Die Bestimmung der Grundbestandteile des Vergleichs nimmt Eggs vor, sie ordnet diese zudem in Bezug auf ihre Vergleichbarkeit ein: Sie geht von der Grundannahme aus, es gebe ein „mit Hilfe des Vergleichs zu charakterisierendes Element und ein zum Vergleich dienendes Element ${ }^{\prime 281}$ (S. 52), die Korrelation sei auf eine gemeinsame Eigenschaft bezogen (das „Tertium Comparationis“), wenn es nichts Gemeinsames gebe, seien die Größen unvergleichbar:

„Zwei Dinge miteinander zu vergleichen bedeutet also immer, dass sie wenigstens eine Gemeinsamkeit aufweisen. Aber auch die Feststellung, dass zwei Dinge nicht miteinander verglichen werden können, können wir erst dann treffen, wenn wir versucht haben, eben dies zu tun und daran gescheitert sind. ${ }^{282}$

Mit einem populären Beispiel ausgedrückt wäre dies die Frage nach der Vergleichbarkeit von Äpfeln und Birnen, die in diesem Zusammenhang diskutiert werden müsste. Sind Äpfel und Birnen vergleichbar? Vermutlich schon, da sie, trotz aller Unterschiede, der Kategorie Obst angehören. Dies bedeutet, dass dieser Kategorisierung ein Vergleich vorausgegangen sein muss.

\footnotetext{
279 Hier sei auf das Konzept von konzeptioneller Mündlichkeit und Schriftlichkeit nach Koch/Oesterreicher verwiesen. Vgl. z.B.: Koch, Peter; Oesterreicher, Wulf (1985): Sprache der Nähe - Sprache der Distanz. Mündlichkeit und Schriftlichkeit im Spannungsfeld von Sprachtheorie und Sprachgeschichte. In: Romanistisches Jahrbuch 36/85, 15-43.

${ }^{280}$ Spanhel, Dieter (1973): Die Sprache des Lehrers. Grundformen didaktischen Sprechens. 2. Aufl. Düsseldorf: Schwann Verlag. S. 211.

${ }^{281}$ Eggs, Frederike (2006b), S. 52.

282 Eggs, Frederike (2006b), S. 46.
} 
Sowohl in den Darstellungen von Eggs, die ihren Vergleichsansatz viel grundsätzlicher wählt als Spanhel, der sich stärker auf das Vergleichen im Unterricht bezieht, finden sich wesentliche Komponenten zur Bestimmung eines fachlichen Vergleichs. Dennoch muss nachfolgend zu dieser allgemeinen Zielzuweisung in Bezug auf das jeweilige schulische Lernfach das Fachspezifische des Vergleichs herausgearbeitet bzw. berücksichtigt werden. Dies soll nachfolgend für die Fachdisziplin bzw. das Unterrichtsfach Geschichte geleistet werden.

\subsection{Vergleichen in der Fachdisziplin Geschichte}

Eine erste grundlegende Bestimmung des historischen Vergleichs nimmt Kaelble im „Lexikon Geschichtswissenschaft“ vor. Dort beschreibt der Autor den Vergleich wie folgt: „Der historische V. [Vergleich, Anm. d. Verf.] dreht sich um die Beschreibung, Analyse und Typisierung von Unterschieden und Gemeinsamkeiten, Divergenzen und Konvergenzen, Transfers und Abschließungen zwischen historischen Gesellschaften. ${ }^{2283}$ In dieser Bestimmung finden sich insbesondere in den von Kaelble gewählten Paaren zentrale Elemente der geschichtswissenschaftlichen Diskussion um den historischen Vergleich. Diese Paare gestaltet Kaelble als Gegensatzpaare aus, die er auf das Feld der historischen Gesellschaften bezieht. Damit kennzeichnet er das Feld historische Gesellschaften als spezifisch historische Vergleichsgrundlage, aus denen die Vergleichselemente hervorgehen. Außerdem weist er über die genannten Gegensatzpaare das Gegenüberstellen als maßgeblichen Teil des Vergleichs aus. Diese Bestimmung legt nahe, dass es insbesondere um die Darstellung von Unterschieden geht. Hier muss allerdings mit einer weiteren, später entstandenen Begriffsbestimmung Kaelbles nachgesteuert werden:

„Unter dem historischen Vergleich versteht man üblicherweise die systematische Gegenüberstellung von zwei oder mehreren historischen Einheiten (von Orten, Regionen, Nationen oder Zivilisationen, auch historische Persönlichkeiten), um Gemeinsamkeiten und Unterschiede, Annäherungen und Auseinanderentwicklungen zu erforschen. ${ }^{2884}$

In dieser Bestimmung werden weitere wesentliche Merkmale des historischen Vergleichs deutlich: Zum einen, dass es sich um einen systematischen Vergleich handelt, dieser Aspekt wird in der zuerst genannten Darstellung zumindest nicht expliziert. Zum anderen differenziert Kaelble nicht nur in Gegensatzpaare, die die Herausarbeitung von Unterschieden zwischen den historischen Vergleichseinheiten nahelegen. Vielmehr nennt er explizit die Erforschung von Gemeinsamkeiten, Unterschieden, Annäherungen und Auseinanderentwicklungen. Es wird nachfolgend zu zeigen sein, dass im Diskurs über den historischen Vergleich die Begriffe Gemeinsamkeiten, Unterschiede und Ähnlichkeiten eine

\footnotetext{
${ }^{283}$ Kaelble, Hartmut (2002): Vergleich, historischer. In: Jordan, Stefan (Hrsg.): Lexikon Geschichtswissenschaft. Hundert Grundbegriffe. Stuttgart: Reclam. S. 303- 306. S. 303.

${ }^{284}$ Kaelble, Hartmut (2012): Historischer Vergleich. Onlineressource: https://docupedia.de/zg/Historischer_Vergleich. S. 4. (Zuletzt eingesehen am 05.04.18). Kaelble spricht von der systematischen Gegenüberstellung „von zwei oder mehreren historischen Einheiten (von Orten, Regionen, Nationen oder Zivilisationen, auch historische Persönlichkeiten), um Gemeinsamkeiten und Unterschiede, Annäherungen und Auseinanderentwicklungen zu erforschen." S. 2.
} 
Rolle spielen. Diese spiegeln sich in den von Kaelble genannten Begriffen wider. Diese zweite Begriffsbestimmung ist ca. 10 Jahre nach der erstgenannten entstanden. Sie verdeutlicht die Bedeutsamkeit der Beschäftigung mit der Entwicklung des Diskurses um den historischen Vergleich. Sie soll nachfolgend aufgezeigt werden. In der zweiten Begriffsbestimmung findet sich ein weiterer Punkt, der ebenfalls als eine Weiterentwicklung der ersten Bestimmung eingeordnet werden kann. Weist Kaelble für den Vergleich zunächst das Feld historischer Gesellschaften als inhaltliche Grundlage aus, nennt er in der zweiten Bestimmung historische Einheiten als Vergleichsfeld. Die Beispiele orientieren sich am Feld der historischen Gesellschaften. Dennoch fällt auf, dass er den Begriff der historischen Einheit in dieser zweiten Bestimmung nicht verwendet, dies spricht für eine Öffnung des Vergleichsbegriffs.

Eine Bestimmung der methodischen Funktion des Vergleichs findet sich bei Haupt/Kocka: Sie unterscheiden „unterschiedliche methodische Zwecke“, auf die der historische Vergleich angelegt sein kann. Sie differenzieren in vier verschiedene Perspektiven oder Verfahren, die sie als heuristisch, deskriptiv, analytisch, paradigmatisch benennen. ${ }^{285}$

Haupt/Kocka subsummieren unter der heuristischen Perspektive solche Vergleiche, die es erlauben, „[...] Probleme und Fragen zu identifizieren, die man ohne inn nicht oder nur schwer erkennen oder stellen würde. ${ }^{\prime 286}$ Der Zweck des Vergleichs ist es also, neue Forschungsfelder aufzudecken und so einen begrenzten Erkenntnisstand weiterzuentwickeln.

Die deskriptive Perspektive wiederum dient der Profilierung einzelner Fälle: „So drängt sich die Einsicht, daß die deutsche Arbeiterbewegung relativ früh als eigenständige Kraft einschließlich zugehöriger Partei auftrat, vor allem dann auf, wenn man sie mit anderen Arbeiterbewegungen vergleicht $[\ldots]$. “287

Die analytische Perspektive dient der „Erklärung historischer Sachverhalte“. ${ }^{288}$ Ebenso sollen darüber gängige Erklärungen kritisiert werden können. Gerade diese Perspektive wird im Hinblick auf den Konstruktionscharakter von Geschichte und das Ziel, Geschichtsbewusstsein auszubilden, bedeutsam für den Geschichtsunterricht sein. Gerade aber auch in Bezug auf die geschichtswissenschaftliche Herstellung oder Prüfung von Urteilen über einen Vergleich spielt die analytische Perspektive eine wesentliche Rolle.

In paradigmatischer Perspektive öffnet der Vergleich „[...] den Blick für andere Konstellationen, er schärft das Möglichkeitsbewußtsein des Historikers und läßt den jeweils interessierenden Fall als eine von mehreren Möglichkeiten erkennbar werden. ${ }^{1289} \mathrm{Er}$ öffnet also den Blick für Alternativen und regt zur Reflexion an.

Es ist davon auszugehen, dass ein Vergleich auf mehrere Zwecke hin angelegt ist. Der Blick auf die Zweckgebundenheit des Vergleichs verbindet sich eng mit dem Ansatz dieser Arbeit, die dem Vergleich

\footnotetext{
${ }^{285} \mathrm{Vgl}$. Haupt, Heinz-Gerhard; Kocka, Jürgen (1996): Historischer Vergleich: Methode, Aufgaben, Probleme. Eine Einleitung. In: Haupt, Heinz-Gerhard; Kocka, Jürgen: Geschichte und Vergleich. Ansätze und Ergebnisse international vergleichender Geschichtsschreibung. New York: Campus Verlag. S. 9-45. S. $12 \mathrm{ff}$.

${ }^{286}$ Ebd. S. 12.

${ }^{287} \mathrm{Ebd}$.

${ }^{288}$ Ebd. S. 13.

289 Ebd. S. 14.
} 
innewohnenden sprachlichen Handlungen zu explizieren. Diese sind grundlegend abhängig vom übergeordneten Zweck des Vergleichs.

Kaelble spricht im Gegensatz zu Haupt/Kocka nicht vom Zweck, sondern von der Intention des Vergleichs und leitet daraus ebenfalls vier Typen ab: den analytischen, den aufklärenden, den verstehenden und den Identitäts-Vergleich. ${ }^{290}$ Diese Vergleichstypen unterscheiden sich von den über den Zweck generierten Perspektiven bei Haupt/Kocka. So soll der aufklärende Vergleich positive und negative Entwicklungen gegenüberstellen und darüber Fehlentwicklungen erklären. Der verstehende Vergleich wiederum soll zu einem besseren Zugang zur Geschichte anderen Gesellschaften ermöglichen, der Identitätsvergleich soll über den Vergleich mit anderen Ländern Mythen über das eigene Land testen. ${ }^{291}$ Lediglich der analytische Vergleich findet sich sowohl bei Haupt/Kocka als auch bei Kaelble. Kaelble bestimmt inn als Vergleich, „[...] der die Ursachen für bestimmte Prozesse, Strukturen, Mentalitäten, Institutionen oder Ereignisse herausfinden will, ohne damit eine Bewertung der verglichenen Gesellschaften zu verbinden. ${ }^{\text {292 }}$ Wie bei Haupt/Kocka geht es also um die Erklärung von Sachverhalten. Diese Begriffsbestimmungen sind sich recht nah, wenngleich Haupt/Kocka auch die Möglichkeit explizieren, über den analytischen Vergleich gängige Erklärungen zu kritisieren. Die Unterschiede in der Typenbildung lassen sich über verschiedene Blickwinkel erklären: Während Kaelble über den Begriff der Intention insbesondere auf die Absicht eines Sprechers oder Schreibers schaut, sprechen Haupt/Kocka von einer Perspektive und klären über den Zweck die unterschiedlichen Erkenntnisziele des Vergleichs. Ihr Blickwinkel ist dadurch auf einen Hörer oder Leser ausgerichtet.

Für diese Arbeit ist der analytische Vergleich maßgeblich, so dass mit beiden Begriffsbestimmungen grundsätzlich weitergearbeitet werden kann, insbesondere aber der Ansatz von Haupt/Kocka ertragreich erscheint.

\subsubsection{Historisches Vergleichen - ein kurzer Blick in die Geschichte}

Um die Begriffsbestimmung und methodische Funktionen des Vergleichs besser einordnen und mit den Anforderungen an das Vergleichen im Geschichtsunterricht zusammenführen zu können, schließt sich an dieser Stelle ein kurzer Rückgriff auf die Entwicklung des Diskurses um das historische Vergleichen an.

\subsubsection{Entwicklung im 18./19. Jahrhundert}

Der Vergleich hat sich bis heute zu einer wesentlichen Methode historischen Arbeitens entwickelt. Zeichnet man diese Entwicklung nach, kann man insbesondere im 18./19. Jahrhundert einen Entwicklungsschub für den Vergleich konstatieren. Die Zunahme der Bedeutsamkeit des Vergleichs geht dabei

\footnotetext{
${ }^{290}$ Kaelble, Hartmut (2002), S. $304 f$.

291 Vgl. ebd.

292 Ebd. S. 304.
} 
einher mit dem Umbruch im Wissenschaftssystem dieser Zeit, der aufkommenden Bedeutung von Empirie und dem Infragestellen des theologischen Weltbildes, wenngleich eine lange Tradition des Vergleichs erkennbar ist:

„Die Wahrheiten sind nun eben nicht mehr prinzipiell vorentschieden, weil die Welt logisch strukturiert und begrifflich erschließbar ist. So ist es dieser historischen Orientierung auch geschuldet, dass etwa die philosophischen Schulen der Logik und Rhetorik, die in ihrer aufklärerischen Ausprägung gerade im deutschen Sprachraum in der ersten Hälfte des 18. Jahrhunderts, und damit unmittelbar vor dem hier in den Blick genommenen Zeitraum, eine Hochzeit hatten, kaum zur Sprache kommen - obwohl Vergleich und Analogie in diesen Traditionen tragende Rollen spielen. ${ }^{\prime 293}$

Der historische Vergleich geht damit auf die Logik als wesentlichen Stützpfeiler der Philosophie zurück. Unter dem Sammelbegriff Logik sind verschiedene Richtungen subsummiert. Dabei entwickelte sich die klassische Logik in unmittelbarer Nähe zur Rhetorik. Als Gemeinsamkeit kann man (als sicherlich sehr grobe Zuordnung) das Argument als wichtiges Grundelement nennen, über das Zusammenhänge hergestellt und begründet werden können. Immer geht es um die Herstellung einer Folgerichtigkeit mittels argumentativer Strukturen. Dies ist auch Aufgabe des Vergleichs, darüber erklärt sich im Weiteren die zuvor vorgenommene Einordnung des Vergleichs als spezielle Form der Argumentation. Über das Gegenüberstellen und Einordnen des Gegenübergestellten können Folgerungen abgeleitet werden. Damit ist der Vergleich jedoch noch nicht als spezifisch historische Form gekennzeichnet. Eggers kennzeichnet den Umbruch im 18./19. Jahrhundert dahingehend, dass die Welt sich als eben nicht logisch erschließbar zeigte. ${ }^{294}$ Dieser Einfluss wird auch in der Ausgestaltung des historischen Vergleichs deutlich: Eggers zeigt auf, dass die Leistung des Vergleichs für die Wissenschaft des 18./19.Jahrhundert neben der Belegbarkeit von Aussagen darin liegt, Strukturen abbilden, also klassifizieren zu können. Er spricht in diesem Zusammenhang vom „klassifizierungswütigen 18. Jahrhundert“. 295 Diese Strömung machte auch nicht vor der Geschichtswissenschaft halt, mehr noch findet sie ihren Niederschlag bis in die Philosophie Kants ${ }^{296}$.

Trotz des Entwicklungsschubs im 18./19. Jahrhundert konstatiert Kocka, dass der systematische Vergleich insgesamt eine vergleichsweise geringe Rolle gespielt habe. Trotz dieser langsamen Entwicklung ist das 18./19.Jahrhundert für den Vergleich bedeutsam, da grundlegende Kategorien (Vergleich und Analogie) ausgebildet wurden. Diese sollen im folgenden Kapitel aufgezeigt werden ${ }^{297}$.

\footnotetext{
${ }^{293}$ Eggers, Michael (2011): Vom Wissen zur Wissenschaft. Vergleich, Analogie, Klassifikation als wissenschaftliche Ordnungsmethoden im 18. Und 19. Jahrhundert -zur Einleitung. In: Eggers, Michael (Hrsg.): Von Ähnlichkeiten und Unterschieden. Vergleich, Analogie und Klassifikation in Wissenschaft und Literatur (18./19. Jahrhundert). Heidelberg: Universitätsverlag Winter. S. 7 - 31. S. 8.

${ }^{294}$ Im Fall des Galileo Galilei zeigt sich dieser Bruch sehr deutlich. Während die kirchliche Position, die Sonne drehe sich um die Erde, rein argumentativ vertreten und damit logisch und auch folgerichtig hergeleitet wurde, wählt Galilei einen anderen Zugang zum Nachweis seiner Theorie, die Erde drehe sich um die Sonne: über die Erforschung und den Nachweis des Phänomens mithilfe des Fernglases. Hier zeigt sich ein ganz anderer LogikAnsatz, der bis heute die Wissenschaft prägt.

${ }^{295}$ Eggers, Michael (2011), S. 22.

${ }^{296}$ Vgl. dazu Eggers, Michael (2011), S. 25.

297 Drüding/Schlutow gehen von fünf Phasen wissenschaftlicher Reflexionen des Vergleichs aus und beginnen ihre Einordnung mit der Frühen Neuzeit. Sie unterscheiden den Vergleich als analytisches und als heuristisches
} 


\subsubsection{Vergleichen versus Analogien bilden: Ausgangspunkte zur Kategorisierung}

Um die Entwicklung und Ausgestaltung des historischen Vergleichs ausgehend vom 18./19. Jahrhundert nachzuvollziehen, ist die Berücksichtigung einer weiteren Perspektive auf den Vergleich notwendig. Dazu müssen der Vergleich bzw. das Vergleichen von Analogie und Analogiebildung als wichtige Strukturierungsmethode des 18./19. Jahrhunderts abgrenzt werden. Eggers betrachtet Vergleich und Analogie im Hinblick auf die Strukturierungsleistung beider Vorgehensweisen:

„Die Analogie behauptet sich trotz der aufklärerischen Standards von Wissenschaftlichkeit und gelangt vor allem durch die Kombination empirischer und metaphysischer Weltwahrnehmung zu neuer Aktualität. Das Vergleichen ist hingegen das im Blick auf das gesamte System der Wissenschaften prominentere und erfolgreichere Modell, da es zahlreiche Disziplinen generiert und erkennbar als methodisches Kriterium einer Wissenschaftlichkeit dient, die sich auf die Evidenz der signifikanten Unterschiede und der daraus entstehenden Ordnungssysteme verlässt. “298

Eggers zeigt, dass es immer wieder um Organisation und Strukturierung von Wissen und in diesem Zusammenhang um Eindeutigkeit bzw. Nachvollziehbarkeit und vor allem Belegbarkeit geht. Der Weg der Wissenschaft dieser Zeit geht weg von normativen wissenschaftlichen Strukturen und hin zu Empirie und Überprüfbarkeit.

Roggenbruck zieht die Grenze zwischen Vergleich, Analogie und Klassifikation, aus Beispielen der Sprachwissenschaft im 19. Jahrhundert hergeleitet, noch trennschärfer als Eggers, wenn sie die Analogie als Ausgangpunkt wissenschaftlicher Annahmen und den Vergleich als Methode zur Überprüfung dieser skizziert, welche in der Folge zur Klassifikation führt. ${ }^{299}$ Sie konstatiert:

„Analogie kann verstanden werden als (1) Homologie von Proportionen oder als (2) Ähnlichkeiten zwischen Elementen. Versteht man sie als Ähnlichkeiten, so ist sie ein Anfangsmoment wissenschaftlicher Induktion, dem sich eine methodische Überprüfung zur Rechtfertigung der angenommenen Analogie anschließt. ${ }^{\text {" }} 00$

Die methodische Überprüfung nimmt dann der Vergleich vor. Diese Abgrenzung macht deutlich, warum gerade dem Vergleich eine hohe Relevanz für die wissenschaftliche Arbeit zugesprochen wurde. Problematisch erscheint in der heutigen Forschung der Ausgangspunkt des Vergleichs aus der Analogiebildung, da der Vergleich sowohl Gemeinsamkeiten als auch Unterschiede darstellen kann und soll. ${ }^{301}$ Dennoch zeigt sich in dieser Abgrenzung die Leistung des Vergleichs.

Mittel, als wissenschaftliche Methode, als Randerscheinung und als Reflexionsrahmen (vgl. Drüding, Markus; Schlutow, Martin (2019): Vergleich(en) im Geschichtsunterricht. Frankfurt a. M.: Wochenschau Verlag. S. 10ff.). Im Rahmen dieser Arbeit wird eine Strukturierung vorgenommen, die die fünf Phasen beginnend mit dem 18./19. Jhd. in 3 Kategorien zusammenfasst.

${ }^{298}$ Eggers, Michael (2011), S. 22.

${ }^{299}$ Roggenbruck, Simone (2011): Analogie als Ausgangspunkt für Vergleich und Klassifikation. Mit Beispielen aus der Sprachwissenschaft des 19. Jahrhunderts. In: Eggers, Michael (Hrsg.): Von Ähnlichkeiten und Unterschieden. Vergleich, Analogie und Klassifikation in Wissenschaft und Literatur (18./19. Jahrhundert). Heidelberg: Universitätsverlag Winter. S. 80-90. S. 87.

${ }^{300}$ Ebd.

${ }^{301}$ Vgl. dazu: Riekenberg, Michael (2008): Der Vergleich. In: Mayer, Ulrich; Pandel, Hans-Jürgen; Schneider, Gerhard (Hrsg.): Handbuch Methoden im Geschichtsunterricht. Schwalbach/Ts. S. 269-285. S. 272. 


\subsubsection{Die Frage nach der Vergleichbarkeit von Geschichte}

In die Diskussion um den historischen Vergleich gehörte gerade im 18./19. Jhd. auch die Frage um die Wiederholbarkeit von Geschichte. Hier können zwei unterschiedliche Positionen festgestellt werden: die Position, Geschichte sei nicht wiederholbar, und demgegenüber die Annahme, dass „[...] die These von der Unwiederholbarkeit geschichtlicher Vorgänge möglicherweise für politische Vorgänge zutreffe, weniger plausibel dagegen für wirtschaftliche und soziale Strukturzusammenhänge oder für kulturelle bzw. mentale Konstellationen sei.“" ${ }^{302}$ Für die Anhänger dieser zweiten Position wurde der Vergleich zu einem bedeutsamen Mittel der Überprüfbarkeit von historischen Aussagen, gerade im Vergleich zu den Naturwissenschaften, deren Ergebnisse wiederholbar und überprüfbar waren. ${ }^{303}$ Allerdings zeigten sich in der Folge Schwierigkeiten, die der Vergleich mit sich brachte. Middell beschreibt vier Probleme: Dazu gehört etwa die Frage nach der Herstellung von Korrelationen, die nur auf der Grundlage von Annahmen herzustellen waren, nicht aber auf einer eindeutigen Datenlage wie in den Naturwissenschaften. ${ }^{304}$ Eine weitere Frage stellte sich hinsichtlich der Vergleichbarkeit kultureller Phänomene: „Wie sollte man kulturelle Konstellationen so formatieren, dass sie sich wie soziale Strukturen vergleichen ließen?“305 Ein drittes Problem zeigte sich hinsichtlich der Interferenz zwischen den Vergleichsobjekten, was bedeutete, dass die Vergleichsobjekte nicht isoliert voneinander zu betrachten waren, sondern voneinander abhingen. Mit diesem dritten sieht Middell das vierte Problem verknüpft, nämlich die Frage, welche Einheiten überhaupt in Beziehung gesetzt werden dürften. ${ }^{306}$ Diese Frage wurde mit den größten Nationalstaaten, Regionen und Zivilisationen beantwortet ${ }^{307}$. Die Weiterentwicklung einer Antwort auf diese Frage spiegelt sich auch im Anfangszitat dieses Kapitels von Kaelble wider, der von historischen Gesellschaften bzw. in der Folge von historischen Einheiten spricht, die er insbesondere auf Orte, Regionen, Nationen oder Zivilisationen und historische Persönlichkeiten bezieht. Die Diskussion des 18./19. Jhd. und die dort aufgeworfenen Fragestellungen wirken also bis in die Gegenwart nach und zeigen die Weiterentwicklung des historischen Vergleichs. Die von Middell dargestellten Probleme hinsichtlich des historischen Vergleichs beschreiben Herausforderungen in allen Grundelementen des Vergleichs: hinsichtlich Vergleichsobjekt, Vergleichsgröße, aber auch, darauf weisen insbesondere das zweite wie auch das dritte Problem hin, in Bezug auf das Tertium Comparationis. $^{308}$

Aus diesen Problemen erwachsen Ansprüche an den historischen Vergleich für die Gegenwart:

\footnotetext{
302 Middell, Matthias (2005): Konjunkturen vergleichender Geschichtswissenschaft. In: Schreiber, Waltraud (Hrsg.): Der Vergleich - Eine Methode zur Förderung historischer Kompetenzen. Ausgewählte Beispiele. Neuried: ars una. S. 11 - 30. S. 12.

${ }^{303}$ Vgl. ebd. S. 13.

${ }^{304}$ Ebd. S. 14.

${ }^{305}$ Ebd. S. 16.

${ }^{306}$ Ebd. S. 18.

${ }^{307} \mathrm{Vgl}$. ebd.

308 Zur Schwierigkeit, ein tragfähiges Tertium Comparationis zu isolieren, vgl. auch Welskopp, Thomas (2007): Erklären, begründen, theoretisch begreifen. In: Goertz, Hans-Jürgen (Hrsg.) (2007): Geschichte. Ein Grundkurs. 3. rev. u. erw. Auflage. Reinbek bei Hamburg: Rowohlt. S. 137-177. S. 158.
} 
„Der vergleichende Historiker ist gezwungen, seine systematischen Begriffe im Forschungsprozess laufend zu revidieren. Weil die abstrahierende Begriffs- und Theoriekonstruktion und die historische Rekonstruktion des ,Materials' Hand in Hand gehen, muss der Komparatist die Verfahren der wissenschaftlichen Begriffs- und Theoriebildung ebenso gut kennen wie die historischen Quellen und die Literatur." 309

Bereits für das 18./19. Jhd. zeigt sich also, wie anspruchsvoll historisches Vergleichen ist. Dieser hohe Anspruch setzt sich auch für die Weiterentwicklung fort.

\subsubsection{Die geschichtswissenschaftliche Debatte um den Vergleich in der neueren und neuesten Zeit - ein kurzer Überblick}

Die Debatte um den Vergleich ist als anhaltend zu kennzeichnen. Einen Sprung in der Chronologie vollziehend, kann ab den 1970er Jahren allerdings ein erneuter Aufschwung des Vergleichs festgestellt werden. Neben amerikanischen historischen Sozialwissenschaftlern ordnet Kaelble Charles Tilly, die Historiker Marc Bloch, Henri Pirenne, Otto Hintze wie auch Karl Lamprecht als Wegweiser für die Komparatistik $^{310}$ ein.

Wesentliche Impulse für den historischen Vergleich ergaben sich aus den Sozialwissenschaften. Tilly bspw. prägte aus sozialwissenschaftlicher Blickrichtung den Begriff des ,encompassing comparison':

„Encompassing comparisons begin with a large structure or process. They select locations within the structure or process and explain similarities or differences among those locations as consequences of their relationship to the whole"311

Tilly stellt damit zunächst einen generalisierenden Zugriff des Vergleichs in den Mittelpunkt, aus dem er im Sinne eines individualisierenden Vergleichs den Blick auf kleinere Einheiten ableitet. Dabei steht die Nationalgeschichte im Mittelpunkt. Dieser Zugriff erscheint kennzeichnend für die Weiterentwicklung des Vergleichs unter Mitwirkung der Sozialwissenschaften.

Mit dem Einfluss der Sozialwissenschaften geht die Entwicklung der Sozialgeschichte und damit eine starke Verbindung von Geschichte und Sozialwissenschaft einher. Allerdings kaufte man sich mit dem damit verbundenen stark nationalgeschichtlichen Zugriff neue Schwierigkeiten ein, woraus insgesamt eine Verengung des Vergleichs resultierte, da es insbesondere um den Vergleich mit Deutschland ging. Welskopp kritisiert den sozialgeschichtlichen Vergleich: „Das auf Deutschland gerichtete Erkenntnisinteresse rechtfertigte sogar so extrem ungleichgewichtige, das heißt ,asymmetrische' Vergleichskonstruktionen, dass der Vergleichspartner eigentlich nur als randständige Kontrastfolie vorkam. “312

Dieses enge Vergleichskorsett führte zu Kritik am historischen Vergleich, die wiederum zu einer Weiterentwicklung weg von der starken Hinwendung zu den Nationalstaaten als Vergleichselemente und so zu einer Öffnung führte.

\footnotetext{
${ }^{309}$ Siegrist, Hannes (2003): Perspektiven der vergleichenden Geschichtswissenschaft. Gesellschaft, Kultur und Raum. In: Kaelble, Hartmut; Schriewer, Jürgen (Hrsg.): Vergleich und Transfer. Komparatistik in den Sozial-, Geschichts- und Kulturwissenschaften. Frankfurt a. M.: Campus Verlag. S. 303-339. S. 320.

310 Vgl. ebd.

${ }^{311}$ Tilly, Charles (1984): Big Structures, Large Prozess, Huge Comparisons. New York: Russel Sage Foundation. S. 125.

312 Welskopp, Thomas (2010): Vergleichende Geschichte. In: Europäische Geschichte Online (EGO), hrsg. vom Institut für Europäische Geschichte (IEG), Mainz 2010-12-03. Online einsehbar unter: http://iegego.eu/de/threads/theorien-und-methoden/vergleichende-geschichte (zuletzt eingesehen am 22.05.2020).
} 
Marc Bloch hatte den Vergleich als historische Operation schon Ende der 1920er Jahre mitgeprägt ${ }^{313}$, auch in der Debatte der 1990er Jahre war seine Perspektive maßgeblich. Bloch macht dabei die Felder auf, die seiner Meinung nach durch den Vergleich abgedeckt werden können:

"Was bedeutet zunächst einmal „vergleichen“ in unserer Wissenschaftsdisziplin? Unbestreitbar das folgende: aus einem oder mehreren verschiedenen sozialen Milieus zwei oder mehr Phänomene auszuwählen, die scheinbar auf den ersten Blick gewisse Analogien aufweisen, den Verlauf ihrer Entwicklung zu beschreiben, Ähnlichkeiten und Unterschiede festzustellen und diese soweit wie möglich zu erklären. ${ }^{\text {3114 }}$

Bloch fokussiert damit die sozialen Milieus als tragbare Vergleichsgrundlage und geht damit ebenfalls weg von einer Nationalgeschichte. Er stellt die Suche nach einer Analogie an den Anfang und nutzt dann den Vergleich zur Klärung dieser.

Als Kritiker der nationalgeschichtlichen Form des Vergleichs ist auch Michel Espagne zu nennen. Er geht über die von Bloch fokussierten sozialen Milieus hinaus. Zur Einordnung der Kritik Espagnes stellt Kaelble fest:

„Er plädierte deshalb dafür, den historischen Vergleich durch die historische Transferuntersuchung, also die Untersuchung der Übertragung von Ideen und Werten, des Austauschs von Waren, der Migration von Menschen von einer Gesellschaft zur anderen zu ersetzen, die die Geschichtswissenschaft für internationale kulturelle Verflechtungen und für die Kulturgeschichte von Erfahrungen und Handlungspraxen öffne. ${ }^{.315}$

Die Kritik Espagnes weist auf einen wesentlichen Aspekt in der Debatte der 1990er Jahre hin: Espagne plädiert statt für einen Vergleich für einen Transfer. Die Diskussion der 90er Jahre treibt von der Diskussion um den Vergleich ausgehende Konzepte wie den Transfer, die "entangled history“ oder die "histoire croisée“ voran, verkürzt gesagt alles Konzepte transkultureller Beziehungsgeschichte, die auf Verbindungen und Austausch zielen und den klassischen Vergleich kritisieren. Ein weiteres Konzept, das dem Ansatz der „histoire croisée“ nahesteht, verknüpft Vergleich und Transfer aus der Idee heraus, die Schwächen der einzelnen Konzepte über die Verbindung beider auszugleichen:

„Darüber hinaus brauchen Transferuntersuchungen und Vergleiche einander und ergänzen sich.

Vergleiche brauchen Transferuntersuchungen, weil Transfers meist ein wichtiger Faktor für Annä-

herungen oder auch für Divergenzen sind. Ohne Transferuntersuchungen übersieht man daher eine wichtige Erklärung für Divergenzen oder Konvergenzen. Umgekehrt brauchen Transferuntersuchungen den Vergleich, weil nur durch den Vergleich festgestellt werden kann, worin sich die Ausgangskultur und die Aufnahmekultur eines Transfers unterscheiden und worin daher der Wandel, also der Kern des Transfers, tatsächlich besteht. ${ }^{\text {“316 }}$

Solcherlei Debatten haben die Entwicklung des historischen Vergleichs beeinflusst und sicherlich zu seiner Öffnung beigetragen. Die stetige Weiterentwicklung zeigt sich in den Begriffsbestimmungen Kaelbles: Hatte Kaelble 1999 den Blick auf das Gegenüberstellen insbesondere von Gesellschaften gerichtet, spricht er 2012 statt von Gesellschaften von historischen Einheiten, die gegenübergestellt werden.

\footnotetext{
${ }^{313} \mathrm{Vgl}$. z. B. Bloch, Marc: Pour une histoire comparée des sociétés européennes (1928), in: Ders., Mélanges historiques, vol. 1, Paris 1983. Zitiert nach: Kaelble, Hartmut (2005): Die Debatte über Vergleich und Transfer und was jetzt? In: H-Soz-u-Kult. Nr. 08. Online einsehbar unter: http://hsozkult.geschichte.hu-berlin.de/index.asp?id=574\&view=pdf\&pn=forum\&type=artikel (zuletzt eingesehen am 12.04.2018).

${ }^{314}$ Bloch, Marc (1994): Für eine vergleichende Geschichtsbetrachtung der europäischen Gesellschaften. In: Middel, Matthias; Sammler, Steffen (Hrsg.): Alles Gewordene hat Geschichte. Die Schule der Annales in ihren Texten. Leipzig: Reclam Verlag. S. 121-167. S. 122.

315 Kaelble, Hartmut (2012): Historischer Vergleich. https://docupedia.de/zg/Historischer_Vergleich (zuletzt eingesehen am 13.04.20209.

${ }^{316}$ Kelble, Hartmut (2005), S. 4.
} 
Mit dieser Einordnung erweitert er das Feld des historischen Vergleichs. Dabei ist nach wie vor der Begriff der Gegenüberstellung, die systematisch zu erfolgen hat, für den Vergleich grundlegend.

Die Debatte um den geschichtswissenschaftlichen Vergleich kann hier nicht weiter vertieft werden, da das Vergleichen im Unterricht im Mittelpunkt der Betrachtung steht, jedoch ist sie nach wie vor nicht abgeschlossen. Middell etwa geht davon aus, dass sich der historische Vergleich auch in Zukunft verändern wird, er vermutet:

Am häufigsten dürften in naher Zukunft Vergleiche sein, wie verschiedene Gesellschaften mit dem Zwang der Verflechtung umgehen: wie sie ihre ehemals nationalen Sozialsysteme unter dem Druck eines weltweiten Kapitalflusses umbauen, wie sie Migration bewältigen, wie sie die Balance zwischen Souveränität und Lernbereitschaft gegenüber Fremden austarieren. ${ }^{\text {(317 }}$

Der Vergleich ist eine wesentliche Methode historischen Arbeitens. Er ist inzwischen als etabliert anzusehen. Er hat sich stark entwickelt, und, folgt man etwa Middell, wird dies auch weiter tun, wenn er für die Geschichtswissenschaft ein bedeutsames Mittel bleiben soll. Kaelble formuliert noch stärker, wenn er aus der Überlegung, was den historischen Vergleich für die gegenwärtige Gesellschaft bedeutsam macht, zu dem Schluss kommt: „Historische Vergleiche aufgeben, hieße, sich einer wichtigen Verantwortung der Geschichtswissenschaft nicht mehr zu stellen. ${ }^{\text {“318 }}$

Auch wenn die Entwicklung des historischen Vergleichs im Rahmen dieser Arbeit nur überblickshaft dargestellt werden kann, dient sie als Grundlage für den Blick auf den Vergleich im Geschichtsunterricht. Selbst wenn Lernende oft von solchen Fachdebatten unbehelligt bleiben, sollte der schulischen Aufgabenstellung der fachwissenschaftliche Blick vorausgegangen sein, da nur darüber seine unterrichtliche Fachspezifik und die damit verbundene epistemische Funktion zu klären ist.

\subsection{Vergleichen im Geschichtsunterricht}

Was leistet ein Vergleich für den schulischen Lernprozess im Allgemeinen und für den Geschichtsunterricht im Besonderen? Am Beispiel eines Auszugs aus einem Schülerforum soll dies expliziert werden. Dabei wird im Folgenden nur der Teil der Frage verwendet, der sich auf das Vergleichen bezieht.

\subsubsection{Ein Beispiel aus einem Schülerforum}

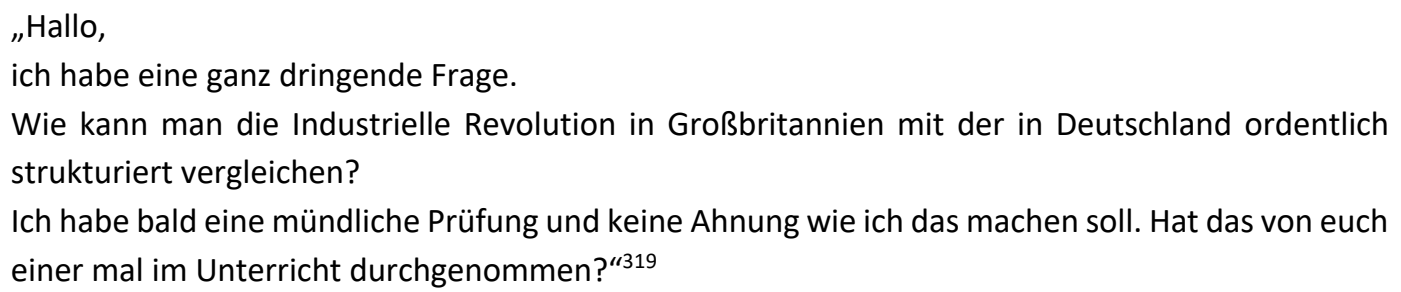

\footnotetext{
317 Middell, Matthias (2005), S. 29.

318 Kaelble, Hartmut (2012), S. 10.

${ }^{319}$ Aus: http://www.schueler-talk.de/geschichte-f25/industrielle-revolution-vergleich-deutschland-engl-

t1737.html (zuletzt eingesehen am 09.10.15).
} 
Diese bereits 2008 im Schülerforum "Schülertalk" gestellte Frage weist darauf hin, dass das Vergleichen bis in die Prüfungsaufgaben hinein eine Rolle im Geschichtsunterricht spielt. ${ }^{320}$

Auch die sich diesem Kapitel anschließende Auswertung von Lehrwerken im Hinblick auf die Häufigkeit von Vergleichsaufgaben bietet ein weiteres Indiz dafür, dass solche Aufgaben im Geschichtsunterricht eine (vermutlich nicht unerhebliche) Rolle spielen. ${ }^{321}$

In weiterer Konsequenz kann das Vergleichen, wenn es überall in der Lebenswirklichkeit der Schüler*innen eine Rolle spielt, auch im Geschichtsunterricht nicht ausgeblendet werden.

Diese Vorüberlegungen führen zu der Annahme, dass im Geschichtsunterricht häufig verglichen wird. Über den Vergleich wird Orientierung (z. B. zwischen verschiedenen Zeiten) hergestellt. Auch Schreiber unterstützt diese These, wenn sie feststellt:

„Der einzelne Geschichtslehrer ist nicht frei mit seiner Entscheidung, sich mit historischen Vergleichen, Transformationen und Beziehungen zu befassen.

Die Lebenspraxis hat ihm diese Entscheidung nämlich längst abgenommen. Vergleiche, mit denen Veränderungen erklärt werden sollen, bzw. Vergleiche, die Beziehungen zwischen den Zeiten herstellen oder Orientierung schaffen sollen, sind überall präsent. “"1322

Wenn Kaelble für die fachwissenschaftliche Diskussion feststellt, dass historisches Vergleichen bedeute, Geschichtswissenschaft verantwortlich zu betreiben, so stellt sich die Geschichtslehrkraft eben der Herausforderung, Vergleiche zu didaktisieren, um die Lernenden kompetenzorientiert an einen fachgerechten Umgang mit historischen Vergleichen heranzuführen. Schreiber argumentiert diesen Zusammenhang über die Bedeutung des Vergleichens in der Lebenspraxis, also als eine Art Reaktion auf die Präsenz von Vergleichen in der Lebenswirklichkeit. Darüber hinaus soll das Vergleichen im Geschichtsunterricht im Sinne des Kernlehrplans „zur kompetenten und kritischen Teilhabe an der Geschichtskultur ${ }^{\prime 323}$ und damit zur Entwicklung von Urteilsfähigkeit ${ }^{324}$ befähigen. Die Begründung für das Vergleichen im Geschichtsunterricht stellt damit nicht nur eine Antwort auf die lebensweltliche Praxis dar, sondern vielmehr ist der Vergleich eine wichtige Methode für die Ausbildung von Geschichtsbewusstsein.

Am Beispiel der Nachfrage aus dem Schülerforum stellt sich die Frage, was den Vergleich im Geschichtsunterricht ausmacht. Gibt es überhaupt eine feste „Form“ des Vergleichens, ähnlich dem Vorgehen bei Analysen im Geschichtsunterricht?

Der Fragende ${ }^{325}$ aus dem Schülerforum weist genau darauf hin: Wie wird ein Vergleich im Geschichtsunterricht aufgebaut?

Hinweis: Die Seite ist inzwischen nicht mehr aktiv, kann aber über folgende Internet-Archiv-Adresse aufgerufen werden: https://web.archive.org/web/20130420021414/http://www.schueler-talk.de/geschichte-f25/industrielle-revolution-vergleich-deutschland-engl-t1737.html (zuletzt eingesehen am 18.07.18).

${ }^{320}$ Hinweis: an diese erste Frage schließt sich eine zweite zum Thema „Argumentation“ an, die in diesem Zusammenhang nicht mehr berücksichtigt werden kann. Es handelt sich hierbei also nur um einen Auszug aus der Gesamtfrage.

321 Inwiefern Vergleichsaufgaben in der Abiturprüfung eingesetzt werden, könnte einen weiteren Untersuchungsaspekt darstellen und die vorgenannten Überlegungen vertiefen, jedoch soll dieser Aspekt in diesem Zusammenhang nicht weiterverfolgt werden.

${ }^{322}$ Schreiber, Waltraud (2005), S. 34.

${ }^{323} \mathrm{Vgl}$. Kernlehrplan Gymnasium Geschichte NRW für die Sekundarstufe I: Ministerium für Schule und Weiterbildung des Landes Nordrhein-Westfalen (Hrsg.) (2007). S.16.

${ }^{324}$ Ebd.

${ }^{225}$ Da nicht ersichtlich ist, ob der Beitrag von einem männlichen oder weiblichen Nutzer geschrieben wurde, wird im Folgenden durchgängig genderunabhängig die männliche Form verwendet. 
Dabei muss der Überlegung, wie ein Vergleich aufgebaut ist, die Frage nach dem Zweck vorangestellt werden. Welchen Zweck erfüllt beispielsweise der Vergleich der Industriellen Revolution in Großbritannien und Deutschland? Erst danach kann auch die Frage nach der Struktur sinnvoll beantwortet werden. Die Frage nach dem Zweck ist wiederum eng verbunden mit der Frage nach dem der Aufgabe zugrundeliegenden Vergleichstyp. Damit ist bereits eine erste Antwort auf die Frage nach einer festen Form des Vergleichs gegeben: Wenn sich der Vergleich am Zweck und damit an einer Typologie orientiert, kann es nicht eine feste Form geben.

Im Fall der Vergleichsaufgabe aus dem Schülerforum handelt es sich um einen analytischen Vergleich, weil er historische Sachverhalte klären soll, also „[...] Erklärungen für ein historisches Phänomen durch die vergleichende Analyse unterschiedlicher Fälle entwickelt". ${ }^{326}$ Dies ist auch zugleich der Zweck. Konkret für dieses Beispiel würde dies bedeuten, über den Vergleich herauszufinden, welche Gemeinsamkeiten, Ähnlichkeiten und Unterschiede sich in der Entwicklung der Industriellen Revolution in beiden Ländern zeigen. Strukturell heißt dies, dass die Entwicklungen nach Kategorien geordnet werden müssen, über die Gemeinsamkeiten, Ähnlichkeiten und Unterschiede gezeigt werden können. Im Hinblick auf die historische Dimension der Zeit müsste neben dem räumlich orientierten Vergleich (Großbritannien/Deutschland) auch ein logischer zeitlicher Zusammenhang hergestellt werden (z. B. chronologisch, dies könnte zu Aussagen führen, dass die Industrielle Revolution in Großbritannien früher gestartet sei). Auf der Grundlage dieser Zuordnung könnte nun eine kleinschrittige Struktur erarbeitet werden. Für die dieser Arbeit zugrundeliegende Vergleichsaufgabe (die der Aufgabe in der Frage sehr ähnlich ist) ist es ein Anliegen, dies nachfolgend zu leisten.

\subsubsection{Eine Antwort auf die Frage im Schülerforum}

Interessant erscheinen in diesem Zusammenhang die Antworten, die auf die Frage im Forum angezeigt werden.

Eine erste Antwort, die knapp vier Stunden nach der Frage gepostet wurde, lautet:

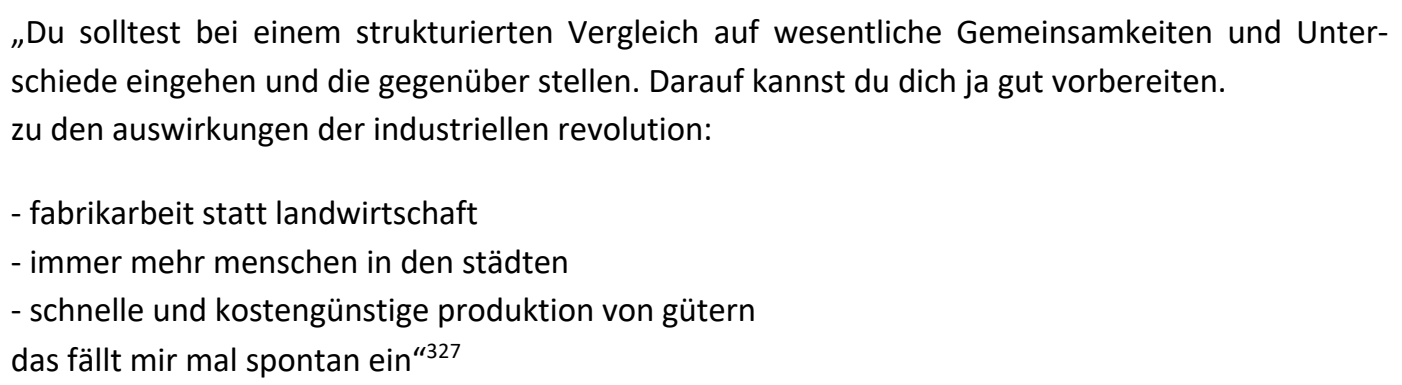

Der oder die Antwortende ${ }^{328}$ benutzt zunächst einmal den Begriff des "strukturierten Vergleichs“, der ja schon in der fachwissenschaftlichen Diskussion, wie zum Beispiel bei Kaelble, auftaucht. Was "strukturiert" in diesem Zusammenhang bedeutet, klärt er jedoch nicht. Dabei müsste expliziert werden, dass es sich um einen analytischen Vergleich handelt, an dem sich die Struktur orientiert, dass es also darum geht, historische Sachverhalte durch ein Gegenüberstellen zu erklären.

\footnotetext{
${ }^{326}$ Kaelble, Hartmut (2002), S. 304.

${ }^{327}$ Aus: http://www.schueler-talk.de/geschichte-f25/industrielle-revolution-vergleich-deutschland-engl-

t1737.html (zuletzt eingesehen am 09.10.15).

${ }^{328}$ Nachfolgend wird wieder genderunabhängig die männliche Form verwendet.
} 
Weiterhin geht er, auch entsprechend dieser Auseinandersetzung, von Gemeinsamkeiten und Unterschieden aus, die gegenübergestellt werden sollen. Es kann vermutet werden, dass er sich mit der Form des historischen Vergleichs beschäftigt hat, wenngleich der Hinweis auf Ähnlichkeiten fehlt. Interessant ist an dieser Stelle, dass dann eine weitere Aufschlüsselung des Vergleichs ausbleibt. Der Antwortende stellt nur fest, dass sich der Fragende darauf gut vorbereiten könne, gibt aber keine Hilfen an, wie dies geschehen könnte bzw. welche Fachliteratur zu Rate gezogen werden könnte. Vielmehr nennt er Aspekte in Bezug auf das Thema, die dem Antwortenden inhaltlich wesentlich erscheinen. Dabei fällt auf, dass er sich nur auf Auswirkungen bezieht, die er in einer Aufzählung darlegt. Es handelt sich also um kein Gegenüberstellen, das er in seiner Antwort als wesentlich herausstellt, sondern um eine Darstellung der Situation nach der Industriellen Revolution.

Eine Strukturhilfe, nach der im Schülerforum gefragt wurde, wie die Formulierung „ordentlich strukturiert" anzeigt, gibt der Antwortende nicht. Auch gibt er keine Vergleichsform vor, sondern reiht Stichpunkte untereinander. Dabei erhält der erste Stichpunkt durch die Präposition "statt“ einen Absolutheitscharakter, da dadurch zwei Größen ins Verhältnis gesetzt werden. Diese Aussage ist fachwissenschaftlich zu hinterfragen, da sie so nicht haltbar ist. Zwar stellt Fabrikarbeit ein wesentliches Element der Industrialisierung dar, die Landwirtschaft vollständig abgelöst hat sie dadurch dennoch nicht. Der Zuzug von immer mehr Menschen in die Städte im zweiten Stichpunkt ist zwar verkürzt, aber zutreffend. Die schnelle und kostengünstige Produktion von Gütern im dritten Punkt müsste durch Modalisierungsformen abgeschwächt werden. Sie ist für den Beginn der Industrialisierung zu allgemein. Da es sich um eine ausdrücklich spontane Zusammenstellung handelt, kann sie vom Leser nicht als vollständig angenommen werden, dies ist sie auch nicht.

Eine zweite Antwort, die hier nicht vertieft betrachtet werden soll ${ }^{329}$, lässt ebenfalls konkrete Strukturhilfen in Bezug auf das historische Vergleichen vermissen.

Wenn dem Schüler die Form eines solchen Vergleichs unklar ist, beide Antwortenden zudem seine Frage weitestgehend nicht lösen können, stellt sich die Frage, ob eine solche Form überhaupt geklärt ist.

Eine erste Antwort kann in der Fachliteratur und im Besonderen bei Riekenberg ${ }^{330}$ gefunden werden, der sich um eine solche Aufschlüsselung bemüht. Eine zweite Antwort wird anschließend in den Methodenseiten der Geschichtslehrwerke gesucht, die eine solche Klärung z. B. in ihren Methodenseiten herbeiführen müssten.

\subsubsection{Merkmale des Vergleichs im Geschichtsunterricht}

Um Merkmale des Vergleichs im Geschichtsunterricht aufzuarbeiten, soll wieder vom Zweck ausgegangen werden, zunächst allerdings im Hinblick auf einen Lernzuwachs im Sinne einer Kompetenzentwicklung bei Schüler*innen. Solche Zwecke (im didaktischen Zugriff wäre an dieser Stelle der Begriff des unterrichtlichen Ziels gängiger) nennt Riekenberg:

„Sie sollen lernen, wie ein systematischer Vergleich aufgebaut ist, welche Funktionen der Vergleich für das historische Lernen auszuüben vermag, welche Erkenntnisziele er zu erreichen mag, welche nicht, und welche Vor- und Nachteile er als Verfahrensweise besitzt. Oder anders formuliert: Aus

\footnotetext{
${ }^{329}$ Aus: http://www.schueler-talk.de/geschichte-f25/industrielle-revolution-vergleich-deutschland-englt1737.html (zuletzt eingesehen am 09.10.15).

${ }^{330}$ Riekenberg, Michael (2008).
} 
der Sicht eines methodenorientierten Geschichtsunterrichts ist das Ziel des Vergleichs das Vergleichen selbst." 331

Ausgehend von diesen Zwecken müssen nun die Merkmale konkretisiert und so der Vergleich in Anbindung an die fachwissenschaftlichen Standards als Methode expliziert werden.

Riekenberg geht davon aus, dass der Vergleich in der spärlichen Forschungslage insbesondere als „Dienstleister für das Transferlernen“332 ausgemacht werde, und schließt die Frage daran, ob er denn im Unterricht wirklich dazu genutzt werde. ${ }^{333}$ Dabei schließt sich der Transferbegriff bei Riekenberg nicht an die fachwissenschaftliche Diskussion an, vielmehr meint er damit Lernübertragungen, also didaktischen Transfer ${ }^{334}$. Da Untersuchungen zur Frage, wie der Vergleich im Unterricht genutzt wird, nicht vorliegen, muss die Frage offenbleiben. Er macht jedoch drei Typen von Vergleichen aus, die in der Praxis des Geschichtsunterrichts seiner Ansicht nach bedeutsam sind. Dazu unterscheidet er in den Vergleich als Thema einer Unterrichtseinheit, Vergleichsaufgaben, die in eine Unterrichtseinheit eingestreut werden, und den Vergleich mit vertrauten Elementen für jüngere Lerner*innen, um diese an den Geschichtsunterricht heranzuführen. ${ }^{335} \mathrm{Er}$ nimmt also eine sehr allgemeine Differenzierung vor. Ggf. könnte der Vergleich in einer weiteren Ausdifferenzierung bspw. als Einstieg in eine Unterrichtseinheit der Kategorisierung hinzugefügt werden, da er sowohl leitend für die Unterrichtseinheit sein kann, aber genauso gut nur einen Aspekt des historischen Lernens darstellen kann. In beiden Fällen sorgt er jedoch dafür, dass eine geschichtsspezifische Lernausganslage geschaffen wird. Diese drei Typen können bereits als Merkmale des Vergleichs ausgemacht werden. Allerdings hat diese Typenbildung nichts mit der Typenbildung der fachwissenschaftlichen Diskussion zu tun, die Riekenberg als Richtschnur für die Ausformung des Vergleichs im Geschichtsunterricht ansieht. ${ }^{336}$ Dabei müsste eine solche Typenbildung der Riekenbergs vorangestellt werden, da die Entscheidung über den Einbezug des Vergleichs in das Unterrichtssetting der Entscheidung über das Vergleichsziel nachgeordnet sein sollte. Das heißt, dass zuerst geklärt werden müsste, ob es sich, legt man Haupt/Kocka zugrunde, um einen analytischen, aufklärenden, verstehenden oder einen Identitäts-Vergleich handelt, bevor eine Entscheidung über das Setting getroffen wird. Ein weiteres Merkmal wäre also der Bezug auf den Vergleichstyp. Für den Geschichtsunterricht kann auf der Grundlage der Lehrwerksanalyse angenommen werden, dass insbesondere der analytische Vergleichstyp eine große Rolle spielt und insofern merkmalsgebend ist. ${ }^{337}$

Bedeutsam ist weiter das didaktische Ziel, das Riekenberg für den historischen Vergleich im Unterricht formuliert und über das sich weitere Merkmale explizieren lassen:

„Der Vergleich im Geschichtsunterricht ist Teil eines gerichteten, methodisch überprüften und kontrollierten Lernvorgangs. Eine Reihe von geschichtsdidaktischen Prinzipien können im Unterricht

\footnotetext{
${ }^{331}$ Riekenberg, Michael (2008), S.269.

${ }^{332}$ Vgl. Riekenberg, Michael (2008), S. 271.

${ }^{333}$ Ebd.

${ }^{334}$ Zum Transferbegriff im Geschichtsunterricht vgl. Schneider, Gerhard (2016): Transfer. In: Mayer, Ulrich; Pandel, Hans-Jürgen; Schneider, Gerhard (Hrsg.): Handbuch Methoden im Geschichtsunterricht. 5. Aufl. Schwalbach/Ts.: Wochenschau Verlag. S. 649-674.

${ }^{335}$ Vgl. Riekenberg, Michael (2008), S. $271 \mathrm{f}$.

${ }^{336}$ Vgl. Riekenberg, Michael (2008), S. 269.

${ }^{337}$ Die folgende Frequenzanalyse berücksichtigt keine Auszählung der Vergleichstypen, es handelt sich hier um eine Annahme, die durch die Beschäftigung mit Vergleichsaufgaben in Lehrwerken entstanden, jedoch nicht empirisch belegt ist.
} 
ohne den Vergleich gar nicht realisiert werden. Dies gilt für den Gegenwartsbezug historischen Lernens wie für das Lernen von Multiperspektivität und Kontroversität [...]. ${ }^{1338}$

Merkmale des Vergleichs sind demnach, dass es sich um einen systematischen Vorgang handelt und dass sich über inn wesentliche Elemente historischen Lernens realisieren lassen. Über die Methodenorientierung lassen sich Riekenberg folgend weitere Merkmale des Vergleichs im Geschichtsunterricht ableiten:

„Zweck des Geschichtsunterrichts ist aus dieser Sicht weniger das Lernen von Inhalten als vielmehr das ,Erlernen der logischen Figuren historischen Denkens'. Der Vergleich ist ein besonders geeigneter Gegenstand, um Methodenlernen wie -reflexion im Geschichtsunterricht zu betreiben, weil er ein stark selektives Verfahren ist. ${ }^{\prime \prime 33}$

Diese Einschätzung ist unmittelbar anschließbar an das didaktische Ziel. Es geht danach um eine Umund Neustrukturierung vorhandener Wissensstrukturen. Hier müssten aber die Vergleichsaufgaben und dem Vergleich zugrundeliegenden Materialien überprüft werden, ob sie genau dies anzuregen in der Lage sind oder Vergleiche vorgeben, so dass es sich letztlich doch „nur“ um die Übernahme vorgegebener Wissensstrukturen handelt.

Die dargestellten Merkmale sind ein Zusammenspiel aus Merkmalen, die inhaltliche Merkmale des didaktisierten Vergleichs im Geschichtsunterricht berücksichtigen, und solchen, die auf den Lern- bzw. Kompetenzzuwachs der Schüler*innen ausgerichtet sind.

Sie sind weiterhin bedeutsam zur Klärung der für diese Arbeit formulierten Frage, welche Fertigkeiten und Kompetenzen Lernende zur Lösung einer historischen Vergleichsaufgabe benötigen. Allerdings geben sie nur erste Hinweise, eine inhaltliche Füllung steht noch aus. Sie soll in Bezug auf die ausgewählte Aufgabe vorgenommen werden.

\subsubsection{Vergleichbares und Unvergleichbares im Geschichtsunterricht?}

Auf die Frage nach Vergleichbarem und Unvergleichbarem, die in der wissenschaftlichen Debatte eine große Rolle spielt, geben Riekenberg und Schreiber in Bezug auf den Geschichtsunterricht Antwort.

Riekenberg diskutiert ausgehend von einer eigenen Typenbildung dazu die Frage, was Vergleiche mit zweien und mit mehreren Vergleichsfällen leisten. Dabei geht er von Inhaltsvergleichen und Medienvergleichen aus, die er dahingehend unterscheidet, dass bei Inhaltsvergleichen historische Inhalte verglichen werden, während der Medienvergleich von unterschiedlichen historischen Medien ausgeht, die verglichen werden, um so zu einer kritischen Reflexion der verwendeten Medien zu gelangen. ${ }^{340}$ In beiden Fällen handelt es sich um einen systematischen Vergleich. Riekenberg nimmt an, dass Inhaltsvergleiche mit nur zwei Fällen den Blick auf Einzelfälle schärfen können, mehrere Fälle hingegen eher eine Allgemeingültigkeit zulassen. Der analytische Vergleichstyp lässt sich in den inhaltlichen Vergleich einordnen. Dies macht ihn relevant für den Geschichtsunterricht und für diese Arbeit, da sich in diese Kategorie der ausgewählte Vergleich einordnen lässt.

Schreiber geht von Beispielen für Zielsetzungen historischer Vergleiche aus und leitet daraus „Parameter des Vergleichens“"341 ab. Sie nimmt eine Typenbildung durch den Vergleich an, indem sie das Her-

\footnotetext{
${ }^{338}$ Riekenberg, Michael (2008), S. 277.

${ }^{339}$ Riekenberg, Michael (2008), S. 277.

${ }^{340}$ Vgl. Riekenberg, Michael (2008), S. $278 \mathrm{ff}$.

${ }^{341} \mathrm{Vgl}$. Schreiber, Waltraud (2005), S. 44.
} 
ausarbeiten von Ähnlichkeiten (über Epochen hinweg) in den Fokus stellt. Weiterhin nennt sie die Abgrenzung und Differenzierung von Vergleichselementen. Dabei können ihrer Meinung nach Vergleiche diachron ansetzen und Entwicklungen in den Blick nehmen oder synchron Epochenspezifik und strukturelle Zusammenhänge erarbeiten. ${ }^{342}$

Ausgehend von diesen Grundprämissen leiten beide Autoren ,Vergleichbares' für den Geschichtsunterricht ab. Dazu nennen sie eher beispielhaft Vergleichsfälle und Arbeitsaufträge für den Unterricht. Die Frage, was nicht verglichen werden kann oder darf, bleibt dagegen weitgehend unbeantwortet. Riekenberg gibt zumindest Ansätze für Nichtvergleichbares und geht in dieser Hinsicht weiter als Schreiber, wenn er unter dem Stichwort „Handreichungen für den Vergleich“343 Überlegungen anstellt, wann ein Vergleich in die Irre führt. Dazu gehört die Annahme, der Vergleichende müsse eine außenstehende Beobachterposition einnehmen oder alle Vergleichsgegenstände hätten das Ziel, die Vergleichsgegenstände gleich intensiv zu vermitteln u.a.m. ${ }^{344}$ Auch nennt er Operationen, die zum Vergleich führen. Dazu gehören seines Erachtens das Beobachten, Fragen, Analysieren, Deuten und Auswerten. ${ }^{345}$ Dies sind vermutlich Beobachtungswerte, die es zu überprüfen gälte.

Insgesamt geht es bei Riekenberg um eine Art „technischer" Aspekte, die nicht in erster Linie als fachspezifisch zu bezeichnen sind, bzw. die Durchführung des Vergleichens im Geschichtsunterricht. Seine Überlegungen sind nur lose angebunden an die fachwissenschaftliche Diskussion um die Frage nach Vergleichbarem und Unvergleichbarem.

Insgesamt ist festzustellen, dass eine empirische Überprüfung fehlt, um zu erkennen, was den Vergleich wirklich ausmacht, was vergleichbar ist, was eher nicht. Es können nur Vermutungen angestellt werden. Außerdem ist festzuhalten, dass keine feste Struktur des Vergleichens auszumachen ist. Eine Art festes Vorgehen benennen beide Autoren ebenfalls nicht. Im Gegenteil stellt Riekenberg fest ${ }^{346}$ :

„Der Vergleich ist keine exakte Methode, weil die Regeln darüber, wie er durchgeführt wird, nicht eindeutig sind. Einzelne historische Vergleiche zum gleichen Thema können ganz unterschiedlich angelegt sein, und entsprechend variieren auch die Ergebnisse, die sie erbringen. ${ }^{\text {3447 }}$

Wenn es aber keine einheitlichen Regeln für den Vergleich zu geben scheint, muss es trotzdem, und gerade diese Überlegung unterstützt Riekenberg in seiner Darstellung ${ }^{348}$, Teilhandlungen geben, die den Vergleich strukturieren. Diese offenzulegen, muss Ziel sein, wenn der Vergleich „Teil eines gerichteten, methodisch überprüften und kontrollierten Lernvorgangs“ sein will. “349

\footnotetext{
${ }^{342}$ Vgl. Schreiber, Waltraud (2005), S. $44 \mathrm{f}$.

${ }^{343}$ Riekenberg, Michael (2008), S. 281.

${ }^{344}$ Vgl. Riekenberg, Michael (2008), S. $281 \mathrm{f}$.

${ }^{345}$ Vgl. Riekenberg, Michael (2008), S. 282.

${ }^{346}$ Für das nachfolgende Zitat ist anzumerken, dass Riekenberg einen mehrdeutigen Methodenbegriff benutzt; zu fragen wäre, ob er den Vergleich in diesem Zusammenhang als Unterrichts- oder Forschungsmethode oder im Sinne der historischen Narration als Erkenntnismethode begreift. Auch danach richtet sich letztlich dessen Ausgestaltung. Zum Methodenbegriff vgl. Mayer, Ulrich, Pandel, Hans-Jürgen; Schneider, Gerhard: Einführung. In: Mayer, Ulrich; Pandel, Hans-Jürgen; Schneider, Gerhard (Hrsg.): Handbuch Methoden im Geschichtsunterricht. 5. Aufl. Schwalbach/Ts.: Wochenschau Verlag. S. 9-11.

${ }^{347}$ Riekenberg, Michael (2008), S. 270.

${ }^{348}$ Vgl. dazu Riekenberg, Michael (S. 277), wenn er von „Prozeduren“ des Vergleichens spricht.

${ }^{349}$ Riekenberg, Michael (2008), S. 277. s.o.
} 


\subsubsection{Das historische Vergleichen in den schulischen Richtlinien}

In Kapitel 2 wurden bereits auf allgemeiner Basis die zugrundeliegenden Richtlinien diskutiert, nun soll ein genauerer Blick auf das Vergleichen in EPA und Kernlehrplänen für die gymnasiale Oberstufe erfolgen.

Exemplarisch soll der Kernlehrplan Geschichte für die Sekundarstufe II/NRW ${ }^{350}$ auf die Frage hin untersucht werden, in welcher Form das Vergleichen dort vorkommt.

In dem 2014 erschienenen Kernlehrplan werden im Kapitel 2.2 die Kompetenzerwartungen an die Lernenden aufgeschlüsselt. Auffällig ist in dieser Zusammenstellung, dass das Vergleichen nur eine untergeordnete Rolle spielt. Unter der Methodenkompetenz wird am Ende der Einführungsphase ${ }^{351}$ von den Lernenden erwartet, dass sie den Unterschied zwischen Quellen und Darstellungen erläutern, Informationen aus ihnen miteinander vergleichen und Bezüge zwischen ihnen herstellen. ${ }^{352}$ Es geht hier also vor allem um einen Vergleich zwischen Quellen und Darstellungen als Teil des Umgangs mit beiden Medien.

Weiterhin wird in einzelnen Inhaltsfeldern das Vergleichen berücksichtigt. So etwa in Inhaltsfeld 2 „Islamische Welt - christliche Welt: Begegnung zweier Kulturen in Mittelalter und früher Neuzeit". ${ }^{353}$ Aus der Formulierung „Die Schülerinnen und Schüler beurteilen den Entwicklungsstand der mittelalterlichen islamischen Wissenschaft und Kultur im Vergleich zu dem des Westens ${ }^{\text {“354 }}$ wird deutlich, dass als Operator das Beurteilen im Vordergrund steht und das Vergleichen dazu Mittel zum Zweck ist. Dies müsste hinterfragt werden. Im Folgenden ist zu zeigen, dass gerade das Beurteilen einen wesentlichen Teil des Vergleichens darstellt.

Im Zusammenhang mit den Kompetenzerwartungen bis zum Ende der Qualifikationsphase für den Grundkurs wird als Teil der zu entwickelnden Sachkompetenz festgelegt: Die Schüler*innen „beziehen historische Situationen exemplarisch durch Fragen, Vergleich und Unterscheidung auf die Gegenwart" ${ }^{355}$ Der Vergleich hat also hier beispielhafte Funktion und soll einen Transfer in die Gegenwart anregen. Unter Methodenkompetenz im selben Abschnitt sollen die Lernenden den Unterschied zwischen Quellen und Darstellungen erläutern, zudem sollen sie Informationen aus ihnen miteinander vergleichen und auch weniger offenkundige Bezüge zwischen ihnen herstellen. ${ }^{356}$ Damit wird für die Schüler*innen ein weiteres Feld des Vergleichens eröffnet, wenn der Unterschied zwischen Quellen und Darstellungen erläutert werden soll. Nach Riekenberg handelt es sich hier um die Kontrastierung unterschiedlicher Repräsentationsformen von Geschichte ${ }^{357}$, der ein Vergleich vorausgegangen sein muss. Dem medialen Vergleich wird der inhaltliche Vergleich hinzugefügt. Interessant erscheint der Verweis auf die weniger offenkundigen Bezüge, da im Unterricht zunächst gezeigt werden müsste, wie

\footnotetext{
350 Ministerium für Schule und Weiterbildung des Landes Nordrhein-Westfalen (2014): Kernlehrplan für die Sekundarstufe II Gymnasium/Gesamtschule in Nordrhein-Westfalen. Geschichte. Online einsehbar unter http://www.schulentwicklung.nrw.de/lehrplaene/upload/klp_SII/ge/KLP_GOSt_Geschichte.pdf (zuletzt eingesehen am 15.10.15).

${ }^{351}$ In NRW ist die gymnasiale Oberstufe in eine Einführungs- und eine Qualifikationsphase gegliedert.

${ }^{352}$ Ebd. S. 21.

${ }^{353}$ Ebd. S. 24.

${ }^{354} \mathrm{Ebd}$.

${ }^{355}$ Ebd. $S 27$.

${ }^{356} \mathrm{Vgl}$. ebd.

${ }^{357}$ Vgl. Riekenberg, Michael (2008), S. 280.
} 
man an solche Bezüge gelangt und auch wie diese bewertet werden müssen (sind diese nur Nebenaspekte oder treiben sie die Erkenntnisse zu einem Thema voran?). Diese Kompetenzerwartung scheint sehr komplex zu sein.

Weiterhin werden die einzelnen Inhaltsfelder dargestellt, die im Unterricht des Grundkurses obligatorisch sind. In Bezug auf das Inhaltsfeld 7 „Friedensschlüsse und Ordnungen des Friedens in der Moderne" sollen die Lernenden vergleichend die Stabilität der Friedensordnungen von 1815 und 1919 beurteilen. ${ }^{358}$ Auch hier wird also der Operator „Beurteilen“ expliziert, das Vergleichen wiederum als Werkzeug zum Beurteilen dargestellt.

Bedeutsam ist auch die Anforderung im Bereich Sachkompetenz für den Leistungskurs. Hier wird formuliert: Die Schüler*innen „,beziehen historische Situationen exemplarisch durch Vergleich, Analogiebildung und Unterscheidung auf die Gegenwart" ${ }^{\prime 359}$ Dadurch wird der schon zuvor diskutierte Zusammenhang zwischen Vergleich und Analogie hergestellt. Da in der Forschung als gängige Formel angenommen werden kann, dass der Vergleich Gemeinsamkeiten und Unterschiede in den Blick nimmt, ist nicht klar, warum der Aspekt der Unterscheidung neben den Kategorien Vergleich und Analogie gesondert hervorgehoben wird.

Sollten im Grundkurs Unterschiede zwischen Quellen und Darstellungen erläutert und Informationen daraus verglichen werden, sollen die Leistungskursschüler*innen in der Kategorie Methodenkompetenz statt , weniger offenkundige Bezüge' herzustellen, wie für die Grundkursschüler*innen formuliert, über diese Herangehensweise den Konstruktcharakter von Geschichte darstellen. ${ }^{360}$ Der Vergleich dient hier also explizit zur Reflexion der Vergleichsgrößen wie auch des Blicks auf Geschichte: „Offenkundig ein Konstrukt, fordert der Vergleich geradezu zum Nachdenken über die methodische Vorgehensweise und die Frage, inwieweit die Methode die Ergebnisse der Untersuchung vorstrukturiert, heraus. ${ }^{1361}$ Auch hier müsste Unterricht wiederum die Methode des Vergleichs explizieren, so dass diese Leistung fassbar wird.

Für den Leistungskurs wird in Inhaltsfeld 6 „Nationalismus, Nationalstaat und deutsche Identität im 19. und 20. Jahrhundert“362 explizit Bezug auf den Vergleich genommen. Im Bereich Sachkompetenz „erläutern [die Schüler*innen. Anm. d. Verf.] die Grundmodelle des Verständnisses von Nation im europäischen Vergleich“. ${ }^{363}$ Es ist wenig überraschend, dass der Blick auf den europäischen Vergleich gerichtet wird, da dies geradezu als klassische Form des Vergleichs erscheint: „Noch heute ist der Bezugspunkt des Vergleichs im Geschichtsunterricht dabei meist die ,Nation'.“364 Nicht eindeutig ist die Formulierung allerdings im Hinblick auf die Frage, ob der Vergleich vorgegeben und ,nur' zu erläutern ist oder ob er ebenfalls durch die Lernenden geleistet werden muss. Der letzte unter die Sachkompetenz fallende Punkt führt jedoch eine Klärung herbei, da die Schüler*innen „nationale und internationale Akteure, ihre Interessen und Konflikte im Prozess 1989/1990" vergleichen sollen. ${ }^{365}$ Hier wird explizit das selbständige Vergleichen herausgehoben, es handelt sich dabei um einen inhaltlichen Vergleich.

\footnotetext{
358 Vgl. Ministerium für Schule und Weiterbildung des Landes Nordrhein-Westfalen (2014), S. 33.

359 ebd. S. 34.

${ }^{360}$ Vgl. ebd. S. 35.

${ }^{361}$ Riekenberg, Michael (2008), S. 277.

${ }^{362}$ Vgl. Ministerium für Schule und Weiterbildung des Landes Nordrhein-Westfalen (2014), S. 40.

363 Ebd. S. 40.

364 Riekenberg, Michael (2008), S. 278.

365 Ministerium für Schule und Weiterbildung des Landes Nordrhein-Westfalen (2014), S. 41.
} 
Auch in Inhaltsfeld 7 taucht das Vergleichen zweimal auf. Es handelt sich thematisch um den Bereich „Friedensschlüsse und Ordnungen des Friedens in der Moderne“. ${ }^{366} \mathrm{Im}$ Bereich Urteilskompetenz lautet die Anforderung an die Lernenden: Sie „beurteilen vergleichend die Stabilität der Friedensordnungen von 1648, 1815 und 1919“367 und sie „beurteilen vergleichend die Bedeutung des Völkerbundes und der UNO für eine internationale Friedenssicherung. “368 Erneut (und hier gleich zweifach) taucht also der Zusammenhang der Operatoren Beurteilen und Vergleichen auf. Wiederum ist in beiden Fällen das Vergleichen dem Beurteilen nachgeordnet, so dass das Vergleichen eine Zubringerfunktion für das Beurteilen erhält.

In der angehängten Progressionstabelle taucht das Vergleichen im Kernlehrplan ein letztes Mal auf. Dabei werden die bereits dargestellten Formulierungen aus den übergeordneten und den Inhaltsfeldern vorangestellten Kompetenzerwartungen wieder aufgegriffen und nebeneinandergestellt. Die Grundaussage lautet, wie bereits zuvor dargestellt, die Schüler*innen „erläutern den Unterschied zwischen Quellen und Darstellungen, vergleichen Informationen aus ihnen miteinander ${ }^{\prime \prime 369}$, dann werden die jeweiligen Differenzierungen angeschlossen. Dies bedeutet, dass der inhaltliche Vergleich innerhalb des Kernlehrplans eine besondere Wertschätzung erhält.

Insgesamt ist festzustellen, dass der Vergleich innerhalb verschiedener Kompetenzkategorien berücksichtigt wird. Er dient als Methode in verschiedenen Inhaltsfeldern und wird auf Einführungs- und Qualifizierungsphase hin ausdifferenziert. Riekenberg stellt zu den Leistungen des Vergleichs fest: „Der historische Vergleich ist geeignet, historisches ,Möglichkeitsbewusstsein'zu schaffen. Er hat Anteil daran, Differenz und Alterität zu konstituieren. Ohne den Vergleich gäbe es keinen Gegenwartsbezug im historischen Lernen. All dies macht ihn didaktisch interessant. “" ${ }^{170}$ Die dargestellten Auszüge aus dem Kernlehrplan zeigen, dass dem Vergleich im Unterricht der Sekundarstufe II die Aufgabe zukommt, die von Riekenberg genannte Alterität und Differenz nicht nur darzustellen, sondern sie, wie er feststellt, auch zu konstruieren. Der Vergleich wird zum Werkzeug, den Konstruktionscharakter von Geschichte erfahrbar zu machen. Auch die Herstellung eines Gegenwartsbezugs wird explizit formuliert. Zudem wird der Vergleich als inhaltlicher und methodischer Vergleich bedeutsam gemacht.

Riekenberg legt in dieser Einordnung wesentliche Kriterien offen, die den Vergleich im Sinne Schriewers zur Denkform ${ }^{371}$ machen. Führt man diese und die Ansprüche des Lehrplans zusammen, lassen sich folgende Kategorien herausarbeiten:

Ein Vergleich als kompetenzorientierte Aufgabe im Geschichtsunterricht

- legt weniger offenkundige Bezüge offen und macht darüber den Konstruktionscharakter von Geschichte erfahrbar

- regt historisches Möglichkeitsbewusstsein an

- $\quad$ konstituiert Differenz und Alterität

- ermöglicht eine Beurteilung

- regt einen Transfer in die Gegenwart an

\footnotetext{
366 Ebd. S. 42.

367 Ebd.

368 Ebd. S. 43.

${ }^{369}$ Ebd. S. 55.

${ }^{370}$ Riekenberg, Michael (2008), S. 283.

${ }^{371}$ Vgl. z. B.: Schriewer, Jürgen: Problemdimensionen sozialwissenschaftlicher Komparatistik. In: Vergleich und Transfer. Kaelble, Hartmut; Schriewer, Jürgen (Hrsg.): Vergleich und Transfer. Komparatistik in den Sozial-, Geschichts- und Kulturwissenschaften. Frankfurt a. M.: Campus Verlag. S. 9-52. S. 24.
} 
Insgesamt kann die Anfangsthese, dass der Vergleich über die Anforderungen im Geschichtsunterricht eine Rolle spielt, durch die Inhalte der Kernlehrpläne gestützt werden.

Allerdings gibt es im Lehrplan keinen Hinweis darauf, ob und vor allem wie der Vergleich expliziert werden soll. Ist die Klärung des Wie ggf. Aufgabe einer Handreichung, findet sich dennoch kein Hinweis darauf, um den Vergleich als Methode zu verdeutlichen, seinen Sinn und Zweck im Unterricht zu vermitteln. Die Beschäftigung mit dem Kernlehrplan zeigt die Notwendigkeit, solche Elemente für Lehrer*innen- und Schüler*innenhand zu explizieren. Eine Offenlegung von Kriterien für den Vergleich im Geschichtsunterricht soll deshalb nachfolgend am Beispiel der ausgewählten Vergleichsaufgabe gezeigt werden.

Weiterhin ist auffällig, dass das Vergleichen mehrfach im Zusammenhang mit dem Beurteilen auftaucht. Der Blick in die „Einheitlichen Prüfungsanforderungen in der Abiturprüfung Geschichte ${ }^{\prime 372}$ kann einen Beitrag zur Klärung dieses Zusammenhangs leisten.

Zuvor sollen jedoch auch die schon zu Anfang befragten weiteren Rahmenvorgaben geprüft werden, ob bzw. wie dort mit dem Vergleichen umgegangen wird. Die „Vorgaben zu den unterrichtlichen Voraussetzungen für die schriftlichen Prüfungen im Abitur in der gymnasialen Oberstufe" (hier exemplarisch für das Jahr 2016 und 2017) (373 $^{373}$ geben keinen Hinweis, wie im Unterricht mit der Methode des Vergleichens umgegangen werden soll bzw. welche Rolle es als Vorbereitung auf die Abiturprüfung spielt. In den "Bildungsplänen zur Erprobung für die Bildungsgänge, die zu einem Berufsabschluss nach Landesrecht und zur allgemeinen Hochschulreife oder zu beruflichen Kenntnissen und zur allgemeinen Hochschulreife führen" (exemplarisch sei hier wieder auf den Lehrplan für den Fachbereich Wirtschaft und Verwaltung verwiesen ${ }^{374}$ ), die auch schon zu Anfang zur Klärung des Rahmenbedingungen herangezogen wurden, gibt es einen einzigen Verweis auf das Vergleichen, der sich aber auf den Vergleich von Lernleistungen bezieht und nicht auf die Methode des historischen Vergleichens. ${ }^{375}$

Um die Struktur des Vergleichens zu prüfen, bleibt, da die oben genannten Rahmenvorgaben keine Strukturhilfe bieten, der Blick in die „Einheitlichen Prüfungsanforderungen in der Abiturprüfung Geschichte" (EPA). ${ }^{376}$

Die EPA ordnen das Vergleichen dem Anforderungsbereich III, also der Reflexion und Problemlösung zu. Zur Aufschlüsselung der Operatoren in drei Anforderungsbereiche in den EPA stellen Schönemann et al. grundsätzlich fest:

„Sie [die nordrhein-westfälischen Lehrpläne, Anm. d. Verf.] orientieren sich im Wesentlichen an der von Jeismann etablierten Trias ,Analyse, Sachurteil, Wertung', um historisches Denken in drei verschiedenen, mit der Jeismann-Trias aber nicht vollkommen identischen Anforderungsbereichen

\footnotetext{
372 Kultusministerkonferenz (2005): EPA Geschichte, S. 4.

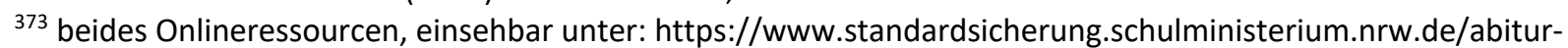
gost/fach.php?fach=12 (zuletzt eingesehen am 21.10.15).

374 Ministerium für Schule und Weiterbildung des Landes Nordrhein-Westfalen (Hrsg.) (2012): Bildungspläne zur Erprobung für die Bildungsgänge, die zu einem Berufsabschluss nach Landesrecht und zur allgemeinen Hochschulreife oder zu beruflichen Kenntnissen und zur allgemeinen Hochschulreife führen. Fachlehrplan Gesellschaftslehre mit Geschichte Fachbereich Wirtschaft und Verwaltung. Grundkurs. Online einsehbar unter: http://www.berufsbildung.schulministerium.nrw.de/cms/upload/_lehrplaene/d/wirtschaft_und_verwaltung/teil3/teil3_glg_wuv_gk.pdf (zuletzt eingesehen am 21.10.15).

375 Ebd. S. 20.

${ }^{376}$ Kultusministerkonferenz (2005): EPA Geschichte.
} 
(kurz: AFB) zu erfassen. [...] Auch die Einheitlichen Prüfungsanforderungen gehen von drei Anforderungsbereichen aus. ${ }^{\text {"377 }}$

Eine solche Zuordnung würde bedeuten, dass für das Vergleichen neben Analyse und Sachurteil auch eine Leistung im Bereich der Wertung erkennbar werden müsste.

Die EPA definieren das Vergleichen als „,auf der Grundlage von Kriterien historische Sachverhalte problembezogen gegenüberzustellen, um Gemeinsamkeiten, Unterschiede, Teil-Identitäten, Ähnlichkeiten, Abweichungen oder Gegensätze zu beurteilen. ${ }^{378}$ Dies bedeutet, dass ein direkter Bezug zum Sachurteil hergestellt wird, weniger jedoch zum Werturteil. Denkbar ist, dass durch eine entsprechende Vergleichsaufgabe ein Werturteil angestoßen werden kann, durch die vorliegende Bestimmung wird dies jedoch nicht zur Voraussetzung für eine historische Vergleichsaufgabe in der Sekundarstufe II. Schaut man sich die in den EPA geforderten Teilhandlungen für das Vergleichen im Einzelnen an, taucht zunächst die Forderung nach einem kriteriengeleiteten Vorgehen auf. Wie zuvor dargestellt, passt dies zu den geschichtswissenschaftlichen Anforderungen an einen Vergleich. ${ }^{379}$ Weiterhin sollen historische Sachverhalte problembezogen gegenübergestellt werden. Der Hinweis auf die historischen Sachverhalte macht die Zuordnung als historischen Lerngegenstand auch für den Laien sichtbar. Die Gegenüberstellung erfolgt anhand eines Problembezugs. Dieser Begriff wird dabei nicht weiter aufgeschlüsselt. Ziel des Vergleichs soll dann das Beurteilen des entsprechenden historischen Sachverhaltes sein. Demnach bedeutet Vergleichen auch Beurteilen. Wenn dies als Anforderung an Schüler*innen der Sekundarstufe II gestellt wird, erscheint fraglich, warum dann im Kernlehrplan für die gymnasiale Oberstufe, wie oben dargestellt, immer wieder das Vergleichen dem Beurteilen explizit vorangestellt wird. Nimmt man die EPA ernst, ergibt sich aus dem Vergleichen immer auch das Beurteilen. Was durch das Vergleichen beurteilt werden soll, wird dann in den EPA aufgeschlüsselt: Gemeinsamkeiten, Unterschiede, Teil-Identitäten, Ähnlichkeiten, Abweichungen oder Gegensätze. Die Aufzählung spiegelt die wissenschaftliche Debatte insofern, als dass das Gemeinsame und das Differente Grundlage für die Beurteilung darstellen.

Bemerkenswert erscheint der in Kapitel 2.2.3 angesprochene Vergleich der Bestimmung des historischen Vergleichens in den EPA Geschichte mit der Bestimmung des Vergleichens in den EPA Sozialkunde/Politik ${ }^{380}$ und ihre unterschiedliche Ausformung hinsichtlich der Zuordnung in die Anforderungsbereiche und der Notwendigkeit eines Sachurteils. Da Geschichte häufig im Fächerverbundfach Gesellschaftslehre unterrichtet wird, soll der Blick auf die EPA Geographie ${ }^{381}$ das Bild ergänzen. Die EPA Geographie sind anders strukturiert als die EPA Geschichte und Sozialkunde/Politik, da die Anforderungsbereiche beschreibend dargestellt sind und weniger (wie in den anderen beiden Fällen) in Form einer Auflistung konkreter Operatoren und ihrer Bestimmungen. Wie in den EPA Sozialkunde/Politik wird das Vergleichen dem Anforderungsbereich II zugeordnet. Verglichen werden sollen Strukturen und Prozesse in verschiedenen Räumen. ${ }^{382}$ Entsprechend der Zuordnung zum Anforderungsbe-

\footnotetext{
${ }^{377}$ Schönemann, Bernd; Thünemann, Holger; Zülsdorf-Kersting, Maik (2010): Was können Abiturienten? Zugleich ein Beitrag zur Debatte über Kompetenzen und Standards im Fach Geschichte. LIT Verlag, Münster. S. 17.

378 Einheitliche Prüfungsanforderungen in der Abiturprüfung Geschichte (2005). S. 8.

${ }^{379}$ Vgl. z. B. Kaelble, Hartmut (1999).

${ }^{380}$ Kultusministerkonferenz (2005): Sozialkunde/Politik. S. 18.

${ }^{381}$ Kultusministerkonferenz (2005): Einheitliche Prüfungsanforderungen in der Abiturprüfung Geographie (Beschluss der Kultusministerkonferenz vom 01.12.1989 i. d. F. vom 10.02.2005). Online verfügbar unter: http://www.kmk.org/fileadmin/veroeffentlichungen_beschluesse/1989/1989_12_01-EPA-Geographie.pdf (zuletzt eingesehen am 22.10.15).

382 Ebd. S. 6.
} 
reich II wird auch hier keine explizite Beurteilung von den Lernenden verlangt. Es kann also festgehalten werden, dass das Beurteilen in der Zeit und damit im Sinne eines Sachurteils ein Fachspezifikum des Vergleichens im Geschichtsunterricht darstellt.

Der Blick auf den Fächerverbund Gesellschaftslehre soll erste fachspezifische Merkmale, aber auch die Schwierigkeiten für die Lernenden verdeutlichen, die sich aus diesen Unterschieden ergeben. Sie müssen demnach die Bestimmung des Vergleichens (und aller anderen vorkommenden Operatoren) für ihr Fach kennen. Es muss klar sein, was der Operator verlangt. Wissen die Lernenden im Fach Geschichte nicht, dass der historische Vergleich in einer Beurteilung münden soll, hätten sie im Unterrichtsfach Geschichte die Aufgabe nur zum Teil gelöst, in den anderen beiden dargestellten Disziplinen wäre dies anders.

Eine weitere Hürde, die die EPA-Bestimmung mit sich bringt, ist die fehlende Aufschlüsselung der für das historische Vergleichen geforderten Teilhandlungen. Zwar wird deutlich, dass das Gegenüberstellen von Sachverhalten eingefordert wird. Aber dies wird nur durch weitere sprachliche Teilhandlungen möglich. Riekenberg nennt, wie zuvor dargestellt, das Beobachten, Fragen, Analysieren, Deuten und Auswerten als Operationen, die zum Vergleich führen. ${ }^{383}$ Es sind weitere Teilhandlungen zu vermuten, die der Vergleich benötigt: Das Gegenüberstellen bringt nachfolgend mit sich, dass eben diese Gegenüberstellungen erklärt und begründet werden. Abschließend müsste die geforderte Beurteilung folgen.

Inwiefern die vermuteten bzw. explizit geforderten Teilhandlungen Eingang in den Schülervergleich finden, muss geprüft werden. Da es sich beim historischen Vergleichen, wie schon die Einordnung in den Anforderungsbereich III zeigt, um eine komplexe Lernaufgabe handelt, müssen die Teilhandlungen und vor allem Möglichkeiten ihrer Versprachlichung den Lernenden bekannt sein. Es schließt sich an dieser Stelle die Frage an, wie in Lehrwerken mit der Explizierung des Vergleichs umgegangen wird. Diese Frage lässt sich über die entsprechenden Methodenseiten beantworten.

\subsubsection{Methodenseiten zum Vergleichen in Geschichtslehrwerken}

Wenn historisches Vergleichen eine Denkform oder Methode für den Geschichtsunterricht darstellt, muss als Konsequenz das Vergleichen auch didaktisch angeleitet werden. Dies gilt umso mehr, wenn Riekenberg mit seiner Annahme Recht hat, dass Vergleiche im Geschichtsunterricht weit verbreitet seien.

Im Anschluss an das Kapitel wird dieser Annahme nachgegangen und eine Überprüfung durch eine Frequenzanalyse vorgenommen. Die Frequenzanalyse bezieht sich auf die zugelassenen GeschichtsLehrwerke der Sekundarstufe II in NRW vor und nach 2014. Ebendiese Lehrwerke wurden auch hinsichtlich ihrer Methodenseiten zum Vergleichen ausgewertet (s. Anhang).

Das Vergleichen taucht als Aufgabentyp bereits in der Sekundarstufe I auf und ist den Lernenden somit in aller Regel bekannt. Im Sinne des wissenschaftspropädeutischen Arbeitens in der Sek. II müssen für die Schüler*innen insbesondere dieser Schulstufe die damit verknüpften Anforderungen geklärt werden, so dass in der Folge Methodenseiten oder andere didaktische Unterstützungen zur Lösung dieses Aufgabentyps in den Lehrwerken vorhanden sein müssten.

${ }^{383}$ Vgl. Riekenberg, Michael (2008), S. 282. 


\subsubsection{Methodenseiten zum Vergleichen im Überblick}

Die Überprüfung der Lehrwerke im Hinblick auf solche Didaktisierungen ergibt ein heterogenes Bild. ${ }^{384}$ Es fällt auf, dass mit einer Ausnahme keines der zwischen 2002 und 2007 in NRW erschienenen Geschichtsschulbücher die Methode des historischen Vergleichens klärt und auch keine Klärung der Operatoren vornimmt ${ }^{385}$, aus denen Lösungshinweise hätten abgeleitet werden können. Lediglich in Band 2 des Lehrwerks „Zeiten und Menschen. Geschichte Oberstufe“ von $2006^{386}$ gibt es eine Erläuterung der Methode unter dem Titel „Historischer Vergleich“. ${ }^{387}$ Dort gibt es Hinweise zur Bedeutung einer Leitfrage, weiterhin werden synchroner und diachroner Vergleich unterschieden und die Bedeutung der sozialen Bedingtheit historischer Phänomene für den Vergleich betont. Im Anschluss werden fünf Schritte des historischen Vergleichs herausgearbeitet: die Klärung des Vergleichstyps, die Beschreibung der Einzelphänomene in ihrer sozialen Bedingtheit, die Festlegung der Vergleichsaspekte, das eigentliche Vergleichen sowie die Beurteilung und Bewertung der Vergleichsergebnisse. Die Darstellung endet also, wie in der EPA-Bestimmung zum Vergleichen vorgegeben, mit einer Sach- oder je nach Aufgabe sogar einer Werturteilsbildung. Der eigentliche Schritt des Vergleichens beschränkt sich dabei auf kurze Hinweise zum historisch-genetischen und zum historisch-typologischen Vergleich. Eine Klärung notwendiger sprachlicher Mittel findet nicht statt. Auch eine Differenzierung der zugrundeliegenden Materialien (etwa Quellen oder Darstellungstexte) wird nicht vorgenommen.

In den nachfolgenden Jahren wandelt sich dann das Bild, in zunehmend mehr Lehrwerken wird eine Klärung des Operators Vergleichen vorgenommen. Wird in nur einem von vier der in 2010 erschienenen und untersuchten Lehrwerken auf die Methode des Vergleichens eingegangen, wird bereits in drei der vier untersuchten und 2011 erschienenen Lehrwerken der Operator erläutert. Auch das in 2012 erschienene Lehrwerk nimmt eine solche Klärung vor. In 2014 finden sich in drei von fünf untersuchten Schulbüchern Hinweise zum Operator. In 2015 gibt es in vier von vier untersuchten Lehrwerken Hinweise zum Operator. Dabei ist festzustellen, dass es sich bei den Lehrwerken durchweg um die Fortführung der 2014 erschienenen Schulbücher zur Einführungsphase handelt, nämlich die Qualifikationsphase. So lässt sich erklären, dass sich in den 2015er Lehrwerken der Qualifikationsphase nicht immer Methodenhinweise zum Vergleichen, sondern manchmal nur die Operatorenbestimmungen der EPA befinden, da diese bereits im Band für die Einführungsphase geklärt wurden.

\subsubsection{Die Aufarbeitung in den Lehrwerken im Einzelnen}

In 2010 gibt es lediglich im „Kursbuch Geschichte“ Methodenseiten zu „Interpretation und Vergleich schriftlicher Quellen“. Auf den Vergleich von Darstellungstexten wird kein Bezug genommen. ${ }^{388}$

\footnotetext{
${ }^{384}$ Im Anhang 3 befindet sich ein tabellarischer Überblick über methodische Hinweise zur Lösung von Vergleichsaufgaben.

${ }^{385}$ Zur besseren Einordnung: Der Beschluss der KMK über die Einheitlichen Prüfungsanforderungen in der Abiturprüfung und damit auch über den Operatorenkatalog stammt aus dem Jahre 1989 und wurde 2005 neu gefasst.

386 Lendzian, Hans-Jürgen (Hrsg.) (2006): Zeiten und Menschen. Geschichte Oberstufe. Band 2. Braunschweig, Paderborn, Darmstadt: Schöningh Verlag. S. $291 \mathrm{f}$.

${ }^{387}$ Vgl. ebd. S. 291.

${ }^{388}$ Vgl. Laschewski-Müller, Karin; Rauh, Robert (Hrsg.) (2010). Kursbuch Geschichte. Neue Ausgabe NordrheinWestfalen. Von der Antike bis zur Gegenwart. Berlin: Cornelsen-Verlag. S. 488.
} 
In 2011 sind in „Zeiten und Menschen. Geschichte Einführungsphase Oberstufe “389 und in „Grundwissen Geschichte. Sekundarstufe II“390 Methodenseiten aufgeführt.

Im erstgenannten Lehrwerk ist ein Methodenkasten zum Thema „Lesekompetenz - Historische Texte aspektorientiert lesen, systematisch auswerten und vergleichen" vorhanden. ${ }^{391}$ Dabei liegt der Hauptaspekt auf dem Lesen und Auswerten; zum Thema werden fünf Schritte angeboten, dabei stellt das Vergleichen den abschließenden fünften Schritt dar. Es ist dazu schlagwortartig in drei Unterpunkte gegliedert, die Untertitelcharakter haben:

„-Prinzipielle Unterschiede und Gegensätze aufzeigen.

-Übereinstimmungen und Ähnlichkeiten aufzeigen.

-Positionen erklären und beurteilen. ${ }^{\text {“392 }}$

Darüber hinaus gibt es einen Methodenkasten zu „Historische Urteile analysieren und vergleichen“393 sowie einen weiteren zum Historischen Vergleich. Dabei handelt es sich um eine Übernahme aus der Auflage von 2006. Auch wenn auf diesen Methodenseiten einzelne sprachliche Handlungen ausgewiesen werden, wie etwa das Erklären und Beurteilen, wird nicht aufgeschlüsselt, was Lernende leisten müssen oder wie sie vorgehen müssen, wenn sie eine Erklärung oder Beurteilung im fachlichen Kontext Geschichte erstellen sollen. Ein sprachliches Repertoire, das sie zur Umsetzung der sprachlichen Teilhandlungen bzw. für das Vergleichen benutzen können, wird ebenfalls nicht aufgeschlüsselt.

In „Grundwissen Geschichte. Sekundarstufe II“ ist ein Teilkapitel zu „Vergleich von Sekundärtexten“394 aufgenommen worden. Im Teilkapitel „Klausurhilfen und Methoden“ sind auf einer Seite Hinweise zur Bedeutung des Vergleichens versammelt. Dazu wird zunächst auf einer halben Seite das Thema „Kontroversität in der historischen Deutung “395 in einem Verfassertext behandelt, dann folgt eine tabellarische Übersicht mit einem 4-Schritt-Schema zur Erarbeitung eines historischen Vergleichs. Es fällt auf, dass es nur wenige Hinweise zum Vergleich an sich gibt und sich die Darstellung eher mit der Vorgehensweise bei der Analyse im Allgemeinen beschäftigt. Die Arbeitsschritte sind unterteilt in die Schlagworte "Leitfragen“, „Analyse der Texte" (inhaltlich und formal), „Historischer Kontext" und "Urtei-

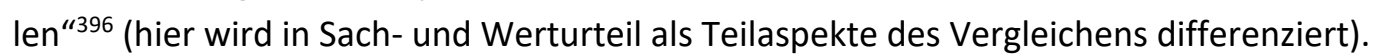

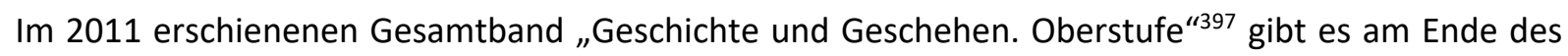
Bandes als tabellarische Übersicht eine Operatorenliste, in der auch Hinweise zum Vergleichen aufgenommen wurden. ${ }^{398}$ Es folgt nach der Nennung des Operators in der zweiten Zeile die EPA-Bestimmung, in der dritten Zeile eine Aufschlüsselung in fünf (Arbeits-)Schritte. Demnach sollen die Lernenden zunächst die zu vergleichenden Sachverhalte wiedergeben, die Vergleichsaspekte benennen, wesentliche von unwesentlichen Vergleichsaspekten unterscheiden, gleiche, ähnliche und unterschiedliche Merkmale herausstellen sowie abschließend eine Einschätzung der Vergleichbarkeit formulieren. In einer dritten Zeile folgt ein Verweis auf ein Aufgabenbeispiel im Lehrwerk, welches als OperatorenTraining ausgewiesen wird. In der vierten Zeile schließt sich eine visuelle Merkhilfe (Darstellung eines

\footnotetext{
389 Lendzian, Hans-Jürgen (Hrsg.) (2014): Zeiten und Menschen. Geschichte Einführungsphase Oberstufe. Nordrhein-Westfalen. Braunschweig, Paderborn, Darmstadt: Schöningh Verlag.

${ }^{390}$ Rauh, Robert (2011): Grundwissen Geschichte Sekundarstufe II. Berlin: Cornelsen Verlag.

${ }^{391}$ Lendzian, Hans-Jürgen (Hrsg.) (2014), S. $40 f$.

$392 \mathrm{Ebd}$.

${ }^{393}$ Ebd. S. 70.

${ }^{394}$ Vgl. Rauh, Robert (2011), S. 273.

${ }^{395} \mathrm{Ebd}$.

${ }^{396}$ Vgl. ebd.

${ }^{397}$ Arand, Tobias et al. (2011): Geschichte und Geschehen Oberstufe NRW. Gesamtband. Stuttgart/Leipzig: Ernst Klett Verlag.

${ }^{398}$ Ebd. S. $640 f$.
} 
Apfels und einer Birne) an. Die Tabelle schließt ab mit übergreifenden Hinweisen zu Leistungen im Anforderungsbereich III allgemein. Schlägt man unter dem angegebenen Aufgabenbeispiel nach, findet man eine Aufgabe zum Vergleich zweier Imperialismustheorien, die durch Hinweise auf Material zur Aufgabe sowie zum Operator ergänzt werden. ${ }^{399}$ Zwar kann man insgesamt feststellen, dass es sich um eine recht ausführliche Darstellung handelt, die zudem durch visuelle Hilfen unterstützt wird. Allerdings sind gerade die visuellen Hilfen ohne Bezug auf einen historischen Kontext, so dass die Nützlichkeit durch die ausgewählten Bilder für das Fach in Frage gestellt werden muss. Ein sprachliches Repertoire zur Realisierung der genannten Teilhandlungen fehlt auch hier.

In dem 2012 erschienenen Lehrwerk ",Geschichtskultur - Module für die Oberstufe “400 befindet sich im Anhang unter dem Kapitel „Arbeitsaufträge in der Abiturklausur" die EPA-Bestimmung des Operators Vergleichen. ${ }^{401}$ Darüber hinaus gibt es zum Vergleich von Texten Satzbausteine zu „Übereinstimmung“ und "Gegensatz“. ${ }^{402}$ Dieses Lehrwerk ist innerhalb der Reihe „Kurshefte Geschichte“ erschienen. Streng genommen könnte es als Kursheft eingeordnet werden, die in der Lehrwerkuntersuchung ansonsten nicht ausgezählt wurden. Es soll jedoch als Beispiel dienen, dass in Lehrwerken auch sprachliche Hinweise zur Erfüllung von Lernaufgaben mit Operatoren vorgenommen werden. Die Autoren haben in einem tabellarischen Überblick verschiedene Arbeitsschritte wie Analyse formaler und inhaltlicher Aspekte, Vergleich von Texten u.a.m. aufgeführt und diesen jeweils Satzbausteine zugeordnet sowie durch ein Formulierungsbeispiel angereichert. Die Operation des Vergleichens wird dadurch allerdings noch nicht geklärt, zumal nur der Vergleich von Texten, also das mediale Vergleichen, berücksichtigt wird. Die Satzbausteine beziehen sich zudem nur auf das Gegenüberstellen, nicht auf das in den EPA für das Vergleichen geforderte Beurteilen.

In 2014 fällt auf, dass in Lehrwerken, in denen zuvor keine Operatorenklärung vorgenommen worden war, diese häufig in der neuen Auflage aufgenommen wurde. Dies hängt sicherlich mit der Umsetzung der neuen Kernlehrpläne zusammen. ${ }^{403}$ Ein Beispiel dafür ist das Lehrwerk „Geschichte und Geschehen Oberstufe Einführungsphase ${ }^{4404}$, da es in der Neuauflage Hinweise zum Vergleichen in einer Operatorenübersicht gibt. ${ }^{405}$ Die Autoren geben hier tabellarisch zunächst die EPA-Bestimmung wieder sowie ein Fünf-Schritt-Schema zum Textaufbau. Eine gesonderte Methodenseite gibt es allerdings nicht, auch keine Unterstützung für die Versprachlichung.

In der Neuauflage des Kursbuch Geschichte von 2014 im Band zur Einführungsphase ${ }^{406}$ gibt es Methodenseiten zum Thema "Schriftliche Quellen vergleichen “407 und damit die Fokussierung auf den medialen Vergleich, die Klärung des historischen Vergleichs wird dann in der Qualifikationsphase (2015) wieder aufgenommen (s.u.). ${ }^{408}$ Nach einem kurzen Einführungstext zur Bedeutung von Quellen und

\footnotetext{
${ }^{399}$ Vgl. ebd. S. 373.

${ }^{400}$ von Reeken, Dietmar (2012): Geschichtskultur - Module für die Oberstufe. Berlin: Cornelsen Verlag.

${ }^{401}$ Ebd. S. 134f.

402 Ebd. S. 136.

${ }^{403}$ Da der Kernlehrplan für die Sekundarstufe II, Geschichte/Gesamtschule in NRW in 2014 veröffentlicht wurde, wurden in der Folge die Lehrwerke überarbeitet, so dass die Geschichtsschulbücher für die Einführungsphase häufig in 2014 erschienen sind und für die Qualifikationsphase in 2015 folgten.

404 Dzubiel, Christiane u.a. (Hrsg.) (2014): Geschichte und Geschehen. Oberstufe Einführungsphase NordrheinWestfalen. Ernst Klett Verlag.

${ }^{405}$ Ebd. S. 247.

406 Laschewski-Müller, Karin/Rauh, Robert (Hrsg.) (2014): Kursbuch Geschichte. Nordrhein-Westfalen Einführungsphase, Berlin: Cornelsen.

407 ebd. S. $146 f$.

408 Laschewski-Müller, Karin/Rauh, Robert (Hrsg.) (2015): Kursbuch Geschichte. Nordrhein-Westfalen Qualifikationsphase, Berlin: Cornelsen.
} 
Multiperspektivität wird auf das Vergleichen übergeleitet. Es folgen Schritte, die das Vorgehen beim Vergleichen aufschlüsseln sollen. Diese beziehen sich in Bezug auf eine vorausgehende Analyse der Quellentexte auf formale und inhaltliche Aspekte. Der eigentliche Vergleich wird dann in zwei Schritten abgehandelt und müsste eigentlich als ,Gegenüberstellen' benannt werden. Die Darstellung schließt ab mit der Einordnung in den historischen Kontext und der Urteilsbildung. Dabei werden sowohl Sachals auch Werturteil angepeilt, obwohl das Werturteil gar nicht über die EPA benannt wird.

Was in der Auflage von 2011 begonnen wurde, wird in der Neuauflage des Lehrwerks „Zeiten und Menschen" fortgesetzt. Der Band für die Einführungsphase von $2014^{409}$ enthält eine Methodenseite $\mathrm{zu}$ „Historische Darstellungen analysieren und kritisch vergleichen“. ${ }^{410}$ Darin werden verschiedene Arbeitsschritte aufgeschlüsselt und mit Textbausteinen, die an das behandelte Thema angepasst sind, versehen. Es handelt sich um eine Weiterentwicklung der Methodenseiten aus 2006 und 2011. Der eigentliche Kern des Vergleichs wird allerdings unter dem Teilschritt „Kritische Auseinandersetzung und Vergleich “411 nur sehr kurz thematisiert, da zunächst allgemeine Analyseaspekte für den Vergleich dargestellt werden (z. B. die Vorstellung des Textauszugs und die strukturierte Textwiedergabe).

Die 2015 erschienenen Lehrwerke zur Qualifikationsphase stellen die Fortführung der 2014 veröffentlichten Schulbüchern zur Einführungsphase dar. In diesem Zusammenhang ist auch die Aufnahme von Methodenseiten zum Vergleichen zu sehen.

Gab es im Lehrwerk „Geschichte und Geschehen“ im Band für die Einführungsphase eine kurze tabellarische Übersicht zum Vergleichen am Ende des Lehrwerks, werden diese Seiten auch im Band für die Qualifikationsphase erneut abgedruckt. Eine weitere Klärung gibt es jedoch nicht.

Im Kursbuch Geschichte für die Qualifikationsphase ${ }^{412}$ sind Methodenseite zum Thema „Darstellungen vergleichen" abgedruckt. ${ }^{413}$ Der mediale Vergleich wird also nur in Bezug auf ein anderes Genre fortgeführt. Es gibt einen Einführungstext mit Hinweisen zu Perspektivität und Kontroversität und der Notwendigkeit der Diskussion, dann folgen Arbeitsschritte. Diese sind eng an die Arbeitsschritte aus der Einführungsphase angelehnt. Es stehen allgemeine Analyseaspekte im Vordergrund (Leitfrage, Analyse von Darstellungsart, Texten, Zielgruppe etc., Historischer Kontext). Auf den eigentlichen Vergleich wird wieder über die Frage: „Unter welchen Gesichtspunkten lassen sich die Texte vergleichen? ${ }^{4114}$ hingewiesen. Auch hier handelt es sich eigentlich wieder um die Teilhandlung ,Gegenüberstellen`. Bezüglich der Urteilsbildung werden, im Unterschied zur EPA-Formulierung, wieder Sach- und Werturteil gefordert.

Im Lehrwerk „Zeiten und Menschen. Geschichte Qualifikationsphase “415 befindet sich im Anhang unter dem Stichwort "Methodenwerkstatt" eine Operatorübersicht mit dem Abdruck der EPA-Bestimmungen. ${ }^{416}$ Unter diesem Stichwort taucht auch die Vergleichs-Bestimmung auf. Weitere Erklärungen gibt es nicht. Jedoch gibt es eine genauere Klärung, wie bereits dargestellt, schon im Einführungsband.

\footnotetext{
${ }^{409}$ Lendzian, Hans-Jürgen (Hrsg.) (2014): Zeiten und Menschen. Geschichte Einführungsphase Oberstufe Nordrhein-Westfalen, Braunschweig u.a.: Schöningh Verlag.

${ }^{410}$ Ebd. S. 101.

${ }^{411} \mathrm{Ebd}$.

${ }^{412}$ Laschewski-Müller, Karin/Rauh, Robert (Hrsg.) (2015): Kursbuch Geschichte. Nordrhein-Westfalen Qualifikationsphase, Berlin: Cornelsen.

${ }^{413}$ Ebd. S. $90 f$.

${ }^{414}$ Ebd.

${ }^{415}$ Lendzian, Hans-Jürgen (Hrsg.) (2015): Zeiten und Menschen. Geschichte Qualifikationsphase Oberstufe Nordrhein-Westfalen. Braunschweig u.a.: Schöningh-Verlag.

${ }^{416}$ Ebd. S. $615 f$.
} 
Fehlte im Lehrwerk „Horizonte. Einführungsphase“ noch eine Klärung des Vergleichs, gibt es im Band zur „Qualifikationsphase ${ }^{\text {“417 }}$ ein Methodentraining zu „Analyse und Vergleich von Darstellungen - Ein Beispiel mit einem Lösungsvorschlag ${ }^{4418}$; dabei werden Darstellungen von zwei Historikern (es handelt sich um Ausschnitte aus Monographien) verglichen. ${ }^{419}$ Das Vorgehen zum Vergleich wird in drei Bearbeitungsschritte aufgeschlüsselt. Im ersten Schritt sollen die Darstellungen analysiert werden, dann folgt im zweiten Schritt eine Erläuterung und historische Einordnung und im dritten Schritt der Vergleich der beiden Darstellungen. Dazu gibt es allgemeine Hinweise zu Inhalten und Aufbau eines Vergleichs, es folgt die Annahme, dass der Vergleich in ein Fazit mündet und in einem abschließenden Urteil die Beurteilungsmaßstäbe und Kriterien offengelegt würden. Dies wird jedoch nicht weiter konkretisiert. Insgesamt geht die Darstellung vom Vergleich mindestens zweier Texte zu einem gemeinsamen Thema aus. ${ }^{420}$

\subsubsection{Zusammenführung der Auswertungsergebnisse aus der Methodenseitenanalyse}

Der Blick in die Methodenseiten der Lehrwerke zeigt, dass zunehmend Anstrengungen unternommen werden, den Operator Vergleichen zu erläutern. Allerdings liegt in vielen Lehrwerken ein Schwerpunkt auf der Analyse, die dem eigentlichen Vergleichen vorausgeht. Was jedoch die Technik des Vergleichens ausmacht, wird sie oft nur kurz und allgemein abgehandelt. Außerdem fallen unterschiedliche Schwerpunktlegungen auf: So beschränkt sich ein Lehrwerk ausschließlich auf die Darstellung des medialen Vergleichs. Außerdem ist eine Schärfung der Begriffszuweisungen nötig, wenn etwa vom Vergleichen die Rede ist, wo eigentlich das Gegenüberstellen gemeint ist.

Nur ein Lehrwerk stellt sprachliche Mittel bereit, um die Lernenden bei der Realisierung des Vergleichs zu unterstützen, und dies auch nur in Bezug auf das Gegenüberstellen.

Es kann insgesamt ein Zusammenhang angenommen werden zwischen der fehlenden Klärung der Teilhandlungen des eigentlichen Vergleichens, der Berücksichtigung nur bestimmter Vergleichstypen und der Bereitstellung sprachlicher Mittel in den Methodenseiten. Solange diese Klärung fehlt, fehlt vermutlich auch die Zuordnung sprachlicher Handlungen.

Hier will diese Arbeit Abhilfe schaffen, der dem empirischen Teil ein Vorschlag für eine Didaktisierung des Vergleichs in fachlicher und sprachlicher Hinsicht für Lernende angefügt ist.

\subsubsection{Zur Didaktisierung des Vergleichs im Geschichtsunterricht: Ein erster Schluss}

Schreiber schlägt vor, die Lernenden „[...] mit einem Instrumentarium auszustatten, um mit historischen Vergleichen, die andere vorlegen, umgehen zu können, bzw. selbst historisch vergleichen zu lernen. ${ }^{4421}$ Dieses Instrumentarium wird jedoch weder durch EPA noch durch Rahmenvorgaben und Lehrpläne expliziert. Auch die Analyse der Methodenseiten in Lehrwerken macht deutlich, dass es derzeit keine ,Instrumente' gibt, mit denen Lernende ausgestattet und das Vergleichen im Geschichtsunterricht didaktisiert werden können. Schreiber nennt drei Begründungen für den Vergleich im Unterricht, aus denen Anforderungen abgeleitet werden können:

\footnotetext{
${ }^{417}$ Baumgärtner, Ulrich et. al (Hrsg.) (2015): Horizonte - Geschichte Qualifikationsphase S II Nordrhein-Westfalen. Braunschweig: Westermann.

${ }^{418}$ Ebd. S. $206 f$.

${ }^{419} \mathrm{Ebd}$.

${ }^{420} \mathrm{Vgl}$. ebd.

${ }^{421}$ Schreiber, Waltraud (2005), S. 39.
} 
- Fähigkeiten und Fertigkeiten im Methodischen (z. B. Auswahl der zu vergleichenden Bereiche, Materialsuche, Darstellung der Ergebnisse),

- Aufbau und Sicherung inhaltsbezogener Erkenntnisse, dabei nennt sie insbesondere grundsätzliche oder kategoriale Erkenntnisse und Einsichten

sowie eine

- theoriebezogene Reflexion zur Erkenntnisbildung, insbesondere im Hinblick auf den Konstruktionscharakter von Geschichte. ${ }^{422}$

Es fällt allerdings auf, dass diese Begründungen kaum vergleichsspezifisch sind, zudem müssen auch aus diesen Begründungen bzw. den daraus ableitbaren Anforderungen sprachliche Teilhandlungen und sprachliche Werkzeuge erst abgeleitet werden.

Insgesamt ist festzustellen, dass Schreiber zwar Begründung, Ziel und Parameter für den Vergleich im Geschichtsunterricht nennt, ein konkretes Instrumentarium gibt sie dem Leser jedoch nicht mit auf den Weg, allenfalls über die ausgewählten Beispiele.

Bei der Generierung sprachlicher Werkzeuge für das Vergleichen im Geschichtsunterricht kann das in Kapitel 2 dargestellte Sprach- und Wissensmodell unterstützen, indem vom kommunikativen Zweck ausgehend mit Hilfe einer empirischen Untersuchung und unter Berücksichtigung normativer Vorgaben wie den EPA solche Mittel abgeleitet werden, die die Textstruktur kennzeichnen. Der funktionale Blick in der Verbindung von Sprache und Fach soll im Folgenden dabei helfen, die Funktion sprachlicher und struktureller Mittel zu verstehen.

\subsection{Ein sprachlicher Blick auf den Vergleich}

Wie bei der fachlichen Betrachtung des Vergleichs und der Klärung fachlicher Strukturen im Geschichtsunterricht sollen auch in diesem Teil zunächst wesentliche Formen dargestellt werden, über die insbesondere kleinere Vergleichseinheiten (ausgehend vom Satz) generiert werden können. So soll zunächst das sprachliche Verständnis vom Vergleich geklärt werden, um anschließend fachliches und sprachliches Verständnis zusammenzufügen und darüber den Vergleich im Geschichtsunterricht insgesamt besser verstehen zu können.

„England und Deutschland hatten unterschiedliche Ausgangsbedingungen für die Industrialisierung im ausgehenden 18. Jahrhundert bzw. zu Beginn des 19. Jahrhunderts." So oder so ähnlich könnte eine Einleitung einer Schüler*innenlösung aussehen. Anhand dieses einfachen Vergleichsbeispiels können erste, zugleich elementare sprachliche Strukturen des Vergleichs in einem deutschen Satz gezeigt werden. Der Satz liefert zunächst, nach Eggs, die Grundbestandteile des Vergleichs: Als solche Grundbestandteile kennzeichnet sie ein durch den Vergleich zu charakterisierendes und ein zum Vergleich dienendes Element. ${ }^{423}$ In der Literatur finden sich dafür unterschiedliche Begriffe wie Vergleichsgröße und Vergleichsobjekt u.a.m. Außerdem muss es eine gemeinsame Eigenschaft, also das ,Tertium Comparationis' geben. Eggs gibt hierzu ein Beispiel und stellt fest, dass das Tertium Compa-

\footnotetext{
${ }^{422} \mathrm{Vgl}$. Schreiber, Waltraud (2005), S. $39 \mathrm{ff}$.

${ }^{423}$ Vgl. Eggs, Frederike (2006b): Vergleiche und Vergleichen. Implikationen der Sprachwissenschaft für die Sprachdidaktik. In: Becker, Tabea und Peschel, Corinna (Hrsg.): Gesteuerter und ungesteuerter Grammatikerwerb. S. 45-62. S. 52.
} 
rationis durch ein Adjektiv ausgedrückt werden kann (so groß wie...), dabei eine Bezugsnorm entstehen muss: Diese kann durch relative Adjektive oder eine Bezugsgröße ausgedrückt werden ${ }^{424} \mathrm{Im}$ Beispiel oben könnten England als Vergleichsgröße und Deutschland als Vergleichsobjekt ausgemacht werden. Das Tertium Comparationis wären die „unterschiedlichen Ausgangsbedingungen für die Industrialisierung im ausgehenden 18. Jahrhundert und Beginn des 19. Jahrhunderts. "Das Tertium Comparationis muss strenggenommen unabhängig von den verglichenen Elementen sein. Es handelt sich um eine komplexe Bezugsgröße. Für das Schülerbeispiel muss festgestellt werden, dass gleich zu Anfang durch die Zuschreibung „unterschiedlich“ eine erste allgemeine Beurteilung vorgenommen wird, die nachfolgend durch Beispiele verifiziert oder falsifiziert werden muss (die Falsifizierung ist für einen schriftlichen Text in diesem Format eher eine theoretische Möglichkeit). Noch nicht geklärt ist, worin sich diese Unterschiedlichkeit zeigt. Im Rahmen des vorliegenden Textkorpus beginnen Vergleichstexte häufig auch mit einem Sachurteil, wie z. B. „Die Industrialisierung in England verlief schneller als in Deutschland." Auch für dieses Beispiel lassen sich die genannten Grundbestandteile des Vergleichs wiederfinden: der Verlauf der Industrialisierung in England als Vergleichsgröße, der Verlauf der Industrialisierung in Deutschland als Vergleichselement, das Adjektiv "schneller (als)“ als Tertium Comparationis. Eggs stellt das Adjektiv als Tertium Comparationis in den Mittelpunkt ihrer Betrachtung. Diesen Eingangsüberlegungen folgend kennzeichnet Eggs als sprachliche Grundbestandteile des Vergleichens das Adjektiv mit seinen Komparationsstufen und der Partikel so sowie den Ausdrücken wie und $a l s$, um einen gleichen oder unterschiedlichen Ausprägungsgrad kennzeichnen zu können. Das als drückt im Beispiel oben also den unterschiedlichen Ausprägungsgrad aus. Das Beispiel liefert nur eine, wenn auch wesentliche Form des Vergleichs. Grundsätzlich besteht eine Vielzahl an Möglichkeiten, Vergleiche auszudrücken, sie sprachlich auszuformen.

Die Bearbeitung der Frage nach den Ausformungen sprachlicher Operationen des Vergleichs muss zunächst über die Forschungsliteratur erfolgen. Dabei bezeichnet Thurmair die linguistische Forschungslage zum Vergleichen als heterogen. Mit ihr kann festgestellt werden:

„So existiert zwar eine kaum überschaubare Reihe von Untersuchungen, die aber insofern sehr beschränkt ist, als fast immer nur ein Teilbereich, nämlich im Wesentlichen die Komparativ-Strukturen, in einer spezifischen Herangehensweise, nämlich Untersuchung der Syntax (hier vor allem satzförmiger Komparative) im Rahmen der generativen Grammatik, betrachtet wird [...].“425

Zwar ist die Blickrichtung auf das Vergleichen im Rahmen dieser Arbeit weniger auf syntaktische Einzelstrukturen, wie der oben gezeigten, als vielmehr auf den Vergleich innerhalb eines komplexen Textes gerichtet, dennoch sollen an dieser Stelle zur besseren Einordnung der Untersuchungsentscheidungen für die Analyse des Schülertextkorpus einige Hinweise zum Forschungsstand folgen.

\subsubsection{Ein Überblick über die Forschungslage}

In den 1970er Jahren ist der Blick insbesondere auf Komparativkonstruktionen über Adjektivphrasen gerichtet. Auf die Diskussion, die auf die Arbeit Dohertys ${ }^{426}$ Bezug nehmend durch Wunderlich und

\footnotetext{
424 Vgl. ebd.

425 Thurmair, Maria (2001): Vergleiche und Vergleichen. Eine Studie zu Form und Funktion der Vergleichsstrukturen im Deutschen. Linguistische Arbeiten, 43. Niemeyer, Tübingen.

${ }^{426}$ Vgl. z. B. Doherty, Monika (1970): Zur Komparation antonymer Adjektive. In: ASG-Bericht 6, Berlin: Deutsche Akademie der Wissenschaft.
} 
Objartel ${ }^{427}$ weitergeführt wird, soll hier nur verwiesen werden. Um den Fokus zu verdeutlichen, soll an dieser Stelle der auch in der Folge häufiger in der Fachdiskussion zitierte Ansatz Wunderlichs in seinen Grundzügen dargestellt werden: Wunderlich untersucht 1973 in der Analyse von Vergleichssätzen Struktur und Bedeutung von Adjektivphrasen. ${ }^{428} \mathrm{Er}$ beschäftigt sich mit Strukturen von Einzelsätzen, in denen über Komparation (Komparativ und Superlativ) die Vergleichsoperation ausgelöst wird. Er beschreibt ihre syntaktischen Strukturen und leitet ihren semantischen Aussagewert ab. Er greift in einem sehr eingegrenzten Rahmen auf Vergleichssätze im Deutschen zu und arbeitet die Kleinstrukturen für den genannten Fokus ab. Einen Bezug zur Einbettung in einen Text nimmt er nicht vor.

Die Arbeit Erbens zu Vergleichsurteilen und Vergleichsstrukturen ${ }^{429}$ entstammt dem Jahr 1988. Auf sie wird sich nachfolgend Eggs stützen, weshalb sie nicht unerwähnt bleiben soll. Sie soll aber vor allem deshalb in ihren Grundzügen dargestellt werden, weil Erben über die offensichtlichen Vergleichsstrukturen mit komparativen Adjektiven hinausgeht und Strukturen mit einer höheren Implizitheit in seine Überlegungen einbezieht, die wiederum in die Betrachtung des Vergleichs im Geschichtsunterricht einbezogen werden müssen. Erben bezieht sich mindestens auf die Debatte der 1970 er Jahre ${ }^{430}$, der Zugriff ist durch die Valenzgrammatik geprägt. Er geht von Vergleichsurteilen aus und nicht von einzelnen „ [...] deutlich strukturierten Vergleichssätzen (eingeleitet durch wie, wie wenn oder als ob) [...] “431, die die Diskussion der 1970er Jahre bestimmt haben: „Es wird dabei auch deutlich werden, daß im neueren Deutsch zunehmend auch bestimmte Muster der Wortbildung genutzt werden, die man (ebenso wie metaphorisch gebrauchte Wörter) als Vergleichsstrukturen in einer bestimmten kommunikativen Funktion erkennen sollte. ${ }^{4332}$ Erben spricht neben den expliziten sprachlichen Strukturen von „verdeckten Vergleichsstrukturen“. ${ }^{433}$ Er geht damit über den zuvor dargestellten Zugriff auf Vergleichsstrukturen hinaus. Die ausgewählten Vergleichssätze sind dabei nicht konstruiert, sondern er bedient sich beispielhafter Vergleichssätze der deutschsprachigen Literatur. Er zeigt anhand von Beispielen, denen die Vergleichsgröße fehlt, dass über die Wahl von Adjektiven und ggf. zugehöriger Begleitwörter Pole der Orientierung entstehen können ${ }^{434}$, und weist darauf hin, dass Adjektive auch außerhalb der Komparation eine Norm ausdrücken können, wie er am Beispiel des,großen Wagen' zeigt, der über der Norm einer angenommenen Durchschnittsgröße liegt. Dies ist gleichzeitig ein Beispiel für einen strukturellen Vergleich. Der strukturelle Vergleich zielt auf die sprachliche Oberfläche ab und kann zeigen, dass sich hinter der Verwendung eines Adjektivs ein Vergleich verstecken kann. Im Beispiel vom großen Wagen zeigt sich, dass es auch einen kleinen Wagen geben muss (Bezug auf das Sternbild) oder (in Bezug auf Autos allgemein) verschiedene Wagengrößen angenommen werden können, wobei der große Wagen im Verhältnis größer ist als die meisten anderen. Solche Vergleiche finden auf einer impliziten Ebene statt und häufig ist man sich ihrer gar nicht bewusst, solche strukturellen Vergleiche können aber auch für die Schülertexte des Vergleichskorpus angenommen

\footnotetext{
427 Objartel, Georg (1970): Zur Semantik einfacherer Vergleichssätze im Deutschen. In: Moser, Hugo (Hrsg.): Linguistische Studien II. Düsseldorf: Schwann. S. 31-49.

${ }_{428}$ Vgl. Wunderlich, Dieter (1973): Vergleichssätze. In: Kiefer, Ferenc; Ruwet, Nicolas (Hrsg.): Generative Grammar in Europe. Dordrecht: Reidel. S. 629-672.

${ }^{429}$ Erben, Johannes (1988): Vergleichsurteile und Vergleichsstrukturen im Deutschen. In: Sprachwissenschaft 13, 1988. S. 309-329.

${ }^{430}$ Dies wird etwa durch seinen Bezug auf die in der Debatte dieser Zeit populären Strukturbäumchen deutlich.

${ }^{431}$ Erben, Johannes (1988). S. 309.

432 Ebd.

${ }^{433}$ Ebd.

${ }^{434}$ Vgl. Erben, Johannes (1988), S. 312.
} 
werden. Allerdings muss der Begriff unterschieden werden von der Überprüfung einer Struktur im Rahmen eines Vergleichstextes.

Erben zeigt weiterhin, dass solche Vergleiche auch über ein Substantiv geleistet werden können. Er stellt dies am Beispiel eines Lehrers dar, der in einem Grimmschen Märchen den Däumling als Grashüpfer bezeichnet: „Auch das ist Ausdruck eines Vergleichsurteils, der Gleichsetzung mit einer Heuschrecke. Wir könnten ergänzen: Du bist ebenso klein und keck wie ein Grashüpfer, bist nicht größer als ein Grashüpfer. “435 Daraus zieht er den Schluss, dass der Hörer oder Leser die Eigenschaften und Größen der genannten Größe kennt und sie einordnen kann. Er folgert: „,[...] das heißt, außersprachliche Weltkenntnis erspart teilweise sprachliche Mittel. ${ }^{4336}$ Erben zeigt dies etwa an der Nutzung von Metaphern und Metaphernkomposita und leitet daraus ein breites sprachliches Repertoire ab, um Vergleichsurteile zu fällen. Erben schließt seine Darstellung mit der Aussage ab:

„Da Vergleichsurteile offenbar zu den wesentlichen geistig-sprachlichen Verständigungsleistungen gehören, überrascht es nicht, daß hierfür auch die deutsche Sprache eine solche Vielfalt von Vergleichssignalen und Vergleichsstrukturen entwickelt hat, wobei das Zusammenwirken von Syntax, Semantik und Wortbildungskonstruktionen wohl besonderes Interesse verdient. ${ }^{4437}$

Auf die Weiterentwicklung der Debatte um den Vergleich aus sprachwissenschaftlicher Sicht sei beispielhaft ein Blick auf die Ausführungen von Bergerová ${ }^{438}$ geworfen. Ihr Fokus sind Vergleichs- bzw. Komparativsätze. Über eine Gegenüberstellung verschiedener Definitionen zum Vergleich in einschlägigen Grammatiken stellt sie die Überlegung an den Anfang ihrer Untersuchungen, dass Vergleichssätze nicht nur, wie in den Definitionen der ausgewählten Grammatiken festgelegt, , [...] einer Klasse der modalen Nebensätze in adverbialer Funktion gleichgesetzt werden. ${ }^{439}$ Sie stellt fest, dass Vergleichssätze auch in anderen Funktionen auftreten können, wie etwa in reinen Hauptsatzgefügen. Gleichzeitig richtet sie ihren Erkenntnisfokus auf Vergleichssätze im Nebensatz oder in nebensatzähnlicher Form. ${ }^{440}$ Ihre Korpusanalyse stützt sie auf journalistische Texte, Literatur und gesprochene Sprache, wissenschaftliche Texte nimmt sie, ausgehend von der Annahme, dass die gesuchten Vergleichsstrukturen dort weniger zu finden sind, nicht in den Blick. Ihr Schwerpunkt liegt in der syntaktischen und semantischen Betrachtung von Einzelsätzen, die sie in grammatikalisch notwendig und nicht notwendige Vergleichssätze differenziert und auf der Basis der Valenzgrammatik in Strukturbäumen aufschlüsselt.

Kurz nach der Analyse Bergerovás erscheinen die Arbeiten zum Vergleich von Hahnemann ${ }^{441}$ und Thurmair ${ }^{442}$.

Hahnemann stellt eine Einordnung des Vergleichsbegriffs an den Anfang ihrer Überlegungen, die im Vergleich der Ausführungen Wunderlichs und Erbens sichtbar wird:

\footnotetext{
435 Erben, Johannes (1988), S. 313.

${ }^{436}$ Erben, Johannes (1988), S. 314.

437 Erben, Johannes (1988), S. 329.

${ }^{438}$ Bergerová, Hana (1997): Vergleichssätze in der deutschen Gegenwartssprache. Frankfurt a. M. u.a.: Peter Lang Verlag.

439 Bergerová, Hana (1997), S. 12.

${ }^{440}$ Vgl. Bergerová, Hana (1997), S. 12.

${ }^{441}$ Hahnemann, Suzan (1999): Vergleiche im Vergleich. Zur Syntax und Semantik ausgewählter Vergleichsstrukturen mit >als< und >wie< im Deutschen. Tübingen: Niemeyer (Linguistische Arbeiten; 397).

442 Thurmair, Maria (2001): Vergleiche und Vergleichen. Eine Studie zu Form und Funktion der Vergleichsstrukturen im Deutschen. Linguistische Arbeiten, 43. Niemeyer, Tübingen.
} 
„[...] der Terminus Vergleich ist nämlich kein primär grammatischer Begriff, sondern entstammt der Rhetorik, wo er als Bezeichnung für die explizite Gegenüberstellung zweier Vergleichsmitglieder anhand eines tertium comparationis im Unterschied zu verwandten Stilmitteln wie Metapher, Gleichnis, Parabel verwendet wird. Dementsprechend wird in der Sprachwissenschaft ein weites Feld unterschiedlicher Phänomene abgedeckt: von metaphorischer Ausdrucksweise bis zur morphologischen Markierung von (komparierten) Adjektiven. ${ }^{1443}$

Gerade für die Auswertung komplexer und zusammenhängender Vergleichstexte erscheint dieser weite Vergleichsbegriff bedeutsam. Hahnemann geht deshalb in ihrem Untersuchungsansatz vom Ergebnis des Vergleichsprozesses aus: der Herstellung von Gleichheit, Ähnlichkeit oder Ungleichheit. ${ }^{444}$ Dieser Bezug passt zu dem in der Geschichtswissenschaft diskutierten und in den Einheitlichen Prüfungsanforderungen für das Fach Geschichte festgelegten Ziel für den historischen Vergleich (vgl. z. B. Einheitliche Prüfungsanforderungen für das Fach Geschichte der KMK).

Für ihre Untersuchung geht Hahnemann von der Anzahl der am Vergleich beteiligten Entitäten und der Prädikate aus, Ziel ihrer Arbeit ist eine Analyse der Formstrukturen von Vergleichen. ${ }^{445}$ Sie beschränkt dabei den Fokus auf Vergleichskonstruktionen mit mindestens drei der genannten Bestandteile und schließt damit bspw. implizite oder metaphorische Strukturen, wie bei Erben thematisiert, bewusst aus. Ihr Fokus liegt damit auf den sprachlich expliziten Strukturen des Vergleichs. ${ }^{466}$ Sie hebt sich von vorausgehenden Arbeiten ab, indem sie statt einer, wie sie es nennt, formelbasierten Beschreibung, eine Bedeutungsanalyse von als und wie leisten will. ${ }^{447}$

Der zuvor dargestellte Blick auf Untersuchungen zum Vergleichen für das Deutsche hat bereits gezeigt, dass immer nur Teilbereiche des Vergleichs untersucht werden. So konstatiert Hahnemann, dass es für das Deutsche keine umfassende Untersuchung gibt. ${ }^{448}$ Unter Bezugnahme auf zuvor benannte Forschungsarbeiten stellt sie fest:

\begin{abstract}
„Aufgrund dieser Auflistung könnte man meinen, daß es sich bei der Thematik Vergleich um ein gut erforschtes Gebiet handelt, wobei höchstens noch in Teilbereichen Präzisierungen möglich sind. Dies ist jedoch nicht der Fall. Obwohl es sich bei der kognitiven Operation des Vergleichens um einen zentralen Bereich innerhalb menschlicher Wahrnehmungs- und Kommunikationsfähigkeit handelt, ist diese Thematik nur stiefmütterlich untersucht. Besonders erstaunt, daß die einzelnen Arbeiten häufig im wissenschaftlichen Vakuum zu stehen scheinen, da vorausgegangene Untersuchungen oft nicht berücksichtigt werden. ${ }^{.449}$
\end{abstract}

Hahnemann unterstützend kann festgestellt werden, dass es insbesondere zahlreiche Arbeiten zu "als" und „wie" als Vergleichspartikeln oder-junktoren gibt, die an dieser Stelle nicht erschöpfend aufgeführt werden können.

\footnotetext{
${ }^{443}$ Ebd.

${ }^{444} \mathrm{Vgl}$. Hahnemann, Suzan (1999), S. 2.

${ }^{445}$ Ebd.

${ }^{446}$ Hahnemann, Suzan (1999), S. 3.

${ }^{447}$ Hahnemann, Suzan (1999), S.10.

${ }^{448}$ Hahnemann, Suzan (1999), S. 9. Hahnemann (ebd.) verweist auf weitere Untersuchungen bspw. bei Erben, Johannes (1988) (s.o.), einzelnen Vergleichsjunktoren bspw. bei Valentin, Paul (1988): Zu Natur, Funktion und Bedeutung der W-Wörter im heutigen Deutsch. In: Askedal, j.o. et al. (Hrsg.): Gedenkschrift für Ingerid Dal. Tübingen: Niemeyer. S. 202-213

oder Bäuerle, Rainer (1997) und zu „irrealen Vergleichssätzen“ etwa bei Oppenrieder, Wilhelm (1991): Aussagesätze im Deutschen. In: Meibauer, Jörg (Hrsg.): Satzmodus zwischen Grammatik und Pragmatik. Tübingen: Niemeyer (=LA 180), S. 161-189.

449 Hahnemann, Suzan (1999), S. 9.
} 
Hahnemann nutzt als empirische Grundlage ein Korpus aus journalistischen Texten. Sie ordnet die verschiedenen Vergleichsstrukturen mit als oder wie den Kategorien Grad-, Analogie-, Satz-, Art-Vergleich und irrealer Vergleichssatz zu. Sie systematisiert diese im Hinblick auf ihre syntaktischen Eigenschaften und schließt die Betrachtung mit der Einordnung semantischer Relationen ab.

Sie schlägt auf der Grundlage dieser Eigenschaftszuordnung für als und wie die folgende Zuordnung vor: „Als syntaktische Funktionen wurden die Kategorien Vergleichspartikel für die Vergleiche und Identifikationspartikel für die Prädikationsrelationen vorgeschlagen, da sich eine Zuordnung zu den Präpositionen oder den Konjunktionen als nicht plausibel erwiesen hat. ${ }^{\text {“450 }}$

Thurmair, die aufgrund der Nähe des Erscheinens ihrer und der Untersuchung Hahnemanns beklagt, nur kaum auf Hahnemann Bezug nehmen zu können, wirft einen textlinguistischen Blick auf den Vergleich. Thurmair geht als grundlegende Operation zunächst von der Komparation aus und nennt, übereinstimmend mit der Darstellung Eggs', als sprachliche Mittel, die eine Vergleichsoperation auslösen, das Komparativmorphem - er und das Superlativmorphem - $e(s t)$, weiterhin die Partikeln so sowie die Vergleichsjunktoren als und wie. Sie ordnet diese sprachlichen Strukturen in das (funktionalpragmatische) Feld der operativen Strukturen (sie lösen eine Vergleichshandlung aus) ein und unterscheidet davon sprachliche Mittel, die das Ergebnis eines Vergleichs darstellen, und strukturelle Vergleiche, die ,nur' aufgrund einer bestimmten Struktur als Vergleich wirken, aber inn nicht benennen. Ihre Blickrichtung ist auf die operativen Mittel des Vergleichs gerichtet. Thurmair taxiert insbesondere Vergleichsstrukturen innerhalb eines Satzes oder einer kleineren syntaktischen oder inhaltlichen Einheit, die sie in Textausschnitte einordnet. Sie kennzeichnet als grundsätzliche Vergleichsstrukturen Äquativvergleiche, Komparationsvergleiche und Superlativvergleiche. Unter Äquativvergleichen fasst sie alle Vergleiche, „,[...], die mit wie gebildet einen Vergleich der Gleichheit zum Ausdruck bringen. ${ }^{\text {“451 }}$ Sie differenziert diese in Grad-, Art-, Modalitäts-, Faktizitäts- und offene Vergleiche. Die Komparativvergleiche beschreibt sie als solche, , „[...] bei denen für Komparandum und Komparationsbasis Ungleichheit hinsichtlich des Ausprägungsgrades einer Eigenschaft angezeigt wird. ${ }^{4552}$ Dabei gehört zu innen immer auch ein komparativisches Adjektiv. Die Superlativvergleiche ordnet sie schließlich als solche ein, ,[...] bei denen der Ausprägungsgrad einer Eigenschaft des Komparandums mit beliebigen anderen Vergleichsentitäten verglichen wird; sie enthalten immer ein Adjektiv im Superlativ [...]. ${ }^{4453}$

Auf der Grundlage dieser Differenzierung leistet Thurmair eine Differenzierung und Einordnung der gängigen Vergleichsstrukturen auf der Grundlage ihres sprachlichen Erscheinungsbildes.

Thurmairs Ergebnisse sind insbesondere in Bezug auf die Strukturen von Komparandum und Komparationsbasis interessant, da sie diese in verschiedene und grundlegende Typen einteilen kann, die sich nicht nur auf Einzelsätze beziehen, sondern einen Text in den Blick nehmen. Die Arbeit Thurmairs kann damit eine Hilfe darstellen, um Kleinstrukturen einzelner Schüler*innen-Lösungen zur vorgegebenen historischen Vergleichsaufgabe herauszuarbeiten. Sie bietet jedoch weniger eine Hilfe, wenn es um die Frage geht, wie die sprachliche Handlung des Vergleichens im Sinne eines Ganztextes konstruiert werden kann.

\footnotetext{
${ }^{450}$ Hahnemann, Suzan (1999), S. 235.

${ }^{451}$ Thurmair, Maria (2001), S. 3.

$452 \mathrm{Ebd}$.

${ }^{453} \mathrm{Ebd}$.
} 
In den bisher chronologischen Zugriff sich einreihend, vom linguistischen Ansatz aber abweichend, sollen die Arbeiten von Eggs zum Vergleich und zu den „Funktionswörtern“454 als und wie in den Blick genommen werden, da sie im Gegensatz etwa zum stark bearbeiteten Ansatz der generativen Grammatik einen funktional-pragmatischen Zugriff wählt. ${ }^{455}$ Zu nennen sind in diesem Zusammenhang insbesondere ihre Dissertationsschrift zu „Die Grammatik von als und wie ${ }^{4456}$ sowie ihr Aufsatz „Vergleichen und Vergleiche - Implikationen der Sprachwissenschaft für die Sprachdidaktik ${ }^{4457}$, der sich auf Erkenntnisse aus ihrer Dissertationsschrift bezieht.

Da es sich beim Vergleichen um einen kommunikativen Prozess handelt, der das Ziel hat, Gemeinsamkeiten und/oder Unterschiede herauszuarbeiten, muss er auch in linguistischer Hinsicht in seiner kommunikativen Funktion wahrgenommen werden. Dazu passt der Blickwinkel, den Eggs auf Grammatik und über die Junktoren (zuvor als Partikel benannt, die Begrifflichkeit muss im Folgenden noch systematisiert werden) als und wie auf den Vergleich wirft:

„Formen müssen hinsichtlich ihrer funktionalen Bestimmung, Funktionen hinsichtlich der dafür in einer Sprache ausgebildeten Funktionen betrachtet werden, und schließlich müssen beide im Zusammenhang ins Blickfeld der Analyse genommen werden. Daraus folgt zwingend, dass Formeinheiten wie Wort, Phrase oder Satz als Grundeinheiten nicht nur syntaktischer, sondern auch funktional-semantischer Kombinatorik anzusehen sind; grammatische Kategorien sind handlungsbezogen zu fundieren. “458

Dieser Ansatz ist auch für das Vergleichen im Geschichtsunterricht und die vorliegenden Schülerschreibprodukte bedeutsam, da diese ebenfalls in ihrer kommunikativen Funktion wahrgenommen werden müssen. Da es sich bei den Lernenden auch in der gymnasialen Oberstufe in mancherlei Hinsicht immer noch um (sicherlich im Vergleich zu jüngeren Lernenden fortgeschrittene) Schreibnovizen handelt, die insbesondere an das wissenschaftliche Schreiben herangeführt werden müssen, kann nicht davon ausgegangen werden, dass Normvorgaben oder als typisch anerkannte Strukturen des Vergleichs immer eingehalten werden. Zudem zeigt die zuvor beschriebene Analyse der Methodenhinweise zum Vergleichen in Lehrwerken, dass gar nicht klar ist, was denn überhaupt typische Strukturen darstellen. Es kann weiterhin angenommen werden, dass die Schüler*innen innerhalb des Schreibprozesses Strukturen und Formen ausprobieren, sie in dieser Hinsicht erst aushandeln müssen, um die komplexe Aufgabe des historischen Vergleichs lösen zu können.

Von welchen Grundannahmen geht Eggs beim Vergleichen aus?

Eggs steckt zunächst einen allgemeinen Rahmen des Vergleichens ab und nimmt Bezug auf Vergleichbares und Unvergleichbares.

Im Verlauf der geschichtswissenschaftlichen Diskussion wurde immer wieder darüber debattiert, was überhaupt verglichen werden kann, während Kaelble von zwei oder mehreren historischen Einheiten

\footnotetext{
${ }^{454}$ Vgl. Eggs, Frederike (2006a): die Grammatik von als und wie. Tübingen: Gunter Narr Verlag. S. 10.

455 Der Vollständigkeit halber sei erwähnt, dass Oppenrieder 1986 einen ähnlichen Ansatz in Bezug auf deutsche Aussagesätze wählt, Vergleichssätze stellen in diesem Zusammenhang jedoch lediglich einen Teilaspekt dar. Vgl. Oppenrieder, Wilhelm (1986): Aussagesätze im Deutschen. In: Meibauer, Jörg (Hrsg.): Satzmodus zwischen Grammatik und Pragmatik. Referate anläßlich der 8. Jahrestagung der Deutschen Gesellschaft für Sprachwissenschaft, Heidelberg 1986. Tübingen: Niemeyer Verlag. S. 161-189.

${ }^{456}$ Eggs, Frederike (2006a).

${ }^{457}$ Eggs, Frederike (2006b).

${ }^{458}$ Eggs, Frederike (2006a), S. 10.
} 
spricht, die miteinander verglichen werden können, und hierunter Orte, Regionen, Nationen, Zivilisationen oder historische Persönlichkeiten versteht ${ }^{459}$, stellt Eggs einen ganz offenen überfachlichen Ansatz dagegen, den sie im weiteren über einen linguistischen Zugriff fundiert:

„Zwei Dinge miteinander zu vergleichen bedeutet also immer, dass sie wenigstens eine Gemeinsamkeit aufweisen. Aber auch die Feststellung, dass zwei Dinge nicht miteinander verglichen werden können, können wir erst dann treffen, wenn wir versucht haben, eben dies zu tun und daran gescheitert sind. ${ }^{\prime 460}$

Dies ist eine pragmatische Aussage, die weit über den geschichtswissenschaftlichen Ansatz der neuesten Zeit, wie er sich bspw. bei Kaelble darstellt, hinausgeht. Auf den Geschichtsunterricht bezogen könnte in Anlehnung an Eggs als weiteres Lern- und Erkenntnisziel formuliert werden, herauszufinden, welche historischen Einheiten sich nicht vergleichen lassen und warum dies so ist.

Eggs Blick ist auf den Unterricht und die Bedeutung des Vergleichens für die Lernenden gerichtet. Sie zeigt beispielhaft, welche Bedeutung das Vergleichen für das Lernen und den Erkenntnisprozess in den Fächern hat. Sie folgert daraus:

„Was läge im Hinblick auf die fächerübergreifende Relevanz des Vergleichens näher, als die mehr oder weniger bewusst stattfindenden Vergleichsprozesse explizit zu machen und im Unterricht die verschiedenen Arten des Vergleichens etwas genauer unter die Lupe zu nehmen, um sich Klarheit über einzelne Ausdrucksformen und deren Zwecke zu verschaffen? “461

Auf den Geschichtsunterricht bezogen, bedeutet dies bspw. für Lernende den Unterschied zwischen einem inhaltlichen und medialen Vergleich und der dahinterliegenden Zwecke zu verdeutlichen.

Die vorliegende Untersuchung widmet sich dem inhaltlichen Vergleich und soll dazu beitragen, dieses Desiderat ein Stück abzubauen, indem gezeigt werden soll, wie Lernende den historischen Vergleich an dem konkreten Beispiel der Industrialisierung realisieren, um daraus Ansätze der Unterstützung erarbeiten zu können.

Nach dem allgemeinen Rahmen für das Vergleichen bezieht sich Eggs dann auf (grammatische) Grundbestandteile des Vergleichens. Hier nimmt sie Adjektive sowie die Partikeln so sowie die Junktoren (oder auch, wie Hahnemann sie klassifiziert, Partikeln) als und wie heraus. Diesen grammatischen Formen ordnet sie im Unterschied zu den meisten der zuvor dargestellten Arbeiten kommunikative Funktionen zu. Im Rahmen ihrer Dissertationsarbeit nennt sie als Ziel:

„Die leitende These ist also, dass die verschiedenen Verwendungen von als und wie nicht auf unterschiedliche Bedeutungen zurückgeführt werden dürfen, sondern dass es eine allen Verwendungen von als und eine allen Verwendungen von wie zugrundeliegende gemeinsame Grundbedeutung gibt, die in jeder Verwendung rekonstruierbar sein muss. Grundlegendes Untersuchungsprinzip ist demnach, die Wörter als und wie als einheitliche Formen zu betrachten und dementsprechend auch einheitliche Funktionen für sie herauszuarbeiten. ${ }^{\text {4462 }}$ [Hervorhebung im Original]

Das so ordnet Eggs als Aspektdeixis ein und kennzeichnet es im Zusammenhang mit einem Adjektiv als Ausprägungsgrad der durch das Adjektiv bezeichneten Eigenschaft. ${ }^{463}$

Sie geht weiterhin darauf ein, dass Adjektive für einen Vergleich grundsätzlich graduierbar sein müssen, dennoch auch absolute Adjektive für einen Vergleich verwendet werden, die auf diese Art und Weise in relative Adjektive umkategorisiert werden.

\footnotetext{
${ }^{459}$ Kaelble, Hartmut (2012), S. 2 (vgl. dazu Kapitel 3.1).

${ }^{460}$ Eggs, Frederike (2006b), S. 46 (vgl. dazu Kapitel 3.1).

${ }^{461}$ Eggs, Frederike (2006b), S. 47.

${ }^{462}$ Eggs, Frederike (2006a), S. 11.

${ }^{463}$ Vgl. Eggs, Frederike (2006b), S. 54.
} 
Formulierungen mit so, als und wie spielen durchaus auch in Vergleichen im Geschichtsunterricht eine Rolle, jedoch lässt sich auch bei diesem funktionalen Zugang die Schwierigkeit feststellen, dass eine von Eggs angenommene gemeinsame Grundbedeutung im Hinblick auf die unterschiedlichen historischen Phänomene in der von ihr geforderten Klarheit nicht feststellbar ist. Auch bei Eggs zeigt sich nachfolgend, dass sich diese gemeinsame Grundbedeutung eher überfachlich als fachspezifisch herleiten lässt. Für den Untersuchungszusammenhang erscheint deshalb ihre Differenzierung in homogene und heterogene Vergleiche ertragreicher. Homogene Vergleiche kennzeichnet sie als solche, „[...] bei denen die beiden Vergleichsgrößen in ein und demselben Wirklichkeitsraum zu lokalisieren sind [...]. “464 Heterogene Vergleiche hingegen charakterisiert sie als solche Vergleiche „[...], die zwischen Dingen vorgenommen werden, die aus verschiedenen Bereichen der Wirklichkeit stammen $[\ldots]^{\prime \prime}{ }^{465}$ Ein Beispiel für einen homogenen Vergleich, der inhaltlich für die historische Vergleichsaufgabe des Textkorpus passt, könnte also lauten: Die Industrielle Revolution in England verlief schneller als in Deutschland. Ein heterogener Vergleich für die Aufgabe könnte sein: Die Industrielle Revolution in England verlief so schnell wie ein D-Zug. Beide Beispiele sind konstruiert und entstammen nicht dem Textkorpus. Die Korpusbeispiele entstammen insbesondere der Kategorie der homogenen Vergleiche. Dies ist sicherlich nicht zuletzt der Aufgabenstellung geschuldet, die einen sachlichen Vergleich innerhalb eines Wirklichkeitsbereichs einfordert. Ein heterogener Vergleich wäre insbesondere als Vergleichsabschluss im Zusammenhang mit der Bildung eines möglichen Werturteils denkbar. Eggs weist darauf hin, dass insbesondere über Metaphern ein heterogener Vergleich gebildet werden kann. ${ }^{466}$ Auf den Bereich der Metaphern als „verkürzte Analogievergleiche ${ }^{\text {4467 }}$ kann an dieser Stelle nur verwiesen werden.

Auf der Grundlage einiger Beispiele charakterisiert Eggs die unterschiedlichen Erkenntnisbereiche der beiden Vergleichstypen:

"Gerade diese Beispiele machen noch einmal sehr gut deutlich, dass der durch den Vergleich zu charakterisierende Wirklichkeitsbereich im Falle des heterogenen Vergleichs neu strukturiert wird, da er gewissermaßen durch die Brille des zum Vergleich herangezogenen Wirklichkeitsbereichs hindurch gesehen wird.

Eben dies geschieht bei einem homogenen Vergleich nicht: Hier werden dem Hörer einfach nur neue Informationen geliefert, die es ihm erlauben, Informationslücken aufzufüllen, die er in einem bestimmten Bereich seines Wissens hatte. Dabei sind diese Informationen konform mit dem jeweiligen Wissensraum und bringen keine Neustrukturierung desselben mit sich. ${ }^{4468}$

Es kann also angenommen werden, dass eine Neustrukturierung des durch den Vergleich angesprochenen Wirklichkeitsbereichs in der Regel erst dann gelingt, wenn das nötige Wissen erschlossen ist. Eggs folgert, wenn sie in Bezug auf Analogievergleiche durch Metaphern feststellt: „Daraus ergibt sich, dass der homogene Vergleich funktional gesehen dazu dient, ein Informationsdefizit zu beheben [Hervorh. i. Orig., Anm. d.Verf.], wohingegen der heterogene Vergleich - ebenso wie eine Metapher - typischerweise dann eingesetzt wird, wenn es darum geht ein Verstehensdefizit zu bearbeiten. ${ }^{4469}$

\footnotetext{
${ }^{464}$ Eggs, Frederike (2006b), S. 58.

465 Ebd.

${ }^{466} \mathrm{Vgl}$. ebd.

${ }^{467}$ Vgl. Eggs, Frederike (2006a), S. 42ff.

${ }^{468}$ Eggs, Frederike (2006b), S. 60.

${ }^{469}$ Eggs, Frederike (2006a), S. 67. Hinzuweisen sei auf ihre Differenzierung: Ein heterogener Vergleich ist nicht nur durch eine Metapher zu realisieren. Zudem sind heterogener Vergleich und Metapher in der Realisierung von Bedeutung unterschiedlich. Eggs beschreibt die Metapher im Vergleich zum heterogenen Vergleich im Allgemeinen als anspruchsvoller. Vgl. S. 71.
} 
Der Begriff eines homogenen Vergleichs ist anschlussfähig an den in den Kernlehrplänen für den Geschichtsunterricht geforderten inhaltlichen Vergleich. Im Hinblick auf den Begriff des heterogenen Vergleichs kann für den Geschichtsunterricht ebenfalls seine Bedeutung festgestellt werden: Über ihn kann die Frage, wann historische Vergleiche sinnvoll oder eben nicht sinnvoll sind, thematisiert werden. In Bezug auf das oben genannte Beispiel kann diskutiert werden, inwiefern die Frage, ob die Industrielle Revolution in England so schnell wie ein D-Zug verlief, zur Diskussion genutzt wird, ob sie zur fachspezifischen Erkenntnisbildung beiträgt. Dem könnte dann ein homogener Vergleich gegenübergestellt werden.

Der bereits angesprochene Schwerpunkt der Arbeit Eggs', die Ableitung der Grundbedeutungen von als und wie, soll an dieser Stelle in Bezug auf homogenen und heterogenen Vergleich kurz beleuchtet werden. Eggs ordnet als und wie der Gruppe der Junktoren zu, die sie insbesondere in Adjunktoren und Subjunktoren teilkategorisiert. Eggs stellt fest, dass als auf eine „Art von Andersartigkeit", wie auf eine „Art von Gleichheit“ verweist, und ordnet diesen Oberkategorien Unterkategorien, Fallbeispiele und syntaktische Strukturen zu. ${ }^{470}$ Sie ordnet die Junktoren in den Bezugsrahmen der homogenen und heterogenen Vergleiche ein. Hahnemann dagegen entscheidet sich für die Klassifizierung als (Vergleichs-)Partikel. Beide Begriffe erscheinen plausibel. Der Begriff des Junktors hat (im Sinne der Funktionalen Pragmatik) den Vorteil, dass er sich auf die Aussagelogik bezieht, damit den Zweck der sprachlichen Mittel als oder wie in den Blick nimmt; Junktor meint die logische Verknüpfung zwischen Aussagen. Im Rahmen dieser Arbeit werden auch aufgrund der Gängigkeit der Zuordnung als Partikel beide Begriffe Verwendung finden. ${ }^{471}$ Angemerkt sei an dieser Stelle, dass Eggs dafür plädiert, die Zuordnung von als und wie insbesondere als Adjunktor vorzunehmen. Sie begründet dies mit der syntaktischen Funktion sowie der semantischen Interpretation von als und wie. ${ }^{472}$ Diese sprachlichen Mittel können im Geschichtsunterricht in Bezug auf das Vergleichen dafür genutzt werden, für Lernende die Konzepte von Andersartigkeit und Gleichheit transparent zu machen.

\subsubsection{Bedeutung für das Vergleichen im Geschichtsunterricht}

Für die Erfassung komplexer Schülertexte bieten die Untersuchungen zum Vergleichen insbesondere über das Feld der generativen Grammatik zwar Ansätze für syntaktische Einzelstrukturen. Die komplexen sprachlichen und fachlichen Teilhandlungen, die die Lernenden für einen Vergleich im Geschichtsunterricht realisieren müssen, wenn sie die Aufgabe beantworten wollen, können so jedoch nicht abgebildet werden. Das sprachliche Feld ist sehr vielfältig, über das Schüler*innen den Vergleich zu realisieren suchen. Gleiches gilt für die mit Sprache zu verknüpfende inhaltliche Dimension.

In manchen Lernendentexten misslingt der Vergleich, weil die sprachlichen Strukturen nicht korrekt produziert werden oder der Vergleich nur teilweise realisiert werden kann. Manchmal ist auch die

\footnotetext{
470 Eggs, Frederike (2006a), S. 517.

${ }^{471}$ Diese „doppelte“ Zuordnung ist nicht zuletzt der Diskussion um als und wie geschuldet. Eggs stellt dazu fest: „Es gibt wohl kaum zwei Ausdrücke des Deutschen, über deren genaue kategoriale Bestimmung sich die germanistische Forschung derart uneins ist wie über die Ausdrücke als und wie." (Eggs, Frederike (2007): Adjunktor. In: Hoffmann, Ludger (Hrsg.): Handbuch der deutschen Wortarten. 2. Aufl. Berlin u.a.: de Gruyter. S. 189-221. S. 189). Diese Diskussion kann im Rahmen dieser Arbeit kaum erschöpfend dargestellt werden, weshalb mit den Begriffen Partikel und Junktor gearbeitet werden soll, wohl wissend, sich damit der wiss. Kritik auszusetzen. 472 Die Zuordnung als Adjunktor kann nachvollzogen werden in: Eggs, Frederike (2007).
} 
sprachliche Norm inkorrekt oder es treffen mehrere der aufgezählten Aspekte zu. Manchmal wird das Gemeinte vielleicht dennoch deutlich. Gerade in diesen Fällen braucht es ein Instrument, über das dargestellt werden kann, mit welchen Mitteln die Lernenden versuchen, den Vergleich zu realisieren. Hierüber können im Weiteren Strategien entstehen, die die Lernenden in der Aufgabenlösung unterstützen. Zudem macht das Textkorpus deutlich, dass nicht vorrangig Formulierungen durch Komparativadjektive mit als und wie eine Rolle spielen. Dies bedeutet, dass die Analyse über solche offensichtlichen sprachlichen Vergleichsstrukturen hinausgehen muss. Eggs wählt mit Hilfe der Funktionalen Pragmatik einen Zugriff über die kommunikative Funktion. Dieser Zugriff wird auch für die nachfolgende Untersuchung gewählt, muss aber über die von Eggs benannten und untersuchten sprachlichen Mittel hinausgehen. Lernende, das wird die Auswertung zeigen, benötigen und verwenden weitere sprachliche Teilhandlungen, die das historische Vergleichen ausmachen. Diese können im Sinne der Funktionalität von Sprache über den kommunikativen Zweck des Schreibanlasses eingeordnet werden.

Wie lassen sich also konkret Teilhandlungen, Strukturen etc. kategorisieren, die die Lernenden in ihren Texten verwenden, und wie lässt sich beurteilen, ob diese erfolgreich für den Vergleich sind? Zu diesen Fragestellungen soll das nachfolgend vorzustellende und zu diskutierende Kategoriensystem einen Beitrag leisten. Die Basis der weiterführenden Überlegungen stellt das Korpus der Schülerarbeiten dar. Außerdem werden die Vorgaben und Richtlinien sowie die forschungsbasierten Ansätze, die zuvor vorgestellt wurden, herangezogen Es handelt sich also um ein Kategoriensystem, das sowohl corpusbased als auch corpus-driven abgeleitet wurde. 


\subsection{Sprachliche Teilhandlungen als wesentliche Elemente des Vergleichens im Geschichtsunterricht}

In das Kategoriensystem sind die sprachlichen Teilhandlungen eingegangen, die sich aus den EPA ableiten lassen und die sich in den Texten der Lernenden finden lassen. Dabei fällt auf, dass die in den Schüler*innentexten vorkommenden sprachlichen Teilhandlungen häufig gleich sind, sich also bestimmte Teilhandlungen als wiederkehrend ableiten lassen. ${ }^{473}$ Dies kann zum einen mit dem der Aufgabe zugrundeliegenden Darstellungstext zusammenhängen, zum anderen mit einem vermutlich impliziten Wissen über den Zweck des Vergleichens im Geschichtsunterricht.

Die nachfolgende Darstellung gibt einen Überblick über die durch EPA und Schüler*innentexte generierten sprachlichen Teilhandlungen.

\section{Vergleichen im Geschichtsunterricht}

\section{Sprachliche Teilhandlungen:}

Gegenüberstellen

Erklären

Begründen

Beurteilen
Diese können sich zeigen:

-im Strukturkonzept („Bauplan“)

-in textkompositionellen Elementen (z. B. „Rahmung“, Absätze etc.)

-in Textelementen oder Begriffen

-in der Herstellung sprachlicher Zusammenhänge ...

Abbildung 11: Sprachliche Teilhandlungen des Vergleichens im Geschichtsunterricht (eigene Darstellung)

Gegenüberstellen und Beurteilen als sprachliche Teilhandlungen werden explizit in den EPA benannt. Darüber hinaus finden sich in den Texten der Lernenden Begründungsstrukturen, die sich daraus erklären, dass Beurteilungen Begründungen benötigen, und Erklärungen, die für das Verständnis des historischen Kontextes benötigt werden. Diese Teilhandlungen werden nachfolgend auf der Grundlage ihrer Bestimmungen in den EPA aufgeschlüsselt und auf das Vergleichen im Geschichtsunterricht bezogen. Darüber hinaus konnte insbesondere eine weitere sprachliche Teilhandlung aus dem Textkorpus herausgefiltert werden: die Folge. Sie wird in Bezug auf die Vorstellung des Kriterienrasters thematisiert. Es wird jedoch zu zeigen sein, dass sie keine im engen Sinne vergleichsspezifische sprachliche Teilhandlung darstellt. Sie findet deshalb an dieser Stelle noch keine Berücksichtigung.

\footnotetext{
${ }^{473}$ Mit Hilfe eines kleinen Korpus an Texten von Lehrenden, die ebf. die Vergleichsaufgabe gelöst haben, konnten die in den Schüler*innentexten auftretenden Strukturen zudem mit den Strukturen in ,Expert*innentexten' abgeglichen werden.
} 
Die für das Vergleichen im Geschichtsunterricht als relevant erkannten Teilhandlungen werden nachfolgend hinsichtlich ihrer Strukturen hinterfragt. Es geht noch nicht um eine Aufschlüsselung der zu ihrer Realisierung benötigten sprachlichen Mittel. Diese werden im Zusammenhang in dem sich aus diesen Vorüberlegungen ergebenden Kriterienraster expliziert.

\subsubsection{Gegenüberstellen als Teilhandlung des historischen Vergleichs}

Das Gegenüberstellen stellt ein wesentliches Element des Vergleichs dar. Ohne mindestens zwei Elemente gegenüberzustellen, ist Vergleichen nicht möglich. Entsprechend wird auch in den EPA das Gegenüberstellen als wesentliches Element zur Bestimmung des Vergleichs aufgenommen.

In den EPA Geschichte wird das Gegenüberstellen als „wie skizzieren, aber zusätzlich argumentierend gewichten ${ }^{4774}$ bestimmt. Skizzieren bedeutet dort: „historische Sachverhalte, Probleme oder Aussagen erkennen und zutreffend formulieren. ${ }^{4775}$ Problematisch erscheint jedoch, dass aus dieser Bestimmung nicht erkennbar wird, dass historische Gegenstände hinsichtlich ihrer Gemeinsamkeiten, Unterschiede oder Ähnlichkeiten untersucht werden müssen, um ,zutreffende Aussagen' formulieren zu können. ${ }^{476}$

Das in den EPA für das Gegenüberstellen angeführte Gewichten ist nicht als Operator für den Geschichtsunterricht bestimmt, es meint jedoch überfachlich, dass der zu gewichtende Gegenstand im Hinblick auf einen übergeordneten Gegenstand bewertet wird (etwa als Einflussgröße auf diesen), er kann damit ein Gegenüberstellen implizieren, muss dies aber nicht zwangsläufig, weil Gewichten auch einen Anteil an einer Gesamtgröße meinen kann. Insofern ist der Begriff für das Gegenüberstellen unscharf. Eine konkrete Klärung, was Gegenüberstellen meint, wird durch den Operator nicht geleistet. Über das im folgenden Kapitel vorzustellende Kriterienraster soll deshalb herausgefunden werden, wie Lernende das Gegenüberstellen realisieren, da aufgrund der sehr allgemeinen EPA-Bestimmung nicht davon ausgegangen werden kann, dass es in Bezug auf den Geschichtsunterricht im Hinblick auf seine Teilhandlungen expliziert wurde.

Der Zweck des Gegenüberstellens kann über die EPA hinaus als Konfrontation eines (historischen) Gegenstandes mit einem anderen beschrieben werden, um so mögliche Unterschiede, Gemeinsamkeiten oder Ähnlichkeiten zu erkennen. Entsprechend werden für das Gegenüberstellen überfachliche sprachliche Mittel benötigt, die einen Gegensatz kennzeichnen. Dazu gehören beispielsweise adversative Konjunktionen wie der Subjunktor während oder Konstruktionen mit anstatt, die in sogenannte Konfrontativsätze eingebettet werden. ${ }^{477}$ Neben der Nutzung solcher expliziter sprachlicher Mittel zur Kennzeichnung des Gegenüberstellens ist außerdem der Kontext bzw. die verwendete Lexik bedeutsam: „Diese Gegensätzlichkeit wird in der Regel an bestimmten lexikalischen Oppositionen erkennbar, z. B. auf staatlicher Ebene versus Parteibeziehungen [...]“ ${ }^{4}{ }^{478}$ Erst der Kontext und die verwendete Fachlexik lassen das Gegenüberstellen fachspezifisch werden.

\footnotetext{
${ }^{474}$ Kultusministerkonferenz (2005): EPA Geschichte, S. 8.

475 Ebd. S.7.

476 Da der Begriff des Gegenüberstellens in erster Linie die Unterschiede impliziert, stehen sie hier im Mittelpunkt. Dabei sind auch Gemeinsamkeiten und Ähnlichkeiten mitgedacht, die aber erst im Vergleich explizit werden.

${ }^{477}$ Vgl. dazu: Zifonum, Gisela; Hoffmann, Ludger; Strecker, Bruno (1997): Grammatik der deutschen Sprache. Bd. 3. Berlin, New York: de Gruyter. S. $2324 f$.

478 Ebd. S. 2324.
} 
Laut EPA benötigt das Gegenüberstellen Argumentationsstrukturen („argumentierend gewichten“). Grundsätzlich muss festgestellt werden, dass ein wesentliches Werkzeug des Geschichtsunterrichts das Argumentieren darstellt. Dies liegt am Konstruktionscharakter von Geschichte. Jedoch sind argumentative Strukturen zu unterscheiden von der historischen Argumentation als übergeordneter Operation, wie Brauch/Mierwald sie am Beispiel des „How To Write Your Essay“-Plans beschreiben. ${ }^{479}$ Die für das Vergleichen ausgearbeiteten Teilhandlungen, wie das Gegenüberstellen, verstehen sich als argumentativ, sind aber von einer historischen Argumentation im engeren Sinne divergent, da für den Vergleich Strukturen über das Argumentieren hinaus verwendet werden. Gemeinsam ist beiden Formen wiederum, dass die für Vergleich und Argumentation geforderten Strukturen am Ende in einem Sachurteil zusammengeführt werden. ${ }^{480}$

\subsubsection{Erklärungen als Elemente des historischen Vergleichs}

Ohne Erklärungen kann kein Vergleichen im Geschichtsunterricht stattfinden, zumindest dann nicht, wenn, wie in den EPA gefordert, historische Sachverhalte problembezogen gegenübergestellt werden sollen. Auch die vielen inhaltlichen Aspekte, die durch den Darstellungstext der ausgewählten Aufgabe angeschnitten, aber nicht ausgeführt werden, fordern Erklärungen ein, wenn sie in den Vergleich transferiert werden. Das Erklären von historischen Zusammenhängen ist damit in Abgrenzung vom alltagssprachlichen Erklären ein fachliches Konzept des Erklärens.

Im Folgenden soll gezeigt werden, wodurch das fachliche Erklären gekennzeichnet ist. Da die EPABestimmung für das Erklären auch das Begründen einfordert, werden die beiden Operationen nachfolgend immer wieder aufeinander bezogen.

Aus funktionalpragmatischer Perspektive sind Begründen und Erklären trennscharf hinsichtlich des innen zugeordneten Zwecks zu unterscheiden. In den EPA Geschichte wird das Begründen als Teil des Erklärens bestimmt. Dem historischen Erklären und Begründen wird jeweils eine eigene fachspezifische Funktion zugeordnet, die von der linguistischen zu unterscheiden ist. Für die Geschichtswissenschaft stellt das historische Erklären ein fachliches Werkzeug dar, das zur Realisierung Begründungen benötigt. Aus linguistischer Sicht passt diese Einordnung nicht zusammen. Die unterschiedlichen Ansätze von Geschichtswissenschaft und funktional-pragmatischer Blickrichtung sollen deshalb nachfolgend dargestellt und für eine unterrichtliche Didaktisierung zusammengeführt werden.

\footnotetext{
479 Vgl. Mierwald, Marcel; Brauch, Nicola (2015).

${ }^{480}$ Eine exakte Abgrenzung des Argumentierens vom Vergleichen kann im Rahmen dieser Arbeit nicht vorgenommen werden, schon deshalb nicht, weil das Argumentieren nicht ausreichend untersucht ist: „Wer glaubt, Argumentieren sei als eine Redepraxis oder Sprechhandlungsweise ,da' und könne einfach empirisch auf seine Eigentümlichkeiten untersucht werden, irrt sich. Immer wird irgendein Begriff vom Argument mitgebracht, der die Strukturen ,vorgibt'. [...] Freilich haben wir ein Vorverständnis. Wörter wie ,Argument', ,Argumentation', Argumentieren kommen in der Umgangssprache vor, haben eine gewisse Bedeutung. Doch dieses Vorverständnis genügt nicht für eine präzise Ab- oder Ausgrenzung gewisser Aktivitäten. Vielmehr erscheint diese eingebettet in ein mannigfaltiges sprachliches (und nichtsprachliches) Geschehen." (Wohlrapp, Harald, 2009: Der Begriff des Arguments. Über die Beziehungen zwischen Wissen, Forschen, Glauben, Subjektivität und Vernunft. 2. Aufl. Würzburg: Verlag Königshausen \& Neumann. S. 7.) Im Folgenden werden zwar immer wieder argumentative Strukturen thematisiert. Es muss aber mit Wohlrapp davon ausgegangen werde, dass eine Struktur des Argumentierens auch für das Argumentieren im Geschichtsunterricht nicht umfassend dargestellt werden kann. Insofern erscheint es konsequent, dass das Argumentieren in den EPA nicht als eigenständiger Operator dargestellt wird.

Im Rahmen dieser Arbeit wird deshalb auf den Versuch einer Aufschlüsselung verzichtet.
} 
Was macht historisches Erklären aus? Welskopp bestimmt es wie folgt:

„Etwas geschichtlich zu e. [erklären. Anm. d. Verf.] heißt, ein historisches Phänomen auf seine Ursachen und Bedingungen zurückzuführen. Es gilt als e., wenn es als notwendige Folge von (auch zeitlich) vor ihm liegenden Einflussfaktoren bestimmt worden ist, d.h., wenn eine kausale ( $\rightarrow$ Kau-

salität) Beziehung zwischen Ursachen und Folgen identifiziert werden kann. ${ }^{4481}$

Im Mittelpunkt steht damit die Herstellung kausaler Beziehungen, diese bestimmt Welskopp insbesondere über die historische Dimension der Zeit und macht sie so zu einem historischen Erklären. Hinter dieser Oberfläche verbergen sich aber weitere Aspekte, die nur über den Blick auf die historische Entwicklung und die damit verbundene geschichtswissenschaftliche Diskussion um das Erklären deutlich wird und so auch verdeutlichen kann, warum historisches Erklären mit dem Begründen zusammenhängt.

Die Debatte um das Erklären ist unmittelbar verknüpft mit der Frage, wie man überhaupt zu geschichtswissenschaftlicher Erkenntnis gelangt:

„Zu ,erklären' hieß also immer auch, Argumente, Kategorien, Modelle und zusammenhängende Darstellungen zu begründen, Konstruktionen einer Vergangenheit diskursiv abzusichern, die man nun einmal nicht beweisen konnte, indem man sich zurückversetzte oder sie in einer experimentalen Reihe unter Laborbedingungen wiederholte. ${ }^{4482}$

Welskopp stellt hier die Auseinandersetzung der Fachwissenschaft Geschichte mit den Methoden der Naturwissenschaften in den Mittelpunkt, durch die der Diskurs um das Erklären nicht unerheblich geprägt wurde und aus dem sich letztlich eine Form des historischen Erklärens ableitet.

Die Debatte wurde im 19. und zu Beginn des 20. Jahrhunderts innerhalb der Fachdisziplin insbesondere geprägt durch das Gegensatzpaar Erklären und Verstehen, die einem spezifischen Blickwinkel auf Geschichte entspringen. Hinter dem Erklären in der Tradition des Historismus stand die Vorstellung, historische Entwicklung sei durch die Merkmale Fortschritt und Ideen geprägt. Aufgabe der Historiker war es damit, diese Ideen zu rekonstruieren, sie also zu erklären. ${ }^{483}$ Dieser Ansatz war damit von einer Art Fortschrittsoptimismus geprägt und geriet mit aufkommender Forschungsskepsis um die Jahrhundertwende in die Kritik. Dem Erklären wurde der Ansatz des Verstehens gegenübergestellt, der die Einmaligkeit historischer Ereignisse und das Verstehen ihrer Individualität als Erkenntnisziel hatte. ${ }^{484}$ Die Historische Sozialwissenschaft der 1970er Jahre setzte (in Anlehnung an den strukturgeschichtlichen Ansatz der 1950er Jahre) dem Ansatz des Verstehens ein Konzept entgegen, das „von der prägenden Macht von Strukturen und Prozessen in den industrialisierenden und industriellen Gesellschaften der Neuzeit ${ }^{\prime 485}$ ausging und dadurch den Ansatz des Erklärens hervorhob und diesen weiterentwickelte. Die Streitfragen um das Erklären kennzeichnet Welskopp als Problem des Verhältnisses zwischen Kausalität und Hermeneutik und der Rolle nomologischen Wissens ${ }^{486}$, also Wissen auf der Grundlage von Gesetzmäßigkeiten (dieser Ansatz ist stark durch die Naturwissenschaften geprägt, zu denen sich die Geschichtswissenschaft stellen bzw. sich von ihnen abgrenzen musste). Der konstruktivistische Ansatz in der Debatte der 1990er Jahre, der kritisch auf die bisherige Diskussion reagierte

\footnotetext{
${ }^{481}$ Welskopp, Thomas (2002): Erklären. In: Jordan, Stefan (Hrsg.): Lexikon Geschichtswissenschaft. Hundert Grundbegriffe. Stuttgart: Reclam. S. 81-84. S. 81.

482 Welskopp, Thomas (2007), S. 138.

${ }^{483}$ Diese verkürzte Darstellung kann ausführlicher nachvollzogen werden bei: Welskopp, Thomas (2007). S. 146ff.

${ }^{484} \mathrm{Vgl}$. Welskopp, Thomas (2007), S. 148ff.

${ }^{485}$ Vgl. ebd. S. 152.

${ }^{486}$ Vgl. ebd. S. 164.
} 
und als besondere Bedingung der Geschichtswissenschaft herausstellte, dass die Herstellung historischer Realität unmöglich sei, bot gleichzeitig die Chance, Geschichte als Konstruktion wahrzunehmen und daraus Schlüsse für die Beschäftigung mit Vergangenem zu ziehen.

Aus der skizzierten Debatte filtert Rüsen drei Ansätze für historisches Erklären heraus: das nomologische, intentionale und das narrative Erklären. ${ }^{487}$ Das nomologische Erklären folgt dem rationalen Erklärungsansatz der Naturwissenschaften und stellt auf der Grundlage erkannter Gesetzmäßigkeiten eine Prognose auf. Dieses Vorgehen ist nicht typisch für die Geschichtswissenschaft, verwendete Gesetzmäßigkeiten entstammen häufig einer anderen Fachdisziplin. Das intentionale Erklären geht dagegen nicht von einer Gesetzmäßigkeit aus. Intentionales Erklären greift auf die Hermeneutik zurück und versucht einen subjektiven Sinnzusammenhang herzustellen, um so Handlungen und Absichten von Personen zu erklären. Rüsen kritisiert diesen Ansatz aber als nicht geschichtsspezifisch, weil Geschichte nicht als Resultat bestimmter Absichten verstanden werden könne. ${ }^{488}$ Das narrative Erklären schließlich ist ein geschichtsspezifisches Verfahren: „Das historische Erzählen selbst stellt eine elementare und fundamentale Erklärungsform dar, die ihre eigenen Plausibilitätskriterien, also auch ihre

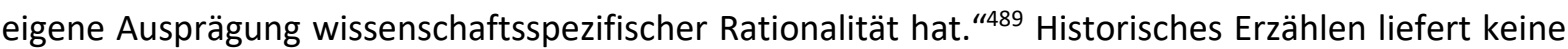
Prognosen, es erklärt vielmehr Geschichte in der Form erzählender Erklärung. Solche narrativen Erklärungen bedienen sich als Teilerklärungen nomologischer oder intentionaler Erklärungen, haben durch den rückblickenden Erklärungsansatz aber eine eigene Struktur. ${ }^{490}$

Für den Geschichtsunterricht stellt Pandel fest, dass die Geschichtsdidaktik nichts vom theoretischen Diskurs um das Erklären antizipiert habe. Insbesondere kritisiert er die Bestimmung für das Erklären in den EPA als fachunspezifisch. ${ }^{491} \mathrm{Er}$ entfaltet für den Geschichtsunterricht vier Arten von Erklären: das kausale, das nomologische, das probabilistische und das narrative Erklären. Das kausale Erklären schließt er an die Ursache-Wirkungs-Ketten der Naturwissenschaften an, grenzt es aber wiederum davon ab, indem er konstatiert, dass es in der Geschichtswissenschaft immer komplizierte UrsacheWirkungs-Ketten oder -Netze gebe, aber nie einfache Kausalzusammenhänge. Das nomologische Erklären schließt er an den bereits dargestellten Erklärtyp an: Ein Sachverhalt würde beim nomologischen Erklären auf ein universell gültiges Gesetz zurückgeführt, wobei die Geschichtswissenschaft keine solchen eigentlich naturwissenschaftlichen Gesetze kenne. Das probabilistische Erklären wiederum beschreibt er als eng mit dem nomologischen verwandt, weil es auf statistischen Wahrscheinlichkeitsgesetzen beruhe. ${ }^{492}$ Das narrative Erklären ordnet Pandel dann als geschichtsspezifischen Erklärungstyp ein: „In einer historischen Darstellung fallen Erzählung und Erklärung zusammen. Die erzählenden Aussagen sind gleichzeitig die erklärenden. Das bloße Aufzählen von Kausalfaktoren stellt noch keine Erklärung dar. [...] Narrationen haben die Aufgabe, historische Ereignisse in eine Ablauffolge zu bringen. ${ }^{4493}$ Diese Aussage schließt sich an den fachwissenschaftlichen Erklärbegriff an und klärt auch, warum eine Reihung, die durchaus häufig im vorliegenden Textkorpus zu finden sein wird, zwar als Mittel zur Lösung der Vergleichsaufgabe genutzt wird, aber letztlich den Vergleich nicht vorantreibt, weil sie eben nichts erklärt.

\footnotetext{
${ }^{487}$ Vgl. Rüsen, Jörn (1997): Gesetze, Erklärungen. In: Bergmann, Klaus; Fröhlich, Klaus; Kuhn, Annette, Rüsen, Jörn; Schneider, Gerhard: Handbuch der Geschichtsdidaktik. 5. Überarb. Aufl. Seelze-Velber: Kallmeyer. S. 164169. S. $165 f f$.

${ }^{488}$ Vgl. ebd. S. 167.

${ }^{489}$ Ebd. S. 167.

${ }^{490}$ Vgl. ebd.

${ }^{491}$ Vgl. Pandel, Hans-Jürgen (2013), S. 408.

${ }^{492}$ Vgl. ebd. S. 408ff.

${ }^{493}$ Ebd. S. 110.
} 
Pandel bemängelt, dass das fachspezifische narrative Erklären im Geschichtsunterricht derzeit kaum vorkomme, stattdessen tatsächlich eher die Wiedergabe mit eigenen Worten gemeint sei. ${ }^{494} \mathrm{Um}$ einem solchen fachunspezifischen Zugang zu entgehen, der zudem kaum fachliches Lernen erzeugt, weil er eben nicht zur Sinnbildung anregt, muss sowohl für Lehrende als auch Lernende zunächst einmal geklärt sein, welcher Zweck hinter dem Erklären (gerade auch in der Abgrenzung zum Begründen) steckt. Erst wenn dies geklärt ist, kann Erklären zu einer fachspezifischen Operation werden, welche die fachliche Debatte aufnimmt, ohne Lernende damit zu überfordern.

An dieser Stelle kann ein funktionalpragmatischer Zugang Klärung bewirken.

Bettet man das Erklären in das in Kapitel 2.6 dargestellte Sprach- und Wissensmodell von Ehlich/Rehbein ein, kann man als Zweck des Erklärens das Transferieren von Wissensstrukturen ableiten. Das bedeutet, dass beim Hörer oder Leser ein Verstehen bestimmter fachlicher Strukturen erreicht werden soll:

„Gerade dieses Verstehen, das Verstehen als eine mentale Aktivität des Hörers, ist nun aber der zentrale Zielpunkt des Erklärens. Dieses Verstehen in einem substantiellen Sinn ist zugleich eine zentrale mentale Aktivität, deren Ergebnisse in $\mathrm{H}$ [gemeint ist der Hörer, Anm. d. Verf.] charakteristische Veränderungen von dessen mentalen Bereich bedeuten. " 495 [Hervorh. im Original]

Im Falle eines Textes muss der Schreibende mögliche Wissensstrukturen vorausdenken, von denen er ausgeht, dass diese beim Leser noch nicht vorhanden sind.

Ehlich beschreibt den Zweck des Erklärens als „Expansion der Sinnstrukturen “496 beim Hörer (oder Leser), es geht also darum, Wissensstrukturen zu übertragen oder Wissenslücken zu füllen. ${ }^{497}$

Die EPA bestimmen das Erklären als „historische Sachverhalte durch Wissen und Einsichten in einen Zusammenhang (Theorie, Modell, Regel, Gesetz, Funktionszusammenhang) einordnen und begründen". ${ }^{498}$ Diese Einordnung geht deutlich über den dargestellten Zweck des Wissenstransfers hinaus, da Begründungen gefordert sind. Es werden damit verschiedene Anforderungen zusammengeführt. Eine klare Trennung der sprachlichen Handlungen fehlt. Dieses Zusammenspiel ist zu erklären auf der Grundlage der geschichtswissenschaftlichen Debatte, die gezeigt hat, dass in der Geschichte immer komplexe Zusammenhänge rückblickend erklärt werden müssen. Damit die dargestellten Zusammenhänge nicht in Zweifel gezogen werden, sind Begründestrukturen notwendig. Das bedeutet aber auch, dass ein kompetenter Leser oder eine kompetente Leserin solche Strukturen erkennen können muss, um die Konstruktion der dargelegten Geschichte kritisch hinterfragen zu können. Beim Sprechen oder Schreiben müssen solche Strukturen außerdem produziert werden können. Diesen Aufgaben geht das Wissen um solche Strukturen und ihre unterschiedlichen Zwecke voraus. Gerade für Lernende ist es deshalb notwendig, diese Strukturen explizit zu klären.

\footnotetext{
${ }^{494} \mathrm{Vgl}$. ebd. S. 411 und 408.

${ }^{495}$ Ehlich, Konrad (2009): Erklären verstehen - Erklären und Verstehen. In: Vogt, Rüdiger (Hrsg.): Erklären Gesprächsanalytische und fachdidaktische Perspektiven. Tübingen: Stauffenburg Verlag. S. 11-24. S. 16.

${ }^{496} \mathrm{Vgl}$. ebd. S. $18 \mathrm{ff}$.

497 "Mit einer Erklärung macht eine Person Anderen oder sich selbst einen Zusammenhang von Sachverhalten oder Sachverhaltselementen so klar, dass er ins Wissen integriert und als allgemeine Orientierung des Handelns genommen werden kann." aus: Hoffmann, Ludger (2014): Deutsche Grammatik. Grundlagen für Lehrerausbildung, Schule, Deutsch als Zweitsprache und Deutsch als Fremdsprache. Berlin: Erich Schmidt Verlag. S. 517. ${ }^{498}$ Kultusministerkonferenz (2005): EPA Geschichte, S. 8.
} 
Für das Kriterienraster wird auf die funktionale Beschreibung des Erklärens zurückgegriffen und auf den Geschichtsunterricht bezogen, um so eine klare Abgrenzung zum Begründen herbeizuführen (Anforderungen durch das Begründen werden im folgenden Teilkapitel expliziert). Im Rückgriff auf das Sprach- und Wissensmodell nach Ehlich/Rehbein muss davon ausgegangen werden, dass Erklären und Begründen unterschiedliche Arten von Wissensveränderung beim Hörer oder Leser bewirken, wenn die Operationen erfolgreich sind. Wissenstransfer (im Sinne des Erklärens) und Bearbeitung eines (vorweggenommenen) Einverständnismangels können in einer dritten übergeordneten Operation, dem komplexen historischen Erklären, zusammenfallen, nicht jedoch in sich selber.

Es bleibt anzumerken, dass über das Erklären hinaus auch Erläuterungen notwendig sein können, um den übergeordneten Vergleich im Geschichtsunterricht erfolgreich zu realisieren. Ausgehend vom Ansatz der Funktionalpragmatik stellt das Erläutern eine reparative Sprechhandlung dar, , [[...] mit der unklare Diskurselemente sachlich oder perspektivisch ergänzt werden. ${ }^{4999}$ Für das Schreiben bedeutet dies, dass der/die Schreibende in seinen/ihren Erklärungen mögliche Wissenslücken des Lesers vorwegnehmen muss, diese Erklärungen also durch Erläuterungen anreichern muss. Solche Strukturen können ggf. notwendig sein für das Erklären innerhalb eines Vergleichs, es ist aber eben nicht konstitutiv, weshalb es in diesem Rahmen nicht weiterverfolgt wird.

\subsubsection{Begründen als Teil des Vergleichens}

Das Begründen spielt insbesondere für das Beurteilen eine Rolle, welches im Sachurteil gefordert ist. Ohne Begründen kann kein historisch fachgerechtes Sachurteil vorgenommen werden. Aber es spielt auch neben dem abschließenden Sachurteil in Teilbegründungen eine Rolle, wenn etwa begründet werden muss, warum bspw. die Gesellschaftsstruktur in England zu Beginn der Industrialisierung offener gewesen sei als in Deutschland.

An dieser Stelle soll nun zunächst geklärt werden, wie das Begründen in den EPA bestimmt und verwendet wird und welchen funktionalen Zweck das Begründen ausfüllt.

Begründen und Nachweisen haben in den EPA eine gemeinsame Bestimmung: „Aussagen (z. B. Urteil, These, Wertung) durch Argumente stützen, die auf historischen Beispielen und anderen Belegen grün-

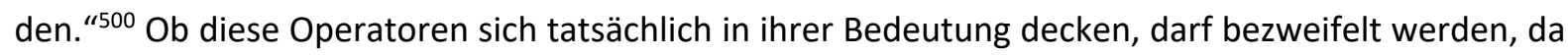
dem Nachweisen zumindest im alltäglichen oder normativen ${ }^{501}$ Verwendungszusammenhang viel stärker das Beweisen (z. B. durch eine bestimmte Unterlage, wie ein Nachweis über die Berechtigung zum Erhalt bestimmter Leistungen) zugrunde liegt als dem Begründen. Für den Geschichtsunterricht erscheint das Nachweisen im Zusammenhang mit einer legitimierenden Grundlage bedeutsam, während das Begründen (ableitbar aus dem Wortstamm Grund) die Gründe für eine bestimmte Haltung, Meinung etc. in den Vordergrund stellt. Insofern ist für das Beurteilen das Begründen die passende Teilhandlung und nicht das Nachweisen.

Auf Grundlage der EPA-Bestimmung spielen wieder Argumentationsstrukturen eine Rolle. Das Begründen soll Aussagen argumentativ stützen. Historisch wird das Begründen erst durch die Art der

\footnotetext{
${ }^{499}$ Graefen, Gabriele; Moll, Melanie (2007), Das Handlungsmuster Begründen: Wege zum Unterricht „Deutsch als fremde Wissenschaftssprache“. In: Redder, Angelika (Hrsg.): Diskurse und Texte. Festschrift für Konrad Ehlich zum 65. Geburtstag. Tübingen: Stauffenburg Verlag. S. 419-515. S. 495.

${ }^{500}$ Ebd.

${ }^{501} \mathrm{Im}$ Sinne eines bürokratischen Gebrauchszusammenhangs.
} 
Argumente, die auf historischen Beispielen und Belegen gründen sollen. Damit ist der Zweck der Handlung „Begründen“ jedoch nur vordergründig erfasst. ${ }^{502}$

Um die Funktion des Begründens (zunächst überfachlich) klarer darstellen zu können, hilft wieder die funktionale Pragmatik weiter. Ehlich/Rehbein schlüsseln die Operation des Begründens hinsichtlich ihres Zwecks auf, dieser Ansatz führt trotz des zunächst überfachlichen Ansatzes zu viel höherer Eindeutigkeit als die EPA-Bestimmung und damit zu einem viel deutlicheren Verständnis für den Zweck und in der Folge den Umgang mit dem Begründen. Ehlich/Rehbein unterscheiden in verschiedene Arten des Begründens, von denen für den geschichtsunterrichtlichen Zusammenhang insbesondere eine in Betracht zu ziehen ist, nämlich das , kognitive Begründen' ${ }^{503} \mathrm{Im}$ Rückgriff auf das Sprach- und Wissensmodell von Ehlich/Rehbein (vgl. Kap. 2.5.1) geht es bei dieser Form des Begründens darum, dass ein Hörer (oder Leser) die Aussage eines Sprechers (oder Schreibers) versteht:

„Für diesen Typ ist es charakteristisch, daß die Handlungssequenz des Begründens ihren Zweck in sich selbst hat, nicht in einer Anschlußhandlung. Vielmehr soll $H$ [der Hörer, Anm. d. Verf.] hier lediglich den propositionalen Gehalt der Assertion des Sprechers S [Hervorh. im Original] verstehen. Das bedeutet: das gemeinsame Handlungssystem ist hier das einer kognitiven Übereinstimmung. “504

Dabei ist jedoch nicht gemeint, dass Erklärungen im Sinne von weiteren Wissensstrukturen ,nachgeschoben' werden, um ein Verständnis zu erzielen (dies würde das Erläutern leisten), sondern es geht um die Bearbeitung eines Einverständnismangels, also um eine mögliche Kritik. Graefen/Moll führen die Grundlegung von Ehlich/Rehbein aus, indem sie das kognitive Begründen vom mündlichen Diskurs auf eine zerdehnte Sprechsituation, also auf die Kommunikation durch einen Text übertragen. Sie stellen in Bezug auf mündliche und schriftliche Kommunikationssituation dazu fest: „In beiden Fällen, also im Text wie in der mündlichen Gesprächssituation, ist das Ziel des Sprechers beim Begründen, eine Beurteilung oder Einstellung des Hörers mit Hilfe eines geeigneten Wissenselements mit dem eigenen Denken zu ,synchronisieren'. " 505 In der Schreibsituation muss der Schreibende einen möglichen Einverständnismangel vorwegnehmen, er bearbeitet damit eine erwartete Störung. Dies ist die anspruchsvolle Aufgabe, die die Lernenden leisten müssen, um erfolgreich zu begründen und damit schließlich den historischen Vergleich zu realisieren.

Gerade im Hinblick auf den Konstruktionscharakter von Geschichte ist das kognitive Begründen im Sinne Ehlich/Rehbeins von hoher Bedeutung. Die Fachlichkeit dieses Ansatzes wird dabei über die historische Kontextualisierung hergestellt. Die Operation des Begründens wird damit zu einem wichtigen Element für historische Sinnbildung, weil darüber Deutungsmuster fundiert werden und diese im Sinne der Kompetenzorientierung reflektiert werden können.

\footnotetext{
502 Zum Begründen als Aufgabenformate im Geschichtsunterricht vgl.: Altun, Tülay; Günther, Katrin (2018): Begründen als Arbeitsauftrag im Geschichtsunterricht. In: Grannemann, Katharina; Kuchler, Christian; Oleschko, Sven (Hrsg.): Sprachbildung im Geschichtsunterricht. Waxmann Verlag.

${ }^{503} \mathrm{Vgl}$. Ehlich, Konrad; Rehbein, Jochen (1986), S. 112.

${ }^{504}$ Ebd. (mit H wird in diesem Auszug der Hörer gekennzeichnet)

505 Graefen, Gabriele; Moll, Melanie (2007): S. 493.
} 


\subsubsection{Beurteilen als zentrale sprachliche Teilhandlung des Vergleichs im Geschichtsun-} terricht

Das Beurteilen stellt, ein zentrales Element für das historische Lernen dar. Aufgeschlüsselt in Sachund Werturteil ist es ein wesentliches Lernziel des Geschichtsunterrichts. In Anlehnung an die Jeismannsche Trias Analyse, Sachurteil und Werturteil ${ }^{506}$, deren Elemente nicht unabhängig voneinander zu denken sind, ist laut EPA das Ziel des Vergleichs das Erreichen eines Sachurteils. Dabei müssen Urteile im Geschichtsunterricht „,widerspruchsfrei, intersubjektiv überprüfbar und begründet sein.“507 Dennoch ist dabei zu berücksichtigen, dass es eine eindeutige Trennung zwischen Sach- und Werturteil nicht geben kann. Becker argumentiert, dass nach Jeismann ein solch künstlicher Dreischritt notwendig für Analyse und Reflexion sei, sich die drei Operationen jedoch ununterscheidbar verbänden. ${ }^{508}$ Er folgert daraus: „Es ist gerade diese Einsicht, die das Modell theoretisch wertvoll macht und den Irrglauben unterbindet, es gäbe ein nachgeordnetes Werturteil losgelöst von einem objektiven Sachurteil oder unabhängig von einer wertfreien Analyse. ${ }^{4509}$ Das Wissen um diese Verbindung ist ausgehend von der Annahme, Urteile sind zugleich Narrationen, bedeutsam für die Unterrichtsgestaltung, denn:

„Das Problem von Wahrheit und Objektivität ist durch diese Einteilung aufgelöst, bzw. es wird anders definiert. Der entscheidende Punkt hinsichtlich historischer Urteilsbildung liegt in der Annahme, dass ein Urteil qualitativ nicht mehr ausschließlich an den historischen Fakten, der empirischen Triftigkeit zu messen ist. In den Blickpunkt rückt vielmehr die Komposition der Fakten, die die/der Urteilende vornimmt. Dies ist zugleich ein Bekenntnis für eine diskursive Lernsituation, also Urteile danach zu bewerten, wie sie sich im Diskussionsprozess bewähren, wie sie sich von anderen Urteilen unterscheiden und welche Perspektiven sie aufnehmen bzw. auslassen. ${ }^{4510}$

Diese Annahme bedeutet in der Konsequenz, dass diskursive Handlungen, die zu einer Urteilsbildung führen wie das Vergleichen im Geschichtsunterricht, narrativen Charakter haben oder sogar zu einer eigenständigen Narration führen, auch wenn sie von der klassischen story-grammar im Sinne Barricellis abweichen. ${ }^{511}$ Ein solcher Schluss hebt die Bedeutsamkeit des Urteilens an sich heraus und schließt die Forderung an, Strukturen der Urteilsbildung im Unterricht explizit zu machen ${ }^{512}$. Dennoch ist es für Schreibnovizen sinnvoll, eine solche Unterscheidung für den Geschichtsunterricht zunächst zu treffen, Eine solche Unterscheidung von Sach- und Werturteil, so wie Becker sie auch vertritt, kann über

\footnotetext{
506 Jeismann, Karl-Ernst (2000).

507 Becker, Axel (2017): Historische Urteilsbildung Barricelli, Michele; Lücke, Martin (Hrsg.): Handbuch Praxis des Geschichtsunterrichts. Bd. 1. 2. Aufl. Schwalbach/Ts.: Wochenschau Verlag. S. 316-325. S. 321.

508 Vgl. ebd. S. 320.

${ }^{509} \mathrm{Ebd}$.

510 Ebd. S. 324.

${ }^{511}$ Vgl. Barricelli, Michele (2017), S. 259f.

512 Diese Forderung erheben auch Schönemann et al. in ihrer Untersuchung: „Wir empfehlen für den gesamten Bereich der Urteilsbildung (historische Sach- und Werturteile) ein bewussteres Vorgehen und die Einübung der für das historische Denken konstitutiven Urteilstypen." (Schönemann et al., 2011. S. 125)
} 
die Klärung des jeweiligen Zwecks für Lernende offengelegt werden. ${ }^{513}$ Das Beurteilen ist entsprechend seiner hohen Bedeutung für den Geschichtsunterricht als eigener Operator in den EPA definiert als „den Stellenwert historischer Sachverhalte in einem Zusammenhang bestimmen, um ohne persönlichen Wertebezug zu einem begründeten Sachurteil zu gelangen “514, und dem Anforderungsbereich III zugehörig. Das Beurteilen wird also durch die Kultusministerkonferenz entsprechend dieser Bestimmung dem Sachurteil zugeordnet. Diese Formulierung überrascht, da das Beurteilen auch die Bestimmung eines Werturteils einschließen müsste. Immerhin lautet der Operator ,Beurteilen', er ist durch seinen Wortsinn nicht auf das Sachurteil eingeschränkt. Erst die Operatoren Bewerten und Stellungnehmen fordern laut EPA ein Werturteil ein.

Explizit eingefordert wird im Zusammenhang mit dem Beurteilen das zuvor dargestellte Begründen.

Entsprechend dieser Einordnung wird von den Schüler*innen eine Interpretation aus der Sachanalyse verlangt. Gautschi beschreibt den Weg zum Sachurteil, den die Lernenden gehen müssen: „Im nächsten Schritt interpretieren sie das Beschriebene, stellen Bezüge zu anderen historischen Zeugnissen her und ordnen es auf diese Weise in einen größeren Zusammenhang von Ursachen und Wirkungen, ins Universum des Historischen ein. Sie gewinnen dadurch [...] ein ,historisches Sachurteil' . “515 Es handelt sich also um ein kriteriengeleitetes Beurteilen.

Als sprachliche Teilhandlung des Vergleichens ist das Beurteilen ebenfalls als Sachurteil gefordert, da die gegenübergestellten historischen Elemente hinsichtlich ihrer Gemeinsamkeiten, Unterschiede, Teil-Identitäten, Ähnlichkeiten, Abweichungen oder Gegensätze ${ }^{516}$ beurteilt werden sollen.

Beim Einsatz von Begründestrukturen wird, wie zuvor dargestellt, davon ausgegangen, dass Aussagen auf einen Einverständnismangel stoßen können, dem durch die Nutzung von Begründestrukturen vorgebeugt werden soll, um so das Einverständnis des Lesers herbeizuführen. Das Sachurteil (wie auch das Werturteil) muss also argumentativ abgesichert werden, denn gerade die Beurteilung kann auf Wiederspruch stoßen, weil sie einordnend ist. Deshalb ist sie nur vollständig im Zusammenhang mit Begründestrukturen, die einen möglichen Prä-Einverständnismangel bearbeiten.

\subsubsection{Historisches Vergleichen als besondere Form des Beweisens}

Die Analyse der sprachlichen Teilhandlungen zeigt, dass dem Vergleichen stark argumentative Strukturen zugrunde liegen. Das Argumentieren stellt wiederum ein wesentliches Werkzeug des Geschichtsunterrichts dar. Dies liegt am Konstruktionscharakter von Geschichte. Jedoch sind argumen-

\footnotetext{
${ }^{513}$ Eine Aufschlüsselung des Sachurteils als Textsorte für den Geschichtsunterricht findet sich bei Wickner (vgl. dazu bspw.: Wickner, Mareike-Cathrine (2018): So schließt sich der Kreis. Textsortenspezifische Schreibförderung im Geschichtsunterricht mit dem ,Genre Cycle'. In: Geschichte lernen 31 (2018). H. 182, S. 38-45.

${ }^{514}$ Einheitliche Prüfungsanforderungen in der Abiturprüfung Geschichte (2005), S. 8.

515 Gautschi, Peter (2011), S. 44.

${ }^{516}$ Vgl. Einheitliche Prüfungsanforderungen in der Abiturprüfung Geschichte (2005), S. 8.
} 
tative Strukturen zu unterscheiden von der historischen Argumentation als übergeordneter Operation. Die für das Vergleichen ausgearbeiteten Teilhandlungen, wie das Gegenüberstellen, verstehen sich als argumentativ, allerdings werden für den Vergleich Strukturen über das Argumentieren hinaus verwendet. Gemeinsam ist beiden Formen wiederum, dass die für Vergleich und Argumentation geforderten Strukturen am Ende in einem Sachurteil zusammengeführt werden.

Die dargestellten Teilhandlungen des Vergleichens im Geschichtsunterricht stehen nicht nur in engem Verhältnis zueinander, vielmehr wirken sie aufeinander. Aus diesem Zusammenspiel lässt sich die folgende Strukturskizze ableiten:

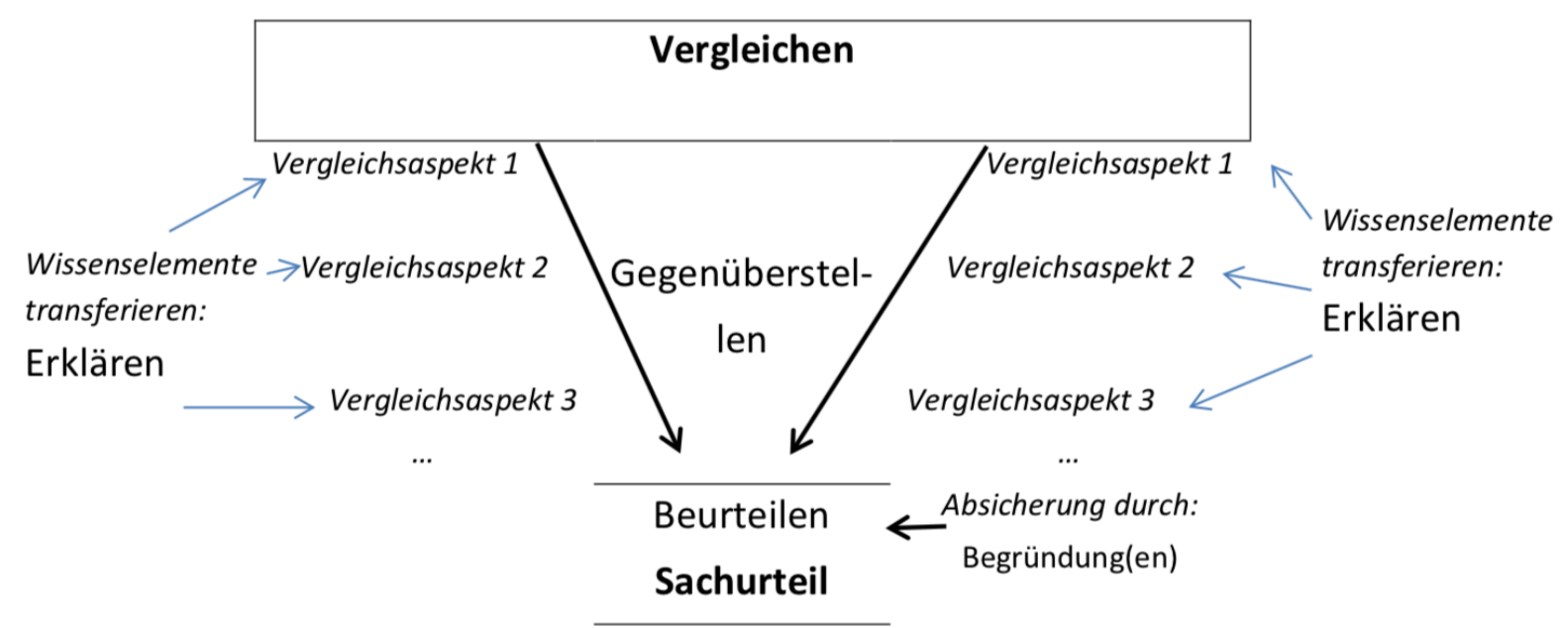

Abbildung 12: Struktur des Vergleichens im Geschichtsunterricht (eigene Darstellung)

Die sprachlichen Teilhandlungen münden im Sachurteil und machen so in ihrer Gesamtheit den Vergleich aus. Insbesondere das Erklären und Begründen sind dazu eingebettet in einen argumentativen Rahmen, der durch das Gegenüberstellen strukturiert wird. Man kann deshalb vom Vergleichen als einer Form ,historischer Beweisführung' sprechen. Das abschließende Sachurteil wird herbeigeführt (und damit bewiesen) durch die Wissenselemente, die gegenübergestellt werden. Damit der Leser diese Wissenselemente in sein Leserwissen integrieren, sie also verstehen kann, müssen sie erklärt werden. Die notwendigen Erklärungen werden in den argumentativen Kontext eingebettet, indem sie durch das Tertium Comparationis zusammengeführt werden. Es ist die Grundlage für die Herleitung von Unterschieden, Gemeinsamkeiten und Ähnlichkeiten. Erklärungen und Begründungen sichern das Sachurteil und damit den Vergleich diskursiv ab. 


\section{Vergleichsaufgaben in Geschichtslehrwerken: eine Frequenz- analyse}

Die Überlegungen zum Vergleichen als Operation des Geschichtsunterrichts zeigen, dass das Vergleichen eine wichtige Aufgabe darstellt. Ob und wie sie damit tatsächlich Eingang in den Unterricht findet, kann darüber allerdings nicht erschlossen werden. Zwar kann eine Untersuchung von Unterricht im Rahmen dieser Arbeit nicht geleistet werden, wohl kann aber eine Analyse von gängigen Lehrwerken Aufschluss darüber geben, ob Vergleichsaufgaben Lehrenden und Lernenden überhaupt angeboten werden. Dahinter steht die Frage, ob durch Lehrwerke dazu beigetragen wird, das historische Vergleichen zu vermitteln. Dieser Frage kann über eine Frequenzuntersuchung von Geschichtslehrwerken nachgegangen werden.

Die Frequenz- oder Häufigkeitsanalyse gilt als Grundtechnik inhaltsanalytischer Verfahren ${ }^{517}$ : „Die einfachste Art inhaltsanalytischen Arbeitens besteht darin, bestimmte Elemente des Materials auszuzählen und in ihrer Häufigkeit mit dem Auftreten anderer Elemente zu vergleichen. “518 Die Frequenzanalyse wird im Rahmen ihrer Nutzung zur Analyse von Lehrwerken für den Geschichtsunterricht aus geschichtsdidaktischer Sicht als ein verfeinertes Verfahren der Raumanalyse eingeordnet. ${ }^{519}$ Überfachliche Hinweise zur Nutzung einer Frequenzanalyse in Bezug auf Schulbücher finden sich außerdem bei Pingel. ${ }^{520}$

Es handelt sich bei diesem Analyseansatz um einen Ausschnitt einer Schulbuchanalyse. ${ }^{521}$ Dies hat zwar den Nachteil einer fragmentarischen Abbildung ${ }^{522}$ der im Folgenden untersuchten Aufgaben, da diese lediglich auf ihre Formulierung über Operator oder W-Frage hin analysiert werden, nicht aber hinsichtlich ihres Kontextes, ihrer Kompetenzorientierung etc. Der gewählte Analyseansatz ist im Rahmen dieser Untersuchung jedoch passend, da der Fokus auch nur auf einem Ausschnitt von Geschichtslehrwerken, nämlich der Verwendung von Operatoren, liegt. Nachfolgend soll deshalb der Vorteil einer solchen Herangehensweise genutzt werden: „Gleichwohl haben frequenzorientierte An-

\footnotetext{
517 Vgl. Mayring, Philipp (2010), S. 13.

518 Ebd. Zum Ablauf einer Frequenzanalyse vgl. ebd. S. 15.

${ }^{519}$ vgl. dazu: Scholle, Dietrich (1997): Schulbuchanalyse. In: Bergmann, Klaus; Fröhlich, Klaus; Kuhn, Annette, Rüsen, Jörn; Schneider, Gerhard: Handbuch der Geschichtsdidaktik. 5. überarb. Aufl. Seelze-Velber: Kallmeyersche Verlagsbuchhandlung. S. 369-375. S. 370.

${ }^{520}$ Pingel berücksichtigt in seinen Überlegungen die Grenzen quantitativer Schulbucherforschung, stellt aber die Chancen heraus: „A simple frequency analysis, however, can tell us a lot, for example, about the room for manoeuvre that authors have to design their textbooks." Pingel, Falk (2010): UNESCO Guidebook on Textbook Research and Textbook Revision. 2. überarb. Auflage. Paris/Braunschweig. S. 67.

${ }^{521}$ Hinweise zu einer umfassenden Evaluation von Geschichts-Schulbüchern finden sich bspw. bei Pohl, Karl Heinrich: Wie evaluiert man Schulbücher? In: Fuchs, Eckhardt; Kahlert, Joachim; Sandfuchs, Uwe (Hrsg.) (2010): Schulbuch konkret. Kontexte - Produktion - Unterricht. Bad Heilbrunn: Julius Klinkhardt Verlag. S. 18-133.

${ }^{522}$ Vgl. dazu: Bubenhofer, Noah; Lange, Willi; Okamura, Saburo; Scharloth, Joachim (2015): Wortschätze in Lehrbüchern für Deutsch als Fremdsprache - Möglichkeiten und Grenzen frequenzorientierter Ansätze. In: Ott, Christine; Kiesendahl, Jana (Hrsg.): Linguistik und Schulbuchforschung: Gegenstände - Methoden - Perspektiven. Göttingen: V\&R unipress. S. 85-107. S. 86.
} 
sätze den Vorteil, dass sie überhaupt eine empirische Grundlage haben, ihre Ergebnisse folglich reproduzierbar sein müssen und somit die Möglichkeit eröffnen, intersubjektiv nachvollziehbare Maßstäbe in die Lehrwerkerstellung einzubringen. ${ }^{4523}$

Dieser Wert soll weiterhin dazu dienen, um neben der Häufigkeit des Operators Vergleichen zu untersuchen, wie häufig solche Operatoren verwendet werden, die für das Vergleichen zu Teiloperationen werden. So kann darauf geschlossen werden, ob auch diese Teiloperationen durch die Lehrwerke vermittelt werden.

\subsection{Zur Auszählung}

Die folgende Frequenzanalyse basiert auf den in NRW zugelassenen Lehrwerken. ${ }^{524}$ Themenhefte wurden hingegen nicht berücksichtigt. Die Analyse ist in zwei Teile untergliedert: In Teil 1 werden die Lehrwerke, die vor 2014, also vor der Umstellung auf die neuen Kernlehrpläne für die Sekundarstufe II, erschienen sind, untersucht. Dabei werden bei Teilbänden immer alle Bände ausgezählt. Die Lehrwerke umfassen einen Zeitraum von 2002 bis 2012, also von zehn Jahren.

Im zweiten Teil werden die Lehrwerke ab $2014^{525}$ aufgeführt (2013 fehlt, da in diesem Jahr kein weiteres zugelassenes Lehrwerk erschienen ist). Da die Lehrwerke seit 2014 jeweils in einen Teil für die Einführungsphase und einen (im Vergleich deutlich umfangreicheren) Teil für die Qualifikationsphase unterteilt sind, werden bei der Auszählung immer beide Teile berücksichtigt.

Die Frequenzanalyse stellt damit einen Querschnitt der zwischen 2002 und 2012 erschienenen Lehrwerke und einen weiteren Querschnitt der seit 2014 erschienenen Geschichtsschulbücher dar, damit ist sie in begrenztem Maße zugleich auch ein Längsschnitt.

Zuvor wurde bereits in schulorganisatorischer Hinsicht der Fokus auf Nordrhein-Westfalen gelegt, aus Gründen der Praktikabilität soll dieser Zugriff auch in der Frequenzanalyse fortgeführt werden.

Im Rahmen dieser Schulbuchauszählung werden Aufgabenstellungen, die mittels Operatoren und WFragen formuliert werden, berücksichtigt. Diese Formate tauchen am häufigsten auf und werden deshalb als gängig angenommen. Dabei liegt der Fokus auf Lernaufgaben, andere Formate wie Testaufgaben werden nicht berücksichtigt.

Der Auswertung liegt eine Tabelle (s. Anhang 4.1) zugrunde, die im ersten Teil den Operatoren der EPA für das Fach Geschichte folgt und außerdem die Operatoren aufnimmt, die im Schulbuch verwendet werden, aber nicht in den EPA genannt werden. Im 2. Teil wird dargestellt, welche W-Fragen verwendet werden und in welcher Frequenz diese auftauchen. ${ }^{526}$

\footnotetext{
${ }^{523}$ Bubenhofer, Noah; Lange, Willi; Okamura, Saburo; Scharloth, Joachim (2015): S. 86.

${ }^{524} \mathrm{Vgl}$. https://www.schulministerium.nrw.de/docs/Schulsystem/Unterricht/Lernmittel/index.html

${ }^{525}$ Berücksichtigt sind die Lehrwerke ab 2014 bis zum Abschluss dieser Arbeit Mitte 2018.

${ }^{526}$ Die Tabelle befindet sich im Anhang 4.1.
} 
4.1.1 Teil 1: Frequenzanalyse der verwendeten Operatoren - Entscheidungen für die Auszählung

Im ersten Teil werden zunächst die in den EPA genannten Operatoren gezählt und entsprechend der EPA-Zuordnung entlang der verschiedenen Anforderungsbereiche eingeordnet. Dabei werden in diesem Teil nur Operatoren gezählt, jedoch keine W-Fragen. Wenn in einer Aufgabe zwei Operatoren gleichwertig genannt werden, werden beide gezählt.

Ein besonderer Fokus stellt die Berücksichtigung von Aufgaben in Bezug auf Darstellungstexte dar, da die nachfolgende Untersuchung die Kombination aus Darstellungstext und Aufgabe in den Mittelpunkt stellt.

Der Auswertungsbogen enthält im Hinblick auf die Operatoren die nachfolgenden Kategorien:

Tabelle 1: Kategorien des Auswertungsbogens (Operatoren)

\begin{tabular}{|c|c|c|c|c|}
\hline Operatoren & $\begin{array}{l}\text { Nennung allge- } \\
\text { mein }\end{array}$ & $\begin{array}{l}\text { Nennung in Be- } \\
\text { zug auf Darstel- } \\
\text { lungstexte in } \\
\text { Verbindung mit } \\
\text { anderen Materi- } \\
\text { alien }\end{array}$ & $\begin{array}{l}\text { Nennung in Be- } \\
\text { zug auf Darstel- } \\
\text { lungstexte ohne } \\
\text { Bezug auf an- } \\
\text { dere Materialien }\end{array}$ & Bemerkungen \\
\hline
\end{tabular}

In Spalte 2 des Auswertungsbogens werden zunächst alle Operatoren gezählt, unabhängig davon, welcher Materialgrundlage sie zuzuordnen sind. Wenn Aufgaben sich ausschließlich auf Darstellungstexte beziehen, werden sie auch in Spalte 4 gezählt, wenn sie sich auf Darstellungstext und andere Materialien beziehen, sind sie außer in Spalte 2 auch in Spalte 3 gezählt.

Da die Lehrwerke die Zugehörigkeit zu Darstellungstexten und/oder anderen Materialien unterschiedlich deutlich ausweisen, ist eine eindeutige Zuordnung in die Spalten 3 und 4 nicht für alle Schulbücher möglich gewesen. Immer dann, wenn die Zuordnung nicht eindeutig war, wurden die Operatoren nur in Spalte 2 gezählt.

Darüber hinaus werden im ersten Teil nach dem oben genannten Schema auch alle Aufgabenstellungen gezählt, die durch handlungsinitiierende Verben (Operatoren) formuliert werden, aber nicht in den EPA genannt werden. Diese werden im Anschluss aufgeführt. Sie werden jedoch nicht einem Anforderungsbereich zugeordnet, da eine solche Zuordnung auf der Grundlage der gesamten Aufgabenformulierung geschehen müsste, um der Aufgabe gerecht zu werden. Der isolierte Blick auf das handlungsinitiierende Verb erscheint dabei nicht ausreichend. 


\subsubsection{Teil 2: Frequenzanalyse der W-Fragen - Entscheidungen für die Auszählung}

Im zweiten Teil werden in einer weiteren Auszählung darüber hinaus Aufgabenstellungen erfasst, die statt durch einen Operator durch eine W-Fragen getragen werden ${ }^{527}$.

Zu einzelnen Fragewörtern können dabei unterschiedliche Präpositionen gehören. Diese werden nicht gesondert aufgeschlüsselt, sondern es wird nur das Fragewort gezählt (Bsp.: „welche“ und „mit welcher" wird als ein Type gezählt, ebenso unterschiedliche Flexionen und Genusausformungen). Z. T. sind Operatoren durch angehängte W-Fragen aspektiert. Diese Aspektierungen wurden im ersten Teil nur mitgezählt, wenn der Operator als eigenständige Aufgabe erscheint, also nicht nur als Aspektierung wahrgenommen werden kann.

Der Auswertungsbogen enthält im Hinblick auf die W-Fragen die nachfolgenden Kategorien:

Tabelle 2: Kategorien des Auswertungsbogens (W-Fragen)

\begin{tabular}{|l|ll|l|l|l|}
\hline W-Frage & $\begin{array}{l}\text { Nennung allgemein } \\
\text { (Gesamtzahl der }\end{array}$ & $\begin{array}{l}\text { Nennung in Bezug } \\
\text { auf Darstellungs- } \\
\text { Aufgaben mit W- }\end{array}$ & $\begin{array}{l}\text { Nennung in Bezug } \\
\text { auf Darstellungs- } \\
\text { texte in Verbindung } \\
\text { mit anderen Materi- } \\
\text { alien }\end{array}$ & $\begin{array}{l}\text { texte ohne Bezug } \\
\text { auf andere Materia- } \\
\text { lien }\end{array}$ & Ban- \\
\hline
\end{tabular}

Alle Aufgaben mit W-Fragen sind in Spalte 2 gezählt. Wenn W-Fragen sich nur auf die Materialgrundlage ,Darstellungstext' beziehen, werden sie außerdem in Spalte 4 gezählt, beziehen sie sich auf Darstellungstext und auf andere Materialien, sind sie in Spalte 3 zugeordnet.

Abschließend wird das Verhältnis von Aufgabenformulierungen mit Operator oder W-Frage für das ausgezählte Schulbuch dargestellt. Aufgabenformulierungen ohne Operator oder W-Frage werden nicht berücksichtigt, da sie kaum vorkommen und dadurch eine sehr untergeordnete Rolle spielen.

\subsubsection{Allgemeine Hinweise und Entscheidungen}

Am Ende jeder Auszählung schließen sich auf dem Auswertungsbogen Anmerkungen zum Lehrwerk an. Da die Lehrwerke häufig durch einen eigenen Schulbuchstil gekennzeichnet sind, sollen diese Anmerkungen auf die jeweiligen Besonderheiten hinweisen.

Da in jedem Lehrwerk auf eine eigene Art und Weise mit Aufgabenstellungen umgegangen wird, muss sich dies auch in der Auszählung abbilden. Wenn ein Schulbuch neben Lernaufgaben außerdem mit Testaufgaben arbeitet, werden diese zwar in der Auszählung nicht berücksichtigt, dennoch stellt dies eine Besonderheit dar und ist jeweils vermerkt.

\footnotetext{
${ }^{527}$ Vgl. ebf. Anhang 4.1.
} 
Grundsätzlich sind bei jedem Schulbuch Einzelentscheidungen nötig. So arbeitet bspw. ein Schulbuch (Bahr, 2006: Horizonte) häufiger mit W-Fragen, denen ein Operator folgt. Hier musste im Einzelfall entschieden werden, ob die W-Frage oder der Operator bestimmend für die Aufgabe ist.

Wenn Teilaufgaben als eigenständig gewertet werden müssen (z. B. wenn zwei Operatoren durch ein „und" verbunden und so als gleichrangig anzusehen sind), werden sie auch in der Statistik als zwei eigenständige Aufgaben gewertet. Folgt einem Operator durch ein „und“ verbunden eine W-Frage, wird diese in der Regel nicht als gleichwertig zum Operator gezählt, da sie eher aspektiert, aber keine grundsätzlich neue Aufgabe erzeugt.

Die Analyse folgt, wie oben schon beschrieben, einer zeitlichen Zäsur, den Schulbüchern vor und ab 2014. Für die gymnasiale Oberstufe ist dies der Zeitpunkt der Einführung der neuen Kernlehrpläne. Nur so konnte überprüft werden, ob die Veränderung der unterrichtlichen Rahmenbedingungen im Sinne einer Kompetenzorientierung auch Veränderungen in den Aufgabenstellungen bedeuten.

Notwendige Einzelfallentscheidungen etwa im Hinblick auf die Wertung von Teilaufgaben können dazu führen, dass eine Nachzählung etwas differente Werte erzeugt, je nach den zugrunde gelegten Prämissen.

Da die Auszählungen sehr zeitaufwändig sind, mussten sie über einen Zeitraum von mehreren Jahren (2012-2016) erfolgen, auch dies kann im Einzelfall zu leicht abweichenden Werten geführt haben, weil die Analyse durchgängig händisch erfolgen musste. Insgesamt zeigen die Einzelauswertungen aber für das Schulbuch jeweils typische Merkmale im Umgang mit Aufgaben, die für einen anschließenden Vergleich genutzt werden können.

Aufgrund der großen Zahl an zugelassenen Lehrwerken und dem damit verbundenen hohen Zeitbedarf für die Auswertung erhebt diese keinen Anspruch auf Vollständigkeit der Berücksichtigung aller für NRW zugelassenen Schulbücher vor und ab 2014. Gleichwohl wird die Mehrzahl der zugelassenen Lehrwerke erfasst (die ausgewerteten Schulbücher sind tabellarisch im Anhang 4.2 erfasst).

Die Einschränkung liegt insbesondere am Verzicht auf die Berücksichtigung von Themenheften und an einer Besonderheit in der Erfassung der Lehrwerke, die für die Sekundarstufe II des Berufskollegs zugelassen sind: In der Liste der zugelassenen Lehrwerke "Geschichte und Gesellschaftslehre für das Berufskolleg" finden sich Lehrwerke mit unterschiedlicher Zuordnung zu den Teildisziplinen. Titel, die zwar unter dem Stichwort "Geschichte und Gesellschaftslehre" in der Liste subsumiert sind, aber als Schwerpunkt Politik und/oder Sozialkunde im Titel ausweisen, werden wegen des fehlenden Fokus auf Geschichte im Titel in Teil 1 und 2 der Auszählung nicht berücksichtigt.

Im 2. Teil werden dann nur die Lehrwerke der Geschichte bzw. der Gesellschaftslehre mit ausgewiesenem Bezug auf Geschichte (im Titel) für die Sek. II berücksichtigt, die unter den Wechsel zum Kernlehrplan fallen. Die Entscheidung, nur Lehrwerke, die Geschichte als Teildisziplin im Titel ausweisen, zu berücksichtigen, hat insbesondere Auswirkungen auf das Lehrwerk „Anstöße Politik“, für das es in 2014 eine Neuauflage gibt. Gab es in der 1. Auflage 2010 eine eindeutige Zuordnung zur Geschichte im Titel (1. Auflage: Gesellschaftslehre mit Geschichte), wird in der Auflage von 2014 lediglich im Titel der Fokus auf die Politik expliziert, Geschichte findet hier keine ausdrückliche Berücksichtigung. 
Ebenso bleiben Lehrwerke, die mit dem Schwerpunkt „Sozialkunde“ ausgewiesen sind, unberücksichtigt.

\subsection{Ergebnisse}

Im Folgenden werden insbesondere solche Ergebnisse in den Blick genommen, die sich auf das Vergleichen beziehen. Das bedeutet, dass die Häufigkeit von Vergleichsaufgaben untersucht wird, dabei solche Operatoren besonders in den Blick genommen werden, die als Teilhandlungen des Vergleichens einbezogen werden müssen. Aber auch allgemeine Auffälligkeiten der Frequenzanalyse werden nachfolgend berücksichtigt, wenn davon auszugehen ist, dass ein Bezug zu den gesuchten Vergleichsaufgaben festzustellen ist.

Dazu sollen die untersuchten Lehrwerke zunächst gekennzeichnet werden.

Nachfolgend werden deshalb zuerst allgemeine Daten zu den ausgezählten Lehrwerken vorgestellt, die sich insbesondere auf die Häufigkeit von Lernaufgaben insgesamt beziehen, aber auch zeigen, wie diese formuliert werden (über einen Operator oder eine W-Frage ${ }^{528}$ ) und auf welche Textgattung sie sich beziehen. Dieser Blickwinkel erscheint sinnvoll für die Einordnung der nachfolgenden Operatoren und insbesondere für das Vergleichen. Ein besonderer Fokus gilt dann dem Vergleichen und seinen in den EPA festgelegten Teilhandlungen, wie etwa dem Erklären, das sich ebenfalls als Operator in der Liste der Kultusministerkonferenz wiederfindet.

Abschließend werden die Ergebnisse der Auszählungen vor und ab 2014 zusammengeführt. Sie sollen die Folie für die nachfolgende Diskussion des Operators, insbesondere im Hinblick auf seine Teilhandlungen, bilden.

Um die Ergebnisse im Überblick anschauen zu können, werden im Folgenden Daten zusammengefasst.

\subsubsection{Ergebnisse der vor 2014 erschienenen Lehrwerke}

Für Teil 1 der Auswertung, der die vor 2014 erschienen Lehrwerke berücksichtigt, wurden 16 unterschiedliche Lehrwerke ausgewertet. Sie sind den unterschiedlichen Schulbuchverlagen zuzuordnen und sind entweder ein- oder mehrbändig. Im Gegensatz zu den Lehrwerken, die ab 2014 erschienen sind und durchgängig in Einführungs- und Qualifikationsphase unterteilt sind, folgen die Lehrwerke aus Teil 1 keinem solch festen Muster.

\footnotetext{
${ }^{528}$ Vereinzelt finden sich in den Lehrwerken Aufgaben, die ohne Interrogativum oder Operator, also über die Umstellung der Satzglieder und das Fragezeichen am Ende, gebildet werden. Diese werden nicht mitgezählt, da sie vergleichsweise selten vorkommen und in der geschichtsdidaktischen Diskussion um Aufgabenformate keine Rolle spielen. Sie zielen i.d.R. eher auf deklaratives Wissen ab.
} 


\subsubsection{Die Nutzung von Aufgaben in den Lehrwerken im statistischen Überblick}

Die folgende Grafik zeigt das Verhältnis von Aufgabenformulierungen durch Operatoren und W-Fragen in den vor 2014 erschienenen Lehrwerke:

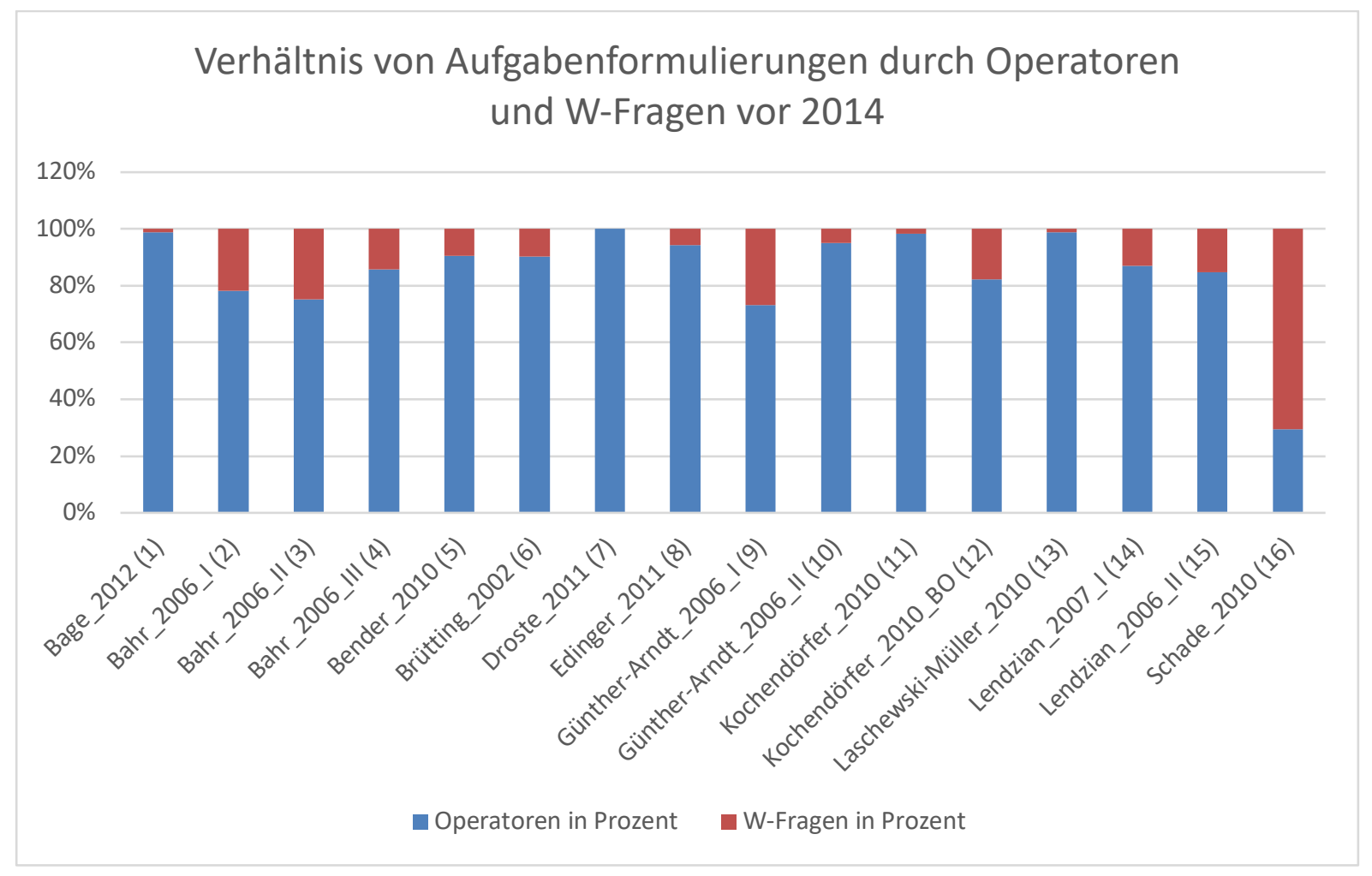

Abbildung 13: Anteile der Aufgaben mit Operator und W-Fragen vor 2014

Bis auf ein Lehrwerk werden in allen Lehrwerken Aufgaben hauptsächlich über Operatoren formuliert.

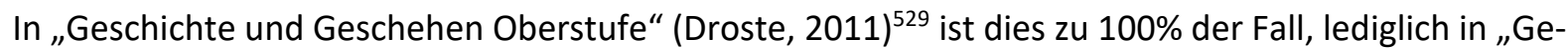
schichte in der Gegenwart" (Schade et al., 2010) werden nur zu 30 \% Operatoren, sonst Formulierungen durch W-Fragen verwendet. Bei diesem Schulbuch handelt es sich um eine Neuauflage eines älteren Lehrwerks (Erstveröffentlichung: 1988), dies könnte die Abweichung erklären.

Das Lehrwerk, in dem die ausgewählte Vergleichsaufgabe abgedruckt ist, entstammt dem Jahre 2010 und gehört damit in die Generation vor der Umstellung auf die neuen Kernlehrpläne. Es handelt sich um das Lehrwerk „Kursbuch Geschichte“ (Laschewski-Müller, 2010). Es berücksichtigt zu 99\%, also fast vollständig Aufgabenformulierungen durch Operatoren.

Betrachtet man umgekehrt die Häufigkeit der Aufgabenformulierungen durch W-Fragen, spiegeln sich die Ergebnisse: Sie zeigen das Bemühen der Autor*innen um Aufgabenformulierungen über Operatoren, auch wenn die Ergebnisse uneinheitlich sind: Bis auf die genannte Ausnahme (Schade et al., 2010)

\footnotetext{
${ }^{529} \mathrm{Im}$ Folgenden wird zur besseren Wiedererkennung und für den besseren Lesefluss mit Kurztiteln gearbeitet. Die vollständige Titel-Nennung befindet sich im Anhang 4.2.
} 
werden in keinem Lehrwerk 30\% an Aufgabenformulierungen durch W-Fragen verwendet. Das Kursbuch Geschichte berücksichtigt nur zu 1\% Formulierungen durch W-Fragen und erreicht damit den zweitniedrigsten Anteil der Vergleichslehrwerke.

Die Annahme, dass Kompetenzorientierung über Aufgabenformulierungen mit Operatoren zu erreichen ist, bildet sich also bereits in den Lehrwerken vor der Umstellung auf die Kernlehrpläne 2014 ab. Damit bieten bereits die meisten Lehrwerke der älteren Generation grundsätzlich die Möglichkeit, Aufgaben, die die Operation Vergleichen beinhalten, zu berücksichtigen, dazu gehört auch das Kursbuch Geschichte.

Weitere Aufschlüsse im Sinne eines allgemeinen Überblicks bietet der Blick auf die Platzierung von Aufgaben: Legt man verschiedene Geschichtslehrwerke nebeneinander und vergleicht die Anordnung von Aufgaben, fällt auch ohne systematische Analyse auf, dass Aufgaben nicht nur sehr unterschiedlich häufig auf den Schulbuchseiten vorkommen, sondern die Aufgabenverteilung auch individuellen Anordnungsprinzipien folgt. Nicht nur ihre Anordnung ist individuell, auch ihre zahlenmäßige Berücksichtigung. Dies ist bedeutsam, da Lernende eine ausreichende Zahl an Lernanlässen benötigen, um sich mit historischen Operationen auseinanderzusetzen und diese zudem üben zu können.

Eine Frequenzanalyse der durchschnittlichen Zahl der Lernaufgaben pro Seite ergibt das nachfolgende Bild:

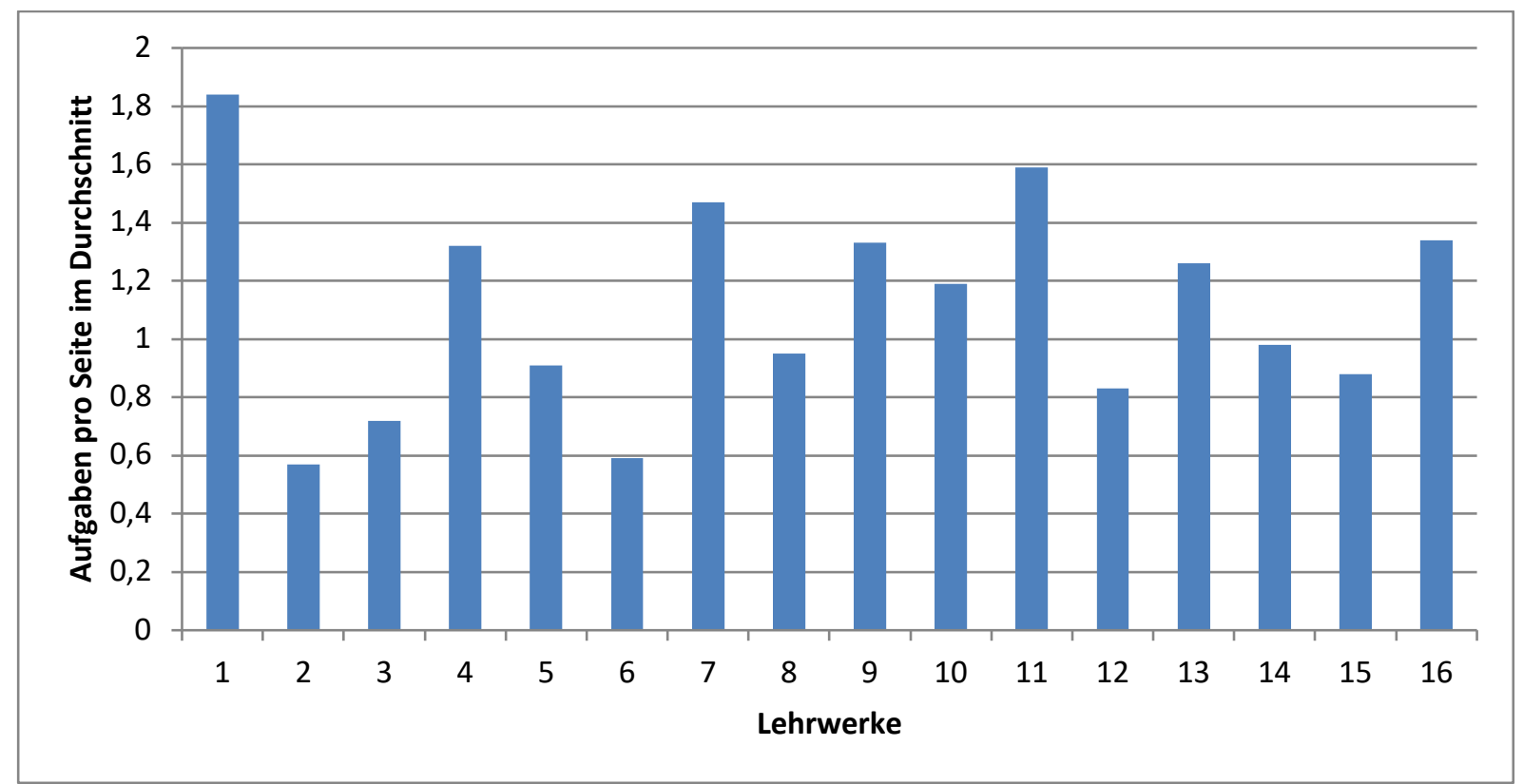

Abbildung 14: Anteile Aufgaben pro Seiten (vor 2014) 530

Aufgaben (hier sind ausschließlich Lernaufgaben gemeint) werden sowohl sehr unterschiedlich angeordnet als auch, dies zeigt die Abbildung, in sehr unterschiedlicher Zahl verwendet.

\footnotetext{
${ }^{530}$ Die Nummerierung der Lehrwerke wird hier für den besseren Lesefluss vorgenommen, sie entspricht der Reihenfolge in Tabelle 4 und folgt der Auflistung in Tabelle 3.
} 
Bezüglich der Anordnung der Aufgaben ist zunächst festzustellen, dass sie in manchen Lehrwerken am Ende eines Kapitels angefügt sind, in anderen sind sie direkt dem Material nachgestellt, auf das sie sich vornehmlich beziehen.

Hinsichtlich der Häufigkeit von Aufgaben ist zu konstatieren, dass sich im Durchschnitt in den ausgezählten Lehrwerken vor 2014 1,11 Aufgaben pro Seite (Mittelwert) befinden. Rechnet man aufgrund der unterschiedlichen Ergebnisse pro Lehrwerk den Median dazu, ergibt sich ein Wert von 1, 09. Kein Schulbuch stellt im Schnitt 2 Aufgaben pro Seite zur Verfügung, alle ausgewerteten Lehrwerke liegen mehr oder weniger knapp unter diesem Wert. Manches Lehrwerk liegt dabei weit unter dem Mittelwert von 1,1 Aufgabe pro Seite und stellt ca. eine Aufgabe pro Doppelseite zur Verfügung. Das Kursbuch Geschichte liegt mit 1,26 Aufgaben pro Seite knapp über dem Mittelwert. Dazu ist anzumerken, dass in diesem Lehrwerk die Aufgaben unter den jeweiligen Materialien, auf die sie Bezug nehmen, angeordnet sind.

Bei einer solchen Betrachtung ist außerdem zu berücksichtigen, dass sich sämtliche der ausgezählten Lehrwerke oft recht langer Darstellungstexte zu den einzelnen Themen bedienen. Da auch die ausgewählte Vergleichsaufgabe auf einem längeren Darstellungstext aufbaut, ist bedeutsam nachzuprüfen, in welchem Maße sich Aufgaben überhaupt auf solche Darstellungstexte beziehen, um daraus Schlüsse ziehen zu können, inwiefern Lernende mit einer solchen Aufgabenkonstellation vertraut sind:

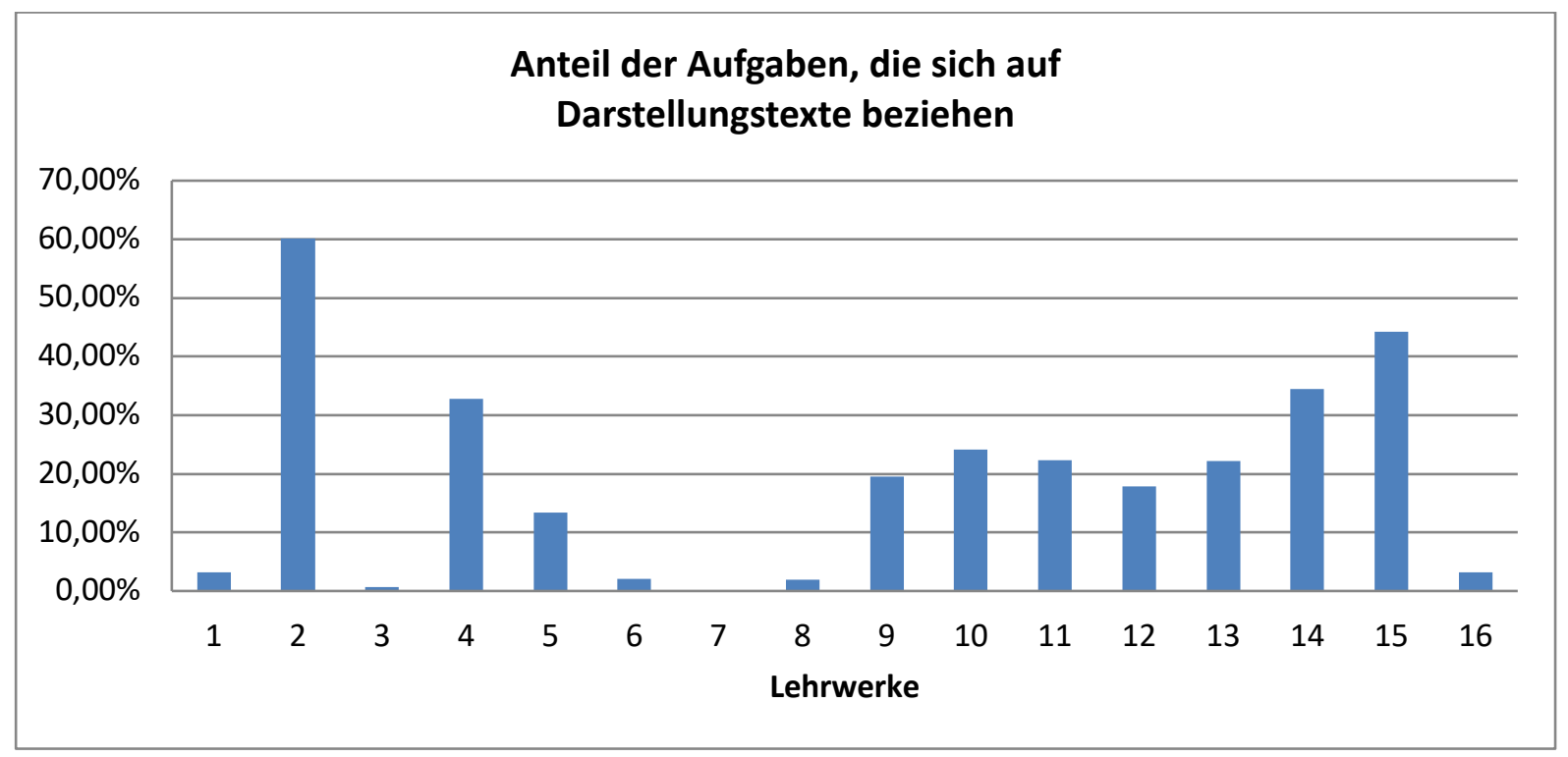

Abbildung 15: Anteil der Aufgaben, die sich auf Darstellungstexte beziehen (vor 2014)

Die Grafik zeigt eine enorme Spannbreite des Bezugs auf Darstellungstexte. In „Geschichte und Geschehen Oberstufe“ (Lehrwerk 7: Droste, 2011) gibt es fast keine Aufgabe, die sich eindeutig einem Darstellungstext zuordnen ließe. In „Horizonte“ Band 1 (Lehrwerk 2: Bahr, 2006) liegt der Wert dagegen bei $60 \%$. Umso erstaunlicher ist, dass in den Bänden 2 und 3 die Werte enorm sinken. Es gibt also nicht nur einen individuellen Schulbuchstil zwischen verschiedenen Lehrwerken, sondern auch innerhalb einer Reihe. Es kann also nicht davon ausgegangen werden, dass in Lehrwerken Aufgaben, die auf einem Darstellungstext basieren, gleichermaßen geübt sind wie Aufgaben, die sich auf Quellen 
und andere Materialien beziehen. Diese Werte müssen mit denen der Erhebung nach 2014 abgeglichen und auch für die Auswertung der eingesetzten Aufgabe berücksichtigt werden.

Das Kursbuch Geschichte erreicht 22,14\% und liegt damit etwas über dem Durchschnitt.

\subsubsection{Die Häufigkeit der Verwendung von EPA-Operatoren}

Zwar gibt der globale Blick auf Aufgaben in Lehrwerken für den Geschichtsunterricht einen ersten Eindruck über den Umgang mit Aufgaben, lässt aber noch keine genaueren Rückschlüsse zu, welche Operatoren für die Aufgabenstellungen eine Rolle spielen und welche Rolle das Vergleichen spielt. Eine Analyse der am häufigsten verwendeten Operatoren entlang der ausgewiesenen Anforderungsbereiche und quer über alle ausgezählten Lehrwerke hinweg soll zur Klärung beitragen und bietet zusammengefasst das nachstehende, sehr eindeutige Bild, das der zuvor dargestellten Heterogenität der Schulbücher entgegensteht:

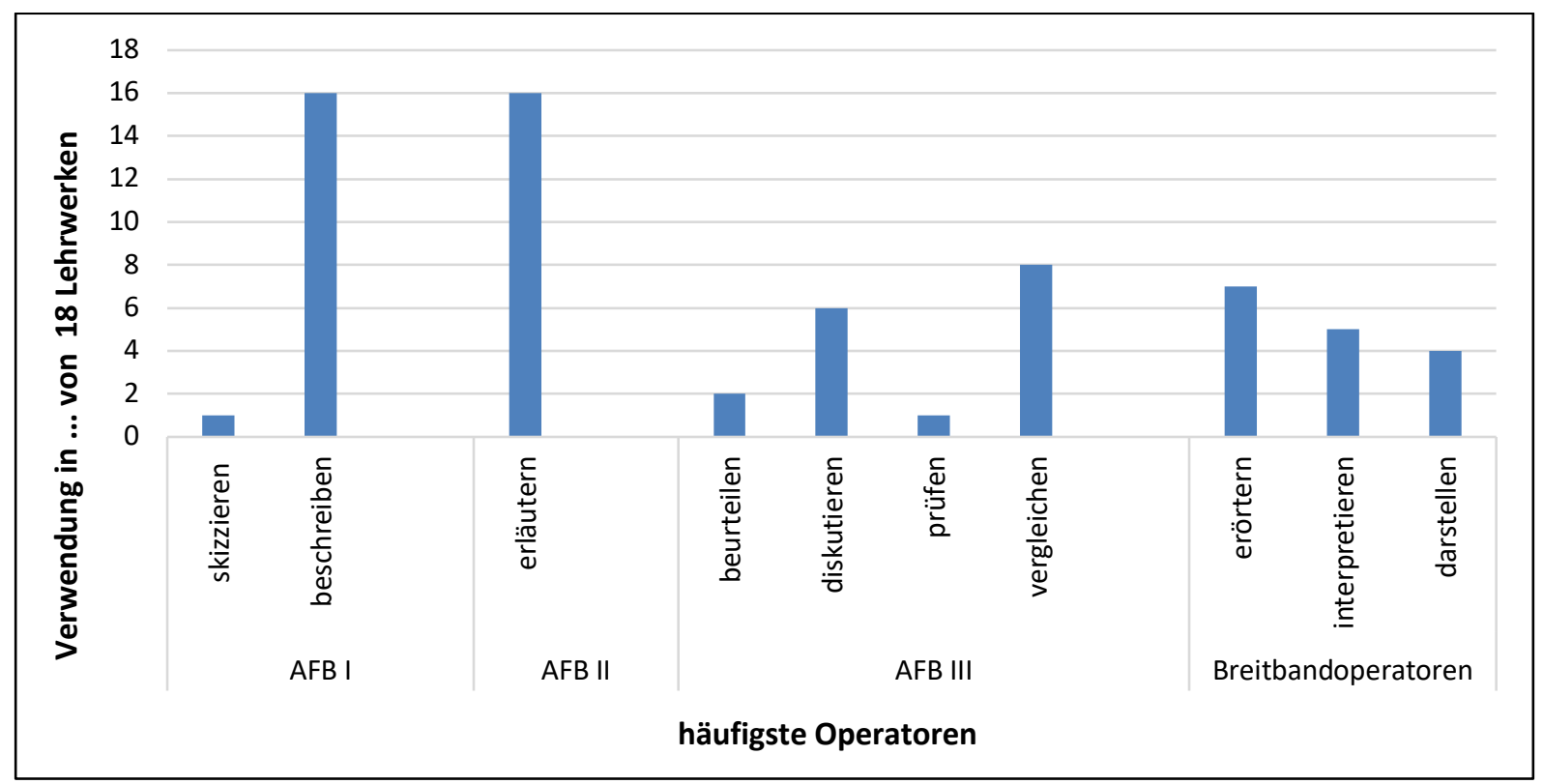

Abbildung 16: Darstellung der am häufigsten verwendeten Operatoren (vor 2014)

Im Anforderungsbereich I ist das Beschreiben der am häufigsten verwendete Operator. 15 von 16 Schulbücher verwenden ihn häufiger als die anderen dem AFB I zugeordneten Operatoren. Lediglich in einem Fall wird das Skizzieren genauso häufig verwendet wie das Beschreiben.

Im Anforderungsbereich II ist das Bild noch eindeutiger: In allen Fällen ist das Erläutern der am häufigsten verwendete Operator.

Im Anforderungsbereich III ist das Bild etwas heterogener, da in zwei Fällen das Beurteilen der am häufigsten verwendete Operator ist, in sechs Fällen das Diskutieren, in einem Fall das Prüfen. Dennoch gibt es auch hier einen Operator, der in acht Lehrwerken am häufigsten verwendet wird und damit die Rangliste anführt: Es handelt sich um das Vergleichen. 
Bei den Breitbandoperatoren kann man feststellen, dass alle drei abwechselnd in den Lehrwerken als häufigster Operator auftauchen. Die Zahlen liegen eng beieinander: Darstellen ist in vier Lehrwerken der am häufigsten verwendete Operator, Interpretieren in fünf Lehrwerken, Erörtern in sieben Lehrwerken.

Wie in Kapitel 3.5 dargestellt, kann angenommen werden, dass als Teilhandlungen für das Vergleichen das Gegenüberstellen und das Beurteilen eine Rolle spielen. Die Darstellung zeigt, dass das Gegenüberstellen und das Erklären als weitere Teilhandlungen nicht zu den am häufigsten genutzten Operatoren zählen. Das dem Anforderungsbereich II zugeordnete Beurteilen wird zumindest in zwei Lehrwerken am häufigsten genutzt.

Da den Anforderungsbereichen eine zunehmende Progression zugeschrieben wird, kann man insgesamt feststellen: Je höher das angenommene Anforderungspotential eines Anforderungsbereiches ist, desto uneinheitlicher wird das Bild der verwendeten Operatoren.

Die Grafik bildet zwar ab, welche Operatoren in einzelnen Lehrwerken häufig genutzt werden, nicht jedoch, inwiefern die übrigen EPA-Operatoren berücksichtigt werden. Daran knüpft sich die Frage, ob für das Vergleichen wichtige Teilhandlungen, die zwar nicht als am häufigsten vorkommend gelistet wurden, dennoch hohe Werte erreichen, so dass Lernende ausreichend Gelegenheit haben, sich mit innen auseinanderzusetzen. Dieser Frage soll nachfolgend, aufgeschlüsselt entsprechend der verschiedenen Anforderungsbereiche, nachgegangen werden.

Die nachfolgende Darstellung zeigt, welche Operatoren in den ausgezählten Lehrwerken im Anforderungsbereich I verwendet werden.

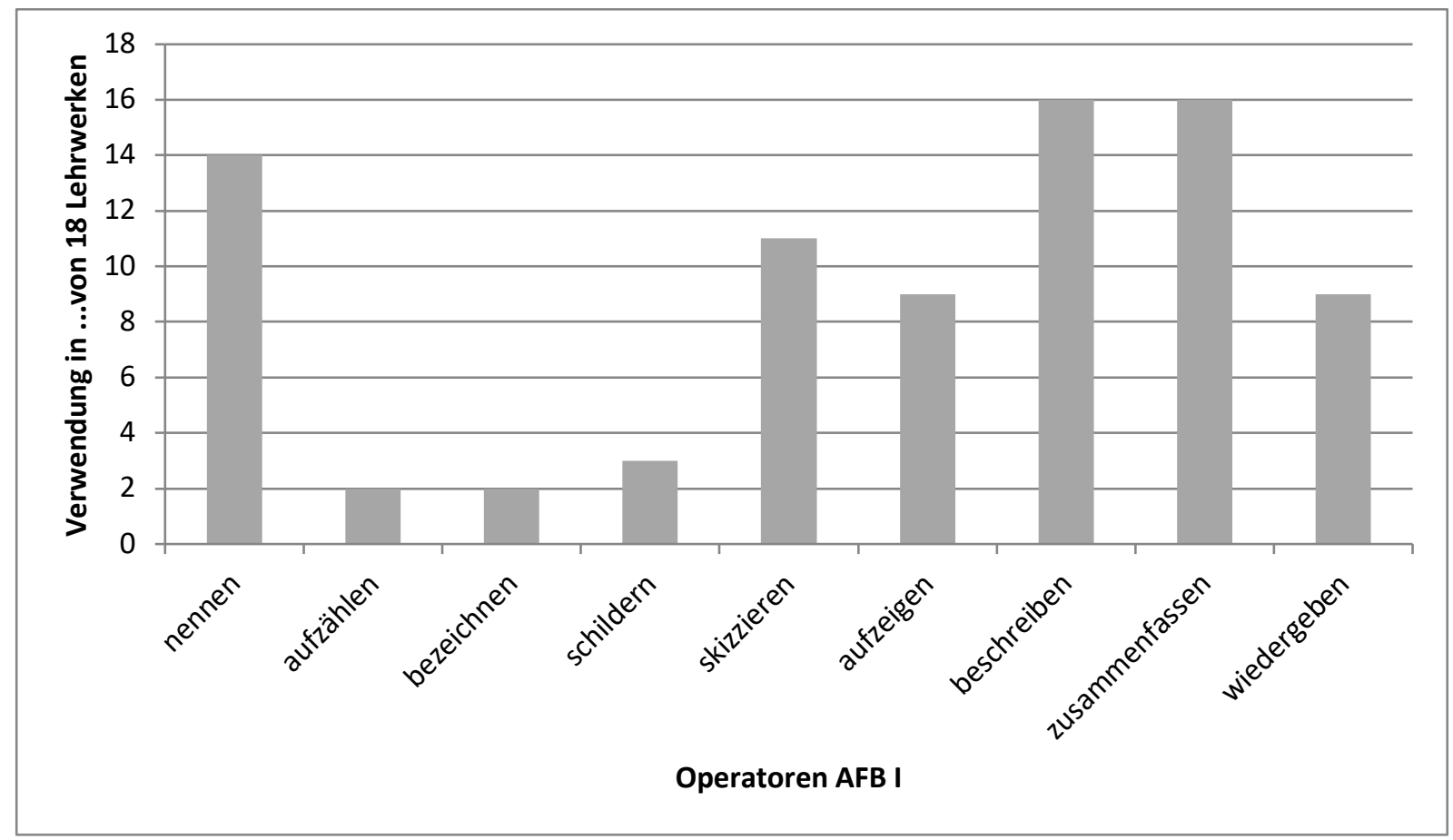

Abbildung 17: Verwendung von Operatoren im Anforderungsbereich I (vor 2014) 
Die Ergebnisse der Grafik zusammenfassend kann festgestellt werden, dass die Operatoren Aufzählen und Schildern in nur 2 von 16 Lehrwerken überhaupt vorkommen, der Operator Schildern taucht sogar insgesamt nur dreimal auf. Beschreiben und Zusammenfassen werden dagegen in allen Lehrwerken verwendet. Schlüsselt man die Verwendungshäufigkeit der einzelnen Operatoren für die einzelnen Lehrwerke auf, ergibt sich dieses Bild:

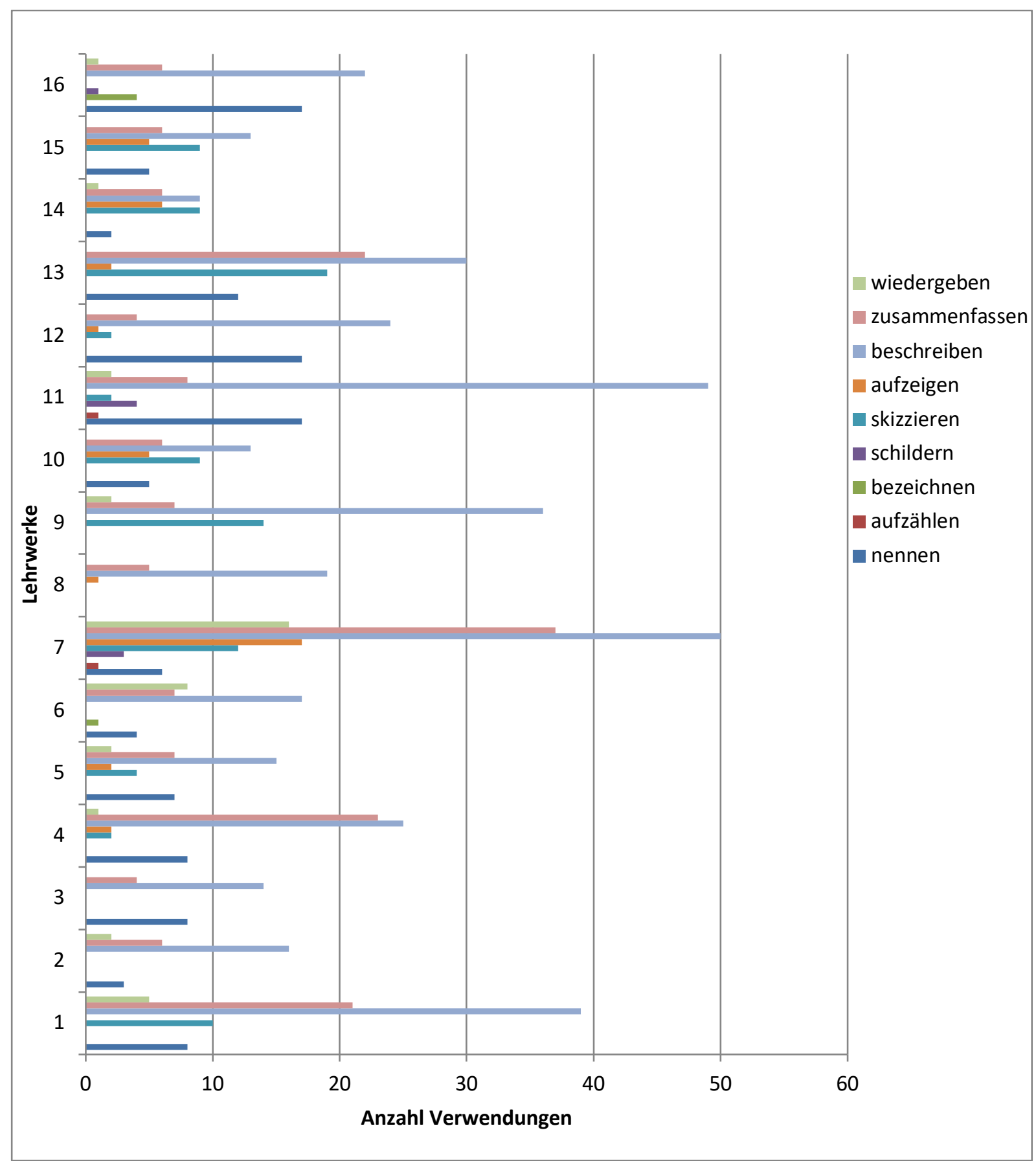

Abbildung 18: Häufigkeit der Verwendung der Operatoren im Anforderungsbereich I in den einzelnen Lehrwerken ${ }^{531}$ (vor 2014)

\footnotetext{
${ }^{531}$ Auf der $x$-Achse sind die einzelnen Lehrwerke aufgeführt, die Aufschlüsselung befindet sich in Anhang 4.3. Auf der $y$-Achse ist die Häufigkeit abzulesen.
} 
Die Grafik veranschaulicht, dass Operatoren, die in allen Lehrwerken verwendet werden, in der Regel auch in mittlerer oder höherer Frequenz gebraucht werden, wie hier die Operatoren Beschreiben und Zusammenfassen zeigen. Operatoren, die nur in wenigen Lehrwerken erscheinen, wie das Aufzählen und das Schildern, werden dort auch nur wenig verwendet. Eine Ausnahme bildet das Bezeichnen, das zumindest in einem Lehrwerk in höherer Frequenz verwendet wird.

Die Schüler*innentexte zur Vergleichsaufgabe werden zeigen, dass sich in den Texten Übernahmen aus dem Darstellungstext wiederfinden, die dem Operator Wiedergeben oder Zusammenfassen zugeordnet werden können. Bei beiden Operationen kann davon ausgegangen werden, dass diese früh geübt werden und deshalb als Strategie eher nutzbar gemacht werden können als andere Operationen, um die Schreibaufgabe zu lösen. Unter Umständen stellen Sie eine Art „Ersatzhandlung“ dar.

\section{Häufigkeiten im Anforderungsbereich II}

Im Anschluss an die Aufschlüsselung des Anforderungsbereichs I wird nun für den Anforderungsbereich II dargestellt, welche Operatoren in den Lehrwerken überhaupt Verwendung finden.

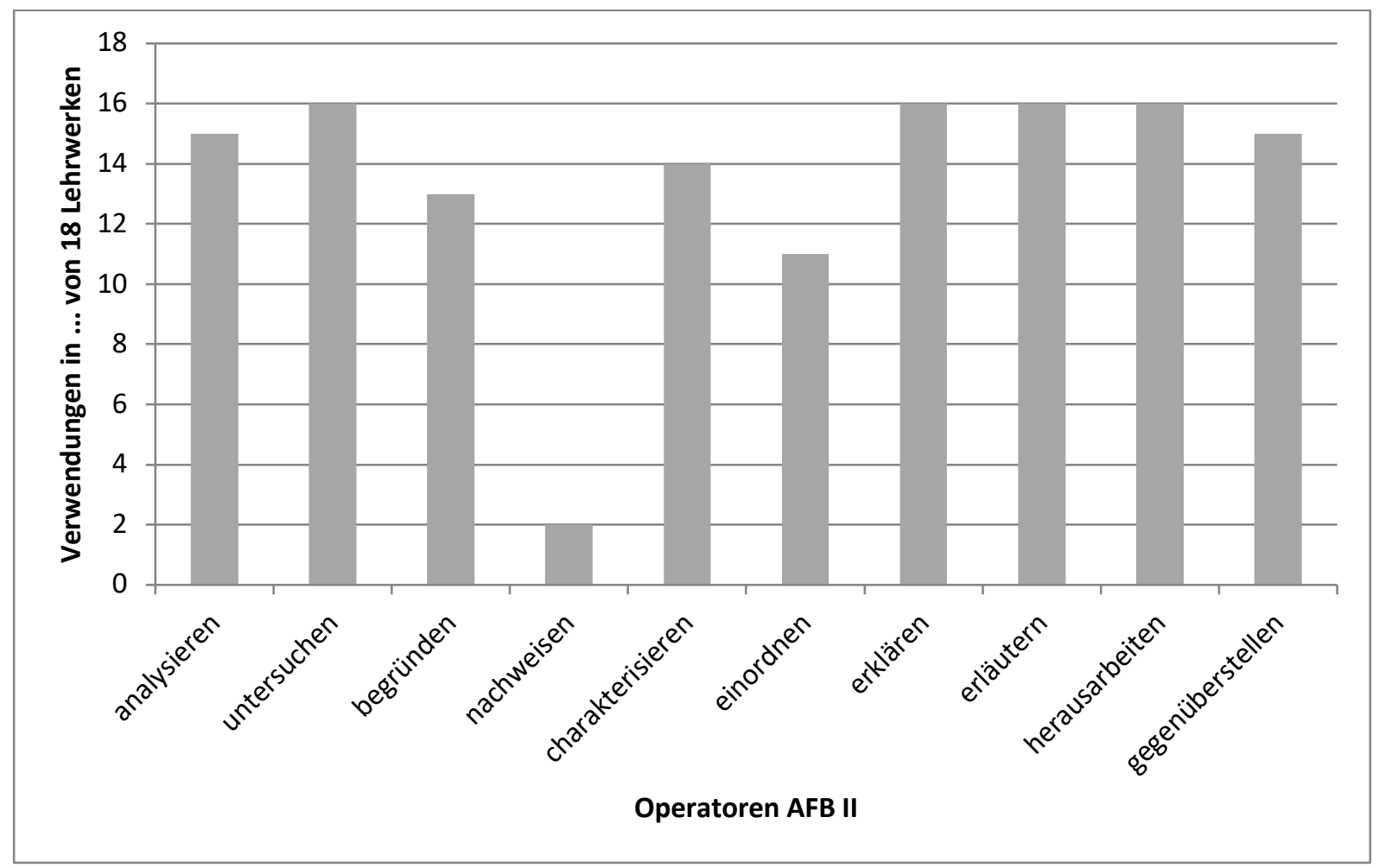

Abbildung 19: Verwendung von Operatoren im Anforderungsbereich II (vor 2014)

Im Anforderungsbereich II ist das Bild etwas homogener als im Anforderungsbereich I, da im Anforderungsbereich II fast alle Operatoren in den meisten Lehrwerken verwendet werden. So tauchen Untersuchen, Erklären, Erläutern und Herausarbeiten in allen Lehrwerken auf. Analysieren und Gegenüberstellen (entspr. den EPA als wesentlicher Teil des Vergleichens) immerhin in 15 von 16 analysierten Lehrwerken. Eine Ausnahme stellt das Nachweisen dar, das nur in zwei Lehrwerken verwendet 
wird. Dabei fällt auf, dass insbesondere das Erläutern häufig verwendet wird. In 14 Lehrwerken ist Erläutern der am häufigsten verwendete Operator.

Wie für den Anforderungsbereich I, wird auch für den Anforderungsbereich II die Frequenz, mit der die Operatoren in den einzelnen Lehrwerken auftauchen, aufgeschlüsselt:

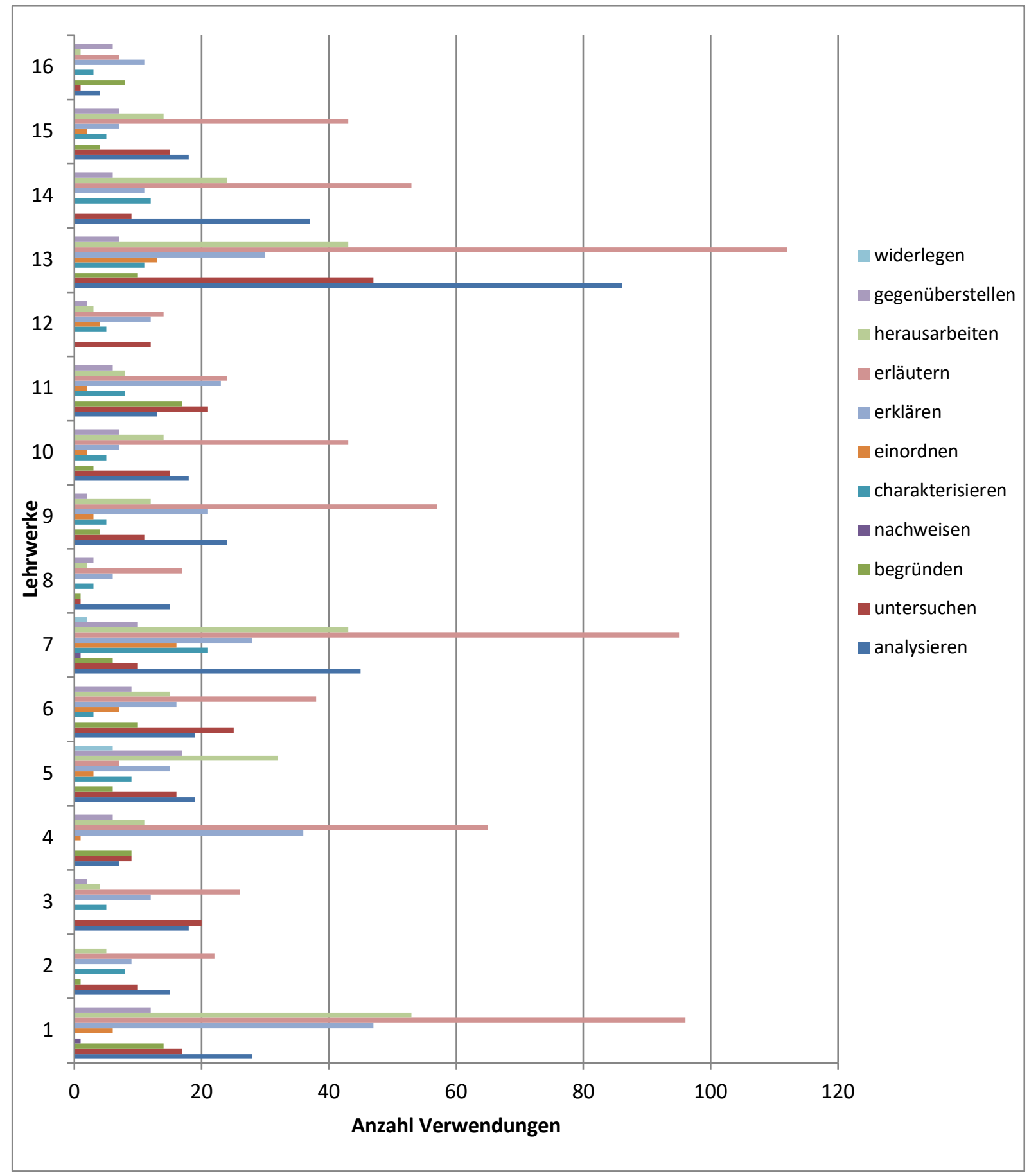

Abbildung 20: Häufigkeit der Verwendung der Operatoren im Anforderungsbereich II in den einzelnen Lehrwerken ${ }^{532}$ (vor 2014)

\footnotetext{
${ }^{532}$ Auf der $x$-Achse sind die einzelnen Lehrwerke aufgeführt, die Aufschlüsselung befindet sich in Anhang 4.3. Auf der $y$-Achse ist die Häufigkeit abzulesen.
} 
Im Anforderungsbereich II sind die für das Vergleichen relevanten Operatoren Begründen, Erklären und Gegenüberstellen eingeordnet. Das Begründen wird, wie in Kapitel 3.5. dargelegt, direkt durch die EPA eingefordert. ${ }^{533}$ Es wird auf der Grundlage der Überlegungen in Kapitel 3.5 außerdem davon ausgegangen, dass dem Vergleichen Erklär-Strukturen zugrunde liegen, da zum einen in der EPA-Bestimmung das Vergleichen auf der Grundlage von Kriterien durchgeführt werden soll, zum anderen das Beurteilen eine Einordnung in einen historischen Zusammenhang verlangt und schließlich der Darstellungstext auf Zusammenhänge zurückgreift, die in einem neuen Zusammenhang wie dem Begründen erklärungsbedürftig sind. Das Gegenüberstellen ergibt sich ebf. direkt aus der EPA-Bestimmung ${ }^{534}$ und ist grundlegend für einen Vergleich.

Die Auswertungen zeigen, dass das Begründen nur in $80 \%$ der Lehrwerke verwendet wird und in diesen Lehrwerken im Mittel siebenmal. Auch hier setzt sich die Annahme fort, dass Operatoren, die nur in wenigen Lehrwerken verwendet werden, in diesen auch nur in geringer Häufigkeit benutzt werden. Ähnliches ergibt sich für das Gegenüberstellen. Es wird immerhin in fast allen Lehrwerken verwendet, aber auch hier im Mittel siebenmal. Beide Operatoren bieten also nur eine begrenzte Anzahl an Gelegenheiten, diese anzuwenden und zu üben. Das Erklären dagegen, das in allen Lehrwerken vorkommt, zeigt zwar in einigen Lehrwerken deutlich höhere Frequenzen, aber auch eine größere Spannbreite der Verwendung von 6- bis 47mal. Der Mittelwert liegt bei 13,5 Verwendungen. Er kann viel eher als bekannt und geübt angenommen werden. Allerdings muss in diesem Zusammenhang der Operator Erläutern mitbetrachtet werden, da insbesondere in Lehrwerken, in denen das Erklären nur selten verwendet wird, das Erläutern in deutlich höherer Frequenz auftaucht. Aufgrund dieser Zahlen und der inhaltlichen Nähe der beiden Operatoren (das Erläutern teilt sich mit den Erklären eine Bestimmung, angefügt wird lediglich der Hinweis, dass weitere Beispiele und Informationen hinzugefügt wer$\operatorname{den}^{535}$ ) muss davon ausgegangen werden, dass die fehlende klare Abgrenzung von Erklären und Erläutern zu einem uneinheitlichen Gebrauch von Erklären und Erläutern führt, also da, wo das Erklären wenig gebraucht wird, der Operator durch das Erläutern ersetzt wird.

\section{Häufigkeiten im Anforderungsbereich III}

Im Anforderungsbereich III tauchen nur zwei der EPA-Operatoren auch in allen Lehrwerken auf. Es handelt sich um das Beurteilen und das Vergleichen. Gerade diese beiden Operatoren sind jedoch im Rahmen dieser Arbeit von besonderem Interesse. Das Entwickeln wird nur in 3 Lehrwerken verwendet, das Prüfen in knapp mehr als die Hälfte der Schulbücher, nämlich in sieben.

\footnotetext{
${ }^{533}$ Vgl. Kultusministerkonferenz (2005): EPA Geschichte, S. 8.

${ }^{534}$ Ebd.

535 Vgl. ebd.
} 


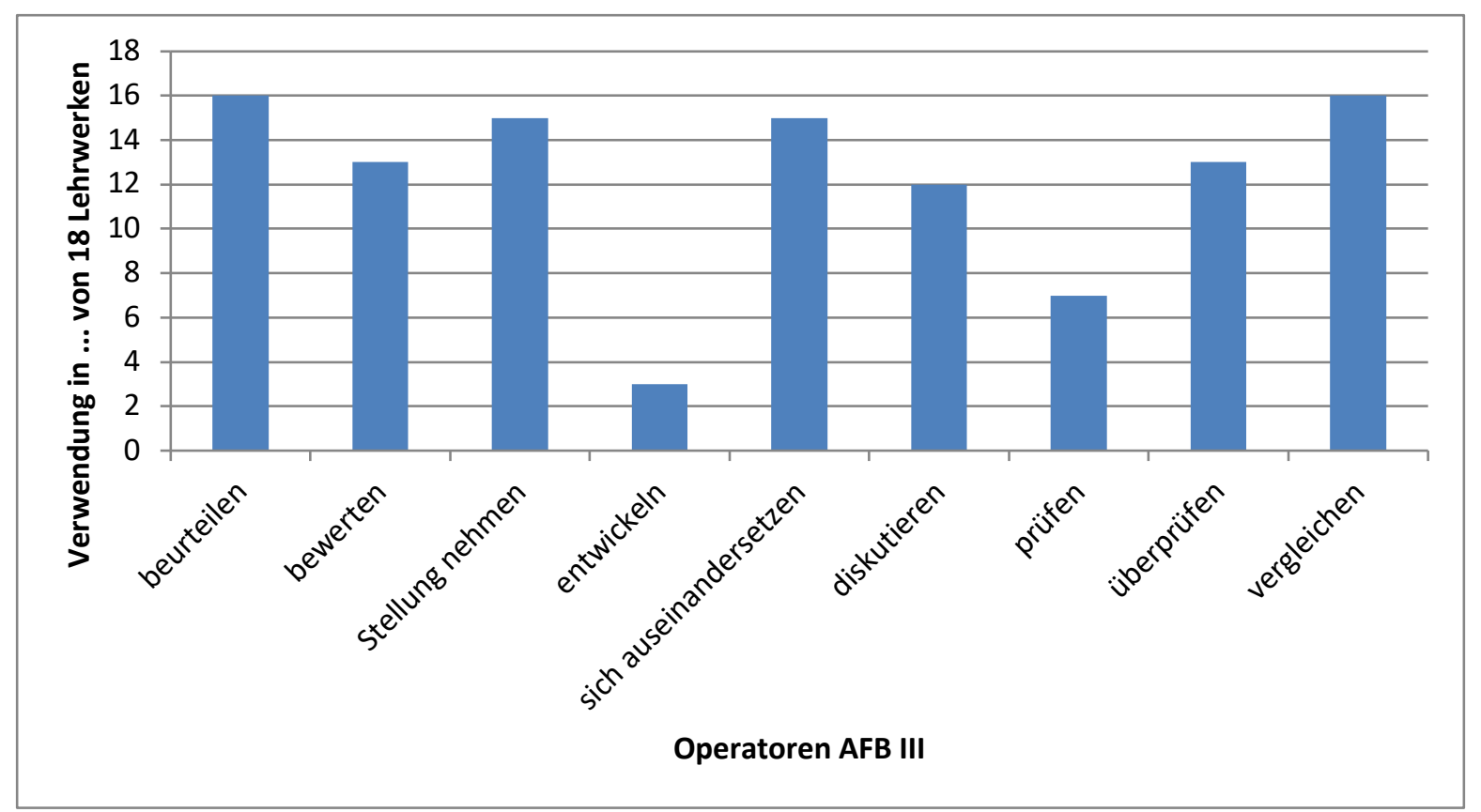

Abbildung 21: Verwendung von Operatoren im Anforderungsbereich II

Dafür, dass alle Operatoren der Vorbereitung auf die Abiturprüfung dienen, ist dies ein sehr heterogenes Bild. Zu prüfen wäre allerdings, ob die in den Schulbüchern nur kaum verwendeten Operatoren auch nur kaum oder gar nicht in den Abiturprüfungen verwendet werden.

Wie häufig werden die in allen Schulbüchern vorkommenden Operatoren verwendet? Wie für den Anforderungsbereich I und II wird an dieser Stelle wieder die Frequenz, mit der die Operatoren des Anforderungsbereichs III in den einzelnen Lehrwerken auftauchen, aufgeschlüsselt: 


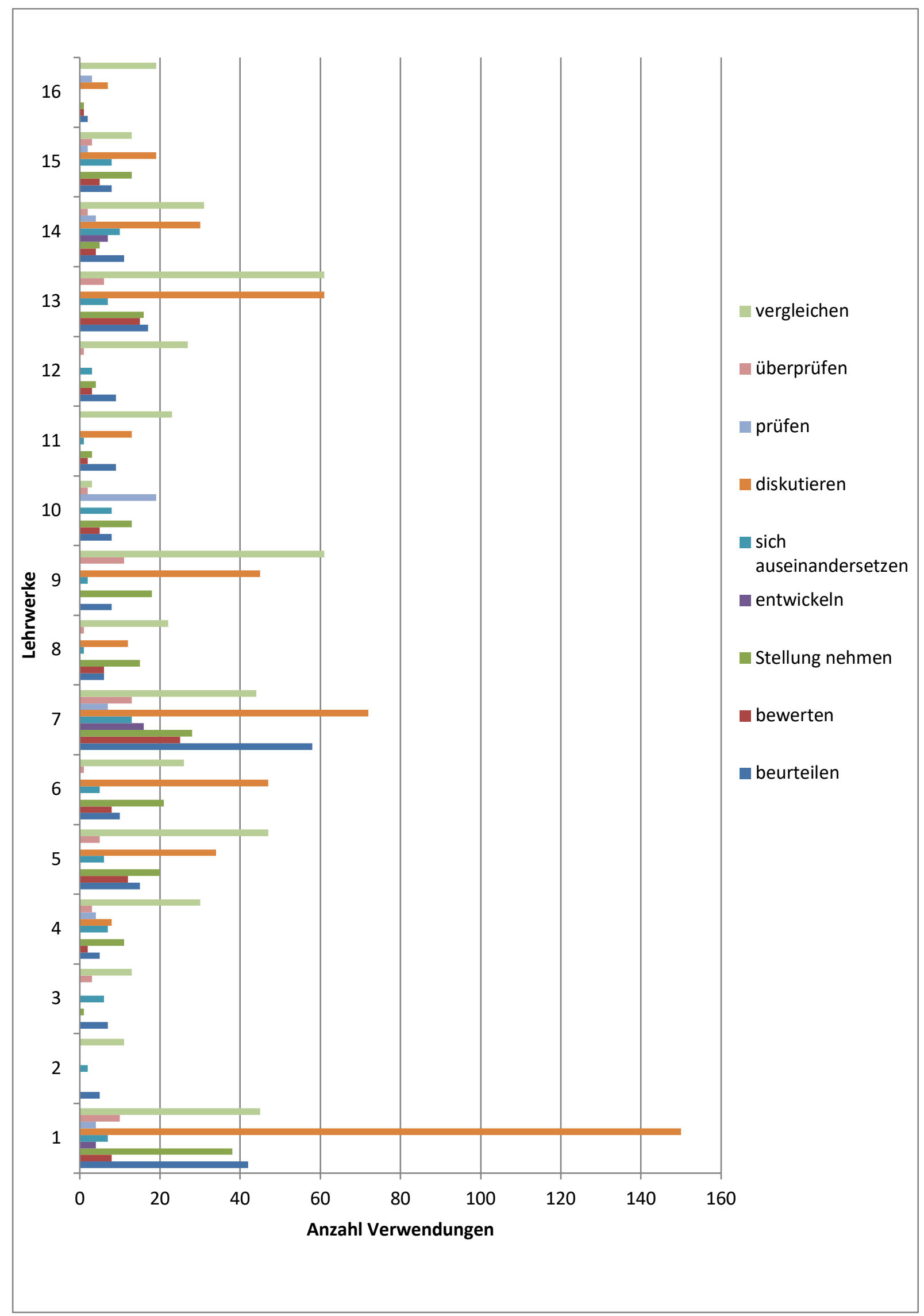

Abbildung 22: Häufigkeit der Verwendung der Operatoren im Anforderungsbereich III in den einzelnen Lehrwerken ${ }^{536}$ (vor 2014) 
Insbesondere die Operatoren Diskutieren und Vergleichen erreichen vergleichsweise hohe Frequenzwerte, obwohl das Diskutieren nur in 12 von 16 Lehrwerken überhaupt vorkommt. Für das Vergleichen kann festgehalten werden, dass es in allen Lehrwerken vorkommt und durchschnittlich in jedem zweiten Lehrwerk sogar den meist verwendeten Operator im Anforderungsbereich III darstellt. Damit gibt es im Verhältnis zu den anderen Operatoren viele Lerngelegenheiten für das Vergleichen im Geschichtsunterricht. Das Beurteilen hingegen kommt zwar in allen Lehrwerken vor, ist aber nur in zwei Lehrwerken von der Frequenz am häufigsten. Die Spannbreite der Verwendungshäufigkeit ist zudem sehr hoch (zwischen 2 und 58 Verwendungen).

Für das Vergleichen kann also festgestellt werden, dass es in allen untersuchten Lehrwerken vor 2014 starke Berücksichtigung findet. Zu prüfen ist im Weiteren, ob dies auch für die Lehrwerke, die nach der Umstellung auf die Kernlehrpläne erschienen sind, gilt. Auch die für das Vergleichen notwendige (Teil-)Operation des Beurteilens findet Berücksichtigung in den Lehrwerken.

\section{Nutzung der,Breitbandoperatoren'}

Unter dem Begriff „Breitbandoperatoren“ sind solche Operatoren subsummiert, die Leistungen in allen drei Anforderungsbereichen verlangen. ${ }^{537}$ Für das Vergleichen konnte bereits gezeigt werden, dass es ebenfalls Leistungen aus mind. zwei Anforderungsbereichen benötigt, so dass eine solche Trennung künstlich ist, weil dies auch für andere Operatoren gilt und zudem abhängig vom Kontext ist. Zu den Breitbandoperatoren gehören das Erörtern, Interpretieren und Darstellen. Die Verwendung dieser Breitbandoperatoren sei der Vollständigkeit halber berücksichtigt, auch wenn ein Zusammenhang zum Vergleichen nur indirekt festgestellt werden kann.

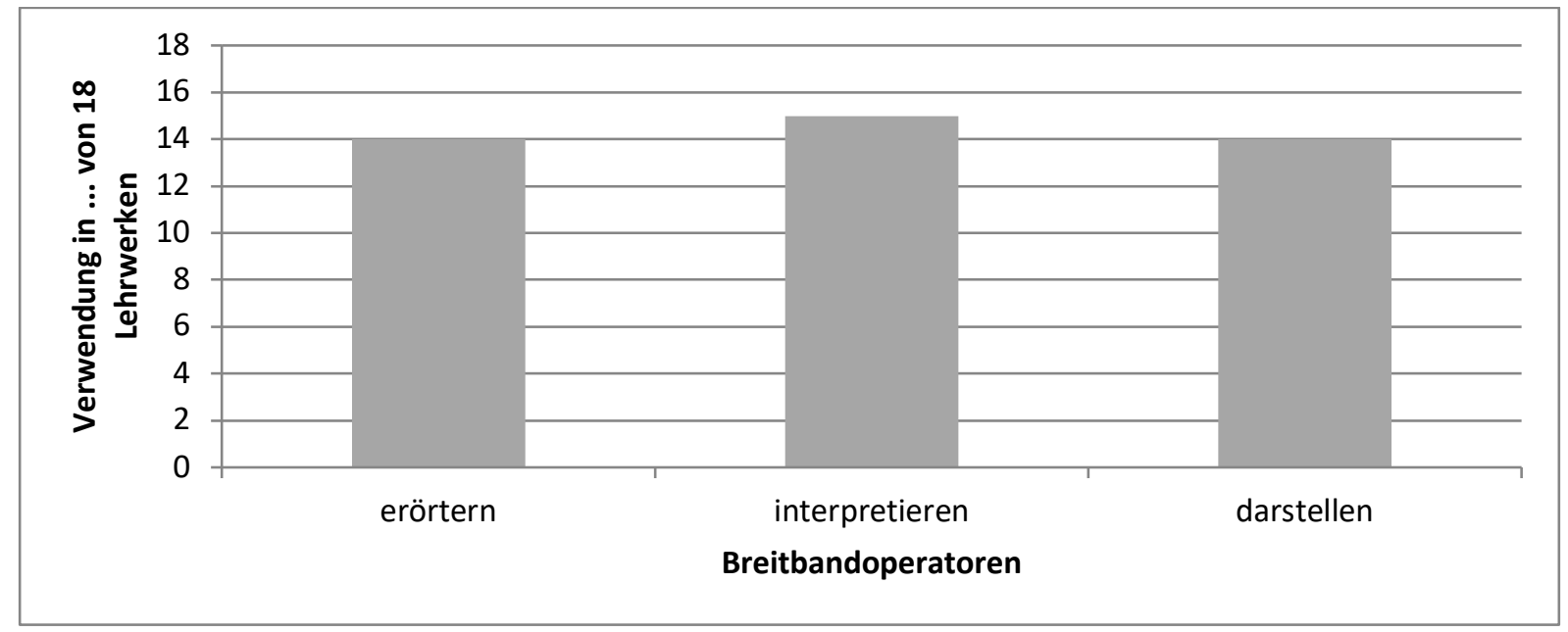

Abbildung 23: Verwendung der Breitbandoperatoren (vor 2014)

537 Ebd. S. 7. 
Das Balkendiagramm zeigt, dass die drei genannten Operatoren in den meisten Lehrwerken vorkommen, aber eben nicht in allen. Dies ist insofern bedeutsam, als dass die Sekundarstufe II auf wissenschaftspropädeutisches Arbeiten vorbereiten soll und das Interpretieren und Erörtern wichtige geschichtswissenschaftliche Arbeitstechniken darstellen. ${ }^{538}$

\subsubsection{Verwendung von Operatoren über den EPA-Katalog hinaus}

Über die in den EPA genannten Operatoren hinaus verwenden alle untersuchten Lehrwerke auch eigene Operatoren. Dies geschieht je nach Schulbuch in sehr unterschiedlicher Häufigkeit. Da sie für das Vergleichen nur eine untergeordnete Rolle spielen, sei im Folgenden nur auf das Verhältnis von EPAund nicht EPA-Operatoren verwiesen, um ein genaueres Bild zu bekommen, welche Bedeutung die EPA-Operatoren und damit auch das Vergleichen einnehmen. ${ }^{539}$ Es ist an dieser Stelle anzumerken, dass der Begriff Operator in diesem Zusammenhang ganz allgemein als handlungsinitiierendes Verb gebraucht wird. Dabei kann es sich sowohl um sprachliche Handlungen, aber auch um andere Aufforderungen, wie bspw. etwas zu erstellen, handeln.

\footnotetext{
${ }^{538}$ Hinweise zu den Einzelauswertungen: Bei dem Lehrwerk, dem gleich zwei der Breitbandoperatoren fehlen, nämlich das Interpretieren und das Erörtern, handelt es sich um Schade (2010). Dieses hat, wie zuvor dargestellt, eine Sonderstellung inne, da es als einziges hauptsächlich über W-Fragen arbeitet. Bei Edinger (2011) und Bender (2010) fehlt das Darstellen. Dies ist insofern interessant, weil anhand konkreter Aufgaben diskutiert werden müsste, ob das Darstellen immer den Anforderungsbereich III abdeckt. Z. B. findet sich in Lendzian_2006_II die Aufgabe „Stellen Sie die Ursachen der Französischen Revolution in einer Mindmap dar“. Die Darstellung der Ursachen kann auch zusammenfassend geschehen, wenn sie z. B. im Unterricht vorbereitet wurden. Dann stünde der Anforderungsbereich I im Vordergrund, Bezüge auf die Anforderungsbereiche II und III wären nicht zwangsläufig nötig. Es wäre insbesondere bei diesem Aufgabeformat zu prüfen, welche Rolle die Situierung der Aufgabe für die Zuordnung zu den Anforderungsbereichen spielt.

${ }^{539}$ Dabei verwenden die verschiedenen Lehrwerke eigene „Operatoren“ und diese in unterschiedlicher Häufigkeit. Die Zusammenführung der Auszählung zeigt bspw. auch, dass in den Lehrwerken mindestens 20 unterschiedliche weitere Verben als Operatoren verwendet werden. Die maximale Anzahl liegt bei 84 Verben. Definitionen oder Erklärungen gibt es in den Lehrwerken dazu i.d.R. nicht. Die Frequenzliste (Token-Auszählung) führt Droste/2011 (Geschichte und Geschehen) mit 981 Nicht-EPA-Operatoren an. Bahr 1/2006 (Horizonte) verwenden dagegen nur 37 eigene Operatoren. Auch hier zeigt sich wieder ein stark individueller Schulbuchstil.
} 


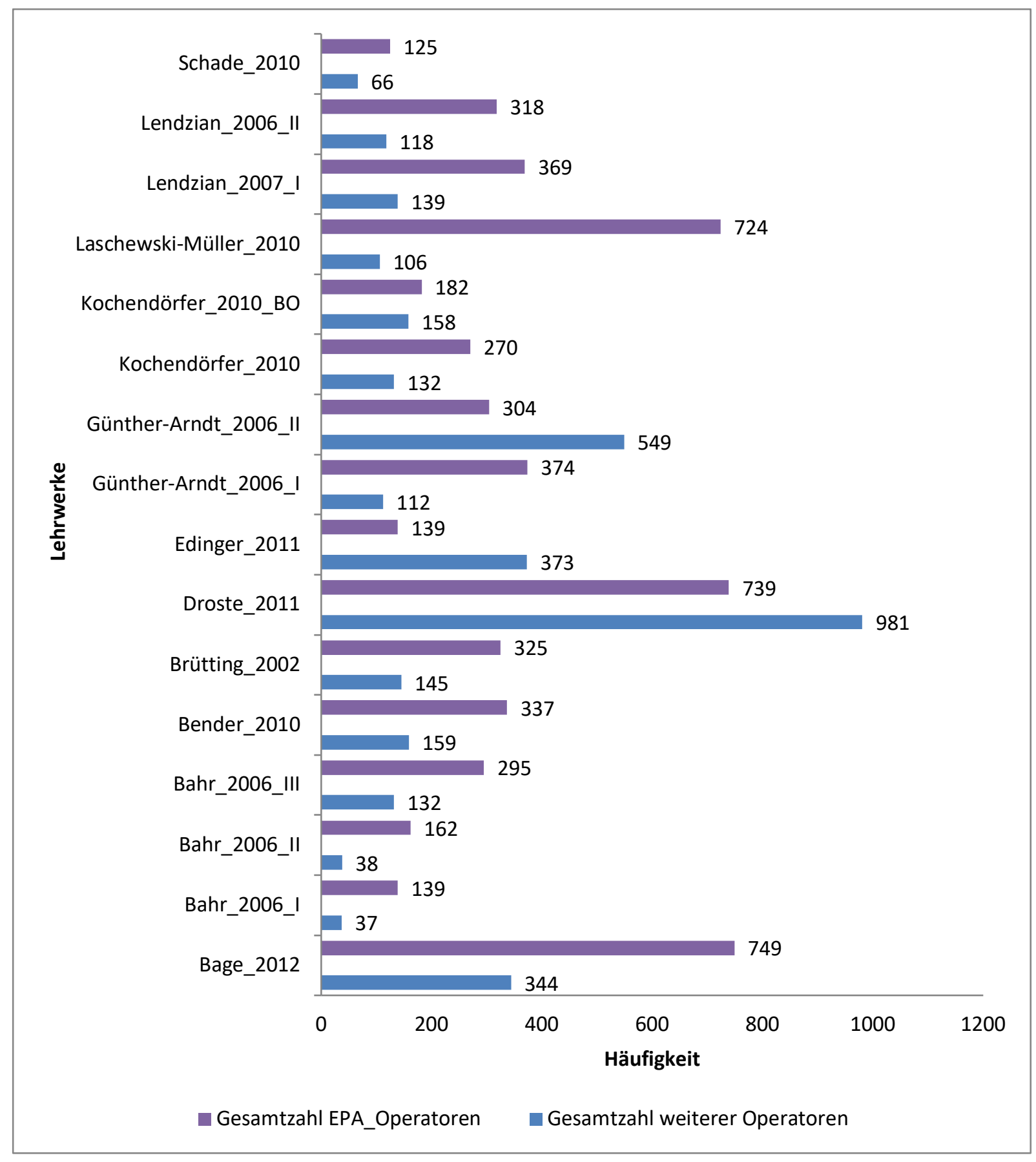

Abbildung 24: Verwendungshäufigkeit im Vergleich von Nicht-EPA-Operatoren und EPA-Operatoren (vor 2014)

Die Darstellung zeigt eine sehr heterogene Verwendungshäufigkeit von Nicht-EPA-Operatoren. Es ist zwar insgesamt festzustellen, dass in den meisten Lehrwerken die Verwendung der EPA-Operatoren überwiegt, aber immerhin in zwei Lehrwerken mehr Nicht-EPA-Operatoren genutzt werden als EPAOperatoren. Hier muss überlegt werden, was diese Vielzahl an Operatoren für die Lernenden bedeutet. Für das Vergleichen konnte festgestellt werden, dass es sich um ein historisches Werkzeug handelt. Schlüsselt man die Nicht-EPA-Operatoren auf, lässt sich feststellen, dass bestimmte Nicht-EPAOperatoren in mehreren der untersuchten Lehrwerke auftauchen. Auffällig ist bspw. das Vorkommen von Zusammenstellen, Erarbeiten und (sich) Informieren. Innerhalb der ersten drei Plätze der Rangliste 
tauchen das Erarbeiten und das (sich) Informieren elfmal auf den ersten drei Plätzen auf, das Zusammenstellen immerhin noch sechsmal. Hier wäre zu klären, warum diese Operatoren so häufig verwendet werden. Erweisen sie sich als wichtiges Hilfsmittel zur Anbahnung historischen Lernens, bliebe die Frage offen, warum sie dann nicht im EPA-Katalog auftauchen.

Diese Frage soll im Rahmen dieser Arbeit nicht vertieft werden. Die Sammlung dieser vielen verschiedenen Operatoren zeigt jedoch einmal mehr, wie bedeutsam methodisches Wissen in Kombination mit der Kenntnis des Zwecks der Operatoren für die Lernenden ist, da sie nur so zu einer sinnvollen Aufgabenlösung gelangen können.

\subsubsection{Einordnung des Operators Vergleichen und seiner Teilhandlungen}

An dieser Stelle soll ein vorläufiges Fazit gezogen und eine erste Antwort auf die Frage gegeben werden, welchen Aussagewert die vorgestellten Daten und Ergebnisse für die zu untersuchende Vergleichsaufgabe besitzen.

Es konnte bereits festgestellt werden, dass das Vergleichen in den Lehrwerken vor 2014 einen häufig gewählten Operator, für den Anforderungsbereich III sogar den am häufigsten gewählten Operator darstellt.

Wie wird in den Lehrwerken mit den für das Vergleichen herausgearbeiteten Teiloperatoren umgegangen: Wie häufig erscheinen diese Strukturen als Aufgaben in den Schulbüchern? Kann davon ausgegangen werden, dass diese Operatoren geübt sind? Ihre Verwendung soll nachfolgend in einer Übersicht zusammengefasst werden. Dabei stellt die Übersicht die Häufigkeiten der für das Vergleichen als wesentlich angenommenen Operatoren im Zusammenhang dar. Es wird nicht zwischen den verschiedenen Materialgrundlagen (Bezug auf Quelle oder Darstellungstext) differenziert, um die Lesbarkeit der Grafik zu erhalten. 


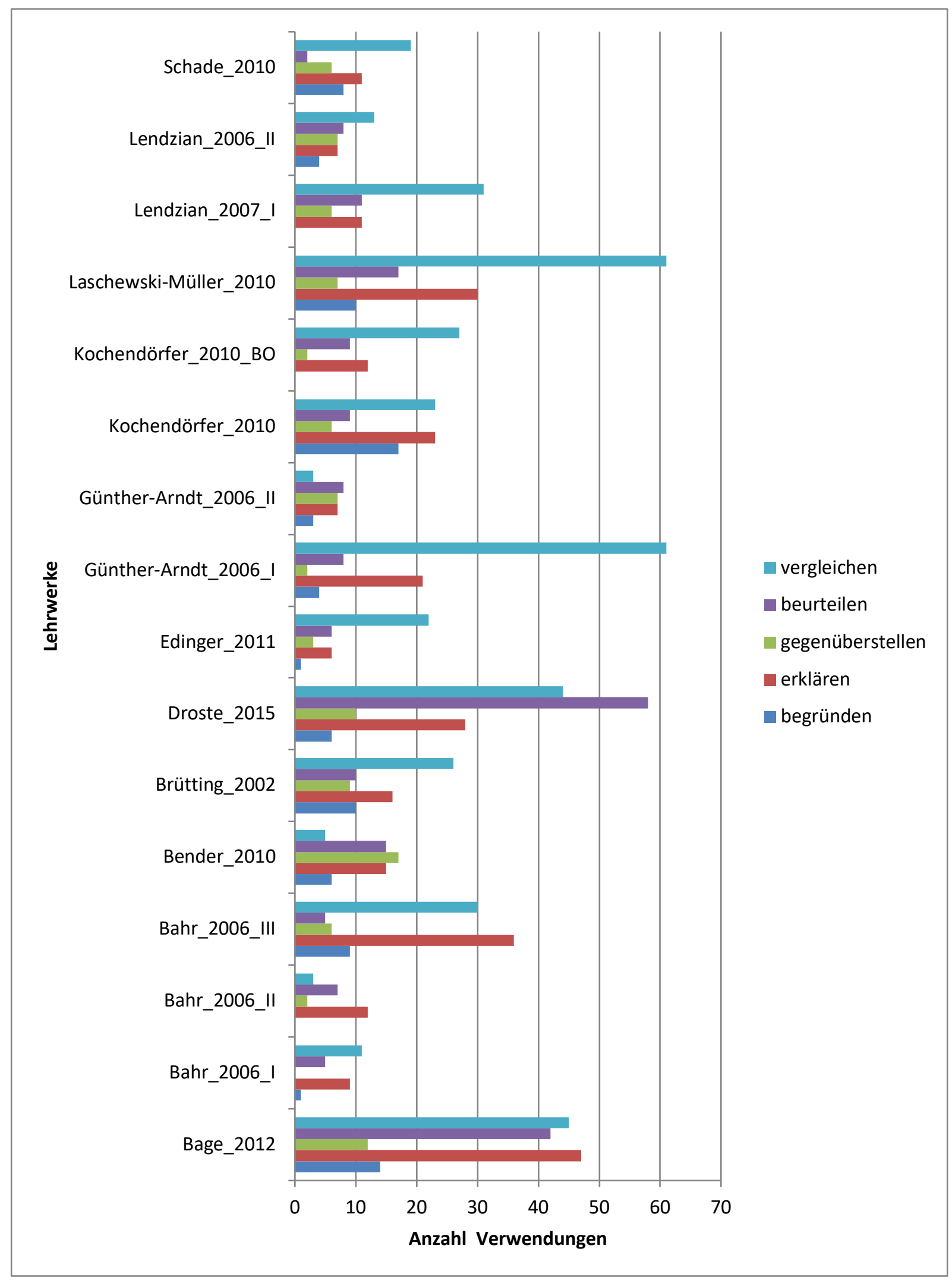

Abbildung 25: Der Operator 'Vergleichen' und seine Teiloperationen: Frequenzen in den Lehrwerken vor 2014

Die Darstellung zeigt zunächst die Häufigkeit des Aufgabentyps Vergleichen. Insgesamt stellt das Vergleichen einen häufig vorkommenden Aufgabentyp dar. Zwar lassen sich Schwankungen zwischen verschiedenen Bänden einer Lehrwerkreihe feststellen, in mindestens einem Band wird das Vergleichen 
jedoch in einer Frequenz von mind. 30 Aufgaben berücksichtigt, so dass angenommen werden kann, dass Lernenden ausreichend Gelegenheit geboten wird, mit dem Operator umzugehen. Einzig in Bender (2010) gibt es nur 5 Aufgaben, die das Vergleichen berücksichtigen, und damit die niedrigste Verwendungshäufigkeit.

Die Grafik zeigt weiterhin, dass das Beurteilen in allen Lehrwerken vorkommt. Allerdings sind hier sehr unterschiedliche Frequenzen festzustellen, die nicht wie beim Vergleichen durch die unterschiedliche Verwendungshäufigkeit in verschiedenen Bänden einer Reihe erklärt werden können. Gerade dieses Ergebnis ist erstaunlich, stellt die Urteilsbildung doch eine wesentliche historische Kompetenz dar. Die Frequenzanalyse unterscheidet nicht in Beurteilen, das auf ein Sachurteil abzielt, oder Beurteilen, das in einem Werturteil endet (streng genommen durch den Operator bewerten abgebildet). Gerade deshalb sind die Frequenzen in manchen Lehrwerken als zu niedrig einzustufen, als dass Lernende sich ausreichend mit diesen elementaren Operationen auseinandersetzen können. Wenn in Schade (2010) das Beurteilen nur zweimal vorkommt, ist dieser Wert deutlich zu niedrig.

Das Gegenüberstellen als unverzichtbare Teiloperation des Vergleichens kommt, wie die Darstellung zeigt, mit einer Ausnahme in allen Lehrwerk vor. Es zeigt sich jedoch insgesamt, dass das komplexere Vergleichen deutlich häufiger eingefordert wird als das Gegenüberstellen. Da es sich bei dem Lehrwerk ohne den Operator Gegenüberstellen um Band 1 der Horizonte-Reihe handelt, bleibt es kein ungeübter Operator, sondern es taucht in den nachfolgenden Bänden, wenngleich in relativ niedriger Frequenz, wieder auf.

Das Erklären stellt einen durchgängig und in den meisten Lehrwerken auch häufig vorkommenden Aufgabentyp dar ${ }^{540}$, so dass Lernende für das Vergleichen hierauf zurückgreifen können müssten, wenn ihnen klar ist, dass er hierfür bedeutsam ist.

Es fällt auf, dass das Begründen als Teiloperation für das Beurteilen, das explizit für das Vergleichen durch den EPA-Standard eingefordert wird, in drei Lehrwerken ganz fehlt. In zwei Lehrwerken handelt es sich um Teilbände (Lendzian, 2006: Zeiten und Menschen Bd. 1 und Bahr, 2006: Horizonte II), so dass davon ausgegangen werden kann, dass der Operator in den Folgebänden geübt werden kann. Anders ist dies in Geschichte und Geschehen für die berufliche Oberstufe, da es sich um einen Gesamtband handelt. In fast allen untersuchten Lehrwerken gibt es weniger Aufgaben zum Begründen als zum Beurteilen, so dass davon ausgegangen werden kann, dass Lernende das Begründen durch die Operation des Beurteilens kennenlernen und anwenden.

\subsubsection{Ergebnisse der ab 2014 erschienenen Lehrwerke}

Für die Untersuchung der ab 2014 erschienen Lehrwerke wurden 10 unterschiedliche Lehrwerke ausgewertet. Dabei handelt es sich um 5 Reihentitel, die alle in Einführungs- (EP) und Qualifikationsphase

\footnotetext{
${ }^{540}$ An dieser Stelle bleibt die Schwierigkeit der Differenzierung von Erklären und Erläutern in den Lehrwerken unberücksichtigt.
} 
(QP) untergliedert sind. Diese entstammen der aktuellen Liste der in Nordrhein-Westfalen zugelassenen Lehrwerke. ${ }^{541}$

\subsubsection{Die Nutzung von Aufgaben in den Lehrwerken im statistischen Überblick}

In allen Lehrwerken werden Aufgaben hauptsächlich durch Operatoren formuliert, in vier der untersuchten Lehrwerke sogar zu 100\%. Der geringste Anteil an Operatoren findet sich im Lehrwerk „Zeiten und Menschen, Qualifikationsphase“. Er liegt bei 89,3\%.

Wie schon in der Auswertung zuvor werden Aufgaben, die weder über Operatoren noch über W-Fragen formuliert werden, nicht berücksichtigt, da sie so gut wie nicht vorkommen (rechnerisch max. einstelliger Bereich).

W-Fragen werden häufig nur noch für die Formulierung übergeordneter Leitfragen benutzt. Da häufig von der Annahme ausgegangen wird, dass W-Fragen stark auf Inhaltswissen rekurrieren, ist auffällig, dass ausgerechnet diese Aufgabenform für Leitfragen ausgewählt wird. W-Fragen als Leitfragen wurden nicht gezählt, da Leitfragen den Lernaufgaben übergeordnet sind.

Die nachfolgende Grafik stellt das Verhältnis von Operatoren und W-Fragen dar. Auf der X-Achse befinden sich wieder die einzelnen Lehrwerke (durch Zahlen gekennzeichnet) ${ }^{542}$, auf der Y-Achse die Verwendungsfrequenzen. Anders als in der Auswertung der vor 2014 erschienenen Lehrwerke werden nun z.T. Frequenzen um bzw. über 1000 Aufgaben erreicht.

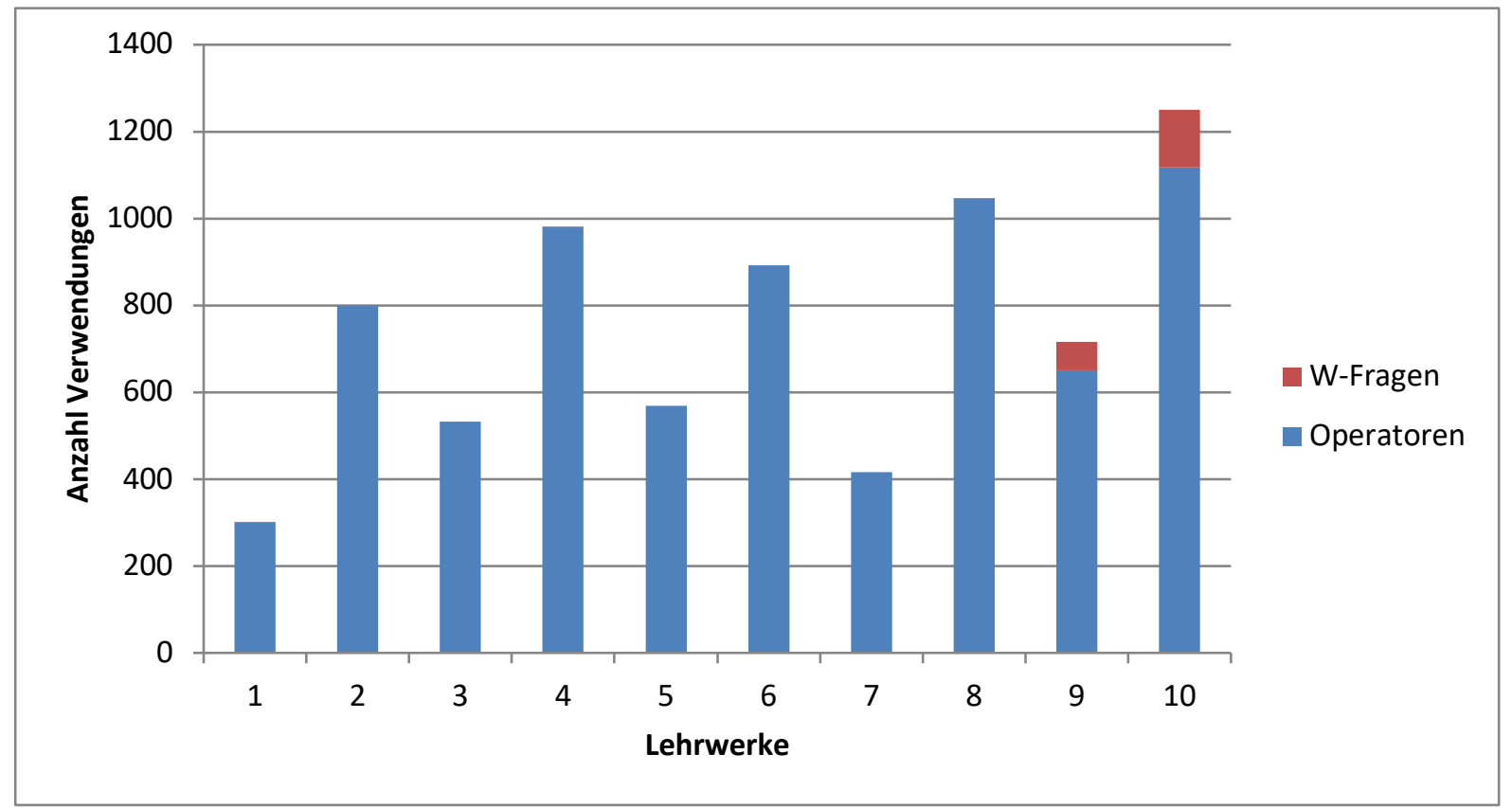

Abbildung 26: Verhältnis von Aufgabenformulierungen durch W-Fragen und Operatoren (ab 2014)

\footnotetext{
541 Letzter Zugriff: 02.07.2016.

542 Hinter den Zahlen verbergen sich die in Anhang 4.4 aufgeschlüsselten Lehrwerke.
} 
Die Vermutung, dass Kompetenzorientierung über Aufgabenformulierungen mit Operatoren erreicht werden soll, wird durch die Auswertung der Lehrwerke ab 2014 verstärkt.

Auch die Anzahl der Lernaufgaben hat sich insgesamt erhöht (Mittelwert: 1,96, Median: 1,88): Lag die Anzahl der Aufgaben in Lehrwerken vor 2014 bei ca. einer Aufgabe pro Seite, sind es nun schon fast zwei Aufgaben pro Seite im Durchschnitt.

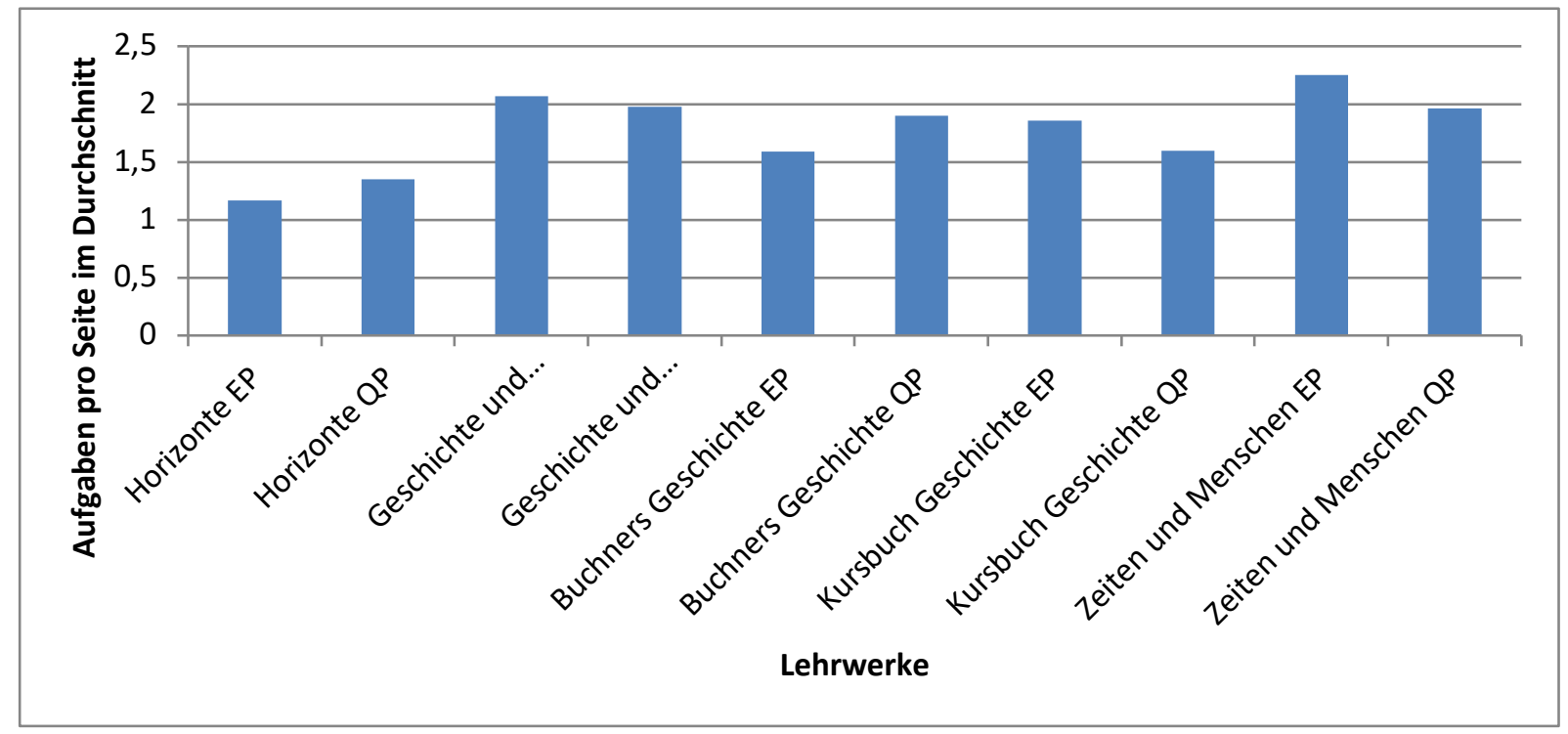

Abbildung 27: Aufgaben pro Seite (ab 2014)

Insgesamt ist festzustellen, dass die Frequenzen der einzelnen Lehrwerke viel enger beieinanderliegen als in den Lehrwerken vor 2014. Das Bild ist in Bezug auf den Gebrauch von Operatoren und die Anzahl von Aufgaben pro Seite viel homogener. Es gibt keinen (so deutlichen) Ausreißer wie im ersten Teil der Analyse. Für die Lernenden bedeutet dies, dass innen mehr Aufgaben bereitgestellt werden, die in hohem Maße über handlungsinitiierende Verben formuliert werden. Allerdings sagen die Zahlen noch nichts darüber aus, in welchem Maße Aufgaben zu Darstellungstexten bereitgestellt werden. Dies kann die folgende Grafik veranschaulichen: 


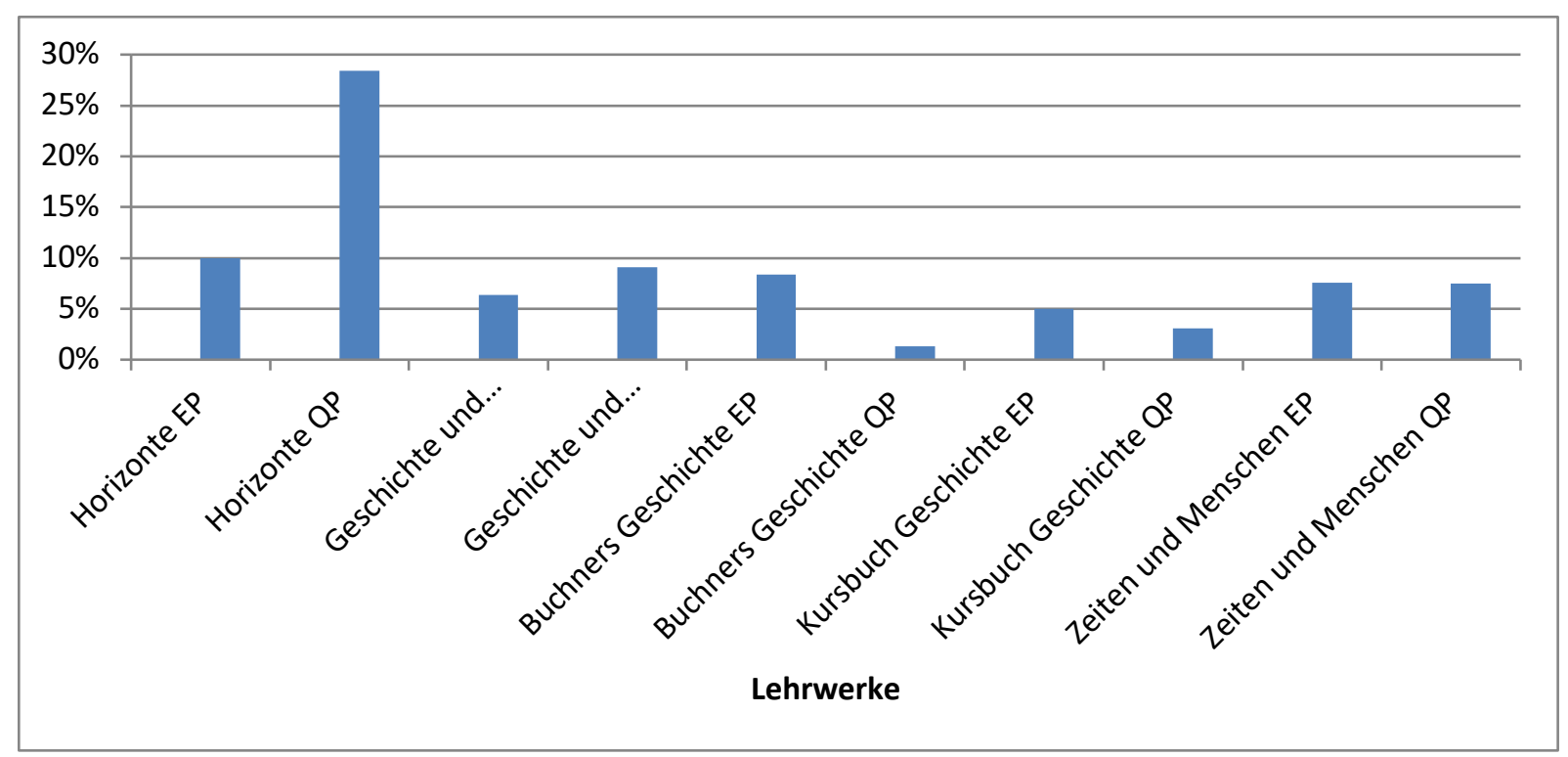

Abbildung 28: Anteil der Aufgaben, die sich auf Darstellungstexte beziehen (ab 2014)

Es fällt auf, dass der Anteil der Aufgaben, die sich eindeutig einem Darstellungstest (und ggf. weiterem Material) zuordnen lassen, im Vergleich zu den Lehrwerken vor 2014 abgenommen hat. Dies gilt auch für die neuen Kursbücher Geschichte (EP und QP) als Nachfolger des Kursbuch Geschichte von 2010, aus dem die untersuchte Vergleichsaufgabe entnommen wurde. Allerdings kann dieser Wert nicht weiter gedeutet werden, da in den Lehrwerken z. T. Angaben fehlen, auf welche Materialgrundlage sich die Aufgaben beziehen (dies gilt z. T. auch für das Kursbuch Geschichte EP und QP), so dass die oben angegebenen Werte aufgrund der fehlenden Angabe möglicherweise einen falschen Eindruck hervorrufen. Auf der anderen Seite sind diese Werte wiederum bedeutsam, da die Lernenden durch die Aufgaben aufgefordert sind, die zutreffende Materialgrundlage auszuwählen. Ob dies ohne eindeutige Angabe im Lehrbuch gelingt, kann an dieser Stelle nicht geklärt werden, ist aber eine wesentliche Fragestellung für den allgemeinen Umgang mit Lernaufgaben in den neueren Geschichts- und Gesellschaftslehrelehrwerken.

\subsubsection{Die Häufigkeit der Verwendung von EPA-Operatoren}

Auch in diesem Teil soll wieder eine Analyse der am häufigsten verwendeten Operatoren entlang der ausgewiesenen Anforderungsbereiche und quer über alle ausgezählten Lehrwerke hinweg vorgenommen werden. Die Grafik für die ab 2014 eingeführten Lehrwerke bietet das folgende Bild: 


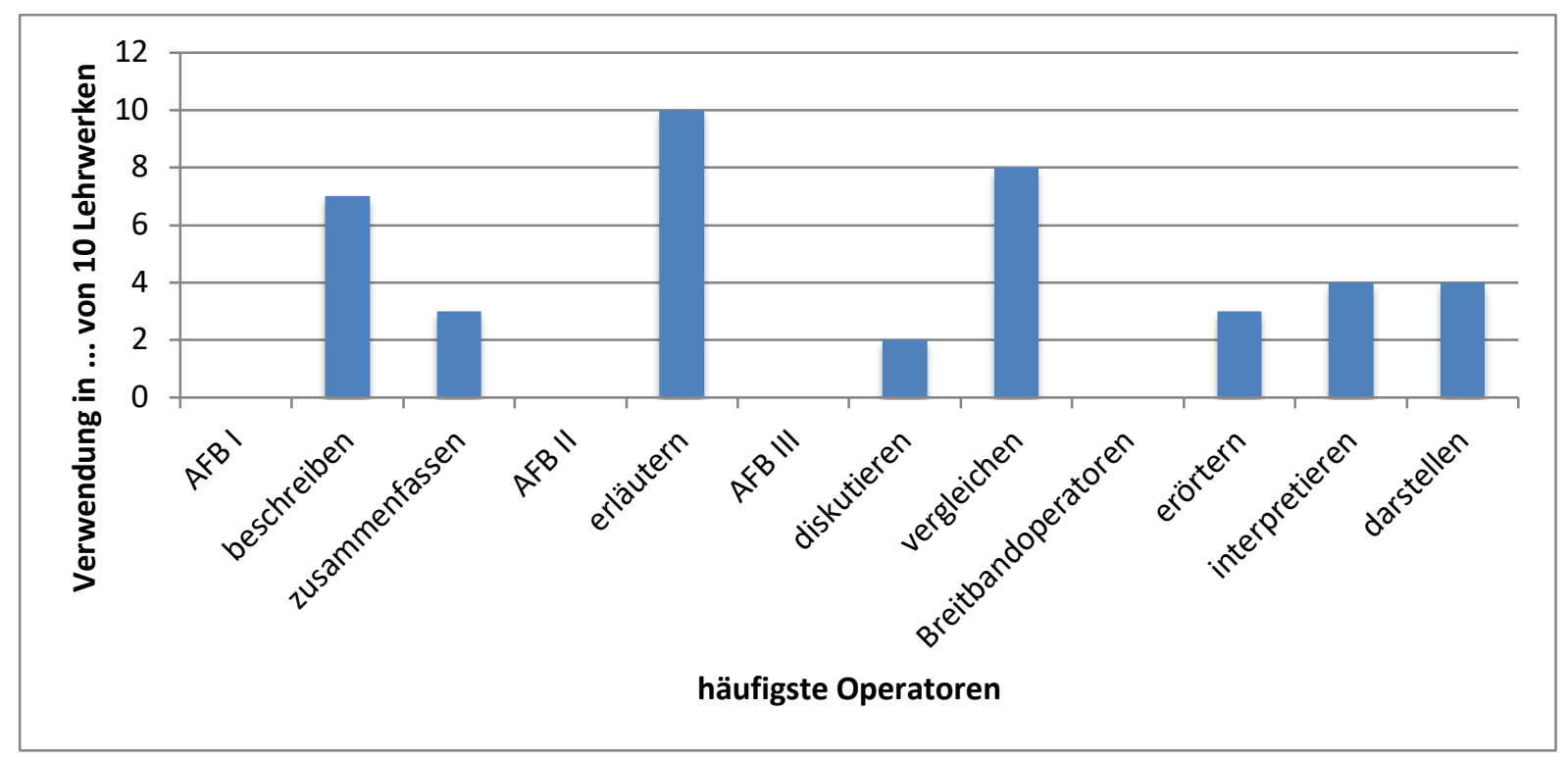

Abbildung 29: Darstellung der am häufigsten verwendeten Operatoren (ab 2014)

In 7 von 10 untersuchten Lehrwerken wird im Anforderungsbereich I der Operator Beschreiben am häufigsten genutzt, in drei Lehrwerken immerhin noch das Zusammenfassen. In den Lehrwerken vor 2014 war das Bild noch eindeutiger, dennoch ist auch in dieser zweiten Auswertung das Beschreiben immer noch der dominante Operator, wenngleich das Zusammenfassen eine deutlich größere Rolle spielt, da es nun in drei Lehrwerken am häufigsten benutzt wird.

Im Anforderungsbereich II ist wie auch schon in der Auswertung der Lehrwerke vor 2014 das Erläutern der in allen Lehrwerken am häufigsten gebrauchte Operator.

Im Anforderungsbereich III ist das Bild eindeutiger als in der Auswertung der Lehrwerke vor 2014, da in 8 Fällen das Vergleichen den am häufigsten gebrauchten Operator darstellt. Danach folgt in zwei Fällen das Diskutieren. Das Beurteilen und Prüfen spielen im Vergleich zur ersten Auswertung in dieser zweiten Frequenzanalyse keine Rolle.

Bei den Breitbandoperatoren kann man wie auch schon in der ersten Auszählung feststellen, dass alle drei in ähnlichem Maße in den Lehrwerken als häufigster Operator auftauchen: das Interpretieren und Darstellen jeweils viermal, das Erörtern dreimal (in einem Schulbuch werden das Darstellen und Interpretieren gleich häufig verwendet).

Insgesamt kann man also erkennen, dass die in der ersten Auszählung besonders häufig genutzten Operatoren auch in dieser zweiten Auszählung wieder sehr häufig vorkommen. In einzelnen Fällen, wie etwa im Anforderungsbereich II, hat sich dieses Bild noch weiter verdichtet. Operatoren, die vorher noch nicht besonders häufig aufgetreten sind, tun dies auch nicht in der zweiten Zählung. Insgesamt hat die Umstellung auf die Kernlehrpläne in den Schulbüchern der neuesten Generation nicht zu einer Umstellung der Nutzung bestimmter Operatoren geführt.

Allerdings darf die Sortierung nach in Schulbüchern am häufigsten genutzten Operatoren nicht darüber hinwegtäuschen, dass die Frequenzzahlen häufig nicht sehr weit auseinanderliegen. Deshalb soll 
die folgende Grafik angefügt werden, die etwas über die absoluten Zahlen aussagt. Dargestellt werden die drei in absoluten Zahlen am häufigsten verwendeten Operatoren.

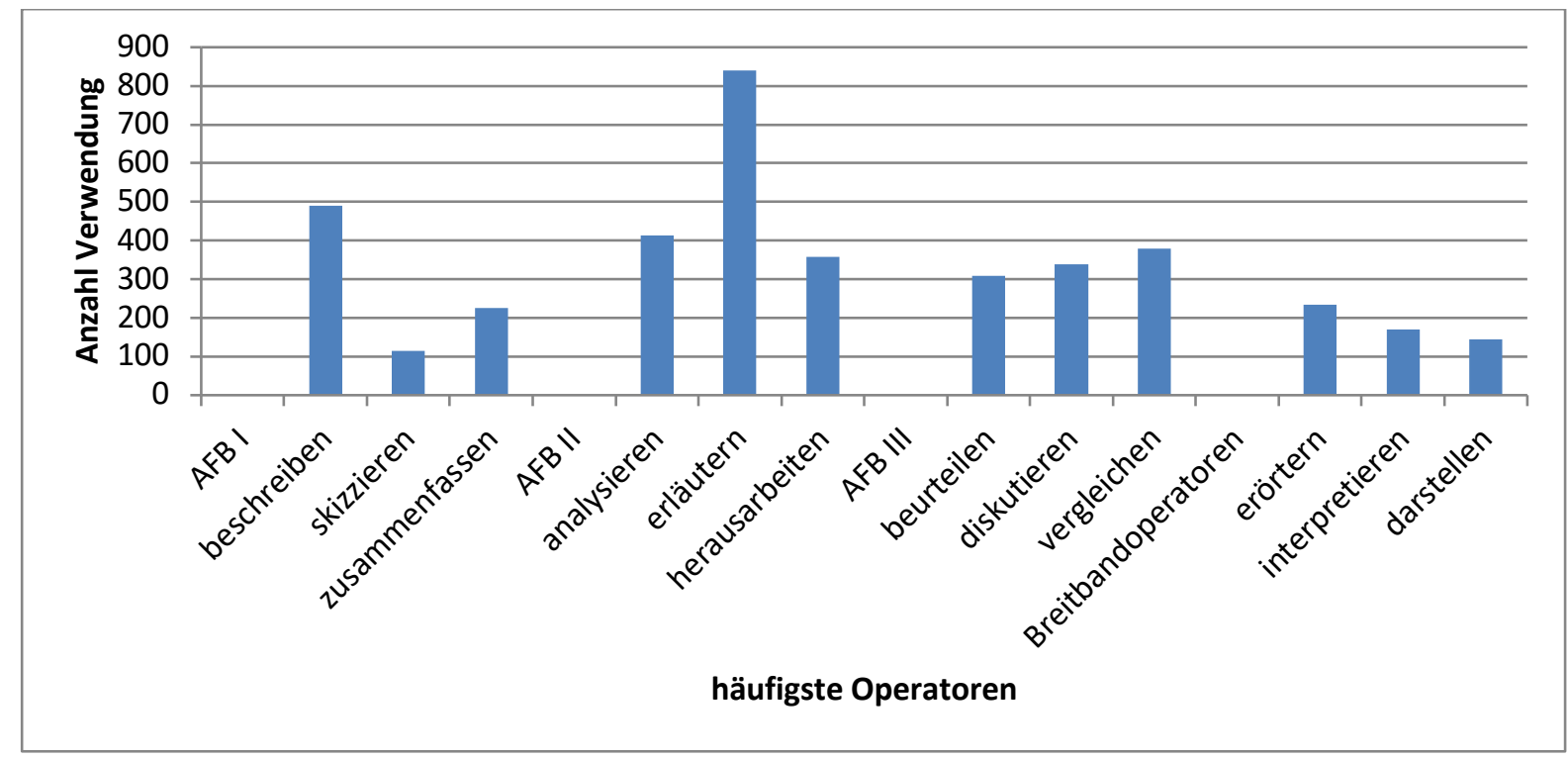

Abbildung 30: Die am häufigsten verwendeten Operatoren in absoluten Zahlen (ab 2014)

Es zeigt sich, dass bestimmte Operatoren, die in der vorigen Grafik nicht abgebildet wurden, sehr wohl eine größere Rolle spielen als die erste Grafik dies zeigen kann, wie etwa im Anforderungsbereich I das Skizzieren und Zusammenfassen und im Anforderungsbereich II das Analysieren und das Herausarbeiten. Besonders interessant ist diese Darstellung für den Anforderungsbereich III: Zwar wird das Vergleichen am häufigsten genutzt, jedoch dicht gefolgt vom Diskutieren und Beurteilen. Das sich in der Auswertung 1 abzeichnende Bild spiegelt sich in diesen Zahlen wider: je komplexer der Anforderungsbereich, desto heterogener die Nutzung der diesem Bereich zugeordneten Operatoren.

Insgesamt kann aber auch in den Lehrwerken ab 2014 davon ausgegangen werden, dass das Vergleichen eine wichtige Rolle spielt. Weiterhin zeigt die Auszählung, dass das Beurteilen als Teilhandlung des Vergleichens eine wichtige Rolle spielt, wenn es auch nicht am häufigsten genutzt wird.

Nachfolgend soll neben den häufig genutzten Operatoren wieder die Nutzung der übrigen Operatoren, die dem jeweiligen Anforderungsbereich zugeordnet sind, abgebildet und gefragt werden, welche Rolle die weiteren dem Vergleichen als Teilhandlungen dienenden Operatoren spielen. 


\section{Häufigkeiten im Anforderungsbereich I}

Nachfolgend wird wieder gezeigt, welche Operatoren des Anforderungsbereichs I in den ausgezählten Lehrwerken verwendet werden.

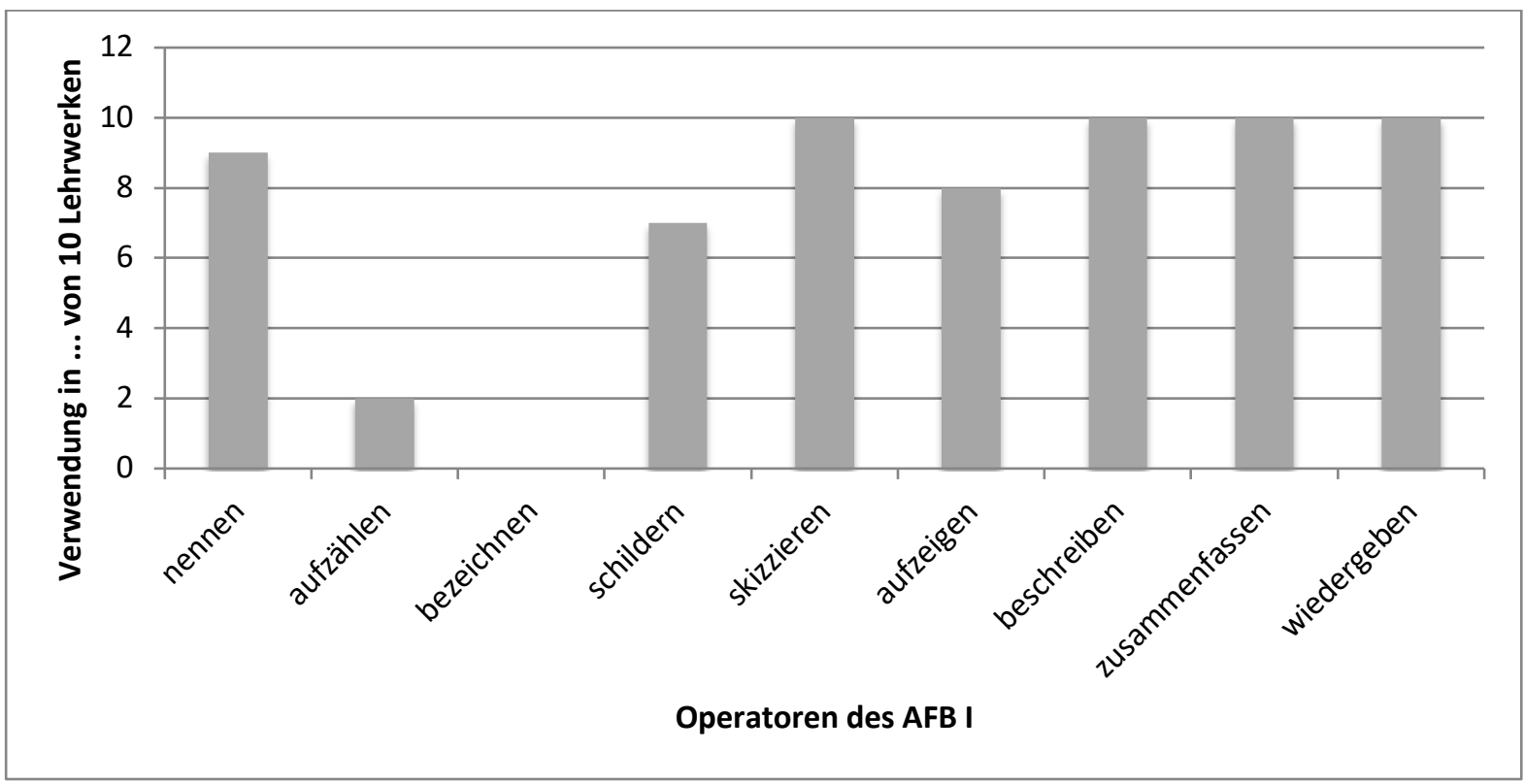

Abbildung 31: Verwendung von Operatoren im Anforderungsbereich I (ab 2014)

Die Darstellung zeigt, dass das Aufzählen nur in zwei Lehrwerken überhaupt vorkommt, das Bezeichnen wird in keinem Lehrwerk verwendet. Diese Ergebnisse passen zur Auswertung der Lehrwerke vor 2014, da diese beiden Operatoren auch in den 16 ausgezählten Lehrwerken kaum verwendet wurden. Hier wäre zu prüfen, welche Frequenzen sich bei einer Auszählung der in der Sekundarstufe I verwendeten Lehrwerke ergeben, da sich die Frage stellt, warum dies so ist bzw. ob diese Operatoren, wenn sie übereinstimmend so wenig genutzt werden, überhaupt sinnvoll für den EPA-Katalog sind.

Es fällt aber auch auf, dass viel mehr Operatoren in allen Lehrwerken genutzt werden. Waren das in der ersten Auszählung nur das Beschreiben und Zusammenfassen, sind es in der zweiten Auszählung außerdem noch das Skizzieren und Wiedergeben. Sicherlich ist das Ergebnis insofern nur bedingt vergleichbar, da es sich zwar um 10 Lehrwerke, jedoch nur um 5 Lehrwerkreihen handelt (jeweils ein Band zur Einführungs- und ein Band zur Qualifikationsphase). Da es aber die für NRW zugelassenen Lehrwerke für die gymnasiale Oberstufe sind, sind die Daten relevant.

Eine Analyse der absoluten Frequenzen, mit der die genannten Operatoren in den Lehrwerken auftauchen, ergibt das nachfolgende Bild: 


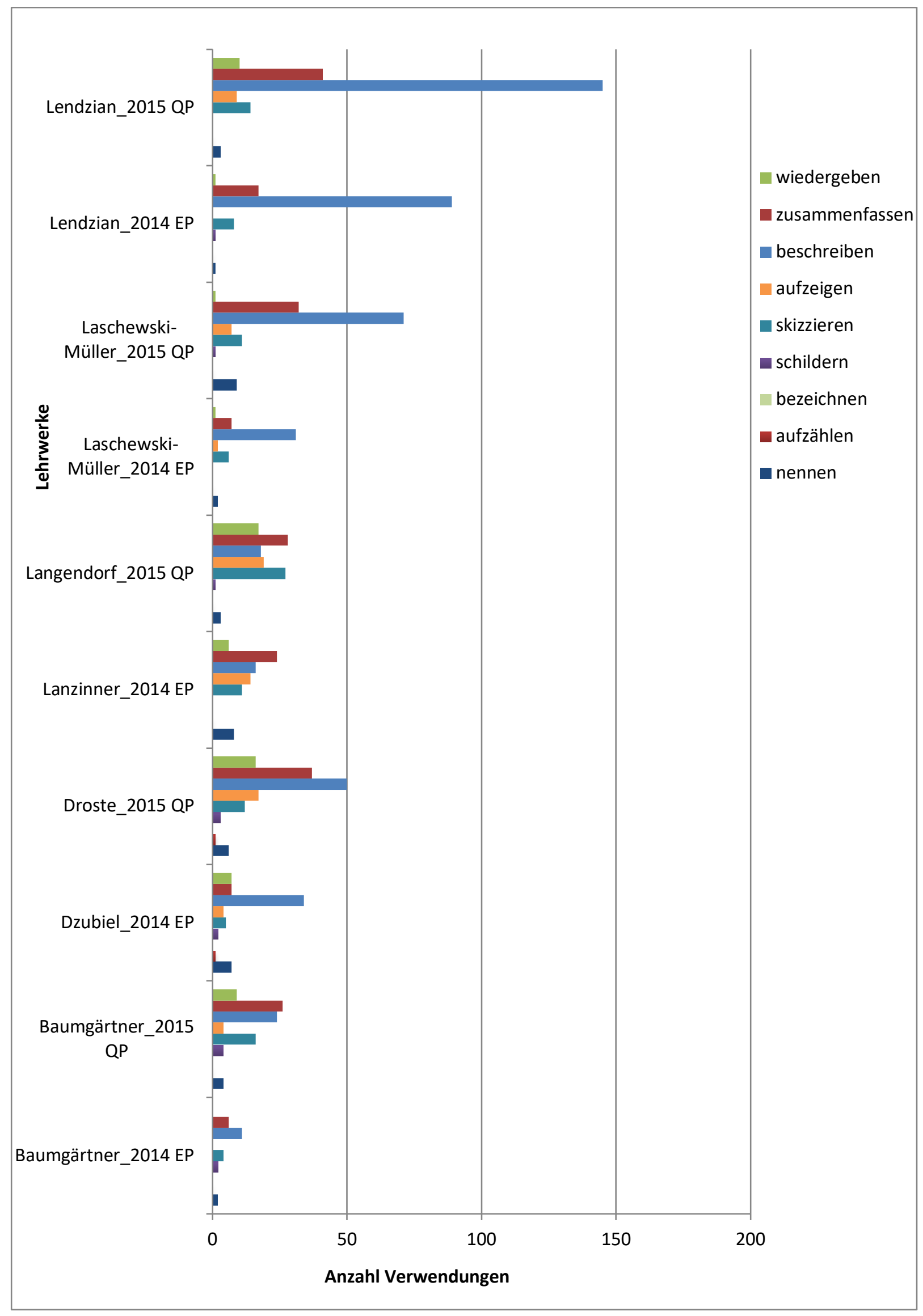

Abbildung 32: Häufigkeit der Verwendung von Operatoren im Anforderungsbereich I in den einzelnen Lehrwerken (ab 2014) 
Die Grafik bildet nicht ab, dass die Lehrwerke sehr unterschiedliche Umfänge haben, so ist Band 1 zur Einführungsphase in allen Fällen in etwa halb so umfangreich wie Band 2 zur Qualifikationsphase (Band 1 ist in der Grafik durch EP, Band 2 durch QP gekennzeichnet). Es zeigt sich jedoch sehr deutlich, dass insbesondere das Beschreiben in drei Lehrwerken sehr hohe Frequenzwerte erreicht, gefolgt vom Zusammenfassen. Wiedergeben und Skizzieren werden zwar auch in allen Lehrwerken der neuesten Generation verwendet, aber nicht unbedingt in höheren Frequenzen, hier verschiebt sich also das Bild zur ersten Auswertung. Es kann also auch für die Lehrwerke ab 2014 davon ausgegangen werden, dass den Lernenden wiedergebende Operationen vertraut sein sollten und diese für die Lösung der Vergleichsaufgabe genutzt werden können.

\section{Häufigkeiten im Anforderungsbereich II}

Eine Aufschlüsselung der einzelnen Operatoren für den Anforderungsbereich II ergibt das nachfolgende Bild:

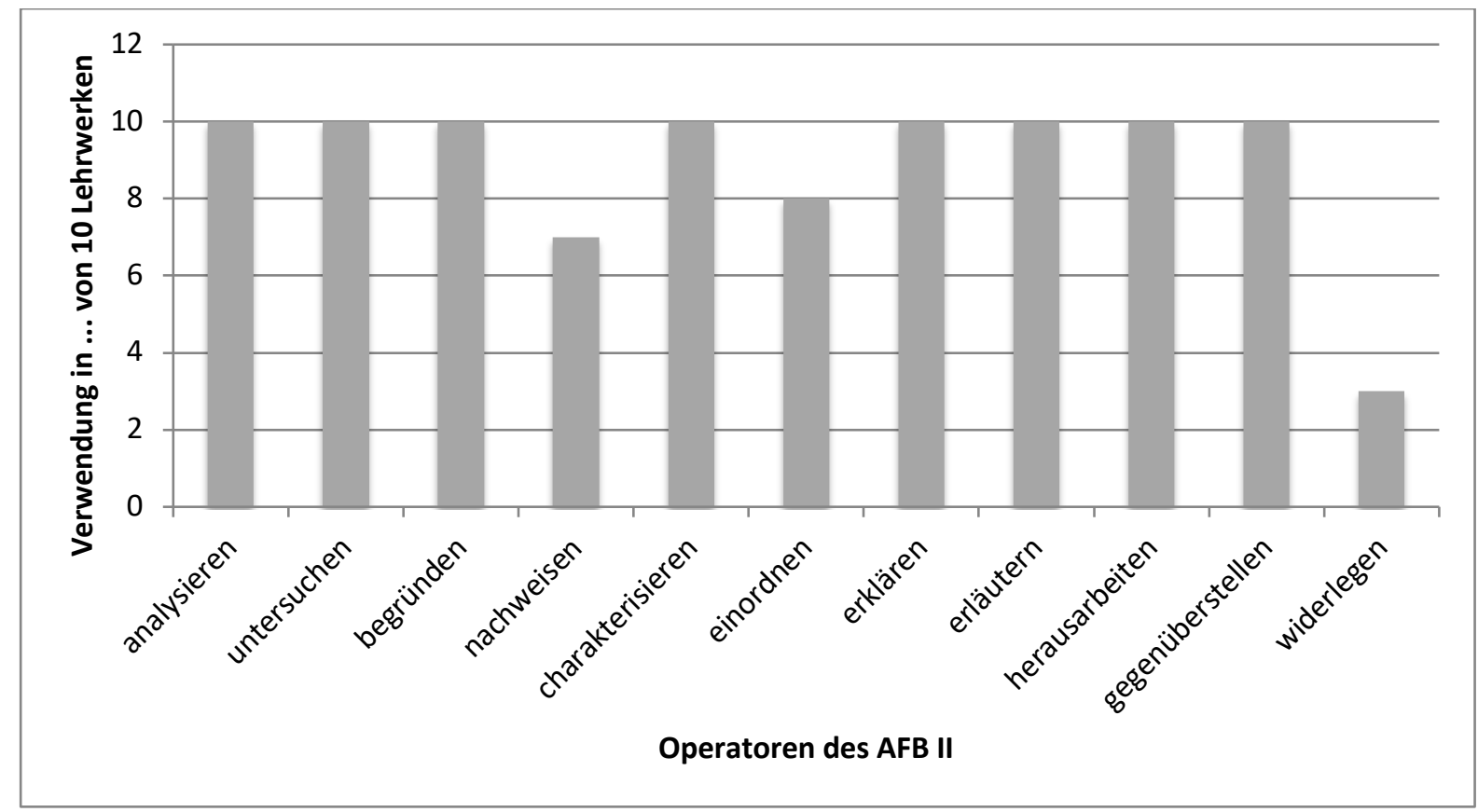

Abbildung 33: Verwendung von Operatoren im Anforderungsbereich II (ab 2014)

Im Anforderungsbereich II werden, wie auch schon in der Auswertung der Lehrwerke vor 2014 zu sehen war, fast alle Operatoren in den meisten Lehrwerken verwendet. Nur Einordnen, Nachweisen und Widerlegen bilden hier Ausnahmen. Dabei fällt das Widerlegen besonders auf, da es nur in drei Lehrwerken überhaupt verwendet wird. Die für das Vergleichen bedeutsamen Operatoren Erklären und Gegenüberstellen werden hingegen in allen Lehrwerken verwendet.

Die Aufschlüsselung der Frequenzen der Operatoren des Anforderungsbereichs II für die einzelnen Lehrwerke ergibt die nachstehende differenzierte Darstellung: 


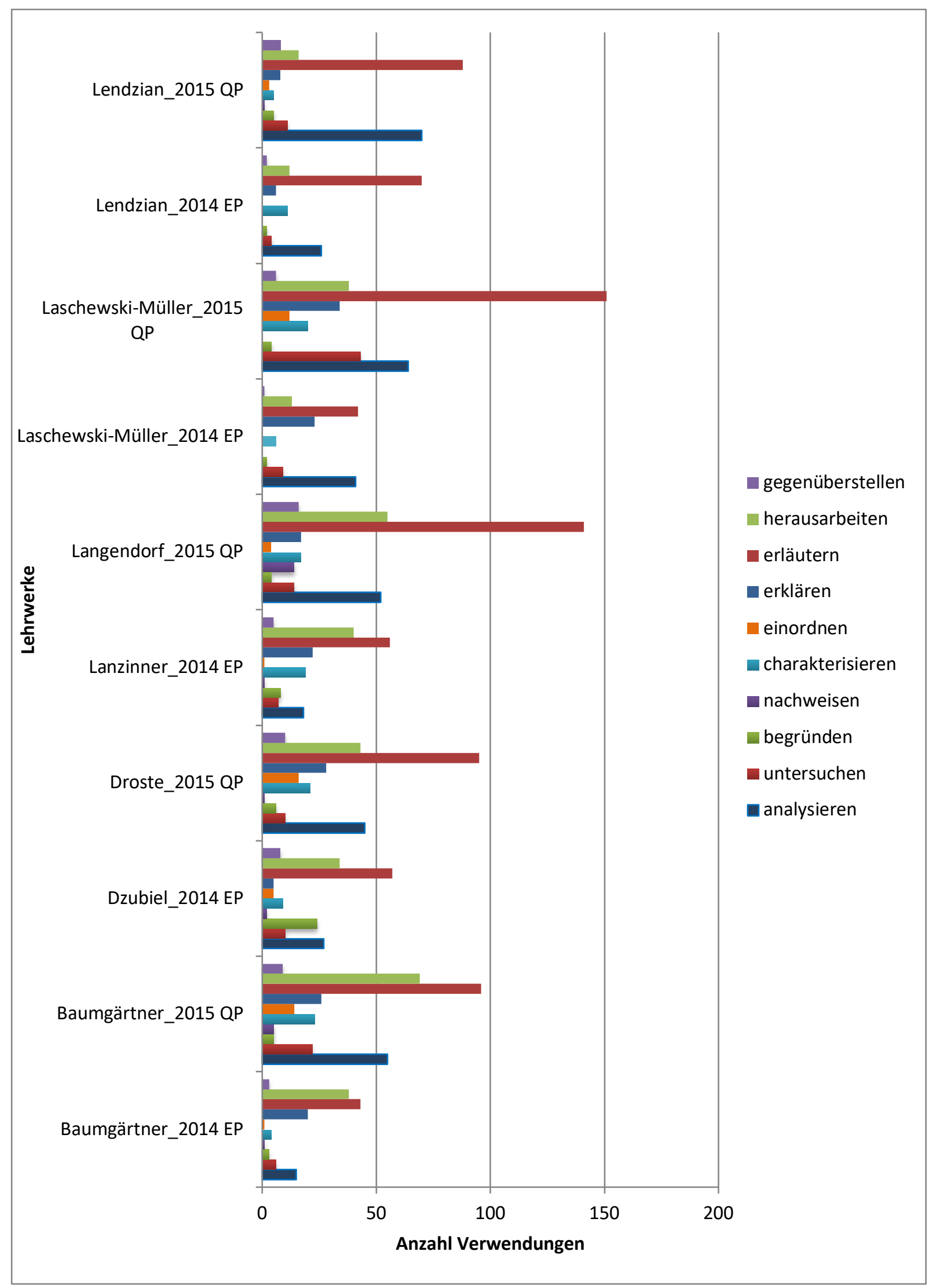

Abbildung 34: Häufigkeit der Verwendung von Operatoren im Anforderungsbereich II in den einzelnen Lehrwerken (ab 2014) 
Die Frequenzen in absoluten Zahlen zeigen, dass einige Operatoren besonders stark für Aufgabenstellungen benutzt werden. Das Erläutern zeigt im Verhältnis zu den weiteren in einem Lehrwerk verwendeten Operatoren durchweg hohe Frequenzwerte. Dies passt zur Überlegung im ersten Teil zur Abgrenzung von Erklären und Erläutern. Während das Erläutern häufig gebraucht wird, fallen die Frequenzwerte für das Erklären deutlich ab. Dabei ist es wesentlich für das Vergleichen. Es kann jedoch angenommen werden, dass Erklären und Erläutern weitgehend synonym gebraucht werden.

Interessant erscheint, dass insbesondere das Begründen sehr unterschiedliche, insgesamt jedoch eher niedrige Frequenzen erreicht.

Eine Aussage, dass es einen Zusammenhang zwischen der Nutzung einzelner Operatoren im ersten und zweiten Band gibt, ist nicht möglich.

\section{Häufigkeiten im Anforderungsbereich III}

Musste im ersten Auswertungsteil festgestellt werden, dass im Anforderungsbereich III nur zwei der EPA-Operatoren in allen Lehrwerken auftauchen, zeigt sich in diesem zweiten Auswertungsteil ein anderes Bild. Bis auf drei Operatoren werden nun alle Operatoren in allen 10 ausgewerteten Lehrwerken verwendet. Ausnahmen bilden lediglich das Bewerten und Überprüfen, die in 9 von 10 Lehrwerken verwendet werden, und das Prüfen, das immerhin in 8 Lehrwerken verwendet wird.

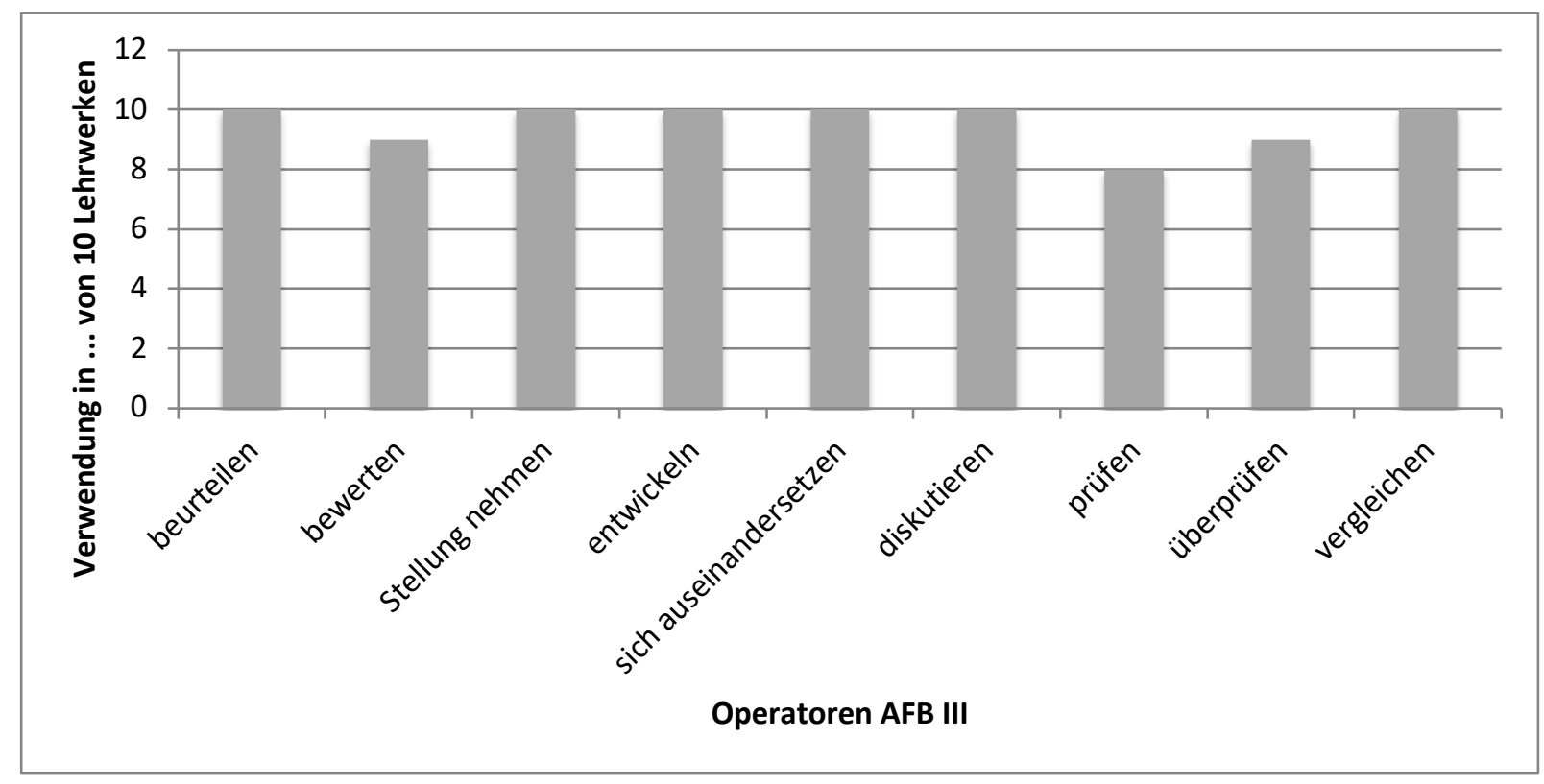

Abbildung 35: Verwendung von Operatoren im Anforderungsbereich III (ab 2014)

Die nachfolgende Aufschlüsselung zeigt wiederum die Unterschiede in der Verwendungshäufigkeit. Die Grafik verdeutlicht, dass zwar fast alle Operatoren in den meisten Lehrwerken verwendet werden, die Frequenzen aber insgesamt heterogen sind: 


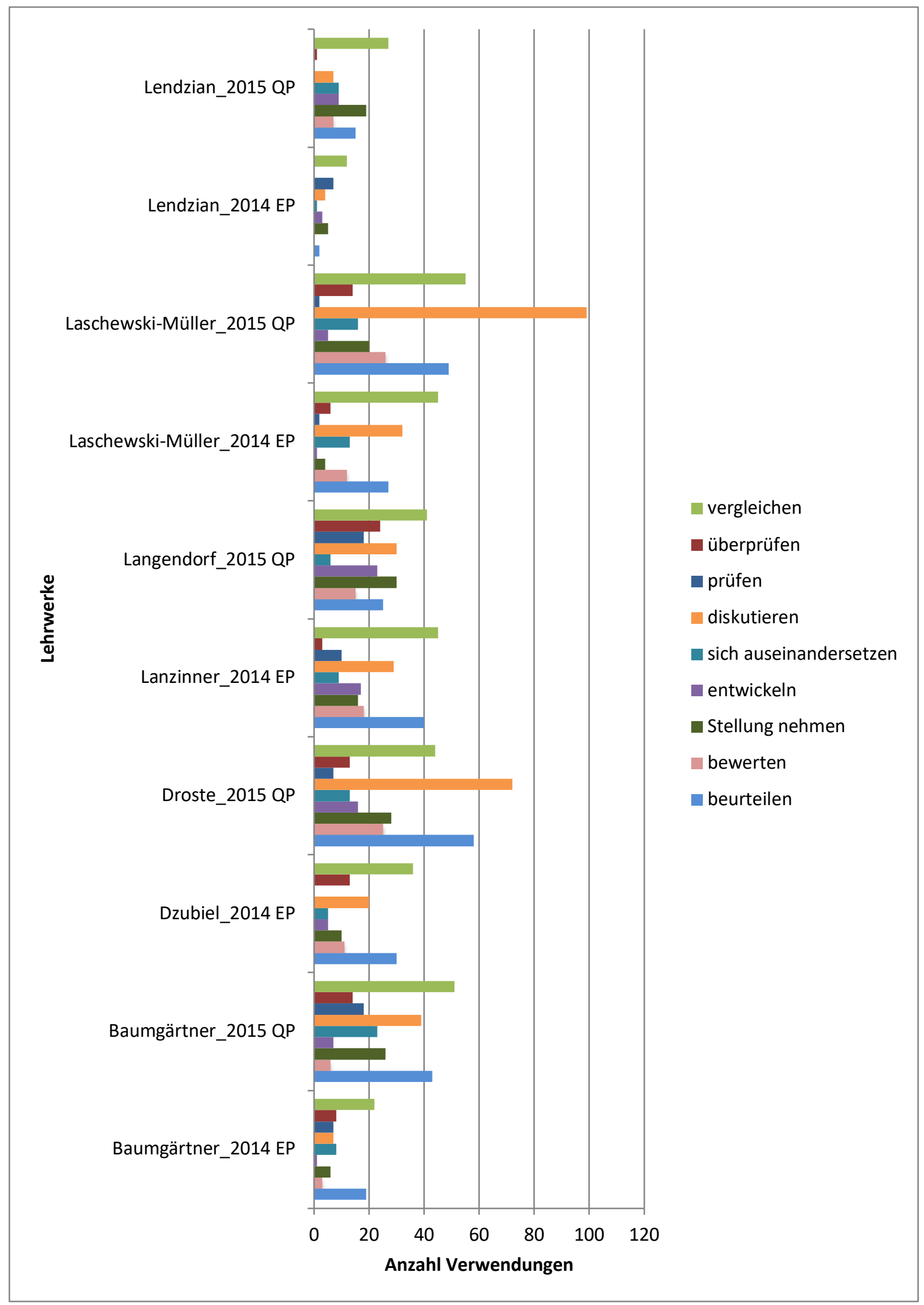

Abbildung 36: Häufigkeit der Verwendung der Operatoren im Anforderungsbereich III in den einzelnen Lehrwerken (ab 2014) 
Auch in der zweiten Auszählung erreicht das Diskutieren in einzelnen Lehrwerken sehr hohe Frequenzwerte, das Vergleichen wird ebenfalls in allen Lehrwerken häufig gebraucht. Dies passt zur ersten Auszählung. Weiterhin wird das Diskutieren nun auch in allen Lehrwerken verwendet, wobei man feststellen kann, dass die Verwendungsfrequenz dieses Operators recht heterogen ist.

Als Teilhandlungen des häufig vorkommenden Vergleichens kann für das Beurteilen festgestellt werden, dass es mit Ausnahme der Lehrwerkreihe „Zeiten und Menschen“ häufiger (mind. 19x) oder sogar häufig (bis zu 58x) verwendet wird. Das bedeutet, dass das Begründen über den Operator Beurteilen vertraut sein könnte. Voraussetzung dazu wäre allerdings die Explizitmachung der Begründestruktur für das Beurteilen.

\section{Nutzung der, Breitbandoperatoren}

Alle sogenannten Breitbandoperatoren werden auch in allen Lehrwerken verwendet. Das Bild ist damit deutlich homogener als im ersten Auszählungsteil, in dem es Lehrwerke gab, die einzelne der drei übergreifenden Operatoren nicht verwendeten.

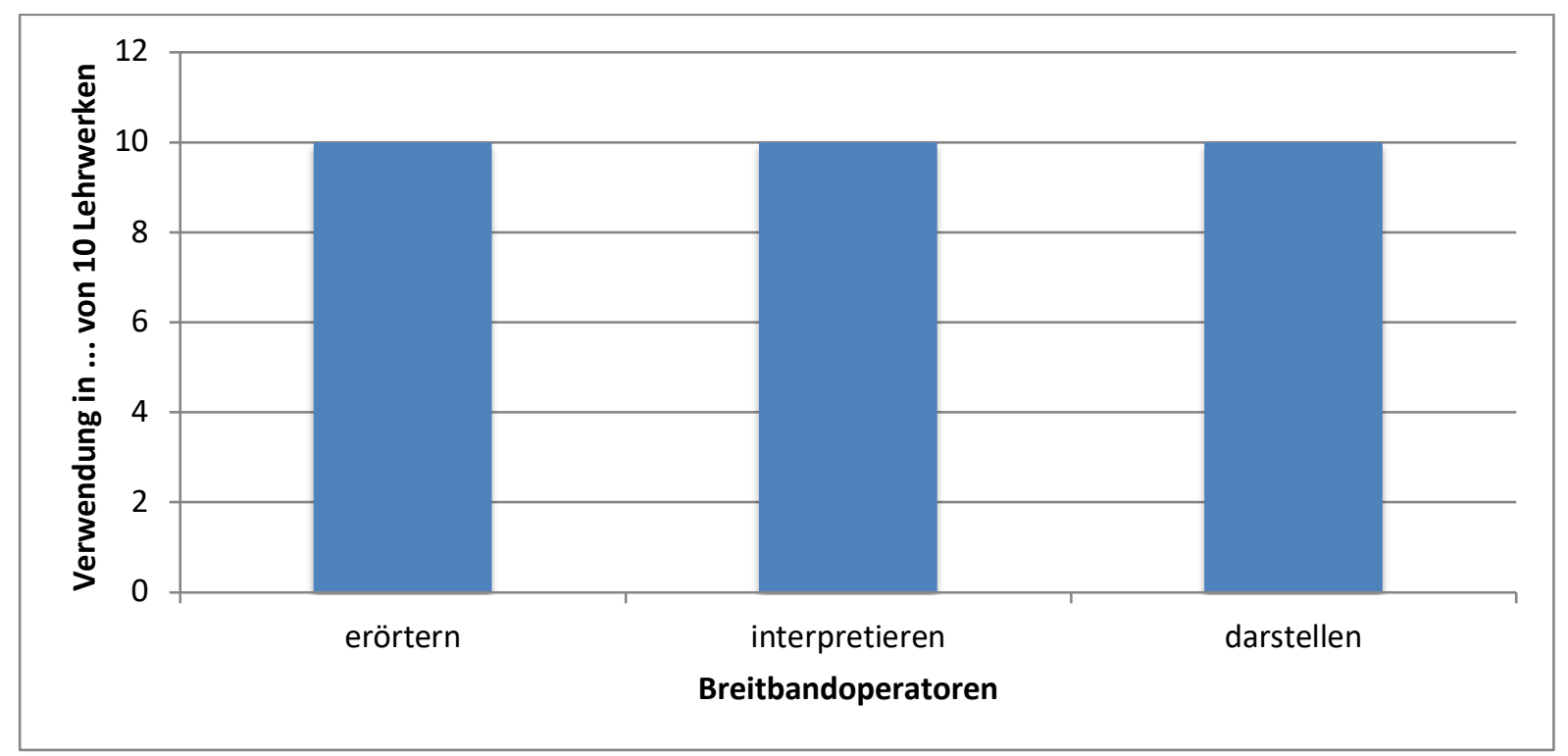

Abbildung 37: Verwendung der Breitbandoperatoren (ab 2014)

Allerdings liegt die Verwendungshäufigkeit dieser Operatoren zum Teil weit auseinander. Insbesondere bei Lanzinner (2014/EP), Langendorf (2015/QP) und Laschewski/Müller (2015/QP) erreicht das Erörtern hohe Frequenzwerte, bei Laschewski/Müller (2015/QP) zudem das Interpretieren, während nur bei Lendzian (2015/QP) das Darstellen eine hohe Frequenz erhält. Durch die kompetenzorientierten Lehrpläne sind den Lehrwerken bestimmte Inhaltsfelder vorgegeben. ${ }^{543}$ Die unterschiedliche Verwendungshäufigkeit lässt sich damit nicht durch die heterogene Themenwahl erklären. Auch für die ,Breitbandoperatoren' ist also wieder ein individueller Schulbuchstil festzustellen.

${ }^{543} \mathrm{Vgl}$. Ministerium für Schule und Weiterbildung des Landes Nordrhein-Westfalen (2014): 


\subsubsection{Verwendung von Operatoren über den EPA-Katalog hinaus}

Auch in diesem zweiten Teil der Frequenzanalyse sollen die Operatoren berücksichtigt werden, die nicht im EPA-Katalog aufgeführt sind. Dabei wird der Begriff Operator wieder in einem weiten Verwendungszusammenhang benutzt. Eine genaue Aufschlüsselung, ob es sich im echten Sinne um ,Operatoren' im Sinne von sprachlichen Handlungen handelt oder um andere Aufforderungen, steht noch aus, so dass wie im ersten Teil nicht differenziert wird.

Die Zahl der unterschiedlichen Operatorentypen für die Lehrwerke ab 2014 ist, wie in der Auswertung der Lehrwerke vor 2014, sehr heterogen:

In dieser zweiten Analyse gibt es ein einziges Lehrwerk, das ,nur' 17 weitere unterschiedliche Verben benutzt. Die Werte der übrigen Lehrwerke liegen zwischen 23 und 49 nicht in den EPA gelisteter Operatoren. Damit liegt die maximale Höchstzahl deutlich unter der max. Anzahl der ersten Auszählung (ein vor 2014 erschienenes Lehrwerk lag bei 84 weiteren unterschiedlichen handlungsinitiierenden Verben).

Im Folgenden werden wieder die Frequenzen in den einzelnen Lehrwerken aufgeschlüsselt und die Frequenzen der verwendeten Nicht-EPA-Operatoren im Vergleich zu den EPA-Operatoren dargestellt.

Kernlehrplan für die Sekundarstufe II Gymnasium/Gesamtschule in Nordrhein-Westfalen Geschichte. OnlineRessource: http://www.schulentwicklung.nrw.de/lehrplaene/upload/klp_SII/ge/KLP_GOSt_Geschichte.pdf (zuletzt eingesehen am 08.07.16). 


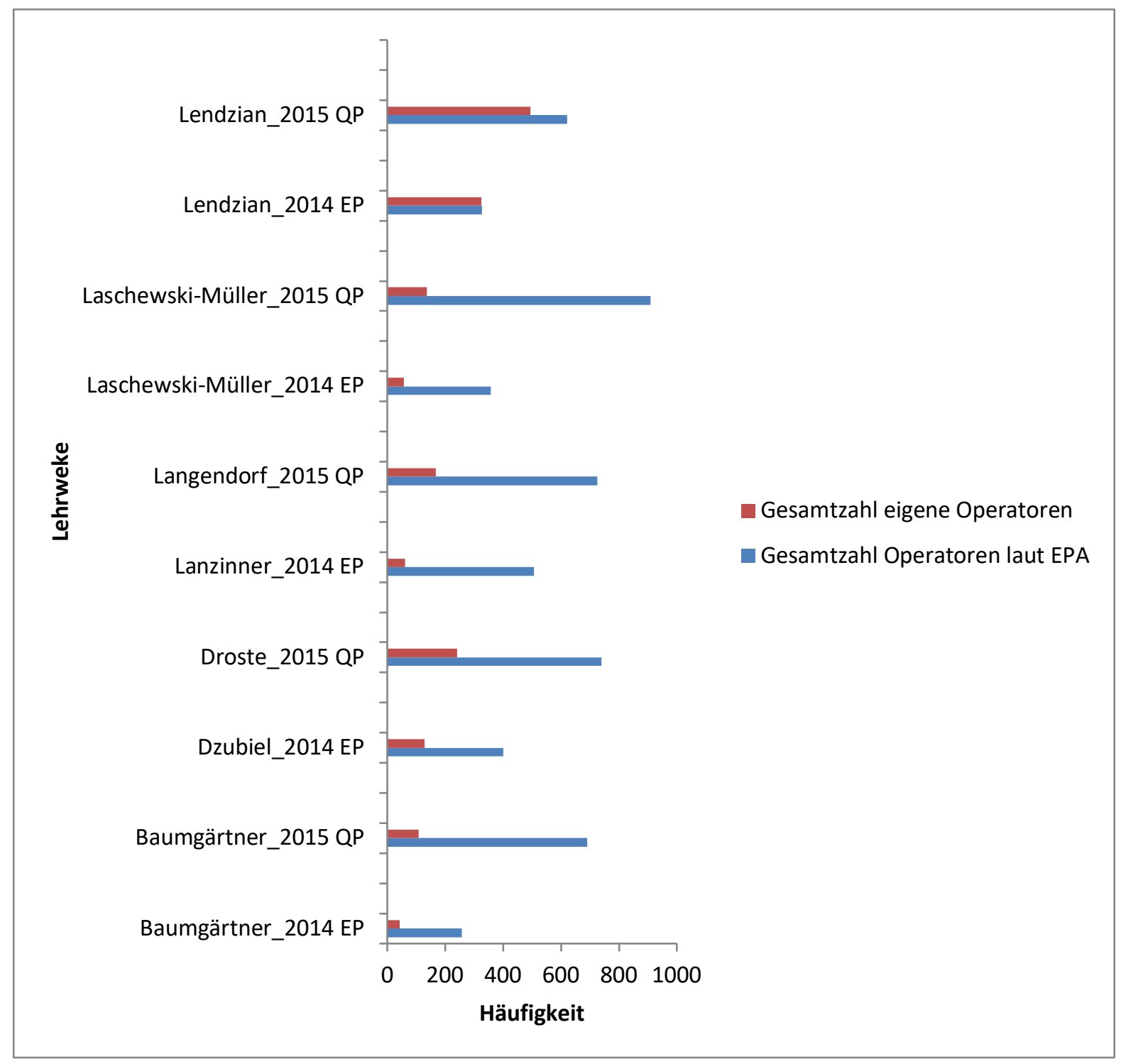

Abbildung 38: Verwendungshäufigkeit im Vergleich von Nicht-EPA-Operatoren und EPA-Operatoren (ab 2014)

Ebenso wie die sehr unterschiedlichen Zahlen der für Aufgabenstellungen benutzten Verben ist auch ihre Verwendungshäufigkeit divergent. Bei der Einordnung der Ergebnisse ist wiederum zu bedenken, dass die Lehrwerke der Einführungsphase deutlich geringer im Umfang sind als die der Qualifikationsphase. Legt man für den Vergleich nur die Lehrwerke der Qualifikationsphase zugrunde, kann man feststellen, dass die Verwendungshäufigkeiten, die zwischen 109 und 495 Verwendungen weiterer Verben liegen, stark schwanken und wieder auf einen stark individuellen Schulbuchstil hinweisen. Insgesamt lässt sich dennoch feststellen, dass im Gegensatz zu den vor 2014 erschienenen Lehrwerken in allen neuen Lehrwerken die Zahl der EPA-Operatoren überwiegt. Bis auf eine Ausnahme (in Lendzian_2014_EP ist die Gesamtzahl von EPA- und Nicht-EPA-Operatoren gleich hoch, in Lendzian_2014_QP liegt die Anzahl der EPA-Operatoren nur 11\% über der Zahl der Nicht-EPA-Operatoren) ist die Zahl der EPA-Operatoren deutlich höher als die der schulbucheigenen Aufgabenformulierungen. 


\subsubsection{Bedeutung der Daten in Bezug auf historische Vergleichsaufgaben}

Wie schon in den Lehrwerken vor 2014 ist das Vergleichen auch in den Lehrwerken ab 2014 ein wichtiger, da häufig vorkommender Operator. Vergleichen kommt in allen Lehrwerken als Lernaufgabe vor und stellt für den Anforderungsbereich III weiterhin den am häufigsten gewählten Operator dar.

Entsprechend des Vorgehens im ersten Teil werden im Folgenden wieder die für das Vergleichen relevanten und in den EPA genannten Teiloperatoren Gegenüberstellen, Beurteilen, Erklären, Begründen und Beurteilen überprüft. Die Bedeutung dieser Operatoren als Teiloperatoren für das Vergleichen sollen nachfolgend noch ausführlich diskutiert werden.

Im ersten Teil standen die folgenden Fragen im Mittelpunkt der Betrachtung: Wie häufig erscheinen die Teiloperatoren als Aufgaben in den Schulbüchern und kann davon ausgegangen werden, dass es genügend Lerngelegenheiten für den Umgang mit ihnen gibt? Sie sollen auch jetzt wieder zum Ausgangspunkt der Betrachtung werden.

Die folgende Grafik stellt, wie im ersten Teil, die Häufigkeiten der genannten Operatoren dar. Sie geht wiederum von den Gesamtnennungen aus. 


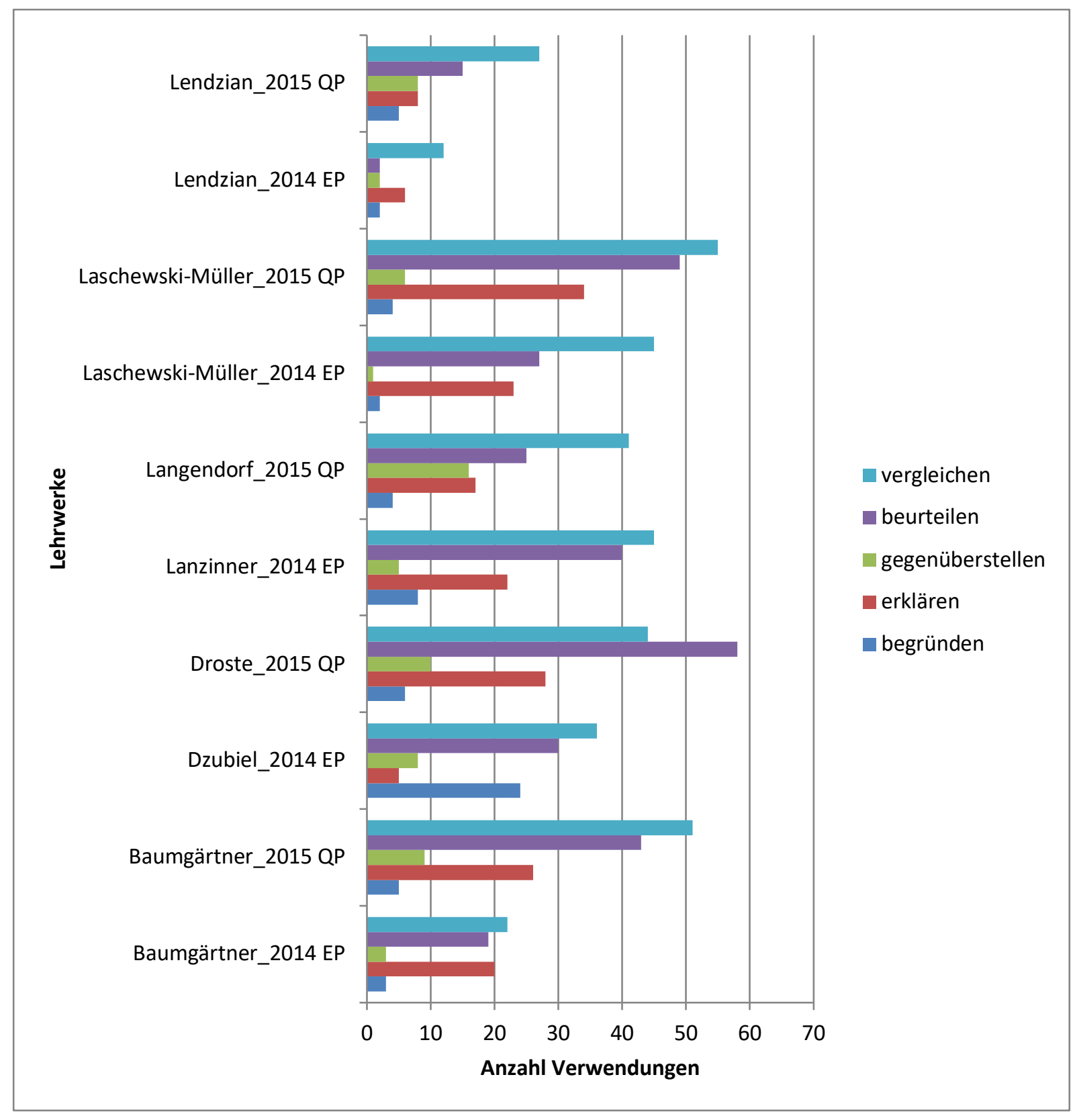

Abbildung 39: Der Operator 'Vergleichen' und seine Teiloperationen: Frequenzen in den Lehrwerken ab 2014

Die Grafik zeigt ähnliche Ergebnisse wie im ersten Teil. Sie macht deutlich, dass das Gegenüberstellen als Lernaufgabe deutlich weniger häufig gestellt wird als das komplexere Vergleichen, das etwa explizit eine Beurteilung des Gegenübergestellten verlangt.

Erkläre-Aufgaben werden häufig gestellt, sie können als geübt angesehen werden. Auch BeurteileAufgaben tauchen relativ häufig auf. Der Operator Begründen hingegen wird eher selten verwendet. Hier kann wieder vermutet werden, dass das Begründen implizit durch das Beurteilen und ggf. durch das Erklären, wenn diese Strukturen bekannt sind und offengelegt werden, eingeübt wird.

Grundsätzlich wäre zur besseren Einordnung der Ergebnisse ein Vergleich mit den Frequenzen in Lehrwerken der Sek. I vonnöten, um besser einschätzen zu können, ob etwa Strukturen wie das Gegenüberstellen dort bereits ausreichend als Aufgaben angeboten wurden. Erste Hinweise darauf finden 
sich in Altun/Günther (2015) ${ }^{544}$ : In der Auswertung von 10 Lehrwerken der Sek. I und II taucht das Gegenüberstellen nicht unter den drei häufigsten Operatoren auf. Dieses sicherlich ausschnitthafte Ergebnis verstärkt den Eindruck, dass Lehrwerke nicht nur einem sehr individuellen Stil folgen, sondern dass Aufgaben und somit Operatoren zwar in Bezug auf das zu bearbeitende Thema gewählt werden, ein Gesamtkonzept zum Einsatz verschiedener Operatoren aber fehlt. Dazu sind jedoch Überlegungen nötig, da Aufgabenstellungen, die durch Operatoren formuliert werden, spätestens in der Abiturprüfung (schriftlich) gelöst werden müssen. Hierauf müssen Lernende vorbereitet werden.

\subsection{Einordnung der Ergebnisse in der Gesamtschau}

Zunächst kann festgestellt werden, dass in den Geschichtslehrwerken ab 2014 Lernaufgaben fast durchgehend über Operatoren formuliert werden. Ist dieser Umstand, wie bereits vermutet, der Kompetenzorientierung geschuldet, muss allerdings auf das unklare Verhältnis zwischen Anforderungsbereichen und Kompetenzorientierung hingewiesen werden. ${ }^{545}$

Der Anteil der Aufgaben pro Seite hat sich in den Lehrwerken ab 2014 auf fast zwei Aufgaben erhöht. Zwar sagt der Anteil der Aufgaben nichts über die Qualität dieser aus und auch nichts darüber, inwiefern es sich um historische Lernaufgaben handelt. Dennoch kann er einen ersten Indikator dafür darstellen, wie stark in Lehrwerken zum Umgang mit dem dargebotenen Stoff angeregt wird. Hier ist zu vermuten, dass Aufgaben durch den Fokus auf die Kompetenzorientierung in den neuen Kernlehrplänen NRW Lernaufgaben stärker in den Blick der Schulbuchautoren gerückt sind. Dabei ist zunächst noch nicht differenziert, welche Rolle unterschiedliche Materialgrundlagen für die Aufgabengestaltung spielen. Da allerdings gerade im zweiten Frequenzanalyseteil (Lehrwerke ab 2014) in einigen Lehrwerken eindeutige Hinweise fehlen, auf welche Materialgrundlage sich Aufgaben beziehen, kann hierüber keine eindeutige Aussage gemacht werden.

Die Nutzung einzelner Operatoren aus der EPA-Liste für das Fach Geschichte ist bereits beschrieben und eingeordnet worden. Die Ergebnisse sollen an dieser Stelle nicht wiederholt werden. Es ist jedoch interessant, dass einzelne Operatoren kaum genutzt werden, während andere sehr häufig vorkommen. Zwar werden in der Lehrwerkgeneration ab 2014 mehr der Operatoren aus dem EPA-Katalog genutzt als in den Lehrwerken vor 2014, dennoch muss gefragt werden, welchen Wert diese kaum genutzten Operatoren besitzen, um historisches Denken anzuregen, oder konkreter, inwiefern sie ein historisches Werkzeug darstellen können. Am Beispiel des Vergleichens soll dies im Folgenden gezeigt werden. Eine solche Untersuchung erscheint wesentlich für den EPA-Katalog, der, wenn Operatoren

\footnotetext{
${ }^{544}$ Altun Tülay; Günther, Katrin (2015): Operatoren am Übergang von der Sekundarstufe I zur Sekundarstufe II als Vorbereitung auf wissenschaftspropädeutisches Arbeiten in der Sekundarstufe II? Eine Auszählung von Aufgabenstellungen in 10 Schulbüchern der Sekundarstufen I und II. Verfügbar unter: https://www.uni-due.de/imperia/md/content/prodaz/altun_g\%C3\%BCnther_operatoren.pdf. S. $6 f$ (zuletzt eingesehen am 01.08.2018). ${ }^{545} \mathrm{Vgl}$. Schönemann, Bernd et al., 2011. 17. Im Rahmen dieser Arbeit wurde dieser Aspekt in Kapitel 3.3.3 dargelegt.
} 
nicht den gewünschten Mehrwert für den Unterricht besitzen, überarbeitet werden müsste. So fällt die in einigen Lehrwerken gehäufte Verwendung der Operatoren Erklären und Erläutern auf. Diese müssten viel schärfer voneinander abgegrenzt werden. Beide Operatoren, das zeigt die Frequenzanalyse, werden sehr individuell eingesetzt, so dass sich die Annahme verstärkt, dass eine klare Profilierung der beiden Operatoren vorgenommen werden muss. ${ }^{546}$ Ein festes Verwendungsschema kann nicht erkannt werden, wäre aber auch als Teilhandlung für das Vergleichen von Bedeutung.

Unterscheiden sich Erklären und Erläutern, wie im EPA-Katalog definiert, nur durch die im Vergleich zum Erklären zusätzlichen Informationen und Beispiele, die für das Erläutern notwendig sind? Dann muss dies bei den Lerner*innen eine große Verunsicherung hervorrufen: Ab wann haben sie so viele Beispiele und Informationen gegeben, dass es sich um ein Erläutern und nicht mehr um ein Erklären handelt? Diese Operatoren müssen deshalb viel stärker hinsichtlich ihres Anforderungspotentials expliziert werden, damit die Aufgabenbearbeitung erfolgreich gelingen kann. Dabei bietet der Zugriff über die Funktionalität von Sprache eine Lösung, da über den kommunikativen Zweck Operatoren in ihrer Funktion begriffen werden können.

Weiterhin muss über die Anzahl der Operatoren nachgedacht werden. In diesem Zusammenhang beschreiben Schönemann et al. die Defizite der derzeitigen Operatorenverwendung und stellen fest, Lernende dürften [...] „,in der Regel damit überfordert sein, sich alle Operatoren und die mit ihnen verbundenen Operationen zutreffend einzuprägen. Das lässt erhebliche Zweifel an der behaupteten Signalwirkung aufkommen. Eine Beschränkung auf maximal zehn Operatoren wäre daher dringend zu diskutieren. ${ }^{4547}$ Da auch die übrigen Fächer in ihren EPA-Katalogen zum Teil die hier genannten, zum Teil aber auch andere Operatoren auflisten, die zudem den Fachdisziplinen entsprechend unterschiedlich definiert oder zumindest prononciert sein können, stellt sich die Frage nach der Handhabbarkeit des EPA-Katalogs (oder besser der Kataloge) noch schärfer: Sind Lernende in der Lage die Vielzahl der von ihnen geforderten Operatoren den Fachdisziplinen entsprechend einzusetzen? Hier sind Zweifel angebracht. So ist beispielsweise das Vergleichen im Geschichtsunterricht ein anderes als das im Biologieunterricht.

Um den Fächern gerecht zu werden, könnten die von Redder et al. vorgeschlagenen Basisqualifikationen ${ }^{548}$ als Grundlage für den Umgang mit Aufgabenformaten dienen. Die Autoren schlagen auf der Grundlage einer Untersuchung an Grundschulkindern vor, Sprachhandlungen zu kategorisieren. Für die 1. und 2. Klasse der Grundschule benennen sie das Beschreiben, Instruieren, Erklären, Reformulieren und Erläutern als zentrale Anforderungen. ${ }^{549}$ Eine solche Kategorisierung und Beschränkung auf wenige Operatoren kann auch für die Sek. II relevant sein. Das Wissen um für die Fächer besonders relevante Operatoren kann dann dazu genutzt werden, diese fachspezifisch auszudifferenzieren. Am Beispiel des Vergleichens wird deutlich, dass Operatoren andere Operatoren als „Teiloperatoren“ oder anders ausgedrückt als sprachliche Teilhandlungen benötigen. Die Kenntnis dieser Teiloperatio-

\footnotetext{
${ }^{546}$ Vgl. hierzu auch Altun, Tülay; Günther, Katrin (2015), S. 13.

547 Schönemann, Bernd; Thünemann, Holger; Zülsdorf-Kersting, Maik (2010), S. $17 f$.

548 Redder, Angelika; Guckelsberger, Susanne, Graßer, Barbara (2013).

${ }^{549}$ Vgl. ebd. S. 14.
} 
nen ermöglicht es den Lernenden, durch ihr Wissen um diese Teilhandlungen und die Kenntnis zentraler Operatoren, die insbesondere diese Teilhandlungen repräsentieren, Lernaufgaben erfolgreich zu lösen. Diese Herangehensweise stellt zudem einen stark kompetenzorientierten Zugriff dar. Die Ausdifferenzierung der Operatoren in den Fachdisziplinen kann dann als Mehrwert und weniger als Belastung für Lernende wahrgenommen werden. Die zuvor dargestellte Funktion des Vergleichens im Fach Geschichte (einhergehend mit der herausgearbeiteten hohen Zahl an Vergleichsaufgaben in Lehrwerken) macht das Vergleichen zu einem Basis-Werkzeug für den Geschichtsunterricht. Schönemann et al. nennen als weitere Hürden im Umgang mit den EPA-Operatoren:

„Zweitens ist es systematisch kaum überzeugend, nicht nur spezifische, sondern auch übergeordnete Operatoren auszuweisen. Wenn bei diesen Operatoren Leistungen in allen Anforderungsbereichen erforderlich sind, dann sollte man sie - im Sinne der Vereinheitlichung - dem AFB III zuordnen. Denn anderenfalls müsste man Operatoren für den AFB II auch als übergeordnete Operatoren für den ersten Anforderungsbereich klassifizieren. Und drittens ist ein Mangel an Präzision bei der Verwendung der Operatoren zu kritisieren. [...] Nimmt man diese Formulierung ernst, dann kann eine Leistung im AFB I (Operator: wiedergeben) nur dadurch erbracht werden, dass man in den AFB II (Operator: herausarbeiten) wechselt. ${ }^{4550}$

Der Zugriff über die von Redder et al. vorgeschlagenen Basisqualifikationen und die daraus resultierende Ausweisung grundlegender sprachlicher Handlungen könnte das Problem der Zuordnung zu den ausgewiesenen Anforderungsbereichen lösen, zumal bereits die Analyse einzelner Aufgaben Grund zu der Annahme gibt, dass die Zuordnung zu den Anforderungen auch durch die Situierung der Aufgabe beeinflusst werden kann. Am Beispiel des sogenannten „Breitbandoperators“ Darstellen kann dies gezeigt werden. Je nach Aufgabenformulierung kann Darstellen als Wiedergeben oder als ein ordnendes und interpretierendes Darstellen verstanden werden.

Eine klare Abgrenzung der Operatoren fehlt. Dies zeigt auch das Beispiel der Kombination des komplexen und dem Anforderungsbereich III zugehörenden Operators Vergleichen mit einer vorangestellten W-Frage: „In welcher Weise unterschieden sich die Wege zum modernen Staat? Vergleichen Sie die Entwicklung in Preußen, Russland und England“. ${ }^{551}$ Das Vergleichen wird hier zur Teilhandlung der W-Frage. Das Unterscheiden stellt, wie im Kapitel zum Vergleichen gezeigt, einen Teil des Vergleichens dar, die Frage nach der "Weise“ gibt einen Perspektivierungshinweis. Strenggenommen hätte die WFrage also der Vergleichsoperation nachgestellt werden müssen.

Insgesamt ist festzustellen, dass für einen Operator notwendige Teiloperationen in der Didaktisierung stärker aufeinander abgestimmt bzw. durch Aufgabenformate geübt werden müssen, damit sie für die Lernenden nachvollziehbar und als Instrument handhabbar werden. Auch im zweiten Teil der Frequenzanalyse bleibt festzustellen, dass die für das Vergleichen benötigten Teilhandlungen in sehr unterschiedlicher Häufigkeit verwendet werden. Es ist zu vermuten, dass diese Annahme auch für andere komplexe Operatoren gilt.

\footnotetext{
550 Schönemann, Bernd et al. (2010), S. 18.

${ }^{551}$ Bahr, Frank (Hrsg.) (2006): Horizonte II. Geschichte für die Oberstufe. Vom Absolutismus bis zum Ersten Weltkrieg. Braunschweig: Westermann. S. 40.
} 


\section{Analyse von Lernendentexten zum historischen Vergleich: Vorstellung der ausgewählten Vergleichsaufgabe}

Nachdem durch die Frequenzanalyse gezeigt werden konnte, dass der Operator Vergleichen eine wichtige Rolle in Geschichtslehrwerken spielt, soll nun überprüft werden, wie Schüler*innen eine Vergleichsaufgabe lösen und welche Teilfertigkeiten zur Anwendung kommen.

Im Folgenden sollen dazu zunächst die ausgewählte Aufgabe und der zugehörige Darstellungstext vorgestellt und analysiert werden.

Das Thema der Vergleichsaufgabe "Beginn der Industrialisierung in England und Deutschland" kann als grundlegend und gängig für den Geschichtsunterricht gelten. Die Lernenden sind in der Regel das erste Mal in der Sekundarstufe I damit in Berührung gekommen, bevor es dann in der Sekundarstufe II wieder aufgegriffen wird. Im Kernlehrplan für die Sekundarstufe I/Gesamtschule ist es bspw. im Inhaltsfeld 2 „Wirtschaft und Arbeit“ mit dem Schwerpunkt "Wirtschaft und Arbeit in der industriellen Gesellschaft" für die Klassen 7-10 verankert. ${ }^{552}$ In der Sekundarstufe II ist das Thema sowohl für die gymnasiale Oberstufe an Gesamtschulen und Gymnasien ${ }^{553}$ relevant als auch für die Bildungsgänge der am Berufskolleg angesiedelten Bildungsgänge der Fachoberschule. Für die Fachoberschule wird explizit der Themenbereich „Industrialisierung und staatliche Arbeitsverwaltung" genannt. ${ }^{554}$ Dieser Bereich ist gerade auch schon in den Klassen 12 angesiedelt. ${ }^{555}$

\subsection{Vorstellung und Einordnung der ausgewählten Vergleichsaufgabe}

Die ausgewählte Aufgabe ist grundsätzlich als Lernaufgabe einzuordnen, die nur im Zusammenhang mit dem zugehörigen Darstellungstext zu lösen ist. Sie ist dem Darstellungstext nachgestellt.

Die Aufgabe wurde auf der Grundlage der Annahme ausgewählt, dass das Thema Industrialisierung, wie zuvor dargestellt, als bekannt vorausgesetzt werden kann. Außerdem wurde auf der Basis der Frequenzanalyse und den insbesondere in Kapitel 2 und 4 dargestellten Zusammenhängen gezielt nach einer Vergleichsaufgabe gesucht. Diese sollte zumindest einem Teilkapitel vorangestellt sein, damit sie inhaltlich möglichst breit angelegt ist und somit von möglichst vielen Lernenden bearbeitet

\footnotetext{
552 Ministerium für Schule und Weiterbildung des Landes Nordrhein-Westfalen (Hrsg.) (2011): Kernlehrplan für die Gesamtschule - Sekundarstufe I in Nordrhein-Westfalen: Gesellschaftslehre Erdkunde, Geschichte, Politik. S. $34 \mathrm{ff}$.

${ }^{553} \mathrm{Vgl}$. z. B.: Inhaltsfeld 4: „Die moderne Industriegesellschaft zwischen Fortschritt und Krise“, in: Ministerium für Schule und Weiterbildung des Landes Nordrhein-Westfalen (Hrsg.) (2014): Kernlehrplan für die Sekundarstufe II Gymnasium/Gesamtschule in Nordrhein-Westfalen: Geschichte. S. 18.

${ }^{554}$ Ministerium für Schule und Weiterbildung des Landes Nordrhein-Westfalen (Hrsg.) (2007): Lehrplan für das Berufskolleg in Nordrhein-Westfalen. Politik/Gesellschaftslehre bzw. Gesellschaftslehre mit Geschichte. Bildungsgänge der Fachoberschule (Anlage C9 bis C11 und D29). S. 12.

555 Untersucht werden Klassen 12 und 13 des Berufskollegs, weitere Hinweise zur Schülergruppe folgen in Kapitel 6.
} 
werden kann. Die Wahl fiel aus den genannten Gründen auf die folgende Aufgabe im „Kursbuch Geschichte ${ }^{\text {“556: }}$

Aufgabe: "Vergleichen Sie die unterschiedlichen Ausgangsbedingungen für die Industrialisierung in England und Deutschland im ausgehenden 18. und beginnenden 19. Jahrhundert. Arbeiten Sie dabei besonders heraus, welche Strukturen und Prozesse fördernd oder hemmend auf die Industrialisierung wirkten. ${ }^{1557}$

Im Mittelpunkt des Vergleichs stehen die Ausgangsbedingungen der Industrialisierung in Deutschland und England, die in der Aufgabenformulierung als unterschiedlich gekennzeichnet werden. Dies bedeutet, dass der Blick auf die Unterschiede, nicht auf die Gemeinsamkeiten gerichtet ist (vgl. dazu Kapitel 2). Der Hinweis auf den zeitlichen Rahmen grenzt die Aufgabe weiter ein und gibt damit zugleich den wesentlichen Zeitraum vor. Weiterhin erhält die Aufgabe eine Aspektierung, die durch den Teiloperator "Herausarbeiten“ eingeleitet wird. Er fungiert hier als eine Art ,Filter', indem er (in allgemeiner Form) die wesentlichen Merkmale, die verglichen werden sollen, in den Mittelpunkt stellt: Strukturen und Prozesse. Kritisch ist zu sehen, dass diese hinsichtlich der Frage eingeordnet werden sollen, ob sie hemmend oder förderlich für die Industrialisierung waren. Damit wird bereits eine Norm und damit letztlich eine Beurteilung vorgegeben, die durch den Vergleich auch hätte erarbeitet werden können.

Die Berücksichtigung des in der Aufgabe vorgegebenen zeitlichen Rahmens kann zur Anregung von Zeitkompetenz beitragen: „Zeitkompetente Narration erörtert Vorstellungen wie Fortschritt, Aufstieg und Karriere auf der einen und Stagnation, Abstieg, Rückschritt, Verfall und Niedergang auf der anderen Seite, gleichermaßen wendet sie diese Begriffe kritisch und differenziert an. [...] Kompetenz zeigt sich auch darin, Zeitvorstellungen als Konstrukt zu erkennen, sei es, dass sie in Texten wahrgenommen werden, sei es, dass man sie selbst verwendet. ${ }^{1558}$ Sowohl durch den zugrundeliegenden Text als auch durch das in der Aufgabe angelegte Tertium Comparationis werden Zeitkompetenzen berücksichtigt. Darüber hinaus werden explizit durch den Bezug in der Aufgabenstellung (fördernd oder hemmend) Vorstellungen von Fortschritt oder Stagnation angesprochen.

Im Sinne der EPA-Bestimmung gibt die Aufgabe bereits Kriterien vor, die gegenübergestellt werden sollen: die Länder, die verglichen werden sollen, den oben erwähnten Zeitrahmen, explizit den Beginn der Industriellen Revolution. Dazu sollen konkret Strukturen und Prozesse untersucht werden. Durch den Hinweis auf die Unterschiede der Entwicklungen wird weiterhin der Beurteilungsrahmen vorgegeben. Insgesamt kann festgestellt werden, dass die Aufgabenformulierung wesentliche Aspekte der EPA-Bestimmung aufgreift und als Strukturhilfe den Lernenden mit an die Hand gibt.

\footnotetext{
556 Laschewski-Müller, Karin; Rauh, Robert (Hrsg.) (2010).

557 Laschewski-Müller, Karin; Rauh, Robert (Hrsg.) (2010), S. 259.

558 Van Norden, Jörg (2011): Was machst du für Geschichten? Didaktik eines narrativen Konstruktivismus. Freiburg: Centaurus Verlag, S. 221.
} 
Damit ist allerdings weder eine umfassende Aussage über die Qualität der Vergleichsaufgabe (im Sinne einer kompetenzorientierten Lernaufgabe) noch über die konkreten Anforderungen, die die Aufgabe an die Lernenden stellt, getroffen. Dies kann erst im Zusammenhang mit der Analyse des Darstellungstextes geschehen,

Im Rückgriff auf die Frequenzanalyse kann jedoch festgestellt werden, dass es sich um eine Aufgabe handelt, die auch in den Lehrwerken der neueren Generation ab 2014 vorzufinden ist. Es gibt keinen Hinweis, ob die Aufgabe mündlich oder schriftlich gelöst werden soll. Aufgrund ihrer Komplexität, die sich nicht zuletzt durch den langen Darstellungstext ergibt, ist allerdings von einer schriftlichen Lösung auszugehen. Die Aufgabe bezieht sich auf eine Textgrundlage und ist damit dem Grundtyp des textbezogenen Schreibens zuzuordnen (im Gegensatz zum materialgestützten Schreiben, vgl. Kapitel 2.7.3).

\subsection{Der Darstellungstext zur Vergleichsaufgabe}

Um die Aufgabe und die Leistungen, die sie den Lernenden abverlangt, besser einschätzen zu können, folgt nun die Analyse des Darstellungstextes, in dessen Kontext die Aufgabe eingebettet ist. Dabei werden die geschichtsdidaktische Sicht und damit die fachlichen Anforderungen betrachtet und mit den sprachlichen Anforderungen verknüpft.

Auch wenn den Schüler*innen im Rahmen der Untersuchung nur der Darstellungstext mit der Aufgabe vorgelegt wird, kann die Aufgabe in ihrer Gesamtheit nur im Zusammenhang mit dem sie umgebenden Material eingeschätzt werden.

Die Wahl eines Darstellungstextes statt einer Aufgabe auf Grundlage einer Quelle kann mit Drüding/ Schlutow begründet werden: „Je komplexer der historische Vergleich angelegt ist, desto schwieriger wird es, quellennah zu arbeiten: Der moderne Geschichtsunterricht kommt ohne Quelleninterpretation nicht aus. [...] Richtet man das Augenmerk jedoch auf komplexere Vergleiche [...], ist man für die Durchführung primär auf historische Darstellungen angewiesen [...], da nur so das benötigte Abstraktionsniveau des Vergleichs erreicht werden kann. “559

Zur besseren Einschätzung der Leistungen eines Darstellungstextes im Geschichtsunterricht bieten sich vorab einige grundsätzliche Überlegungen zur Funktion dieser Textsorte im Hinblick auf das historische Lernen an. Pandel ordnet sie in seiner Aufschlüsselung historischer Erzählhandlungen als „Nacherzählung“560 ein. Dabei kennzeichnet er das Nacherzählen als „[...] eine Reformulierung eines erzählten Geschehens nach dem Muster einer Ausgangserzählung. Dieses Muster ist kein produktives Verknüpfen der Ereignisse, da die Ereignisse und der Erzählplan vorgegeben sind. Es handelt sich um einen reproduktiven Sinngebrauch“561. Es ist zu prüfen, ob diese Einordnung auch für den vorliegenden Text gilt. Ausgehend von einer Nacherzählung im Sinne Pandels sollen Lernende über die Aufgabe

\footnotetext{
559 Drüding, Markus; Schlutow, Martin (2019), S. 34.

560 Pandel, Hans-Jürgen (2010), S. 154.

561 Ebd.
} 
einen diskursiven Text (nämlich den Vergleich) erstellen. Es ist im Weiteren zu prüfen, inwiefern ein Wechsel der Erzählhandlung für Lernende möglich ist.

\subsubsection{Analyse des Darstellungstextes zur Vergleichsaufgabe}

Weder Vergleichsaufgabe noch zugehöriger Darstellungstext können isoliert betrachtet werden. Deshalb erfolgt zunächst eine Einordnung in den Lehrwerkkontext, in dem Aufgabe und Text auftauchen, zunächst kurz im Hinblick auf das gesamte Lehrwerk und dann auf das zugehörige Kapitel. Erst dann folgt die Analyse des eigentlichen Darstellungstextes.

\subsubsection{Einordnung des Darstellungstextes in den Kontext des Lehrwerkes}

Der Darstellungstext „Die Anfänge der Industrialisierung“562 ist innerhalb des „Kursbuch Geschichte“ in das Kapitel 6 „Entstehung und Wandel der modernen Industriegesellschaft" eingebettet. Er steht am Beginn des zweiten Teilkapitels „Die Anfänge der Industrialisierung“. Eingeleitet wird dieses Themenfeld durch das Teilkapitel 1 „Die Industrialisierung: Begriffe, Probleme, Grundzüge“.

Im Anschluss an das Teilkapitel 2 folgen die Themenbereiche "Die Entwicklung der Industriewirtschaft", "Gesellschaftlicher Wandel und ,soziale Frage'“ sowie "Industrialisierung und Globalisierung" als nachfolgende Teilkapitel. Das Kapitel 6 wird abgeschlossen durch die für das Lehrwerk obligatorischen Methodenseiten, hier zum Umgang mit Statistiken, und eine unter dem Titel "Grundwissen“ jeweils am Ende der Kapitel angefügten Zusammenfassung.

Hinzuweisen ist zudem auf den Epochenüberblick, der den Kapiteln 5, 6 und 7 vorausgeht und hier mit dem Titel „Zur Aktualität des 19. Jahrhunderts“ überschrieben ist. In diesem Zusammenhang gibt es ein Teilkapitel von knapp einer Seite Länge, worin ein Gegenwartsbezug hergestellt wird, in dem die Industrialisierung (im Sinne eines Werturteils) als Basis für die heutigen modernen Gesellschaften dargestellt wird.

Die Struktur der Kapitel und Teilkapitel ist immer gleich aufgebaut. Den Anfang des Kapitels macht eine Doppelseite, die etwa mit Hilfe eines ganz kurzen Darstellungstextes und einem ersten Material knappe Informationen zum Thema gibt, außerdem werden darüber der Kompetenzerwerb benannt und durch eine Zeitleiste am unteren Rand der Doppelseite Beginn und Ende des zu behandelnden Themas abgegrenzt. In Kapitel 6 befindet sich auf der linken Seite ein Ausschnitt eines Gemäldeentwurfs, das Industriegebäude in Berlin zeigt („Die Stadtbahnanlagen an der Jannowitzbrücke“ von Julius Jacob und Wilhelm Herwarth um $1891^{563}$ ). Auf der rechten Seite informiert ein kurzer Text über Friedrich Engels' Einordnung der Industrialisierung in Manchester als epochalen Umbruch und die zeitliche Eingrenzung der Industriellen Revolution. Es wird also schon an dieser Stelle über das Erleben Engels'

\footnotetext{
562 Laschewski-Müller, Karin; Rauh, Robert (Hrsg.) (2010), S. $257 f$.

563 Ebd. S. 250.
} 
die Zuordnung der Industrialisierung als Revolution vorgenommen. Anschließend werden im Aufzählungsstil die zu erwerbenden Kompetenzen benannt. Da diese Kompetenznennung einen Gradmesser für den Darstellungstext und die zugehörige Aufgabe darstellt, soll sie im Folgenden zitiert werden:

„Kompetenzerwerb: Nach Bearbeitung des Kapitels 6 können Sie...

- Die Ursachen und Vorbedingungen der Industrialisierung darstellen und erläutern,

- Strukturen und Prozesse der industriellen Revolution erklären,

- sich mit wissenschaftlichen Deutungen der Industrialisierung auseinandersetzen,

- die sozialen Auswirkungen der Industriewirtschaft analysieren und beurteilen,

- Statistiken interpretieren. “564

Für das zu bearbeitende Kapitel 2 sind insbesondere die Ziele aus Punkt 1, in Ansätzen auch schon aus Punkt 2 relevant. Eine explizite Auseinandersetzung mit wissenschaftlichen Deutungen der Industrialisierung aus Punkt drei wird durch die Aufgabe nicht angeregt. Auch im Darstellungstext wird nicht ausgewiesen, inwiefern er sich auf wissenschaftliche Grundlagen bezieht.

Eine Karte weist bereits auf dieser Eingangsdoppelseite England (vermutlich Manchester) und ,Deutschland' (vermutlich das Ruhrgebiet) als Betrachtungspunkte des Kapitels aus. Die Umkreisung von Manchester in dieser Karte mit einer Art „Radarlinien“ bewertet England als besonders bedeutsam für das Themenfeld. Der Darstellungstext untermauert die Grafik durch den Hinweis, dass die Industrielle Revolution in England einsetzte.

Die Zeitleiste am Seitenende ordnet die Industrialisierung in den Zeitraum von 1750 bis 1930 ein. Die fachliche Richtigkeit soll hier nicht weiter untersucht werden. Bedeutsam erscheint jedoch, dass die Einführungsdoppelseite bereits einige Vorannahmen als gegeben vermittelt, wie zum Beispiel die Zuordnung der Industrialisierung als Revolution und den besonderen Status Englands als Ausgangspunkt der Industriellen Revolution.

Das auf die Eingangsdoppelseite folgende erste Teilkapitel stellt sodann für das Thema als wichtig erachtete Aspekte in den Mittelpunkt. Als allgemeines Schema kann festgestellt werden, dass die Teilkapitel jeweils durch einen (unterschiedlich langen) Darstellungstext eingeleitet werden. Dieser Text wird begleitet durch kleinere Materialien, wie etwa bildliche Darstellungen. Am Seitenrand gibt es z. T. Begriffserklärungen, Hinweise auf relevante Internetseiten und andere Tipps. Zum Darstellungstext gehören in der Regel ein oder mehrere Aufgaben. Dann folgt ein Materialteil. Die unterschiedlichen Materialien werden dabei nicht gattungsspezifisch gekennzeichnet. An die einzelnen Materialien sind jeweils Aufgaben angefügt.

Kapitel 1 folgt diesem Schema. ${ }^{565}$ Der vorangestellte zweiseitige Darstellungstext thematisiert unter dem Untertitel „Eine oder drei Revolutionen?“ zunächst den Begriff „Industrialisierung“. Als theoretische Grundlage wird Hobsbawm zitiert. Darauf aufbauend ordnen die Autoren den Begriff der „Industriellen Revolution" ein und problematisieren seine Verwendung. Weiterhin gehen die Autoren von einer Aufschlüsselung in drei Teil-Revolutionen aus. Zur weiteren Fundierung wird die Definition

\footnotetext{
564 Ebd.

${ }^{565}$ Vgl. hier wie auch im Weiteren: ebd. S. $252 \mathrm{ff}$.
} 
Kockas von 2001 angeführt. Außerdem wird der Begriff „Kapitalismus“ als bedeutsam dargestellt und von der „Industrialisierung“ abgegrenzt. Unter dem Untertitel „Regionale Ungleichheiten“ wird angenommen, dass die Industrialisierung in England begann, Deutschland seinen Rückstand später jedoch aufholen konnte. Hierzu werden verschiedene Beispiele angefügt. Unter dem Untertitel „Schrittmacherindustrie" werden im letzten Teil des Darstellungstextes Leitsektoren in den verschiedenen Phasen der Industrialisierung benannt. Die Doppelseite schließt mit einer Aufgabe zum Darstellungstext. Auf der nächsten Doppelseite schließen sich Historikertexte von Landes und Hobsbawm an, die kontrovers die Bedeutung der technischen Innovationen einschätzen, und weitere Texte, die den Begriff ,Kapitalismus' (Kocka) und den Verlauf der Industrialisierung (ebf. Kocka) einordnen. Hieran werden jeweils Aufgaben angefügt. Auf einer nachfolgenden Einzelseite sind zwei Karten abgedruckt, welche die Industrialisierung bis 1850 und zwischen 1850 und 1914 darstellen. Zwei Teilaufgaben zu den Karten beenden das Teilkapitel 1.

Die Einführungsdoppelseite und Teilkapitel 1 können, wenn man einen progressiven und lerndidaktischen Aufbau des Lehrwerkes annimmt, als Vorwissen angesehen werden, das sich die Lernenden entweder schon angeeignet haben oder auf das sie bei Bedarf zurückgreifen können.

Es ist kritisch anzumerken, dass im Sinne des Konstruktionscharakters von Geschichte nicht festgestellt wird, aus welcher Zeit das Konzept der Industrialisierung bzw. der Industriellen Revolution ursprünglich stammt. Dass es sich um einen in der Forschung diskutierten Begriff handelt, weil es davon abhängig ist, auf welchen Bereich er bezogen wird, bzw. hinsichtlich der Frage, ob er überhaupt Berechtigung hat, wird kaum deutlich. ${ }^{566}$ Ziegler erläutert die Problematik des Begriffs: „In der jüngeren Forschung wird aber gleichzeitig immer mehr die graduelle Veränderung betont und damit der evolutionäre Charakter der Industrialisierung. So stellt sich die Frage, ob der Umwälzungsprozess nicht sehr viel früher eingesetzt hatte, als man lange Zeit annahm [...]. “567

Diese Diskussion um den Begriff der Industriellen Revolution ist bedeutsam für die Debatte um die deutsche Entwicklung, da im Lehrwerk Deutschland als "Nachzügler“568 eingeordnet wird. Das Lehrwerk nutzt damit zum einen den Begriff „Deutschland“569, der jedoch einen Nationalstaatscharakter impliziert, den es zwischen 1800 und 1850 so nicht gegeben hat. Zum anderen wird durch den Begriff ,Nachzügler' eine Deutung vorgegeben, aber nicht offengelegt, die so den Charakter einer Norm erhält.

Zwar wird der Begriff, Industrielle Revolution' seit den 60er Jahren auch in Deutschland zunehmend verwendet, eine einheitliche Definition liegt aber nicht vor. ${ }^{570} \mathrm{Immerhin}$ gibt es einen Hinweis im Lehrwerk, dass der Begriff nicht einheitlich gebraucht wird.

\footnotetext{
${ }^{566} \mathrm{Vgl} \mathrm{hierzu:} \mathrm{Hahn,} \mathrm{Hans} \mathrm{Werner} \mathrm{(2011):} \mathrm{Die} \mathrm{industrielle} \mathrm{Revolution} \mathrm{in} \mathrm{Deutschland} \mathrm{(Enzyklopädie} \mathrm{deut-}$ scher Geschichte 49), 3. Auflage München. S. 51.

${ }^{567}$ Ziegler, Dieter (2009): Die Industrielle Revolution in Deutschland, 2. bibliographisch aktualisierte Auflage Darmstadt. S. 1.

568 Laschewski-Müller, Karin; Rauh, Robert (Hrsg.) (2010), S. $257 f$.

${ }^{569}$ Ebd.

${ }^{570}$ Vgl. Ziegler, Dieter (2009). S. 57f.
} 
Des Weiteren wird England als Land, in dem die Industrialisierung begann, eingeordnet, eine eigenständige Erarbeitung durch die Lernenden ist hier nicht vorgesehen.

Zusammenfassend kann festgestellt werden, dass das Konzept der Industrialisierung bzw. der Industriellen Revolution vor dem Darstellungstext und der Vergleichsaufgabe thematisiert, jedoch nicht kritisch hinterfragt wird (dies könnte bei einem Lehrwerk für die Sekundarstufe II im Hinblick auf wissenschaftspropädeutisches Arbeiten angenommen werden). Weiterhin kann vermutet werden, dass die Lernenden aufgrund fehlender Hinweise im Lehrwerk die Annahme, dass die Industrialisierung in England begann, vermutlich als gegeben annehmen und die Bezeichnung ,Deutschland' nicht hinterfragen werden.

\subsubsection{Einordnung des Darstellungstextes in das Teilkapitel 2}

Kapitel 2 ist überschrieben mit dem Titel „Die Anfänge der Industrialisierung“. Auf diese Überschrift folgt der zu überprüfende Darstellungstext. ${ }^{571} \mathrm{Er}$ ist zwei und eine viertel Seite lang und in verschiedene Abschnitte untergliedert. Am äußeren Seitenrand befinden sich jeweils unterschiedliche Materialien, wie ein Bild einer Spinnerei in England, ein englisches Lied über die wachsende Eisenindustrie auf der ersten Textseite, ein zeitgenössischer Kupferstich von Adam Smith mit kurzem Untertext zu seiner Lehre und ein Literaturtipp auf der zweiten Textseite sowie ein Internettipp auf der dritten Textseite. Die Vergleichsaufgabe schließt den Text ab.

Im Anschluss an den Text folgen verschiedene Materialien. Der Aufbau folgt damit insgesamt dem zuvor beschriebenen Schema. Bei den sich anschließenden Materialien handelt es sich um einen Text des Historikers Barrington Moore von 1969 über den Wandel in der englischen Landwirtschaft, einen weiteren Text von Hagen Schulze von 1998 über die Ursachen der englischen Industrialisierung, einen Kupferstich aus dem 18. Jahrhundert, der Erfindungen in der englischen Landwirtschaft zeigt, verschiedene Statistiken über die wirtschaftliche Entwicklung in Deutschland und England in Bezug auf die Steinkohleförderung, Roheisenproduktion, den Rohbaumwollverbrauch und die Kapazität aller Dampfmaschinen. Es folgen weiter ein Textausschnitt von Adam Smith aus seinem Werk „Über den Wohlstand der Nationen“ von 1776 sowie ein Auszug aus dem preußischen Oktoberedikt von 1807. Eine Karikatur von 1848, welche die Schwierigkeiten für die Wirtschaft durch die vielen Zollgrenzen aufs Korn nimmt, schließt das Teilkapitel ab.

Da der Darstellungstext das Kapitel einleitet, kann eine chronologische Bearbeitung von den Autoren als geplant angenommen werden. Es ist davon auszugehen, dass Auftaktdoppelseite und Kapitel 1 bekannt sind und der Darstellungstext in Kapitel 2 in der Bearbeitung vorausgeht (auch wenn die Lehrperson im Unterricht davon abweichen kann). Dies bedeutet, dass die weiteren Materialien an dieser Stelle zunächst nicht im Fokus stehen und der Darstellungstext wesentliche Aspekte im Hinblick auf die Anfänge der Industrialisierung klären muss.

${ }^{571} \mathrm{Vgl}$. hier wie auch im Weiteren: ebd. S. $257 \mathrm{ff}$. 


\subsubsection{Analyse des Darstellungstextes aus geschichtsdidaktischer und sprachlicher Perspektive}

Zur besseren Nachvollziehbarkeit wird der Darstellungstext im Folgenden für die Analyse in seine verschiedenen Abschnitte zerlegt.

Zunächst verdeutlichen die Teilüberschriften den Fokus, den die Autoren setzen: Sie beschreiben nicht übergeordnet die Anfänge der Industrialisierung (es wird nur in den ersten drei Sätzen auf Europa, Asien und Nordamerika verwiesen), sondern nehmen gezielt England und Deutschland als Vergleichsobjekte in den Blick. Ohne dies im Titel schon auszuweisen, handelt es sich um einen auf eine nationalstaatliche Perspektive zugespitzten Blick auf die Industrialisierung. Ein französisches Lehrwerk hätte vermutlich ebenso das eigene Land als Vergleichsgröße ausgewählt, ein ganzheitlicher Blick auf die Anfänge der Industriellen Revolution ist durch den Text nicht vorgesehen.

Nachfolgend werden die Inhalte des Darstellungstextes durch ein textchronologisches Vorgehen entlang der einzelnen Textabschnitte im Sinne einer Bestandsaufnahme genauer analysiert und im Hinblick auf die Anforderungen an die Lerner*innen eingeordnet. Für eine bessere Nachvollziehbarkeit werden die einzelnen Abschnitte zunächst zitiert und anschließend analysiert. Analyse und Einordnung werden sogleich miteinander verknüpft, da es nicht darum geht, additiv kleinste sprachliche Bestandteile zu kennzeichnen, sondern den Text im Hinblick auf seine Funktionalität für die Schüler*innen zu überprüfen.

Der Text ist untergliedert in drei Teilkapitel, die jeweils durch eine Überschrift gekennzeichnet sind. Diese werden nachfolgend abschnittweise betrachtet. Farbige Kennzeichnungen entstammen der Originalvorlage und wurden übernommen.

\section{Teilkapitel 1 (3 Abschnitte):}

\section{Abschnitt 1:}

„Pionierland England

Die Industrialisierung begann im England des ausgehenden 18. Jahrhunderts und erfasste im beginnenden 19. Jahrhundert den westlichen Teil des europäischen Kontinents sowie Nordamerika. Während der zweiten Hälfte des 19. Jahrhunderts strahlte sie auf Osteuropa und Japan aus. Im 20. Jahrhundert entfaltete sie ihre Wirksamkeit in der gesamten Welt - dieser Prozess dauert bis in die Gegenwart an. ${ }^{\text {5572 }}$

Der Untertitel weist bereits vor Textbeginn Englands Vorreiterrolle aus; insgesamt kann man davon ausgehen, dass diese Zuschreibung in der wissenschaftlichen Diskussion weitgehend akzeptiert ist, dennoch handelt es sich um eine Zuschreibung bereits in der Überschrift.

572 Laschewski-Müller, Karin; Rauh, Robert (Hrsg.) (2010). S. 257. 
Die einleitende und zunächst nüchterne Beschreibung wechselt sehr schnell durch die Verwendung des Verbs „ausstrahlen“ in eine implizite Wertung mithilfe einer Metapher. Die Verwendung von Metaphern ist dem Bereich der Alltäglichen Wissenschaftssprache zuzuordnen. ${ }^{573}$ Statt eines sachlichen „verbreiten“ oder „ausbreiten“ schließt „ausstrahlen“ ein positives „,mit Licht erfüllen“ ein, so dass die Annahme naheliegt, dass der Prozess der Industrialisierung positiv zu werten sei. Das Beispiel zeigt die Bedeutung bildhafter Sprache für die Vermittlung historischer Inhalte. Zwar stellt Lucas dazu zunächst fest: „Eine wertneutrale Sprache, die doch aussagekräftig wäre, gibt es nicht. “574 und stellt die Bedeutung bildhafter Sprache heraus, die er als figurative Sprache benennt. Dabei geht er davon aus, dass die Absicht hinter der Verwendung solcher bildhafter Sprache die Schaffung höherer Anschaulichkeit sei. ${ }^{575}$ Gleichzeitig kritisiert er dieses Vorgehen deutlich:

„Halten wir fest, daß im Bemühen um konkrete statt abstrakter Sprache allzuleicht figurative Ausdrucksweise statt instruktiver Vorstellungen vom gewesenen nur allegorische Bilder schafft, die, statt im Besonderen das Allgemeine dem Nachdenken anzubieten, in der Regel eher irreführend oder ebenso verschleiernd und ob ihrer quasi abstrakten Bildhaftigkeit tendenziell verharmlosend wirken. ${ }^{4576}$

Gerade die oben gezeigte Verwendung der Metapher "ausstrahlen“, die thematisch als Lichtmetapher ${ }^{577}$ eingeordnet werden kann, erschwert oder verhindert sogar das Nachdenken über den Prozess der Ausbreitung der Industriellen Revolution, indem sie eine positive Wertung vorwegnimmt, die jedoch nicht offengelegt wird.

Zudem legen die verwendeten Verben insgesamt nahe, dass die einzelnen Länder ohne England nicht zu einer Industrialisierung gefunden hätten. Dies wird in der Forschung aber zum Beispiel für Deutschland diskutiert. Dazu formuliert Hahn:

"Gerade die neuere Forschung hat verstärkt darauf hingewiesen, daß auch in Deutschland schon vor 1800 ein nicht zu unterschätzendes wirtschaftliches Entwicklungspotential vorhanden war. Es ist daher mit gutem Grund sogar vermutet worden, daß Deutschland auch ohne die englische Vorreiterrolle zum Schauplatz einer autochthonen Industriellen Revolution hätte werden können [...]. ${ }^{\text {“578 }}$

Eine solche Blickrichtung wird durch das Lehrwerk nicht angeregt, böte sich aber im Hinblick auf die neuere Rezeption des Themas und im Hinblick auf das zentrale Element der Multiperspektivität an.

\footnotetext{
573 "Vorweg lässt sich sagen, dass Metaphorik in der deutschen AWS eine sehr große Rolle spielt. Es sind keineswegs nur Übertragungen auf mentale Vorgänge, die hier zu beobachten sind. Eine große Menge ursprünglich konkreter Verben, deren Grundbedeutungen entweder sinnlich oder räumlich zu verstehen sind, werden auf das wissenschaftliche Handeln mit seinen prozessualen Aspekten und seinen Ergebnissen, bis in die Textproduktion hinein, bezogen und dabei in verschiedene Richtungen expandiert." Aus: Graefen, Gabriele (2008) Versteckte Metaphorik - ein Problem im Umgang mit der fremden deutschen Wissenschaftssprache. In: Dalmas, Martine; Foschi-Albert, Marina; Neuland, Eva (Hrg.) Wissenschaftliche Textsorten im Germanistikstudium deutsch-italienisch-französisch kontrastiv. Trilaterales Forschungsprojekt in der Villa Vigoni (2007-2008). 2. Auflage (2013), Teil 2, 151-168. S. 153. Online verfügbar unter: www.villavigoni.it/contents/editions/ VV_Gesamtmanuskript_nuova_edizione_04.03.13.pdf (zuletzt eingesehen am 02.06.18).

${ }^{574}$ Lucas, Friedrich J. (1975), S. 331.

${ }^{575}$ Ebd.

${ }^{576}$ Ebd.

577 Vgl. dazu: Graefen, Gabriele (2008), S. 158.

578 Hahn, Hans Werner (2011), S. 7.
} 
Der Hinweis im Darstellungstext auf den bis in die Gegenwart andauernden Prozess der Industrialisierung deutet im Zusammenhang mit der Einschätzung ihrer Wirksamkeit ein Werturteil an.

Direkt Handelnde werden in diesem Abschnitt nicht genannt, es gibt weiterhin keine Distanzierungsmittel, so dass der Textausschnitt kaum als Rekonstruktion von Geschichte bzw. historische Narration erkennbar ist.

Abschnitt 2:

„Die Beantwortung der Frage, warum England eine Pionierrolle im Industrialisierungsprozess einnahm, ist also eng verknüpft mit den Ursachen der Industriellen Revolution. Historiker nennen mehrere begünstigende Umstände und Vorgänge, die unabhängig voneinander das englische Wirtschaftswachstum beschleunigt haben. Zu den wichtigsten gehören die schnellen Fortschritte in der Landwirtschaft (M 4, M 6), die die stark wachsende Bevölkerung ernähren und ihr einen gewissen Wohlstand garantieren konnte. Die Bevölkerungszunahme und die Tatsache, dass die Landbevölkerung nicht an die Scholle gebunden war, sondern in den Städten nach neuen Betätigungsfeldern suchte, sorgten sowohl für ein großes Angebot an Arbeitskräften als auch für eine steigende Güternachfrage auf dem englischen Binnenmarkt. England besaß außerdem große und leicht abzubauende Kohlevorkommen, kurze und kostengünstige Verkehrswege und ausreichend Kapital zum Investieren. Hinzu kam, dass der Staat den Unternehmern durch innere Reformen die für die Betätigung notwendigen Freiräume verschaffte. Zudem erlaubte die relativ offene Gesellschaftsstruktur des Königreichs flexible Reaktionen auf die unterschiedlichsten wirtschaftlichen Herausforderungen. ${ }^{\text {"579 }}$

In diesem Abschnitt fallen die von Autoren- oder Verlagsseite blau markierten Begriffe auf, die durch diese farbliche Heraushebung als inhaltlich besonders bedeutsam herausgestellt werden. Auf sie wird im Folgenden noch näher eingegangen.

Zunächst wird in diesem Textausschnitt die Annahme einer englischen Pionierrolle mit den Ursachen für den frühen englischen Start der Industriellen Revolution zusammengeführt. Da der Begriff ,Ursachen' blau markiert ist, kann er für die Leser als Strukturhilfe dienen. Auf der Basis von Aussagen nicht näher genannter Historiker werden nun solche Ursachen benannt. Sprachlich ist der allgemeine Verweis auf ,Historiker' als Behauptung zu werten, auch wenn er durch Fachliteratur sicherlich fundiert werden könnte. Zwar kann davon ausgegangen werden, dass die Nennung einzelner Historiker, auf die sich die folgenden Aussagen stützen, für die Lernenden in diesem unterrichtlichen Zusammenhang weniger von Bedeutung ist. Da die Sekundarstufe II jedoch wissenschaftspropädeutisches Lernen anbahnen soll, wäre aus didaktischer Sicht eine solche direkte Bezugnahme konsequent.

579 Laschewski-Müller, Karin; Rauh, Robert (Hrsg.) (2010), S. 257. 
Die nachfolgenden Ursachen sind ebenfalls blau markiert, so dass sie optisch schnell erfasst werden können. Die Einordnung „zu den wichtigsten gehörten“580 verweist auf ein Urteil, dessen Grundlage vermutlich die Historikeraussagen sind. Zunächst werden die „Fortschritte in der Landwirtschaft" genannt. Sie werden nicht weiter aufgeschlüsselt, der Hinweis auf die nachfolgenden Materialien überlässt dies der Aufarbeitung durch die Lernenden, die durch die konkreten Materialangaben fokussiert auf die genannten Materialien zugreifen können. Weiterhin wird auf die stark wachsende Bevölkerung verwiesen. Hier gibt es weder eine Aufschlüsselung in konkrete Zahlen noch einen Verweis auf weiterführende Materialien, so dass diese Aussage für die Lernenden ungeprüft bleiben muss. Als weitere Ursachen werden das "große Angebot an Arbeitskräften“ und „eine steigende Güternachfrage" benannt und ebenfalls blau markiert. Die Begründung für diese Ursachen wird den Begriffen vorangestellt, was zu einem inhaltlich sehr dichten Aussagegefüge in einem Hauptsatz führt, der zudem durch ein zweigliedriges Nebensatzgefüge unterbrochen und so inhaltlich noch stärker verdichtet wird. Bedeutsam erscheint die im Nebensatz verwendete metaphorische Wendung „(nicht) an die Scholle gebunden", die nicht erklärt wird und nicht in den alltäglichen Sprachgebrauch gehört, aber wesentlich für das Verständnis des Zusammenhangs ist. Immerhin gibt sie Aufschluss über Besitz- und Lebensverhältnisse und erklärt die fehlende Bindung an ein Stück Land bzw. an einen Herrn. Der Ausdruck beinhaltet damit ein Konzept, das auf die Spätantike (Latifundienbesitz), in diesem Zusammenhang aber eher auf die mittelalterliche Leibeigenschaft zurückgeht. Wieso die fehlende Bindung an die Scholle jedoch zu einer steigenden Güternachfrage führt (beim Bezug auf die wachsende Bevölkerung ist dies über Weltwissen erschließbar), muss vom Leser indirekt erschlossen werden. Hierüber gibt der Text keinen konkreten Aufschluss.

Im Folgenden werden in einem additiven Stil weitere Gründe für die Pionierstellung Englands gegeben. Dazu gehören Kohlevorkommen, Verkehrswege und Kapital sowie die Reformbereitschaft des Staates. Diese Aspekte werden nicht weiter ausgeführt. Abschließend wird auf die Gesellschaftsstruktur Englands verwiesen, die als offen bezeichnet wird und als bedeutsam für die Wirtschaft herausgestellt wird. Der Begriff ,offene Gesellschaftsstruktur' ist wieder blau markiert. Inwiefern von Offenheit die Rede sein kann, wird nicht ausgeführt.

In diesem Abschnitt erscheint mit dem Verweis auf „Historiker" die erste, wenn auch allgemeine, Personalisierung, die allerdings durch die fehlende Namensnennung eher verschleiert als erklärt.

Es fällt gerade in Bezug auf den Aufzählungsstil eine starke und gehäufte Verwendung von Attribuierungen auf, die die Bezugswörter ausdifferenzieren. Hinter ihnen verstecken sich zum Teil komplexe Konzepte, wie etwa im Falle der „offenen Gesellschaftsstrukturen“.

\footnotetext{
${ }^{580}$ Die in den Erläuterungen in Anführungsstrichen gekennzeichneten Wiederaufnahmen aus dem Darstellungstext werden als bereits den Autoren zugeordnete Textteilen angesehen und nicht erneut zitiert. Dies dient dem besseren Lesefluss, soll aber keinesfalls die Urheberschaft infrage stellen.
} 
Abschnitt 3:

"Aufsehenerregende Erfindungen wie die Dampfmaschine von Watt (1765/69) oder die Spinnmaschinen von Hargreaves („Spinning Jenny", 1764) und Arkwright (1769) beschleunigten das englische Wirtschaftswachstum. Mit der Mechanisierung der Baumwollspinnerei, die zum ersten Führungssektor in der englischen Industriegeschichte aufstieg, begann das Zeitalter der Massenproduktion im Textilgewerbe. Für die Entfesselung der Wirtschaftskräfte noch bedeutsamer wurde jedoch das Vordringen des Energieträgers Kohle und der damit einhergehende Ausbau der Eisenindustrie. Die Verbilligung und Verbesserung des Eisens sowie die Modernisierung der Produktionsverfahren schufen die Voraussetzungen für die Entstehung einer leistungsfähigen Maschinenindustrie und später für den Eisenbahnbau. Damit wirkte die Eisenindustrie in viele andere Wirtschaftszweige hinein und veränderte vom Verkehrswesen bis zu individuellen Reisen alle Bereiche des gesellschaftlichen Lebens. " ${ }^{281}$

Der dritte Abschnitt schließt mit konkreten Erfindungen an, die die Industrialisierung angetrieben haben. Es handelt sich um die Dampfmaschine und die Spinnmaschinen. Ziegler erläutert die Berechtigung, gerade diese Erfindungen herauszuheben:

„Selbstverständlich ist der europäische Weg der Industrialisierung nicht ohne Kohle und Koks, die Dampfmaschine, die ,Spinning Jenny als erste Baumwollspinnmaschine oder die Stahlgewinnung durch das ,Puddeln` vorstellbar. Diese und andere technische Errungenschaften stellen insofern eine notwendige Bedingung für die Industrialisierung dar, aber hinreichend sind sie noch lange nicht. ${ }^{4582}$

Dem Gedanken, dass diese Erfindungen noch keine hinreichende Bedingung für die Industrialisierung gewesen seien, folgen auch die Autoren des Darstellungstextes, wenn sie mit weiteren Voraussetzungen für die Entfaltung dieser anschließen. Als weitere Ursache wird so der Ausdruck der „Massenproduktion im Textilgewerbe“ blau hervorgehoben, da diese erst durch die Spinnmaschinen ermöglicht wurde. Die Autoren ordnen jedoch die Bedeutung von Kohle- und Eisenindustrie als bedeutsamer ein. Sie sprechen in diesem Zusammenhang von der "Entfesselung der Wirtschaftskräfte“. Dabei handelt es sich wieder um eine figurative oder metaphorische Sprache ${ }^{583}$, die eine emotionalisierende Wirkung und Einordnung des Beginns der Industrialisierung schafft, indem sie den Eindruck eines haltlosen Ausbrechens vermittelt, das als Befreiung einzuordnen ist.

Insgesamt wird die Beschleunigung englischen Wirtschaftswachstums, die durch den Ausdruck der ,entfesselten Wirtschaftskräfte' besonders hervorgehoben wird, als entscheidendes Merkmal in der Forschung jedoch inzwischen kritisch gesehen:

„Insbesondere für Großbritannien konnte nachgewiesen werden, dass das Wachstum dank einer entwickelten handwerklichen Produktion und heimgewerblicher Protoindustrie während der Jahrzehnte vor dem Beginn des "Maschinenzeitalters", der "industriellen Revolution", bisher unterschätzt worden war. Da das Ausgangsniveau des Bruttosozialprodukts in den sechziger Jahren des 18. Jahrhunderts demzufolge höher war, als bisher angenommen, konnte das Wachstum nicht

\footnotetext{
581 Ebd.

582 Ziegler, Dieter (2009), S. 1.

${ }^{583}$ Vgl. hierzu: Lucas, Friedrich J. (1975), S. 330.
} 
so rasant gewesen sein, wie es die älteren Arbeiten noch angenommen hatten. Man geht deshalb von einer graduellen Beschleunigung des Wirtschaftswachstums aus. " 584

Dieser Ansatz findet im Schulbuchtext keine Berücksichtigung.

Im Darstellungstext werden dann die Faktoren benannt, die für die Entfesselung als Auslöser gesehen werden. Zusammenfassend wird die Eisenindustrie (als Schlagwort ebf. blau markiert) als wesentlicher Einflussfaktor für die Industrialisierung gewertet, die bis in die Gesellschaft hineingewirkt habe. Hier handelt es sich um eine Einordnung der zuvor genannten Faktoren im Sinne einer Beurteilung.

Die Argumentation wird gerade in diesem Abschnitt über einen starken Nominalstil versprachlicht, Distanzierungsmittel werden nicht genutzt, so dass ein hoher Faktizitätsanspruch vermittelt wird.

\section{Unterkapitel 2 (1 Abschnitt):}

„Nachzügler' Deutschland

Im Vergleich zu England war Deutschland ein "Nachzügler" in der Industrialisierungsgeschichte (M 7 a-d). Es war noch im Stadium der Frühindustrialisierung, als in England seit den 1770er-Jahren die Indus-trielle Revolution einsetzte (s. M 5, S. 255). Die Unterschiede erklären sich aus verschiedenartigen Ausgangssituationen. Bis zum Ende des Heiligen Römischen Reiches Deutscher Nation im Jahre 1806 war Deutschland in 300 zum Teil ausgesprochen kleine Territorialstaaten zersplittert. Eine Vielzahl von Zollschranken, abweichende Maß-, Münz- und Gewichtssysteme, Handelsmonopole sowie schlecht erschlossene Verkehrsverbindungen hemmten die wirtschaftliche Expansion. Trotz mancher Fortschritte bei der Agrarproduktion blieb die deutsche Landwirtschaft weit hinter den Leistungen der englischen zurück. Es überwogen ertragsschwache Kleinbetriebe (ca. 70-80\% aller Höfe), deren Betreiber oft einem Nebenerwerb nachgehen mussten, um ihre Existenz zu sichern. Die Abhängigkeiten der bäuerlichen Bevölkerung von ihren Gutsherren waren häufig noch so stark, dass dadurch die zur Bildung freier Arbeitsmärkte notwendige individuelle Mobilität eingeschränkt war. Feudale Abgaben, staatliche Steuern und große Unterschiede bei der Verteilung des Wohlstandes behinderten die Entstehung von Massenkaufkraft, die der gewerblichen Wirtschaft hätten zugute kommen können. Auch war die deutsche Gesellschaftsstruktur nicht so offen wie in England. Starre Standesschranken und konservative Grundeinstellungen engten den Spielraum für innovatorisches Denken und Handeln ein. Und im Handwerk bildete das Festhalten an der überkommenen Zunftverfassung ein zentrales Hindernis für individuelles Erfolgsstreben und wirtschaftliche Neuerungen. Im Gegensatz zu England gängelten die absolutistischen deutschen Fürsten mit ihren merkantilistischen Konzepten (s. S. 164 f.) die wirtschaftliche Entwicklung durch massive Eingriffe und Beschränkungen; staatsfreie Märkte für Kapital, Boden und Waren konnten sich daher nur schwer entfalten." "585

Der zweite Untertitel führt den ersten fort, nach der Ausweisung Englands als Pionierland wird ,Deutschland' hier als Nachzügler benannt. Damit wird zugleich ein Vergleich angelegt, der im ersten Satz des Abschnittes expliziert wird. Die Angabe der zugehörigen Materialien zu dieser Aussage erhält

\footnotetext{
${ }^{584}$ Ziegler, Dieter (2009), S. 5.

585 Laschewski-Müller, Karin; Rauh, Robert (Hrsg.) (2010), S. $257 f$.
} 
so eine Belegfunktion. Es fehlt dabei ein Hinweis darauf, dass mit dem Begriff ,Deutschland' noch kein Nationalstaat gemeint ist.

Insgesamt wird das Pionier-Nachzügler-Konzept in der Forschung diskutiert. Hahn bezeichnet es in Anlehnung an Ziegler dennoch als ertragreich, auch wenn in der neueren Forschung die Industrialisierung stärker als zusammenhängender Prozess untersucht würde. ${ }^{586}$ Diese Debatte bildet der Darstellungstext nicht $a b$, vielmehr wird hierin eine Setzung vorgenommen. Dabei könnte dieses Konzept erneut dazu dienen, den Konstruktionscharakter von Geschichte zu thematisieren.

Um den Vergleich auszuführen, wird im Darstellungstext auf das Konzept der dreiphasigen Industrialisierung zurückgegriffen. Dann folgt die Erklärung für die unterschiedliche Entfaltung der Industrialisierung. Dazu wird in erster Linie die Struktur ,Deutschlands' benannt. In Analogie zu den besonderen Voraussetzungen Englands werden nun die ,deutschen' Gegebenheiten dargestellt, wie Verkehrswege, Zollschranken etc., dies schafft eine direkte strukturelle Vergleichbarkeit. Besonders werden dann die landwirtschaftlichen Bedingungen herausgestellt. War in Bezug auf England von einer fehlenden Bindung an die Scholle die Rede, heben die Autoren nun die bäuerliche Abhängigkeit vom Grundherrn hervor. So wird ein direkter Gegensatz aufgebaut. Sehr abstrakt benennen die Autoren Faktoren, die den Aufbau einer Massenkaufkraft behindert hätten. Ihr Fokus liegt im Weiteren auf dem Gesellschaftssystem, das (auch hier lässt sich der direkte Gegensatz zu England herstellen) sie als nicht offen, sondern durch starre Standesschranken gekennzeichnet beschreiben. Diese Annahme wird durch den Verweis auf die Zunftstrukturen untermauert. Insbesondere das Adjektiv „überkommen “ wertet die ,deutschen' Gesellschaftsstrukturen im Sinne eines Sachurteils als nicht mehr zeitgemäß.

Durch die Herstellung eines direkten Gegensatzes („Im Gegensatz zu England“) wird der Vergleich nicht nur am deutlichsten in diesem Abschnitt expliziert, sondern durch die Verwendung des Verbs „gängeln“ auch stark wertend. Die Einordnung der unterschiedlichen Strukturen wird dem Leser damit abgenommen.

\footnotetext{
${ }^{586}$ Hahn, Hans-Werner (2011), S. 63.
} 


\section{Unterkapitel 3 (4 Abschnitte):}

\section{Abschnitt 1:}

\section{"Staatliche Reformen}

Erst im Verlauf des 19. Jahrhunderts wurden diese Hemmnisse für eine dynamische Industriegesellschaft und -wirtschaft allmählich beseitigt. Dabei nahm der Staat eine herausragende Rolle ein. Durch die Liberalisierung der Agrar- und Gewerbeverfassung, den Abbau von Zollschranken oder die Vereinheitlichung des Rechts- und Finanzwesens schuf er die Voraussetzungen zur Entfesselung einer modernen Wirtschafts- und für den Übergang zur modernen Marktgesellschaft. ${ }^{1587}$

Der erste Abschnitt thematisiert die Bedeutung der staatlichen Voraussetzungen für den Verlauf des Industrialisierungsprozesses. Die Bedeutung des Staates wird im Text als „herausragend" bewertet. Eine nachfolgende Aufzählung soll diese Einschätzung belegen. Durch die Wertung mithilfe des Adjektivs „herausragend“ wird wiederum eine Beurteilung vorgenommen. Als erster Grund wird die Liberalisierung im Agrar- und Gewerbesektor benannt. Der Begriff „Liberalisierung“ ist blau unterlegt. Dies kann entweder mit der besonderen Bedeutung der Liberalisierung erklärt werden oder mit der Überlegung, dass es sich ggf. um einen erklärungswürdigen Begriff handelt. Dies kann jedoch durch den Text selber nicht geklärt werden. Die Aufhebung feudaler Strukturen ist für ,Deutschland' von besonderer Bedeutung gewesen, so dass die Erstnennung der veränderten Agrarverfassung im Darstellungstext sinnvoll ist, ebenso kann die Bedeutung der Gewerbereform eingeschätzt werden. ${ }^{588}$ Der Länge des Darstellungstextes ist vermutlich geschuldet, dass dieser bedeutsame Bereich nur benannt wird. Die Erschließung, warum gerade diese Bereiche wichtig für die Entwicklung waren, wird dadurch allerdings erschwert.

Für die Darstellung der Reformen wird auf die Nennung konkreter Personen, die den Reformprozess vorantrieben, verzichtet, Handelnde also nicht personalisiert. Stattdessen wird der Staat im Text zur handelnden Person gemacht.

Es folgt die Nennung der Reformen in den weiteren Sektoren. Diese Faktoren werden abschließend als Voraussetzung für die Industrialisierung eingeschätzt. In diesem Zusammenhang fällt erneut der Ausdruck „Entfesselung“ auf, der zuvor schon im Text aufgetaucht ist. Seine Bedeutung wird so verstärkt. Hier liegt der Schluss nahe, dass es sich um den bewussten Einsatz dieses Begriffs handelt. Zudem wird im letzten (durch das Bindestrichwort für das Verständnis nicht einfachen) Satz des Abschnitts der Eindruck erweckt, dass durch die Reformbestrebungen für die moderne Wirtschafts- und Marktgesellschaft die Voraussetzungen geschaffen wurden. Der Gebrauch des Begriffs „Entfesselung“ lässt erneut den Eindruck entstehen, dass es sich um einen von da ab unumkehrbaren, ,Naturgewalten' gleichkommenden Prozess der Befreiung gehandelt habe.

\footnotetext{
587 Ebd. S. 258.

${ }^{588}$ Vgl. Ziegler, Dieter (2009), S. 16.
} 
Abschnitt 2:

„Entscheidende Grundlagen dafür wurden in den preußischen Reformen während der ersten beiden Jahrzehnte des 19. Jahrhunderts gelegt, die das Überleben und den Wiederaufbau des von den napoleonischen Armeen besiegten Landes garantieren sollten (s. S. 232). Bei ihren Wirtschafts- und Gesellschaftsreformen orientierten sich Staat und Bürokratie Preußens auch am englischen Vorbild. Die Reformbeamten, die sich seit 1807 an die Modernisierung des preußischen Staates begaben, kannten nicht nur die englische Entwicklung. Auch "die Bibel des Kapitalismus", Adam Smiths Buch über den "Wohlstand der Nationen" ("The Wealth of Nations", M 8) hatte bei den Reformkräften in Deutschland begeisterte Aufnahme gefunden. Die Leitbegriffe dieses Werks, allen voran "Besitzindividualismus", "Leistungsprinzip", „Arbeitsteilung", „freie Märkte" und „Konkurrenz" prägten daher die Wirtsschaftsund Gesellschaftsreformen auf dem Kontinent. “589

In Abschnitt 2 fällt ein Wechsel in der Terminologisierung auf. War bisher von ,Deutschland' die Rede, musste dem Leser klar sein, dass es sich zum Beginn der Industrialisierung nicht um ein Nationalstaatsgebilde handelte (s.o.). In diesem Abschnitt ist nun von Preußen die Rede. Der Wechsel wird nicht thematisiert, sondern als bekannt vorausgesetzt. Die für die Industrialisierung bedeutsamen Inhalte der Reformen werden nachfolgend angesprochen. Sie werden in diesem ersten Satz des Abschnitts zudem in den Zusammenhang der Unterwerfung Preußens durch Napoleon und die anschließenden Befreiungskriege eingeordnet. Allerdings ist hier eher implizit vom „Wiederaufbau des von den napoleonischen Armeen besiegten Landes" die Rede, so dass der Leser den Zusammenhang (unterstützt durch den Verweis auf eine Seite im Lehrwerk) selbst herstellen muss.

Ganz allgemein bleibt der Hinweis im nachfolgenden Satz, dass sich die preußischen Reformer "auch" am englischen Vorbild orientierten, offen. Inwiefern dies geschah, wird nicht dargestellt. Der Verweis auf England dient vor allem der Überleitung auf das Hauptwerk Adam Smiths. Hieraus werden die Leitmotive benannt, welche die preußischen Reformer für sich übernahmen. Bei diesen Begriffen handelt es sich um Fachvokabular, mehrheitlich in Form von zweigliedrigen Komposita, wie z. B. „Besitzindividualismus". Die Begriffe weisen durchweg ein hohes Abstraktionsniveau auf, werden aber nur benannt und nicht weiter erklärt.

Zwar werden Handelnde in diesem Abschnitt vergleichsweise stärker personalisiert als in den Abschnitten zuvor, jedoch erweisen sich die Verweisstrukturen in Bezug auf die Reformer als divergent. So wird zunächst über die Nutzung des Passivs kein Handelnder genannt, dann ist als abstrakte Größe von „Staat und Bürokratie Preußens" die Rede, danach von den „Reformbeamten“. Der Bezug auf die Reformer wird im nächsten Satz anschließend über die „Reformkräfte“ hergestellt. Damit bleiben die Reformer nicht nur eine weitgehend abstrakte Größe, der Text bezieht sich zudem auf unterschiedliche Bevölkerungsgruppen, die erkannt werden müssen: im engeren Sinne auf die Reformbeamten, im

${ }^{589}$ Laschewski-Müller, Karin; Rauh, Robert (Hrsg.) (2010), S. 258. 
weiteren Sinne auf die staatlichen Entscheidungsträger (,Staat“) und zum Schluss auf alle gesellschaftlichen Reformkräfte.

Insgesamt weist der Abschnitt eine sehr hohe Informationsdichte auf, die vor allem durch allgemeine und konkrete Verweise entsteht und dem Leser ein hohes Verständnis für die Textverweise und ein gutes geschichtliches Wissen abverlangt.

\section{Abschnitt 3:}

„Das zeigte sich am preußischen Oktoberedikt von 1807 (M 9), das eine Mischung aus politischem Manifest und national-ökonomischem Programm darstellte. An die Stelle einer gebundenen Ständegesellschaft, die jedem Menschen eine feste, durch Geburt und Recht erworbene soziale Position zuwies, sollte nun eine mobile Marktgesellschaft treten. Dazu passten auch die Ablösung sozialer Abhängigkeitsverhältnisse auf dem Land und der Erlass der Gewerbefreiheit im Jahre 1810. Die Reformbürokratie wollte vor allem durch die Einführung der allgemeinen Gewerbefreiheit die Wirtschaftskraft des Landes stärken und damit zugleich die Steuereinnahmen des Staates erhöhen. Am Ideal einer Gesellschaft freier Wirtschaftssubjekte waren zudem die Bemühungen ausgerichtet, die die Macht der Zünfte brechen und die traditionellen Begrenzungen gewerblicher Produktion aufheben sollten. Die Reformer wollten damit alle kreativen Energien des Landes mobilisieren und eine dynamische Konkurrenzwirtschaft begründen, die den Wohlstand der Einwohner und die Macht des Staates garantierte. ${ }^{1590}$

Der Abschnitt wird mit dem Verweis auf das Oktoberedikt eingeleitet, die besondere Bedeutung wird durch die blaue Markierung herausgestellt. Der Begriff selber wird nicht weiter erläutert, im Nebensatz werden nur kurz die übergeordneten Gestaltungsprinzipien benannt. Diese Prinzipien werden durch weiteres Fachvokabular (z. B. "Manifest") dargestellt, das als bekannt vorausgesetzt wird. Die Veränderungen, die dieses Edikt forderte, werden in einem Vergleich (alt-neu) dargestellt. Dabei wird das zuvor bereits am Beispiel der Feudalherrschaft als überkommen beschriebene Prinzip der Ständegesellschaft einer neuen „mobilen Marktgesellschaft“ gegenübergestellt. Daraus ist eine Gegensätzlichkeit ableitbar, nicht weiter erläutert wird jedoch die Verwendung des Begriffs „mobil“ in diesem Zusammenhang. Im folgenden Satz wird das Prinzip an weiteren Forderungen des Edikts ausgeführt, dazu wird die „Ablösung sozialer Abhängigkeitsverhältnisse“ genannt. Auf der Grundlage des Textes kann dieser Sachverhalt abgeleitet werden, allerdings kann die nachfolgende Forderung in diesem Zusammenhang ein Missverständnis produzieren: Es ist im Folgenden vom „Erlass der Gewerbefreiheit" die Rede, der Begriff "Gewerbefreiheit" ist wiederum als besonders bedeutsam blau markiert. Nach der Verwendung des Ausdrucks „Ablösung" kann der „Erlass" in der logischen Folge im Sinne von ,entbinden von' verstanden werden, er meint jedoch hier eine Anordnung (im Sinne von ,Edikt'). Diejenigen, die die Reform vorantrieben, werden weiter als „Reformbürokratie“ benannt. Dieser Begriff kann aufgrund seines hohen Abstraktionsgrades und der Tatsache, dass dadurch die Handelnden nicht direkt benannt werden, Lucas entsprechend in den Bereich der "verschleiernden Nomina“ 591

\footnotetext{
590 Ebd.

${ }^{591}$ Vgl. Lucas, Friedrich J. (1975), S. 329.
} 
eingeordnet werden. Zudem wird die Haltung dieser Gruppe als einheitlich beschrieben, wenn ausgesagt wird, dass diese vor allem etwa die Wirtschaftskraft erhöhen wollte. Der Ausdruck „Ideal einer Gesellschaft freier Wirtschaftssubjekte" im folgenden Satz erhöht die Abstraktion weiter. Zwar soll am Beispiel des Versuchs, die Macht der Zünfte zu brechen, der Ausdruck konkretisiert werden, jedoch muss dazu zunächst die Bedeutung des Ideals in diesem Zusammenhang geklärt sein. Abschließend wird die Zielsetzung der Reformer für die Bevölkerung und den Staat auf den Punkt gebracht.

\section{Abschnitt 4:}

„Der Staat beseitigte zum einen die rechtlichen Hindernisse, die den Wirtschaftsaufschwung behinderten. Zu diesem Zweck schuf er Bedingungen für die Entstehung freier Arbeits-, Kapital- und Bodenmärkte. Zudem wollten die Reformer infrastrukturelle Voraussetzungen schaffen, die eine Verbindung einzelner Wirtschaftsfaktoren ermöglichten. Mithilfe staatlicher Investitionen wurde das Verkehrsnetz erweitert und leistungsfähiger gemacht. Durch die Abschaffung von Zollschranken wurde der Binnenmarkt ausgebaut - ein Prozess, der mit dem 1834 gegründeten Zollverein seinen vorläufigen Höhepunkt erreichte. Staatliche Gewerbeschulen und-akademien wurden errichtet, mit denen Preußen seinen technologischen Rückstand gegenüber der englischen Industriekonkurrenz aufzuholen hoffte. "592

In Abschnitt 4 wird eingangs die Bezeichnung „der Staat“ verwendet. Auf der Grundlage des vorigen Abschnitts ist hier der Bezug zu Preußen als Staat im Deutschen Bund bzw. im 1834 gegründeten Deutschen Zollverein herzustellen, erst im letzten Satz wird dieser Bezug erneut expliziert. Zudem erscheint der Staat wieder als handelnde Person im Sinne des rhetorischen Stilmittels der Personifikation. Die tatsächlich handelnden Personen (zunächst die Reformer) werden hier nicht benannt. Die Formulierung durch eine Personifikation wird im folgenden Satz fortgesetzt, bevor im nachfolgenden Satz die Bezeichnung erneut zu den Reformern (ohne diese beim Namen zu nennen) wechselt. Dies muss durch den Leser als Synonym verstanden werden.

In der Grundstruktur werden zwei Hauptleistungen ,des Staates' genannt, diese werden eingeleitet durch ,zum einen' und ,zudem'. Der Abschnitt weist verschiedene Verweisstrukturen auf, die mit der Leistung des Staates in Zusammenhang gebracht werden müssen oder synonymhaft auf den Staat rückverweisen. Die zwei Hauptleistungen werden als Beseitigung rechtlicher Hindernisse und Schaffung infrastruktureller Voraussetzungen benannt. Beide Punkte werden durch die Nennung von Beispielen unterstützt. Diese Beispiele haben Aufzählungscharakter und werden den Leser*innen durch den Nominalstil in hochabstrakter Form vermittelt, wenn etwa von „Bedingungen für die Entstehung freier Arbeits-, Kapital- und Bodenmärkte" die Rede ist. War in den ersten Teilen die Darstellung auf historische Zusammenhänge fokussiert, sind in diesem Teil für das Textverständnis insgesamt vermehrt wirtschaftliche Kenntnisse von Bedeutung. Durch den Verweis auf den Zollverein, der der letzte durch blaue Farbe hervorgehobene Begriff ist, wird der hauptsächlich wirtschaftsfachliche Wissensbereich in den historischen Erkenntnisbereich zurückgeführt. Abschließend ist erneut von Preußen die Rede, das durch die dargestellten Maßnahmen den Rückstand aufzuholen hoffte. Von ,Deutschland'

\footnotetext{
$592 \mathrm{Ebd}$
} 
ist nicht mehr die Rede. Die Frage, ob bzw. inwiefern ,Deutschland' den dargestellten Rückstand aufholen konnte, wird hier nicht mehr beantwortet, allerdings beschäftigt sich das nachfolgende Kapitel mit der Entwicklung der Industriewirtschaft.

\subsubsection{Fazit}

Der vorliegende Darstellungstext nimmt Bezug auf die Entstehung der Industriegesellschaft, indem er im Vergleich von England und Deutschland unterschiedliche Entwicklungen aufzeigt. Pandel ist einerseits recht zu geben, wenn er Schulbücher und damit auch die darin befindlichen Darstellungstexte als „kondensierte Nacherzählung “593 kennzeichnet: „Dass solche verdichteten Erzählungen hohe Verständnisschwierigkeiten bei Schülerinnen und Schülern bewirken, ist offensichtlich. " ${ }^{594}$ Gleichzeitig muss aber auch in Anlehnung an Onken festgestellt werden, dass im Text auf der Grundlage eines erweiterten Erzählbegriffs narrative Strukturen festzustellen sind, da er bspw. über vorweggenommene Vergleichsaspekte und Kausalbeziehungen Kohärenzen herstellt ${ }^{595}$. Er birgt, hier ist Pandel zuzustimmen, einige Herausforderungen: Aufgrund der Länge von mehr als zwei Seiten wird auf viele Aspekte Bezug genommen, die aber zum Teil nur schlagwortartig angerissen werden. Der Text ist für eine Oberstufe vorgesehen, was den relativ hohen Komplexitätsgrad erklärt. ${ }^{596}$ Dies ist mit Fortschreiten des Textes insbesondere an der zunehmenden Häufung von Fachbegriffen festzumachen, die aus dem historischen, aber auch aus dem wirtschaftlichen Kontext stammen.

Beispielhaft konnte gezeigt werden, welchen Einfluss die Verwendung von Metaphern als Konstruktionsform der Alläglichen Wissenschaftssprache auf die Textaussage hat. Dieser Zusammenhang könnte sicherlich an weiteren Beispielen vervollständigt werden, gerade aber das Beispiel der ,Entfesselung der Marktwirtschaft' zeigt die hohe Bedeutung der Lexik und der Konzepte, die hinter einzelnen Begriffen stehen und zu einer versteckten Wertung führen. ${ }^{597}$

Für die nachfolgende Lösung der Aufgabe ist bedeutsam, dass der Text den geforderten Vergleich von England und ,Deutschland' zum Teil vorwegnimmt, da darin schon sprachliche Vergleichsstrukturen aufgebaut werden. Dies wird erreicht, indem im ersten Unterkapitel die Entwicklung Englands an bestimmten Schlagwörtern aufgezeigt wird. Diese Schlagwörter werden insbesondere im zweiten Unterkapitel wiederaufgenommen, indem an ihrem Beispiel die Entwicklung ,Deutschlands' aufgezeigt

\footnotetext{
593 Pandel, Hans-Jürgen (2010). S. 154.

${ }^{594} \mathrm{Ebd}$.

${ }^{595} \mathrm{Vgl}$. Onken, Björn (2016): Theorie und Praxis im Konflikt. Überlegungen zur Narrativität von Verfassertexten in Geschichtsschulbüchern. In: Buchsteiner, Martin; Nitsche, Martin (Hrsg.): Historisches Erzählen und Lernen: Historische, theoretische, empirische und pragmatische Erkundungen. Wiesbaden: Springer VS. S. 69- 84. S. 78.

${ }^{596}$ Schrader ordnet im Rahmen ihrer Korpusanalyse Darstellungen als ,im wesentlichen ausformulierte Ereignisdarstellungen mit leichten, oft implizit-einseitigen, Argumentationssätzen“ ein (vgl. Schrader, 2013, S. 95). Für den vorliegenden Text ist die Einordnung insgesamt übertragbar, wenngleich festgestellt werden muss, dass die Formulierung der Argumentationssätze nicht als, leicht', sondern als durchaus komplex zu bezeichnen ist.

${ }^{597}$ Zur Bedeutung von Metaphern für die Geschichte vgl. auch Demandt, Alexander (1987): Metaphern für Geschichte. Sprachbilder und Gleichnisse im historisch-politischen Denken. München: Beck Verlag. Demandt ordnet Metaphern (er versteht den Metaphernbegriff in einem weiten Sinne) als Werkzeug ein (vgl. ebd. S. 9), er bewertet sie kritisch, wenn er feststellt: Bilder ziehen Bilanz. Sie zeigen, wo der Autor zu denken aufgehört hat." (ebd., S. 448).
} 
wird. Die Aussagen könnten also übernommen und direkt in eine andere Darstellungsform (z. B. eine Tabelle) überführt werden. Dabei werden an manchen Stellen bereits Beurteilungen vorgenommen, die, ohne sie zu hinterfragen, ebenfalls für die Lösung der Vergleichsaufgabe übernommen werden könnten; solche Beurteilungen verstecken sich in wertenden Adjektiven und Verben.

Eine weitere Herausforderung stellt der Wechsel der Blickrichtung (von ,Deutschland' zu Preußen) dar, da das Konzept des Begriffs ,Deutschland' nicht thematisiert wird.

Insgesamt bietet der Text eine Vielzahl an Informationen, die eher schlaglichtartig aufgenommen werden, ohne sie zu erklären. Mit diesen können Lernende unterschiedlich umgehen: Sie können diese unkritisch übernehmen oder bestimmte Schlagwörter als Ausgangspunkt nehmen, weitere und vertiefte Zusammenhänge zu erarbeiten. Wie sie damit umgehen, kann in der Folge der Umgang mit der Vergleichsaufgabe zeigen.

Der Text geht mit bestimmten Begriffen, wie etwa dem der Industriellen Revolution, recht unkritisch um. An manchen Stellen hätten, wie bereits dargestellt, Ergebnisse oder Diskussionen im Rahmen der neueren Forschung genauer aufgenommen werden können. Hier ist eine Bezugnahme auf Kontroversen in der wissenschaftlichen Debatte wünschenswert, um so Lernenden gerade der Sekundarstufe II den Konstruktionscharakter von Geschichte und die Bedeutung einer wissenschaftsbasierten Herangehensweise zu verdeutlichen.

Layout und Aufbau des Textes sind insgesamt als übersichtlich einzuordnen, so dass Lernende die verschiedenen Teilkapitel über Teilüberschriften und Absätze des Textes schon optisch erfassen können (ein wichtiger Aspekt bei einem langen Text). Dennoch fügt sich das letzte Unterkapitel inhaltlich in die Darstellung zuvor nur schwer ein. Dies liegt zum einen am expliziten Blick auf Preußen, obwohl nicht geklärt wird, warum ausgerechnet Preußen als Beispiel für die deutsche Entwicklung dient, und an der Verwendung einer Vielzahl an Fachbegriffen. Für die Lösung der Vergleichsaufgabe ist dieser Teil nicht nötig.

Insgesamt muss festgestellt werden, dass der Darstellungstext viele mögliche unterschiedliche und zum Teil sehr anspruchsvolle Herausforderungen birgt, mit denen die Lernenden umgehen müssen, wenn sie die Aufgabe lösen wollen.

\subsection{Einordnung von Text und Aufgabe}

Unter Bezugnahme auf Text und Vergleichsaufgabe soll nun im Hinblick auf die Anforderungen an eine historische Lernaufgabe hinterfragt werden, zu welchen Leistungen die Aufgabe konkret herausfordert. Dazu werden nachfolgend in Bezug auf die für den Vergleich relevanten Aspekte das ,Prozessmodell sprachlichen Handelns' im Geschichtsunterricht von Handro ${ }^{598}$ mit dem Kompetenzmodell ,Schreiben' nach Becker-Mrotzek/Schindler ${ }^{599}$ zusammengeführt ${ }^{600}$ und auf die vorliegende Aufgabe hin angewandt:

\footnotetext{
${ }^{598}$ Vgl. Handro, Saskia (2013), S. 325.

${ }^{599}$ Vgl. Becker-Mrotzek, Michael; Schindler, Kirsten (2007), S. 24.

${ }^{600}$ Vgl. dazu Kap. 2.7.2.
} 
Tabelle 3: Zusammenführung der Prozessmodelle für die Einordung der Vergleichsaufgabe 601

\begin{tabular}{|c|c|c|}
\hline \multicolumn{2}{|c|}{$\begin{array}{l}\text { Darstellung (Erkenntnispräsentation u. Sinnstiftung/ } \\
\text {-reflexion) }\end{array}$} & \multirow{2}{*}{$\begin{array}{l}\text { Anforderungen durch die unter- } \\
\text { suchte Vergleichsaufgabe } \\
\text { Bildung eines abschließenden Sach- } \\
\text { urteils, das über eine Gegenüberstel- } \\
\text { lung argumentiert wird. }\end{array}$} \\
\hline & $\begin{array}{l}\text { Historische Narrationen im Dis- } \\
\underline{\text { kursprozess }}\end{array}$ & \\
\hline \multicolumn{3}{|l|}{ Textmuster } \\
\hline $\begin{array}{l}\text { Kenntnisse über Text- } \\
\text { muster }\end{array}$ & $\begin{array}{l}\text { Welche Teilhandlungen machen } \\
\text { das zugrundeliegende Textmuster } \\
\text { als besondere Form des histor. Ar- } \\
\text { gumentieren (Erklären und Be- } \\
\text { gründen) aus? }\end{array}$ & $\begin{array}{l}\text { Der historische Vergleich als beson- } \\
\text { dere Form des Argumentierens bein- } \\
\text { haltet nachfolgende Teilhandlungen: } \\
\text { Gegenüberstellen, Erklären, Beurtei- } \\
\text { len, Begründen }\end{array}$ \\
\hline $\begin{array}{l}\text { Inhalte elizitieren } \\
\text { und passende Text- } \\
\text { muster realisieren }\end{array}$ & $\begin{array}{l}\text { Welche Textmuster werden in Ab- } \\
\text { hängigkeit vom fachlichen/histor. } \\
\text { Kontext und der geforderten Teil- } \\
\text { handlung benötigt (z. B. Begrün- } \\
\text { den, Erklären...)? }\end{array}$ & $\begin{array}{l}\text { Wissen über: Konzept, Industrielle } \\
\text { Revolution'; Beginn der Industriellen } \\
\text { Revolution in Bezug auf England und } \\
\text { 'Deutschland' (Gesellschaftssysteme, } \\
\text { wirtschaftl. Voraussetzungen etc.), } \\
\text { da diese durch den Text nur angeris- } \\
\text { sen, aber nicht erklärt werden. } \\
\text { Zu den genannten Teilhandlungen } \\
\text { müssen Lernende außerdem Folgen } \\
\text { erkennen können, da der Darstel- } \\
\text { lungstext damit arbeitet. }\end{array}$ \\
\hline $\begin{array}{l}\text { Routinen zum Reali- } \\
\text { sieren der Textmus- } \\
\text { ter }\end{array}$ & $\begin{array}{l}\text { Wie ist ein histor. Argument aufge- } \\
\text { baut? Wie eine Beurteilung? Wie } \\
\text { werden Begründungen und Erklä- } \\
\text { rungen realisiert? Welche sprachli- } \\
\text { chen Mittel werden benötigt? }\end{array}$ & $\begin{array}{l}\text { Das historische ,Argument' im ,Ver- } \\
\text { gleich' funktioniert über das Gegen- } \\
\text { überstellen und das Ableiten von Ge- } \\
\text { meinsamkeiten, Ähnlichkeiten und } \\
\text { Unterschieden. } \\
\text { Ggf. müssen Wissenslücken des Le- } \\
\text { sers über Erklärungen aufgefüllt wer- } \\
\text { den. } \\
\text { Der Vergleich schließt mit einem } \\
\text { Sachurteil. }\end{array}$ \\
\hline
\end{tabular}

${ }^{601}$ Kategorien (Auszüge) nach: Handro, Saskia (2013), S. 321 und Becker-Mrotzek, Michael; Schindler, Kirsten (2007), S. 24. 


\begin{tabular}{|c|c|c|}
\hline & $\begin{array}{l}\text { Welche sprachlichen Mittel wer- } \\
\text { den benötigt? }\end{array}$ & $\begin{array}{l}\text { Sprachliche Mittel des Gegenüber- } \\
\text { stellens, Erklärens, Begründens und } \\
\text { Beurteilens } \\
\text { Bsp.: Für das Gegenüberstellen zur } \\
\text { Darstellung von Unterschieden z. B.: } \\
\text { anders als, gegensätzlich, im Gegen- } \\
\text { satz zu, hingegen, dagegen, begüns- } \\
\text { tigen...behindern, } \\
\text { (noch) nicht (vergleichend ge- } \\
\text { braucht) } \\
\text { Weitere sprachliche Mittel für das } \\
\text { Gegenüberstellen sowie für das Er- } \\
\text { klären, Begründen und Urteilen wer- } \\
\text { den ausführlich dargestellt und auf- } \\
\text { geschlüsselt in Kapitel } 6.2\end{array}$ \\
\hline $\begin{array}{l}\text { Überwachen und Re- } \\
\text { flektieren der Pro- } \\
\text { duktionsprozesse }\end{array}$ & $\begin{array}{l}\text { (z. B. auf der Grundlage einer } \\
\text { Checkliste, die die Fragen aus den } \\
\text { genannten Anforderungen berück- } \\
\text { sichtigt) }\end{array}$ & $\begin{array}{l}\text { Wird durch die vorliegende Aufgabe } \\
\text { nicht angeregt. }\end{array}$ \\
\hline \multicolumn{3}{|l|}{ Leserorientierung } \\
\hline $\begin{array}{l}\text { Kenntnisse über Le- } \\
\text { serorientierung }\end{array}$ & $\begin{array}{l}\text { Welches Interesse hat der Leser } \\
\text { vermutlich? } \\
\text { Welches Vorwissen kann ange- } \\
\text { nommen werden? }\end{array}$ & $\begin{array}{l}\text { Hier müsste im Vorfeld geklärt wer- } \\
\text { den, wer der Leser ist: Lehrkraft? Ler- } \\
\text { nende? } \\
\text { Klärung der Ausgangsbedingungen } \\
\text { und Herausarbeitung hemmender } \\
\text { und fördernder Strukturen für die In- } \\
\text { dustrialisierung } \\
\text { Je nach Leser: } \\
\text { Lehrkraft: Wissensvorsprung; } \\
\text { Lernende: Vorwissen durch unter- } \\
\text { richtliche Beschäftigung. }\end{array}$ \\
\hline $\begin{array}{l}\text { Ermitteln und Reali- } \\
\text { sieren der Leserbe- } \\
\text { dürfnisse }\end{array}$ & $\begin{array}{l}\text { Welche Inhalte, Zusammenhänge } \\
\text { etc. müssen erklärt, welche } \\
\text { Schlüsse begründet werden? }\end{array}$ & $\begin{array}{l}\text { Insbesondere die im Text angeschnit- } \\
\text { tenen Strukturen und Prozesse müs- } \\
\text { sen erklärt werden. } \\
\text { Begründung des abschließenden }\end{array}$ \\
\hline
\end{tabular}




\begin{tabular}{|c|c|c|}
\hline & & Sachurteils nötig. \\
\hline $\begin{array}{l}\text { Routinen zum Reali- } \\
\text { sieren der Leserbe- } \\
\text { dürfnisse }\end{array}$ & $\begin{array}{l}\text { Mit welchen sprachlichen Mitteln } \\
\text { kann eine histor. Begründung so } \\
\text { formuliert werden, dass sie für den } \\
\text { Leser verständlich ist? }\end{array}$ & $\begin{array}{l}\text { Über die Herstellung kausaler Zu- } \\
\text { sammenhänge (z. B: Ein Grund dafür, } \\
\text { dass ..., ist...) } \\
\text { (ausführlich dargestellt und aufge- } \\
\text { schlüsselt in Kapitel 6.2) }\end{array}$ \\
\hline $\begin{array}{l}\text { Überwachen und Re- } \\
\text { flektieren der Leser- } \\
\text { orientierung }\end{array}$ & $\begin{array}{l}\text { (s.o.: z. B. auf der Grundlage einer } \\
\text { Checkliste, die die Fragen aus den } \\
\text { genannten Anforderungen berück- } \\
\text { sichtigt) }\end{array}$ & $\begin{array}{l}\text { Wird durch die vorliegende Aufgabe } \\
\text { nicht angeregt. }\end{array}$ \\
\hline
\end{tabular}

Über das Modell können die Anforderungen an die Lernenden sichtbar gemacht werden. Es wird deutlich, wie vielfältig diese sind, weil das Vergleichen unterschiedliche Teilhandlungen berücksichtigt, die wieder besondere sprachliche Muster zu ihrer Realisierung nach sich ziehen. Diese müssen zudem auf den inhaltlichen Kontext bezogen werden. Soll der Zweck des historischen Vergleichs erreicht werden, kann dies außerdem nur unter Berücksichtigung der Leserschaft geschehen, die in diesem schulischen Aufgabenkontext eine besondere Herausforderung darstellt, da es sich nicht um eine reale Schreibaufgabe handelt.

Neben der Überprüfung der Aufgabe auf ihre Herausforderungen für den Lösungs- bzw. Schreibprozess soll außerdem die Aufgabenqualität im Sinne einer kompetenzorientierten historischen Lernaufgabe überprüft werden. Dies geschieht im Folgenden anhand der in Kapitel 2.2 dargestellten Qualitätskriterien historischer Lernaufgaben, wie Thünemann sie vorschlägt ${ }^{602}$, sowie der dort vorgestellten Checkliste nach Köster/Bernhardt/Thünemann ${ }^{603}$.

Die bereits in Kapitel 2.2.1 vorgestellten Kriterien Thünemanns werden nachfolgend aufgenommen und die vorliegende Vergleichsaufgabe daraufhin geprüft: Als Kriterium I bestimmt Thünemann, dass die Aufgabe problemorientierte Lernimpulse gibt, die den gesamten Lernprozess strukturieren, und grenzt diese vom reinen Faktenwissen $a b .{ }^{604}$ Zwar ermöglicht die vorliegende Vergleichsaufgabe eine Problemorientierung, rekurriert aber gleichzeitig auf die Fakten des Darstellungstextes, da kein weiterer Bezug auf andere Materialien genommen wird. Insofern bezieht sie sich stark, wenn auch nicht ausschließlich, auf Faktenwissen.

Als Kriterium II benennt Thünemann die Anregung eines Werturteils durch die Aufgabe. Hier ist festzustellen, dass der Operator Vergleichen entsprechend der EPA-Bestimmung eine Sachurteilsbildung einfordert (vgl. dazu Kapitel 3). Ein Werturteil kann auch für das Vergleichen durch eine entsprechende

\footnotetext{
602 Thünemann, Holger (2013): Historische Lernaufgaben. Theoretische Überlegungen, empirische Befunde und forschungspragmatische Perspektiven. In: Zeitschrift für Geschichtsdidaktik 12, S. 141-155.

${ }^{603}$ Köster, Manuel; Bernhardt, Markus; Thünemann, Holger (2016): Aufgaben im Geschichtsunterricht. Typen, Gütekriterien und Konstruktionsprinzipien, In: Geschichte lernen, 174. S. 7.

${ }^{604} \mathrm{Vgl}$. hier wie auch für die nachfolgenden Kriterien: Thünemann (2013), ebd.
} 
Aufgabenformulierung angeregt werden. Die vorliegende Aufgabenstellung geht darüber jedoch nicht hinaus. Auch die Aspektierungen sind auf eine Sachurteilsbildung hin angelegt.

Das Kriterium III definiert Thünemann als Anregung zur Selbstreflexion. Da diese Aufgabe auf die Umstrukturierung des im Darstellungstext angelegten Inhaltswissens hin ausgelegt ist und weder dieses Inhaltswissen noch die Operation des historischen Vergleichens reflektiert, regt sie aus sich heraus nicht zur Selbstreflexion an.

Insgesamt muss also festgestellt werden, dass die vorliegende Aufgabe die von Thünemann benannten Kriterien in weiten Teilen nicht erfüllt.

Legt man im Anschluss an diese übergreifenden Merkmale die Kriterien der Checkliste nach Köster/Bernhardt/Thünemann ${ }^{605}$ an, lassen sich (im tabellarischen Überblick) auf die dort aufgeworfenen Fragen die nachfolgenden Antworten geben:

Tabelle 4: Anwendung der Checkliste nach Köster/Bernhardt/Thünemann (2016) für die Vergleichsaufgabe 606

\begin{tabular}{|l|l|}
\hline $\begin{array}{l}\text { Wird mit der Aufgabe bzw. den Aufgaben ein } \\
\text { historisches Problem bearbeitet? }\end{array}$ & $\begin{array}{l}\text { Ja, der Vergleich des Beginns der Industrialisierung } \\
\text { in England und Deutschland weist auf die Frage hin, } \\
\text { ob und inwiefern die Industrielle Revolution in } \\
\text { Deutschland später begann }\end{array}$ \\
\hline $\begin{array}{l}\text { Wird die Problembearbeitung durch eine } \\
\text { historische Leitfrage strukturiert? }\end{array}$ & $\begin{array}{l}\text { Keine offene Leitfrage, da die Frage nach dem Ver- } \\
\text { gleich in Bezug auf das gewählte Tertium Compara- } \\
\text { tionis („unterschiedliche Ausgangsbedingungen“) } \\
\text { eine Normierung beinhaltet. }\end{array}$ \\
\hline $\begin{array}{l}\text { Werden die Lernenden zur Bildung eigener } \\
\text { Sach- und Werturteile aufgefordert? }\end{array}$ & $\begin{array}{l}\text { Der Vergleich zielt aufgrund der Aufgabenformulie- } \\
\text { rung auf ein Sachurteil ab, jedoch nicht auf ein } \\
\text { Werturteil. }\end{array}$ \\
\hline $\begin{array}{l}\text { Werden die Lernenden angeregt, ihren eige- } \\
\text { unterschiedlicher Antworten zu reflektie- } \\
\text { ren? }\end{array}$ & $\begin{array}{l}\text { Nein, die Aufgabe zielt auf rein inhaltliche Aspekte } \\
\text { ab. }\end{array}$ \\
\hline Ist die Aufgabe verständlich formuliert? & $\begin{array}{l}\text { Sie enthält eine hohe Informationsdichte. Die As- } \\
\text { pekte, die bearbeitet werden sollen, werden so } \\
\text { strukturiert, dass der übergeordnete Operator zu- } \\
\text { erst genannt wird und die einzelnen Aspekte folgen. } \\
\text { Ist das Prinzip bekannt, kann dadurch eine höhere } \\
\text { Übersichtlichkeit erreicht werden. Gleichzeitig sind } \\
\text { Operator und Aspektierungen in komprimierter } \\
\text { Form formuliert. }\end{array}$ \\
\hline
\end{tabular}

${ }^{605}$ Vgl. Köster, Manuel; Bernhardt, Markus; Thünemann, Holger (2016), S. 7.

${ }^{606}$ Vgl. die Wiedergabe der Checkliste auf S. 199 nach Köster/Bernhardt/Thünemann, (2016), S. 7. 


\begin{tabular}{|l|l|}
\hline $\begin{array}{l}\text { Ist die Aufgabe inhaltlich offen und nicht auf } \\
\text { eine spezifische Lösung festgelegt? }\end{array}$ & $\begin{array}{l}\text { Durch den Darstellungstext als einzige Material- } \\
\text { grundlage, der zudem schon Vergleichsstrukturen } \\
\text { vorgibt, sowie die Normvorgabe in Aufgabe und } \\
\text { Text ist die Offenheit eingeschränkt, wenngleich } \\
\text { vorhandenes Wissen der Lernenden in die Aufga- } \\
\text { benlösung eingebracht werden kann. }\end{array}$ \\
\hline $\begin{array}{l}\text { Liegt das Anforderungsniveau der Aufgabe } \\
\text { in der „Zone der nächsten Entwicklung“ der } \\
\text { Mehrzahl der Lernenden? }\end{array}$ & $\begin{array}{l}\text { Diese Frage muss individuell auf eine konkrete Lern- } \\
\text { gruppe hin beantwortet werden. }\end{array}$ \\
\hline $\begin{array}{l}\text { Kann die Aufgabe von Lernenden unter- } \\
\text { schiedlichen Niveaus auf verschiedene Art } \\
\text { und Weise bearbeitet werden? }\end{array}$ & $\begin{array}{l}\text { Eine Differenzierung ist in der Aufgabe nicht ange- } \\
\text { legt. }\end{array}$ \\
\hline $\begin{array}{l}\text { Regt die Aufgabe die Lernenden zur Koope- } \\
\text { ration an? }\end{array}$ & $\begin{array}{l}\text { Die Aufgabe kann in Partner- oder Gruppenarbeit } \\
\text { bearbeitet werden, jedoch gibt es dazu in der Auf- } \\
\text { gabenformulierung keine Hinweise. }\end{array}$ \\
\hline $\begin{array}{l}\text { Finden standardisierte Operatoren Verwen- } \\
\text { dung? }\end{array}$ & $\begin{array}{l}\text { Ja, das Vergleichen ist die übergeordnete Opera- } \\
\text { tion. Der Operator ist in den EPA definiert. }\end{array}$ \\
\hline $\begin{array}{l}\text { Werden die Lernenden zur Produktion eige- } \\
\text { ner Darstellungen aufgefordert? }\end{array}$ & $\begin{array}{l}\text { Die Lernenden werden zur Produktion des Ver- } \\
\text { gleichs aufgefordert. Dabei ist die Darstellung inso- } \\
\text { fern gelenkt, als dass der zugrundeliegende Darstel- } \\
\text { lungstext Teilbeurteilungen vorgibt. }\end{array}$ \\
\hline
\end{tabular}

Die Checkliste zusammenfassend muss festgestellt werden, dass die Aufgabe nur einem geringen Teil der genannten Kriterien gerecht wird. Eine Hürde für einen eigenständigen Vergleich scheinen darüber hinaus vor allem die vorgegebenen Vergleichsstrukturen im Text zu sein, die eher zur Wiedergabe statt zu einem eigenständigen Vergleich anregen. Die Texte insbesondere aus den ersten Erhebungen, in denen Text und Aufgabe unverändert eingesetzt wurden, werden dies zeigen. Inhaltliche Strukturen wurden von den Lernenden häufig übernommen, statt sie für einen eigenständigen Vergleich zu nutzen.

Zwar stellt das historische Vergleichen und damit auch die vorliegende Aufgabe, wie im Prozessmodell dargelegt, eine Vielzahl an Anforderungen für die Realisierung dieser, gleichwohl regt die Form dieser textbezogenen Schreibaufgabe zum historischen Vergleich in der Kombination von Aufgabe (und den entsprechenden Aspektierungen) und inhaltlich dichtem Darstellungstext, der auf manche Erklärstruktur verzichtet, insgesamt zur Reproduktion statt zur kritischen Auseinandersetzung mit einer vorgegebenen Narration an, die in einem eigenständigen Vergleich münden sollte. 


\subsection{Die Vergleichsaufgabe als typische Aufgabe}

Die ausgewertete Vergleichsaufgabe entstammt einem Lehrwerk vor der Umstellung auf die kompetenzorientierten Kernlehrpläne. Es stellt sich daher die Frage, inwiefern sie als typisch auch für die Lehrwerkgeneration nach 2014 gelten kann.

Zur Klärung dieser Frage bietet sich zunächst ein Vergleich mit dem „Kursbuch Geschichte“ von $2015^{607}$ an, das also nach dem Inkrafttreten des neuen Kernlehrplans erschienen ist.

In der Neuauflage ist die Vergleichsaufgabe nicht mehr enthalten. Der Darstellungstext wurde deutlich überarbeitet, das Grundkonzept „Pionierland England“ und „Nachzügler“ Deutschland“ bleibt hingegen erhalten, jedoch auf zwei unterschiedliche Themenkomplexe verteilt ${ }^{608}$. Die Darstellung Englands fällt in das Unterkapitel „Die Anfänge der Industrialisierung“, die Darstellung der Entwicklung „Deutschlands" (das Konzept wird auch hier im entsprechenden Kapitel nicht thematisiert) wird im Folgekapitel „Die ,Zweite Industrielle Revolution“" aufgearbeitet. Der Bezug auf Preußen wird in der Neubearbeitung aufgegriffen, aber durch den Bezug auf Bayern ergänzt und in einen expliziten Zusammenhang zur Entwicklung Deutschlands gebracht. Die Kernaussage des in der älteren Auflage initiierten Vergleichs wird also auch durch die Darstellungstexte der genannten Kapitel hergestellt. Die Vergleichsaufgabe wurde hingegen ersetzt durch mehrere Aufgaben, die jeweils unter den Kapiteln zu finden sind und entsprechend der drei Anforderungsbereiche ausgewählte Operatoren berücksichtigen. Für diese Aufgaben kommt man bei einer Einzelprüfung zu ähnlichen Ergebnissen wie für die zuvor untersuchte Vergleichsaufgabe. Auch diese Aufgaben sind weder materialbasiert, nur über das Zusammenspiel verschiedener Aufgaben differenzierend (jedoch nicht innerhalb der einzelnen Aufgabe), noch regen sie zur Reflexion auf der Metaebene an.

Stichproben aus anderen Lehrwerken zeigen ein ähnliches Bild. Eine echte Lernaufgabe wird aus den Aufgaben häufig nur, wenn sie durch die Lehrkräfte, die die Aufgaben einsetzen, in einen entsprechenden Kontext eingebettet werden.

Da die Ergebnisse der Frequenzanalyse gezeigt haben, dass das Vergleichen eine häufig gebrauchte Operation darstellt, kann die ausgewählte Aufgabe auch für die neuere Generation als typisch eingeschätzt werden.

\footnotetext{
${ }^{607}$ Laschewski-Müller, Karin / Rauh, Robert (Hrsg.) (2015): Kursbuch Geschichte. Nordrhein-Westfalen Qualifikationsphase. Berlin: Cornelsen. Thematisch verortet im Inhaltsfeld 4 und damit dem Band 2 (Qualifikationsphase) zugeordnet.

${ }^{608}$ Ebd. S. $16 \mathrm{ff}$.
} 


\section{Analyse von Lernendentexten zum historischen Vergleich: Untersuchungsdesign und Methodik}

Nach der Einordnung der ausgewählten Vergleichsaufgabe soll in diesem Kapitel das Untersuchungsdesign vorgestellt werden, das der Auswertung der Lernendentexte zugrunde liegt. Im Anschluss daran wird das für die Analyse der Schreibprodukte entwickelte Kriterienraster vorgestellt.

\subsection{Untersuchungsdesign}

Aus den vorherigen Ausführungen wurde deutlich, dass das Vergleichen sowohl eine grundlegende historische Methode als auch ein spezifisches historisches Textmuster darstellt. Die in Kapitel 4 dargestellte Frequenzanalyse zeigt darüber hinaus, dass das Vergleichen eine häufig verwendete Aufgabenstellung in Geschichtslehrwerken der Sekundarstufe II darstellt. Dennoch wurden Strukturen für den Vergleich im Geschichtsunterricht bisher nicht aufgeschlüsselt.

Die Untersuchung soll auf der Grundlage der in Kapitel 5 bereits dargestellten Schreibaufgabe die eingangs gestellte Forschungsfrage beantworten, welche Fertigkeiten und welche Kompetenzen Lernende zur Lösung der historischen Vergleichsaufgabe benötigen. Diese Frage soll auf der Grundlage eines Textkorpus beantwortet und mithilfe eines auf einem Kriterienraster basierenden Ratings ausgewertet werden, worüber Strukturen des Vergleichs im Geschichtsunterricht aufgeschlüsselt werden können. Die Arbeit schließt dabei an die empirische Schreibdidaktik an.

\subsubsection{Forschungsmethodischer Zugang}

Die empirische Schreibdidaktik ist eine noch junge Disziplin, die sowohl empirisch als auch anwendungsorientiert ausgerichtet ist. ${ }^{609}$ Sie erforscht als übergeordnete Arbeitsfelder Schreibprozess, Schreibentwicklung, Methoden der Schreibentwicklung, schulische Textsorten, Schreibaufgaben u.a.m. ${ }^{610}$ Steinhoff et al. systematisieren die Untersuchungsgegenstände der empirischen Schreibdidaktik in einem ,didaktischen Dreieck', das durch den Bezug auf Lernende, Lerngegenstände und Lehrende bestimmt wird: „Bezieht man diesen Zusammenhang auf die empirische Schreibdidaktik, so lässt sich sagen, dass sie die komplexe Interaktion zwischen (a) den Merkmalen der Lernenden, (b) den Schreibprodukten und -prozessen als Lerngegenstände und (c) den Merkmalen und Konzepten der Lehrenden untersucht." 611

\footnotetext{
${ }^{609}$ Vgl. dazu: Bachmann, Thomas; Becker-Mrotzek, Michael (2017), S. 25.

${ }^{610}$ Vgl. dazu: Steinhoff, Torsten; Grabowski, Joachim; Becker-Mrotzek, Michael (2017): Herausforderungen der empirischen Schreibdidaktik. In: Becker-Mrotzek, Michael; Grabowski, Joachim; Steinhoff, Torsten (Hrsg.): Forschungshandbuch empirische Schreibdidaktik. Münster: Waxmann. S. 9-24. S. 10.

${ }^{611}$ Vgl. ebd. S. 11.
} 
Im Mittelpunkt der vorliegenden Untersuchung steht die Untersuchung von Textprodukten auf der Grundlage einer Schreibaufgabe. Im Rahmen des genannten didaktischen Dreiecks sollen Zusammenhänge zu den Lernenden dahingehend erfasst werden, als dass Vergleichstexte als Lernaufgabe aufgefasst werden und erforscht wird, wie Lernende eine historische Vergleichsaufgabe lösen bzw. durch welche sprachlichen und fachlichen Teilhandlungen sie den Zweck des Textmusters modellieren.

Im Rückgriff auf das didaktische Dreieck ist das Erkenntnisinteresse auf den Lerngegenstand und damit das Schreibprodukt ausgerichtet („Sachdimension“). ${ }^{612}$ Es geht um die Erfassung spezifischer fachlicher und sprachlicher Merkmale des historischen Vergleichs und damit letztlich um die Herausarbeitung von Merkmalen spezifischer Textkompetenz zum historischen Vergleich.

Einen wesentlichen Ansatz für die vorliegende Arbeit stellt die Untersuchung von Lernphänomenen in realen Situationen dar, der Schwerpunkt liegt deshalb nicht in erster Linie auf Design und Ziel, nicht die Methode steht im Mittelpunkt, sondern ihr Einsatz: Die Arbeit verbindet die Untersuchung von Textprodukten mit der Erfassung von ausgewählten Merkmalen des Schreibprozesses, indem die ausgesuchte authentische Schreibaufgabe variiert wird. Durch dieses Design-Experiment sollen erste Hinweise ermöglicht werden, welchen Einfluss Änderungen im Unterrichtsdesign auf die Textproduktion der Lernenden nehmen können. Der Zugang der empirischen Schreibdidaktik wird an dieser Stelle verknüpft mit dem Ansatz fachdidaktischer Entwicklungsforschung. Dieser Zugang ist der Vielfalt geschuldet, die unterrichtliche Prozesse mit sich bringen und in denen es für die Lehrkräfte auch immer darum geht, Entwicklungspotentiale hinsichtlich der Lehr-/Lernmaterialien auszumachen. ${ }^{613}$

Zwar kann diese Arbeit kein vollständiges Bild der Entwicklungspotentiale für Vergleichsaufgaben im Geschichtsunterricht zeigen, aber zumindest erste Hinweise geben.

Gerade das Forschungsfeld Schule birgt unterschiedliche Herausforderungen hinsichtlich des Zugangs zum Forschungsfeld, der Heterogenität der erforschten Schüler*innen, unterrichtlicher Vorbedingungen, dem Zugriff auf möglichst gleich große Gruppen, dem Zeitpunkt der Durchführung der Forschung u.a.m., denen begegnet werden muss.

\subsubsection{Das Textkorpus}

Die Entscheidungen für die Gestaltung der dieser Arbeit zugrundeliegenden Stichprobe und das daraus resultierende Textkorpus ergeben sich aus dem zuvor skizzierten Erkenntnisinteresse, aber auch aus

\footnotetext{
${ }^{612}$ Vgl. dazu: Steinhoff, Torsten (2017), S. 356.

613 „In der Tradition des Design Research mit seinen verschiedenen Spielarten [...] umfasst das Forschungsfeld der Fachdidaktischen Entwicklungsforschung nicht nur deskriptive Analysen von (defizitären) Lernständen, sondern bearbeitet auch systematisch die Frage, in welcher Strukturierung die Lerngegenstände erarbeitet werden sollen, und wie dies erfolgen kann." Aus: Prediger, Susanne (2013): Sprachmittel für mathematische Verstehensprozesse - Einblicke in Probleme, Vorgehensweisen und Ergebnisse von Entwicklungsforschungsstudien. In: Pallack, Andres (Hrsg.): Impulse für eine zeitgemäße Mathematiklehrer-Ausbildung. MNU-Dokumentation der 16. Fachleitertagung Mathematik. Neuss: Seeberger. Online verfügbar unter: http://www.mathematik.uni-dortmund.de/ prediger/veroeff/13-Prediger-MNU-FL-MuM_Sprachmittel.pdf. S. 5 (letzter Zugriff am 28.11.17).
} 
den schulischen Gegebenheiten, da es sich um eine praxisnahe Forschung handelt, die die im Feld gebotenen Bedingungen berücksichtigen muss.

\subsubsection{Zur Auswahl der Stichprobe}

Die Stichprobe umfasst 88 Texte, von denen nach Bereinigung 84 Texte ausgewertet werden konnten, von Schüler*innen des Berufskollegs in NRW, die zwischen 2012 und 2016 gesammelt wurden.

Die Anzahl der Texte ist zum einen der Möglichkeit geschuldet, auf solche Texte zugreifen zu können (dies hat nicht zuletzt mit den Organisationsstrukturen des Berufskollegs zu tun, wie im Folgenden noch erläutert wird), und zum anderen dem Zeitrahmen, welcher für eine sinnvolle Auswertung zur Verfügung stand und somit eine natürliche Begrenzung der Textmenge darstellt.

Zwar stellt das Korpus eine methodische Herausforderung dar, dennoch kann davon ausgegangen werden, dass eine erste Erfassung wiederkehrender (und damit für die Textsorte grundlegender) fachlicher und sprachlicher Merkmale des Vergleichs im Geschichtsunterricht auch über die zunächst kleine Zahl an Texten geleistet werden kann, da qualitative und quantitative Ansätze in der anschließenden Auswertung verbunden werden.

Hinsichtlich der Repräsentativität in der Korpuslinguistik, konstatiert Steinhoff, werde inzwischen mehrheitlich davon ausgegangen, dass auch sehr umfangreiche Textkorpora Bedingungen der Repräsentativität im strikten Sinne nicht erfüllen können ${ }^{614}$ :

„Dies führt man darauf zurück, dass die erforderliche, sowohl merkmalsgerechte als auch proportionsgetreue Bestimmung der Grundgesamtheit der Daten bei einer derart komplexen Materie wie einer Sprache bzw. eines Sprachausschnitts weder theoretisch noch praktisch erreicht werden kann. ${ }^{\prime 615}$

Ausgehend von dieser Annahme ist die Auswahl der Schüler*innen, die die in das Korpus eingegangen Texte produziert haben, relevant: Bei einer heterogenen Schülerschaft kann davon ausgegangen werden, dass die Lernenden unterschiedliche Zugänge und unterschiedliches Wissen zum bzw. über den Vergleich im Geschichtsunterricht besitzen. Für die Herausarbeitung konstitutiver Strukturen ist dies ein wichtiger Faktor. Eine solche Lerner*innenschaft kann für das Berufskolleg in mehrfacher Hinsicht angenommen werden, da Lernende aus unterschiedlichen Schulen der Sekundarstufe I mit sehr unterschiedlichen bildungsbiographischen Werdegängen in den verschiedenen Bildungsgängen des Berufskollegs vereint sind.

Zudem kann hinsichtlich empirischer Fundierung von Fachunterricht (dies gilt nicht zuletzt für den Gesellschaftslehre- und Geschichtsunterrichts ${ }^{616}$ ) vom Berufskolleg als von einer vernachlässigten Schulform gesprochen werden. Es wurde in der Vergangenheit wenig bis kaum für die Erforschung geschichtsdidaktischer Fragestellungen berücksichtigt, so dass der Blick in diese Schulform im Rahmen dieser Arbeit zum Abbau dieses Desiderats beitragen möchte.

\footnotetext{
${ }^{614}$ Vgl. ebd. S. 358.

615 Ebd.

${ }^{616}$ Das Fach Geschichte ist in NRW in der Regel in ein Fächerkonglomerat (z. B. Gesellschaftslehre) integriert, in dem anteilig Geschichte unterrichtet wird.
} 
Erforscht wurden für diese Untersuchung Lernende der Klassen 12 und 13 von drei Berufskollegs mit unterschiedlichen beruflichen Schwerpunkten. Es handelt sich konkret um sechs Klassen aus Berufskollegs in der Region zwischen Rhein und Ruhr. ${ }^{617}$ Dabei wurden ausschließlich Bildungsgänge berücksichtigt, die auf den Erwerb der Fachhochschulreife bzw. der Allgemeinen Hochschulreife zielen, um so hinsichtlich der fachlichen Bildungsziele eine möglichst große Übereinstimmung auch über verschiedene Schulen hinweg zu erreichen.

Die Beschränkung auf drei Schulen wie auch die Entscheidung, die Anleitung der Schreibaufgabe in die Hände der Lehrkräfte zu legen, ist schulorganisatorischen Gründen geschuldet.

Auf der Grundlage von Expert*innengesprächen ${ }^{618}$ können zumindest für einen Teil der untersuchten Schüler*innen die Voraussetzungen zur Lösung der Vergleichsaufgabe umrissen werden: Die Lernenden bringen insbesondere sehr unterschiedliche thematische Kenntnisse mit. In drei der untersuchten Klassen haben sich die Lernenden bereits mit dem Thema Industrialisierung beschäftigt, in den anderen Klassen hat es dazu entweder keine Unterrichtseinheit gegeben oder Angaben darüber fehlen. Kontrollierte einheitliche Bedingungen für alle Klassen waren, auch dies ist dem Schulalltag geschuldet, nicht möglich. Durch den Themeneinstiegscharakter der ausgewählten Aufgabe kann jedoch davon ausgegangen werden, dass die Lösung der Aufgabe für alle Lernenden grundsätzlich möglich ist. Gerade für die Klassen, die sich nicht zuvor mit dem Inhalt, Industrialisierung' beschäftigt haben, bedeutet dies, dass die Aufgabenlösung nicht zuvor ,erlernt' ist, sondern die Schüler*innen sich auf zuvor erworbene Kompetenzen stützen und diese transferieren müssen.

\subsubsection{Zur Korpusgestaltung}

Den Lernendentexten liegt die in Kapitel 5 vorgestellte Aufgabe mit dem zugehörigen Darstellungstext zugrunde. Das Korpus basiert auf der ursprünglichen Schreibaufgabe sowie zweier Variationen. In der Auswertung der dem Korpus zugrundeliegenden ersten Klassensätze hat sich bereits gezeigt, dass Lernende häufig vorgegebene Strukturen aus dem zugrundeliegenden Darstellungstext für ihre Texte übernommen haben. Daraus resultierte die Entscheidung, die Schreibaufgabe zu variieren. Ziel der Variationen ist zu untersuchen, welche Variante möglicherweise hilfreich für die Textproduktion ist. Die Varianten sollen vermeiden helfen, Inhalte des Darstellungstextes nur wiederzugeben anstatt sie in eine Vergleichsstruktur zu überführen.

Die Erhebung ist deshalb dreiteilig. Es handelt sich dabei um eine zufällige Stichprobe.

\footnotetext{
${ }^{617}$ Der Zugang zum Feld stellte sich unterschiedlich schwierig dar. Insbesondere die Tatsache, dass Geschichte am Berufskolleg immer als Teildisziplin unterrichtet wird, hat zur Folge, dass Bedenken von Lehrpersonen zu berücksichtigen waren, die Teildisziplin für eine solche Untersuchung sei nicht genug ausgeprägt. In solchen Fällen wurde in solchen Lerngruppen nicht erhoben.

Eine weitere Schwierigkeiten bei der Erhebung ergibt sich daraus, dass Lehrkräfte inzwischen mit einer Vielzahl an Erhebungsanfragen konfrontiert werden, so dass der Feldzugang oft nur mit Hürden möglich ist.

618 Hierunter fallen Gespräche mit den entspr. Fachlehrkräften.
} 
Dem Textkorpus liegen auf der Grundlage der Variationen drei Teile zugrunde ${ }^{619}$, die durch eine leicht differenzierte Text- oder Aufgabengrundlage definiert sind:

Der erste Teil geht auf den unveränderten Darstellungstext zurück. Auch die Aufgabe wurde unverändert dem Lehrwerk entnommen. Darstellungstext und Aufgabe werden nachfolgend noch ausführlich dargestellt.

Im zweiten Teil wurden dann im Darstellungstext bereits vorgegebene Vergleichsaspekte entfernt, aus dem einen Text wurden zwei Texte, die jeweils auf ein Vergleichselement eingehen (da der Ursprungstext in jeweils einen Teil zu England und Deutschland vorstrukturiert war, handelt es sich nur um kleinere Eingriffe). Da es hier um die Frage geht, wie oder ob sich Schüler*innentexte verändern, wenn Vergleichselemente weitgehend fehlen, beschränkt sich dieser Teil der Erhebung auf die Auswertung von Schüler*innentexten.

Dem dritten Teil liegen die Texte aus der Erhebung 2 zugrunde. Darüber hinaus erhalten die Lernenden als Scaffold eine Tabelle, um die Vergleichselemente gegenüberzustellen und so den Vergleich vorzustrukturieren. Diese Tabelle ist der Vergleichsaufgabe vorangestellt und soll so die Vergleichselemente gegenüberstellen, bevor der Vergleich als Ganzes gelöst wird. Alle Textvarianten sind dem Anhang angefügt. ${ }^{620}$

Tabelle 5: Erhebungsteile: Variationen im Überblick

\begin{tabular}{|l|l|l|l|}
\hline Erhebung & & & \\
\hline Teil 1 & $\begin{array}{l}\text { unveränderte Schreib- } \\
\text { aufgabe }\end{array}$ & unveränderter Text & keine zusätzliche Hilfe \\
\hline Teil 2 & $\begin{array}{l}\text { unveränderte Schreib- } \\
\text { aufgabe }\end{array}$ & $\begin{array}{l}\text { veränderter Text: jeweils ein } \\
\text { Text zu England und Deutsch- } \\
\text { land, Entfernung vorgegebener } \\
\text { Vergleichselemente }\end{array}$ & keine zusätzliche Hilfe \\
\hline Teil 3 & $\begin{array}{l}\text { unveränderte Schreib- } \\
\text { aufgabe }\end{array}$ & $\begin{array}{l}\text { veränderter Text: jeweils ein } \\
\text { Text zu England und Deutsch- } \\
\text { land, Entfernung vorgegebener } \\
\text { Vergleichselemente }\end{array}$ & $\begin{array}{l}\text { Aufgabe (,Gegenüber- } \\
\text { stellen'), vor der Textpro- } \\
\text { duktion eine Tabelle aus- } \\
\text { zufüllen }\end{array}$ \\
\hline
\end{tabular}

Während für den ersten Teil drei Klassensätze für die gelöste Vergleichsaufgabe vorliegen, konnten für Erhebungsteil 2 nur zwei, für Erhebungsteil drei nur ein Klassensatz erhoben werden. Diese Ungleichgewichtung muss zunächst als Störfaktor angenommen werden. Der Vergleich der drei Untersuchungsteile hat damit zwar nur eine eingegrenzte Aussagekraft, es werden sich dennoch erste Rückschlüsse ableiten lassen, ob bzw. wie Textgrundlage und die Unterstützung bei der Realisierung einer zentralen Vergleichsoperation (dem Gegenüberstellen) einen Einfluss auf das Textprodukt haben.

\footnotetext{
${ }^{619}$ Neben den Texten haben die Schüler*innen zunächst einen Erhebungsbogen erhalten, der allgemeine Merkmale zu Alter, Geschlecht und Sprachbiographie abfragt. Des Weiteren lagen ihnen Darstellungstext und Aufgabe vor.

${ }^{620} \mathrm{Vgl}$. Anhang 6, 6.1. und 6.3.
} 
Die Textprodukte werden mit Knopp et al. als „drafts“ oder „textproduced so far" aufgefasst, „[...] weil sie aufgrund der Erhebungssituation keine fertigen überarbeiteten Texte darstellen können. “621 Dennoch ermöglicht das Rating Aussagen über die Realisierung der Vergleichsaufgabe und damit auch über die Schreibkompetenz der Lernenden. ${ }^{622}$

\subsubsection{Zur Korpusaufbereitung}

Alle Texte des Vergleichskorpus wurden zunächst digitalisiert und codiert. Markierungen, Streichungen etc., die die Lernenden selber vorgenommen haben, wurden übernommen, um eine möglichst hohe Originalität der Texte zu erhalten. Alle Rater konnten für die Auswertung so jederzeit auf die Daten zugreifen. Ein Vergleich mit den handschriftlichen Originaltexten war ebf. möglich. Auf eine Anreicherung durch Metadaten oder Annotationen konnte verzichtet werden. Es wurde lediglich eine Zuordnung zur zugrundeliegenden Variante der Schreibaufgabe vorgenommen.

\subsubsection{Zur Korpusauswertung}

Durch die Variation der Schreibaufgabe im realen schulischen Kontext und unter Verzicht auf eine Randomisierung kann von einem quasi-experimentellen Design gesprochen werden (wenn auch unter Berücksichtigung der dem Forschungsfeld Schule zugehörigen Störfaktoren).

Im Sinne eines Mixed-Method-Verfahren wurden die Texte in einer ersten Analyse über einen quantitativen Zugang mittels eines selbst entwickelten Kriterienrasters geratet. In einer zweiten Analyse wurden über einen qualitativen Zugang einzelne Lernendentexte auf der Grundlage der durch das Kriterienraster und textanalytisch überprüft, um so (im Gegensatz zur Auswertung durch das Kriterienraster) individuelle sprachliche und fachliche Strukturen berücksichtigen zu können.

Die Auswertungskriterien des Kriterienrasters werden nachfolgend noch dargelegt, sie entstanden sowohl corpus-driven als auch corpus-based. ${ }^{623}$

Die Texte wurden zunächst in einer Pilotierung von drei zuvor geschulten Ratern auf der Grundlage des Kriterienrasters mit Hilfe eines Manuals geratet und mit Hilfe von SPSS ausgewertet. Aufgabenrückläufer, in denen eine Lösung fehlte, wurden dem Korpus entnommen. ${ }^{624}$

\footnotetext{
${ }^{621}$ Knopp et al. 2014, S. 112.

622 „Die Feststellung von Textqualität durch sogenannte Ratings stellt u.E. eine geeignete Methode dar, um Aussagen über die Schreibkompetenz des jeweiligen Schreibers zu machen (Grundannahme). die solchermaßen festgestellte Textqualität fungiert als Indikator für Schreibkompetenz." (Knopp, Matthias; Jost, Jörg; Linnemann, Markus; Becker-Mrotzek, Michael (2014): Textprozeduren als Indikatoren von Schreibkompetenz - ein empirischer Zugriff. In: Bachmann, Thomas; Feilke, Helmuth (2014): Werkzeuge des Schreibens. Stuttgart: Fillibach bei Klett. S. 111-128. S. 111f).

${ }^{623} \mathrm{Vgl}$. dazu Bubenhofer, Noah (2009).

${ }^{624}$ Da es sich nur um wenige Texte handelt, die dem Korpus aufgrund fehlender Bearbeitung entnommen werden mussten, wird aufgrund der geringen Zahl auf eine weitere Auswertung verzichtet.
} 
Um Rückschlüsse auf die Interraterreliabilität der Auswertungen zu erhalten, wurden die Rater einer doppelten Reliabilitätsprüfung unterzogen, indem zunächst paarweise für alle Varianten der Cohen's Kappa-Koeffizient errechnet wurde und anschließend für alle drei Rater zugleich Krippendorffs Alpha. Anschließend wurde für die Gesamterhebung Krippendorffs Alpha errechnet.

Die Messgenauigkeit des Instruments wurde über die interne Konsistenz der Items untereinander mit Hilfe von Cronbachs Alpha ermittelt.

Anschließend konnten die einzelnen Items korreliert und einer Faktorenanalyse unterzogen werden.

Aus den mittels des Kriterienrasters gerateten Texten wurden in einem weiteren Schritt drei Schüler*innentexte einer Feinanalyse unterzogen, die erfolgreiche und weniger erfolgreiche Strukturen des Vergleichs im Geschichtsunterricht genauer hinterfragen soll. Dazu wurden zunächst die erreichten Gesamtpunktzahlen (gerechnet mit SPSS) der Korpustexte errechnet und dann die Texte mit der höchsten bzw. der niedrigsten Gesamtpunktzahl sowie ein Text mit mittlerer Gesamtpunktzahl ausgewählt und auf ihre Realisierung der im Kriterienraster angeführten Strukturen hin qualitativ untersucht.

\subsection{Darstellung des erarbeiteten Erhebungsinstruments}

Das vorliegende Textkorpus wurde mithilfe eines Kriterienrasters ${ }^{625}$ ausgewertet, welches die für das Vergleichen im Geschichtsunterricht benötigten Textkomponenten berücksichtigt.

Aufgrund der Überlegungen in Kapitel 3.5 kann davon ausgegangen werden, dass dem Vergleichen im Geschichtsunterricht wiederkehrende Textkomponenten zugrunde liegen. Das Vorhaben schließt durch den Fokus auf solche Textkomponenten an die empirische Schreibdidaktik an: „Eine erste Anforderung an Forschungsprojekte besteht deshalb darin, Texte als konventionalisierte Textmuster auf der Grundlage einer entsprechenden linguistischen Expertise zu verstehen und zu untersuchen. Dafür wird üblicherweise eine Reihe verschiedener Textkomponenten in den Blick genommen. ${ }^{\text {“626 }}$

Für das Vergleichen im Geschichtsunterricht relevante wiederkehrende Komponenten konnten für die Verwendung im Kriterienraster auf der Grundlage geschichtsdidaktischer Forschungsliteratur in Verbindung mit aktuellen Textanalyserastern und in den Texten vorgefundenen Strukturen hergeleitet werden. Dabei finden sowohl geschichtsdidaktische als auch sprachliche Komponenten Berücksichtigung. Durch das vorliegende Kriterienraster werden also zunächst Schreibkompetenzen für den Vergleich im Geschichtsunterricht modellhaft dargestellt. Es handelt sich damit um eine sowohl corpusbased als auch corpus-driven angelegte Untersuchung.

\footnotetext{
${ }^{625}$ Das Kriterienraster ist in Manualform in Anhang 6.4 einzusehen.

${ }^{626}$ Steinhoff, Thorsten; Grabowski, Joachim; Becker-Mrotzek, Michael (2017), S. 14.
} 


\subsubsection{Zum Aufbau des Erhebungsinstruments}

In Bezug auf die empirische Schreibdidaktik kann das Vorgehen als produktbezogene Auswertung durch operationalisierte Kriterien beschrieben werden, sprachprozessbezogene Kriterien spielen für die Beantwortung der Frage, welche Textkomponenten sich in den Texten der Schüler*innen finden lassen, eine untergeordnete Rolle.

Das Kriterienraster orientiert sich am Zürcher Textanalyseraster ${ }^{627}$, darüber hinaus wurde das Diagnoseinstrument Fast Catch Bumerang ${ }^{628}$ einbezogen. Es nimmt sowohl formale Kriterien als auch vergleichsspezifische inhaltliche Elemente in den Blick. Da die Untersuchung in der Sekundarstufe II angesiedelt ist, finden ebenso Elemente, die (vor-)wissenschaftliche Textkompetenz abbilden, Berücksichtigung. Ein Hauptaugenmerk liegt auf der Operationalisierung sprachlicher Teilhandlungen, die für das historische Vergleichen realisiert werden müssen. Anhand dieser Leitkategorien sollen nachfolgend die einzelnen Inhalte des Kriterienrasters betrachtet werden.

Nicht berücksichtigt wird durch das Kriterienraster das Vorwissen der Schüler*innen der Stichprobe. Dies hängt zum einen mit den zuvor dargestellten heterogenen Bedingungen der Lerngruppen und der Erhebungssituation zusammen. Zum anderen ist das Anliegen dieser Arbeit, Strukturen des historischen Vergleichs in den Schülertexten herauszuarbeiten. Die Frage nach dem Vorwissen stellt eine eigene Studie dar, in der erhoben würde, welchen Einfluss das Vorwissen auf die Schreibprodukte hat. Darum geht es im Rahmen dieser Untersuchung jedoch nicht.

Die nachfolgende Darstellung gibt einen Überblick über die im Kriterienraster berücksichtigten Elemente. Es ist allerdings darauf hinzuweisen, dass in diesem Kapitel versucht wird, die Items unter thematischen Oberbegriffen darzustellen, statt sie additiv entlang ihrer Position im Kriterienraster abzuarbeiten. Dies kann dazu führen, dass die einzelnen Items thematisch im Raster unter anderen Teilüberschriften subsummiert sind.

\footnotetext{
${ }^{627}$ Hanser, Cornelia/Nussbaumer, Markus/Sieber, Peter (1996): Zürcher Textanalyseraster. In: Nussbaumer, Markus: Lernerorientierte Textanalyse - Eine Hilfe zum Textverfassen? In: Feilke, Helmuth / Portmann, Paul R. (Hrsg.): Schreiben im Umbruch. Stuttgart: Klett und Nussbaumer, Markus; Sieber, Peter (1994) Texte analysieren mit dem Zürcher Textanalyseraster. In: Sieber, Peter (Hrsg.): Sprachfähigkeiten - Besser als ihr Ruf und nötiger denn je! Aarau u.a., 141-186.

${ }^{628}$ Reich, Hans H.; Roth, Hans-Joachim; Döll, Marion (2009): Fast Catch Bumerang - Auswertungshinweise, Schreibimpuls und Auswertungsbogen. In: Lengyel, Drorit; Reich, Hans H.; Roth, Hans-Joachim; Döll, Marion (Hrsg.): Von der Sprachdiagnose zur Sprachförderung. FörMig Edition Band 5. Münster, S. 209-241.
} 


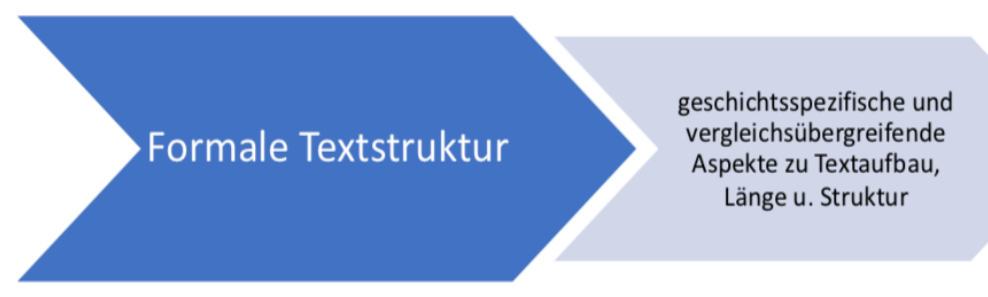

Textlänge, mittlere

Satzlänge,

textstrukturierende

Elemente (Einleitung,

Hauptteil, Schluss,

Überschrift)
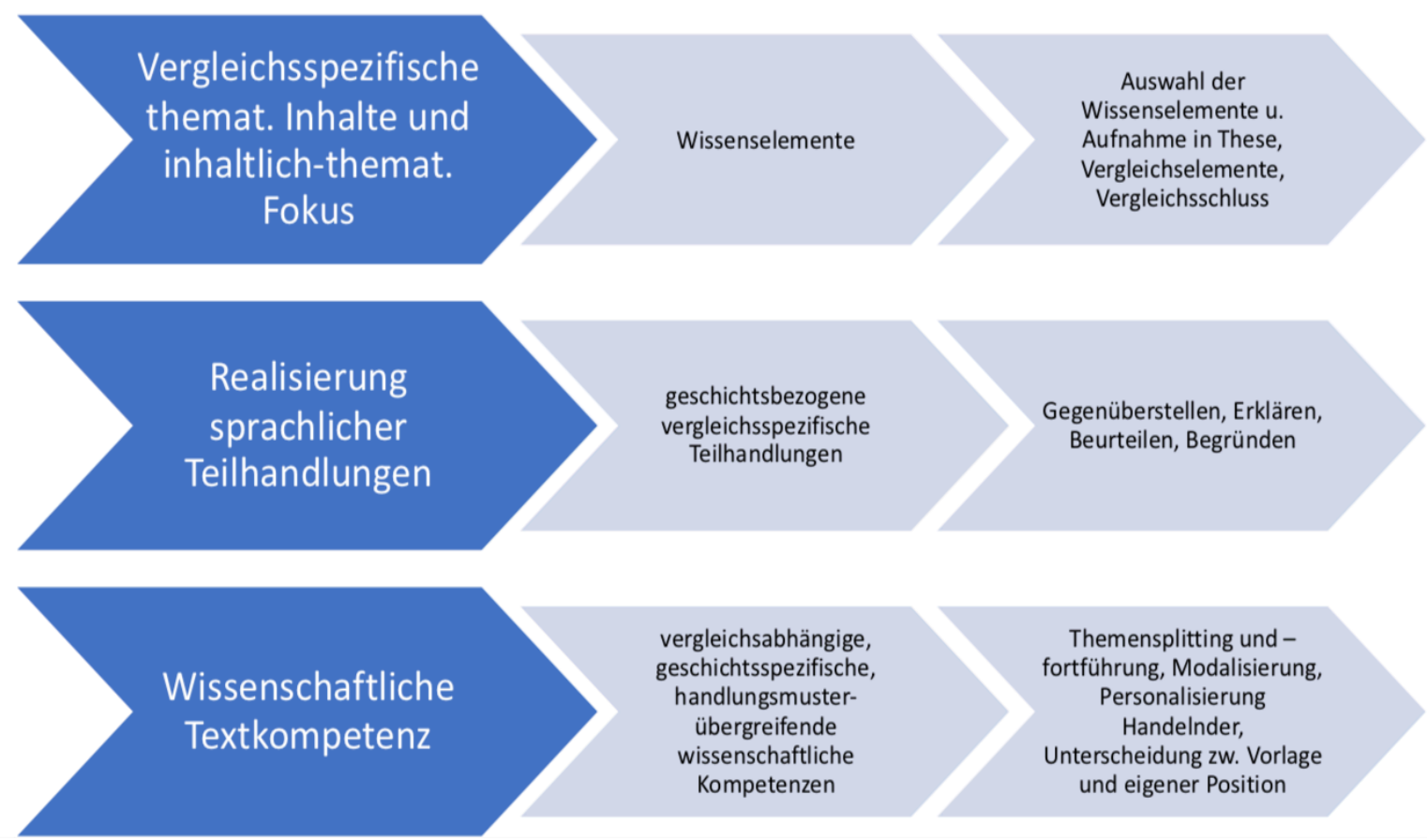

Abbildung 40: Kriterien für die Analyse von Vergleichstexten im Geschichtsunterricht (eigene Darstellung)

Die Darstellung zeigt vier Hauptelemente, auf deren Grundlage die für die Auswertung relevanten Items dargestellt und kategorisiert werden können. Diese Hauptelemente werden durch den mittleren Pfeil genauer charakterisiert, während im rechten Pfeil die Mittel genannt sind, die in der konkreten Auswertung berücksichtigt wurden.

Die genannten Hauptelemente sollen nachfolgend aufgeschlüsselt werden.

Da im Kriterienraster der Erfassung solcher Items, die unter den genannten Hauptelementen zu subsumieren sind, zwei Items allgemeiner Art vorausgehen, sollen diese kurz thematisiert werden:

Dem Kriterienraster vorangestellt ist die Zuordnung der Bearbeitungsvariante, die lediglich der Nachvollziehbarkeit dient, dem eigentlichen Rating aber noch nicht zuzurechnen ist. Außerdem wird aufgenommen, ob die Lernenden ihren Text zum Beispiel durch eine Tabelle oder ähnliches vorstrukturieren, ihn also planen, bevor sie in die Produktion gehen. Dies ist eines der wenigen Items, die auch den Schreibprozess berücksichtigen. Es ist insbesondere für die Erhebung 3 bedeutsam, da die Lernenden als Teil der Aufgabe dazu angehalten werden, erst eine Tabelle als Gegenüberstellung auszufüllen und dann den Text zu produzieren. Für die Erhebung 3 wird angenommen, dass die dem Schreiben vorausgehende Aufbereitung der im Darstellungstext dargestellten Aspekte als Gegenüberstellung die Lösung der Vergleichsaufgabe unterstützen kann. Die Annahme begründet sich in denen in Kapitel 2.7 vorgestellten Überlegungen zum Schreiben und zum Schreibprozess. Die tabellarische Gegenüberstellung 
ist dem ersten Teilprozess, also dem Planen, zuzuordnen. Wenn Lernende also eine Vorstrukturierung vornehmen, kann sie dies in der Formulierung unterstützen. Da auch andere Formen der Vorstrukturierung denkbar sind (z. B. eine stichwortartige Zusammenstellung von für wichtig erachteten Inhalten), schließt die Formulierung „Nutzung von Mitteln zur Vorstrukturierung" die Berücksichtigung solcher Strukturen explizit mit ein. ${ }^{629}$

\subsubsection{Formale Textstruktur}

Das Element „Formale Textstruktur" beinhaltet vergleichsbezogene und vergleichsmusterübergreifende Strukturen und gibt erste Anhaltspunkte zur Art des Textaufbaus, zu Länge und Strukturierung des Textes. Als textsortenübergreifende Strukturen werden Textlänge und mittlere Satzlänge erfasst. Zudem wird durch das Item „Bearbeitung der Aufgabe“ eingeordnet, ob es sich überhaupt um eine ernsthafte Aufgabenbearbeitung handelt.

Als weiteres Element des Textaufbaus wird über das Kriterienraster erfasst, ob die Vergleichsaufgabe als Fließtext oder in einer anderen Form aufbereitet wird. Das vorliegende Kriterienraster beschränkt sich bzgl. vergleichsabhängiger Elemente auf die Form des Textaufbaus. Zwar nehmen andere Textanalyseinstrumente, wie bspw. „Fast Catch Bumerang "6630 weitere formelle Strukturelemente wie z. B. die Adressierung hinzu. Jedoch handelt es sich beim historischen Vergleich um ein (vor-)wissenschaftliches Textprodukt, worin (etwa im Gegensatz zur Adressierung der für ,Fast Catch Bumerang' zu produzierenden Bauanleitung an Jugendliche) auf eine direkte Leseransprache in der Regel verzichtet wird. Im Gegensatz zur genannten Bumerang-Bauanleitung sprechen die Lernenden als Textproduzenten ihre Leser nicht direkt an:

„In den meisten Texten, besonders aber in wissenschaftlichen Artikeln, entfällt der Gebrauch der Mittel des Lenkfeldes, z.B. der Interjektionen, da sie ihre spezifische Funktion im Diskurs erfüllen [...]. Auch der Imperativ [...] entfällt bzw. ist reduziert auf eine kleine Liste formalisierter Anweisungen, die dem Verweisen im Text dienen, meist in abgekürzter Form: vgl., siehe. ${ }^{4631}$

Indirekte Formen im Sinne einer Metakommunikation ${ }^{632}$, in der der Autor Hinweise auf die Textgestaltung gibt und darüber eine Leserlenkung herstellt, werden im Rahmen dieser Arbeit zwar nicht überprüft, da sie wissenschaftliche Schreiberfahrung erfordern, wenn sie wirkungsvoll sein sollen, und keine Voraussetzung zur Realisierung des Vergleichs im Geschichtsunterricht darstellen. Dafür wird aber über das Item „Themenfortführung" der Gebrauch deiktischer Mittel berücksichtigt, die ebf. ein wichtiges Mittel im Sinne einer Adressatenorientierung darstellen.

Es wird vermutet, dass Textlänge und durchschnittliche Satzlänge Hinweise auf Textqualität geben. Hinsichtlich der Bedeutung der Textlänge stellen Reich et al. fest:

\footnotetext{
${ }^{629}$ Die in der Erhebung 6 geforderte Tabelle wird dabei als ein Mittel berücksichtigt.

${ }^{630} \mathrm{Vgl}$. Reich, Hans H.; Roth, Hans-Joachim; Döll, Marion (2009).

${ }^{631}$ Graefen, Gabriele (1997), S. 121.

${ }^{632}$ Vgl. ebd. S. 158.
} 
„Die Erfassung des Textumfangs ist ein grobes quantitatives Maß, das jedoch häufig eben nicht unabhängig von der Qualität der Texte ist. In der Regel sind längere Texte gelungener als kürzere. Im Einzelfall ist der Zusammenhang leider nicht vollkommen stabil, so dass das Zählen der Wörter die Berücksichtigung von Qualitätskriterien nicht ersetzen kann. “633

Ein weiteres quantitatives Maß stellt die Erfassung der durchschnittlichen Satzlänge dar. Ihre Bedeutung schätzen Reich et al. wie folgt ein:

„Neben der Anzahl der Wörter ist die Anzahl der Sätze ein Größenmaß, das Auskunft über den Textumfang geben kann. Es ist für sich allein genommen allerdings nicht sehr aussagefähig, da Sätze eben unterschiedlich lang ausfallen; allerdings gilt auch hier durchaus wieder ein Zusammenhang mit der Textqualität." ${ }^{634}$

Beide Maße sind zwar nicht unumstritten, da bisher ein eindeutiger Zusammenhang zur Textqualität nicht hergestellt werden konnte, er wird aber auch für die vorliegende Arbeit vermutet. Deshalb soll der hier angesprochene mögliche Zusammenhang im Weiteren für die ausgewählte historische Vergleichsaufgabe überprüft werden.

Hinsichtlich des Kriteriums „Bearbeitung der Aufgabe“ geht es um die holistische Einschätzung, inwiefern eine ernsthafte Aufgabenbeantwortung vorliegt, da mögliche Spaßantworten so schneller herausgefiltert werden können.

In Bezug auf vergleichsabhängige formale Kriterien wird über das Item „Texttaufbau“ in eine Realisierung als Stichwörter, Tabelle oder Fließtext unterschieden. Diese Differenzierung ist deshalb als vergleichsabhängig einzuordnen, weil das Vergleichen im Geschichtsunterricht inhaltliche Aspekte benötigt, die wiederum Auswirkung auf die Textstruktur haben. Ein Urteil etwa ist vom meist vorausgehenden Gegenüberstellen abzuheben und gehört nicht in eine tabellarische Form (außer es wird auch hier wieder von der restlichen Tabelle abgehoben als eine Art Einleitung oder Schluss).

Weiterhin ist unter dem Strukturelement „Formale Textstruktur“ die Kategorie „Inhaltliche Gestaltung" subsummiert. Unter dieser Kategorie sind textstrukturierende Elemente gefasst. Neben der Frage nach einer eigenständigen Überschrift werden solche textkompositionellen Elemente aufgenommen, die den klassischen Dreischritt ${ }^{635}$ der Textstrukturierung widerspiegeln: Einleitung, Hauptteil und Schluss. Durch diese Items soll die Funktion dieser Elemente im Hinblick auf den historischen Vergleich untersucht werden. Es geht also nicht um das allgemeine Vorhandensein dieser Elemente, sondern um den Nutzen für die Realisierung der Vergleichsaufgabe.

\footnotetext{
${ }^{633}$ Reich, Hans H.; Roth, Hans-Joachim; Döll, Marion (2009), S. 220.

634 Ebd.

635 Zur Bedeutung von Dreierschemata auch in der wissenschaftlichen Textproduktion: vgl. Feilke, Helmuth (2010): „Aller guten Dinge sind drei“ - Überlegungen zu Textroutinen \& literalen Prozeduren. In: Fest-Platte für Gerd Fritz. Hrsg. und betreut von Iris Bons, Thomas Gloning und Dennis Kaltwasser. Gießen 17.05.2010. OnlineZugriff unter: http://www.festschrift-gerd-fritz.de/files/feilke_2010_literale-prozeduren-und-textroutinen.pdf (zuletzt eingesehen am 10.08.2017).
} 
Insbesondere die Überschrift ist im Sinne der Leserlenkung ${ }^{636}$ bedeutsam für einen fachlichen Text, wie er durch die schriftliche Lösung der Vergleichsaufgabe gefordert wird. Rehbein benennt die Überschrift als textartspezifische Ankündigung ${ }^{637}$, also als Vorankündigung auf die Struktur, die den Leser erwartet, und die immer mit dem Inhalt verknüpft ist. Graefen führt dazu unter Rückgriff auf Rehbein aus:

„Über die Funktionalität von Überschriften im Text besteht im allgemeinen Einigkeit. Sie geben dem Leser durch die inhaltliche Klassifizierung von Textteilen nicht nur einen Überblick über den Text und seine inhaltliche Organisation, sondern auch die Möglichkeit eines selektiven Zugriffs auf inn interessierende Subeinheiten des Artikels - unterstellt, daß dieser folgerichtig aufgebaut ist, wie es textartadäquat wäre. ${ }^{6638}$

Graefen differenziert die Überschrift in Haupt- und Teilüberschriften aus. Diese werden für die Lernendentexte nicht vorausgesetzt, allerdings findet sich eine solche Strukturierung im der Aufgabe zugrundeliegenden Darstellungstext. Im Zusammenspiel mit dem historischen Gegenstand, der der Vergleichsaufgabe zugrunde liegt, kann eine (Haupt-)Überschrift allerdings den Zusammenhang von historischem Gegenstand und historischem Vergleich herstellen. Dieser Aspekt ist neben der Wahrnehmung der Überschrift als textstrukturelles auch als wissenschaftspropädeutisches Element einzuordnen. Feilke stellt dazu fest:

„Die möglichen Ausdrucksmuster für Überschriften sind jeweils nur die ,Spitze eines Eisbergs', d.h. eines komplexen, sozial stabilen Gebrauchszusammenhangs. Die Muster sind in hohem Maß von Gebrauchskonfigurationen (z.B. der jeweiligen Domäne und der Textsorten) abhängig, die erst einmal verstanden sein müssen. Es ist Ergebnis einiger Schrift- und Texterfahrung, wenn wir in der Lage sind, aus einem Überschriftentyp zuverlässig auf die Domäne, Gattungsmerkmale und sogar erwartbare Inhalte zu schließen. Nicht das Muster an sich, sondern der Gebrauchszusammenhang ist kompetenzrelevant, und der ist über ein Memorieren von Ausdrucksmustern gerade nicht zu vermitteln. ${ }^{4639}$

Die von Feilke angesprochenen Muster, die für die Realisierung einer Überschrift verstanden sein müssen, sind dabei im Sinne der Leserorientierung wiederum abhängig vom Zweck. Denn der Gebrauch konfiguriert sich ja gerade über die Aussageabsicht, also den Zweck.

Aus diesen Überlegungen kann gefolgert werden, dass das Vergleichen als Textsorte eine eigene Überschriftenstruktur einfordert, die den Leser auf den im Text folgenden Vergleich vorbereitet. Deshalb wird davon ausgegangen, dass eine Überschrift, wenn sie bspw. nicht gerade vom Darstellungstext übernommen wurde, Einfluss auf die Textqualität hat.

Gerade für das Textmuster historischer Vergleich spielt der Textaufbau eine wichtige Rolle, da zunächst die Vergleichselemente und das Tertium Comparationis einleitend benannt werden müssen und der Vergleich abschließend in einem Sachurteil zusammengeführt werden soll. Deshalb berücksichtigt das Kriterienraster die Strukturierung in Einleitung, Hauptteil und Schluss. Zudem weist die Untersuchung

\footnotetext{
${ }^{636}$ Vgl. dazu: Becker-Mrotzek, Michael; Böttcher, Ingrid (2012): Schreibkompetenz entwickeln und beurteilen. 4. Aufl. Berlin: Cornelsen. S. 59.

${ }^{637}$ Rehbein, Jochen (1978): Ankündigungen. In: Germanistische Linguistik (Varia V) 2-5, 340-387.

${ }^{638}$ Graefen, Gabriele (1997), S. 167.

${ }^{639}$ Ebd. S. 3.
} 
von Knopp et al. auf einen deutlichen Zusammenhang von Textqualität und der Realisierung einer Texteröffnung (Einleitung) und eines Textschlusses hin, in Anlehnung an das Konzept Feilkes sprechen sie in diesem Zusammenhang von Textprozeduren:

„Die deutlichsten Effekte weisen die Realisierungen von texteröffnenden und textschließenden Prozeduren auf [...]. Hier zeigt sich möglicherweise besonders klar, inwiefern ein Schreiber bereits zu Beginn des Textproduktionsprozesses eine gewisse Vorstellung von dem Text, der realisiert werden soll, besitzt. ${ }^{\prime 640}$

Aufgrund dieser deutlichen Relevanz werden Einleitung und Schluss als Textstrukturelemente auch für das Kriterienraster zum historischen Vergleichen aufgenommen und durch den Hauptteil als weitere Struktur ergänzt, da damit zu rechnen ist, dass die Textproduktionen der Lernenden unvollständig sein können. Diese Strukturelemente sind dabei in ihrer inhaltlichen Realisierung auf den historischen Vergleich bezogen. Nach der Nennung der zu vergleichenden Elemente und dem Tertium Comparationis in der Einleitung müssten diese Elemente dann im Hauptteil im Hinblick auf Gemeinsamkeiten, Unterschiede und Ähnlichkeiten ausgeführt werden. Im Schluss wäre dann entsprechend der EPA-Bestimmung das abschließende Sachurteil erwartbar. Dies wird jedoch im Kriterienraster als eigene Kategorie formuliert, so dass unter dem Hauptelement ,Formale Textstruktur' das Kriterium ,Schluss' bereits erfüllt ist, wenn ein gedanklicher Abschluss erfolgt; dies kann auch durch eine Zusammenfassung geleistet werden. Diese Festlegung folgt auch der in Kapitel 3 dargestellten Überlegung, dass es keine feste Form des historischen Vergleichens gibt. Insgesamt geht es bei den genannten Strukturelementen noch nicht um ihre Erkennbarkeit, sachliche Richtigkeit oder fachspezifische Ausführung.

\subsubsection{Vergleichsspezifische thematische Inhalte und inhaltlich-thematischer Fokus}

Unter diesem Strukturierungselement werden die zuvor beschriebenen Textstrukturmerkmale Einleitung, Hauptteil und Schluss wiederaufgenommen und inhaltlich ausgeführt.

Die folgende Strukturskizze soll die unter diesem Strukturierungselement subsummierten Merkmale veranschaulichen:

${ }^{640}$ Knopp, Matthias et al. (2014), S. 125. 
Einleitung

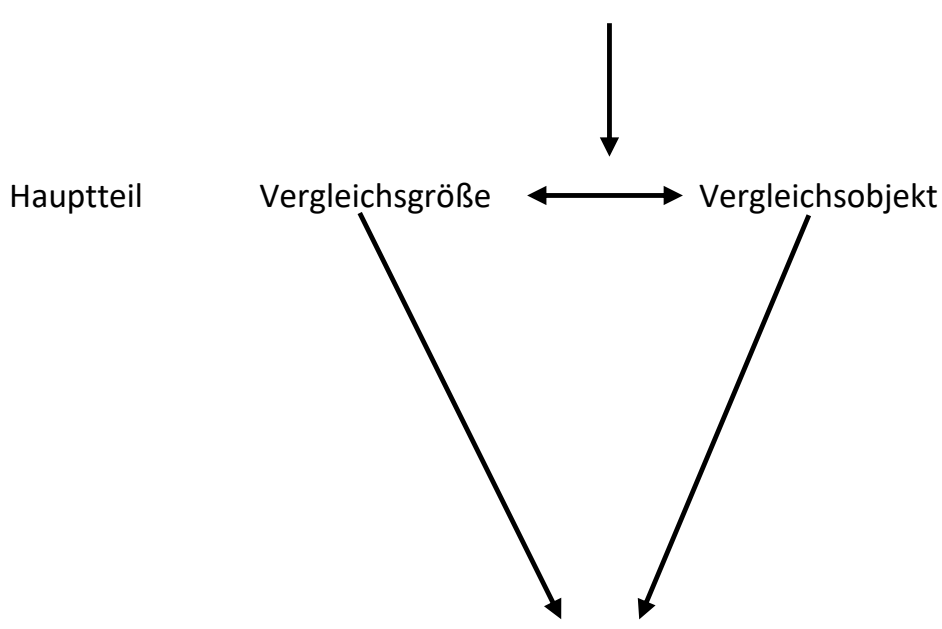

Schluss zentrale These

Aufnahme der Vergleichselemente

Vergleichsabschluss mit abschließendem Sachurteil
Vergleichsgröße (England)

Vergleichsobjekt

(,Deutschland)

tertium comparationis

historisch-inhaltliche Aspekte (aus dem zugrundeliegenden Darstellungstext, ggf. eigene Ergänzungen, z. B. zu Entwicklung in der Landwirtschaft, polit. System etc.)

\section{Abbildung 41: Textstrukturskizze 'Vergleich' (eigene Darstellung)}

Durch das Kriterienraster wird für die Einleitung überprüft, ob im Text eine zentrale These vorhanden ist, die beide Vergleichsgrößen erfasst und Bezug auf das Tertium Comparationis nimmt. Dies kann auch indirekt geschehen. Der Darstellungstext gibt solche Strukturen vor, sie können also übernommen werden. Dabei muss keine Begründung vorliegen, da diese schon ein Sachurteil implizieren würde, welches unter einer anderen Kategorie erfasst wird. Die These wird als Strukturelement der Textsorte Vergleich angenommen, weil sie die Elemente der Gegenüberstellung im Hinblick auf ein Ergebnis einordnet. Weiterhin werden hier die Hauptelemente des Vergleichs aufgenommen und nach den Grundelementen des Vergleichs gefragt. Dem liegt die Annahme zugrunde, dass ein Vergleich nicht vollständig sein kann, wenn Vergleichsgröße, Vergleichsobjekt und Tertium Comparationis nicht oder nur teilweise berücksichtigt werden. Deshalb wird an dieser Stelle überprüft, ob alle drei Elemente explizit genannt werden.

Da ein weiteres wesentliches Element des Vergleichs das Gegenüberstellen darstellt, sind im Hauptteil Wissenselemente unabdingbar, die gegenübergestellt werden können. Diese liefert der Darstellungstext in den ersten beiden Abschnitten, der für beide Länder jeweils Faktoren für den Beginn der Industrialisierung aufschlüsselt. Im dritten Abschnitt werden dann darüber hinaus Veränderungen in Bezug auf die genannten Elemente aufgezeigt, die, so die Annahme im Text, Voraussetzung für die Entwicklung der Industrialisierung waren. Auch im veränderten Text der Erhebungen 4, 5 und 6 bleiben diese Strukturen erhalten, nur dass Vergleichsgröße und Vergleichsobjekt stärker voneinander getrennt wurden. Der dritte Teil des Darstellungstextes, der die Veränderungen in Deutschland skizziert, bleibt ebenfalls erhalten. Die Herausforderung dieses dritten Textteils ist, dass er für die Lösung der 
Vergleichsaufgabe nicht nötig ist, da nicht nach der weiteren Entwicklung gefragt wird. Allerdings ist zu fragen, ob Elemente, die die Weiterentwicklung in Deutschland betreffen, ggf. einen positiven Einfluss auf die Textqualität haben. Deshalb werden sie miterfasst.

Im Schluss muss dann das Sachurteil realisiert werden. Es hat, wie auch die übrigen Elemente, eine sprachliche und inhaltliche Dimension. Die sprachliche Dimension wird in der nachfolgenden Kategorie in den Elementen "Begründen“ und „Beurteilen“ berücksichtigt. Das Sachurteil muss die Vergleichsthese verifizieren oder falsifizieren und die Elemente des Gegenüberstellens im Urteil zusammenführen. Deshalb ist es textstrukturell am Ende anzuordnen. Mögliche weitere Beurteilungselemente werden nachfolgend noch berücksichtigt. Unter diesem Strukturierungselement geht es zunächst um einen inhaltlich konsistenten Abschluss des Textes im Hinblick auf den Vergleich. ${ }^{641}$

\subsubsection{Realisierung sprachlicher Teilhandlungen}

Unter diesem dritten Strukturierungselement werden solche sprachlichen wie fachlichen Teilhandlungen berücksichtigt, die den Vergleich im Geschichtsunterricht ausmachen. Es handelt sich dabei um die in Kapitel 3.5 dargestellten sprachlichen Teilhandlungen:
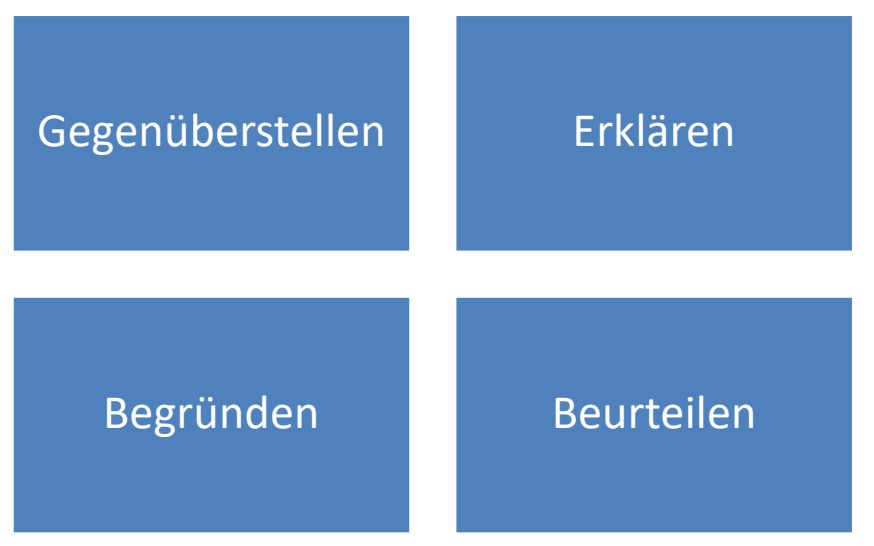

Abbildung 42: Sprachliche Teilhandlungen des Vergleichs (eigene Darstellung)

\footnotetext{
${ }^{641}$ Das Vorkommen eines Sachurteils ist deshalb in der Kategorie „Thematische Fokussierung“ eingebunden, weil dazu inhaltliches Wissen eine wesentliche Voraussetzung darstellt:

„Deklaratives Wissen ist vor allem für die Interpretationskompetenz wichtig. Je mehr Begriffe und Konzepte vorhanden sind, und je reicher diese ausdifferenziert sind, umso mehr Verknüpfungen und Vergleiche sind möglich, umso eher lassen sich Ursachen und Wirkungen bestimmen, umso besser sind Ereignisse, Sachverhalte und Personen zeitlich zu ordnen und zueinander in Beziehung zu setzen.", aus: Bernhardt, Markus, Gautschi, Peter; Meyer, Ulrich (2011): Historisches Lernen angesichts neuer Kerncurricula. Von Bildungsstandards und Inhaltsfeldern zur Themenbestimmung und Unterrichtsplanung im Geschichtsunterricht. Wiesbaden: Institut für Qualitätsentwicklung. S. 14. Online einsehbar unter:

https://www.google.com/url?sa=t\&rct=j\&q=\&esrc=s\&source=web\&cd=1\&ved=2ahUKEwiP17Ljx7vpAhUS3hoKHVTnBBIQFjAAegQIAhAB\&url=https\%3A\%2F\%2Fdieter-vaupel.jimdofree.com\%2Fapp\%2Fdownload\%2F7230429781\%2FBernhardt\%2BGautschi\%2BMayer\%2BHistorisches\%2BLernen\%2B-Kerncurricula.pdf\%3Ft\%3D1586423116\&usg=AOvVaw0VGaNTSmVz4lvSTjiNp8c- (zuletzt eingesehen am 17.05.2020).
} 
Über das Kriterienraster soll zunächst herausgefunden werden, ob Lernende das Gegenüberstellen als wesentliches Element des Vergleichens realisieren. Weiterhin soll überprüft werden, wie sie das Gegenüberstellen in ihren Texten ausführen, ob Elemente in Verbindung gesetzt und so auf Gemeinsamkeiten, Ähnlichkeiten und Unterschiede überprüft werden oder ob sie unverbunden nebeneinanderstehen.

Wie auch bei den folgenden Teilhandlungen wird das Gegenüberstellen aufgeschlüsselt in einen fachlich/pragmatischen und einen sprachlichen Aspekt. Auch wenn davon ausgegangen werden muss, dass beides untrennbar zusammenhängt, kann nur über diese Trennung im Kriterienraster erfasst werden, ob und wie Strukturen des Gegenüberstellens in den Texten der Schüler*innen expliziert werden.

Im ersten Teil werden solche Strukturen erfasst, die den Zusammenhang zwischen zwei Vergleichselementen herstellen; dabei können hier auch implizite Strukturen berücksichtigt werden. Im zweiten Teil, in dem es um die Versprachlichung des Gegenüberstellens geht, werden dann nur die expliziten sprachlichen Mittel berücksichtigt, wie bspw. Komparation, Antonyme, die Partikeln als und wie u.a.m., also solche Mittel, die auf eine Bezugsgröße verweisen. Nicht berücksichtigt werden dagegen isolierte relative Adjektive, die auf eine Bezugsnorm statt auf eine Bezugsgröße verweisen (z. B. „die Industrialisierung verlief schnell“), da die Gegenüberstellung entsprechend der Aufgabe konkret auf den Beginn der Industrialisierung von England und Deutschland bezogen sein soll und nicht eine Verbindung zu einem Abstraktum herstellen soll.

Auch für das Begründen als weitere sprachliche Teilhandlung wird im Manual wieder unterschieden zwischen dem fachlich/pragmatischen und den sprachlichen Formen für das Begründen. Für das fachlich/pragmatische Begründen soll eingeschätzt werden, ob bzw. inwiefern Begründungsstrukturen vorhanden sind. Für die Vergleichsaufgabe könnte eine mögliche Begründung wie folgt formuliert lauten: „Ein Grund für die Annahme, dass England ein Pionierland war, ist...".

Das Begründen stellt eine hohe Kompetenzanforderung dar, soll es nicht mit dem Erklären vermischt werden. Deshalb wird im Kriterienraster mit dem Rating "2"als höchster Rang nach eindeutigen Begründungsstrukturen gefragt. Nicht eindeutig vom Erklären abgrenzbare Strukturen werden als mittlerer Rang erfasst.

Die sprachliche Realisierung des Begründens wird gesondert erfasst, dazu wird die Anzahl der sprachlichen Mittel ausgezählt. Um diese von sprachlichen Mitteln des Erklärens abzugrenzen, werden ausschließlich Sachverhalte und Beziehungen über Lexeme erfasst (z. B. „A begründet sich aus B“). Diese Eingrenzung kann mit Graefen/Moll fundiert werden: „Wie lässt sich das Begründen vom Erklären unterscheiden [...]? Wir denken, dass der Autor selbst das Bedürfnis hat, seine Begründungen sprachlich zu kennzeichnen. ${ }^{\text {“642 }}$ Über Lexeme wiederum können Begründungen gekennzeichnet werden. ${ }^{643}$

Für das Erklären als neben dem Gegenüberstellen und Begründen zu berücksichtigender sprachlicher Teilhandlung wird im Kriterienraster in Abgrenzung zum Begründen auf die funktionale Beschreibung

\footnotetext{
${ }^{642}$ Graefen, Gabriele; Moll, Melanie (2007). S. 495.

${ }^{643}$ Die im Manual aufgeführten lexikalischen Mittel sind entnommen aus: ebd. S. 499.
} 
des Erklärens zurückgegriffen (vgl. dazu Kap. 5.3.3) und auf den Geschichtsunterricht bezogen. Begründe-Strukturen werden in diesem Ansatz vom Erklären unterschieden.

Um das Erklären eindeutig von anderen Formen abgrenzen zu können, wird nach Erklären-warum gefragt. ${ }^{644}$ Hoffmann geht davon aus, dass eine Erklärung immer ein erklärendes Element und ein zu erklärendes Element verbindet. ${ }^{645}$ Über die Verbindung solcher Elemente mit der ,Warum'-Frage sollen die Erklärungen in den Lernendentexten ausgewertet werden.

Die Versprachlichung des Erklärens wird ebenfalls über das Grundelement Erklären-warum ausgewertet, um so kausale Mittel $^{646}$, die das Erklären kennzeichnen von anderen Operationen wie dem Begründen abzugrenzen, das für die Versprachlichung ebenfalls kausale Strukturen benötigt, aber aus funktionalpragmatischer Sicht zu einem unterschiedlichen Zweck verwendet wird .

Als weitere wichtige sprachliche Teilhandlung für das Vergleichen berücksichtigt das Kriterienraster Beurteilungsstrukturen. Von den Lernenden wird durch eine solche Teilhandlung für die Lösung der vorliegenden Vergleichsaufgabe eine möglichst differenzierte analytisch-sachliche historische Beurteilung auf der Grundlage des Darstellungstextes verlangt. Dabei können diese Beurteilungen sowohl als Teilurteile wie auch als abschließendes Sachurteil in Erscheinung treten. Deshalb werden Beurteilungsstrukturen zum einen durch das Item „abschließendes Sachurteil“ (vgl. TF 04) erfasst. Darüber hinaus muss aber davon ausgegangen werden, dass auch „Teilurteile“ in Bezug auf einzelne Aspekte vorliegen, die in diesem Teilkriterium erfasst werden sollen. Diese spielen für die abschließende Beurteilung im Sinne eines Sachurteils eine vorbereitende Rolle. Deshalb werden solche Beurteilungen zudem durch die Kategorie „Beurteilen“ erfasst, die sich in die Items „Beurteilen fachlich/ pragmatisch“ und „Versprachlichung des Beurteilens" aufschlüsselt.

Auf fachlich/pragmatischer Ebene werden sachliche Wertungen erfasst. Diese können aus der Gegenüberstellung erwachsen. Entsprechend werden für das Item "Versprachlichung des Beurteilens“ sprachliche Mittel, die das Beurteilen explizieren, berücksichtigt (z. B. Verben, Adjektive, Nomen, die eine Wertung einschließen, wie etwa macht deutlich, ist festzustellen u.a.m.).

Über die dem Vergleichen direkt zuzuordnenden Teilhandlungen hinaus ist davon auszugehen (und dies zeigen bereits die Auswertungen aus der Pilotierung), dass auch solche sprachlichen Handlungen auftreten, die nicht direkt zum Vergleichen gehören, aber von den Lernenden dennoch aus verschiedenen Gründen realisiert werden. Da diese Strukturen in der Darstellung sprachlicher Teilhandlungen als Elemente des Vergleichs (Kap. 5.3) noch nicht thematisiert wurden, werden sie an dieser Stelle etwas ausführlicher behandelt:

\footnotetext{
644 "Aufzuklären ist stets eine Warum-Frage“ aus: Hoffmann, Ludger (2014), S. 518. Damit ist der Blick auf Grund und Ursache gerichtet (z. B. in Abgrenzung zu „Wozu“, das die Zweckgebundenheit fokussiert). 645 Ebd.

${ }^{646}$ Als sprachliche Mittel sind hier Konjunktionen wie z. B. weil, da, daher, Adverbien wie darum, daher etc. und feststehende Wendungen: ...liegt daran, dass zu nennen.
} 
Hierzu gehört die Folge (im Sinne einer Folgerung). Ihr Zweck ist die Herstellung einer Grund-FolgeRelation. Sie ist damit das Ergebnis von etwas Vorausgehendem, die Wirkung einer vorausgehenden Handlung. Hoffmann spricht von einer "Abfolge entlang einer Chronologie“. ${ }^{647}$ Dieser Gedanke der chronologischen Abfolge ist gerade für die Geschichte und für das zugrundeliegende Thema relevant, da der Beginn der Industrialisierung aus Entwicklungen entsteht, die wiederum zu weiteren Entwicklungen führen. Solche Folgen legt auch der Darstellungstext nahe bzw. gibt sie vor. Die Folge ist eng mit der Kausalstruktur verbunden, funktioniert jedoch entgegengesetzt. ${ }^{648}$ Für die vorliegende Aufgabe kann angenommen werden, dass die Formulierung von Folgen durch die Übernahme von Textteilen entsteht, die eine solche Folge vorgeben (z. B.: „Die Verbilligung und Verbesserung des Eisens sowie die Modernisierung der Produktionsverfahren schufen die Voraussetzungen für die Entstehung einer leistungsfähigen Maschinenindustrie und später für den Eisenbahnbau. Damit wirkte die Eisenindustrie in viele andere Wirtschaftszweige hinein [...]. $\left.{ }^{4649}\right)$. Eine solche logische Relation kann durch sprachliche Mittel wie so dass, deshalb, infolge u.a.m. realisiert werden oder auch ganz ohne explizite sprachliche Mittel. Im genannten Textbeispiel übernimmt das „damit“ (im Sinne von mit der Folge) die Explizierung der Folge.

Genauer kann die Grund-Folge-Relation in Folge und Folgerung ausdifferenziert werden:

„Wie wir bei Kausalverhältnissen zwischen Grund und Begründung unterscheiden, unterscheiden wir bei Konsekutivverhältnissen zwischen Folge und Folgerung. Bei adjazentem [also: direkt aufeinander folgendem; Anm. d. Verf.] Gebrauch nun scheint stets eine Formulierung eines Folgerungsverhältnisses vorzuliegen [...]. Bei nicht-adjazentem Gebrauch, also bei konsekutiven Grundsätzen, hingegen wird auf propositionaler Ebene eine Ursache-Folge-Relation formuliert. ${ }^{4650}$

Insbesondere die Folge kann in den Texten der Schüler*innen angenommen werden. Es ist jedoch zu fragen, inwiefern solche Folge-Strukturen für den Vergleich zuträglich sein können, da die Folge allein nur Strukturen innerhalb eines Vergleichsobjektes veranschaulicht, damit also nur implizit zum Vergleich beitragen kann. Erst durch Kausalverbindungen werden beide Vergleichsobjekte in Verbindung gebracht.

Für das oben angeführte Beispiel aus dem Text eines Schülers oder einer Schülerin ist zudem festzustellen, dass es sich nicht um eine eindeutige Folge handelt, sondern diese durch den Text- bzw. Satzzusammenhang angenommen werden kann. Dies liegt am Gebrauch des Konnektors „damit“. Erwartbar wäre für die Kennzeichnung einer Grund-Folge-Relation bspw. ein „dadurch“. Der Subjunktor „damit" hingegen markiert einen Finalzusammenhang. ${ }^{651}$

\footnotetext{
${ }^{647} \mathrm{Vgl}$. Hoffmann, Ludger (2014), S. 520.

${ }^{648}$ Zur logisch-semantischen Struktur kann festgestellt werden: „Es liegt dieselbe Schlussfigur vor wie beim kausalen Verhältnis. Allerdings sind Antezedens und Konsequens nun in umgekehrter Weise auf Haupt- und Nebensatz verteilt. Der Nebensatz liefert das Konsequens, der Hauptsatz das Antezedens." aus: Zifonum, Gisela; Hoffmann, Ludger; Strecker, Bruno u. a. (1997), S. 2293.

${ }^{649}$ Laschewski-Müller, Karin; Rauh, Robert (2011), S. 257.

${ }^{650}$ Zifonum, Gisela; Hoffmann, Ludger; Strecker, Bruno u. a. (1997), S. 2311.

${ }^{651} \mathrm{Vgl}$. Zifonum, Gisela; Hoffmann, Ludger; Strecker, Bruno u. a. (1997), S. 828f. Eine solche unsichere Nutzung von Konnektoren zeigt die Relevanz, sprachliche Mittel im Hinblick auf den Zweck des dahinterstehenden Handlungsmusters zu klären. Dazu gehört auch die Klärung des Gebrauchs von Konnektoren als wichtigen Aspekt für Textkompetenz.
} 
Folge-Strukturen sollen zumindest holistisch hinsichtlich ihres Beitrags zur Lösung der vorliegenden Vergleichsaufgabe erfasst werden. Es ist anzunehmen, dass es sich im Vergleich zu den zuvor genannten Teilhandlungen um eine kompetenzniedrigere Struktur handelt, die zum Teil aus dem Darstellungstext übernommen werden kann. Bereits die Analyse der Texte aus der Erhebung 1 weist zudem darauf hin, dass Folge-Strukturen häufiger in den Schüler*innentexten vorkommen.

Die Reihung ist ebenfalls eine Struktur, die sich in einigen Korpustexten zeigt. Auch sie wurde in Kapitel 3.5 nicht als wesentliches Element zur Konstruktion eines historischen Vergleichs angenommen.

Mit einer Reihung ist ein Hintereinanderschalten von Assertionen ${ }^{652}$ gemeint, also eine strukturierte oder unstrukturierte Wiedergabe von verschiedenen Wissenselementen. ${ }^{653}$ Für die Lösung der vorliegenden Vergleichsaufgabe kann angenommen werden, dass diese Wissenselemente dem Darstellungstext entstammen. Die Reihung kommt dem Operator Aufzählen am nächsten. Entsprechend den EPA Geschichte ist das Aufzählen als „zielgerichtet Informationen zusammentragen, ohne diese zu kommentieren " 654 bestimmt. Die Reihung wird im Rahmen dieser Auswertung darüber hinaus so verstanden, dass Informationen (aus dem Darstellungstext) zusammengetragen werden, aber diese auch ohne Verbindung hintereinandergeschaltet werden können (also weniger zielgerichtet). Es handelt sich dabei u. U. um eine Wiedergabe von Inhalten aus dem Darstellungstext ohne die Zielrichtung des Vergleichs.

Kameyama folgend kann die der Reihung zugrundeliegende sprachliche Handlung als vor allem dem Rephrasieren oder der Planstörungsexothese zugehörig eingeordnet werden. Das bedeutet, dass das Rephrasieren, das über eine zielgerichtete Reihung geleistet werden kann, beispielsweise das Erklären unterstützen kann, indem über die Reihung Wissenselemente transferiert werden. Es kann aber auch sein, dass die nicht zielgerichtete Reihung anzeigt, dass Inhalte des Darstellungstextes nicht verstanden wurden und damit nicht in den Vergleich übertragen werden können. Dieses Nichtverstehen könnte auch über eine Leerstelle im Text realisiert werden oder den Abbruch der Textproduktion insgesamt. Die Reihung kann aber die Funktion einer Art ,Ablenkungs- oder Verschleierungshandlung' übernehmen, die das Nichtverstehen eigentlich verdecken soll.

Damit muss auch für das Vergleichen gerechnet werden, zumal durch den Darstellungstext komplexes inhaltliches Wissen vermittelt wird, das es zunächst einmal zu entschlüsseln gilt.

Zwar kann die Reihung im Rahmen einer übergeordneten sprachlichen (Teil-)Handlung auftauchen. Sie stellt aber keine eigenständige sprachliche Handlung oder Teilhandlung dar, sondern kann eher als Konzept ${ }^{655}$ aufgefasst werden, das hier eine bestimmte Teilfunktion erhält. ${ }^{656}$

${ }^{652}$ Gemeint sind damit Aussagen, die die Wirklichkeit eines Sprechers versprachlichen, über Assertionen soll Wissen transferiert werden; vgl. dazu z. B: Hoffmann, Ludger (2014), S. 502.

${ }^{653} \mathrm{im}$ schulischen Kontext ist häufig von ,Informationen' die Rede.

${ }^{654}$ Kultusministerkonferenz (2005): EPA Geschichte, S. 7.

${ }^{655} \mathrm{Vgl}$. dazu: Kameyama, Sinichi (1999): Wiederholen. In: Bührig, Kristin; Matras, Yaron (Hrsg.): Sprachtheorie und sprachliches Handeln. Festschrift für Jochen Rehbein zum 60. Geburtstag. Tübingen: Stauffenburg Verlag. S. 187-202. S. 193.

${ }^{656}$ Vgl. ebd. S. 194. 
Aufgrund der Komplexität des Darstellungstextes können solche Strukturen auch für die Gesamterhebung angenommen werden. Über die holistische Erhebung hinsichtlich der Frage, ob solche Reihungen zum Vergleich beitragen, können die dargestellten Konzepte der Reihung berücksichtigt und anschlieBend ausgewertet werden.

\subsubsection{Wissenschaftliche Textkompetenz}

Unter diesem vierten und letzten Strukturierungselement werden solche Kompetenzen berücksichtigt, die Lernende im Sinne wissenschaftspropädeutischen Arbeitens in der Sekundarstufe II erreichen sollen. Diese Elemente sind hinsichtlich ihrer Bedeutung für historisches Arbeiten allgemein und für den historischen Vergleich ausgewählt.

Das erste Item bezieht sich auf den Umgang mit der Textgrundlage. Hierdurch wird hinterfragt, ob Lernende Übernahmen anderer Positionen, also insbesondere aus dem Vorlagentext, als Aussagen anderer gekennzeichnet haben. Dies ist eine Kompetenz, die in allen Fächern erwartet wird und gleichzeitig ein ganz wesentliches Element historischen Arbeitens darstellt, will man dem Konstruktionscharakter von Geschichte gerecht werden. Gerade der korrekte Umgang mit Quellen stellt eine Kernkompetenz im Geschichtsunterricht dar. Dazu gehört auch das fachgerechte Zitieren. Was für Quellentexte gilt, gilt jedoch ebenso für Darstellungstexte. Dabei ist es gerade in Darstellungstexten für Schüler*innen nicht leicht zu erkennen, welche Passagen gekennzeichnet werden müssen und an welchen Stellen im Text Wissenselemente ein Allgemeingut darstellen, die ungekennzeichnet bleiben dürfen. Da der Lehrplan hinsichtlich historischer Zitiertechniken keine nähere Bestimmung vornimmt, sondern lediglich von sach- und fachgerechter Analyse und Interpretation von Quellen und Darstellungstexten ${ }^{657}$ spricht, werden darin nur allgemeine Zitiertechniken vorausgesetzt. Dabei wird berücksichtigt, ob die Lernenden, wenn sie Textpassagen oder Teilsätze aus dem Darstellungstext übernehmen, diese vollständig und korrekt kennzeichnen (etwa durch Anführungsstriche, Umformulierung in die indirekte Rede oder sprachliche Kennzeichnungen wie „der Autor sagt..."). Dazu gehört auch die Kennzeichnung übernommener Konzepte und Zuschreibungen, wie etwa bei den Begriffen „Pionier“ und „Nachzügler ${ }^{\prime 658}$, oder Wertungen, die im Darstellungstext vorgenommen und in den Texten der Lernenden übernommen werden.

Als weiteres Merkmal gehört zum historischen Handwerkszeug der Gebrauch von Distanzierungsmitteln. Geschichte ist konstruiert und verlangt deshalb die Reflexion vorgegebener Aussagen, Standpunkte (auch des eigenen) und damit Distanz. Mit Hilfe von Distanzierungsmitteln kann (im Falle des Textes) ein Schreiber seinem Leser verdeutlichen, ob er eine Aussage für ,faktisch' hält oder ob sie nur

\footnotetext{
${ }^{657}$ Vgl. Ministerium für Schule und Weiterbildung des Landes Nordrhein-Westfalen (Hrsg.) (2014): Kernlehrplan für die Sekundarstufe II Gymnasium/Gesamtschule in Nordrhein-Westfalen. Geschichte. Online verfügbar unter: http://www.standardsicherung.schulministerium.nrw.de/lehrplaene/upload/klp_SII/ge/KLP_GOSt_Geschichte.pdf (zuletzt eingesehen am 04.10.17). Z. B. S. 35.

${ }^{658}$ Vgl. Laschewski-Müller, Karin; Rauh, Robert (Hrsg.) (2010). S. $257 f$.
} 
zu einem bestimmten Grad als faktisch angenommen werden kann, z. B. weil sie von einem anderen Autor stammt und nicht überprüft wurde ${ }^{659}$. Im Deutschen wichtige Strukturen für die Darstellung verschiedener Grade von Faktizität sind insbesondere Verbmodi (Verwendung von Indikativ oder Konjunktiv) oder Modalverben (Verwendung von Modalverben wie können, müssen etc.). ${ }^{660}$

Auch um die Vergleichsaufgabe zu lösen, müssen die Lernenden verschiedene Grade von Faktizität bzw. unsicherer Faktizität versprachlichen können. Der Schreibende/die Schreibende nimmt dadurch eine Bewertung vor.

In dem der Vergleichsaufgabe zugrunde gelegten Darstellungstext kann man ein hohes Maß an Faktizität feststellen, die den Lesern vermittelt wird. Nur in wenigen Formulierungen wird überhaupt eine Distanzierung vorgenommen. Es muss deshalb angenommen werden, dass der Einbezug von Distanzierungsmitteln für Lernende in Bezug auf diese Aufgabe eine hohe Kompetenz abverlangt, da sie zunächst einmal reflektieren müssen, welche Aussagen überhaupt distanzierungsbedürftig sind und wie sie dann mit aus dem Text übernommenen Aussagen in ihren eigenen Texten umgehen. Für die Auswertung auf der Grundlage des Kriterienrasters werden als grundlegende Strukturen insbesondere Modalverben, Verbmodi, darüber hinaus modale Satzadverbialia (z. B. wahrscheinlich, eventuell etc.) und Modalpartikeln angenommen, es werden aber auch weitere Strukturen wie bspw. formelhafte Verkürzungen (z. B. wie angenommen etc.) berücksichtigt, sofern sie eine inhaltliche Distanzierung markieren, also modal abschwächend sind.

Als drittes Merkmal für den Umgang mit der Textgrundlage wird überprüft, ob bzw. inwiefern Lernende eine Personalisierung von Handelnden vornehmen.

Bergmann grenzt den Begriff der Personalisierung deutlich von der Personifizierung ab, indem er die Personalisierung als „[...] eine Form der Wirklichkeitserfassung, bei der die Wirklichkeit als das Entscheidungsfeld und als das Resultat des Handelns weniger Einzelpersonen begriffen wird“661, beschreibt und kritisiert. Im Gegensatz dazu definiert er die Personifizierung als „Darstellung von Geschichte an ,namenlosen' handelnden und leidenden Personen und aus der Sicht dieser Personen, die immer gesellschaftliche Gruppierungen vertreten " ${ }^{\prime 662}$ und der er entsprechend didaktische Vorteile zuschreibt, weil die Personifizierung nicht von der ,Geschichte der Großen' ausgeht. Schneider unterzieht in Bezugnahme auf die Differenzierung Bergmanns beide Begriffe einer kritischen Prüfung. Zwar unterstützt er Bergmanns Prinzip der Personifizierung, nennt aber auch Hürden beim Gebrauch des Konzepts. Er macht insbesondere Schwierigkeiten im Auffinden personifizierender Quellen für bestimmte

${ }^{659}$ Zu den Herausforderungen durch Modalverben im Geschichtsunterricht vgl. auch: Peuschel, Kristina, Burkhard, Anne (2019): Sprachliche Bildung und Deutsch als Zweitsprache in den geistes- und gesellschaftswissenschaftlichen Fächern. Tübingen: Narr Studienbücher.

${ }^{660} \mathrm{vgl}$. hierzu wie auch im Folgenden: Diewald, Gabriele (2013): Modus und Modalverben - Kategorisierungsoptionen im grammatischen Kernbereich der Modalität. In: Abraham, Werner; Leiss, Elisabeth (Hrsg.): Funktionen von Modalität. Berlin/Boston: de Gruyter. S. 77-112. S. 89ff.

${ }^{661}$ Bergmann, Klaus (1997): Personalisierung, Personifizierung in: Bergmann, Klaus; Fröhlich, Klaus; Kuhn, Annette; Rüsen, Jörn; Schneider, Gerhard (Hrsg.), Handbuch der Geschichtsdidaktik, Seelze, S. 298-300, S. 298.

662 Ebd. S. 299. 
Zeiträume aus und bemerkt, im Geschichtsunterricht müsse „[...] heute auch unbefangen und offen über menschliche Größe [Hervorh. i. Orig.] gesprochen werden können. “663

Es wird deutlich, dass hinter den Begriffen Personalisierung und Personifizierung ein grundlegendes Konzept für den Geschichtsunterricht steckt. Wenn in Bezug auf das genannte Item die ,Personalisierung Handelnder' untersucht werden soll, ist damit ein eingegrenzter Begriff von Personalisierung gemeint, der lediglich prüft, ob die Lernenden sich auf allgemeine Zuschreibungen zurückziehen oder historische Akteure beim Namen nennen. Die Sichtbarmachung der Handelnden ist ein Prinzip von Geschichtsschreibung. In Bezug auf den der Vergleichsaufgabe zugrundeliegenden Darstellungstext ist allerdings festzustellen, dass der Text HandeInde kaum benennt. Handelnde werden stark typisiert (z. B. „der Staat“ als Handelnder statt Benennung tatsächlich Handelnder) oder verschleiert (z. B.: „Dazu passten auch [...] der Erlass der Gewerbefreiheit im Jahr 1810“664). Auch hier ist von einer hohen geschichts-wissenschaftlichen Textkompetenz auszugehen, wenn Lernende solche abstrakten Formulierungen für ihre eigenen Texte nutzbar machen wollen. Dennoch ist die Nennung der Akteure als wesentlich für (vor-)wissenschaftliches Schreiben einzuschätzen, so dass trotz der Hürden durch die Textvorlage auf die Erfassung solcher Strukturen nicht verzichtet werden kann. Allerdings wird bereits die Nennung der im Text genannten Typisierungen wie Bevölkerung, Reformkräfte, Betreiber etc. als hinreichend gewertet, um der schwierigen Textstruktur Rechnung zu tragen.

Als vergleichsspezifische Mittel wissenschaftlichen Schreibens können die Mittel zur Themenentwicklung bzw. zur Themafortführung gefasst werden, die das 4. Strukturierungselement abrunden sollen. Solche Mittel ${ }^{665}$ sind zentral für das Sprechen oder Schreiben über einen Redegegenstand ${ }^{666}$ und maßgeblich, um einen Vergleich zu realisieren. Im Gegensatz zur Themafortführung, die nachfolgend noch dargestellt wird, werden über die Themenentwicklung verschiedene Themen miteinander verbunden. ${ }^{667}$ Die Themenentwicklung kann in unterschiedliche Typen differenziert werden ${ }^{668}$, den für den historischen Vergleich entscheidenden Typ stellt das Themensplitting dar. Zifonum et al. definieren das Themensplitting wie folgt: „Unter THEMENSPLITTING [Hervorh. i. Orig., Anm. d. Verf.] verstehen wir den Fall, daß von einem mehrere Gegenstände/Sachverhalte umfassenden Thema zu Einzelthemen, die jeweils einen dieser Gegenstände/Sachverhalte umfassen, übergegangen wird. “669 Das bedeutet nicht, dass die Einzelthemen durch das Themensplitting keine Berührungspunkte (mehr) haben. Hierunter sind vielmehr solche Mittel gefasst, die das Vergleichsthema weiterentwickeln, indem sie

\footnotetext{
${ }^{663}$ Schneider, Gerhard (2012): Personalisierung/Personifizierung, in: Barricelli, Michele; Lücke, Martin (Hrsg.): Handbuch Praxis des Geschichtsunterrichts, Bd. 1, Schwalbach/Ts. S. 302-315, S. 314.

${ }^{664}$ Laschewski-Müller, Karin; Rauh, Robert (2010), S. 258.

${ }^{665}$ Eine Aufführung weiterer Mittel zur Thematisierung des Redegegenstands findet sich Bsp. bei Hoffmann, Ludger (2013): Deutsche Grammatik. Grundlagen für Lehrerausbildung, Schule, Deutsch als Zweitsprache und Deutsch als Fremdsprache. Berlin: Erich Schmidt Verlag.

${ }^{666}$ Vgl. dazu: Hoffmann, Ludger (2013), S. 75.

${ }^{667}$ Das Themensplitting definieren Zifonum et al. entsprechend: „Unter einer THEMENENTWICKLUNG [Hervorh. im Original] verstehen wir einen unselbständigen sprachlichen Akt, mit dem ein Sprecher/Autor von einem Thema TH1 zu einem Thema TH2 bzw. Themen TH2 ... THn übergeht“. Aus: Zifonum, Gisela; Hoffmann, Ludger, Strecker, Bruno (1997). S. 536.

${ }^{668} \mathrm{Vgl}$. ebd. S 536f.

${ }^{669}$ Vgl. ebd. S. 537.
} 
mindestens ein Paar enthalten, das über eine Teilung (und damit letztlich über den Gegensatz) versprachlicht wird. Es setzt voraus, „[...] dass ein Redegegenstand eine Gruppe, mindestens ein Paar enthält. Daraus können einzelne Elemente gewählt und thematisch weiterentwickelt werden. ${ }^{1670 ~ I m ~ v o r-~}$ liegenden Darstellungstext wird ein solches Paar bereits vorgegeben: England - Deutschland. Es handelt sich also um die grundlegenden Vergleichsobjekte für den historischen Vergleich. Insbesondere diese Ausdrucks- oder im Hinblick auf den Vergleich besser Gegensatzpaare werden im Kriterienraster erfasst. Weitere solcher Gegensatzpaare können z. B. „die eine - die andere“, „er - sie“, "der - der", „das erste - das zweite“, „einer - ein weiterer“ darstellen.

Es handelt sich beim Themensplitting insofern um ein Mittel wissenschaftlicher Textkompetenz, als dass über sprachliche Mittel der Teilung der Zweck des historischen Vergleichs angebahnt werden kann. Dies gilt insbesondere, weil Oberflächenstrukturen (wie hier das Teilen) durch die Handlungsstrukturen vorgeprägt werden. ${ }^{671}$ Damit handelt es sich beim Themensplitting nicht mehr um ein alltägliches Mittel, sondern um ein fachliches, das wiederum durch alltägliche Wissenschaftssprache ${ }^{672}$ versprachlicht werden kann. Das Themensplitting stellt damit ein wesentliches Mittel zur Entwicklung eines roten Fadens dar, weil die Ausdrucks- oder Gegensatzpaare nicht nur getrennt, sondern dem Zweck entsprechend, vergleichend zu einem Sachurteil zu gelangen, immer wieder aufeinander bezogen werden müssen.

Ein weiteres Mittel zur Entwicklung eines roten Fadens stellt die Themafortführung dar. Im Unterschied zur Themenentwicklung versteht man unter Themafortführung „,[...] einen unselbständigen sprachlichen Akt, mit dem ein Sprecher/Autor sich auf einen vorgängig eingeführten Gegenstand bzw. Sachverhalt bezieht. ${ }^{4673}$ Im Falle des Vergleichs des Beginns der Industriellen Revolution in England und Deutschland bedeutet dies die Orientierung auf das übergeordnete Thema „Beginn der Industriellen Revolution“, das fortgeführt wird, während im vorliegenden Fall gleichzeitig über das Themensplitting das Vergleichspaar entwickelt wird. Schließlich werden die Vergleichsobjekte spätestens im Sachurteil zusammengeführt und auf das übergeordnete Thema zurückgeführt. Die Themafortführung stellt damit ein wesentliches Mittel zur Leserführung dar und ist als solches unter wissenschaftlicher Textkompetenz zu subsummieren: „Textverstehen kommt nur dann zustande, wenn die einzelnen inhaltlichen Elemente sukzessiv und in kohärenter Weise zu dessen Aufbau beitragen und wenn dem Leser klar wird, an welchen Textstellen Inferenzen nötig sind. ${ }^{4674}$ Sie sind damit ein wesentliches Mittel der Leserführung und damit der Adressatenorientierung. Dabei muss es einem Autor/einer Autorin gelingen, die Leser mit Hilfe solcher Strukturen durch den Text zu leiten.

Über das Kriterienraster werden solche sprachlichen Mittel zur Themafortführung betrachtet, die im Hinblick auf wissenschaftliche Textkompetenz relevant sind. Dabei handelt es sich um Anaphern und Zeigwörter (deiktische Ausdrücke). Zeigwörter verweisen auf Außersprachliches:

„Vom Sprecher aus gesehen, ist sie [die deiktische Prozedur, Anm. d. Verf.] Ausdruck einer vorgenommenen Fokussierung; bei $\mathrm{H}$ [Hörer, Anm. d. Verf.] wird dadurch eine Orientierungshandlung

\footnotetext{
670 Hoffmann, Ludger (2013), S. 197.

${ }^{671}$ Vgl. Steinhoff, Torsten (2007), S. 82.

${ }^{672}$ Vgl. dazu Kapitel 2.7.

673 Zifonum, Gisela et al. (1997), S. 536.

${ }^{674}$ Graefen, Gabriele (1997), S. 114.
} 
initiiert. Das geschieht dadurch, daß der Sprecher die Aufmerksamkeit von $\mathrm{H}$ ohne symbolische Mittel auf das von inm mental fokussierte Element der Sprechsituation fixiert. S überträgt damit (s)einen Fokus auf H. Dies unterstellt ein jeweils in der Sprechsituation vorgegebenes kommunikatives "setting", welches eine schnelle Orientierung erlaubt. “675

Für den vorliegenden textlichen Zusammenhang werden solche sprachlichen Elemente fokussiert, die eine Objektdeixis repräsentieren: „Mit der OBJEKTDEIXIS (der, dieser, $\underline{\text { er }}$ ) [alle Hervorh. i. Orig., Anm. d. Verf.] kann auf Objekte im weitesten Sinn und Personen verwiesen werden, die sich in einem Verweisraum (Wahrnehmungsfeld, Text-/Rederaum) befinden." ${ }^{\text {}} 76$

Durch das Kriterienraster werden die Zeigwörter wie der, die, das, dieser, diese, dieses (angepasst an Numerus und Kasus), dieses, welches, jenes, da, darauf, hier, dort oder in zusammengesetzten Ausdrücken: damit, hiermit, dadurch, dafür, hierdurch erfasst. Es handelt sich um Ausdrücke, von denen für Lernende der Sekundarstufe II angenommen werden kann, dass sie über das Alltagswissen verfügbar und durch schulische Kontexte ihre Verwendung auch in fachlichen Kontexten geläufig ist. Gerade die Verwendung solcher objektdeiktischen Mittel für die eigene Textproduktion stellt jedoch eine hohe Anforderung an Schreibnovizen dar. Kann in einer direkten Gesprächssituation, also innerhalb eines gemeinsamen Wahrnehmungsraums, der Gebrauch eines deiktischen Mittels (wie "diese da" o.ä.) durch eine Zeighilfe unterstützt werden, ist dies im Text nicht möglich. Der Schreiber muss an das Wissen eines nicht bekannten Lesers anschließen und deiktische Mittel so wählen, dass der Leser die Verweisobjekte, an die der Autor anschließt, identifizieren kann. Dem Autor wird eine hohe Kompetenz zur Leserführung abverlangt, da er über deiktische Verweise die Aufmerksamkeit des Lesers steuert:

„Festzustellen ist, daß die deiktische Verweisung mit der Entfernung vom Wahrnehmungsraum zunehmend abstrakter wird. Die Ausweitung des Deixisgebrauchs ist daher nicht risikolos, und es ist zu vermuten, daß gerade für wissenschaftliche Texte besondere Vorkehrungen und Restriktionen existieren, um die Gefahr von Vagheit und Mißverstehen einzuschränken. ${ }^{4677}$

Eine funktionierende Verweisstruktur zeichnet einen wissenschaftlichen Text aus. Deiktische Verweisstrukturen sind dabei zu unterscheiden von anaphorischen Strukturen, die ebenfalls die Leserführung steuern. Die Leistung der Anapher stellt Graefen im Unterschied zur Deixis dar: „Ähnlich wie bei der Deixis geht es auch bei der Anapher um die Aufmerksamkeit des Hörers, aber nicht im Sinne einer Lenkung oder Steuerung, wie das bei der Deixis der Fall ist. Stattdessen könnte man von einem Verzicht des Sprechers auf eine Steuerung sprechen, weil der Aufmerksamkeitsfokus nicht geändert werden soll. ${ }^{4678}$ Sprachlich realisiert wird die Anapher über die Personalpronomen ${ }^{679} \mathrm{er} / \mathrm{sie} / \mathrm{es}$. Dadurch, dass ein bestimmtes Element schon im Fokus des Lesers ist und wieder aufgenommen wird, ist eine anaphorische Struktur leichter nachzuvollziehen als eine deiktische. Gleichwohl bietet sie eine wichtige

\footnotetext{
675 Ebd. S. $123 f$.

676 Zifonum et al. 1997, S. 41.

${ }^{677}$ Graefen, 1997, S. 131.

678 Ebd.138.

${ }^{679}$ Hoffmann stellt im Hinblick auf den Begriff des Pronomens heraus, dass es sich nicht um Stellvertreter von Nomen handele, sondern diese eine eigene Funktionalität bergen. Vgl. Hoffmann, Ludger (2013), S. 548.
} 
wissenschaftliche Struktur. Anaphorische Mittel können z. B. im Zusammenspiel mit Vergleichspartikeln Verwendung finden ${ }^{680}$ (,Sie begann in Deutschland später als in England': Das sie in diesem konstruierten Beispiel verweist auf die Industrielle Revolution zurück, die, wird die Anapher korrekt gebraucht, zuvor genannt worden sein muss).

Inwiefern deiktische und anaphorische Strukturen in den vorwissenschaftlichen Texten der Schüler*innen produziert werden, soll überprüft werden. ${ }^{681}$

Das Kriterienraster bildet insgesamt Elemente des historischen Vergleichs ab, die als konstitutiv erscheinen. Dabei musste schon aus auswertungsökonomischen Gründen eine Fokussierung stattfinden, so dass kein Anspruch auf Vollständigkeit erhoben wird. Auch können nur solche Elemente abgebildet werden, die Bezug auf den historischen Vergleich im Allgemeinen sowie den zugrundeliegenden Aufgabenkontext nehmen.

Im folgenden Kapitel sollen die Ergebnisse des Textratings dargestellt und darüber die Funktion und Bedeutung der Items eingeordnet werden.

680 Vgl. dazu: Hoffmann, Ludger (2013), S. 221.

${ }^{681}$ Sicherlich wäre an dieser Stelle eine Ausweitung der Untersuchung und Differenzierung in ana- und katadeiktische bzw. ana- und kataphorische Strukturen denkbar. Insbesondere vorausweisende (kataphorische) Strukturen stellen eine noch höhere Anforderung an den Leser dar, was wiederum bedeutet, dass der Autor seine Leserschaft noch genauer vorhersehen und fokussieren muss. Aufgrund der Tatsache, dass die Vergleichstexte von Schreibnovizen eingefordert wurden, sind solche Strukturen weniger erwartbar und werden deshalb nicht berücksichtigt. Graefen stellt selbst für wissenschaftliche Texte fest, dass kataphorische Strukturen (sie spricht von Prozeduren) in dem von ihr untersuchten Korpus nur ausnahmsweise vorkommen (vgl. Graefen, 1997. S.290). 


\section{$7 \quad$ Auswertung der Lernendentexte}

Die Auswertung der Lernendentexte erfolgte auf der Basis des in Kapitel 6 vorgestellten Kriterienrasters mit zugehörigem Manual. Darin ist fast durchgängig eine Zuordnung der Ratingentscheidung in eine dreistufige Skala vorgesehen. Diese Vorgabe ist darüber zu erklären, dass die Mehrzahl der Kategorien eine Rangordnung abbildet, weil eine qualitative Einschätzung vorgenommen wird $(0=$ nicht erkennbar, $1=$ teilweise erkennbar, $2=e r k e n n b a r)$. Deshalb wurde hier mit ordinalen Skalierungen gearbeitet. Für einige wenige Items, in denen es um das Auszählen bestimmter Merkmale aus dem Text und damit um eine Häufigkeitszählung ging, wurde eine metrische Skala als Vergleichswert zugrunde gelegt Die Skalenzuordnung kann im Anhang ${ }^{682}$ nachvollzogen werden.

Das Rating wurde zunächst pilotiert und im Anschluss eine Prüfung der Interraterreliabilität sowie der internen Konsistenz vorgenommen. Diese Prüfung wurde sowohl für die Pilotierung als auch für das gesamte Rating durchgeführt.

\subsection{Interraterreliabilität}

Die Lernendentexte wurden mit dem im letzten Kapitel vorgestellten Manual durch drei Rater beurteilt. Dazu wurden zunächst für eine Pilotbeurteilung 22 Texte ausgewählt. Um die Messgenauigkeit des Ratings mithilfe des zugrundeliegenden Kriterienrasters bestimmen zu können, wurde für diese Texte die Interraterreliabilität bestimmt. Wirtz/Caspar bestimmen diese in der Unterscheidung von Reliabilität und Übereinstimmung wie folgt: „Maße der Interraterreliabilität quantifizieren das Aus$\mathrm{ma}$, in dem jede einzelne Person von den verschiedenen Ratern ähnlich weit unter beziehungsweise über dem Durchschnitt der untersuchten Stichprobe liegend eingeschätzt wird. “683 Eine InterraterÜbereinstimmung ist dagegen nach dieser Betrachtungsweise nur gegeben, wenn unterschiedliche Rater ein Objekt genau gleich beurteilen. ${ }^{684}$

\subsubsection{Zum Vorgehen bei der Ermittlung der Interraterreliabilität}

Für die vorliegende Interraterreliabilitätsprüfung wurden sowohl der Cohens-Kappa-Koeffizient als auch Krippendorffs Alpha ermittelt. Während Cohens Kappa ein Maß für die Interraterreliabilität darstellt, das die Wahrscheinlichkeit ermittelt, mit der ein Rater Elementen eine bestimmte Kategorie zuordnet, wird bei der Ermittlung von Krippendorffs Alpha das erwartete Maß von (Nicht-)Übereinstimmung ermittelt. Durch die Verwendung beider Maße kann ein differenzierter Blick auf die Reliabilität

\footnotetext{
682 Vgl. Manual in Anhang 6.

${ }^{683}$ Wirtz, Markus; Caspar, Franz (2002): Beurteilerübereinstimmung und Beurteilerreliabilität. Methoden zur Bestimmung und Verbesserung der Zuverlässigkeit von Einschätzungen mittels Kategoriensystemen und Ratingskalen. Göttingen, Bern, Toronto, Seattle: Hogrefe. S. 36.

${ }^{684}$ Vgl. ebd. S. 34.
} 
der vorliegenden Beurteilung durch ein Kriterienraster geworfen werden. Außerdem erweitert Krippendorffs Alpha das Spektrum auf beliebig viele Rater, während Cohens Kappa in der Regel von der Berechnung zweier Raterurteile ausgeht. Krippendorffs Alpha erlaubt zudem selbst für kleine Stichproben verschiedene Skalierungen. ${ }^{685}$ Für die Bestimmung von Cohens Kappa (Auswertung über SPSS) wurden jeweils zwei Rater miteinander verglichen (dazu wurden alle drei Varianten durchgespielt). Krippendorffs Alpha wurde über das Online-Tool der Website http://dfreelon.org/utils/recalfront/ ermittelt.

Für eine gute Interraterreliabilität nehmen Bortz/Döring in Bezug auf Cohens Kappa einen Wert zwischen 0,60 und 0,75 an. ${ }^{686} \mathrm{Im}$ Folgenden wird von 0,7 als guter Wert ausgegangen, ein durchaus gängiges Maß, das auch für Krippendorffs Alpha angewandt werden kann. ${ }^{687}$

Die Interraterreliabilitäts-Koeffizienten wurden für jedes Item einzeln berechnet. Die Einzelergebnisse sind ausführlich im Anhang dargestellt (sowohl für Cohens Kappa als auch für Krippendorffs Alpha), so dass nun überblickshaft auf das Gesamtergebnis eingegangen wird und folgend auffallende Einzelergebnisse aufgeschlüsselt und eingeordnet werden sollen.

\subsubsection{Ergebnisse aus der Pilotierung}

Um Rater nachschulen zu können oder nicht tragfähige Items zu identifizieren und anpassen zu können, wurden als Pilotierung $25 \%{ }^{688}$ der Texte aus dem Gesamtkorpus ausgewählt und geratet.

Zunächst sollen die Ergebnisse aus der Errechnung des Cohens-Kappa-Koeffizienten dargestellt werden.

Aus den drei Ratern wurden die Paare Rater 1 und 2, Rater 1 und 3, Rater 2 und 3 gebildet, um paarweise die Cohens-Kappa-Koeffizienten errechnen zu können.

Für die Auswertung des Raterpaares 1 und 2 kann festgestellt werden, dass bis auf eine Ausnahme alle Werte bei oder über dem Wert von 0,7 liegen. Die Ausnahme erklärt sich durch eine Differenz in der Zuordnung, die in der Nachschulung besprochen werden konnte. Die Einzelwerte können im Anhang 7.1 eingesehen werden.

Für das Raterpaar 1 und 3 ergeben sich in der Pilotierung Kappa-Werte unter der Grenze von 0,7 für die Items VS („Vorstrukturierung“), „Textlänge“ und „Satzlänge“, UT („Unterscheidung zwischen Vorlagentext und eigener Position“) und MT 02 („Themenfortführung“).

Für das Item VS wurde festgelegt, dass Tabellen, denen kein Text folgt, die ,Hauptbearbeitung' der Aufgaben darstellen, sie jedoch kein Mittel zur Vorstrukturierung sind, da dann davon ausgegangen

\footnotetext{
${ }^{685}$ Hayes, Andrew F.; Krippendorff, Klaus (2007): Answering the Call for a Standard reliability Measure for Coding data. In: Communication Methods and Measures 1 Jg., Nr. 1, 77-89. Online verfügbar unter: http://www.afhayes.com/public/kalpha.pdf (zuletzt eingesehen am 25.06.2018).

${ }^{686}$ Vgl. Bortz, Jürgen; Döring, Nicola (2009): Forschungsmethoden und Evaluation in den Sozial- und Humanwissenschaften. 4. Aufl. Heidelberg: Springer Verlag. S. 277.

${ }^{687}$ Vgl. Hayes, Andrew F., Krippendorff, Klaus (2007), S. 87.

${ }^{688}$ Ausgehend von der Gesamtzahl der Korpustexte von 88, bereinigt liegt der Anteil bei $26 \%$.
} 
werden müsste, dass der Schüler/die Schülerin die Hauptaufgabe unbeantwortet lässt. Diese wurden jedoch zum Teil in der Piloterhebung unterschiedlich erfasst.

Die Abweichung der Textlängenwerte setzt sich zum Teil im Nachfolgeitem "Satzlänge" fort; die Abweichungen können darüber erklärt werden, dass sich ein Rater zunächst einer Satzerkennungssoftware bedient hatte, die einer manuellen Nachprüfung nicht standhält.

Für die Items UT und MT 02 kann kein klarer Grund für die Abweichungen erkannt werden. Die differierenden Werte könnten zu Anfang noch nicht ausreichend ausgeführten Beispielen geschuldet sein. Das Manual wurde durch Beispiele angereichert, alle auffälligen Items nachgeschult. Insgesamt zeigen die Ergebnisse der unauffälligen Items hohe Übereinstimmungswerte.

Die Rechnung der Kappa-Werte für die Raterkombination 2 und 3 zeigt etwas größere Unterschiede als die beiden zuvor gezeigten Kombinationen. Dabei ergeben sich niedrige Werte in der Regel aus den zuvor dargestellten Ergebnissen. Erklärungsbedürftig erscheinen die Kappawerte für die Items ST_01.1 und ST 02_2. Diese waren in den Rechnungen für die vorhergehenden Paare unauffällig. Dies bedeutet, dass die Einschätzungen des letzten Raterpaares weiter voneinander abweichen als dies bei den anderen Paarungen der Fall ist. Dieser Punkt kann erst besser eingeschätzt werden, wenn alle drei Ratings ins Verhältnis gesetzt werden können. An dieser Stelle wird die anschließende Rechnung von Krippendorffs Alpha bedeutsam.

Die Rechnung von Krippendorffs Alpha für alle drei Rater weist nur auf zwei Werte hin, die näher zu betrachten sind: Es handelt sich dabei um die Werte der Items VS und ST 05_1. Alle übrigen Items (und eben auch die Werte der oben angesprochenen Items ST_01.1 und ST 02_2) erreichen in der Vergleichsrechnung Werte von mindestens 0,7. Die Gründe für die Unterschiede beim Rating des Items VS wurden bereits für die Kappa-Werte besprochen.

Das Item ST 05.1 zeigt in den paarweisen Auswertungen der Kappa-Werte nur bei den Paarungen 1/3 und 2/3 abweichende Werte, so dass auch hier davon ausgegangen werden kann, dass das Nachschulen eines Raters ausreichend ist. Da zwei Rater unauffällige Werte zeigen, kann davon ausgegangen werden, dass das Item grundsätzlich funktioniert.

Es kann nach der Rechnung der Kappa- bzw. Krippendorffs-Alpha-Werte insgesamt angenommen werden, dass die Items genügend spezifiziert und durch Beispiele und Erklärungen untermauert sind, das Auswertungsraster also grundsätzlich funktioniert, so dass die Überprüfung der Interraterreliabilität nun für die Gesamterhebung vorgenommen wird.

\subsubsection{Die Interraterreliabilität für die gesamte Erhebung}

Für die gesamte Erhebung wurde Krippendorffs Alpha in Bezug auf alle drei Rater für jedes einzelne Item ermittelt.

Lediglich ein Item liegt unter dem geforderten Reliabilitätswert von 0,7. Dabei handelt es sich um Item ST 04_2 („Einschätzung der sprachlichen Mittel für das Beurteilen“). Im Gegensatz zur Erfassung des 
Beurteilens auf fachlich-pragmatischer Ebene (ST 04_1), das einen Interraterreliabilitätswert von 0,849 zeigt, liegt das Item ST 04_2 bei einem Wert von 0,623. Hier bildet sich möglicherweise die Schwierigkeit für die Rater ab, sprachliche Mittel als eindeutig für das Beurteilen einzuschätzen bzw. diese von Erklärstrukturen abzugrenzen, obwohl grundsätzlich Begründestrukturen als solche identifiziert werden können, wie das Item St 04_1 zeigt. Da jedoch der Wert immer noch im 0,6-Bereich liegt und das Begründen wesentlicher Teil des Vergleichens ist, findet das Item für die nachfolgende Berechnung Berücksichtigung.

Der Reliabilitätskoeffizient Krippendorffs Alpha wird als strenger Wert eingeschätzt. ${ }^{689} \mathrm{Im}$ Rahmen dieser Auswertung wird deshalb davon ausgegangen, dass das erarbeitete Kriterienraster für den historischen Vergleich als tragfähig angesehen werden kann. Eine zu große Gleichheit der Raterwerte kann im umgekehrten Fall dafürsprechen, dass die Items zu stark vereinfacht wurden und so an Aussagekraft verlieren.

Da die Werte gut sind und zum Teil sehr hohe Interraterreliabilitätswerte zeigen, kann mit dem Instrument weitergearbeitet werden.

\subsection{Die interne Konsistenz}

Über die Berechnung von Cronbachs Alpha soll auch für die relativ kleine Stichprobe die interne Konsistenz als weiteres Reliabilitätsmaß für die Items des Kriterienrasters geprüft werden ${ }^{690}$.

Für die Messung wurden nur solche Items analysiert, die als gemeinsamer Nenner die Textqualität abbilden. Deshalb fielen Items heraus, über die nur indirekt auf die Textqualität geschlossen werden kann, wie das Item VS („Nutzung von Mitteln zur Vorstrukturierung, die nicht in der Aufgabe gefordert werden"), da es hier nur um eine quantitative Erfassung von Tabellen, Stichpunkten etc. geht, die vor der Verfassung des Textes angelegt wurden; solche Mittel wurden aber nicht in ihrer Qualität erfasst. Zudem wurden solche Items nicht berücksichtigt, denen metrische Skalen zugrunde liegen. Diese bilden nur indirekt Textqualität ab, da zu diesem Zeitpunkt nicht klar ist, ob eine höhere Quantität auch mehr Qualität erzeugt oder indirekte Mittel ebenso zielführend sein können. Zu solchen Items zählen Textlänge, mittlere Satzlänge, ST 02.2 („Versprachlichung des Begründens“), ST 03.2 („Versprachlichung des Erklärens“), ST 04.2 („Versprachlichung des Beurteilens“), MT 01 („Themensplitting“), MT 02 („,Themenfortführung“) und DM 01 („Modalisierung“). Da zu den meisten Items immer auch die

\footnotetext{
${ }^{689}$ Aus: Raupp, Juliana; Vogelsang, Jens (2009): Medienresonanzanalyse. Eine Einführung in Theorie und Praxis. Wiesbaden: VS Verlag für Sozialwissenschaften Onlinezusatzmaterial. Springer. Online verfügbar unter: http://www.springer.com/cda/content/document/cda_downloaddocument/Berechnung-von-Reliabi-

lita\%CC\%88tskoeffizienten-Anhang.pdf?SGWID=0-0-45-1508109-p174296378 (zuletzt eingesehen am 08.08.17). S. XXI: „Die statistischen Eigenschaften von Krippendorff's erlauben die Berechnung eines 95\%-Konfidenzintervalls (95\%-KI). Das Konfidenzintervall wiederum gibt Auskunft über die Präzision der Reliabilitätsschätzung."

${ }^{690}$ Ergebnisse in Anhang 7.3.
} 
inhaltliche Qualität ausgewertet wurde, ist davon auszugehen, dass darüber Textqualität ausreichend erfasst werden kann. Die übrigen 20 Items bleiben für die Rechnung von Cronbachs Alpha erhalten.

Auf der Grundlage eines Cronbachs-Alpha-Koeffizient von 0,9 wird mit einem Rater weitergerechnet. Nach Bortz/Döring sind Werte über $\alpha 0,8$ anzustreben. Auch Schnell et al. bezeichnen Werte über $\alpha$ 0,8 als akzeptabel. In manchen Veröffentlichungen werden bereits Werte über $\alpha 0,7$ als gut eingestuft. Der vorliegende Cronbachs-Alpha-Koeffizient ist als gut einzustufen, es ist insgesamt von einer hohen internen Konsistenz auszugehen.

\subsection{Analyse und Interpretation der Ergebnisse}

Für die Analyse und anschließende Interpretation der Ergebnisse wurden zunächst Korrelationen errechnet, um Aussagen über lineare Zusammenhänge zwischen den Items machen zu können. In einem weiteren Schritt wurde eine explorative Faktorenanalyse durchgeführt ${ }^{691}$, um zu prüfen, ob sich die verschiedenen Variablen in wenigen Faktoren zusammenfassen lassen.

\subsubsection{Korrelationen}

Um Aussagen über Textqualität machen zu können, wurden (bivariate) Korrelationen errechnet. Über den zu errechnenden Korrelationskoeffizienten werden „Enge und Richtung des Zusammenhangs zwischen zwei Merkmalen " ${ }^{\prime \prime 92}$ bestimmt. Dabei sind für diese Arbeit die folgenden Korrelationskoeffizienten maßgeblich: Während mit Hilfe des Korrelationskoeffizienten nach Pearson ein linearer Zusammenhang zwischen zwei Variablen für nominal skalierte Zahlen bestimmt werden kann, werden mit dem Rangkorrelationskoeffizienten nach Spearman Rangzahlen berechnet. Da das Kriterienraster weitgehend qualitative Einschätzung erfragt, ist die Errechnung der Rangkorrelationskoeffizienten nach Spearman bestimmend. Für die Erfassung von Häufigkeiten, die ebf. vereinzelt abgefragt werden, ist dagegen aufgrund der Nominalskalierung der Zahlen die Bestimmung des Korrelationskoeffizienten nach Pearson folgerichtig.

Bei einem Korrelationskoeffizienten von 0 besteht kein linearer Zusammenhang, bei 1 ein perfekter Zusammenhang. Ein mittlerer Zusammenhang besteht bei einem Korrelationskoeffizienten zwischen 0.5 bis $0.8{ }^{693}$ Die Korrelation wird bei einem $p$-Wert, der kleiner oder gleich dem Signifikanzniveau ist (ein Signifikanzniveau von 0,05 gilt als gut geeignet), als signifikant angenommen.

Für die Auswertung der Ratingergebnisse wurden zunächst für jeden Text die Punktzahlen zusammengerechnet. Damit eine hohe Anzahl verwendeter sprachlicher Mittel die Gesamteinschätzung der Ein-

\footnotetext{
${ }^{691}$ Ergebnisse im Anhang 7.4.

${ }^{692}$ Vgl. Bortz, Jürgen; Döring, Nicola (2006), S. 507.

693 Die Einordnung orientiert sich am Online-Kurs „Neue Statistik“ der Fernuniversität Hagen: http://www.fernuni-hagen.de/ksw/neuestatistik/content/MOD_23196/html/comp_23414.html (zuletzt eingesehen am 20.06.2018).
} 
zeltexte über die Gesamtpunktzahl nicht verfälschen kann, indem z. B. viele sprachliche Mittel verwendet werden, die Textqualität über die übrigen Items aber sehr niedrig eingeschätzt wird und der Text dadurch eine insgesamt hohe Punktzahl erhält, die der Qualität nicht entspricht, wurde mit umkodierten Items gearbeitet. Die metrischen Items, die über eine Punktzahl von " 2 " hinausgingen, wurden deshalb in ein Ordinalschema von 0, 1 und 2 umkodiert. Textlänge und mittlere Satzlänge wurden nicht in die Berechnung der Gesamtpunktzahl einbezogen.

\subsubsection{Zusammenhang von Textlänge und mittlere Satzlänge zur Textqualität}

Da auf die erreichten Gesamtpunktzahlen noch in Bezug auf die Unterscheidung in drei Gruppen Bezug genommen wird, geht es hier zunächst nur um einen möglichen Zusammenhang von Textlänge und mittlere Satzlänge und Qualität der Vergleichstexte.

Korreliert man zunächst die Textlänge (Auszählung der Anzahl der Wörter im Text) mit der Gesamtpunktzahl (Korrelation nach Pearson), ergibt sich ein Korrelationskoeffizient von 0,76, so dass ein Zusammenhang angenommen werden kann. Die Korrelation ist auf einem Niveau von $>.001$ zweiseitig signifikant.

Die Darstellung in einer Punktwolke zeigt dieses Bild:

Tabelle 6: Korrelation Textlänge/Gesamtpunktzahl

\section{Korrelationen}

\begin{tabular}{lll|l} 
& & Textlänge & Gesamtpunktzahl \\
\hline Textlänge & Korrelation nach Pearson & 1 & $\mathbf{, 7 5 8}^{\text {** }}$ \\
\cline { 2 - 4 } & Signifikanz (2-seitig) & & $\mathbf{, 0 0 0}$ \\
\cline { 2 - 4 } & $\mathrm{N}$ & 84 & 84 \\
\hline Gesamtpunktzahl & Korrelation nach Pearson &, $758^{* *}$ & 1 \\
\cline { 2 - 4 } & Signifikanz (2-seitig) &, 000 & \\
\cline { 2 - 4 } & $\mathrm{N}$ & 84 & 84 \\
\hline
\end{tabular}

**. Die Korrelation ist auf dem Niveau von 0,01 (2-seitig) signifikant. 


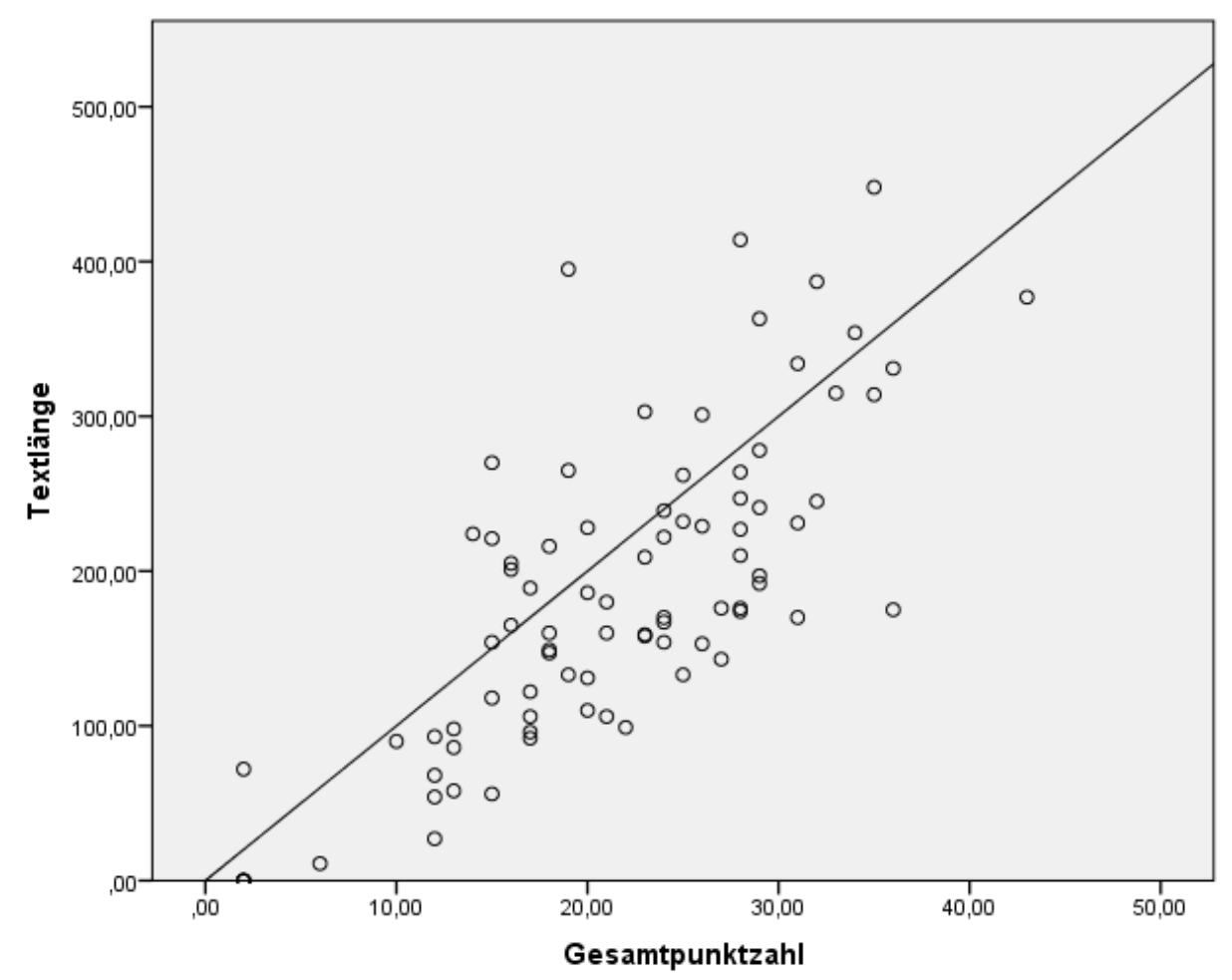

Abbildung 43: Punktwolke Textlänge/Gesamtpunktzahl

Es kann also festgestellt werden, dass qualitativ bessere Texte in der Regel auch längere Texte sind. Der Zusammenhang erscheint insbesondere aufgrund der Menge der zu verarbeitenden Informationen durch den langen Darstellungstext und die zugehörige komplexe Aufgabe nachvollziehbar. Für andere Arbeitsaufträge könnte ein solcher Zusammenhang anders ausfallen (z. B. für die Produktion der Textsorte Definition).

Für einen möglichen Zusammenhang von mittlerer Satzlänge und Gesamtpunktzahl fällt das Ergebnis wie folgt aus:

Tabelle 7: Korrelation Satzlänge/Gesamtpunktzahl

\section{Korrelationen}

\begin{tabular}{|c|c|c|c|}
\hline & & $\begin{array}{l}\text { Satz- } \\
\text { läng } \\
\text { e }\end{array}$ & $\begin{array}{l}\text { Gesamt- } \\
\text { punktzahl }\end{array}$ \\
\hline \multirow[t]{3}{*}{ Satzlänge } & $\begin{array}{l}\text { Korrelation nach } \\
\text { Pearson }\end{array}$ & 1 &, $590^{* *}$ \\
\hline & Signifikanz (2-seitig) & & ,000 \\
\hline & $\mathrm{N}$ & 84 & 84 \\
\hline \multirow[t]{3}{*}{$\begin{array}{l}\text { Gesamt- } \\
\text { punktzahl }\end{array}$} & $\begin{array}{l}\text { Korrelation nach } \\
\text { Pearson }\end{array}$ & $\begin{array}{l}, 590^{*} \\
*\end{array}$ & 1 \\
\hline & Signifikanz (2-seitig) &, 000 & \\
\hline & $\mathrm{N}$ & 84 & 84 \\
\hline
\end{tabular}

**. Die Korrelation ist auf dem Niveau von 0,01 (2-seitig) signifikant. 


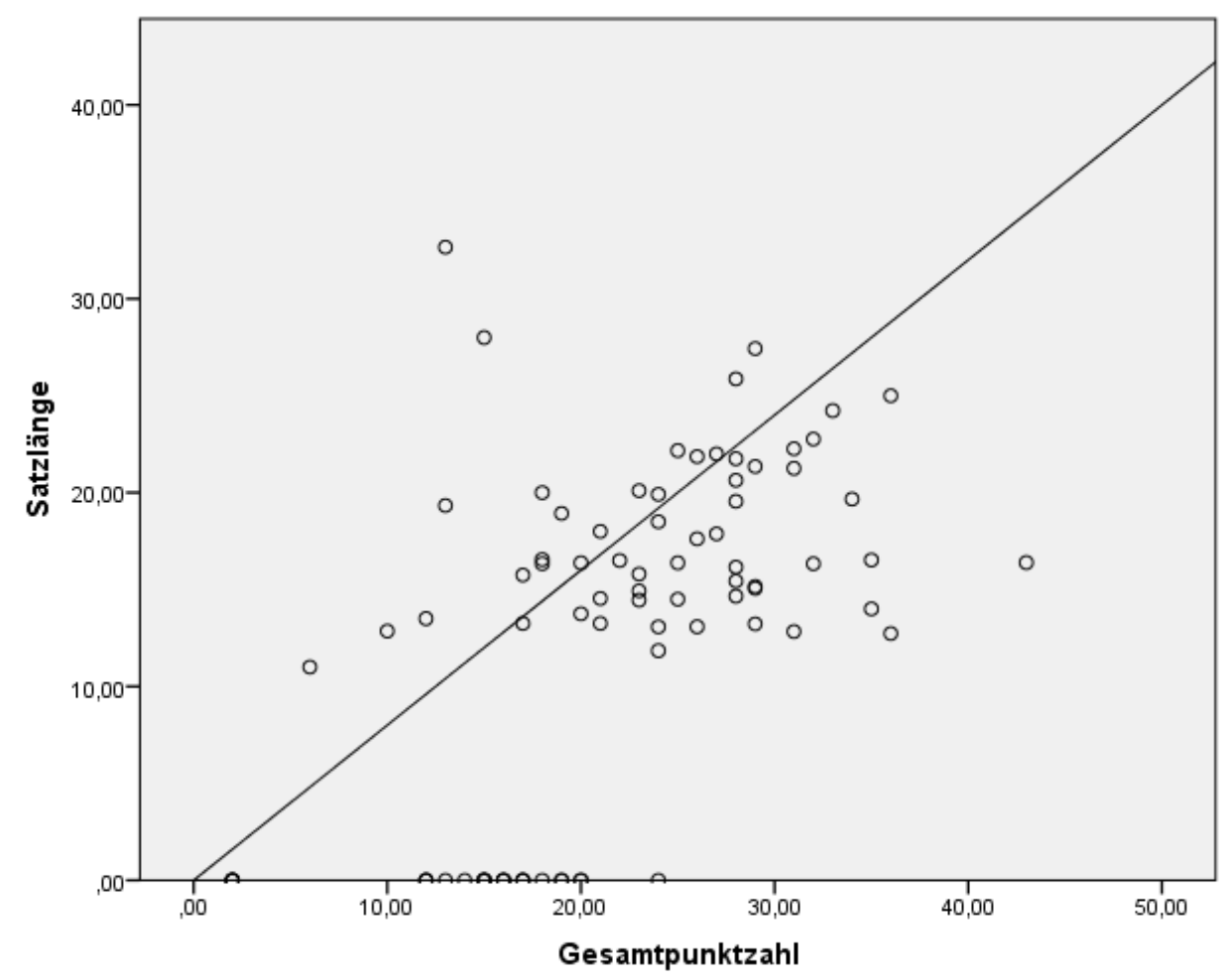

Abbildung 44: Punktwolke Satzlänge/Gesamtpunktzahl

Zwar liegt der Korrelationskoeffizient (nach Pearson) niedriger als in Bezug auf Textlänge und Gesamtpunktzahl (er liegt in Bezug auf die mittlere Satzlänge bei 0,59), dennoch muss schon aufgrund des signifikanten Korrelationsniveaus von 0,01 ein linearer Zusammenhang angenommen werden. Dieser kann als mittlerer Zusammenhang eingeordnet werden. Die Punktewolke zeigt entsprechend eine etwas größere Streuung als im ersten Beispiel.

Auch längere Sätze sind demnach ein Qualitätskriterium für gute Vergleichstexte.

Die Punktewolke beinhaltet ebenfalls einige Schüler*innenlösungen, in denen es keine Sätze gibt. Das sind solche Texte, die als Tabelle realisiert wurden und nur Stichworte enthalten. Keiner dieser Bearbeitungen erreicht eine Qualität über einen mittleren Bereich hinaus. ${ }^{694}$ Insofern muss schon deshalb ein Zusammenhang zwischen Satzlänge und Gesamtpunktzahl angenommen werden.

7.3.2 Zum Zusammenhang von Textqualität und der Nutzung expliziter sprachlicher Mittel

Für die nachfolgende Faktorenanalyse sollen Items festgelegt werden, mit denen man Textqualität möglichst eindeutig bestimmen kann. Dies ist jedoch nicht für alle Items ohne Prüfung möglich.

Es kann insbesondere für einige metrische Items nicht ohne Prüfung davon ausgegangen werden, dass eine große Häufigkeit der Nutzung expliziter sprachlicher Mittel die Qualität der Teilhandlung verbessert. Das bedeutet zum Beispiel, dass ein Text viele explizite sprachliche Mittel, die z. B. das Begründen

${ }^{694}$ Dies gilt nur für die Kombination „Tabelle“ und „Stichwörter“, nicht für „Tabelle“ und „Ganzsätze“. 
kennzeichnen, beinhalten kann und trotzdem qualitativ schlechte Begründung aufweist. Dagegen ist es möglich, dass ein Text gute Begründestrukturen zeigt, obwohl dafür nur wenige explizite sprachliche Mittel genutzt werden.

Grundsätzlich wird zunächst jedoch vermutet, je besser die sprachlichen Teilhandlungen in einem Text realisiert werden, desto mehr explizite sprachliche Mittel werden auch verwendet. Ob es in den Korpustexten tatsächlich einen solchen Zusammenhang gibt, muss jedoch zunächst überprüft werden. Es geht dabei um die Items ST02.2 („Versprachlichung des Begründens“), ST03.2 („Versprachlichung des Erklärens“), ST04.2 („Versprachlichung des Beurteilens“). Bei diesen Items bietet sich eine Prüfung an, weil in einem vorausgehenden Item zunächst immer die Qualität der zu realisierenden Teilhandlung geratet wurde.

Dazu wurde der Faktorenanalyse eine Häufigkeitsanalyse vorangestellt, die Aufschlüsse darüber gibt, wie oft sprachliche Mittel der jeweiligen Teilhandlung benutzt wurden.

7.3.2.1 Begründen, Erklären und Beurteilen: Realisierung der sprachlichen Teilhandlungen und Nutzung expliziter sprachlicher Strukturen

In Bezug auf die Teilhandlung Begründen (ST02.2) kann zunächst festgestellt werden, dass zur Realisierung des Begründens in 73 Texten kein explizites sprachliches Mittel benutzt wurde, in 8 Texten wurde ein und in 3 Texten wurden 2 sprachliche Mittel realisiert.

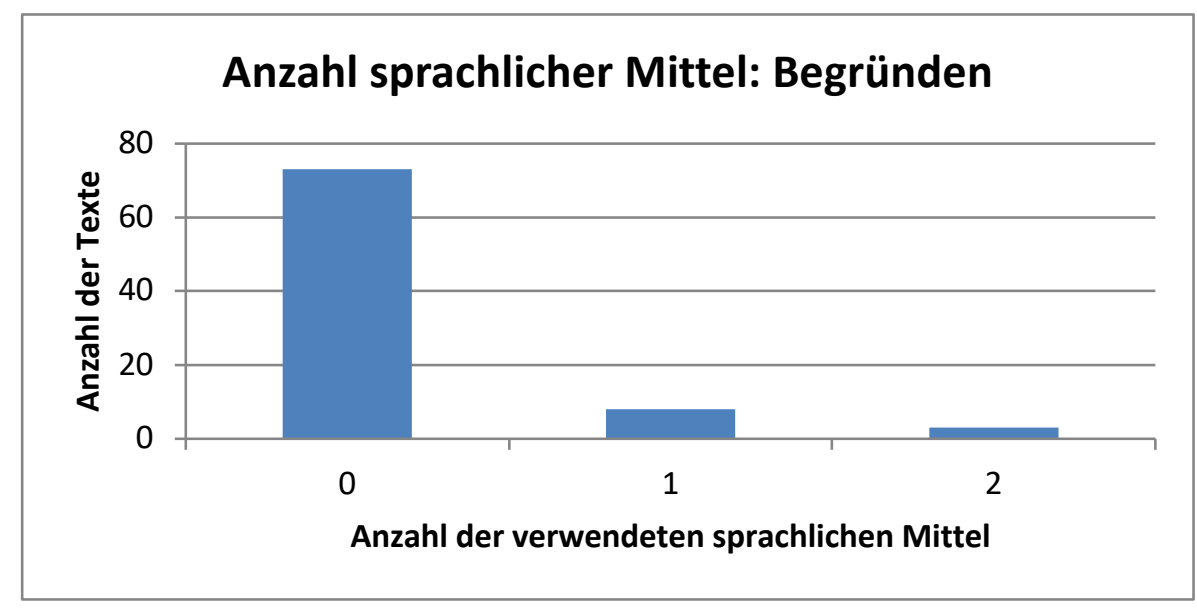

Abbildung 45: Häufigkeiten sprachlicher Mittel: Begründen

Die Darstellung zeigt, dass dieses Item einen hohen Bodeneffekt aufzeigt, da es nur den wenigsten Lernenden gelingt, eine sprachlich explizite Begründung zu formulieren. Dennoch zeigt die qualitative Einschätzung von Begründungsstrukturen auch, dass diese zumindest zum Teil realisiert werden. Fehlende sprachliche Mittel sind damit noch kein Indiz für fehlende Begründungsstrukturen, wie das nachfolgende Diagramm zeigt: 


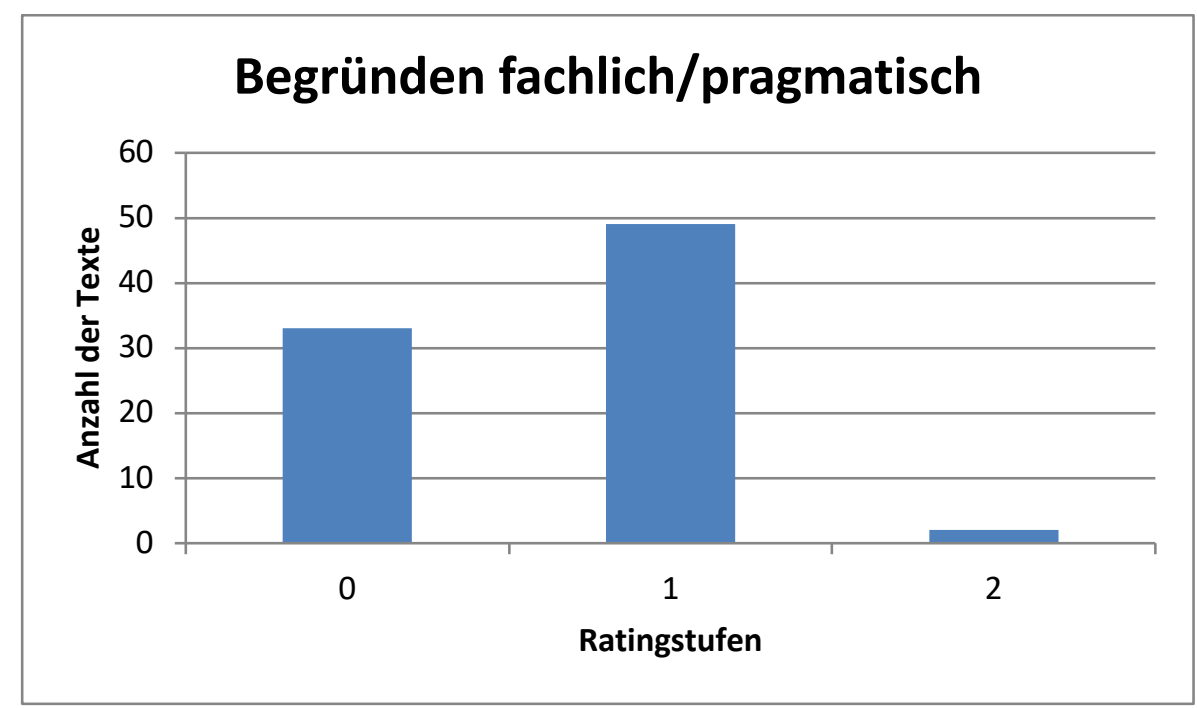

Abbildung 46: Realisierung der sprachlichen Teilhandlung: Begründen

Die hohe Anzahl der als „1“ gewerteten Texte verdeutlicht, dass Begründungen häufig nicht vollständig sind, weil sie sich zum Beispiel nur auf ein Vergleichsobjekt beziehen oder nur implizit bleiben. Es ist also zu vermuten, dass das explizite Formulieren von Begründungen für die Lernenden eine hohe Anforderung darstellt.

Betrachtet man außerdem die mit "2" (Begründungen sind teilweise erkennbar) gerateten Texte (es handelt sich um 2 Texte), fällt in der Einzelbetrachtung auf, dass diese nur ein oder kein sprachliches Mittel enthalten. Damit kann keine direkte Zuordnung zwischen Qualität der Begründungsstrukturen und Anzahl der sprachlichen Mittel in Bezug auf das Begründen festgestellt werden. Dafür spricht auch der hohe Bodeneffekt. Entsprechend zeigt eine Korrelationsrechnung einen niedrigen bis mittleren Zusammenhang von $0,302 .{ }^{695}$ Dennoch ist die Korrelation zweiseitig signifikant:

Tabelle 8: Korrelationen: Begründen

\section{Korrelationen}

ST 02.1 ST 02.2

\begin{tabular}{llll}
\hline ST 02.1 & Korrelation nach Pearson & 1 &, $302^{* *}$ \\
\cline { 2 - 4 } & Signifikanz (2-seitig) & &, 005 \\
\cline { 2 - 4 } & $\mathrm{N}$ & 84 & 84 \\
\hline \multirow{2}{*}{ ST 02.2 } & Korrelation nach Pearson &, $302^{* *}$ & 1 \\
& Signifikanz (2-seitig) &, 005 & \\
\cline { 2 - 4 } & $\mathrm{N}$ & 84 & 84 \\
\hline
\end{tabular}

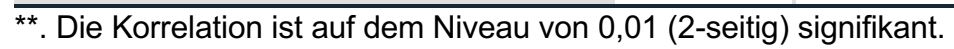

\footnotetext{
${ }^{695}$ Da es sich um gemischte Skalen handelt (ordinal und nominal), wird das , niedrigere' Skalenniveau angesetzt und deshalb mit dem Korrelationskoeffizienten nach Pearson gerechnet. Der Vergleich mit Spearman-Rho bestätigt die Gesamteinschätzung des Zusammenhangs: 0,329.
} 
Eine Kreuztabelle kann diesen Zusammenhang auflösen:

Tabelle 9: Realisierung der Teilhandlung und Nutzung sprachlicher Mittel: Begründen

ST 02.1 * ST 02.2 Kreuztabelle

Anzahl

\begin{tabular}{lll|l|l|l} 
& \multicolumn{3}{c}{ ST 02.2 } & & \\
& & 0 & 1 & 2 & Gesamt \\
\hline ST 02.1 & 0 & 33 & 0 & 0 & 33 \\
\cline { 2 - 6 } & 1 & 39 & 7 & 3 & 49 \\
\cline { 2 - 6 } & 2 & 1 & 1 & 0 & 2 \\
\hline Gesamt & 73 & 8 & 3 & 84 \\
\hline
\end{tabular}

Sie zeigt, dass fehlende Begründungen auch mit fehlenden sprachlichen Mitteln einhergehen.

Es kann für das Begründen festgestellt werden, dass mit Begründungen, die gelingen oder teilweise gelingen, nicht unbedingt die Verwendung expliziter sprachlicher Strukturen verbunden ist. Dies widerspricht noch nicht der eingangs dargestellten Vermutung, dass die Verwendung expliziter sprachlicher Mittel die Textqualität verbessert, sondern zeigt zunächst nur, dass Begründungen auch ohne explizite sprachliche Mittel funktionieren können. Es stellt sich hieran anschließend die Frage, ob die große Zahl nur teilweise gelingender Begründungen in gute Begründungen überführt werden könnte, wenn im Unterricht die sprachliche Handlung des Begründens und zugehörige sprachliche Mittel explizit gemacht würden.

Im Folgenden soll die Eingangshypothese für die Teilhandlung des Erklärens überprüft werden. In Bezug auf das Erklären verschiebt sich das Bild leicht:

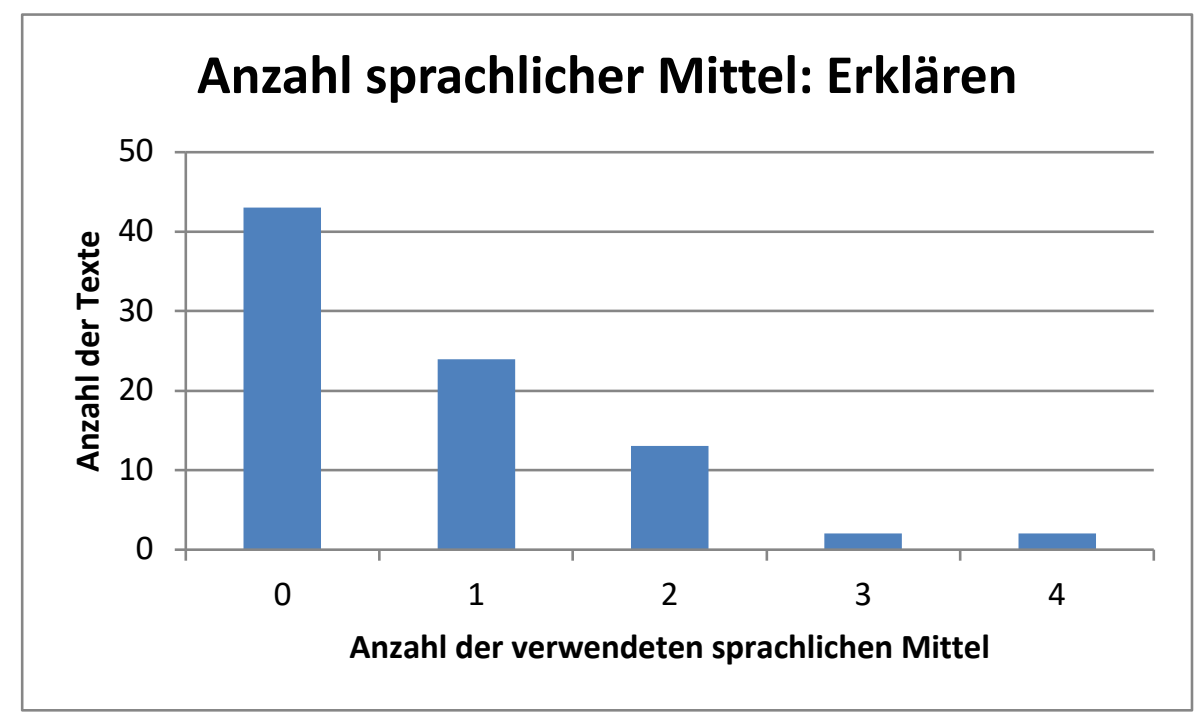

Abbildung 47: Häufigkeiten sprachlicher Mittel: Erklären

Immerhin in knapp der Hälfte der Texte findet sich mindestens ein sprachliches Mittel. Nur in 2 Texten lassen sich 3 und in zwei weiteren Texten 4 sprachliche Mittel finden. Setzt man für diese vier Texte 
nun die Anzahl der verwendeten sprachlichen Mittel (ST 03_2) mit der Realisierung der Teilhandlung (ST 03_1) in Bezug ergibt sich folgendes Bild:

Tabelle 10: Texte mit den meisten sprachlichen Mitteln: Realisierung der Teilhandlung

\begin{tabular}{|l|l|l|}
\hline Text & $\begin{array}{l}\text { Realisierung der sprachlichen } \\
\text { Teilhandlung,Erklären' (Ra- } \\
\text { tingstufe) }\end{array}$ & $\begin{array}{l}\text { Anzahl der verwendeten } \\
\text { sprachlichen Mittel }\end{array}$ \\
\hline 1 & 1 & 3 \\
\hline 2 & 1 & 4 \\
\hline 3 & 2 & 3 \\
\hline 4 & 2 & 4 \\
\hline
\end{tabular}

Die Nutzer der meisten sprachlichen Mittel für das Erklären schaffen es zwar, mindestens teilweise Erklärstrukturen zu realisieren. Die Quantität der sprachlichen Mittel ist aber umgekehrt kein Garant für gute und vollständige Erklärungen.

In der qualitativen Bewertung der Erklärstrukturen in den Lernendentexten des Gesamtkorpus zeigt sich zunächst im Vergleich zu den Beurteilungsstrukturen ein deutlich höherer Anteil von Lernenden, die zumindest zum Teil Erklärstrukturen in ihren Texten realisieren, indem sie Antworten auf WarumFragen geben. Da auch in der Zählung der sprachlichen Mittel kein so deutlicher Bodeneffekt wie beim Begründen festgestellt werden kann, kann vermutet werden, dass den Schüler*innen Erklärstrukturen etwas vertrauter sind als Begründungen. Dies kann daran liegen, dass Begründestrukturen eine höhere Anforderung darstellen, da das Erklären auf den Wissensaufbau bei einem Hörer oder Leser hin angelegt ist, das Begründen aber „[...] auf die Umorganisation des hörerseitigen Wissens entsprechend der sprecherseitigen (verbalisierten) Wissensstrukturen als Grundlage der weiteren Kooperation von Sprecher und Hörer abzielt. ${ }^{4{ }^{696}}$ Begründen fordert also eine Einstellungsveränderung von Hörer oder Leser.

\footnotetext{
${ }^{696}$ Schramm, Karen; Hardy, llonca; Saalbach, Henry; Gadow, Anne (2013): Wissenschaftliches Begründen im Sachunterricht. S. 299. In: Becker-Mrotzek, Michael; Schramm, Karen; Thürmann, Eike; Vollmer, Helmut Johannes Hrsg.): Sprache im Fach. Sprachlichkeit und fachliches Lernen. Münster: Waxmann. S. 295-316.
} 


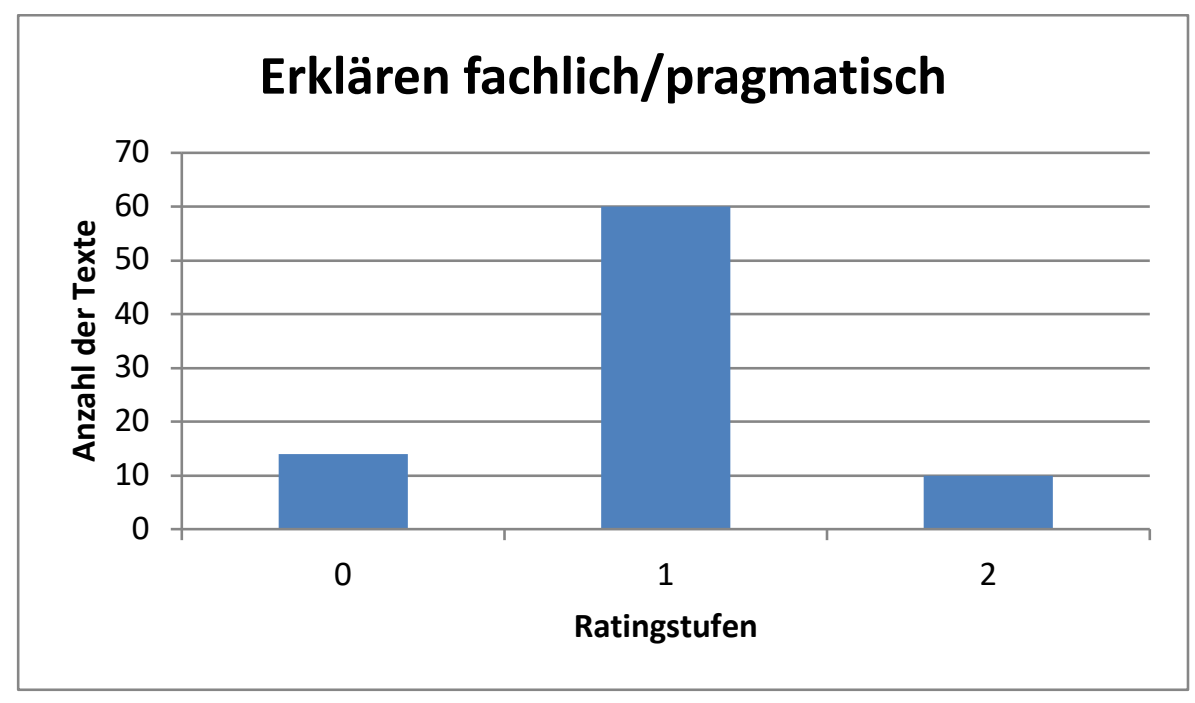

Abbildung 48: Realisierung der sprachlichen Teilhandlung: Erklären

In nur 10 Texten tauchen Erklärstrukturen explizit ${ }^{697}$ und fachlich korrekt auf.

Zum Vergleich folgt nun die Darstellung für die Verwendung expliziter sprachlicher Mittel.

In der Darstellung sind zunächst nur diese 10 mit "2" und damit qualitativ gut gerateten Texte (mit eindeutigen und korrekten Erklärstrukturen) aufgenommen, um zu prüfen, ob gute Erklärungen durchgängig sprachliche Mittel benötigen:

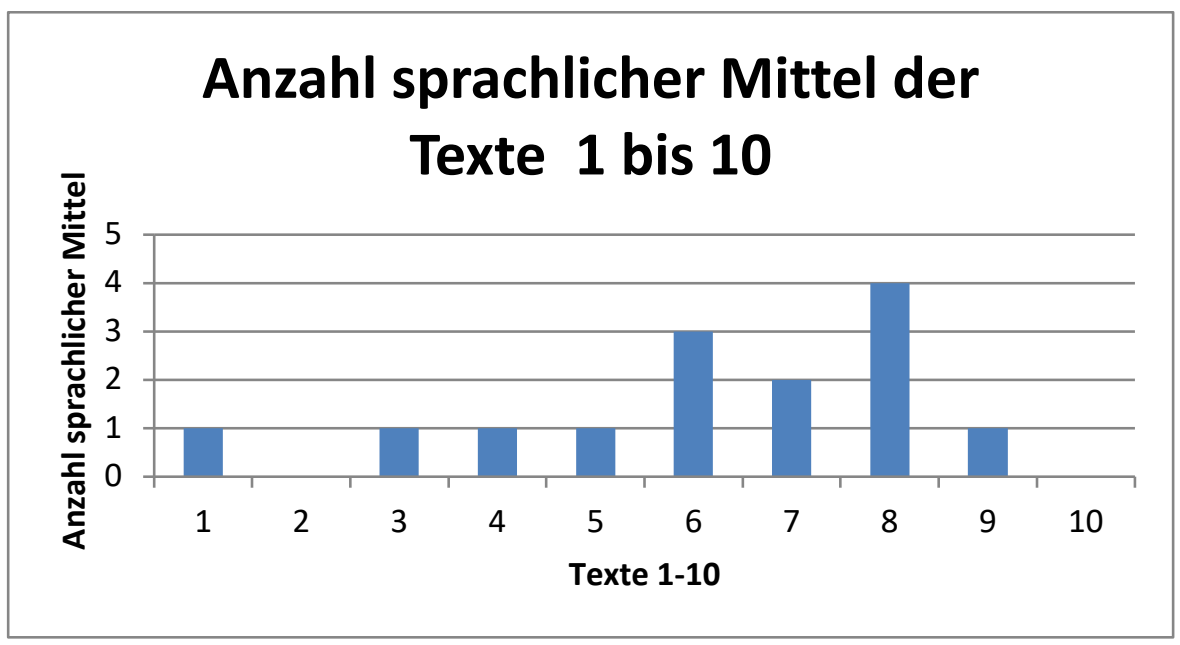

Abbildung 49: Häufigkeiten sprachlicher Mittel von Texten mit guten Erklärstrukturen

Es zeigt sich, dass 5 der 10 als „gut" gewerteten Texte nur ein explizites sprachliches Mittel beinhalten, zwei Texte gar keine und nur in drei Texten mehr als ein sprachliches Mittel vorhanden ist. Da Texte nur mit einer "2", also mit erkennbaren Erklärstrukturen geratet wurden, wenn das Erklären mind.

\footnotetext{
${ }^{697}$ Mit explizit sind solche Strukturen gemeint, die eindeutig dem Erklären zuzuweisen sind und sich von anderen Strukturen wie bspw. dem, Erläutern oder Begründen abgrenzen lassen. Es geht hier noch nicht um die expliziten sprachlichen Mittel, auch wenn beides einhergehen kann.
} 
dreimal ausführlich expliziert wurde, kann also für die Texte mit guten Erklärstrukturen kein eindeutiger Zusammenhang zwischen Qualität der Erklärstrukturen und der Nutzung expliziter sprachlicher Mittel zur Beantwortung der Frage „Warum“ festgestellt werden.

Für das Gesamtkorpus ergibt sich folgendes Bild ${ }^{698}$ :

Tabelle 11: Korrelationen: Erklären

\section{Korrelationen}

\begin{tabular}{llll} 
& & ST 03.1 & ST 03.2 \\
\hline ST 03.1 & Korrelation nach Pearson & 1 &, $398^{* *}$ \\
\cline { 2 - 4 } & Signifikanz (2-seitig) &, 000 \\
\cline { 2 - 4 } & $\mathrm{N}$ & 84 & 84 \\
\hline ST 03.2 & Korrelation nach Pearson &, $398^{* *}$ & 1 \\
& Signifikanz (2-seitig) &, 000 & \\
\cline { 2 - 4 } & $\mathrm{N}$ & 84 & 84 \\
\hline
\end{tabular}

**. Die Korrelation ist auf dem Niveau von 0,01 (2-seitig) signifikant.

Die Korrelation ist (zweiseitig) signifikant, auch wenn sie noch knapp unter einem mittleren Zusammenhang liegt. Eine Kreuztabelle kann die Zusammenhänge wiederum aufschlüsseln:

Tabelle 12: Realisierung der Teilhandlung und Nutzung sprachlicher Mittel: Erklären

ST 03.1 * ST 03.2 Kreuztabelle

Anzahl

\begin{tabular}{|c|c|c|c|c|c|c|c|}
\hline & & & & & & & \\
\hline & & 0 & 1 & 2 & 3 & 4 & Gesamt \\
\hline ST 03.1 & 0 & 14 & 0 & 0 & 0 & 0 & 14 \\
\hline & 1 & 27 & 19 & 12 & 1 & 1 & 60 \\
\hline & 2 & 2 & 5 & 1 & 1 & 1 & 10 \\
\hline Gesamt & & 43 & 24 & 13 & 2 & 2 & 84 \\
\hline
\end{tabular}

Es zeigt sich, dass für gute Erklärungen in der Regel zumindest teilweise explizite sprachliche Mittel verwendet werden, allerdings ist die Verwendung solcher Mittel kein Garant für gute Erklärstrukturen: In 12 Texten, die teilweise erkennbare Erklärstrukturen vorweisen können, werden immerhin zwei sprachliche Mittel verwendet, dennoch hat die Nutzung solcher Strukturen nicht gleichzeitig zu gut erkennbaren Strukturen geführt.

${ }^{698}$ Auch hier wurde wieder mit Spearman-Rho gegen gerechnet: 0,428. 
Wie beim Begründen ist also wiederum zusammenfassend festzuhalten, dass die Realisierung von Erklärungen auch ohne die Verwendung expliziter sprachlicher Mittel möglich ist, gleichzeitig das Erklären für viele Lernende sehr herausforderungsvoll ist.

Die Häufigkeitszählung der sprachlichen Mittel zum Beurteilen zeigt einen noch stärkeren Bodeneffekt als das Beurteilen. Da Begründen und Beurteilen zusammenhängen, mehr noch das Begründen im Beurteilen mündet, ist dies nicht verwunderlich. Das Formulieren expliziter Beurteilungen scheint eine hohe Hürde, im Vergleich zu Begründen und Erklären die höchste Hürde für die Lernenden zu sein:

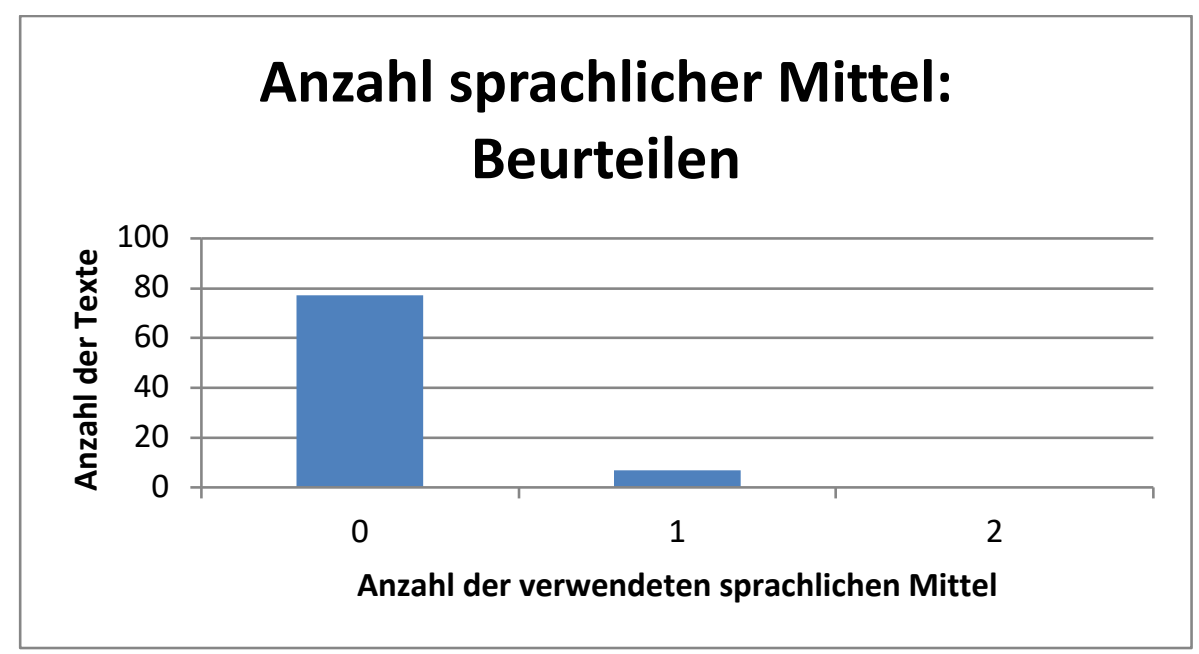

Abbildung 50: Häufigkeiten sprachlicher Mittel: Beurteilen

Die Auswertung der fachlich/pragmatischen Beurteilungsstrukturen zeigt wie beim Erklären, dass zumindest angedeutete Beurteilungen oder Teilbeurteilungen vorhanden sind. Ein richtiges Urteil im Sinne eines historischen Sach- (oder ggf. auch Wert-) Urteils wird nur in 3 Fällen realisiert:

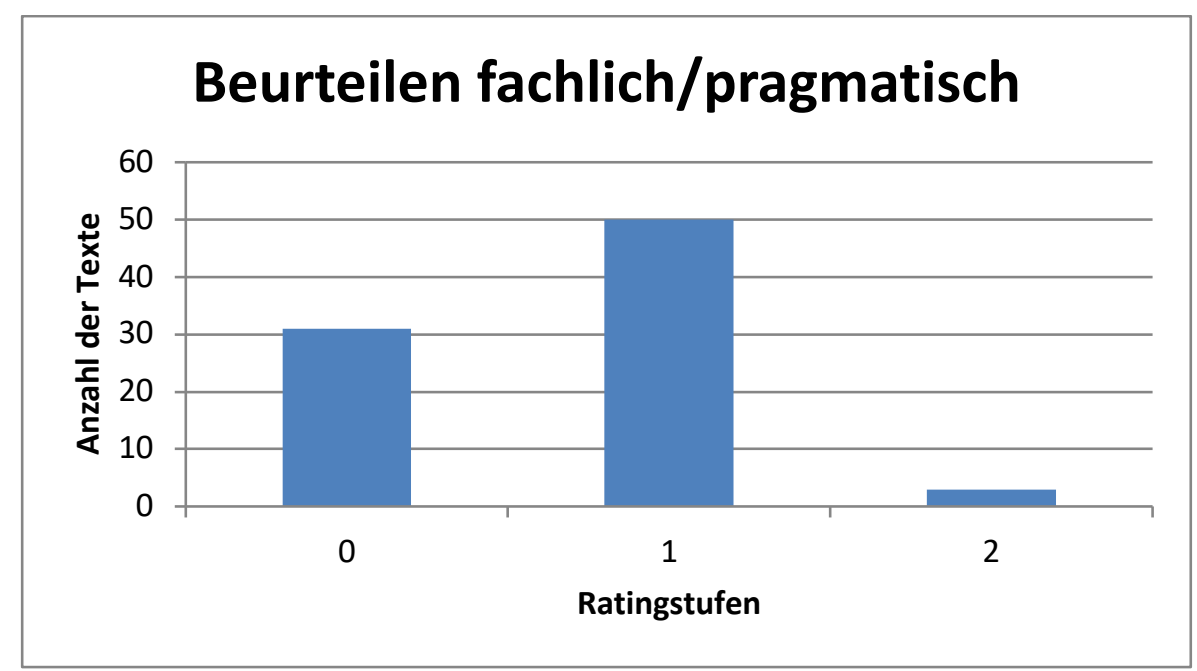

Abbildung 51: Realisierung der sprachlichen Teilhandlung: Beurteilen 
Dabei finden sich in denen als „2“ (Kriterium erfüllt) gerateten Texten in einem Fall kein, in den übrigen beiden Fällen nur ein explizites sprachliches Mittel zur Beurteilung. Der ermittelte Korrelationskoeffizient ist entsprechend schwach ausgeprägt ${ }^{699}$ :

Tabelle 13: Korrelationen: Beurteilen

Korrelationen

\begin{tabular}{lll|l} 
& & ST 04.1 & ST 04.2 \\
\hline ST 04.1 & Korrelation nach Pearson & 1 &, $344^{* *}$ \\
\cline { 2 - 4 } & Signifikanz (2-seitig) & &, 001 \\
\cline { 2 - 4 } & $\mathrm{N}$ & 84 & 84 \\
\hline \multirow{2}{*}{ ST 04.2 } & Korrelation nach Pearson &, $344^{* *}$ & 1 \\
\cline { 2 - 4 } & Signifikanz (2-seitig) &, 001 & \\
\cline { 2 - 4 } & $\mathrm{N}$ & 84 & 84 \\
\hline
\end{tabular}

**. Die Korrelation ist auf dem Niveau von 0,01 (2-seitig) signifikant.

Eine Kreuztabelle spezifiziert das Bild wie folgt:

Tabelle 14: Realisierung der Teilhandlung und Nutzung sprachlicher Mittel: Beurteilen

ST 04.1 * ST 04.2 Kreuztabelle

Anzahl

\begin{tabular}{lll|l|l} 
& \multicolumn{3}{c}{ ST 04.2 } & \\
& & 0 & 1 & Gesamt \\
\hline ST 04.1 & 0 & 31 & 0 & 31 \\
\cline { 2 - 5 } & 1 & 45 & 5 & 50 \\
\cline { 2 - 5 } & 2 & 1 & 2 & 3 \\
\hline Gesamt & 77 & 7 & 84 \\
\hline
\end{tabular}

Neben dem Befund, dass die Realisierung des Beurteilens eine große Anforderung für Lernende darstellt, ist wieder festzustellen, dass das Beurteilen auch ohne die Nutzung expliziter sprachlicher Mittel möglich ist. Dieser Befund stärkt die Ergebnisse aus der Analyse von Begründen und Erklären.

Zwar konnte die Hypothese, dass eine eindeutige Realisierung der vorgestellten Teilhandlungen auch mit der Nutzung expliziter sprachlicher Mittel einhergeht, nicht bestätigt werden. Der Befund lässt aber den nachfolgenden Schluss zu: Nur wenn Lernende wissen, welchen Zweck eine sprachliche (Teil-)Handlung verfolgt, können sie diesen mithilfe zugehöriger sprachlicher Mittel in ihren Texten generieren. Zwar ist es möglich, den Zweck einer Teilhandlung zu realisieren, ohne ein explizites sprachliches Mittel zu nutzen, umgekehrt funktioniert dies aber nicht.

Die Grafiken zeigen zunächst insgesamt, dass die Nutzung expliziter sprachlicher Mittel für die Lernenden eine hohe Anforderung darstellt. Wie bereits dargestellt, handelt es sich bei den Textproduzent*innen um eine sehr heterogene Schülerschaft, bei der zumindest zum Teil davon ausgegangen

${ }^{699}$ Zum Vergleich: Spearman-Rho: 0,312. 
werden muss, dass sprachliche Teilhandlungen ungeübt sein können. Bei einigen Lernenden kann zudem davon ausgegangen werden, dass die Schulzeit durch berufliche Tätigkeit unterbrochen wurde. ${ }^{700}$ Es ist damit nicht klar, wie vertraut den Lernenden die geforderten Teilhandlungen und ihre sprachliche Realisierung im Hinblick auf das Fach Geschichte sind. In einer Folgeuntersuchung müsste deshalb untersucht werden, wie gut Schüler*innen die Teilhandlungen des Vergleichens realisieren, wenn Strukturen hinsichtlich ihres Zwecks geklärt sind und passende Redemittel zur Verfügung stehen. ${ }^{701}$

Summiert man abschließend die Punktzahl der Items, die die fachlich/pragmatischen Leistungen erfassen (ST02.1, ST03.1, ST04.1) und korreliert diese mit der Summe der Items, die die Häufigkeit der sprachlichen Mittel wiedergeben (ST02.2, ST03.2, ST04.2) ${ }^{702}$, ergeben sich folgende Werte ${ }^{703}$ :

Tabelle 15: Korrelation Realisierung sprachliche Teilhandlungen/Nutzung sprachlicher Mittel gesamt

\section{Korrelationen}

\begin{tabular}{|c|c|c|c|c|}
\hline & & & $\begin{array}{l}\text { Funktionalprag- } \\
\text { matisch }\end{array}$ & sprachlich \\
\hline \multirow[t]{6}{*}{ Spearman-Rho } & \multirow[t]{3}{*}{ Funktionalpragmatisch } & Korrelationskoeffizient & 1,000 &, $629^{* *}$ \\
\hline & & Sig. (2-seitig) & . &, 000 \\
\hline & & $\mathrm{N}$ & 84 & 84 \\
\hline & \multirow[t]{3}{*}{ sprachlich } & Korrelationskoeffizient &, $629^{* *}$ & 1,000 \\
\hline & & Sig. (2-seitig) &, 000 & . \\
\hline & & $\mathrm{N}$ & 84 & 84 \\
\hline
\end{tabular}

**. Die Korrelation ist auf dem 0,01 Niveau signifikant (zweiseitig).

Es lässt sich eine Korrelation (Spearman-Rho), von 0,629 sowie eine (zweiseitige) Signifikanz zeigen. Damit kann man insgesamt einen deutlichen Zusammenhang zwischen qualitativer und sprachlicher Realisierung der genannten Teilhandlungen feststellen. Allerdings konnte oben gezeigt werden, dass der Zusammenhang nicht eindeutig ist, wenn man nur die qualitativ als gelungen eingeschätzten sprachlichen Teilhandlungen der Anzahl der genutzten sprachlichen Mittel gegenüberstellt. Zudem zeigen die Daten einen direkten Zusammenhang zwischen dem Fehlen einer sprachlichen Teilhandlung und der Nichtnutzung passender sprachlicher Mittel. Einer erkennbaren Realisierung einer sprachlichen Teilhandlung steht aber nicht eine vermehrte Nutzung sprachlicher Mittel gegenüber.

\footnotetext{
${ }^{700}$ Einige Texte stammen aus den einjährigen Klassen 13, einer Sonderform des Berufskollegs, die in einem Jahr zur Allgemeinen Hochschulreife führt. In dieser Klasse halten sich Lernende auf, die nach Abschluss der Fachhochschulreife aus den Klassen 12 in die Klasse 13 wechseln, aber aufgrund des Erlasses auch solche Lernende, die eine fehlende Fachhochschulreife durch entsprechende berufliche Tätigkeiten ausgleichen können. Zudem wird diese Form von solchen Schüler*innen genutzt, die sich nach Ausbildung und Beruf zu einem Studium entschließen und deshalb das Abitur nachholen müssen. In diesen Klassen kann also von einer besonders hohen Heterogenität ausgegangen werden.

${ }^{701}$ Vermutlich erleichtert Übung und Kenntnis des Zwecks der sprachlichen (Teil-)Handlungen die Realisierung dieser.

702 Dies lässt sich durch die Umwandlung in Z-Faktorwerte (jeweils für ST02.1, ST03.1, ST04.1 und ST02.2, ST03.2, ST04.2) darstellen, die dann in einem weiteren Schritt miteinander korreliert werden.

${ }^{703}$ Die Werte wurden dazu in ein Ordinalsystem überführt.
} 
Dies zeigt, dass zwar die Items, die die Realisierung der Teilhandlungen messen, Textqualität abbilden, dies für die Auszählung der sprachlichen Mittel aber nur begrenzt gilt. Deshalb werden die Items, die die Nutzung sprachlicher Mittel für das Begründen, Erklären und Beurteilen messen, nicht in die nachfolgende Faktorenanalyse einbezogen.

\subsubsection{Die Items Themensplitting, Themenfortführung und Modalisierung}

Für die Items MT01, MT02 und DM01, die ebenfalls Häufigkeiten messen, ist dieses vergleichende Vorgehen nicht möglich, da die dahinterstehenden Kategorien für sich stehen und nicht in ein qualitatives und quantitatives Rating aufgesplittet sind. Dennoch können nachfolgend zumindest die Häufigkeiten der sprachlichen Mittel erfasst und die Konsequenzen daraus für die anschließende Faktorenanalyse dargestellt werden.

Das Item MT01 („Themensplitting: Entwicklung durch Teilung“) zeigt folgende Häufigkeitsverteilungen:

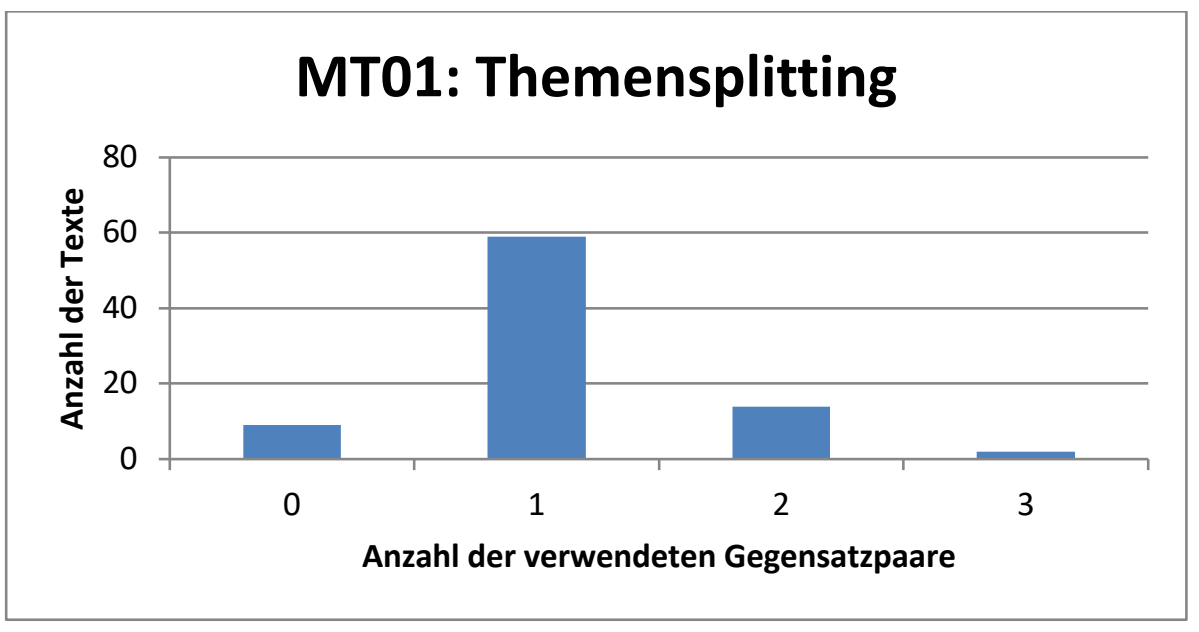

Abbildung 52: Themensplitting

Dieses Bild lässt sich mit Blick auf die einzelnen Vergleichstexte schnell erklären: Das Item nimmt auf, wie viele Gegensatzpaare sich in den Lernendentexten befinden. Viele Lernende haben, um dem Vergleichsthema gerecht zu werden, Deutschland und England als Gegensatzpaar verwendet. Weitere klar identifizierbare Gegensatzpaare, die sich auf Teilstrukturen des Gesamtvergleichs beziehen, fehlen jedoch in den meisten Texten. Die Texte, in denen sich kein Gegensatzpaar befindet, realisieren schließlich auch keinen Vergleich, weil zum Beispiel nur Vergleichsobjekt oder Vergleichsgröße berücksichtigt werden, nicht jedoch beide Elemente, oder beide Elemente wiedergebend nebeneinanderstehen, ohne dass ein Vergleich geleistet wird. Für dieses Item ist also festzustellen, dass die Entwicklung des Vergleichs durch Teilung (also eben ein Themensplitting) Voraussetzung für die Realisierung eines Vergleichs ist, in den meisten Texten jedoch auf die Verwendung des Gegensatzpaares England-Deutschland beschränkt ist.

Das Item MT02 („Themenfortführung“) evaluiert, inwiefern es den Lernenden gelungen ist, die in ihren Texten vorkommenden Einzelelemente zu einem Gesamttext zu verbinden. Dazu wurden, wie zuvor 
dargestellt, insbesondere solche sprachlichen Mittel berücksichtigt, die bedeutsam für die Entwicklung wissenschaftlicher Textkompetenz sind.

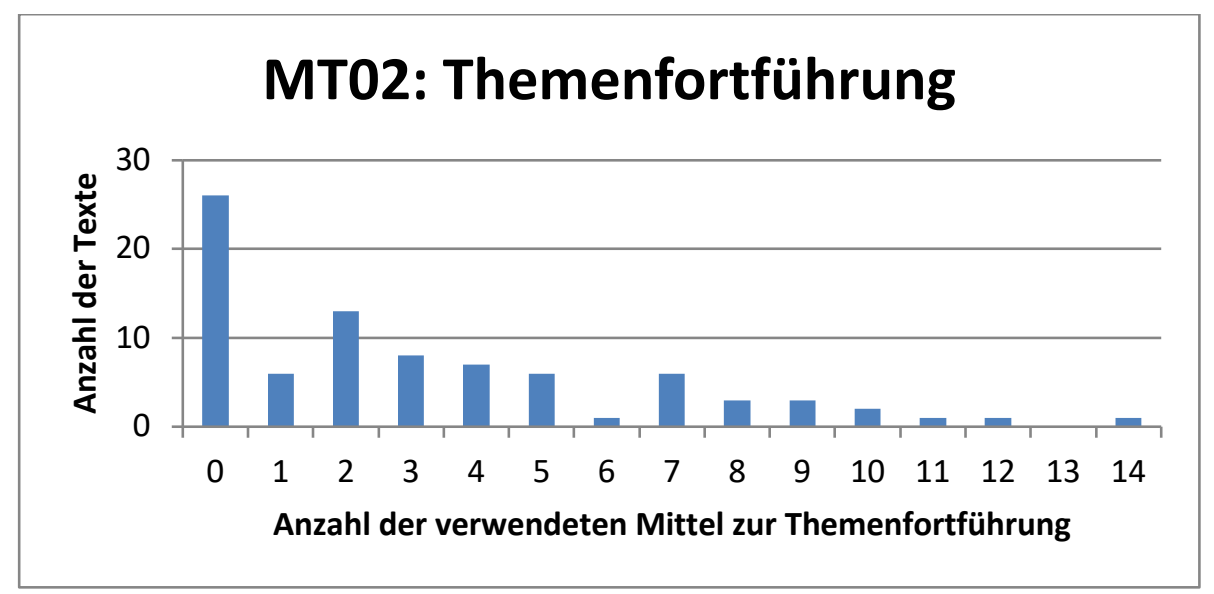

Abbildung 53: Häufigkeiten: Themenfortführung

Das Bild zeigt eine große Bandbreite der Nutzung solcher Mittel. Auffallend ist vor allem, dass in immerhin 31\% der vorliegenden Texte keine für wissenschaftliche oder wissenschaftspropädeutische Texte bedeutsamen sprachlichen Verweismittel benutzt werden. Das bedeutet noch nicht, dass diese Texte völlig zusammenhanglos sind, aber eben andere basalere Mittel zur Themenfortführung genutzt werden (z. B. „und“ o.ä.). Allerdings steht dieses Ergebnis auch im Zusammenhang mit denen von Lernenden häufig genutzten Reihungen oder der Entscheidung für Stichworte und/oder Tabellen statt eines Fließtextes.

Auch das Item "Modalisierung" wurde quantitativ erfasst. Dabei steht im Fokus, ob bzw. wie häufig Lernende Mittel gebrauchen, die anzeigen, dass Inhalte des eigenen Textes aus der Vorlage übernommen wurden. Gerade im Hinblick auf historisches und wissenschaftspropädeutisches Arbeiten ist das Anzeigen einer anderen Urheberschaft von hoher Bedeutung. Allerdings ist dieses Vorgehen gerade dann eine hohe Anforderung, wenn ein Darstellungstext aus einem Schulbuch zugrunde liegt, aus dem kaum hervorgeht, welche allgemeingültigen Aussagen ohne Hinweis auf eine andere Urheberschaft übernommen werden dürfen und welche unbedingt zu kennzeichnen sind, z. B. weil sie eine Beurteilung oder Wertung beinhalten. 


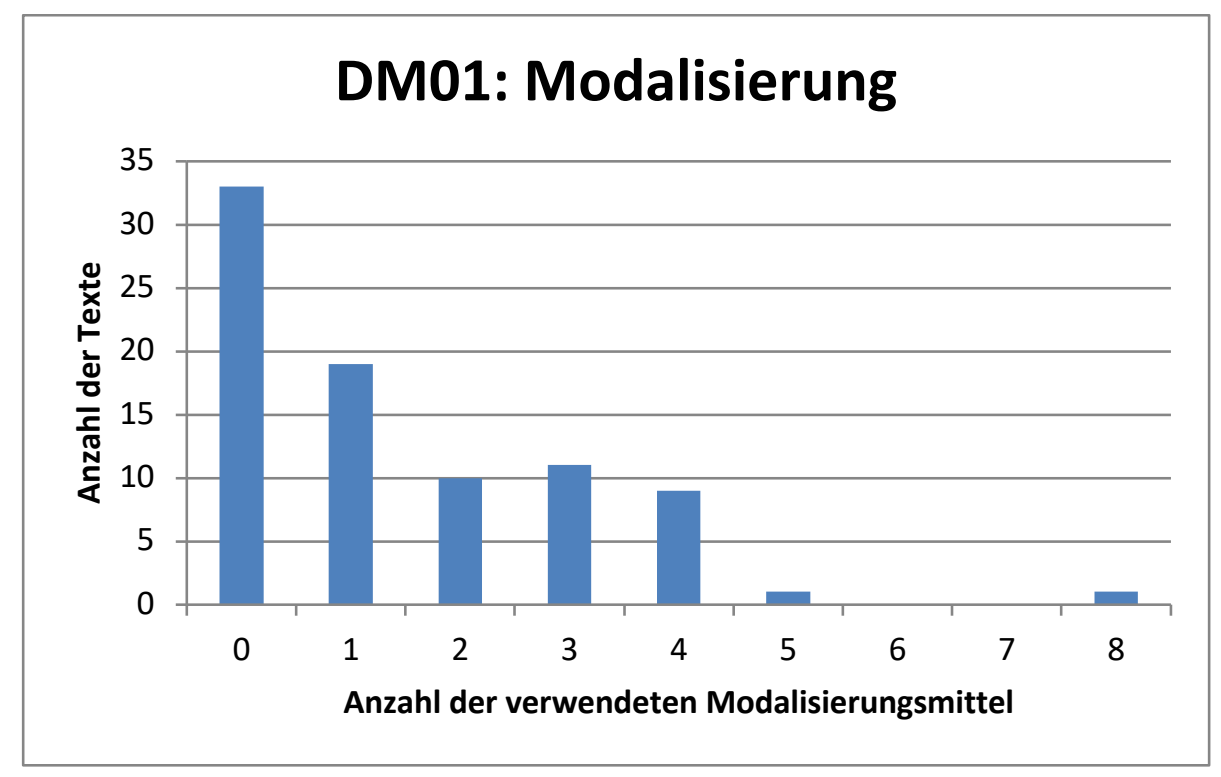

Abbildung 54: Häufigkeiten: Modalisierung

Entsprechend der hohen Anforderung an die Lernenden bei der Umsetzung der Kennzeichnungen anderer Urheberschaften überrascht es kaum, dass in knapp 40\% der Texte solche Distanzierungen nicht auftauchen. Demgegenüber steht, dass der Gebrauch von Modalisierungsmitteln aufgrund des Konstruktionscharakters von Geschichte grundlegend für historisches Arbeiten ist. In immerhin 23\% der Fälle taucht zumindest ein Modalisierungsmittel auf, häufig handelt es sich dabei um die Kennzeichnung eines Ausdrucks (insbesondere der im Text vorkommenden Zuschreibung Englands als „Pionierland“ oder „Pionierrolle“) in Anführungsstrichen. Danach fällt die Kurve schnell ab. Ein Text fällt mit 8 Modalisierungsformen aus der Reihe. Dies liegt zum einen an der dreifachen Kennzeichnung des Begriffs Pionierland in Anführungszeichen sowie der Verwendung von Modalverben (z. B. ,sich erklären lassen 1.

Insgesamt muss auch für diese Items angenommen werden, dass die explizite sprachliche Realisierung von Themensplitting, Themenfortführung und Modalisierung eine hohe Anforderung an die Lernenden darstellt. Nachfolgend werden die Items mit der durch das Rating zu erreichenden Gesamtpunktzahl korreliert. Darüber lässt sich der Anteil der Items an der Gesamtpunktzahl berechnen. Es lassen sich folgende Zusammenhänge feststellen (Korrelation nach Pearsons Rho):

Tabelle 16: Anzahl der Items Themensplitting, Themenfortführung und Modalisierung an der Gesamtpunktzahl

\begin{tabular}{|l|l|l|}
\hline MT01/Gesamtpunktzahl & 0,527 & $\begin{array}{l}\text { mittlerer linearer Zusam- } \\
\text { menhang }\end{array}$ \\
\hline MT02/Gesamtpunktzahl & 0,731 & $\begin{array}{l}\text { mittlerer linearer Zusam- } \\
\text { menhang }\end{array}$ \\
\hline DM01/Gesamtpunktzahl & 0,633 & $\begin{array}{l}\text { mittlerer linearer Zusam- } \\
\text { menhang }\end{array}$ \\
\hline
\end{tabular}


Die Ergebnisse zeigen, dass es insgesamt einen mittleren Zusammenhang zwischen der Güte der Textproduktionen und der Häufigkeit der verwendeten sprachlichen Mittel gibt. Für den in Bezug auf die Modalisierungsformen angesprochenen Text heißt das, dass der/die Schüler*in, der/die 8 Modalisierungsformen verwendet hat, außerdem 9 der untersuchten sprachlichen Mittel zur Themenfortführung und immerhin 2 unterschiedliche sprachliche Mittel zum Themensplitting verwendet hat. In der Gesamtpunktzahl erreicht er/sie 35 von höchstens 43 Punkten. Seine/Ihre Textqualität ist damit als vergleichsweise hoch zu bezeichnen.

Für die Items kann insgesamt angenommen werden, dass sie Textqualität abbilden, so dass sie in die folgende Faktorenanalyse einbezogen werden.

\subsubsection{Befunde aus der Analyse - Zusammenfassung und erste Ableitungen}

An dieser Stelle können folgende erste Befunde festgehalten werden:

In vielen der ausgewerteten Lernendentexte werden die Teilhandlungen Begründen, Erklären und Beurteilen nicht oder nur teilweise realisiert, so dass gefolgert werden kann, dass diese Teilhandlungen eine Herausforderung für die Lernenden darstellen. Zudem werden in den meisten Texten, in denen die genannten Teilhandlungen zumindest teilweise vorkommen, kaum oder nur wenige explizite sprachliche Mittel genutzt.

Die Hypothese, dass eine eindeutige Realisierung der vorgestellten Teilhandlungen auch mit der Nutzung expliziter sprachlicher Mittel einhergeht, konnte zwar nicht bestätigt werden, es kann aber ein Zusammenhang zwischen dem Wissen um den Zweck dieser sprachlichen Teilhandlungen und der Kenntnis zugehöriger sprachlicher Mittel vermutet werden. Denn nur wenn Lernende den Zweck einer sprachliche (Teil-)Handlung kennen, können sie diesen mithilfe zugehöriger sprachlicher Mittel in ihren Texten generieren. Zwar ist es möglich, den Zweck einer Teilhandlung zu realisieren, ohne ein explizites sprachliches Mittel zu nutzen, umgekehrt funktioniert dies aber nicht.

Die Aufnahme von Gegensatzpaaren, die für das Gegenüberstellen und damit für das Vergleichen unverzichtbar sind, beschränken sich in den meisten Korpustexten auf das im Darstellungstext genannte Gegensatzpaar 'Deutschland'/'England'. Denkbar wäre gewesen, außerdem die aufgenommenen Teilaspekte in der Gegenüberstellung als Gegensatzpaare darzustellen (zum Beispiel in einer Tabelle, in der die Gegensätze wirklich gegenübergestellt und nicht nur untereinander aufgereiht werden). Für Lernende wäre im Hinblick auf eine Didaktisierung im Unterricht zu verdeutlichen, welche Funktion Gegensatzpaare haben, damit ein solches Gegenüberstellen auch gelingen kann.

In Bezug auf das Item ,Themenfortführung' fällt auf, dass in etwa einem Drittel der vorliegenden Texte keine für wissenschaftliche oder wissenschaftspropädeutische Texte bedeutsamen sprachlichen Verweismittel benutzt werden. Auch hier müsste Unterricht ansetzen, und für das historische Vergleichen funktionale Satzverknüpfungsmittel für Lernende klären.

Modalisierungsformen werden kaum genutzt. Dies stellt sicherlich ein Problem dar, das auf der Grundlage des Darstellungstextes zu betrachten ist, der ebenfalls kaum mit solchen Mitteln arbeitet. Diese 
sind aber grundlegend, um Geschichte als Konstruktion nachvollziehbar zu machen. Sie sind Voraussetzung für eigene Narrationen der Lernenden.

\subsubsection{Durchführung einer explorativen Faktorenanalyse}

Es bietet sich an, zu prüfen, ob für den vorliegenden Datensatz die Durchführung einer explorativen Faktorenanalyse geeignet ist, um so die unterschiedlichen Items in verschiedene Oberkategorien zusammenfassen zu können:

„Die explorative Faktorenanalyse geht von den wechselseitigen Zusammenhängen der Einzelindikatoren aus, die als Korrelationen quantifizierbar sind. [...] Die Faktorenanalyse extrahiert nun aus der Korrelationsmatrix einen sog. Faktor, der inhaltlich das Gemeinsame der Faktoren erfasst. “704

Für die Beantwortung der Frage, ob eine Faktorenanalyse sinnvoll ist, wurden zunächst die Kommunalitäten ${ }^{705}$ geprüft, um den Grad des Zusammenhangs einer Variablen mit den anderen Variablen aufzuklären. Dafür wurde im Falle der Häufigkeitsauszählungen mit umkodierten Variablen weitergearbeitet. ${ }^{706}$ Die Items „Textlänge“ und „mittlere Satzlänge“ wurden ausgeschlossen, sie sollen im Anschluss an die Faktorenanalyse in Bezug auf die Gesamtqualität der Texte geprüft werden. Da kein Item unter dem unteren Maß von 0.4 liegt (der niedrigste gemessene Wert liegt bei 0,58$)^{707}$, kann von einer guten Eignung der Items ausgegangen werden, so dass alle Items in der weiteren Analyse berücksichtigt werden können. Nach Prüfung der Items durch KMO- und Bartlett-Test kann festgestellt werden, dass sich die Daten sehr gut für eine Faktorenanalyse eignen. ${ }^{708}$ Daher kann nun eine explorative Faktorenanalyse ${ }^{709}$ folgen.

Zur Durchführung der Faktorenanalyse wurde Varimax als Rotationsverfahren ausgewählt. Die Anwendung einer Rotation ermöglicht es, „[...] möglichst gut differenzierende Faktoren extrahieren zu können, und Subjektivität bei der Interpretation der Faktoren weitestgehend auszuschließen. ${ }^{\text {"710 }}$ Varimax stellt (im Unterschied zum schiefen) ein orthogonales Rotationsverfahren dar, bei dem die Faktoren unkorreliert sind. Ziel dieses Verfahrens ist, die Faktoren zu vereinfachen:

„Die Anzahl von Variablen mit hoher Ladung auf einem Faktor wird minimiert, d.h. pro Faktor sollen einige Variablen hoch, alle übrigen aber möglichst gering laden. Durch die Reduktion auf wenige

\footnotetext{
${ }^{704}$ Bortz, Jürgen; Döring, Nicola (2006). S. 147.

705 Die Ergebnisse der Kommunalitätsprüfung wie auch der weiteren Prüfungen und der Faktorenanalyse sind Anhang 7.4 einzusehen.

706 Dies betrifft die Items TF03.2, MT01, MT02, DM01.

${ }^{707}$ Vgl. Schendera, Christian (2010): Clusteranalyse mit SPSS, Mit Faktorenanalyse. München: Oldenburg Wissenschaftsverlag. S. 225.

708 Der KMO-Wert kann mit 0,827 angegeben werden (Werte ab 0.8 können als gut eingeordnet werden, vgl. dazu z.B. Leonhardt, Rainer, 2010: Datenanalyse mit SPSS. Göttingen: Hogrefe. S. 223.), die Daten eignen sich also sehr gut für eine Faktorenanalyse; das Ergebnis des Bartlett-Test kann mit 0,000 als signifikant eingeordnet werden.

${ }^{709}$ Da Faktoren und Komponenten nicht bekannt sind, handelt es sich um eine explorative Faktorenanalyse.

${ }^{710}$ Schendera, Christian F. G. (2010), S. 184.
} 
Variablen mit hohen Faktorladungen auf einem Faktor erhöht Varimax die Interpretierbarkeit des

jeweiligen Faktors [Hervorh. i. Orig.]. “711

Das Ergebnis der rotierten Komponentenmatrix aller zugelassenen Items zeigt, dass einige Items nicht eindeutig zuzuordnen sind, also auf mehrere Faktoren laden. Diese wurden für die Analyse ausgeschlossen und mit den übrigen Items weitergerechnet. Die Faktorenanalyse ${ }^{712}$ (s. Anhang) ergibt 4 Komponenten (im Folgenden wird mit dem Begriff Faktoren weitergearbeitet), zu denen die Items zusammengefasst werden können. Ein Item steht für sich, weil es auf keinen anderen Faktor lädt. Auch dieses Item soll nachfolgend auf seine isolierte Stellung hin interpretiert werden.

\subsubsection{Interpretation der Rotierten Komponentenmatrix}

Die Items IG01 („Eigenständige Überschrift“), TF 03.3 („Darstellung entspr. Vorlage“), ST04.1 („Beurteilen fachlich/pragmatisch“), MT02 („Themenfortführung“), DM01 („Modalisierung“) und DM02 („Personalisierung Handelnder") mussten aus der Faktorenanalyse ausgeschlossen werden, weil sie auf mehrere Faktoren luden und damit nicht eindeutig interpretierbar sind.

Nach Ausschluss der oben genannten Items ergeben sich die nachfolgend dargestellten Faktoren:

Die Matrix zeigt 4 Faktoren, ein Item lädt nicht auf andere Faktoren und wird von der Faktorenbildung ausgeschlossen. Es handelt sich dabei um das Item „Unterscheidung zwischen Vorlage und eigener Position", das nachfolgend noch besprochen und eingeordnet werden soll. Daher ist im Weiteren von 4 Faktoren auszugehen. Diese werden in der folgenden Tabelle aufgeschlüsselt, die zugehörigen Items benannt und die Eigenschaften der Faktoren beschrieben. Dabei bleiben die Items in der Reihenfolge, in der sie in der Analyse auftauchen. ${ }^{713}$

Tabelle 17: Modell aus der Faktorenanalyse

\begin{tabular}{|c|c|c|c|c|}
\hline Faktor & Eigenschaft & Item & Inhalt & Beschreibung \\
\hline $\begin{array}{l}\text { Faktor } 1 \\
\text { Vergleichs- } \\
\text { kompetenz }\end{array}$ & $\begin{array}{l}\text { Aufnahme der Grund- } \\
\text { elemente des Ver- } \\
\text { gleichs und Einbettung } \\
\text { inhaltlicher Vergleichs- }\end{array}$ & TF03.2 & Deutschland & $\begin{array}{l}\text { Nennung von wesentli- } \\
\text { chen historisch-inhalt- } \\
\text { lichen Vergleichsas- } \\
\text { pekten }\end{array}$ \\
\hline I: & $\begin{array}{l}\text { elemente (Einbettung } \\
\text { in den historischen } \\
\text { Kontext) }\end{array}$ & TF03.1 & England & $\begin{array}{l}\text { Nennung von wesentli- } \\
\text { chen historisch-inhalt- } \\
\text { lichen Vergleichsas- } \\
\text { pekten }\end{array}$ \\
\hline
\end{tabular}

\footnotetext{
711 Ebd. S. 206.

712 Dieser Faktorenanalyse liegt eine Gesamtvarianz von 67\% zugrunde, das bedeutet, dass diese 4 Faktoren bereits $67 \%$ der Gesamtvarianz aller Untersuchungsitems aufklären.

713 Die Faktorenanalyse wurde mit SPSS errechnet. Das Programm sortiert die Items zu einem Faktor entsprechend der Faktorwerte, mit dem die einzelnen Items auf einen Faktor laden (vom ranghöchsten zum niedrigsten Faktor).
} 


\begin{tabular}{|c|c|c|c|c|}
\hline & & \multirow{2}{*}{$\begin{array}{l}\text { ST05.2 } \\
\text { ST01.1 }\end{array}$} & \multirow{2}{*}{$\begin{array}{l}\text { Reihung } \\
\text { Gegenüberstellen fach- } \\
\text { lich/pragmatisch }\end{array}$} & \multirow{2}{*}{$\begin{array}{l}\text { qualitative Einschät- } \\
\text { zung } \\
\text { Herstellung eines Zu- } \\
\text { sammenhanges zwi- } \\
\text { schen mind. zwei Ver- } \\
\text { gleichselementen }\end{array}$} \\
\hline & & & & \\
\hline & & MT01 & $\begin{array}{l}\text { Themensplitting (Ent- } \\
\text { wicklung durch Teilung) }\end{array}$ & $\begin{array}{l}\text { Häufigkeitsauszählung } \\
\text { sprachlicher Mittel }\end{array}$ \\
\hline & & ST05.1 & $\begin{array}{l}\text { Die sprachliche Hand- } \\
\text { lung Folge }\end{array}$ & $\begin{array}{l}\text { qualitative Einschät- } \\
\text { zung }\end{array}$ \\
\hline & & IG03 & Hauptteil & $\begin{array}{l}\text { Textstrukturierung: } \\
\text { Aufnahme der Ver- } \\
\text { gleichsobjekte }\end{array}$ \\
\hline & & ST01.2 & $\begin{array}{l}\text { Versprachlichung des } \\
\text { Gegenüberstellens }\end{array}$ & $\begin{array}{l}\text { Verwendung expliziter } \\
\text { sprachlicher Mittel für } \\
\text { das Gegenüberstellen } \\
\text { (qualitative Erhebung) }\end{array}$ \\
\hline \multirow{6}{*}{$\begin{array}{l}\text { Faktor } 2 \\
\text { Vergleichs- } \\
\text { kompetenz } \\
\text { II: }\end{array}$} & \multirow{6}{*}{$\begin{array}{l}\text { Anlage eines problem- } \\
\text { bezogenen und kriteri- } \\
\text { enorientierten Ver- } \\
\text { gleichs }\end{array}$} & IG02 & Einleitung & $\begin{array}{l}\text { Textstrukturierung: } \\
\text { Texteröffnung }\end{array}$ \\
\hline & & TA & Textaufbau & $\begin{array}{l}\text { Stichwörter/Ta- } \\
\text { belle/Fließtext }\end{array}$ \\
\hline & & TF01 & $\begin{array}{l}\text { Nennung einer zentra- } \\
\text { len These }\end{array}$ & $\begin{array}{l}\text { Bezug auf beide Ver- } \\
\text { gleichsobjekte }\end{array}$ \\
\hline & & TF02 & $\begin{array}{l}\text { Explizite Fokussierung } \\
\text { auf den Vergleich durch } \\
\text { Aufnahme direkter Ver- } \\
\text { gleichselemente }\end{array}$ & $\begin{array}{l}\text { Nennung von Ver- } \\
\text { gleichsgröße, Ver- } \\
\text { gleichsobjekt und Ter- } \\
\text { tium Comparationis }\end{array}$ \\
\hline & & ST02.1 & $\begin{array}{l}\text { Begründen fach- } \\
\text { lich/pragmatisch }\end{array}$ & $\begin{array}{l}\text { Vorwegnahme von Ein- } \\
\text { wänden }\end{array}$ \\
\hline & & ST03.2 & $\begin{array}{l}\text { Versprachlichung des Er- } \\
\text { klärens (Erklären-wa- } \\
\text { rum) }\end{array}$ & $\begin{array}{l}\text { Häufigkeitsauszählung } \\
\text { sprachlicher Mittel }\end{array}$ \\
\hline \multirow[t]{2}{*}{ Faktor 3} & \multirow[t]{2}{*}{$\begin{array}{l}\text { (Sach-) Urteilsbildung } \\
\text { als Vergleichsziel }\end{array}$} & TF04 & Vergleichsabschluss & $\begin{array}{l}\text { abschließendes } \\
\text { Sachurteil }\end{array}$ \\
\hline & & IG04 & Schluss & $\begin{array}{l}\text { Textstrukturierung: } \\
\text { Textabschluss }\end{array}$ \\
\hline
\end{tabular}




\begin{tabular}{|c|c|c|c|c|}
\hline $\begin{array}{l}\text { Vergleichs- } \\
\text { kompetenz } \\
\text { III: }\end{array}$ & & ST04.2 & $\begin{array}{l}\text { Versprachlichung des } \\
\text { Beurteilens }\end{array}$ & $\begin{array}{l}\text { Häufigkeitsauszählung } \\
\text { sprachlicher Mittel }\end{array}$ \\
\hline $\begin{array}{l}\text { Faktor } 4 \\
\text { sprachliche } \\
\text { Handlungs- }\end{array}$ & $\begin{array}{l}\text { sprachliche Hand- } \\
\text { lungskompetenzen: } \\
\text { Kernelemente des Ver- } \\
\text { gleichs }\end{array}$ & ST02.2 & $\begin{array}{l}\text { Versprachlichung des } \\
\text { Begründens } \\
\text { Begründen von Sachver- } \\
\text { haltsbeziehungen }\end{array}$ & $\begin{array}{l}\text { Häufigkeitsauszählung } \\
\text { sprachlicher Mittel }\end{array}$ \\
\hline kompetenz & & ST03.1 & $\begin{array}{l}\text { Erklären fachlich/prag- } \\
\text { matisch }\end{array}$ & Erklären-warum \\
\hline
\end{tabular}

Die 4 durch die Faktorenanalyse errechneten Faktoren wurden in der Tabelle bereits auf gemeinsame Eigenschaften zurückgeführt, sie lassen sich in einem Kompetenzmodell darstellen.

\subsubsection{Faktor 1: „Aufnahme der Grundelemente des Vergleichs und Einbettung inhaltlicher Vergleichselemente"}

Unter Faktor 1 lassen sich die Items subsumieren, die die basalen Strukturen des (historischen) Vergleichs darstellen. Dazu gehört insbesondere das Gegenüberstellen (ST01.1 und ST01.2). Es stellt einen Teil des historischen Vergleichs ${ }^{714}$ dar, füllt jedoch nicht den historischen Vergleich im Ganzen aus. Das Gegenüberstellen repräsentiert einen elementaren Vergleichsteil, der zunächst aus dem Wissen um ein alltägliches Vergleichskonzept übertragen werden kann, indem ein Zusammenhang zwischen zwei Vergleichselementen hergestellt und versprachlicht wird. Auch die Versprachlichung des Gegenüberstellens kann zunächst mit aus dem alltäglichen Vergleichskonzept bekannten sprachlichen Mitteln realisiert werden (z. B. über Komparation mit Hilfe der Adjunktoren „als" und „wie“). In diesen Zusammenhang ist auch das Item "Themensplitting (Entwicklung durch Teilung)" (MT01) einzuordnen. Zwar ist es ein Mittel, das wissenschaftlicher Textkompetenz zuzurechnen ist, weil es zur Entwicklung von Textlogik beiträgt, gleichzeitig stellt es ein Mittel dar, das als bekannt vorausgesetzt werden kann, weil die im entsprechenden Item erhobenen Gegensatzpaare auch im alltäglichen Vergleich realisiert werden müssen. Die Herstellung von Gegensatzpaaren ist darüber hinaus notwendig für die Komparation (,Jan läuft schneller als Erik“). ${ }^{715}$ Einen historischen Bezug erhält der Vergleich auf dieser basalen Ebene nur durch die entsprechende historische Kontextualisierung, im Beispiel also den Bezug auf Deutschland und England zu Beginn der Industrialisierung. Diese thematischen Aspekte werden über die Items „England“ (TF03.1) und „Deutschland“ (TF03.2) erfasst. Dabei geht es in diesen Items nur um die Nennung historischer Vergleichsaspekte aus dem Darstellungstext für die jeweiligen Vergleichselemente, noch nicht um einen differenzierten Bezug aufeinander, so dass auch diese Items als Grundelemente des historischen Vergleichs eingeordnet werden können. Der historische Vergleich endet in einem Sachurteil, das zuvor argumentiert, das heißt begründet und erklärt werden muss. Eine solche Struktur

\footnotetext{
${ }^{714}$ Gemeint ist hier jeweils der didaktisierte historische Vergleich im Geschichtsunterricht.

715 Zwar können Gegensatzpaare in der Komparation auch unausgesprochen bleiben, wie im Fall von „Jan ist größer", dennoch beziehen sie sich auf eine Vergleichsgröße, die dem Hörer/Leser bekannt sein muss.
} 
ist in diesem ersten Faktor allerdings noch nicht gemeint. Es geht hier noch nicht um eine historische „Beweisführung“ in dem Sinne, dass ein Urteil gebildet oder argumentativ vorbereitet wird, sondern nur um die Wiedergabe von Inhalten aus dem zugrundeliegenden Darstellungstext (wie dies auch im Anforderungsbereich I der EPA eingefordert wird). Diese Wiedergabe bilden auch die Items „Sprachliche Handlung Folge“ und „Reihung" ab, über die die historischen Inhalte transportiert werden. Über sie werden ebenfalls Inhalte des Vergleichs auf einer basalen Ebene vermittelt, da durch sie für die Wiedergabe gesorgt werden kann. Jedoch tragen Reihung und Folge nicht oder (im Falle der Folge) wenig zu einer Strukturierung im Sinne einer Beweisführung bei, die kausale Zusammenhänge transportieren muss.

Das Item „Hauptteil“ passt zu den zuvor genannten Items, da es bei dieser Kategorie um die Verarbeitung der grundlegenden Vergleichselemente im Hauptteil des Textes geht.

Fasst man die Eigenschaften der für diesen Faktor genannten Items zusammen, können sie als „Aufnahme der Grundelemente des Vergleichs und Einbettung inhaltlicher Vergleichselemente" benannt werden. Im Sinne einer Einordnung der für diese Items zu leistenden Kompetenzen werden sie als „Vergleichskompetenz I“ gekennzeichnet.

\subsubsection{Faktor 2: Anlage eines problembezogenen und kriterienorientierten Vergleichs}

Unter Faktor 2 fallen zunächst sehr divergent erscheinende Items. Zu diesem Faktor gehört insbesondere das Item "Nennung einer zentralen These" (TF01). Es ist maßgeblich für den historischen Vergleich, bahnt eine solche These doch ein Sachurteil an oder nimmt es ggf. schon vorweg. An einer dem Vergleich vorausgehenden These können etwa die einzelnen Vergleichsaspekte abgearbeitet werden und Vergleichsgröße und Vergleichsobjekt anhand des Tertium Comparationis gegenübergestellt werden. Die These ist ein wesentliches Strukturelement des Vergleichs und dem Gegenüberstellen überoder sogar vorgeordnet, weil sie, im Sinne der EPA-Bestimmung zum Vergleichen, einen Problembezug ermöglicht. ${ }^{716}$ Hiermit eng verbunden ist das Item „Explizite Fokussierung auf den Vergleich durch Aufnahme direkter Vergleichselemente" (TF02). Rückten unter Faktor 1 insbesondere Vergleichsgröße und Vergleichsobjekt (England und Deutschland) in den Fokus, findet in diesem Item zudem das Tertium Comparationis, also das Gemeinsame der beiden genannten Vergleichselemente, woraufhin Gemeinsamkeiten, Ähnlichkeiten und Unterschiede hin untersucht werden sollen, eine stärkere Berücksichtigung. Es stellt die Grundlage für einen kriterienorientierten Vergleich dar.

Zu diesen Items passt die Zugehörigkeit des Items „Einleitung“ (IG02). These und Berücksichtigung dieser Vergleichselemente können als die Einleitung strukturierende Aspekte des Vergleichs eingeordnet werden, da dem Leser darüber Thema und Absicht und zugleich die Kontextgebundenheit des historischen Vergleichs vermittelt werden.

Auch die Items „Begründen fachlich/pragmatisch“ (ST02.1) und „Versprachlichung des Erklärens" (ST03.2) passen zu diesem Faktor, weil auch sie einen problembezogenen und kriterienorientierten Vergleich ermöglichen. Das Begründen stellt einen Teil des Beurteilens und damit für das historische

\footnotetext{
${ }^{716}$ Vgl.: Kultusministerkonferenz (2005): EPA Geschichte, S. 8: „,auf der Grundlage von Kriterien historische Sachverhalte problembezogen gegenüberzustellen $[\ldots]^{\prime}$.
} 
Vergleichen einen Teil der Sachurteilsbildung dar, da Urteile, wollen sie nachvollziehbar und überzeugend sein, immer einer Begründung und damit der Offenlegung der dahinterstehenden Kriterien bedürfen. Ähnliches gilt für das Erklären. Es hat das Transferieren von Wissenselementen zum Ziel. Gerade für den der Aufgabe zugrundeliegenden Darstellungstext sind Erklärstrukturen von hoher Bedeutung, da inhaltliche Aspekte, die für den Vergleich nutzbar gemacht werden sollen, einiges Vorwissen voraussetzen. Ist dies nicht vorhanden, müssen solche Aspekte erklärt werden, bevor sie beurteilt und eingeordnet werden können. Interessant ist, dass die Versprachlichung des Erklärens Faktor 2 zugehört, nicht jedoch der fachlich/pragmatische Schwerpunkt. Umgekehrt stellen nachfolgend das fachlich/pragmatische Erklären und die Versprachlichung des Begründens einen eigenen Faktor (Faktor 4) dar. Deshalb sollen mögliche Zusammenhänge an späterer Stelle dargestellt werden.

Als letztes Item gehört der „Textaufbau“ (TA) zu Faktor 2. Hier wird erfasst, ob der Vergleich aus einer Stichwortsammlung besteht, ohne eine Struktur der Gegenüberstellung zu berücksichtigen, ob er als Tabelle oder als Fließtext realisiert wird. Zu Anfang wurde bereits gezeigt, dass die Auswahl des Textaufbaus einen Einfluss auf die Güte des Gesamttextes hat, da über eine Tabelle ein strukturiertes Gegenüberstellen klarer realisiert werden kann als über eine Stichwortsammlung (sicherlich gibt es Ausnahmen, über das Kriterienraster wurde aber explizit nach Stichwörtern ohne die Struktur des Gegenüberstellens gefragt), der Fließtext wiederum eine differenziertere Möglichkeit zur Ausformulierung sprachlicher Teilhandlungen bietet. Korreliert man das Item „Textaufbau“ mit der Gesamtpunktzahl, ergibt sich ein Korrelationskoeffizient (nach Pearson, da sich die Gesamtpunktzahl metrisch darstellt) von 0,486 und damit ein mittlerer linearer Zusammenhang zwischen Gesamtpunktzahl und Realisierung des Textaufbaus. Betrachtet man die Einzelfälle, ist zunächst festzustellen, dass nur in drei Fällen Stichwörter als Mittel der Textrealisierung gewählt wurden, in 35 Fällen Tabelle oder Kombination aus Tabelle und einer anderen Struktur und in 46 Fällen ein zusammenhängender Text. Für den stichwortartigen Textaufbau werden höchstens 19 von 43 Punkten erreicht. Dies festigt den Verdacht, dass durch Tabelle oder Ganztext ein differenzierterer kriterienorientierter, problembezogener historischer Vergleich ausgeführt werden kann als durch Stichworte. Insofern passt das Item ebenfalls zu den übrigen Items in Faktor 2, da es immer wieder um Problembezug und Kriterienorientierung geht. Deshalb werden die Eigenschaften von Faktor 2 als „Anlage eines problembezogenen und kriterienorientierten Vergleichs" benannt. Da sie über die in Faktor 1 dargestellten basalen Elemente des Vergleichs hinausgehen, werden sie als „Vergleichskompetenz II“ eingeordnet.

\subsubsection{Faktor 3: (Sach-) Urteilsbildung als Vergleichsziel}

In Faktor 3 vereinigen sich drei inhaltlich sehr homogene Items: „Vergleichsabschluss" (TF04), "Schluss" (IG04) und „Versprachlichung des Beurteilens" (ST04.2). Allen Items ist gemeinsam, dass sie sich innerhalb der Textstruktur auf den Schluss beziehen und das Sachurteil repräsentieren. Im Hinblick auf den „Schluss" wird nach einer abschließenden Textstruktur (im Sinne einer textschließenden Handlung) gefragt, die den Zweck des Vergleichs berücksichtigt. Dieses Item ist noch sehr offengehalten, da es sich zunächst auf die Textstrukturierung bezieht. Der Text kann also durch eine Zusammenfassung, ein Fazit oder eben durch eine Beurteilung im Sinne eines Sachurteils abgeschlossen werden. Inhaltlich 
differenzierter wird der Zusammenhang zwischen Textabschluss und Sachurteil durch das Item „Vergleichsabschluss" erhoben. An dieser Stelle wird explizit die Bildung eines abschließenden Sachurteils erwartet. Es führt über die Beurteilung des historischen Kontextes zur Sinnbildung als wesentliches Element des historischen Vergleichs, weshalb es hier in dieser Explizitheit erwartet und gemessen wird. Im Sinne einer logischen Textstrukturierung wird es am Ende angenommen. ${ }^{717}$ Dazu passt das Item „Versprachlichung des Beurteilens". Es erhebt solche sprachlichen Mittel, die eine Wertung beinhalten. Diese können im Sinne von Teilurteilen über den ganzen Text verteilt sein. Da aber abschließend ein Sachurteil erwartet wird, ergibt sich eine enge Verknüpfung zwischen Textabschluss und Beurteilungsstrukturen. Auffallend ist allerdings, dass es die Versprachlichung des Beurteilens ist, während das Item „Beurteilen fachlich/pragmatisch“ keinem Faktor eindeutig zugeordnet werden konnte, weil es auf mehrere Faktoren lädt. Dies könnte mit der Eindeutigkeit solcher Strukturen zusammenhängen, da implizite Beurteilungen über den ganzen Text verteilt auftauchen können. Für ein eindeutiges abschließendes Sachurteil müssen hingegen explizite sprachliche Strukturen des Beurteilens realisiert werden. Fasst man die Eigenschaften dieser drei Items zusammen, können sie als „(Sach-)Urteilsbildung als Vergleichsziel“ benannt werden. Da das Ziel bzw. der Zweck des Vergleichs im Mittelpunkt steht, kann davon ausgegangen werden, dass das Item eine weitere Kompetenzstufe darstellt. Es beinhaltet zudem eine wesentliche Kompetenzstufe, die in Gautschis Kompetenzmodell "Guter Geschichtsunterricht" dem Werturteil vorangestellt ist. Wird diese Kompetenzstufe erreicht, kann der Vergleich entsprechend den EPA als abgeschlossen gelten. Faktor 3 wird deshalb als „Vergleichskompetenz III" benannt.

\subsubsection{Sprachliche Handlungskompetenzen: Kernelemente des Vergleichs}

Unter Faktor 4 fallen die Items „Versprachlichung des Begründens" und „Erklären fachlich/pragmatisch“. Wie schon für Faktor 2 festgestellt, finden sich hier die Gegenstücke zu den unter „Vergleichskompetenz II: Anlage eines problembezogenen Vergleichs“ fallenden Items „Begründen fachlich/pragmatisch" und "Versprachlichung des Erklärens". Wie ist diese Trennung zu erklären und welche Eigenschaft kommt diesem Faktor 4 zu? Die sprachlichen Teilhandlungen Begründen und Erklären können als konstitutiv für den historischen Vergleich gelten. Sie sind es gerade, die das Beurteilen vorbereiten und das Vergleichen über das Gegenüberstellen heben. Sie geben dem Vergleich die argumentative Struktur. Insofern ist es sinnvoll, diese sprachlichen Teilhandlungen herauszuheben. Dies klärt noch nicht, dass in Faktor 4 nur der sprachliche Teil des Begründens und nur der fachlich/pragmatische Teil des Erklärens auftauchen. Es kann aber vermutet werden, dass das Erklären in Faktor 2 über die sprachlichen Mittel (im Gegensatz etwa zum Beurteilen) nicht klar vom Begründen abzugrenzen ist, da sich die sprachlichen Mittel überschneiden können. Kann das Erklären eindeutig ausgemacht und etwa vom Beurteilen abgegrenzt werden, gilt die Anforderung als erfüllt. Die Auswertung sprachlicher Mittel sowie der Qualität des Erklärens zu Beginn der Analyse hat bereits gezeigt, dass die Qualität des fachlich/pragmatischen Erklärens bei den meisten Texten in einem mittleren Bereich liegt, das Item also

\footnotetext{
${ }^{717}$ Ein Sachurteil zu Anfang des Textes wäre theoretisch denkbar, ist aber gerade bei Schreibnovizen weniger erwartbar. Es könnte aber über das Item TF01 „Nennung einer zentralen These“ erfasst werden.
} 
meist nur teilweise erfüllt ist. Dies lässt darauf schließen, dass das Einbringen von Erklärstrukturen im Vergleichstext eine sehr hohe Anforderung an die Lernenden darstellt, sicherlich nicht zuletzt, weil diese sich in die mögliche Leserschaft hineinversetzten und möglicherweise nicht vorhandene Wissensstrukturen berücksichtigen bzw. durch ihren Text transferieren müssen.

Explizite Mittel für das Begründen, auch das hat die vorangestellte Analyse bereits gezeigt, kommen kaum vor, das Begründen wird wie das Erklären häufig nur teilweise realisiert. Auch hier muss von einer hohen Anforderung an die Lernenden ausgegangen werden.

Ohne diese sprachlichen Teilhandlungen könnte der historische Vergleich jedoch nicht ausgeführt werden.

Auch wenn nur teilweise aufgeklärt werden kann, wieso es zu der Differenzierung von sprachlich bzw. fachlich/pragmatisch bei den Faktorzuordnungen einer sprachlichen Teilhandlung kommt, kann mit Hilfe dieses Faktors jedoch festgestellt werden, wo für die Lernenden besonderer Klärungsbedarf liegt. Der Faktor 4 wird, weil er keine höhere Kompetenzstufe, aber wesentliche sprachliche Teilhandlungen vereinigt, als "Sprachliche Handlungskompetenzen: Kernelemente des Vergleichs" benannt und als übergeordnete „sprachliche Handlungskompetenz" eingeordnet.

\subsubsection{Ausgeschlossene Items}

Die Items IG01 („Eigenständige Überschrift“), TF 03.3 („Darstellung entspr. Vorlage“), ST04.1 („Beurteilen fachlich/pragmatisch“), MT02 („Themenfortführung“), DM01 („Modalisierung“) und DM02 („Personalisierung Handelnder“) wurden aus den zu Anfang dargestellten Gründen ausgeschlossen. Nach der Zuordnung der Faktoren in das Kompetenzmodell muss festgestellt werden, dass gerade die Kompetenzen, die sich in einem hohen Grad auf geschichts-wissenschaftspropädeutisches Arbeiten beziehen, aus der Analyse herausfallen. Diese Items sollen deshalb nachfolgend besprochen werden: Insbesondere DM01 und DM02 stellen wichtige geschichtswissenschaftliche Werkzeuge dar. Durch die Modalisierung (DM01) kann der Konstruktionscharakter von Geschichte berücksichtigt und versprachlicht werden, indem Aussagen anderer gekennzeichnet, deren Aussagewerte schließlich kritisiert oder bestätigt und ggf. in eine eigene Deutung überführt werden können. Die Personalisierung Handelnder (DM02) wiederum ist als ein Aspekt von Multiperspektivität einzuordnen ${ }^{718}$ und damit ein wesentliches Lernziel von Geschichtsunterricht, aber gleichzeitig auch ein Werkzeug des geschichtswissenschaftlich Schreibenden. Mittel der Themenfortführung (MT02) sind ebenfalls als solche wissenschaftspropädeutische Mittel einzuordnen, da explizit (deiktische und anaphorische) Verweisstrukturen erhoben wurden, durch die sich wissenschaftliche Texte auszeichnen. ${ }^{719}$ Insofern gehören diese Aspekte zusammen.

Auch IG01 („Eigenständige Überschrift“) fällt aus der Faktorenanalyse heraus. Dieses Item lädt auf nichts. Da der Überschrift eines Sach- oder (vor-)wissenschaftlichen Textes eine eigene Struktur innewohnt, kann davon ausgegangen werden, dass die Realisierung einer Überschrift für den Vergleich

\footnotetext{
${ }^{718} \mathrm{Vgl}$. dazu Schneider, Gerhard (2012): Personalisierung/Personifizierung. In: Barricelli, Michele; Lücke, Martin (Hrsg.), Handbuch Praxis des Geschichtsunterrichts, Bd. 1, Schwalbach/Ts. 2012, S. 302-315, S.308.

${ }^{719}$ Vgl. dazu: Graefen, Gabriele (1997): Der wissenschaftliche Artikel - Textart und Textorganisation. Frankfurt am Main, Berlin, Bern, New York, Paris, Wien: Lang Verlag.
} 
eine hohe Anforderung für die Lernenden darstellt. Es fällt auf, dass häufig die Textüberschrift in den Grundzügen übernommen oder eine Überschrift ganz ausgelassen wurde. In Sachtexten kann davon ausgegangen werden, dass die Überschrift wesentliche Hinweise auf den Inhalt gibt (im Unterschied bspw. zu literarischen Textsorten). Für den Vergleichstext bedeutet dies, dass die wesentlichen Vergleichskomponenten zumindest teilweise Berücksichtigung finden müssen (z. B.: Deutschland zu Beginn der Industriellen Revolution: ein Vergleich; Der Beginn der Industriellen Revolution: Deutschland und England im Vergleich o.ä.). Über den Zweck der Überschrift, nämlich die Leser über das Grundanliegen (und damit wiederum den Zweck des Textes) zu informieren, und die damit verbundenen Strukturen müssen Lernende zunächst Klarheit haben, bevor sie solche Strukturen selbständig realisieren können. Insofern ist auch dieses Item aufgrund seiner besonderen Zweckgebundenheit als wissenschaftspropädeutisches Mittel einzuordnen.

ST04.1 (Beurteilen fachlich/pragmatisch) lädt auf mehrere Faktoren und wird deshalb nicht berücksichtigt. Es kann aufgrund der Faktorladungen am ehesten den Faktoren 2 oder 3 zugeordnet werden und ist damit im Zusammenhang mit den Kompetenzstufen II und III einzuordnen. Unter der Kompetenzstufe II sind sprachliche Teilhandlungen wie das Erklären und Begründen subsumiert, sodass das Beurteilen hierhin passt. Unter der Kompetenzstufe III sind jedoch Items vereinigt, die das Sachurteil widerspiegeln. Beide Zuordnungen erscheinen sinnvoll, da das Beurteilen, wie zuvor dargestellt, eine wesentliche Kompetenz für den Geschichtsunterricht darstellt, gleichzeitig aber auf eine Teilbeurteilung im Sinne der Kompetenzstufe II reduziert werden kann. Für die eindeutige Zuordnung wurde deshalb das Item „Versprachlichung des Beurteilens" herangezogen.

TF03.3 als letztes nicht berücksichtigtes Item versammelt solche Inhaltselemente, die über den eigentlichen Vergleich hinausgehen, weil es sich auf Textteil 3 des Darstellungstextes bezieht, worin die Entwicklung Deutschlands skizziert wird. Es wurde erfasst, weil sich viele Lernende in ihren Texten darauf bezogen haben. Es fällt, da es nicht eindeutig zuzuordnen ist, aber aus der Analyse heraus, so dass die Einschätzung verstärkt wird, dass dieser Teil als optional für die Vergleichsaufgabe anzunehmen ist, aber keinen notwendigen Teil darstellt. Es ist nur in Bezug auf die vorliegende Aufgabe zu untersuchen und spielt für die Übertragung auf andere Vergleichsaufgaben keine Rolle.

Aus diesen Ergebnissen können im Weiteren Kriterien herausgearbeitet werden, die anschließend in ein verkürztes Kriterienraster zur Auswertung von Vergleichsaufgaben für Lehrkräfte übertragen werden (Kap. 9). Bevor dies geleistet werden kann, sind jedoch weitere Untersuchungsaspekte vorauszuschalten, um ein solches Modell auszudifferenzieren. Deshalb sollen zunächst die Rating-Ergebnisse in Bezug zu den drei Textgrundlagen gestellt werden, um die Frage zu beantworten, ob bzw. welchen Einfluss die Textgrundlage auf die Textproduktionen der Schüler*innen hatte. Daraus können erste Rückschlüsse für die Bearbeitung von Vergleichsaufgaben auf der Basis von Darstellungstexten gezogen werden. Außerdem soll ein möglicher Zusammenhang von Textlänge und Textqualität überprüft werden. Im nachfolgenden Kapitel werden dann drei Schüler*innentexte auf der Grundlage des Kriterienrasters untersucht, um so individuelle Strukturen sichtbar zu machen. Die Ergebnisse dieses und des folgenden Kapitels werden im Weiteren in die Überlegungen zur Modellierung des Kriterienraster für Lehrkräfte einbezogen. 


\subsubsection{Zusammenfassung der zentralen Befunde}

Das Ergebnis der Faktorenanalyse mit Varimax ermöglicht die Einordnung der zuvor beschriebenen Items in vier Faktoren, die wiederum in vier Kompetenzstufen zuzuordnen sind. Das Vergleichen im Geschichtsunterricht kann dadurch detailliert aufgeschlüsselt werden in drei Vergleichskompetenzstufen, die es von einem basalen Vergleichen über einen problembezogenen Vergleich kennzeichenbar machen und auf der dritten Stufe in einer Urteilsbildung als zentrales Ziel von Geschichtsunterricht münden. Die vierte sprachliche Handlungskompetenz verläuft dabei quer zu den drei zuvor genannten Kompetenzstufen.

Dabei konnte Vergleichskompetenz I durch die Aufnahme basaler Vergleichselemente gekennzeichnet werden, zentral ist die Zuordnung des Gegenüberstellens als grundlegende Teilhandlung des Vergleichens im Geschichtsunterricht.

Vergleichskompetenz II erfordert bereits den Rückbezug auf eine zentrale These und textstrukturierende Elemente wie Einleitung und Hauptteil, aber über die Berücksichtigung der sprachlichen Teilhandlungen Erklären und Begründen ein problemorientiertes Vorgehen, da beide Teilhandlungen eine Sachurteilsbildung anbahnen.

Vergleichskompetenz III berücksichtigt schließlich die Sachurteilsbildung als Ziel des Vergleichs im Geschichtsunterricht. Entsprechend beziehen sich alle Items auf den Textschluss und führen die unter Vergleichskompetenz I und II subsummierten Items so zusammen.

Die quer zu den progressiv aufeinander aufbauenden Vergleichskompetenzen I-III liegenden sprachlichen Handlungskompetenzen, die sich vor allem auf das Erklären und Begründen beziehen und damit insbesondere die Vergleichskompetenz II erweitern, können als Kernkompetenzen für das historische Lernen im Geschichtsunterricht beschrieben werden. Es konnte bereits in Kapitel 7.2 gezeigt werden, dass sie eine große Herausforderung für die Lernenden darstellen. Letztlich sind sie bedeutsam für viele der in den EPA genannten Operatoren und damit unabdingbar für das Rezipieren und Produzieren von Texten und damit letztlich das Narrativieren im Geschichtsunterricht.

Das Kompetenzmodell als Ergebnis aus der Faktorenanalyse ermöglicht zum einen die Aufschlüsselung wesentlicher Elemente des Vergleichs im Geschichtsunterricht als auch die Einordnung von Lernendentexten hinsichtlich ihrer Qualität für das Lernen im Geschichtsunterricht. Dieser doppelte Effekt kann nachfolgend für die Anbahnung von Vergleichskompetenzen im Geschichtsunterricht nutzbar gemacht werden. 


\subsubsection{Zum Einfluss der Textgrundlage auf die Aufgabenlösung}

Um die Frage beantworten zu können, ob die Textgrundlage einen Einfluss auf die Textqualität hatte, werden die verschiedenen Erhebungen drei Gruppen zugeordnet. Zu Gruppe 1 gehören die Erhebungen 1 bis 3 . Hier haben die Lernenden mit der unveränderten Textgrundlage und Aufgabe gearbeitet. Zu Gruppe 2 zählen die Erhebungen 4 und 5. Hier haben die Schüler*innen zwei Texte bekommen (einer zu England, einer zu Deutschland), inhaltlich wurden dafür nur im Ursprungstext vorgegebene Vergleichselemente entnommen und der Text entsprechend der schon bestehenden Teilüberschriften geteilt, die Aufgabe blieb unverändert. Zu Gruppe 3 zählt die Erhebung 6, die Lernenden haben dabei mit der schon für Gruppe 2 veränderten Textgrundlage gearbeitet. Außerdem sollten sie eine vorgeschaltete Tabelle ausfüllen, bevor sie die eigentliche Vergleichsaufgabe lösen sollten. Die Tabelle war als Scaffold gedacht, wodurch das Gegenüberstehen als Teilhandlung des Vergleichs vorbereitet werden sollte. Über ein Histogramm in Form eines Stapeldiagramms kann deutlich gemacht werden, wie Textgrundlage und Bearbeitung zusammenhängen:

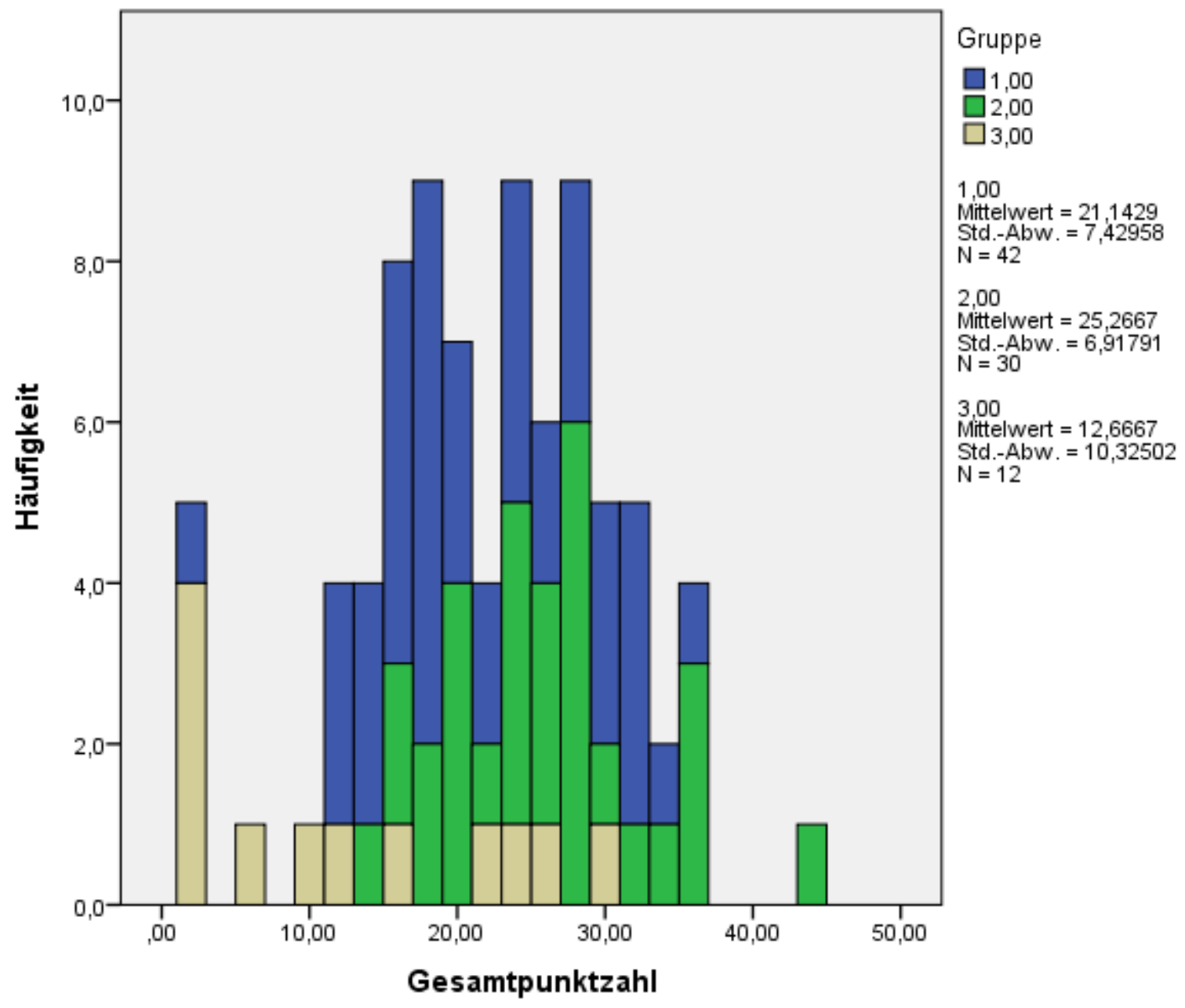

Abbildung 55: Ergebnisse aus den unterschiedlichen Erhebungsteilen

Das Histogramm zeigt drei gestaffelte Balken an. Fasst man diese gestapelten Balken als einen gesamten Balken auf, gibt das Diagramm Auskunft über die Verteilung der erreichten Gesamtpunktzahlen 
ohne eine Gruppendifferenzierung. Mit Ausnahme der fünf Schüler*innentexte, die lediglich zwei Punkte erreicht haben, zeigt das Diagramm weitestgehend eine Normalverteilung an. Der Mittelwert für die Gesamtpunktzahl aller Texte liegt bei 21,40, die Standardabweichung bei 8,63.

In Blau sind die Texte der Gruppe 1 gekennzeichnet, die von allen Gruppen die größte Bandbreite anzeigt. Dies erscheint insofern folgerichtig, da in Gruppe 1 die meisten Schüler*innentexte vereinigt sind. Es fällt aber auch auf, dass der beste Text nicht in die Gruppe 1 gehört. Gruppe 1 hat mit dem authentischen Schulbuchtext gearbeitet. Die vorhergehende Analyse hat gezeigt, welche sprachlichen und fachlichen Anforderungen dieser Text enthält. Zudem werden durch den Darstellungstext Vergleichsstrukturen vorgegeben, die für die Aufgabenlösungen im Sinne einer Wiedergabe übernommen werden können. Dies haben viele Lernende getan, woraus in diesen Texten Reihungen entstanden sind, nicht aber für die Lösung der Aufgabe zweckorientierte eigenständige Vergleichsstrukturen.

Die Ergebnisse von Gruppe 2 werden durch die grünen Balken gekennzeichnet. Die Spannbreite ist geringer als die der Gruppe 1 (vgl. Standardabweichung im Diagramm), im Mittel sind die Punktwerte höher als in Gruppe 1. Die Texte der Gruppe 2 zeigen also insgesamt eine höhere Textqualität als die Texte der Gruppe 1. Da die hinter den Texten stehenden Lernenden nicht bekannt sind, kann hier nicht geklärt werden, ob die Gruppe 2 leistungsstärker gewesen sein könnte. Da aber an verschiedenen Schulen in den Klassen 12 und 13 erhoben wurde, erscheint diese Annahme eher unwahrscheinlich. Es ist wahrscheinlicher, dass die Umstrukturierung der Texte zu dem besseren Ergebnis geführt hat. In Gruppe 2 wurden dem authentischen Darstellungstext die vorgegebenen Vergleichsstrukturen entnommen und die Texte in zwei Textteile aufgeteilt. Es kann angenommen werden, dass die Leistung der Lernenden bei der Beantwortung der Vergleichsaufgabe zwar höher einzuschätzen ist als die der Gruppe 1, weil die Vergleichsaspekte selbständig gefunden werden mussten, aber gleichzeitig dieser Umstand dazu geführt hat, dass die Lernenden weniger reine Wiedergabestrukturen produziert haben. In Gruppe 3, im Diagramm durch die beigen Säulen gekennzeichnet, lagen die veränderten Texte vor, die auch für die Gruppe 2 verwendet wurden, und das Scaffold durch die Vorabaufgabe. Gerade diese Gruppe hat jedoch am schlechtesten abgeschnitten. Sicherlich sind diese Ergebnisse mit Vorsicht zu lesen, weil nur in einer Klasse erhoben werden konnte. Zudem wurde die Erhebung kurz vor den Ferien durchgeführt, ein Umstand, der dazu geführt haben könnte, dass die Aufgabe mit geringerer Motivation als bei den übrigen Teilerhebungen bearbeitet wurde. Dennoch zeigen die Ergebnisse auch, dass es selbst für Lernende der Sekundarstufe II nicht reicht, ein Scaffold hinzuzufügen, auf der Metaebene jedoch nicht den Zweck zu thematisieren. Sicherlich hätte den Lernenden erst deutlich werden müssen, welche Bedeutung das Gegenüberstellen für das Vergleichen hat und wie dies strukturiert durchzuführen ist (dies war von der durchführenden Lehrkraft jedoch nicht gefordert und wurde nicht in der Aufgabe angelegt). Dieses Ergebnis ist ein wichtiger Hinweis auf den Umgang mit solchen Scaffolds eben auch in Bezug auf das historische Vergleichen. 


\section{Qualitative Analyse ausgewählter Lerner*innentexte: Drei}

\section{Beispiele}

Nachdem die statistische Auswertung in Kapitel 7 ein Kompetenzmodell für die Realisierung von Vergleichstexten ergeben hat, soll nun anhand von drei Beispieltexten aus dem Korpus überprüft werden, wie in diesen Texten das historische Vergleichen realisiert wurde und welche Kompetenzen des zuvor dargestellten Kompetenzmodells in den Texten sichtbar werden.

Dazu wurde jeweils ein Text mit der geringsten, einer mittleren und der höchsten Gesamtpunktzahl ausgewählt ${ }^{720}$ :

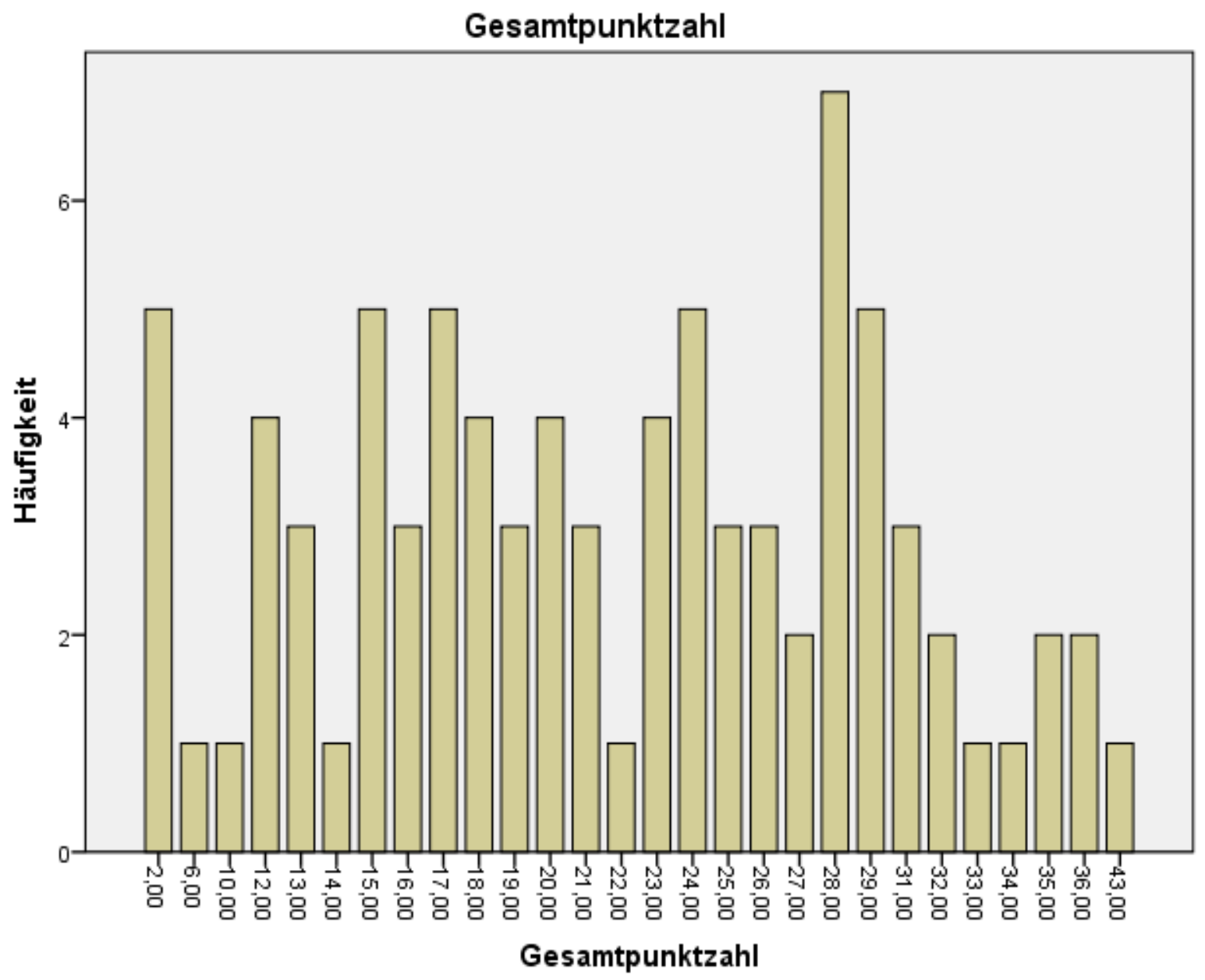

Abbildung 56: Gesamtpunktzahlen der Korpustexte

Nur ein Text erreicht mit 43 Punkten die höchste Punktzahl, dieser wurde entsprechend für die nachfolgende Analyse ausgewählt.

\footnotetext{
${ }^{720}$ Es wäre auch eine Auswahl von Einzeltexten über die Zuordnung zu den Kompetenzen denkbar gewesen, die auf der Grundlage der Faktorenrechnung entstanden sind. Da in der Schulpraxis in der Regel Texte auf der Grundlage des bestehenden Notensystems eingestuft werden, ist die Auswahl über die Gesamtpunktzahl diesem Vorgehen näher.
} 
Als mittlere Punktzahl wurde der Text ausgewählt, der 22 Punkte erreicht. Da kein Text exakt mit dem arithmetischen Mittel ${ }^{721}$ von 21,5, bepunktet wurde, wurde der Text ausgewählt, der direkt darüber liegt.

Die Grafik zeigt, dass 5 Texte mit nur 2 Punkten am punktzahlniedrigsten sind. Schlüsselt man diese 5 Texte hinsichtlich der zugehörigen Erhebung auf, fällt auf, dass nur ein Text der Erhebung 2 und damit Erhebungsteil 1 mit authentischer Aufgabe und unverändertem Text angehört, aber 4 Texte der Erhebung 6 und damit Erhebungsteil 3 mit leicht verändertem Text und der Tabelle als Scaffold. ${ }^{722}$ Da zudem die Mehrheit der punktniedrigen Aufgabenlösungen aus einer Tabelle besteht, wurde eine Realisierung in Form einer Tabelle aus Erhebung 6 ausgewählt (zum Vergleich: die punktniedrigste Aufgabenlösung aus Erhebung 2 wurde in Form von Stichwörtern ausgeführt).

\subsection{Text 1: Eine Tabelle als Vergleichslösung}

Die Schüler*innen der Erhebung 6 hatten zur Aufgabe, eine Tabelle mit wichtigen Vergleichselementen anzufertigen und dann einen Text mit der Gesamtaufgabenlösung zu verfassen. Der Verfasser ${ }^{723}$ der vorliegenden Lösung beschränkt sich auf eine Tabelle, die Bearbeitung des Aufgabenteils 2 (und damit der Hauptaufgabe) fehlt. ${ }^{724}$ Die Tabelle enthält die folgenden Inhalte ${ }^{725}$ :

\footnotetext{
${ }^{721}$ Der Median liegt bei 21,4, so dass aufgrund der Ähnlichkeit der Werte das arithmetische Mittel zugrunde gelegt werden kann.

722 Ausgewählt wurden die Texte 6_04 (2 Punkte), 6_07 (22 Punkte), 4_24 (43 Punkte).

${ }^{723} \mathrm{Im}$ Folgenden wird aufgrund des besseren Leseflusses auf die weibliche Form verzichtet, die immer mitgedacht ist. Eine Aufschlüsselung in männliche oder weibliche Autoren wurde zwar erfasst, spielt für diese Erhebung aber keine Rolle und wird deshalb für alle drei Texte nicht angegeben.

724 Dies ist auch so bei den übrigen punktniedrigsten Texten der Erhebung 6. Ein Ausweichen auf den 5. punktniedrigsten Text aus der Erhebung 2_04 erscheint dennoch nicht geboten, weil diese Stichwortsammlung keine Vergleichselemente erhält.

${ }^{725}$ Auf die Beifügung eines Scans der Original-Schüler*innenlösungen wird hier wie bei den beiden Folgetexten aus Datenschutzgründen verzichtet. Die digitale Umsetzung der Texte folgt dem Layout der Originaltexte.
} 


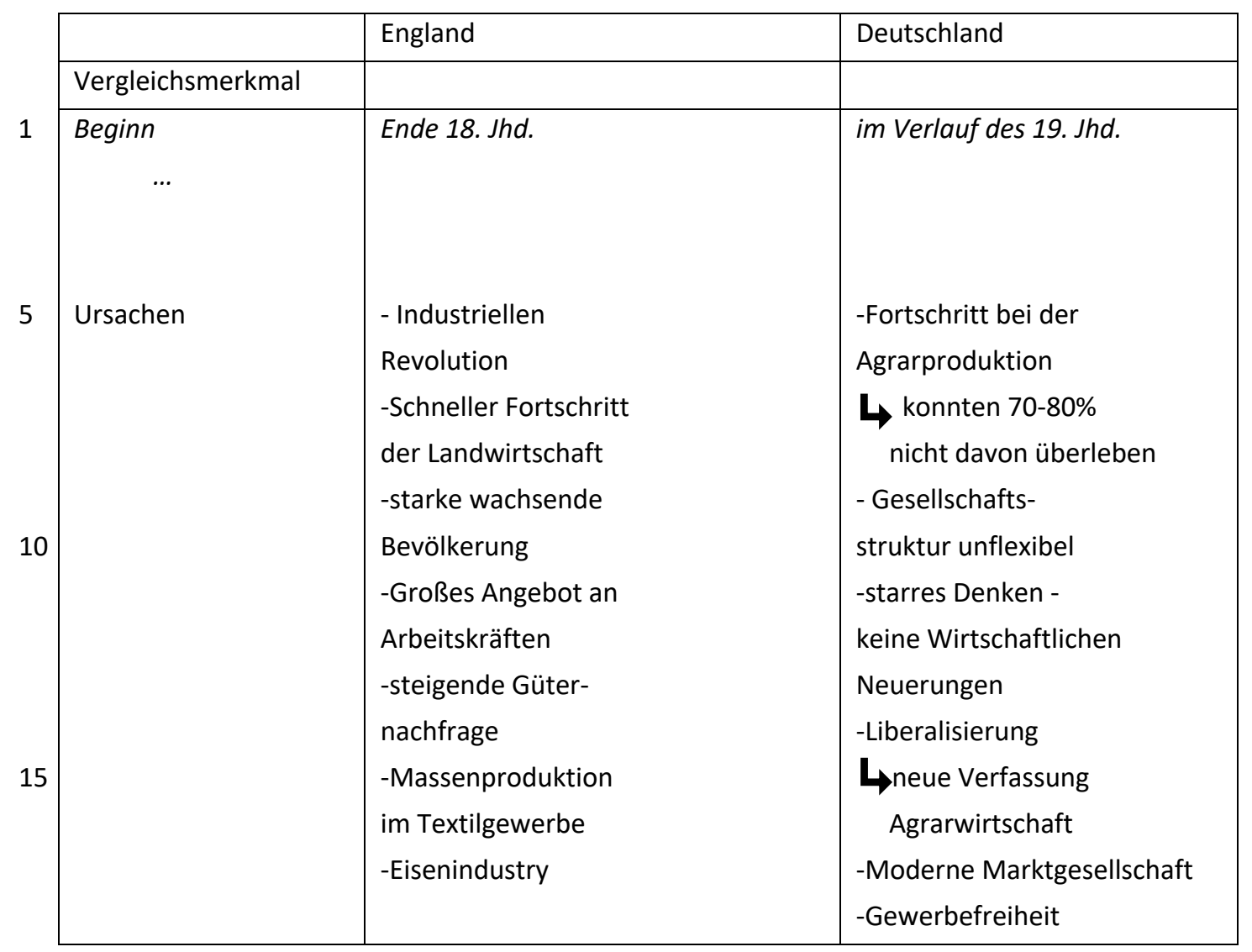

\subsubsection{Analyse der formalen Textstruktur}

Im Rating hat diese Aufgabenbearbeitung durch alle Rater lediglich eine Bewertung der Kategorien „Nutzung eines Mittels zur Vorstrukturierung“, „Bearbeitung der Aufgabe“ und „Textaufbau“ erhalten, da die Hauptaufgabe fehlt. Die Nutzung des Mittels zur Vorstrukturierung war in der Aufgabe gefordert. Die Bearbeitung der Aufgabe konnte trotz des Fehlens der Hauptaufgabe als ernsthafte Aufgabenlösung eingeordnet werden, der Textaufbau als Tabelle. Alle weiteren Kategorien wurden als nicht erfüllt bewertet. Bei der Analyse steht deshalb nun im Mittelpunkt, wie die Tabelle bearbeitet wurde und welche Elemente des Vergleichs hier realisiert wurden.

Aufgrund der stichwortartigen Bearbeitung der Vergleichsaufgabe in der Tabelle kann nur die Anzahl der Wörter, jedoch keine mittlere Satzlänge angegeben werden. Die Anzahl der Wörter ist mit 49 insbesondere in Bezug auf die Länge der Textvorlage und die Bearbeitungsdauer von mind. 45 Min. als gering einzustufen. Eine Strukturierung in Einleitung, Hauptteil und Schluss kann nicht festgestellt werden. Dies ist in einer Tabelle, die Elemente gegenüberstellen soll, auch nicht als passende Strukturierungselemente anzunehmen. Es muss davon ausgegangen werden, dass die Tabelle lediglich den Hauptteil fokussiert. 


\subsubsection{Vergleichsspezifische thematische Inhalte und inhaltlich-thematischer Fokus}

Eine zentrale These wird nicht genannt. Immerhin werden aber beide Tabellenspalten ausgefüllt und darüber zumindest ein basales Themensplitting (entspr. Vergleichskompetenzstufe I) erreicht. Die Bearbeitung der Tabelle beschränkt sich auf ein Merkmal: Der Schüler nennt mit dem Begriff Ursachen ein Vergleichsmerkmal über das vorgegebene Merkmal Beginn hinaus. Das Merkmal Beginn ist für die Vergleichselemente bereits vorausgefüllt, so dass in der Analyse die Zuordnung von Elementen zum Merkmal Ursachen im Mittelpunkt der Betrachtung steht. Für England wird als erstes die Industrielle Revolution den Ursachen zugeordnet. Die Industrielle Revolution kann jedoch nicht gleichzeitig eine Ursache und Teil des Tertium Comparationis darstellen, so dass an dieser Stelle ein logischer Bruch vorliegt. Die nachfolgenden Stichpunkte beziehen sich dann auf einige im Text genannten Faktoren, die für den frühen Beginn der Industriellen Revolution ausgemacht werden. Der Schüler nennt die Fortschritte in der Landwirtschaft, die stark wachsende Bevölkerung, das Angebot an Arbeitskräften, die steigende Güternachfrage, die Massenproduktion sowie die Eisenindustrie. Es handelt sich dabei um die Nennung der im Darstellungstext fett gedruckten Begriffe (mit Ausnahme der „offenen Gesellschaftsstruktur). Auffällig ist, dass der Begriff Eisenindustrie (der Schüler anglisiert den Begriff) alleine steht und so noch nicht als Ursache bestimmt werden kann, da Hinweise, inwiefern dieser eine Ursache darstellt, fehlen.

In Bezug auf Deutschland nennt der Schüler zunächst den Fortschritt bei der Agrarproduktion als Ursache. Dieser Aspekt steht dem Pendant in der Auflistung zu England jedoch nicht direkt gegenüber, so dass für den Leser nicht deutlich wird, wie dieses Merkmal in der Gegenüberstellung zu England zu werten ist. Durch einen Pfeil wird diesem Aspekt ein Hinweis zugefügt: „ davon überleben“ (Z. 5). Dieser Hinweis erklärt sich nur über den Darstellungstext, in dem herausgestellt wird, dass in der deutschen Landwirtschaft zunächst ertragsschwache Kleinbetriebe überwogen, hier wird ein Anteil von $70-80 \%$ an der gesamten Landwirtschaft genannt. ${ }^{726}$ Da der Schüler mit der Tabelle nur eine Aufgabe vor der eigentlichen Vergleichsaufgabe beantwortet, ist denkbar, dass er für sich selbst ein Stichwort notiert hat, dabei noch keinen anderen Leser fokussiert, für den diese Notiz so nicht verständlich wäre.

Weiterhin stellt er eine unflexible Gesellschaftsstruktur und starres Denken für Deutschland fest.

Diese beiden Aspekte zeigen, dass der Schüler zwar keine direkte inhaltliche Gegenüberstellung vergleichbarer Punkte schafft, die Gegenüberstellung dennoch kenntlich macht, indem er sprachlich über Positiv-/Negativ-Zuschreibungen arbeitet: Für England sind die verwendeten Attribuierungen durchweg positiv konnotiert (schnell, stark wachsend, groß, steigend), wohingegen die für Deutschland genutzten Attribuierungen durchweg als negativ, da nachteilig für den Industrialisierungsprozess, einzuordnen sind (nicht, unflexibel, starr, keine). Allerdings sind die Zuschreibungen unterschiedlich, so bilden der erste Stichpunkt sowie die letzten drei Stichpunkte Ausnahmen und durchbrechen diese Art der Gegenüberstellung (die Konnotierung wird über die Auswahl der Fachbegriffe sowie der zugehörigen Attribuierungen geschaffen: Fortschritt, neu, modern). Inhaltlich verbindet der Schüler

${ }^{726}$ Vgl. Laschewski-Müller, Karin; Rauh, Robert (2010), S. 257. 
Elemente aus allen drei Teilen des Darstellungstextes. Es gelingt jedoch nicht, diese in einen systematischen Zusammenhang zu bringen, so dass kein systematisches Gegenüberstellen erreicht wird, aber entspr. Vergleichskompetenzstufe I zumindest die Grundelemente des Vergleichs aufgenommen und unstrukturiert gegenübergestellt werden.

Ein Vergleichsabschluss fehlt, korrespondierend zur fehlenden Strukturierung in Einleitung, Hauptteil und Schluss; allerdings war dies in dieser vorbereitenden Aufgabe auch noch nicht gefordert.

\subsubsection{Realisierung sprachlicher Teilhandlungen}

Da nur die erste der beiden Aufgaben bearbeitet wird, wird entsprechend der Aufgabenstellung in der Hauptsache (wenn auch unstrukturiert) die sprachliche Teilhandlung „Gegenüberstellen“ realisiert. Es lassen sich, gekennzeichnet über die Pfeile (Z. 7 und 15), als Teilhandlung des Gegenüberstellens zumindest in Ansätzen Erklärungen und/oder Folgen feststellen.

Das Gegenüberstellen zeigt sich an der Textoberfläche nur über die Einordnung der ausgewählten inhaltlichen Aspekte in die vorgegebene Tabelle und die in Kapitel 8.1.2 beschriebenen Konnotationen. Im Sinne von Ehlich/Rehbein muss aufgrund der fehlenden visuellen Struktur (gegensätzliche Aspekte werden in der Tabelle nicht, gegenüberstehend' angeordnet) und der Zuordnungen von Wissenselementen, die die weitere Entwicklung Deutschlands, jedoch nicht die geforderten Ausgangsbedingungen thematisieren, von einem Gegenüberstellungs-Versuch gesprochen werden. ${ }^{727}$ Das Gegenüberstellen erfordert laut EPA für den Geschichtsunterricht eine argumentative Gewichtung. Zwar gewichtet der Schüler über die genannten sprachlichen Mittel, die zu einer Konnotation führen, die aufgenommenen Wissenselemente für sich, stellt sie aber nicht im Rahmen der Tabelle in einen argumentativen Gesamtzusammenhang. Deshalb muss davon ausgegangen werden, dass die Darstellung nicht zu einer mentalen Veränderung beim Leser führt.

Eine auffallende weitere Struktur in dieser Tabelle stellen die beiden Pfeile dar, die den Stichworten „Fortschritt bei der Agrarproduktion“ und „Liberalisierung“ angefügt werden. Hier ist zu klären, welche möglichen sprachlichen Teilhandlungen sich dahinter verbergen.

Der Pfeil lässt in beiden Fällen zwei Lesarten zu: Er kann als Folge oder als Erklärung eingeordnet werden:

\footnotetext{
${ }^{727} \mathrm{Vgl}$. dazu Ehlich, Konrad; Rehbein, Jochen (1986). Die Autoren unterscheiden in Bezug auf die sprachliche Handlung des Begründens zwischen dem Begründen und dem Begründungsversuch: „Solange nämlich die Veränderung im Wissen des Hörers nicht erreicht ist, hat man es auf seiten des Sprechers lediglich mit einem Versuch zu tun - einem Versuch, der je nach Qualität der Handlungsmittel gelingt oder mißlingt. Wir unterscheiden deshalb systematisch zwischen dem Begründungsversuch - als dem Versuch des Sprechers, eine mentale Veränderung beim Hörer zu erreichen - und der Begründung als ganzer, die sprecherseitig diesen Versuch und hörerseitig den entsprechenden Vollzug umfaßt [Markierungen im Original]." (ebd. S. 94f).
} 
Für das erste Beispiel wären folgende Lesarten denkbar (fehlende Wissenselemente werden dabei auf der Grundlage des Darstellungstextes eingefügt): Es zeichneten sich Fortschritte bei der Agrarproduktion ab, daraus resultierte, dass $70-80 \%$ der Höfe nicht überleben konnten. ${ }^{728}$ Die hinter dieser Lesart liegende Teilhandlung wäre damit die Folge.

Eine zweite Lesart könnte lauten: Weil 70-80\% der Höfe nicht überleben konnten, zeichneten sich Fortschritte bei der Agrarproduktion ab. In diesem Fall kann eine Erklärung zugrunde gelegt werden, die den EPA-Geschichte entsprechend historische Sachverhalte einordnen soll.

Auch der zweite Pfeil lässt die Lesarten Folge und Erklärung zu: In Zeile 12 führt der Schüler in Bezug auf Deutschland das Stichwort „Liberalisierung“ an. Über den Pfeil in der folgenden Zeile verweist er auf eine neue Verfassung in der Agrarwirtschaft, er spielt dabei vermutlich auf die im Darstellungstext genannte neue Agrarverfassung an. ${ }^{729}$

Entsprechend der Lesart Erklärung kann davon ausgegangen werden, dass über den durch den Pfeil gekennzeichneten Verweis Wissen transportiert werden soll: Dem Leser wird ein Beispiel gegeben, worin sich die Liberalisierung zeigt: Ein Beispiel für die Liberalisierung stellt die neue Agrarverfassung dar. Da eine Erklärung mehr beinhaltet als ein Beispiel, handelt es sich zwar um eine sehr basale Teilstruktur, allerdings kann ihr der Zweck Wissenstransfer bzw. Herstellung eines konkreten Zusammenhanges zugeordnet werden, der eine Erklärung charakterisiert.

Als Folge könnte formuliert werden: Aus der Liberalisierung resultierte eine neue Agrarverfassung.

Schon aufgrund der Unklarheit in der Klärung der dem Pfeil zugrundeliegenden sprachlichen Teilhandlung (möglicherweise werden durch den Pfeil auch beide Teilhandlungen markiert) muss von einem Erklärungsversuch oder dem Versuch der Realisierung einer Folge gesprochen werden, so dass das Vergleichskompetenzniveau II bzw. das entsprechende Item der dazu quer liegende sprachliche Handlungskompetenz nicht erreicht wird. ${ }^{730}$

\footnotetext{
728 Dabei handelt es nicht um eine korrekte Aussage, da der Darstellungstext einen anderen Zusammenhang transportiert: Danach mussten die Betreiber der meisten ertragsschwachen Kleinbetriebe einem Nebenerwerb nachgehen, um ihre Existenz zu sichern. Bei etwa 70-80\% solcher Höfe handelte es sich um solche ertragsschwachen Kleinbetriebe. (vgl. Laschewski-Müller, Karin; Rauh, Robert (Hrsg.) (2010). S. 257f).

${ }^{729}$ Da aber Hinweise auf die Inhalte dieser neuen Verfassung fehlen und die Begriffe „neue Verfassung" und „Agrarwirtschaft" unverbunden untereinanderstehen, kann nur der kundige Leser erfassen, dass die Agrarverfassung aufgrund ihrer inhaltlichen Ausgestaltung ein Beispiel für die Liberalisierung in Deutschland darstellt. Für einen solchen Leser führt dieses Wissenselement jedoch nicht zu einer Integration neuen Wissens über einen Wissenstransfer, so dass davon ausgegangen werden muss, dass es sich um einen Erklärungsversuch handelt.

Dieses Beispiel zeigt sehr deutlich die Schwierigkeit von Schreibaufgaben in der Schule. Nimmt man an, dass sich diese Tabelle an einen Leser richtet, kann sie durch eine Geschichtslehrkraft gelesen werden, nicht jedoch durch eine sachunkundige Person, da Wissen vorausgesetzt wird, von dem die Lernenden annehmen können, dass die Fachlehrkraft es besitzt.

${ }^{730}$ Auch eine Reihung wäre denkbar, dem widerspricht allerdings die explizite Nutzung des Pfeils, auf den ansonsten hätte verzichtet werden können.
} 


\subsubsection{Wissenschaftliche Textkompetenz}

In der Bearbeitung der Tabelle macht der Schüler Bezugnahmen auf den Darstellungstext nicht kenntlich. Allerdings legt die Aufgabe dies auch nicht nahe, da es hier zunächst darum geht, Inhalte auszuwählen und im Hinblick auf den Vergleich vorzustrukturieren. Durch die sehr knappen Inhalte der Tabelle werden zudem Zusammenhänge so sehr verkürzt, dass keine direkte Übernahme von Inhalten aus dem Darstellungstext vorliegen. Durch den Stichwortstil sind ebenfalls keine Modalisierungen vorhanden, so dass an diesen beiden Punkten auf der Grundlage von Tabelle und Aufgabe kaum wissenschaftliche Textkompetenz auszumachen ist. Ein Themensplitting wird in der Tabelle durch die Benennung der Spalten („England“ und „Deutschland“) angelegt, in den Spalten finden sich keine weiteren Versprachlichungen.

Ebenso ist eine Personalisierung Handelnder kaum gegeben, am ehesten finden sie sich in der Aufnahme der Begriffe „Bevölkerung“ und „Arbeitskräfte“; dadurch bleiben die Inhalte jedoch sehr abstrakt.

Insgesamt finden sich kaum Merkmale (vor-)wissenschaftlicher Schreibkompetenz. Hier ist kritisch zu fragen, ob die Tabellenform dazu überhaupt das geeignete Medium darstellt. Auch wenn für diesen Text argumentiert werden kann, dass es sich um eine Vorarbeit für die eigentliche Vergleichsaufgabe handelt, zeigen die übrigen in Tabellenform realisierten Vergleichstexte ähnliche Strukturen. In den meisten Fällen fehlt insbesondere das zentrale Ziel der Sachurteilsbildung.

\subsubsection{Holistische Einordnung des Textes}

Die Bearbeitung der Tabelle zeigt, dass der Schüler einige ausgewählte historisch-inhaltliche Aspekte aus dem Darstellungstext zum Merkmal Ursachen auswählt und über die Tabelle in eine Struktur des Gegenüberstellens überführen kann. Es ist jedoch auch festzustellen, dass die Auswahl von als wesentlich für das Gegenüberstellen erachteten Aspekten nicht systematisch und nicht vollständig erfolgt. Insbesondere in Bezug auf Deutschland fehlt eine Abgrenzung zur Situation bei Beginn der Industrialisierung und zu einem späteren Zeitpunkt. Diesen Zusammenhang muss sich der Leser selbst erschließen. Die Struktur des Gegenüberstellens wird über Konnotationen erreicht, nicht jedoch über eine systematische Gegenüberstellung der Teilaspekte, so dass der Eindruck einer Reihung von Einzelaspekten entsteht. Als sprachliche Teilhandlungen des Gegenüberstellens können die Folge und/oder das Erklären ausgemacht werden.

Merkmale, die das über das Gegenüberstellen hinausgehende historische Vergleichen ausmachen, fehlen. Insgesamt ist der Text entsprechend dem im letzten Kapitel beschriebenen Kompetenzmodell der Kompetenzstufe I und damit einer basalen Vergleichskompetenz (Vergleichskompetenz I: Aufnahme der Grundelemente des Vergleichs und Einbettung inhaltlicher Vergleichselemente (Einbettung in den histor. Kontext)) zuzuordnen. 


\subsection{Text 2: Ein kurzer Vergleich als Fließtext}

Auch diese Aufgabenlösung ist der Erhebung 6 (Erhebungsteil 3) zuzuordnen, die die Aufgabe umfasst, eine Tabelle mit wichtigen Vergleichselementen anzufertigen und dann einen Text mit der Gesamtaufgabenlösung zu verfassen. Der Schüler, der die nachfolgende Lösung angefertigt hat, füllt die Tabelle kaum aus, fügt aber als eine Art Metakommentar eine persönliche Meinung hinzu. Die eigentliche Vergleichsaufgabe löst er in einem knappen Fließtext. Der Text wurde mit einer Gesamtpunktzahl von 22 Punkten geratet und bildet im Vergleich zu den anderen Texten des Korpus damit eine mittlere Gesamtpunktzahl ab.

Im Folgenden werden Tabelle und Text abgebildet:

\section{Lösung der Aufgabe 1:}

Tabelle 19: Schüler*innenlösung 2

1

\begin{tabular}{|l|l|l|}
\hline & England & Deutschland \\
\hline Vergleichsmerkmal & & \\
\hline $\begin{array}{l}\text { Beginn } \\
\quad . .\end{array}$ & Ende 18. Jhd. & im Verlauf des 19. Jhd. \\
-Landwirtschaft & um 18 Jhd. & noch keine \\
\hline
\end{tabular}

teh
Meinung $\rightarrow$ Ich finde den Text zu „zäh“

und verstehe nicht wirklich das

Prinzip dieser Tabelle! 


\section{Lösung der Aufgabe 2:}

2.) England war quasi der Vorreiter der

Industrialisierung. Besonders durch die Landwirtschaft

und Erfindungen wie z.B. die Dampfmaschine oder

5 auch die Spinnmaschinen waren mitunter eine Grund für das Wirtschaftswachstums England's. Dazu kommt noch die Verbilligung von Eisen hinzu, was ermöglichte, das man z.B. Züge bauen konnte und somit das Reisen ermöglicht hatte.

10

Deutschland war ein Nachzügler, da Deutschland zu diesem Zeitpunkt in mehrere Teile zerteilt gewesen ist und durch verschiedenen $u$. unterschiedliche Währungs- und Gewichtssysteme hatte. Dementsprechend lebten die Menschen von ihren Erträgen der Landwirtschaft. Zusätzlich mussten die Bauern nebenbei noch arbeiten gehen, um ihre Existenz zu sichern. 


\subsubsection{Analyse der formalen Textstruktur}

Zwar werden in dieser Vergleichslösung beide Aufgaben bearbeitet, der erste Teil jedoch nur fragmentarisch.

Da im Gegensatz zur ersten Schülerlösung eine Lösung der Vergleichsaufgabe vorliegt, steht im Folgenden die Bearbeitung der Aufgabe 2 im Fokus. Im Verlauf der Analyse wird dabei auch Bezug auf die voranstehende Tabelle genommen.

Mit einer Textlänge von 99 Wörtern kann die Bearbeitung der Hauptaufgabe (Aufgabe 2) insbesondere in Bezug auf die Vielzahl der Informationen und der Länge des Darstellungstextes als kurz bezeichnet werden. Die mittlere Textlänge liegt für diesen Text bei 16,5 Wörtern pro Satz. Unter Ausschluss der Korpus-Texte, für die keine mittlere Satzlänge errechnet werden konnte, liegt der Mittelwert für die mittlere Satzlänge in diesem Korpus bei 17,84. Der vorliegende Text liegt damit etwas unter der durchschnittlichen Satzlänge, aber nahe der von Hoffmann ermittelten durchschnittlichen Satzlänge von 15,9 in wissenschaftlichen Texten. ${ }^{731}$

Eine Strukturierung in Einleitung, Hauptteil und Schluss ist nur teilweise erkennbar, ein Textabschluss fehlt, ebenso eine Überschrift. Der Text ist in zwei Abschnitte gegliedert, die sich auf jeweils ein Vergleichsobjekt fokussieren. Um welches Vergleichsobjekt es dabei geht, wird in der Einleitung gekennzeichnet.

\subsubsection{Vergleichsspezifische thematische Inhalte und inhaltlich-thematischer Fokus}

Eine zentrale These (entspr. Vergleichskompetenzstufe II) wird nicht vollständig formuliert. Zu Beginn jedes Abschnitts wird für jeden Vergleichsaspekt eine Beurteilung vorgenommen, diese aber weder zu Anfang noch am Ende zusammengeführt. Es wird aber grundsätzlich erkennbar, dass England eine Vorreiterrolle zugesprochen wird, während Deutschland als Nachzügler eingeordnet wird. Diese Zuordnung entspricht der Beurteilung durch den Darstellungstext. Da diese Zuordnungen in jedem Abschnitt an erster einleitender Stelle auftreten, erscheinen sie als grundlegend für den Vergleich; sie entsprechen einem Mittel zum Themensplitting entspr. Vergleichskompetenzstufe I.

Nach dieser grundsätzlichen Einordnung werden Wissenselemente aus dem Darstellungstext in den Vergleichstext überführt (entspr. Vergleichskompetenzstufe I: Nennung von wesentlichen historischinhaltlichen Vergleichsaspekten), die die Funktion haben, die Grundannahmen zu belegen. So werden als Beleg für die Vorreiterrolle Englands Landwirtschaft und Erfindungen genannt, die zum

\footnotetext{
${ }^{731}$ Vgl. Hoffmann, Lothar (1998): Syntaktische und morphologische Eigenschaften von Fachsprachen. In: Hoffmann, Lothar; Kalverkämper, Hartwig; Wiegand, Herbert Ernst (Hrsg.): Fachsprachen / Languages for Special Purposes. Ein internationales Handbuch zur Fachsprachenforschung und Terminologiewissenschaft / An International Handbook of Special-Language and Terminology Research. Berlin: de Gruyter. S. 416-427. S. 417.
} 
Wirtschaftswachstum Englands geführt haben. Warum jedoch Landwirtschaft und Erfindungen die Entwicklung der Industriellen Revolution gefördert haben, bleibt ungesagt. Weiterhin wird die Verbilligung des Eisens genannt, wodurch es möglich wurde, Züge zu bauen und in der Folge zu reisen. Als Beleg für die Nachzüglerrolle Deutschlands wird durch den Hinweis darauf, dass Deutschland zu diesem Zeitpunkt in mehrere Teile zerteilt war, Bezug auf die politisch-geographische Situation genommen. Der Hinweis auf die territoriale Zersplitterung wird gleichrangig durch ein ,und' verbunden mit den unterschiedlichen Währungs- und Gewichtssystemen, die streng genommen eher als eine Auswirkung der Zersplitterung zugordnet werden müssen. Der Text verbleibt in Bezug auf die geographische Situation bei dem alltagssprachlichen Hinweis darauf, dass Deutschland in "mehrere Teile“ zersplittert gewesen sei, der Verweis auf "mehrere Teile“ wird jedoch nicht in das dahinterstehende Fachkonzept überführt. Es wird nicht benannt, was für eine Art von Teilen gemeint ist.

Als Folge schließt sich inhaltlich der Hinweis darauf an, dass die Menschen nicht von den Erträgen der Landwirtschaft leben konnten. Durch die fehlende Kontextualisierung wird für den Leser jedoch nicht ersichtlich, inwiefern das Leben von der Landwirtschaft ein Hinderungsgrund für den Beginn der Industrialisierung darstellte. Die Darstellung schließt mit dem Satz: „Zusätzlich mussten die Bauern nebenbei noch arbeiten gehen, um ihre Existenz zu sichern." In der alltagssprachlichen Wendung „nebenbei arbeiten gehen“ verbirgt sich ein Konzept der Gegenwart, nämlich die Ausführung einer Nebentätigkeit, sie verbirgt zudem, dass landwirtschaftliche Arbeit eben auch als (stark körperlich belastende) Arbeit eingeordnet werden muss.

Die den Beginn der Industrialisierung Deutschlands als verzögernd genannten Aspekte stehen nicht im direkten Zusammenhang zu den für die englische Industrialisierung als förderlich herausgestellten Elementen (entspricht Vergleichskompetenzstufe I: Aufnahme der Grundelemente des Vergleichs und Einbettung inhaltlicher Vergleichselemente, aber noch nicht kriteriengeleitet oder problemorientiert). Da ein Textabschluss fehlt, werden sie auch nicht am Ende zusammengeführt.

\subsubsection{Realisierung sprachlicher Teilhandlungen}

Die für das historische Vergleichen wesentliche Teilhandlung des Gegenüberstellens (Vergleichskompetenzstufe I) wird insbesondere optisch über die Einteilung in zwei Abschnitte dargestellt. Die die Abschnitte einleitenden Sätze nehmen jeweils ein Element des Gegensatzpaares Deutschland/England auf und kennzeichnen darüber das Gegenüberstellen. Im Weiteren stehen dann die Abschnitte inhaltlich jeweils für sich. Weitere sprachliche Kennzeichnungen des Gegenüberstellens über das genannte Gegensatzpaar hinaus können nicht ausgemacht werden. Eine eindeutige argumentative Gewichtung der ausgewählten Inhalte entspr. Vergleichskompetenzstufe II kann nur in Ansätzen festgestellt werden. So werden zwar für die englische Entwicklung Landwirtschaft und Erfindungen durch die Intensitätspartikel besonders als maßgeblich herausgestellt, die Begriffe gehen als Argumente aber insofern verloren, als dass sie in eine Reihung überführt werden, statt sie als Begründung nutzbar zu machen. Gerade dieser Zusammenhang führt im Weiteren dazu, dass 
Begründungsstrukturen nur in Ansätzen ausgemacht werden können, die aber nicht als strukturierte Begründung zu Ende geführt werden.

Dies gilt ebenso für Erklär-Strukturen. Ähnlich wie beim Begründen werden sie vollständig beendet, wie das Beispiel zeigt: „Zusätzlich mussten die Bauern nebenbei noch arbeiten gehen, um ihre Existenz zu sichern." (Z. 16f) Der Konnektor um stellt zwar eine Erklär-Struktur sprachlich her (entspr. des Manuals: Aufklärung einer Warum-Frage), die Ausgangsfrage, warum die Bauern nicht von ihrer Landwirtschaft leben konnten, wird jedoch nicht thematisiert. Es bleibt auch offen, warum dieser Aspekt im Sinne einer Nutzung als Begründung die Nachzüglerrolle Deutschlands unterstützen soll (an dieser Stelle wird die fachliche Richtigkeit nicht weiter hinterfragt, weil es um die Analyse der Teilhandlungen geht).

Insgesamt fällt auf, dass die ausgewählten inhaltlichen Elemente weniger gegeneinander abgewogen als vielmehr gleichgesetzt werden. Dieser reihende Charakter wird gestärkt durch Adverbien wie dazu, dementsprechend oder zusätzlich.

Eine Gesamtbeurteilung im Sinne eines Sachurteils (entspr. Vergleichskompetenzstufe III) fehlt. Die jeweiligen Vergleichsobjekte werden lediglich für sich beurteilt, aber nur indirekt über das Gegensatzpaar Vorreiter/Nachzügler in einen Zusammenhang gebracht.

Insgesamt können Ansätze für die Teilhandlungen des historischen Vergleichens festgestellt werden, diese werden jedoch nicht strukturiert zu Ende geführt. Dies kann auf inhaltliche Unklarheiten auf der Grundlage des Darstellungstextes zurückzuführen sein oder darauf, dass explizites Wissen über die Realisierung eines historischen Vergleichs fehlt. Aufgrund des Metakommentars in der Tabelle muss von einer Mischung aus beidem ausgegangen werden.

\subsubsection{Wissenschaftliche Textkompetenz}

Bezugnahmen auf den Darstellungstext werden auch in dieser Bearbeitung nicht vorgenommen. Dabei sind die im Text aufgenommenen Einzelaspekte durchgängig dem Ursprungstext zuzuordnen, ebenso die Zuschreibung Englands als Vorreiter und Deutschlands als Nachzügler. Korrespondierend zur fehlenden Kennzeichnung ist im Text nur eine Modalisierung erkennbar. Eine Distanzierung vom grundlegenden Darstellungstext ist insgesamt kaum erkennbar.

Das Themensplitting beschränkt sich wie in Text 1 auf die Nennung des Gegensatzpaares England/Deutschland, wird allerdings im Unterschied zu Text 1 durch die Übernahme der Zuschreibungen "Vorreiter" und "Nachzügler" sowie optisch durch die Strukturierung in zwei deutlich voneinander getrennten Absätzen gekennzeichnet.

Im Unterschied zur Aufgabenbearbeitung als Tabelle, wie in Text 1, bietet der Fließtext in dieser Aufgabenbearbeitung Elemente der Themenfortführung, wie insbesondere die Verweisstruktur über „ihre“, aber auch Verweise über „dazu“ und „was". Solche Strukturen werden zwar nicht durchgängig geleistet, wie etwa die Wiederholung „Deutschland war ein Nachzügler, da Deutschland [...]“ in Zeile 11 zeigt, sie verweisen aber auf die gedankliche Verknüpfung von Wissenselementen und sind deshalb 
elementare Strukturen wissenschaftlicher Textkompetenz. Insbesondere im Vergleich von Text 1 und 2 zeigt sich die Bedeutung eines Fließtextes, da gerade die Formulierung einzelner Aspekte in einem zusammenhängenden Text zu solchen Strukturen der Themenfortführung anregt. Obwohl der vorliegende Schülertext sich oft alltagssprachlicher Wendungen bedient, kommt er nicht ohne Verweisstrukturen aus.

Wie schon in Text 1 ist auch in diesem Text eine Personalisierung Handelnder kaum gegeben, am ehesten findet sie sich im Begriff „Bauern“ wieder. Im Gegensatz dazu fällt auf, dass England und Deutschland durch die aktivischen Formulierungen „England war quasi der Vorreiter [...]“ (Z. 2 f) und „Deutschland war ein Nachzügler" (Z. 11) personalisiert werden. England und Deutschland werden hier vermutlich als Nationen verstanden (auch wenn dies insbesondere für Deutschland so nicht zutrifft) und darüber wie Personen behandelt.

Insgesamt finden sich auch in diesem Text nur wenige Merkmale (vor-)wissenschaftlicher Schreibkompetenz, obwohl es sich um einen Fließtext handelt, der viel stärker solche Formen einfordert als etwa eine Tabelle.

\subsubsection{Holistische Einordnung des Textes}

Wie schon in Text 1 zeigt auch die Aufgabenbearbeitung in Text 2, dass der Schüler nur einige historisch-inhaltliche Aspekte aus dem Darstellungstext auswählt und für den Vergleichstext nutzbar macht. Auffällig ist insbesondere die nur in Ansätzen oder nur teilweise geleistete Realisierung für das Vergleichen wichtiger sprachlicher Teilhandlungen entsprechend der Vergleichskompetenzstufen I und II, die die Vermutung nahelegen, dass dem Lernenden nicht immer bewusst ist, welche er für die Realisierung des Vergleichs überhaupt benötigt. Da die sprachlichen Teilhandlungen, die sich an der Textoberfläche zumindest in Ansätzen erkennen lassen, nicht ausgeführt werden, muss darüber hinaus davon ausgegangen werden, dass diese eher intuitiv benutzt werden, aber nicht als Strategiewissen vorliegen.

Besonders spannend ist in diesem Zusammenhang der Metakommentar, der durch den Ausdruck "Meinung" und einen Pfeil besonders kenntlich gemacht wird, bevor die eigentliche Meinung dann formuliert wird. ${ }^{732}$ Der Hinweis „Ich finde den Text zu ,zäh““, deutlich erkennbar positioniert auf der Tabelle auf dem Deckblatt, zeigt, dass der Schüler dem Leser (und damit dem/der die Erhebung Auswertenden) seine Erfahrungen mit dem Text auf den Weg geben will. Der Hinweis, dass das Prinzip der Tabelle nicht klar werde, zeigt, dass die Tabelle nicht als Möglichkeit der Strukturierung im Sinne einer Kategorienbildung erfasst wird. Auch dies ist ein deutlicher Hinweis sowohl auf fehlendes explizites Wissen im Umgang mit der Tabelle als Mittel der Gegenüberstellung als auch auf fehlendes Wissen um den Zweck der Tabelle. Gerade dieser Metakommentar ist als eine Art kurzer Erfahrungsbericht im Zusammenhang mit der Textauswertung ein wichtiger Hinweis für eine Didaktisierung der historischen

\footnotetext{
${ }^{732}$ Mit Ehlich kann ein solcher Kommentar als Abduktion beschrieben werden. Vgl. dazu Ehlich, Konrad (1981): Schulischer Diskurs als Dialog? In: Schröder, Peter; Steger, Hugo (Hrsg.): Dialogforschung. Düsseldorf: Schwann, S. 334-369. S. 362.
} 
Vergleichsaufgabe im Unterricht, da er zeigt, an welchen Stellen der Schüler Unterstützung einfordert. Er ist als Aufforderung für die Konzeption von Unterrichtsmaterial einzuordnen.

Im Sinne des durch die Faktorenanalyse erhobenen Kompetenzmodells ist dieser zweite Schülertext ebenfalls in die Kompetenzstufe I (Aufnahme der Grundelemente des Vergleichs und Einbettung inhaltlicher Vergleichselemente) einzuordnen. Die Kompetenzstufe II (Anlage eines problembezogenen und kriterienorientierten Vergleichs) wird auch hier kaum erreicht, obwohl der Text im Rahmen der Erhebung eine auf alle Texte bezogene mittlere Gesamtpunktzahl erreicht.

Im Sinne einer Erfüllung der Merkmale der Kompetenzstufe I werden insbesondere die Vergleichsobjekte Deutschland und England genannt, ein Gegenüberstellen sowie ein Themensplitting zumindest grundlegend angelegt und in einem Hauptteil die Vergleichsobjekte strukturiert aufgenommen. Darüber hinaus werden im Sinne der Anlage eines problembezogenen und kriterienorientierten Vergleichs Ansätze des Erklärens zumindest zum Teil durch sprachliche Mittel gekennzeichnet.

Die erreichte Gesamtpunktzahl des Textes in Bezug auf die Zuordnung in das Kompetenzmodell lässt darauf schließen, dass Text und Aufgabenbearbeitung selbst für Lernende in der Sekundarstufe II, die sich auf eine fachgebundene oder allgemeine Hochschulreife vorbereiten, zu schwierig sind oder das historische Vergleichen zu wenig expliziert ist. 


\subsection{Text 3: Der Text mit der höchsten Gesamtpunktzahl}

Der dritte ausgewählte Text hat die höchste Gesamtpunktzahl erreicht. Er ist der Erhebung 2 zuzuordnen (Veränderung des authentischen Darstellungstextes in jeweils einen Text zu England und Deutschland). Im Ursprungstext vorhandene Vergleichselemente wurden entfernt. Eine Tabelle zur Vorstrukturierung der Aufgabe war nicht gefordert, wird dennoch durch den Schüler geleistet ${ }^{733}$.

\section{Lösung der Aufgabe:}

Tabelle 20: Schüler*innenlösung 3

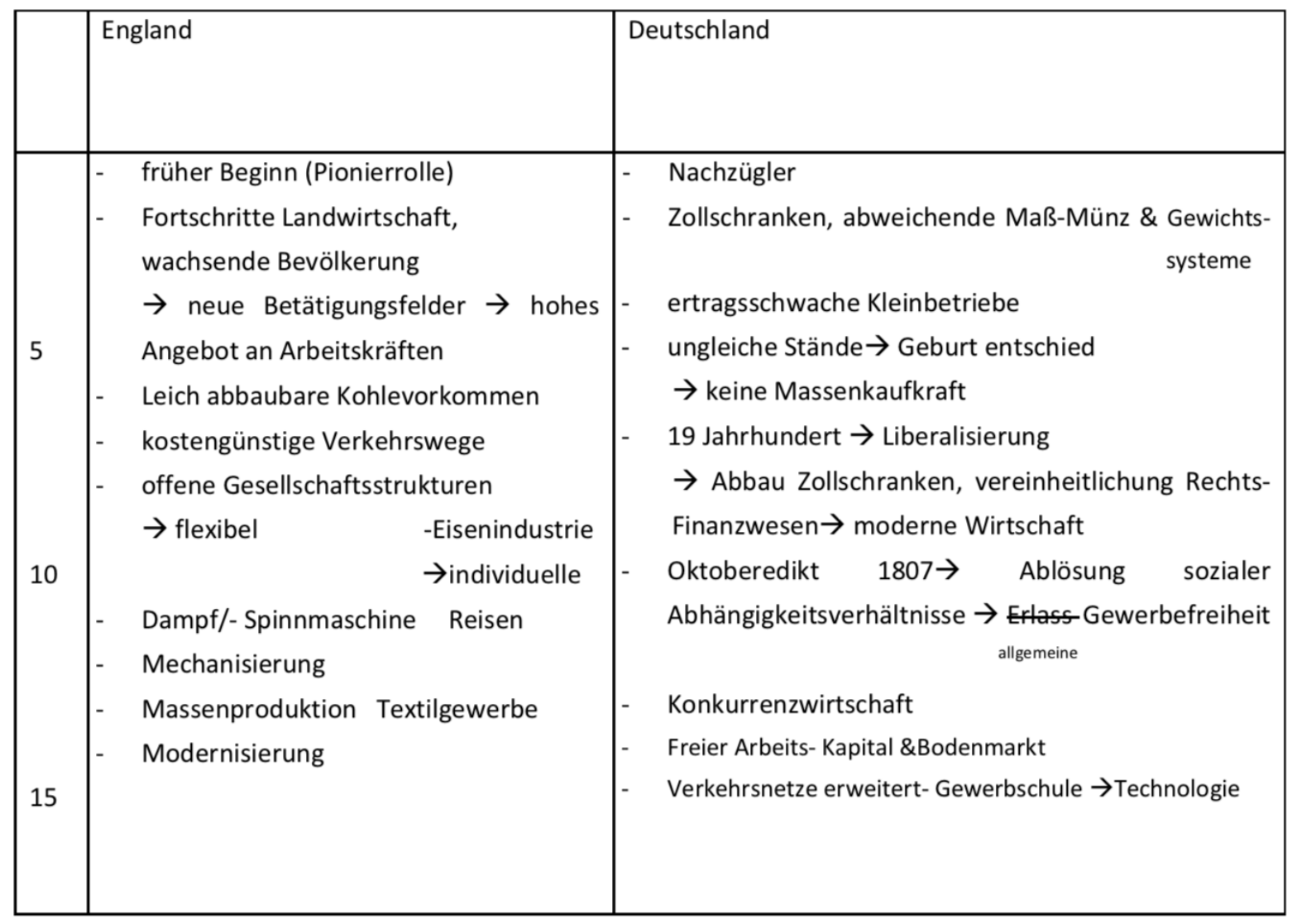

${ }^{733}$ Die Tabelle befindet sich auf dem Aufgabenblatt, die eigentliche Aufgabenlösung auf den beigefügten leeren Seiten. Während die Aufgabenlösung mit einem wasserfesten Stift bearbeitet wurde, wurde die Tabelle mit einem Bleistift verschriftlicht. Sowohl Anordnung als auch Stiftwahl zeigen den Vorläufigkeitscharakter der Tabelle: Sie kann jederzeit verändert werden, da der Bleistift radierbar ist und Veränderungen zulässt. 
Industrialisierung in Deutschland und England

Die zwei Texte handeln von der Industrialisierung in Deutsch-

5 land und England im ausgehenden 18. und beginnenden 19. Jahrhundert.

Die Ausgangsbedingungen für die Prozesse der Industrialisierung waren in Deutschland und England ziemlich gegensätzlich.

In England waren die Voraussetzungen gut, in Deutschland jedoch eher schlecht. Dazu trugen verschiedenste Faktoren bei, die alle ausschlaggebend für die einzelnen Prozesse waren.

England hatte die „Pionierrolle“ und Deutschland die der „Nachzügler". In Deutschland waren die Stände sehr ungleich, man wurde in seine soziale Situation hineingeboren, dies sorgte z.B. dafür, dass es viele Bauern gab, die an ihre Gutsheere gebunden waren und somit die Bildung freier Arbeitsmärkte eingeschränkt war. Dies gab es in England nicht. Die Landwirtschaft schritt fort, die Bevölkerung wuchs, wodurch dass Angebot an Arbeitskräften stieg. Die Kohle war leicht abbaubar und die Kosten der Verkehrswege günstig, diese Faktoren wirkten auch fördernd für die Industrialisierung. In Deutschland hingegen gab es Zollschranken, abweichende Maß- , Münz und Gewichtssysteme und das Verkehrsnetz war nicht ausgebaut, dies wirkte natürlich hemmend

25 für die Prozesse der Industrialisierung. England entfaltete sich im Gegensatz immer schneller, es gab eine offene Gesellschaft, die 
für Flexibilität sorgte. Dampf- und Spinnmaschinen wurden erfunden, die Mechanisierung trat ein, so wie auch die Massenproduktion im Textilgewerbe. Die Eisenindustrie ermöglichte noch schnelle und bessere Verkehrswege und individuelle Reisen. Diese Faktoren haben alle so fördernd gewirkt, dass England schnell modernisiert bzw. industrialisiert war. In Deutschland begann erstmals die Modernisierung, durch die Liberalisierung im 19. Jahrhundert, dort wurden nämlich Zollschranken abgebaut und das Rechtsfinanzwesen vereinheitlicht.

Dies verstärkte den Prozess zur modernen Wirtschaft. Mit dem Eintreten der mobilen Marktgesellschaft wurden soziale Abhängigkeitsverhältnisse abgeschafft. Nun gab es auch eine allgemeine Gewerbefreiheit, die zu einer Art Konkurrenzwirtschaft führte. Weitergehend gab es freie Arbeits- Kapitals und Bodenmärkte, die die Prozesse enorm beschleunigt haben. Die Verbindung einzelner Wirtschaftsfaktoren konnte nun stattfinden. Verkehrsnetze wurde des weiteren ausgebaut und aufgrund der zurückhängender Technologie wurden Gewerbschulen und Akademien errichtet. Somit war Deutschland letztendlich auch modernisiert.

Insgesamt lässt sich also sagen, dass in England die Industrialisierung schneller stattfinden konnte, da die Gegebenheiten besser waren. Durch die Hindernisse in Deutschland hat alles etwas länger gedauert, doch letzten Endes hat auch dort die Industrialisierung erfolgreich stattgefunden, so dass England und Deutschland auf einem ähnlich modernen Stand waren. 


\subsubsection{Analyse der formalen Textstruktur}

Obwohl nicht gefordert, stellt der Schüler der Aufgabenbearbeitung eine Gegenüberstellung mit Hilfe einer Tabelle voran. Nur in zwei weiteren Lernendentexten wird ein Mittel zur Vorstrukturierung genutzt, ohne dass wie in den Aufgabenstellungen des Erhebungsteils 3 explizit danach gefragt wurde. Es fällt jedoch auf, dass alle drei Texte, die ein Mittel zur Vorstrukturierung nutzen, der Erhebung 4 zugehörig sind. Hier ist zu vermuten, dass die Lernenden entweder gelernt haben, ihre Texte vor der Ausformulierung vorzubereiten und zu planen, oder die durchführende Lehrkraft einen entsprechenden Hinweis gegeben hat.

Mit einer Textlänge von 377 Wörtern kann die Bearbeitung der Aufgabe (ohne Tabelle) als lang angesehen werden (der längste Text beinhaltet 486 Wörter, nur 4 Texte sind länger als der vorliegende Text 4_24). Die mittlere Satzlänge kann mit 16,4 Wörtern pro Satz angegeben werden und liegt dabei fast ebenso hoch wie in Text 2.

Der Text weist eine Strukturierung in Einleitung, Hauptteil und Schluss auf (entspricht den Vergleichskompetenzstufen I und III durch die Formulierung eines Schlusses), dem Text wird zudem eine eigenständige Überschrift vorangestellt, wenngleich diese noch nicht auf die Textsorte Vergleich vorausweist.

8.3.2 Vergleichsspezifische thematische Inhalte und inhaltlich-thematischer Fokus

Nach einer Einleitung, die auf die Darstellungstexte hinweist, welche die Grundlage für den Vergleich bilden, wird entsprechend der Vergleichskompetenzstufe II eine zentrale These formuliert („Die Ausgangsbedingungen für die Prozesse der Industrialisierung waren in Deutschland und England ziemlich gegensätzlich. In England waren die Voraussetzungen gut, in Deutschland jedoch eher schlecht.", Z. 7ff). Auch wenn die Einordnung pauschal ausfällt, wird der anschließende Vergleich der Ausgangsbedingungen vorbereitet, indem daran anschließend allgemein auf verschiedene „Faktoren“ hingewiesen wird, von denen der Leser nun ausgehen kann, dass sie im Text aufgegriffen und ausgeführt werden.

Bevor der Schüler nun die in der Tabelle aufgeführten Wissenselemente aufnimmt und in eine ausformulierte Gegenüberstellung überführt, übernimmt er im Sinne eines Themensplittings (Vergleichskompetenzstufe I) aus dem Darstellungstext die Zuschreibungen „Pionierrolle“ für England und „Nachzügler" für Deutschland, welche in den beiden Darstellungstexten in der Einleitung vorgenommen werden. Sie erhalten im Lernendentext die Funktion, den gegensätzlichen Elementen Konzepte zuzuweisen.

Nach dieser Einführung nimmt der Schüler nun einzelne Wissenselemente aus dem Darstellungstext auf. Im Gegensatz zu den vorigen Texten führt er jeweils die Situation in England und Deutschland zusammen und stellt sie explizit gegenüber, z. B.: 
„In Deutschland waren die Stände sehr ungleich, man wurde in seine

soziale Situation hineingeboren, dies sorgte z.B. dafür, dass es viele

Bauern gab, die an ihre Gutsheere gebunden waren und somit die Bildung

freier Arbeitsmärkte eingeschränkt war. Dies gab es in England nicht.“ (Z. 15-18)

Nach der Kennzeichnung der gesellschaftlichen Situation in Deutschland und ihrer Auswirkungen auf den Arbeitsmarkt fügt er die Bemerkung an „Dies gab es in England so nicht.“ und stellt über den Verweis "dies" und die Verneinung den Gegensatz her.

Inhaltlich werden unterschiedliche historische Konzepte sehr verkürzt miteinander verknüpft. Dabei sind die gewählten Verknüpfungen nur teilweise zutreffend. So wird das Ständesystem in Verbindung mit der sozialen Situation gebracht, in die man hineingeboren wird. Dieser Zusammenhang erschließt sich nur über die Kenntnis des Ständesystems und kennzeichnet es nur zum Teil, da man auch heute in eine bestimmte soziale Situation hineingeboren wird, aber eben mit unterschiedlichen Konsequenzen, die durch diesen knappen Hinweis nicht deutlich werden. Das Beispiel zur Veranschaulichung geht davon aus, dass es viele Bauern gab, die an ihren Gutsherrn gebunden waren. Hier entsteht zunächst ein Missverständnis durch den Begriff "Gutsheere“. Durch die korrekte grammatikalische Einbindung in den Satz kann nicht nur von einem Orthographiefehler ausgegangen werden. Es scheint das Konzept von Gutsherrschaft nicht klar zu sein. Diese These passt auch zur unklaren Verknüpfung mit der Bildung freier Arbeitsmärkte. Dieser Zusammenhang kann aus der Folgerung durch „somit“ nicht erschlossen werden. Der Hinweis des Darstellungstextes auf die aus der Gutsherrschaft resultierende fehlende Mobilität wird ausgelassen.

Im Anschluss an den Hinweis, dass es solche Strukturen in England nicht gab, wird die Situation in England ausgeführt:

„Die Landwirtschaft schritt fort, die Bevölkerung wuchs, wodurch dass

Angebot an Arbeitskräften stieg. Die Kohle war leicht abbaubar und

die Kosten der Verkehrswege günstig, diese Faktoren wirkten auch

fördernd für die Industrialisierung." (Z. 19-22)

Eine weitere Kennzeichnung, dass nun die Situation in England aufgenommen wird, erfolgt nicht, erschließt sich aber durch den Zusammenhang.

Die Darstellung beginnt mit dem fachunspezifischen Hinweis auf das Fortschreiten der Landwirtschaft. Vermutlich ist damit der Fortschritt der Landwirtschaft gemeint, es fehlen jedoch Hinweise, woraus dieser sich ergab. Die genannten Faktoren werden sowohl als Reihung als auch zum Teil als Folge aneinandergefügt, wie z. B.: „Die Landwirtschaft schritt fort, die Bevölkerung wuchs, wodurch dass Angebot an Arbeitskräften stieg." Es wird jedoch nicht geklärt, 
inwiefern das Fortschreiten (der Fortschritt?) der Landwirtschaft Einfluss auf das Angebot an Arbeitskräften hat.

Bereits an dieser Stelle zeigt sich, dass der Schüler die in der Tabelle aufgeschlüsselten Faktoren in seinem Text abarbeitet. Obwohl, wie dargestellt, die genannten Faktoren nicht immer in einen fachgerechten Zusammenhang gebracht werden (dabei muss auch berücksichtigt werden, welche hohen Anforderungen der Darstellungstext an die Leserschaft stellt; erst recht bei einer Übertragung der Inhalte in ein anderes Textmuster wie den Vergleich), stellt der Schüler darüber die Vergleichsobjekte in einen engen Zusammenhang. Wie in einem Ping-Pong-Spiel wendet er sich von einem Objekt zum anderen und erreicht dadurch ein strukturiertes Gegenüberstellen. Nachdem er zunächst Gesellschaftsordnung und Agrarstrukturen für Deutschland dargestellt hat, greift er diese Aspekte nun in Bezug auf England auf und fügt die Aspekte Kohleabbau und Verkehrswege an. Über den adversativen Konjunktor „hingegen“ wechselt er nun wieder zur Situation in Deutschland. Diese Struktur hält er im Hauptteil durch, bevor er im Schluss die Einzelaspekte zusammenführt. Dies entspricht sowohl dem in der Vergleichskompetenzstufe I angesiedelten fachlichen Gegenüberstellen als auch der Verwendung zugehöriger expliziter sprachlicher Mittel.

Die Situation in Deutschland wird durch die Reihung weiterer Einzelfaktoren ergänzt:

„In Deutschland hingegen gab es Zoll-

schranken, abweichende Maß-, Münz und Gewichtssysteme und

das Verkehrsnetz war nicht ausgebaut, dies wirkte natürlich hemmend

für die Prozesse der Industrialisierung." (Z. 22-25)

In einer Art Behauptung werden die Einzelfaktoren als „natürlich hemmend“ beschrieben, ohne dass erklärt wird, warum dies so war. Die Modalpartikel „natürlich“ hat dabei die Funktion, den zuvor angeführten Sachverhalt als Tatsache zu kennzeichnen ${ }^{734}$ und so eine fachgerechte Erklärung des Zusammenhangs zu umgehen.

Der sich anschließende Wechsel des Blickwinkels von Deutschland zu England wird wieder sprachlich gekennzeichnet: „England entfaltete sich im Gegensatz immer schneller, [...].“ (Z. 25f), dann folgt eine Begründung über Englands offene Gesellschaft, die für Flexibilität sorgte. Damit wird der eingangs angeführte Faktor Gesellschaft wiederaufgenommen. Die an diesen Hinweis angeschlossene Reihung „Dampf- und Spinnmaschinen wurden erfunden, die Mechanisierung trat ein, so wie auch die Massenproduktion im Textilgewerbe.“ (Z. 27ff) kann der Leser auf die Flexibilität als Auslöser zurückführen, eine sprachliche Kennzeichnung gibt es jedoch nicht. Nach einem Hinweis auf die Bedeutung der Eisenindustrie werden die Einzelfaktoren in eine Gesamteinschätzung für Englands Beginn der Industrialisierung überführt: „Diese Faktoren haben alle so fördernd gewirkt, dass England schnell modernisiert bzw. industrialisiert war." ( $Z$.

${ }^{734}$ Vgl. dazu Hoffmann, Ludger (2014), S. 406. 
30ff). Zwar hält sich der Schüler durchgehend eng an die Textvorlage und arbeitet einzelne Faktoren oft in einem verkürzten Zusammenhang ab, insgesamt gelingt es ihm aber, die Vergleichsobjekte strukturiert gegenüberzustellen. Das schafft er insbesondere über die sprachliche Kennzeichnung des Gegenüberstellens und damit über eine eindeutige Leserführung.

Im Anschluss an die Gegenüberstellung der Situation zu Beginn der Industrialisierung in England und Deutschland fügt der Schüler Informationen über die Weiterentwicklung in Deutschland an. Er orientiert sich damit am Darstellungstext und arbeitet entsprechend dieser Vorlage den letzten Textteil mit dem Titel "Staatliche Reformen“ der Vorlage ab. Im Vergleich zu anderen Korpustexten bearbeitet der Schüler diesen Teil sehr ausführlich und nennt viele unterschiedliche Faktoren, welche die Entwicklung in Deutschland kennzeichnen und die Industrialisierung vorangetrieben haben. Die Darstellung zeigt die sehr enge Orientierung am Darstellungstext, wie das folgende Beispiel zeigt: „Mit dem Eintreten der mobilen Marktgesellschaft wurden soziale Abhängigkeitsverhältnisse abgeschafft.“ (Z. 36ff) Im Ursprungstext heißt es: „An die Stelle einer gebundenen Ständegesellschaft, die jedem Menschen eine feste, durch Geburt und Recht erworbene soziale Position zuwies, sollte nun eine mobile Marktgesellschaft treten. ${ }^{7735}$ Der Vergleich beider Textstellen zeigt ein Missverständnis in der Übernahme der Aussage des Darstellungstextes im Lernendentext, da Ursache und Wirkung vertauscht werden: Während im Lernendentext die mobile Marktgesellschaft Ursache für die Abschaffung sozialer Abhängigkeitsverhältnisse ist, geht der Darstellungstext davon aus, dass die Ständegesellschaft durch eine mobile Marktgesellschaft ersetzt werden sollte, ein viel aktivischer und damit gewollter oder sogar gesteuerter Vorgang. Auffallend ist die Nominalisierung „Eintreten“, die sich kaum in die Gesamtaussage einpasst, weil das dahinterstehende Konzept unklar bleibt. Die Textbeispiele zeigen, dass insbesondere der Umgang mit fachlichen Verben eine hohe Anforderung an Lernende stellt, da das jeweils dahinterstehende Fachkonzept erschlossen werden muss, sei es als Verb, als Nominalisierung oder auch als Adjektivierung. Diesen Textabschnitt, der für die Lösung der Vergleichsaufgabe nicht Voraussetzung ist, sie aber ergänzen kann, wird durch ein Fazit abgeschlossen: „Somit war Deutschland letztendlich auch modernisiert.“ (Z. 44f) Der Schüler bildet ein (Teil-)Urteil und bindet so die Darstellung der weiteren Entwicklung Deutschlands an den eigentlichen Vergleich an. Er schafft darüber erneut Transparenz im Sinne der Leserführung.

Durch einen Absatz und einen größeren Zeilenabstand zum vorhergehenden Textteil auch visuell gekennzeichnet, fügt sich nun der Vergleichsabschluss für den gesamten Text an. Der Schüler formuliert ein abschließendes Sachurteil (vgl. Vergleichskompetenzstufe III). Zwar pauschalisiert er an mehreren Stellen, grundsätzlich scheint er aber mit der Sachurteilsbildung als Vergleichsabschluss vertraut zu sein und kann diese auch sprachlich kennzeichnen (,Insgesamt lässt sich also sagen [...].", Z. 46).

\footnotetext{
735 Geänderte Textversion der Erhebung 2. Das Zitat ist unverändert aus dem unveränderten Darstellungstext nach Laschweski-Müller; Rauh (2010) übernommen: nach Laschweski-Müller; Rauh (2010).
} 


\subsubsection{Realisierung sprachlicher Teilhandlungen}

In Kapitel 8.3.2 konnte bereits über den Inhalt des Lernendentextes gezeigt werden, dass der Schüler explizite sprachliche Mittel nutzt, um das Gegenüberstellen im Fließtext zu markieren. Zwar werden fachlich-inhaltliche Aspekte nicht immer in einen eindeutigen und korrekten Zusammenhang gestellt, dennoch kann der Leser nachvollziehen, inwiefern sich die Voraussetzungen in England und Deutschland zu Beginn der Industriellen Revolution unterschieden. Dieser Zusammenhang zeigt die besondere Bedeutung der Kenntnis für das historische Vergleichen wichtiger sprachlich-kognitiver Teilhandlungen.

Im Gegensatz zu den zuvor besprochenen Texten finden sich in diesem Text Begründe-Strukturen (entspr. Vergleichskompetenzstufe II), die historische Urteile nach sich ziehen bzw. vorbereiten. So wird etwa das abschließende Sachurteil durch eine knappe Begründung abgesichert, die zudem sprachlich (eingeleitet durch „da“; entspr. der übergeordneten sprachlichen Handlungskompetenz) als solche gekennzeichnet wird: „Insgesamt lässt sich also sagen, dass in England die Industrialisierung schneller stattfinden konnte, da die Gegebenheiten besser waren. Durch die Hindernisse in Deutschland hat alles etwas länger gedauert, doch letzten Endes hat auch dort die Industrialisierung erfolgreich stattgefunden, so dass England und Deutschland auf einem ähnlich modernen Stand waren." (Z. 47f) Zwar handelt es sich um eine pauschale Begründung, der Vergleich wird aber darüber zum Abschluss gebracht. Interessant erscheint dabei insbesondere der Bezug auf die weitere Entwicklung in Deutschland, die in den Vergleich einbezogen wird. Der Hinweis auf den „ähnlich modernen Stand“ ist dabei eine Schlussfolgerung des Schülers. Sie ist pauschal und nicht bspw. durch Erklärstrukturen oder Verweise auf den Darstellungstext abgesichert. Dies zeigt sehr deutlich, dass das verwendete Handwerkszeug des historischen Erklärens nicht sicher verwendet werden kann. Dennoch zeugen sowohl die verwendeten thematischen Inhalte wie auch die sprachlichen Teilhandlungen von grundsätzlichen Textsortenkenntnissen, die über das alltägliche Vergleichen hinausgehen und durchaus fachlich fundiert sind, wie der Abschluss durch ein Sachurteil zeigt.

Auch zur Vergleichskompetenzstufe II zugehörige Erklär-Strukturen sind in diesem Text zu finden, die auf die Frage „Warum“ antworten, sie werden aber nicht durchgängig sprachlich gekennzeichnet oder (wie in den Texten zuvor) nicht zu Ende geführt. Stattdessen weicht der Autor auf Reihungs- oder Folgestrukturen aus, ein Vorgehen, das sich schon in den zuvor besprochenen Texten gezeigt hat (z. B. „England entfaltete sich im Gegensatz immer schneller, es gab eine offene Gesellschaft, die für Flexibilität sorgte.“, Z. 25ff. Gerade diese Aspekte hätten mithilfe einer kausalen Struktur in einen Erklärungs-Zusammenhang überführt werden können). Hier kann angenommen werden, dass der Darstellungstext, der Zusammenhänge und Erklärungen bei einer Fülle unterschiedlicher Aspekte nur anreißt, eine Hürde schon im Leseverstehen und damit erst recht für die eigene Textproduktion darstellt.

Insgesamt sind in diesem Text die für das historische Vergleichen wesentlichen Teilhandlungen erkennbar. Im Sinne einer fachgerechten Nutzung als historische Werkzeuge des Vergleichens 
ist ihre Anwendung jedoch noch zu oberflächlich. Dies lässt vermuten, dass der zugrundeliegende Text (bzw. die Texte) zu schwer war (waren) und inhaltliche Kenntnisse fehlten.

\subsubsection{Wissenschaftliche Textkompetenz}

Auch in diesem Text finden sich nur wenige Kennzeichnungen des Ursprungstextes. Immerhin werden die Begriffe ,Pionierrolle' und ,Nachzügler' durch Anführungsstriche markiert. Sie sind also Übernahmen aus dem Darstellungstext, wenngleich darauf nicht explizit hingewiesen wird. Mittel zur Modalisierung werden zumindest zum Teil genutzt, beschränken sich aber auf die Nutzung des Modalverbs ,können“ (z. B. „[...] konnte nun stattfinden“ 'Z. 42) und des komplexen Prädikats , lassen ${ }^{176}$ (,[...]lässt sich also sagen“, Z. 47), das ein Zulassen realisiert, sowie die Modalisierung durch das Pronomen „etwas", das eine Unbestimmtheit herbeiführt. Das Modalverb „können“ ist in diesem Zusammenhang besonders interessant, weil es im Gegensatz zu den beiden anderen Varianten gleich zweimal auftaucht. Nach Redder ist ,können' der Gruppe der 'möglichkeitsbezogenen Modalverben ${ }^{1737}$ zuzurechnen: „[...] sie kennzeichnen das Ergebnis einer ,Einschätzung' [Hervorh. i. Orig.] von Bedingungen des Handlungsraums und den dadurch gegebenen Handlungsmöglichkeiten. ${ }^{\text {"738 }}$ Die Verwendung von ,können' im Lernendentext zeigt distanzierend die Möglichkeit, dass sich durch die Modernisierung Deutschlands die Industrialisierung entwickeln konnte. Zu bemerken ist insbesondere, dass im Fazit und damit in der abschließenden Urteilsbildung gleich zwei Modalisierungsmittel in einem Satz verwendet werden: „Insgesamt lässt sich also sagen, dass in England die Industrialisierung schneller stattfinden konnte, da [...] [Hervorh. d. Verf.]." (Z. 47f). Dem Schüler scheint also sehr bewusst zu sein, dass eine Beurteilung Widerspruch hervorrufen kann, und sichert entsprechend sein Urteil ab, indem er (gekennzeichnet durch das ,da') eine Begründung anfügt und mit Hilfe von Modalisierungsmitteln die Allgemeingültigkeit oder Absolutheit der eigenen Aussage zurücknimmt. Dies ist eine wichtige Kompetenz gerade für wissenschaftliches Schreiben. Im Sinne des historischen Arbeitens kennzeichnet er, dass ein solches Urteil eine Konstruktion darstellt, die ggf. durch das Hinzukommen anderen Wissens in Frage gestellt werden könnte.

Alle vier im Text vorkommenden Modalisierungsmittel sind auf das letzte Textdrittel konzentriert. Solange der Schüler Inhalte des Darstellungstextes übernimmt, fehlen solche Mittel.

Ein Themensplitting ist insbesondere über das Gegensatzpaar England/Deutschland erkennbar. Da im Textverlauf Strukturen des Gegenüberstellens sprachlich gekennzeichnet werden,

\footnotetext{
${ }^{736}$ Vgl. dazu: Zifonum, Gisela et al. (1997), Bd. 1. S. 705.

737 Vgl. Redder, Angelika (1986): Modalverben im Unterrichtsdiskurs. Pragmatik der Modalverben am Beispiel eines institutionellen Diskurses. Tübingen: Niemeyer Verlag. S.6f.

738 Ebd. S.7.
} 
erscheint die Beschränkung auf dieses eine Gegensatzpaar für die Lösung der Vergleichsaufgabe ausreichend.

Der Text bietet eine für die Textlänge relativ hohe Zahl an Mitteln zur Themenfortführung. Dabei handelt es sich insbesondere um Zeigwörter, die auf Personen oder Gegenstände verweisen, wie die oder dies oder die Anapher es, sowie um örtliche Verweise wie dort. Insgesamt kann eine durchgängige Verwendung von Verweisstrukturen festgestellt werden.

Eine Personalisierung Handelnder wird bis auf eine Ausnahme nicht vorgenommen. Im Gegensatz dazu werden die Länder geradezu personalisiert, wenn der Schüler formuliert: „England hatte die ,Pionierrolle‘ und Deutschland die der ,Nachzügler'.“ (Z. 13f)

Insgesamt finden sich in diesem Text mehr Merkmale (vor-)wissenschaftlicher Schreibkompetenz als in den Texten 1 und 2. Dennoch werden Übernahmen aus dem Darstellungstext insgesamt kaum gekennzeichnet, historische Werkzeuge nur punktuell angewandt, obwohl der Schüler gerade in Bezug auf die Formulierung seines Sachurteils zeigt, dass er Werkzeuge des (vor-)wissenschaftlichen historischen Arbeitens grundsätzlich kennt und anwenden kann.

\subsubsection{Holistische Einordnung des Textes}

Die Analyse zeigt, dass auch in dem Text, der beim Rating die meisten Punkte erhalten hat, Strukturen zur Realisierung eines historischen Vergleichs fehlen oder manchmal nur basal vorhanden sind. Um eine bessere Einschätzung der Gesamtleistung des Verfassers dieses Textes zu erhalten, soll auch dieser Text in das durch die Faktorenanalyse erhobene Kompetenzmodell eingeordnet werden:

Die in Faktor 1 aufgeführten Grundelemente des Vergleichs sowie die Einbettung inhaltlicher Vergleichselemente sind in diesem Text durchweg vorzufinden: So werden bspw. im Hauptteil sowohl inhaltlich-thematische Vergleichsaspekte in Bezug auf England wie auch auf Deutschland benannt und gegenübergestellt. Dabei werden die Gegenüberstellungen durchweg auf der sprachlichen Oberfläche gekennzeichnet und durch die Anordnung der Textteile verdeutlicht. In diesem Lernendentext finden sich darüber hinaus solche Elemente des Vergleichens, die dem Faktor 2 zuzuordnen sind und damit einen problembezogenen und kriterienorientierten Vergleich kennzeichnen. Dazu gehört etwa die Formulierung einer Einleitung, die gerade im vorliegenden Text strukturiert formuliert wird. Weiterhin werden eine These genannt und Vergleichsgröße, Vergleichsobjekt und Tertium Comparationis benannt. Erklärstrukturen können z. T. festgestellt werden, hervorzuheben sind insbesondere Begründe-Strukturen, durch die insbesondere das abschließende Sachurteil gestützt wird. Der Text nimmt damit alle zu Faktor II gehörenden Elemente grundsätzlich auf, auch wenn sie unterschiedlich differenziert ausgeführt werden. 
In Faktor 3 wird vor allem die Urteilsbildung fokussiert. Zwar bleibt das im Schülertext formulierte Sachurteil undifferenziert, immerhin ist aber ein solches Urteil grundsätzlich feststellbar. Weiterhin wird es als Textabschluss formuliert und mit Begründungen versehen. Auch wenn ein historisch-korrektes Sachurteil noch nicht erreicht wird, kann der Text aufgrund der vorhandenen Strukturen dem Faktor 3 zugeordnet werden und geht damit weit über die in Text 1 und 2 gefundenen basalen Vergleichsstrukturen hinaus.

Im Hinblick auf die Elemente des Faktors 4 (Sprachliche Handlungskompetenz) zeigen sich in diesem Schülertext sprachliche Kennzeichnungen von Begründestrukturen. Zum anderen fällt als zweites Element das Erklären (fachlich/pragmatisch) unter diesen Faktor. Dieses wird nur zum Teil geleistet, es finden sich nur wenige Erklär-Strukturen, die die Frage ,Warum' beantworten. Zumindest wird der Faktor 4 zum Teil erreicht.

Auch wenn dieser Schülertext nicht als Modelltext einzuordnen ist, so zeigt er dennoch viele Strukturen des Vergleichens, die insgesamt noch stärker auf das Ziel eines historischen Sachurteils hin ausdifferenziert werden müssten. Dies gilt bspw. für Erklärstrukturen, zeigt sich aber gerade auch im Bereich (vor-)wissenschaftlicher historischer Textkompetenz. Gerade in Bezug auf die zuvor dargestellten Modalisierungsformen stellt sich die Frage, wieso der Schüler diese nur am Textende für sich nutzbar macht, wenn er sie doch augenscheinlich zu verwenden weiß. Dies lässt vermuten, dass solche Formen in Bezug auf Darstellungstexte, die als Grundlage für eine Schreibaufgabe dienen, wenig expliziert wurden. Diese Vermutung lässt sich aber auch auf andere Elemente beziehen, bspw. das Formulieren eines Sachurteils einschließlich zugehöriger Begründungsstrukturen. Bereits in Bezug auf den hier an zweiter Stelle dargestellten Schülertext konnte angenommen werden, dass der zugrundeliegende Darstellungstext eine hohe Anforderung darstellt und die dort aufgenommenen Inhalte aufgrund des hohen Abstraktionsniveaus für Lernende nur schwer in eigene Texte übertragbar sind. Darüber hinaus muss aber auch gefragt werden, wie das Vergleichen im Geschichtsunterricht thematisiert wird und ob zumindest grundlegende sprachliche Strukturen expliziert werden.

Da für die vorliegende Vergleichsaufgabe eine Lösung in einer zum Lehrwerk gehörenden Handreichung für Lehr*innen vorliegt, soll auch diese auf realisierte Vergleichsstrukturen untersucht werden. Für Lehrkräfte dienen solche Handreichungen zur Unterrichtsvorbereitung. Deshalb ist der Blick auf die entsprechende Lösung hilfreich, um zu analysieren, welche Elemente des Vergleichens in dieser Handreichung berücksichtigt werden, damit sie Lehrkräften bewusstgemacht werden und so Eingang in den Unterricht finden können.

\subsection{Exkurs: Die Lösung der Aufgabe in der Handreichung für Lehrkräfte}

Die Lösung in der Handreichung ist nicht als Musterlösung einzuordnen, es handelt sich vielmehr um Lösungshinweise für die Hand der Lehrkraft. Deshalb wird die Lösung aus der Handreichung auch keinem Rating unterzogen. Es muss davon ausgegangen werden, dass bereits die 
Erfassung grundlegender Strukturen wie Textlänge oder Textaufbau nicht vergleichbar sein können. Gleichwohl geben die Lösungshinweise in komprimierter Form Aufschluss darüber, welche Aspekte von den Autoren als bedeutsam für die Aufgabenbearbeitung durch die Lernenden angesehen werden. Im Folgenden wird die Lösung aus der Handreichung für die bessere Vergleichbarkeit mit den Lernendentexten in vollständiger Form wiedergegeben:

\section{„Zu Aufgabe 1 (Darstellungstext), S. 259}

In England gab es bereits gegen Ende des 18. Jahrhunderts ein selbstbewusstes Bürgertum, das die Wirtschafts- und Finanzpolitik mitbestimmte. Auch hatten Bürgertum und Adel genügend Geld angesammelt, sodass sie in die Wirtschaft investieren konnten. Die Existenz freier Lohnarbeit, günstige naturräumliche Bedingungen (Energiequellen, Rohstoffe, Verkehr) und die Entwicklung der Kaufkraft (Nachfrage) sorgten für einen kräftigen Entwicklungsschub, der die Industrielle Revolution hervorbrachte. Alle diese Bedingungen waren in Deutschland an der Wende vom 18. zum 19. Jahrhundert noch nicht gegeben. Hier musste der Staat durch Reformen „von oben“ erst die entsprechenden Weichen stellen. Mit der Befreiung der Bauern aus der Erbuntertänigkeit, der Schaffung rechtsstaatlicher Verhältnisse und der Veränderung des Eigentumsrechts, das dem Bürgertum die Verfügungsgewalt über das Eigentum garantierte, leitete das Allgemeine Landrecht von 1794 die Entfesselung der Wirtschaft von ständischen Zwängen ein. Aber erst die Reformen während der napoleonischen Zeit schufen die Voraussetzungen für eine Stärkung der Wirtschaft bzw. für die Industrialisierung. Die Gewerbereform wie auch die Steuer- und Finanzreform stärkten dabei sowohl das Bürgertum als auch seine Investitionsbereitschaft. Und die Gründung des Deutschen Zollvereins sorgte durch die Schaffung eines großen Binnenmarktes für einen besseren Austausch der Waren und Güter. ${ }^{1739}$

Der Text verweist vornehmlich auf inhaltliches Wissen, das in der Lösung der Lernenden aufgenommen werden soll. Einleitung und These werden nicht genannt, dadurch werden die für das Vergleichen grundlegenden Elemente (Vergleichsgröße, Vergleichsobjekt und Tertium Comparationis) nicht expliziert. Wie in dem der Aufgabe zugrundeliegenden Darstellungstext wird zunächst die Situation in England aufgenommen, dann die in Deutschland. In Bezug auf England werden die Bedingungen für die Entwicklung der Industriellen Revolution in den Mittelpunkt gestellt. Dies geschieht als Reihung oder als Folge. Mit der Aussage „Alle diese Bedingungen waren in Deutschland an der Wende vom 18. zum 19. Jahrhundert noch nicht gegeben." (Z. 8f) wird die deutsche Situation der englischen gegenübergestellt und durch die Verneinung sprachlich als Gegensatz markiert. Interessanterweise werden die Bedingungen, die anders waren, nun nicht ausdifferenziert, sondern es bleibt bei der Globalaussage, dass alle für England genannten Bedingungen in Deutschland zunächst nicht gegeben waren. Es wird damit

\footnotetext{
739 Asch, Bettina; Gehrke, Hans-Joachim; Geske-Wolf, Barbara u.a. (2010): Kursbuch Geschichte. Neue
} Ausgabe. Handreichungen für den Unterricht mit Kopiervorlagen, Cornelsen Verlag Berlin. S. 135. 
eine Beurteilung vorgenommen, der jedoch keine Begründung angefügt wird. Was zunächst anders war, muss sich die Lehrkraft selbst erschließen. Stattdessen wird die Entwicklung Deutschlands vorgeführt, die laut des Schulbuchtextes erst vollzogen werden musste, damit die Industrielle Revolution in Deutschland beginnen konnte. Damit stellen die Autoren einen Bezug zum dritten Teil des Darstellungstextes im Lehrwerk her, der, wie zuvor dargestellt, Aspekte über die Vergleichsaufgabe hinaus thematisiert. Dabei werden die genannten Wissenselemente wieder vor allem als Folge aufbereitet oder aneinandergereiht. Außerdem wird über Gradpartikeln wie ,auch' und ,erst' eine Art Gewichtung der genannten Elemente hinsichtlich ihres Einflusses auf die Entwicklung der Industrialisierung hergestellt. ${ }^{740}$

Der Text endet ohne Abschluss mit dem Verweis auf die Bedeutung des Deutschen Zollvereins. Sprachlich fällt ein sowohl assertiver als auch metaphorischer Stil auf, wenn etwa von „Entfesselung der Wirtschaft" (Z. 14), "Weichen“, die gestellt werden müssen (Z. 10), u.a.m. die Rede ist. Erklärungen von Zusammenhängen werden kaum vorgenommen. Personalisierungen Handelnder fehlen weitgehend, ebenso Kennzeichnungen von Übernahmen aus dem Darstellungstext, obwohl bspw. der Ausdruck „Entfesselung der Wirtschaft“ aus dem Darstellungstext übernommen wurde ${ }^{741}$. Ein Rückbezug auf England über den Hinweis hinaus, dass die Bedingungen in Deutschland gegensätzlich waren, wird nicht vorgenommen. Ebenso fehlt ein vollständiges Sachurteil.

Insgesamt kann festgestellt werden, dass die Lösungshinweise für die Vergleichsaufgabe kaum Vergleichsstrukturen aufweisen, insbesondere fehlen wesentliche sprachliche Teilhandlungen. Hinweise, wie ein historischer Vergleich für diese Aufgabe strukturiert werden kann, sind nicht vorhanden. Die Lehrkraft bekommt als wesentlich angenommene historische Inhalte angeboten, die fachliche sowie sprachlich-kognitive Operation des historischen Vergleichens wird hingegen nicht thematisiert.

\subsection{Fazit aus der qualitativen Analyse}

Auch wenn die Lehrerhandreichung keine Musterlösung darstellt, können Parallelen zwischen den Lernendentexten und der Handreichungslösung festgestellt werden. Sowohl die Lernendentexte als auch der Handreichungstext fokussieren Wissenselemente, die für einen historischen Vergleichstext unabdingbar sind. Die Fokussierung auf Wissenselemente geht allerdings in den schwächeren Texten häufig zu Lasten der für das historische Vergleichen wichtigen sprachlich-kognitiven Handlungen. Gerade die als schwächer eingeschätzten Korpustexte zeigen häufig inhaltliche Strukturen, die gereiht oder in Form von Folgen oder nicht miteinander verbunden werden. Sowohl der zuvor analysierte mittlere als auch der als schwächer eingeschätzte Lernendentext nehmen zwar Wissenselemente aus dem Darstellungstext auf, übertragen sie aber kaum in einen historischen Vergleich. Beide untersuchten Texte kommen nicht über die basale Vergleichskompetenz I hinaus. Selbst in dem Lernendentext, der im Rating die höchste Punktzahl erreicht hat, müssen Vergleichsstrukturen und insbesondere sprachliche

\footnotetext{
${ }^{740}$ Vgl. Zifonum, Gisela et al. (1997), S. 881f.

${ }^{741}$ Laschewski-Müller, Karin; Rauh, Robert (2010), S. 257.
} 
Handlungen viel differenzierter ausgeführt werden. Immerhin hat der Autor dieses Textes eine fachbezogene Vorstellung davon, welche Elemente in einen historischen Vergleich gehören.

Wenn aber sogar die Lösungshinweise der Lehrer*innenhandreichung die für das historische Vergleichen benötigten fachlichen und kognitiv-sprachlichen Strukturen nicht thematisieren, liegt der Schluss nahe, dass sowohl Lehrkräften als auch Lernenden benötigtes explizites Handlungswissen für die Aufgabenlösung fehlt. Dabei kann vermutet werden, dass Lehrkräfte aus ihrer unterrichtlichen Erfahrung und der thematischen Auseinandersetzung im Studium (so sie nicht fachfremd unterrichten) erfahrener im Umgang mit dem Vergleichen im Geschichtsunterricht sind als Schüler*innen der Sekundarstufe Il und zumindest implizites Handlungswissen besitzen. Ob es aber Geschichtslehrkräfte leisten können, den Operator Vergleichen für die unterrichtliche Vermittlung differenziert in seine Bestandteile aufzuschlüsseln, ist fraglich, da ein Modell fehlt. Die EPA leisten, wie gezeigt, nur eine allgemeine Begriffsbestimmung. Methodenseiten in Lehrwerken umgehen ebenfalls häufig eine konkrete Aufschlüsselung (vgl. Kap. 3.3.6).

Eine erste Forderung für den Geschichtsunterricht muss also lauten, Operatoren wie das Vergleichen fachlich und sprachlich aufzuschlüsseln und den Lernenden ausgehend vom Zweck des Operators insbesondere die sprachlich-kognitiven Teilhandlungen solcher Operatoren transparent zu machen.

Da die Aufgabenlösung verknüpft ist mit dem zugrundeliegenden Darstellungstext, ist eine weitere Forderung, solche Darstellungstexte auf ihre unterrichtliche Eignung zu überprüfen. Im Falle des untersuchten Darstellungstextes musste wiederholt festgestellt werden, dass der Text zu schwer ist, weil er zu viele Wissenselemente enthält, die inhaltlich kaum aufgeschlüsselt oder erklärt werden. Da es sich um einen diskursiven Text handelt, ordnet dieser ein oder bewertet sogar. Dies geschieht aber eher indirekt etwa durch den Gebrauch metaphorischer Sprache. Dies ist nur mit Leseerfahrung zu erkennen. In der Neuauflage des „Kursbuch Geschichte“ wird der Darstellungstext nicht mehr verwendet. Insgesamt scheint beim Blick in neuere Lehrwerke das Bewusstsein für Anforderungen von Texten gestiegen zu sein. Dennoch muss es Aufgabe gerade von Geschichtsunterricht sein, Strategien zum Verstehen von Texten (Darstellungs- und Quellentexte) zu vermitteln. Dies ist unabdingbar für den Umgang mit historischen Texten.

Weiterhin zeigen sowohl der erste als auch der zweite Lernendentext, die beide der Erhebung 6 zuzuordnen sind, dass es nicht reicht, eine Hilfestellung wie hier die Tabelle bereitzustellen. Vielmehr hätte dieses Mittel auf einer Metaebene reflektiert werden müssen ${ }^{742}$, um es als strategisches Handlungswissen für die Lösung von Vergleichsaufgaben nutzbar zu machen.

Aus der Einsicht um das Fehlen einer Aufschlüsselung der Strukturelemente eines Vergleichs im Geschichtsunterricht schließt sich im folgenden Kapitel auf der Grundlage der untersuchten Vergleichsaufgabe ein Vorschlag an, um einen Vergleich im Geschichtsunterricht zu explizieren.

\footnotetext{
${ }^{742}$ Vgl. dazu bspw. Kilian, Jörg; Brouër, Birgit; Lüttenberg, Dina (2016): Handbuch Sprache in der Bildung. Berlin: de Gruyter. S.282.
} 


\section{Modell „Vergleichen im Geschichtsunterricht“: Übertragungs- möglichkeiten für die Verwendung im Geschichtsunterricht}

In diesem Kapitel sollen Möglichkeiten aufgezeigt werden, wie die Ergebnisse der quantitativen und der qualitativen Analyse für den Unterricht nutzbar gemacht werden können. Dazu soll zum einen ein Vorschlag für ein praxistaugliches Manual vorgelegt werden, das als Unterstützung für die Lehrkraft zur Einschätzung von Vergleichstexten im Geschichtsunterricht dienen kann. Zum anderen wird eine Strukturierungshilfe von Vergleichstexten für die Schülerhand angefügt, um Schüler*innen bei der Formulierung eines Vergleichs im Geschichtsunterricht zu unterstützen. Beides ist fundiert in den Ergebnissen aus Kapitel 7 und 8.

9.1 Vorschlag für ein praxistaugliches Kriterienraster für den Vergleich im Geschichtsunterricht: Eine Lehrer*innenhandreichung

Die Analyse der Lösungshinweise aus der Handreichung für Lehrkräfte in Kapitel 8 hat gezeigt, dass Strukturen des historischen Vergleichens kaum aufgeschlüsselt werden. Die Einzelanalyse der drei Lernendentexte hat ergeben, dass sowohl der mit einer geringen Gesamtpunktzahl als auch der mit einer mittleren Gesamtpunktzahl geratete Text nicht über das Kompetenzniveau I (Grundelemente eines Vergleichs und inhaltliche Einbettung) hinauskommen. Es ist also zu vermuten, dass fachliche und kognitiv-sprachliche Strukturen des Vergleichens im Geschichtsunterricht den Lernenden nicht transparent sind. Nur der dritte untersuchte Text erreicht ein höheres Kompetenzniveau.

Eine Prüfung, welche Texte des Gesamtkorpus überhaupt Vergleichsstrukturen des Kompetenzniveaus III realisieren, das zum Sachurteil als Ziel des historischen Vergleichs führt, ergibt folgendes Bild:

Tabelle 21: Realisierung von Vergleichsstrukturen des Kompetenzniveaus III nach Ausprägung

\begin{tabular}{|l|l|l|l|l|}
\hline $\begin{array}{l}\text { Kompetenzni- } \\
\text { veau III }\end{array}$ & Items & $\begin{array}{l}\mathbf{0} \text { (nicht er- } \\
\text { kennbar) }\end{array}$ & $\begin{array}{l}\mathbf{1} \text { (teilweise er- } \\
\text { kennbar) }\end{array}$ & $\mathbf{2}$ (erkennbar) \\
\hline & $\begin{array}{l}\text { TF04 (Vergleichsab- } \\
\text { schluss) }\end{array}$ & $67(79,8 \%)$ & $14(16,7 \%)$ & $3(3,6 \%)$ \\
\hline & IG04 (Schluss) & $51(60,7 \%)$ & $25(29,8 \%)$ & $8(9,5 \%)$ \\
\hline & $\begin{array}{l}\text { ST04.2 (Versprachlichung } \\
\text { des Beurteilens) }\end{array}$ & $77(91,7 \%)$ & $7(8,3 \%)$ & $0(0 \%)$ \\
\hline
\end{tabular}

Die Tabelle stellt die Ergebnisse des Ratings der Items dar, die aufgrund der Faktorenanalyse dem Kompetenzniveau III zugeordnet werden können. Es zeigt sich, dass nur in acht Texten ein Textschluss realisiert wird, der als vollständig gelten kann (dieses Item gilt bereits als erfüllt, wenn eine textschlieBende Sprachhandlung erkennbar ist), nur in drei Texten ein Vergleichsabschluss in Form eines Sachurteils, der als vollständig eingeordnet werden kann, vorliegt und in keinem Text Strukturen des Beurteilens durchgängig sprachlich gekennzeichnet werden. Dabei wird kein Text in allen drei Kategorien als 
vollständig (mit einer „2“) geratet, nur drei Texte werden in zwei Kategorien mit einer „2“ geratet. Es muss also konstatiert werden, dass das Ziel des historischen Vergleichs, die Bildung eines Sachurteils, insgesamt nicht erreicht wird. ${ }^{743}$

In Kapitel 8.4 konnte zudem gezeigt werden, dass die zur analysierten Vergleichsaufgabe gehörenden Lösungshinweise aus der Lehrerhandreichung Strukturen des Vergleichens im Geschichtsunterricht nicht thematisiert. Deshalb ist es ein Anliegen dieser Arbeit, solche Strukturen für den Umgang im Geschichtsunterricht aufzuschlüsseln. Dies soll in Form eines Manuals geschehen, das auf dem Kriterienraster und dem zugehörigen Auswertungsmanual der Korpusanalyse beruht. Auf der Grundlage der Faktorenanalyse wurde eine Auswahl der als wesentlich erkannten Items getroffen und das sehr umfangreiche Manual für den Praxiseinsatz verkürzt.

Das Kriterienraster ist fundiert in den Befunden der quantitativen und qualitativen Korpusanalyse und wurde zudem durch eine kleine Studierendengruppe angewandt. Die Auswertung durch die Studierenden stimmt mit dem Rating der Texte in hohem Maße überein.

${ }^{743}$ Eine vollständige Aufschlüsselung der Zuordnung zu den einzelnen Kompetenzniveaus befindet sich im Anhang 9.1 . 


\subsubsection{Zur Struktur des Manuals}

Das nachfolgende Indikatorenmodell soll die Struktur veranschaulichen, die das Kriterienraster für die Lehrer*innenhand berücksichtigt:

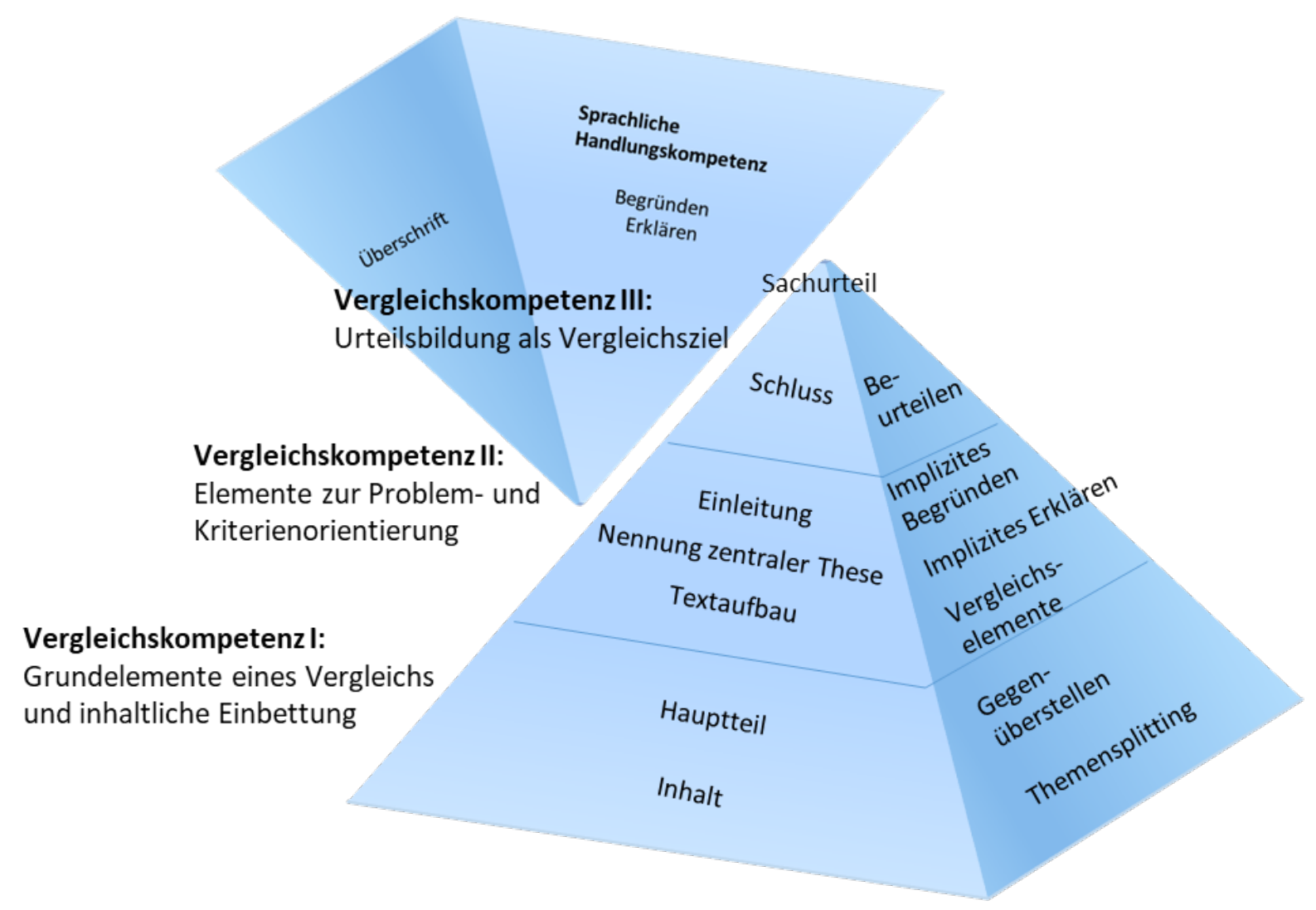

Abbildung 57: Indikatorenmodell "Vergleichen im Geschichtsunterricht" (eigene Darstellung)

Das Modell nimmt die in Kapitel 7 ausführlich dargestellten Kompetenzstufen der Faktorenanalyse durch die Darstellung der Ebenen in der Pyramide auf. Die ,Sprachliche Handlungskompetenz' im Sinne eines ,expliziten Begründens und Erklärens' verläuft als übergeordnete Kompetenz diagonal zu den Kompetenzstufen und ist wesentliches Element der Kompetenzstufen II und III, weshalb es durch das umgekehrte Dreieck in der Grafik besonders kenntlich gemacht wird. Außerdem wird in diesem Dreieck die ,Überschrift' einbezogen. Mit der Aufnahme als eine Seite des umgekehrten Dreiecks soll die Sonderstellung des Items Überschrift (vgl. Kap. 6.2.2), für das in der Faktorenanalyse keine Verbindungen zu anderen Item gefunden werden konnte, dargestellt werden (vgl. dazu Kapitel 7.3.4.5).

Das Kriterienraster ist grundsätzlich darauf ausgelegt, dass eine Vergleichsaufgabe im Geschichtsunterricht in Form eines Fließtextes gelöst wird. Diese Entscheidung resultiert aus dem Ergebnis der Korpusanalyse. Darin konnte gezeigt werden, dass über andere Darstellungsformen wie Tabellen oder Stichwortlisten wichtige Elemente des historischen Vergleichs nicht realisiert wurden. So fehlt einer tabellarischen Darstellung bspw. häufig ein Sachurteil, da über eine Tabelle zwar eine strukturierte Gegenüberstellung vorgenommen werden kann, diese dann aber oft nicht in einem abschließenden Sachurteil zusammengeführt wird. Die Entscheidung für einen zusammenhängenden Text resultiert 
weiterhin aus der in Kapitel 2.7 dargestellten Bedeutung des Schreibens im Geschichtsunterricht und dient letztlich als Vorbereitung auf das wissenschaftliche Schreiben, das entsprechend der Lehrpläne im Unterricht der Sekundarstufe II angeregt werden soll.

Das folgende Inhaltsverzeichnis gibt einen Überblick über die aufgenommenen Indikatoren und ihre Zuordnung in das Kompetenzmodell entspr. der Faktorenanalyse in Kapitel 7, die Aufschlüsselung der einzelnen Items folgt im Anschluss an den Überblick:

Tabelle 22: Inhaltsverzeichnis: Kriterienraster für die Lehrerhand

\begin{tabular}{|c|c|}
\hline Inhalt & Zuordnung zu den Vergleichskompetenzen \\
\hline \multicolumn{2}{|l|}{ Inhaltliche Gestaltung } \\
\hline Einleitung & $\begin{array}{l}\text { II (Elemente zur Problem- und Kriterien- } \\
\text { orientierung) }\end{array}$ \\
\hline Hauptteil & $\begin{array}{l}\text { I (Grundelemente eines Vergleichs und in- } \\
\text { haltliche Einbettung) }\end{array}$ \\
\hline Schluss & III (Urteilsbildung als Vergleichsziel) \\
\hline \multicolumn{2}{|l|}{ (Eigenständige) Überschrift (optional) } \\
\hline \multicolumn{2}{|l|}{ Inhaltliche Fokussierung } \\
\hline Nennung einer zentralen These & $\begin{array}{l}\text { II (Elemente zur Problem- und Kriterien- } \\
\text { orientierung) }\end{array}$ \\
\hline $\begin{array}{l}\text { Explizite Fokussierung auf den Vergleich durch Auf- } \\
\text { nahme direkter Vergleichselemente }\end{array}$ & $\begin{array}{l}\text { II (Elemente zur Problem- und Kriterien- } \\
\text { orientierung) }\end{array}$ \\
\hline $\begin{array}{l}\text { Nennung von wesentlichen historisch-inhaltlichen } \\
\text { Vergleichsaspekten (Orientierung an den Vorgaben } \\
\text { des Darstellungstextes) }\end{array}$ & $\begin{array}{l}\text { I (Grundelemente eines Vergleichs und in- } \\
\text { haltliche Einbettung) }\end{array}$ \\
\hline Vergleichsabschluss & III (Urteilsbildung als Vergleichsziel) \\
\hline \multicolumn{2}{|l|}{$\begin{array}{l}\text { Fokussierung auf den Vergleich durch verwendete } \\
\text { sprachliche Teilhandlungen }\end{array}$} \\
\hline Gegenüberstellen & $\begin{array}{l}\text { I (Grundelemente eines Vergleichs und in- } \\
\text { haltliche Einbettung) }\end{array}$ \\
\hline Begründen & $\begin{array}{l}\text {-II (Elemente zur Problem- und Kriterien- } \\
\text { orientierung) } \\
\text {-Sprachliche Handlungskompetenz }\end{array}$ \\
\hline Erklären & $\begin{array}{l}\text {-II (Elemente zur Problem- und Kriterien- } \\
\text { orientierung) } \\
\text {-sprachliche Handlungskompetenz }\end{array}$ \\
\hline Beurteilen & III (Urteilsbildung als Vergleichsziel) \\
\hline
\end{tabular}




\begin{tabular}{|l|l|}
\hline $\begin{array}{l}\text { Wiss. Textkompetenz I: Umgang mit der Textgrund- } \\
\text { lage }\end{array}$ & \\
\hline $\begin{array}{l}\text { Unterscheidung zwischen Vorlagentext und eigener } \\
\text { Position }\end{array}$ & \\
\hline Modalisierung & \\
\hline Personalisierung Handelnder & \\
\hline $\begin{array}{l}\text { Wiss. Textkompetenz II: Entwicklung eines „Roten } \\
\text { Fadens“ (Mittel zur Themenentwicklung) }\end{array}$ & \\
\hline Themensplitting (Entwicklung durch Teilung) & $\begin{array}{l}\text { I (Elemente zur Problem- und Kriterienori- } \\
\text { entierung) }\end{array}$ \\
\hline Gesamteindruck & \\
\hline
\end{tabular}

Das Kriterienraster berücksichtigt zunächst die grundlegende Textstrukturierung in Einleitung, Hauptteil und Schluss, über die der historische Vergleich bereits kenntlich gemacht werden kann. Weiterhin wurde das Item „(eigenständige) Überschrift“ aufgenommen, obwohl in der Faktorenanalyse ersichtlich wurde, dass dieser Faktor mit keinem anderen zusammenhängt und obwohl Knopp et al. in ihrer Untersuchung zu Indikatoren für die Schreibkompetenz am Beispiel von instruktiven und berichtenden Texten in der Sekundarstufe I für die Überschrift feststellen, dass das Vorhandensein von Überschriften keinen signifikanten Einfluss auf die Textqualität habe. ${ }^{744}$ Für die Sekundarstufe II muss jedoch die Bedeutung einer Überschrift im Rahmen historisch-wissenschaftspropädeutischen Arbeitens berücksichtigt werden. Insbesondere für wissenschaftliche oder vorwissenschaftliche Texte, zu denen die untersuchte Aufgabe herausfordert, stellt die Überschrift ein wesentliches Element zur Lenkung der Lesererwartung dar und ist im Sinne des wissenschaftspropädeutischen Schreibens elementar. Die Auswertung der Korpustexte in Bezug auf dieses Item zeigt, dass die Realisierung einer Überschrift für das Vergleichen eine große Herausforderung darstellt und ohne vorher zu klären, wie eine fach- und sachgerechte Überschrift formuliert werden kann, für die Lernenden zu schwer zu realisieren ist (nur in 10 Texten konnte eine eigenständige Überschrift festgestellt werden, wohingegen in 48 Texten und damit $57 \%$ des Korpus gar keine Überschrift erkennbar ist). Dies erklärt letztlich auch, warum das Item ,Überschrift' nicht mit anderen Items zusammenhängt. Es bedeutet aber nicht, dass das Item nicht berücksichtigt werden sollte, sondern vielmehr, dass das Formulieren einer Überschrift für den historischen Vergleich (und für andere Textsorten) im Unterricht expliziert und geübt werden muss. Spätestens für das wissenschaftliche Schreiben gilt, dass Texten eine passende Überschrift zugeordnet werden muss, weshalb das Item trotz der dargestellten Schwierigkeiten hier Berücksichtigung findet. Da das Item ,Überschrift' auf keinen anderen Faktor lädt, also mit keinem anderen Item zusammenhängt, kann hier jedoch keine eindeutige Zuordnung zu einer Kompetenzstufe des Kriterienrasters vorgenommen werden, es kann aber davon ausgegangen werden, dass es sowohl ein Element der Textstrukturierung als

\footnotetext{
${ }^{744}$ Knopp, Matthias; Jost, Jörg; Linnemann, Markus; Becker-Mrotzek, Michael (2014), S. 125.
} 
auch ein Merkmal des wissenschaftlichen Schreibens darstellt und deshalb mindestens der Vergleichskompetenzstufe II zugeordnet werden muss.

In Bezug auf die inhaltliche Fokussierung des Vergleichs wurden die Elemente ausgewählt, die durch die Aufnahme der zentralen Vergleichselemente eine Problem- und Kriterienorientierung ermöglichen und den Vergleich zu einem Abschluss führen.

Einen weiteren Bestandteil des Kriterienrasters für Lehrkräfte stellen die sprachlich-kognitiven Teilhandlungen dar, die den historischen Vergleich wesentlich als Textsorte bestimmen. Dazu gehören das Gegenüberstellen, Begründen, Erklären und Beurteilen. Im Gegensatz zum Kriterienraster für das Rating wird für das Kriterienraster für Lehrkräfte nicht in die fachlich-pragmatische Realisierung und die sprachlichen Mittel unterschieden, um das Item so handhabbar für den Praxiseinsatz zu machen. Der Fokus liegt für den Unterricht auf dem Wissen um diese für das historische Vergleichen wesentlichen Teilhandlungen und Möglichkeiten der sprachlichen Realisierung. Die Anzahl der benutzten sprachlichen Mittel spielt in diesem Kontext eine untergeordnete Rolle und wird deshalb nicht gesondert erfasst. Auch solche sprachlich-kognitiven Teilhandlungen, die nicht originär für den historischen Vergleich sind, wie bspw. die Reihung, werden (ebf. im Gegensatz zum ursprünglichen Kriterienraster) im Kriterienraster für Lehrkräfte nicht erfasst, wenngleich die Möglichkeit besteht, dass Lernende auf solche Teilhandlungen zurückgreifen. Es werden nur solche Elemente aufgenommen, die nicht verzichtbar für das Textmuster sind.

Die Items der Kategorien „Wissenschaftliche Textkompetenz I und II“, die keinem Faktor der Faktorenanalyse zugeordnet werden konnten, werden teilweise im Kriterienraster berücksichtigt. Diese Entscheidung beruht auf der Bedeutung des fachgerechten historischen Arbeitens. Die angesprochenen Items sind als Werkzeuge des Historikers einzuordnen und machen deshalb einen historischen Vergleich wesentlich aus, auch wenn sie übergreifende Bedeutung für andere geschichtliche bzw. geschichtsdidaktische Textsorten haben. Deshalb müssen Grundfertigkeiten für das geschichtswissenschaftliche Arbeiten wie Bedeutung und Umgang mit Modalisierungen als Mittel der Distanzierung, die Unterscheidung zwischen Vorlagentext und eigener Position sowie die Personalisierung Handelnder im Unterricht thematisiert und für das historische Vergleichen nutzbar gemacht werden können.

Das Item ,Themenfortführung' findet hier hingegen keine Berücksichtigung. Es handelt sich um ein grundlegendes Mittel für das wissenschaftliche Schreiben, ist aber weniger als spezifisch geschichtliches Werkzeug, sondern vielmehr als fächerübergeordnetes Werkzeug anzusehen und entfällt auch aus der ökonomischen Entscheidung heraus, das Kriterienraster benutzbar für den Unterricht zu machen.

Als ein für den historischen Vergleich im Sinne wissenschaftlicher Kompetenz spezifisches Mittel ist das Item Themensplitting (Entwicklung durch Teilung) einzuschätzen und wird entsprechend im Manual berücksichtigt. Es ist ein grundlegendes Mittel des historischen Vergleichs, weil darüber Gegensatzpaare expliziert werden können. Entsprechend der Zuordnung im Ausgangsmanual wird es im Manual für die Lehrerhand der Kategorie „Wiss. Textkompetenz II: Entwicklung eines ,Roten Fadens' (Mittel zur Themenentwicklung)“ zugeordnet. 
Für die Items, die keinem der Faktoren aus der Faktorenanalyse zuzuordnen sind, entfällt eine Einordnung in die Kompetenzstufen.

Das Manual schließt mit der Möglichkeit, einen Gesamteindruck zu formulieren. Diese Möglichkeit bestand im ursprünglichen Kriterienraster nicht, entspricht aber der unterrichtlichen Praxis, eine Möglichkeit für die Berücksichtigung solcher Elemente zu schaffen, die über das Manual hinausgehen, und diese im Hinblick auf ihre Bedeutung für den historischen Vergleich einzuschätzen.

Alle Items sind in drei Stufen skaliert, dies bedeutet zwar einen Verzicht auf eine ausdifferenzierte Einschätzung der Lernendentexte, ermöglicht dafür aber eine möglichst eindeutige Zuordnung der Texte zu den einzelnen Codes und einen geringeren zeitlichen Aufwand für die Texteinschätzung als bei mehrstufigen Skalen. Den einzelnen Codes sind sowohl Erläuterungen als auch in fast allen Fällen Beispiele zugeordnet, die in den meisten Fällen aus dem in dieser Arbeit verwendeten Korpus stammen. Vereinzelte Beispiele sind selbst konstruiert.

Im Folgenden ist das ausführliche Kriterienraster mit der Aufschlüsselung der einzelnen Items angefügt. Die ausgewählten Beispiele beziehen sich auf den Vergleich des Beginns der Industriellen Revolution in England und ,Deutschland': 
9.1.2 Historisches Vergleichen: Ein Kriterienraster zur Einschätzung einer Vergleichsaufgabe im Geschichtsunterricht

Tabelle 23: Kriterienraster für die Hand der Lehrkraft

\begin{tabular}{|c|c|c|c|}
\hline Item & Code & Erläuterungen & Beispiele und Bemerkungen \\
\hline \multicolumn{4}{|l|}{ Inhaltliche Gestaltung } \\
\hline \multirow{3}{*}{$\begin{array}{l}\text { Einleitung } \\
\text { Die Einleitung lenkt den Leser, indem sie auf das Vor- } \\
\text { gehen im Hauptteil vorausweist. } \\
\text { Für den historischen Vergleich sollten neben der ört- } \\
\text { lichen und thematischen Einordnung des Themas be- } \\
\text { reits in der Einleitung Vergleichsobjekt, Vergleichs- } \\
\text { größe und Tertium Comparationis genannt werden. } \\
\text { Darüber hinaus sollte sie eine einleitende These ent- } \\
\text { halten. }\end{array}$} & $\begin{array}{l}0=\text { nicht er- } \\
\text { kennbar }\end{array}$ & $\begin{array}{l}\text { Es ist keine Einleitung im Sinne einer } \\
\text { texteröffnenden Sprachhandlung vor- } \\
\text { handen. }\end{array}$ & $\begin{array}{l}\text { z. B. Start mit Tabelle ohne einleiten- } \\
\text { den Satz }\end{array}$ \\
\hline & $\begin{array}{l}\text { 1= teilweise } \\
\text { erkennbar }\end{array}$ & $\begin{array}{l}\text { Eine Einleitung ist teilweise vorhanden, } \\
\text { das heißt, es werden Teilaspekte ge- } \\
\text { nannt, um die es im Vergleich/Hauptteil } \\
\text { gehen soll. } \\
\text { Sie nimmt nur einen Teil der Aufgaben- } \\
\text { stellung auf (z. B. nur ein Vergleichsob- } \\
\text { jekt und stellt nicht fest, dass es auch } \\
\text { um ein weiteres Objekt bzw. einen Ver- } \\
\text { gleich geht) } \\
\text { und/oder: Die Einleitung ist nur zum Teil } \\
\text { korrekt. }\end{array}$ & $\begin{array}{l}\text { "Die Industrialisierung in England im } \\
\text { ausgehenden 18. Jahrhundert } \\
\text { wurde begünstigt durch Faktoren wie } \\
\text { landwirtschaftliches Wachstum, wel- } \\
\text { ches der wachsenden Bevölkerung } \\
\text { Wohlstand gebracht hatte." } \\
\text { oder } \\
\text { "Zu Beginn der Industrialisierung } \\
\text { spielte England eine große Rolle." }\end{array}$ \\
\hline & 2=erkennbar & $\begin{array}{l}\text { Es ist eine Einleitung im Sinne einer text- } \\
\text { eröffnenden Sprachhandlung vorhan- } \\
\text { den. }\end{array}$ & $\begin{array}{l}\text { „Der Darstellungstext, Die Anfänge der } \\
\text { Industrialisierung' [...] beschäftigt sich } \\
\text { mit den Anfängen der Industrialisie- } \\
\text { rung in England und Deutschland. Im } \\
\text { Folgenden werden nun die unter- } \\
\text { schiedlichen Ausgangsbedingungen }\end{array}$ \\
\hline
\end{tabular}




\begin{tabular}{|c|c|c|c|}
\hline & & & $\begin{array}{l}\text { für die Industrialisierung in England } \\
\text { und Deutschland im ausgehenden } 18 . \\
\text { und beginnenden 19. Jahrhundert ver- } \\
\text { glichen, wobei besonders herausgear- } \\
\text { beitet wird, welche Strukturen und Pro- } \\
\text { zesse sich fördernd oder hemmend auf } \\
\text { die Industrialisierung auswirkten." } \\
\text { oder } \\
\text { "Im vorliegenden Text "Die Anfänge } \\
\text { der Industrialisierung" geht es um die } \\
\text { industrielle Entwicklung in England } \\
\text { und Deutschland vom 18., bis ins } 19 . \\
\text { Jahrhundert." }\end{array}$ \\
\hline \multirow{2}{*}{$\begin{array}{l}\text { Hauptteil } \\
\text { Im Hauptteil wir der Vergleich entfaltet, hier werden } \\
\text { die verschiedenen inhaltlichen Elemente ggf. mit Er- } \\
\text { klärungen versehen und mit Hilfe des Gegenüber- } \\
\text { stellens auf Gemeinsamkeiten, Ähnlichkeiten und } \\
\text { Unterschiede hin untersucht. }\end{array}$} & $\begin{array}{l}0=\text { nicht er- } \\
\text { kennbar }\end{array}$ & $\begin{array}{l}\text { Der Vergleich wird nicht entfaltet, das } \\
\text { heißt: } \\
\text { Es ist kein Hauptteil vorhanden oder } \\
\text { der Hauptteil geht nicht auf mind. ein } \\
\text { Vergleichsobjekt ein. }\end{array}$ & \\
\hline & $\begin{array}{l}1=\text { teilweise } \\
\text { erkennbar }\end{array}$ & $\begin{array}{l}\text { im Hauptteil wird nur auf ein Vergleichs- } \\
\text { objekt eingegangen } \\
\text { oder } \\
\text { die wesentlichen inhaltlichen Ver- } \\
\text { gleichsaspekte werden nur teilweise be- } \\
\text { nannt } \\
\text { oder }\end{array}$ & $\begin{array}{l}\text { „England Ausgangssituation: } \\
+ \text { freier Handel } \\
\text { + landwirtschaftlicher Erfolg } \\
\text { + steigende Güter Nachfrage } \\
\text { + Erfindungen wie Spinnmaschine } \\
\text { + Dampfmaschine } \\
\text { + Wachsende Bevölkerung }\end{array}$ \\
\hline
\end{tabular}




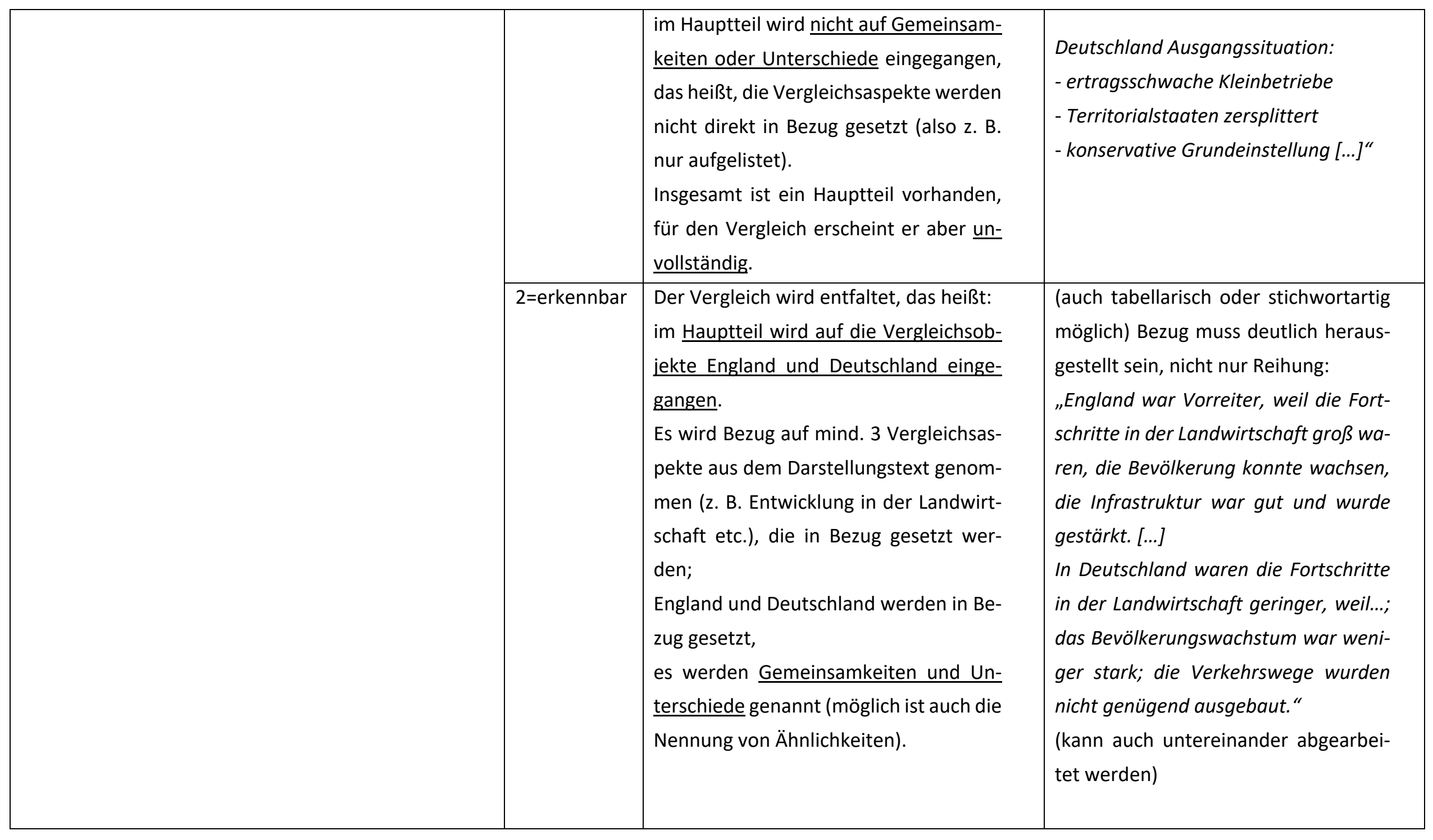




\begin{tabular}{|c|c|c|c|}
\hline \multirow{3}{*}{$\begin{array}{l}\text { Bei diesem Item geht es zunächst darum, dass im } \\
\text { Sinne einer klaren Textstruktur grundsätzlich ein } \\
\text { Textabschluss vorhanden ist. Ein abschließendes } \\
\text { Sachurteil wird gesondert gewertet, deshalb ist an } \\
\text { dieser Stelle das Kriterium bereits erfüllt, wenn ein } \\
\text { Abschluss in Form eines Fazits oder eines Satzes, der } \\
\text { dem Leser den Abschluss des Textes anzeigt, formu- } \\
\text { liert wird. }\end{array}$} & $\begin{array}{l}0=\text { nicht er- } \\
\text { kennbar }\end{array}$ & $\begin{array}{l}\text { Es fehlt eine textschließende Sprach- } \\
\text { handlung ganz, das heißt: } \\
\text { - es fehlt ein Abschluss des Ver- } \\
\text { gleichstextes } \\
\text { - es gibt kein Fazit oder eine Zusammen- } \\
\text { fassung } \\
\text { - ggf. gibt es eine Markierung, dass der } \\
\text { Text nicht fertiggestellt wurde (,....", } \\
\text { etc., usw. o.ä.) }\end{array}$ & $\begin{array}{l}\text { z. B. wenn es nur eine Tabelle gibt, } \\
\text { aber kein Fazit o.ä. daraus } \\
\text { oder } \\
\text { wenn der Fließtext mit der Aufzählung } \\
\text { weiterer Einzelaspekte schließt, die } \\
\text { vorher noch nicht genannt und /oder } \\
\text { nur aufgezählt werden. }\end{array}$ \\
\hline & $\begin{array}{l}\text { 1= teilweise } \\
\text { erkennbar }\end{array}$ & $\begin{array}{l}\text { Der Schluss ist nicht vollständig: } \\
\text { Der Text nimmt nur einen Aspekt oder } \\
\text { ein Vergleichsobjekt auf, ist aber grund- } \\
\text { sätzlich als gedanklicher Abschluss zu er- } \\
\text { kennen. }\end{array}$ & $\begin{array}{l}\text { „Der technologische Rückstand im Ver- } \\
\text { gleich zu England sollte durch staatli- } \\
\text { che Gewerbeschulen aufgeholt wer- } \\
\text { den." } \\
\text { „Das englische Wirtschaftswachstum } \\
\text { wurde durch aufsehenerregende } \\
\text { Erfindungen (z.B. Dampfmaschine } \\
\text { oder Spinnmaschinen) beschleunigt } \\
\text { und durch das Vordringen des Energie- } \\
\text { trägers Kohle konnte die Eisenindustrie } \\
\text { ausgebaut werden." } \\
\text { (in beiden Beispielen fehlt der Bezug } \\
\text { auf das zweite Vergleichsobjekt: } \\
\text { Deutschland) }\end{array}$ \\
\hline & $2=$ =rkennbar & $\begin{array}{l}\text { Textschließende Sprachhandlung (An- } \\
\text { knüpfung an den Hauptteil, Abschluss }\end{array}$ & $\begin{array}{l}\text { z. B. „Deutschland hatte viele hem- } \\
\text { mende Probleme, weshalb es etwas }\end{array}$ \\
\hline
\end{tabular}




\begin{tabular}{|c|c|c|c|}
\hline & & $\begin{array}{l}\text { des Vergleichstextes), verweist durch } \\
\text { Fazit oder Zusammenfassung auf den } \\
\text { Zweck des Vergleichs }\end{array}$ & $\begin{array}{l}\text { schwierig war. England hatte gute Ab- } \\
\text { sichten und Voraussetzungen.": Zwar } \\
\text { wird auf der inhaltlichen Seite nicht er- } \\
\text { klärt, warum England "gute Absichten“ } \\
\text { hatte, aber ein Textschluss im Sinne ei- } \\
\text { ner abschließenden Einordnung ist er- } \\
\text { kennbar. }\end{array}$ \\
\hline \multicolumn{4}{|l|}{ Optional: } \\
\hline \multirow{3}{*}{$\begin{array}{l}\text { (Eigenständige) Überschrift } \\
\text { Da eine Überschrift das erste ist, was ein Leser von } \\
\text { einem Text zur Kenntnis nimmt, ist sie ein wichtiges } \\
\text { Element zur Lenkung der Leseerwartung. Die Über- } \\
\text { schrift sollte also auf das historische Thema, das be- } \\
\text { arbeitet wird, hinweisen und auf den zu erwarten- } \\
\text { den historischen Umgang mit dem Thema (also hier: } \\
\text { Vergleich) }\end{array}$} & $\begin{array}{l}0=\text { nicht er- } \\
\text { kennbar }\end{array}$ & Es gibt keine Überschrift & \\
\hline & $\begin{array}{l}\text { 1= teilweise } \\
\text { erkennbar }\end{array}$ & $\begin{array}{l}\text { Die Überschrift ist eine Übernahme der } \\
\text { vorgegebenen Überschrift im Darstel- } \\
\text { lungstext (also ohne Hinweis auf einen } \\
\text { Vergleich) } \\
\text { oder } \\
\text { es wird die Aufgabenstellung wiederge- } \\
\text { geben } \\
\text { oder } \\
\text { die Überschrift ist nicht eindeutig als } \\
\text { Überschrift zu identifizieren }\end{array}$ & $\begin{array}{l}\text { "Die Anfänge der Industrialisierung" } \\
\text { „England" } \\
\text { „Aufgabe 1" }\end{array}$ \\
\hline & $\begin{array}{l}2=\text { erkenn- } \\
\text { bar }\end{array}$ & $\begin{array}{l}\text { Die Überschrift nimmt Bezug auf die } \\
\text { Vergleichselemente und nennt das Ter- } \\
\text { tium Comparationis, so dass mindestens }\end{array}$ & $\begin{array}{l}\text { "Ausgangsbedingungen der Industriel- } \\
\text { len Revolution in England und Deutsch- } \\
\text { land" } \\
\text { oder }\end{array}$ \\
\hline
\end{tabular}




\begin{tabular}{|c|c|c|c|}
\hline & & $\begin{array}{l}\text { implizit zu erkennen ist, dass es sich um } \\
\text { einen } \underline{\text { Vergleich handelt. }}\end{array}$ & $\begin{array}{l}\text { "Vergleich der Ausgangsbedingungen } \\
\text { der Industriellen Revolution in England } \\
\text { und Deutschland" }\end{array}$ \\
\hline \multicolumn{4}{|l|}{ Inhaltliche Fokussierung } \\
\hline \multirow{3}{*}{$\begin{array}{l}\text { Nennung einer zentralen These } \\
\text { Über die zentrale These werden die Vergleichsele- } \\
\text { mente (Vergleichsgröße und Vergleichsobjekt) auf- } \\
\text { genommen und in Beziehung zueinander gesetzt. Sie } \\
\text { stellt eine erste Beurteilung dar, die am Ende im ab- } \\
\text { schließenden Sachurteil wiederaufgenommen und } \\
\text { abschließend überprüft wird. Dadurch strukturiert } \\
\text { sie den Vergleich vor und lenkt die Lesererwartung. } \\
\text { Die zentrale These ist damit ein wesentliches } \\
\text { Textstrukturierungselement und gehört dadurch in } \\
\text { die Einleitung. }\end{array}$} & $\begin{array}{l}0=\text { nicht er- } \\
\text { kennbar }\end{array}$ & $\begin{array}{l}\text { Es wird keine These formuliert, an der } \\
\text { die Vergleichsaspekte abgearbeitet wer- } \\
\text { den }\end{array}$ & \\
\hline & $\begin{array}{l}1=\text { teilweise } \\
\text { erkennbar }\end{array}$ & $\begin{array}{l}\text { Die These wird nicht vollständig formu- } \\
\text { liert. Sie bezieht sich z. B. nur auf ein } \\
\text { Vergleichsobjekt }\end{array}$ & $\begin{array}{l}\text { "England war der Vorreiter der Indus- } \\
\text { trialisierung, da hier die Ausgangssitu- } \\
\text { ation denkbar gut war." }\end{array}$ \\
\hline & $2=$ erkennbar & $\begin{array}{l}\text { Eine zentrale These wird genannt (sie } \\
\text { muss nicht als solche sprachlich gekenn- } \\
\text { zeichnet sein). } \\
\text { Die zentrale These ist daran erkennbar, } \\
\text { dass sie Bezug auf beide Vergleichsgrö- } \\
\text { ßen nimmt. Alle Vergleichsaspekte soll- } \\
\text { ten sich auf sie beziehen. }\end{array}$ & $\begin{array}{l}\text { "Während England aufgrund des früh- } \\
\text { zeitigen Beginns als ,Pionier' der In- } \\
\text { dustriellen Revolution bezeichnet wer- } \\
\text { den kann, ist Deutschland als Nachzüg- } \\
\text { ler einzuordnen." }\end{array}$ \\
\hline \multirow{3}{*}{$\begin{array}{l}\text { Explizite Fokussierung auf den Vergleich durch Auf- } \\
\text { nahme direkter Vergleichselemente } \\
\text { (kann z. B. schon in der These genannt werden): } \\
\text { a) Nennung des zum Vergleich dienenden Elements } \\
\text { (Vergleichsgröße) }\end{array}$} & $\begin{array}{l}0=\text { nicht er- } \\
\text { kennbar }\end{array}$ & $\begin{array}{l}\text { Weder a) noch b) noch c) werden expli- } \\
\text { zit genannt }\end{array}$ & \\
\hline & $\begin{array}{l}1=\text { teilweise } \\
\text { erkennbar }\end{array}$ & $\begin{array}{l}\text { a), b) oder c) werden nicht vollständig } \\
\text { explizit genannt (z. B. nur a), und/oder } \\
\text { b) etc.) }\end{array}$ & \\
\hline & $2=$ erkennbar & a), b) und c) werden explizit genannt & $\begin{array}{l}\text { "Der Beginn der Industriellen Revolu- } \\
\text { tion (c) in England (a) und Deutschland } \\
\text { (b) verlief sehr unterschiedlich" }\end{array}$ \\
\hline
\end{tabular}




\begin{tabular}{|c|c|c|c|}
\hline $\begin{array}{l}\text { b) Nennung des durch den Vergleich zu charakteri- } \\
\text { sierenden Elements (Vergleichsobjekt) } \\
\text { c) Tertium Comparationis } \\
\text { Hinweis: die Vergleichselemente können auch } \\
\text { im Text verteilt sein. }\end{array}$ & & & \\
\hline $\begin{array}{l}\text { Nennung von wesentlichen historisch-inhaltlichen } \\
\text { Vergleichsaspekten (Orientierung an dem/den zu- } \\
\text { grundeliegenden Text/en) }\end{array}$ & $\begin{array}{l}0=\text { nicht er- } \\
\text { kennbar } \\
1=\text { teilweise } \\
\text { erkennbar } \\
\text { 2=erkennbar }\end{array}$ & $\begin{array}{l}\text { Die Aufschlüsselung, inwiefern dieses } \\
\text { Kriterium erfüllt ist, muss auf der Basis } \\
\text { der Inhalte erfolgen, die für die Lösung } \\
\text { der Vergleichsaufgabe zugrunde gelegt } \\
\text { werden }\end{array}$ & \\
\hline Vergleichsabschluss & $\begin{array}{l}0=\text { nicht er- } \\
\text { kennbar }\end{array}$ & kein abschließendes Sachurteil & $\begin{array}{l}\text { z. B. Tabelle ohne Fazit, auch nicht in } \\
\text { einzelnen Spalten klar erkennbar }\end{array}$ \\
\hline $\begin{array}{l}\text { Bildung eines abschließenden Sachurteils, } \\
\text { Beurteilung im historischen Kontext (entspr. Jeis- } \\
\text { mann): Sinnbildung/Deutung } \\
\text { z. B.: In England konnte sich die Industrielle Revolu- } \\
\text { tion schneller entwickeln/ in Deutschland konnte }\end{array}$ & $\begin{array}{l}\text { 1= teilweise } \\
\text { erkennbar }\end{array}$ & $\begin{array}{l}\text { Das Sachurteil wird angedeutet bzw. } \\
\text { teilweise realisiert } \\
\text { z. B. ist das Urteil nur auf ein Vergleichs- } \\
\text { objekt bezogen oder nimmt nur Teilas- } \\
\text { pekte auf oder es ist pauschal und kaum } \\
\text { fundiert. }\end{array}$ & \\
\hline $\begin{array}{l}\text { ter entwickeln, weil... (Hinweis auf unterschiedliche } \\
\text { Strukturen und Ausgangsbedingungen) }\end{array}$ & $2=$ =rkennbar & $\begin{array}{l}\text { Das Sachurteil wird ausführlich reali- } \\
\text { siert, es ist auf beide Vergleichsobjekte } \\
\text { bezogen. }\end{array}$ & $\begin{array}{l}\text { „Insgesamt lässt sich also sagen, dass } \\
\text { in England die Industrialisierung } \\
\text { schneller stattfinden konnte, da die Ge- } \\
\text { gebenheiten besser waren. }\end{array}$ \\
\hline
\end{tabular}




\begin{tabular}{|c|c|c|c|c|}
\hline & & & \multicolumn{2}{|c|}{$\begin{array}{l}\text { Durch die Hindernisse in Deutschland } \\
\text { hat alles etwas länger gedauert, doch } \\
\text { letzten Endes hat auch dort die Indus- } \\
\text { trialisierung erfolgreich stattgefunden, } \\
\text { so dass England und Deutschland auf } \\
\text { einem ähnlich modernen Stand wa- } \\
\text { ren." }\end{array}$} \\
\hline \multicolumn{5}{|c|}{ Fokussierung auf den Vergleich durch verwendete sprachliche Teilhandlungen } \\
\hline \multirow{3}{*}{$\begin{array}{l}\text { Gegenüberstellen } \\
\text { Herstellung eines Zusammenhanges zwischen mind. } \\
\text { zwei Vergleichselementen: Diese werden so ange- } \\
\text { ordnet, dass Gemeinsamkeiten, Ähnlichkeiten oder } \\
\text { Unterschiede gezeigt werden können. Dies kann z. B. } \\
\text { durch zwei gegenüberliegende Tabellenspalten ge- } \\
\text { schehen oder im Fließtext unter Verwendung ent- } \\
\text { sprechender sprachlicher Mittel. }\end{array}$} & $\begin{array}{l}0=\text { nicht er- } \\
\text { kennbar }\end{array}$ & $\begin{array}{l}\text { Der Text geht auf kein oder nur auf ein } \\
\text { Vergleichsobjekt ein, dadurch kann } \\
\text { nichts gegenübergestellt werden. }\end{array}$ & & \\
\hline & \multirow{3}{*}{$\begin{array}{l}\text { 1= teilweise } \\
\text { erkennbar }\end{array}$} & \multirow[b]{3}{*}{$\begin{array}{l}\text { sprachliche Handlung wird angedeutet } \\
\text { bzw. teilweise realisiert und nur implizit } \\
\text { realisiert: } \\
\text { z. B. gibt es nur eine stichwortartige Auf- } \\
\text { zählung } \\
\text { oder } \\
\text { es gibt „nur" eine Tabelle, die einzelne } \\
\text { Aspekte für jedes Vergleichsobjekt un- } \\
\text { tereinander aufreiht, wie eine Aufzäh- } \\
\text { lung, die Aspekte werden aber nicht ge- } \\
\text { genüber angeordnet } \\
\text { oder }\end{array}$} & \multicolumn{2}{|c|}{ Beispiel für eine Reihung/Aufzählung: } \\
\hline & & & England & Deutschland \\
\hline $\begin{array}{l}\text { durch zwei gegenüberliegende Tabellenspalten ge- } \\
\text { schehen oder im Fließtext unter Verwendung ent- } \\
\text { sprechender sprachlicher Mittel. } \\
\text { Die EPA bestimmen das Gegenüberstellen als: „wie } \\
\text { skizzieren, aber zusätzlich argumentierend gewich- } \\
\text { ten“ (Kultusministerkonferenz, 2005: EPA Ge- } \\
\text { schichte, S. 8). }\end{array}$ & & & $\begin{array}{l}\text {-schneller Fort- } \\
\text { schritt der Land- } \\
\text { wirtschaft } \\
\text {-Landbevölke- } \\
\text { rung nicht an } \\
\text { Scholle gebun- } \\
\text { den } \\
\text {... }\end{array}$ & $\begin{array}{l}\text {-in Territorial- } \\
\text { staaten geglie- } \\
\text { dert } \\
\text {-Vielzahl von } \\
\text { Zollschranken } \\
\text {-abweichende } \\
\text { Maß-, Münz- } \\
\text { und Gewichts- } \\
\text { systeme } \\
\text {... }\end{array}$ \\
\hline
\end{tabular}




\begin{tabular}{|c|c|c|c|}
\hline $\begin{array}{l}\text { Sprachliche Mittel des Gegenüberstellens: } \\
\text { Zur Darstellung von Unterschieden: z. B. anders als, } \\
\text { gegensätzlich, im Gegensatz zu, hingegen, dagegen, } \\
\text { begünstigen...behindern, } \\
\text { (noch) nicht (vergleichend gebraucht) } \\
\text { Gegenüberstellung über Antonyme oder gegensätz- } \\
\text { lich wirkende Begriffe wie z. B. schnell-langsam (ins- } \\
\text { besondere Verben; Adjektive); dabei darf es sich }\end{array}$ & & $\begin{array}{l}\text { es gibt einen Text, der erst das eine, } \\
\text { dann das andere Vergleichsobjekt abar- } \\
\text { beitet, ohne explizite Zusammenhänge } \\
\text { zwischen beiden herzustellen } \\
\text { oder } \\
\text { typographische Markierung: z. B. wird } \\
\text { nur durch einen Absatz kenntlich ge- } \\
\text { macht, dass zwei Elemente gegenüber- } \\
\text { gestellt werden. }\end{array}$ & \\
\hline $\begin{array}{l}\text { auch um unterschiedliche Wortarten handelt, z. B.: } \\
\text { „Freiheit - abhängig“ } \\
\text { über Komparation (größer als...), Steigerung (z. B. } \\
\text { schneller als) } \\
\text { Zur Darstellung von Gemeinsamkeiten: genauso, } \\
\text { gleich wie..., } \\
\text { Zur Darstellung von Ähnlichkeiten: ähnlich wie, fast } \\
\text { so wie... }\end{array}$ & $\begin{array}{l}2=\text { erkenn- } \\
\text { bar }\end{array}$ & $\begin{array}{l}\text { Die sprachliche Handlung wird ausführ- } \\
\text { lich und explizit realisiert: Es gibt einen } \\
\text { gegliederten und strukturierten Text, } \\
\text { der weitgehend einen Zusammenhang } \\
\text { zwischen den Vergleichselementen her- } \\
\text { stellt und die Aspekte nicht nur unterei- } \\
\text { nander aufreiht, sondern sie inhaltlich } \\
\text { gewichtet. } \\
\text { oder: } \\
\text { Eine Tabelle markiert eine Gegenüber- } \\
\text { stellung erkennbar, wenn die einzelnen } \\
\text { Aspekte weitgehend ausdrücklich gge- } \\
\text { genüber' angeordnet sind. }\end{array}$ & $\begin{array}{l}\text { z. B.: } \\
\text { „Als Pionier der Industrialisierung kön- } \\
\text { nen anhand des Beispiels ,England' } \\
\text { mehrere Faktoren dargestellt werden, } \\
\text { die den Beginn dieses Prozesses be- } \\
\text { günstigen und fördern, sogar regel- } \\
\text { recht bedingen. [...] } \\
\text { Demgegenüber konnte aufgrund von } \\
\text { rückständigen Gesetzen und Struktu- } \\
\text { ren die Industrialisierung erst verspä- } \\
\text { tet, nämlich nach Abschaffung dieser, } \\
\text { in Deutschland einsetzen. [...]" }\end{array}$ \\
\hline
\end{tabular}




\section{Begründen}

Beim Begründen werden (mögliche) Einwände eines Lesers oder Hörers bearbeitet/vorweggenommen. Der Autor macht dem Leser eine Aussage/Handlung verständlich: aus Nicht-Akzeptierbarem Akzeptierbares machen (Vgl.: Ehlich/Rehbein, 1989: Begründen IV: kognitives Begründen)

Die EPA bestimmen das Begründen als:

„Aussagen (z. B. Urteil, These, Wertung) durch Argumente stützen, die auf historischen Beispielen und anderen Belegen gründen" (Kultusministerkonferenz, 2005: EPA Geschichte, S. 8.)

\section{Sprachliche Mittel des Begründens:}

Die sprachlichen Mittel des Begründens können sich mit denen des Erklärens überschneiden (z. B. über die Konjunktionen, die kausale Zusammenhänge, wie $d a$, weil etc. anzeigen). Deshalb ist es wichtig, jeweils die Funktion zu klären:

Immer dann, wenn ein möglicher Einwand bearbeitet wird, handelt es sich um eine Begründe-Struktur. Um die sprachlichen Mittel des Begründens vom Erklären besser abgrenzen zu können, werden im Folgenden Lexeme aus dem Argumentationswortschatz

\begin{tabular}{|c|c|c|}
\hline $\begin{array}{l}0=\text { nicht er- } \\
\text { kennbar }\end{array}$ & $\begin{array}{l}\text { Es gibt keine Begründungen, es wird } \\
\text { kein Einwand vorweggenommen. }\end{array}$ & \\
\hline $\begin{array}{l}\text { 1= teilweise } \\
\text { erkennbar }\end{array}$ & $\begin{array}{l}\text { Es gibt nur Begründungen, die sich auf } \\
\text { jeweils ein Vergleichsobjekt beziehen, } \\
\text { aber nicht in direktem Zusammenhang } \\
\text { stehen. } \\
\text { Es gibt implizite Begründungen, die Ab- } \\
\text { grenzung von Erklärstrukturen ist nicht } \\
\text { eindeutig zu erkennen. }\end{array}$ & $\begin{array}{l}\text { z. B.: Aussage, dass England schneller } \\
\text { in der Entwicklung war, wird begrün- } \\
\text { det, aber nicht vergleichend mit } \\
\text { Deutschland: } \\
\text { Bsp.: "Die Ursachen der Industriellen } \\
\text { Revolution spielen eine große Rolle, } \\
\text { wieso England einer der ersten Länder } \\
\text { der Industrialisierung war" (nachfol- } \\
\text { gend werden die Ursachen erklärt) }\end{array}$ \\
\hline $2=$ erkennbar & $\begin{array}{l}\text { Es gibt mind. eine explizite Begründung, } \\
\text { die sich auf beide Vergleichsobjekte be- } \\
\text { zieht. }\end{array}$ & $\begin{array}{l}\text { Es wird z. B. dargelegt, warum von der } \\
\text { Annahme ausgegangen wird, dass ein } \\
\text { Pionierland der Industrialisierung war. } \\
\text { z. B.: „Ein Grund für die Annahme, dass } \\
\text { England ein Pionierland war, ist..." }\end{array}$ \\
\hline
\end{tabular}




\begin{tabular}{|c|c|c|c|}
\hline $\begin{array}{l}\text { für Sachverhaltsbeziehungen aufgelistet (nach Grae- } \\
\text { fen/Moll, } 2007 \text { (S. 499): } \\
\text { „N ist der Grund für } A \text { / } N \text { begründet sich aus } D / N \text { ist } \\
\text { die Grundlage für } A \text { / } N \text { ist die Basis für } A / N \text { basiert } \\
\text { auf } D / N \text { ist die Ursache für } A \text { / } N \text { verursacht } A / N \\
\text { liegt } D \text { zugrunde / } N \text { ist die Folge von } D / N \text { folgt aus } \\
D / N \text { ruft A hervor / } N \text { führt zu } D / N \text { ist die Wirkung } \\
\text { von } D \text { " }\end{array}$ & & & \\
\hline $\begin{array}{l}\text { Erklären (Erklären-warum) } \\
\text { Erklären gewährt Einsicht in funktionale Zusammen- } \\
\underline{\text { hänge. Das Ziel des Erklärens ist die Wissensvermitt- }}\end{array}$ & $\begin{array}{l}0=\text { nicht er- } \\
\text { kennbar }\end{array}$ & $\begin{array}{l}\text { Die sprachliche Handlung wird nicht re- } \\
\text { alisiert, es kommen keine Erklärungen } \\
\text { vor. }\end{array}$ & \\
\hline $\begin{array}{l}\text { „Mng (im Gegensatz zum Begründen): } \\
\text { „Mit einer Erklärung macht eine Person Anderen } \\
\text { oder sich selbst einen Zusammenhang von Sachver- } \\
\text { halten oder Sachverhaltselementen so klar, dass er } \\
\text { ins Wissen integriert und als allgemeine Orientie- } \\
\text { rung des Handelns genommen werden kann.“ (Hof- } \\
\text { mann, 2014; S. 527) } \\
\text { Die EPA bestimmen das Erklären als: „historische } \\
\text { Sachverhalte durch Wissen und Einsichten in einen } \\
\text { Zusammenhang (Theorie, Modell, Regel, Gesetz, }\end{array}$ & $\begin{array}{l}\text { 1= teilweise } \\
\text { erkennbar }\end{array}$ & $\begin{array}{l}\text { teilweise erkennbar: sprachliche Hand- } \\
\text { lung wird angedeutet bzw. teilweise } \\
\text { oder nur implizit realisiert. }\end{array}$ & $\begin{array}{l}\text { Bsp.: anstatt einer ausgeführten Erklä- } \\
\text { rung werden mehrere Aspekte ohne } \\
\text { weitere Ausführung aneinanderge- } \\
\text { reiht, um so einen zuvor genannten } \\
\text { (meist übergeordneten Aspekt) zu er- } \\
\text { klären: } \\
\text { „Weitere hemmende Faktoren sind } \\
\text { schlechte Verkehrswege und die er- } \\
\text { tragsschwache Wirtschaft" (dadurch } \\
\text { soll erklärt werden, warum in Deutsch- } \\
\text { land die Industrialisierung so langsam } \\
\text { und spät startete, statt einer richtigen }\end{array}$ \\
\hline
\end{tabular}




\begin{tabular}{|c|c|c|c|}
\hline \multirow[b]{2}{*}{$\begin{array}{l}\text { Funktionszusammenhang) einordnen und be- } \\
\text { gründen.“ (Kultusministerkonferenz, 2005: EPA Ge- } \\
\text { schichte, S. 8). } \\
\text { „Aufzuklären ist stets eine Warum-Frage“ (Hoff- } \\
\text { mann, 2014; S. 528) } \\
\text { Sprachliche Mittel des Erklärens: } \\
\text { (Erklären-warum: Die sprachlichen Mittel tragen } \\
\text { dazu bei, eine Antwort auf eine Warum-Frage zu ge- } \\
\text { ben.) }\end{array}$} & & & $\begin{array}{l}\text { Erklärung wird hier aber eher aufge- } \\
\text { zählt) }\end{array}$ \\
\hline & 2=erkennbar & $\begin{array}{l}\text { sprachliche Handlung wird mehrfach } \\
\text { (mind. } 2 x \text { ) ausführlich und explizit reali- } \\
\text { siert, dabei ist der fachliche Zusammen- } \\
\text { hang korrekt: Es gibt fachlich richtige } \\
\text { Antworten auf die Frage „Warum“. }\end{array}$ & $\begin{array}{l}\text { "Aufgrund der steigenden Bevölkerung } \\
\text { auf dem Land und dadurch, dass die } \\
\text { Bauern und Arbeitskräfte nicht an die } \\
\text { Höfe gebunden waren, zogen viele von } \\
\text { ihnen in die größeren Städte, um dort } \\
\text { neue Betätigungsfelder sprich Arbeit } \\
\text { zu finden." }\end{array}$ \\
\hline \multicolumn{4}{|l|}{$\begin{array}{l}\text { Konjunktionen: } \\
\text { weil, da, daher, wegen, um, aufgrund, denn } \\
\text { Adverbien: } \\
\text { darum, daher, deswegen, deshalb } \\
\text { warum (im Fragemodus), weshalb } \\
\text { feststehende Wendungen: ...liegt daran, dass..., der } \\
\text { Grund ist..., aus diesem Grund... }\end{array}$} \\
\hline \multirow[t]{2}{*}{ Beurteilen } & $\begin{array}{l}0=\text { nicht er- } \\
\text { kennbar }\end{array}$ & $\begin{array}{l}\text { Ein historisches Sachurteil oder Teilur- } \\
\text { teile werden nicht realisiert }\end{array}$ & \\
\hline & $\begin{array}{l}\text { 1= teilweise } \\
\text { erkennbar }\end{array}$ & $\begin{array}{l}\text { Ein Sachurteil wird angedeutet bzw. teil- } \\
\underline{\text { weise (z. B. nur in Teilbeurteilung) oder }} \\
\text { nur implizit realisiert }\end{array}$ & $\begin{array}{l}\text { "Die Industrialisierung begann wegen } \\
\text { der ganzen Faktoren erst später." }\end{array}$ \\
\hline
\end{tabular}




\begin{tabular}{|c|c|c|c|}
\hline $\begin{array}{l}\text { Es wird eine Beurteilung im Sinne eines historischen } \\
\text { Sachurteils gebildet (möglich ist auch je nach Aufga- } \\
\text { benstellung ein über das Sachurteil hinausgehendes } \\
\text { Werturteil). } \\
\text { Histor. Sachurteil entsteht auf der Basis der vorange- } \\
\text { gangenen Analyse } \\
\text { Die EPA bestimmen das Beurteilen als: } \\
\text { „den Stellenwert historischer Sachverhalte in einem } \\
\text { Zusammenhang bestimmen, um ohne persönlichen } \\
\text { Wertebezug zu einem begründeten Sachurteil zu ge- } \\
\text { langen“ (Kultusministerkonferenz (2005): EPA Ge- } \\
\text { schichte, S. 8.) }\end{array}$ & & $\begin{array}{l}\text { oder } \\
\text { das Sachurteil ist pauschal, es kann nur } \\
\text { oberflächlich auf die Vergleichsaspekte } \\
\text { zurückgeführt werden. } \\
\text { Mögliche Erscheinungsformen: } \\
\text {-es gibt Teilurteile über Positiv-/Negativ- } \\
\text { zuschreibungen } \\
\text {-das Urteil steckt in der zentralen These } \\
\text { in der Textmitte, wird jedoch nicht im } \\
\text { Schluss zu Ende geführt, es gibt außer- } \\
\text { dem keine Teilbeurteilungen } \\
\text {-die Teilurteile sind pauschal und undif- } \\
\text { ferenziert }\end{array}$ & \\
\hline $\begin{array}{l}\text { Hier können auch „Teilurteile“ berücksichtigt wer- } \\
\text { den. } \\
\text {-sachliches Beurteilen: kriteriengeleitete Conclusio } \\
\text {-Das Urteil steckt im Vergleich bzw. erwächst aus } \\
\text { dem Vergleich }\end{array}$ & 2=erkennbar & $\begin{array}{l}\text { Ein Sachurteil ist klar erkennbar, das } \\
\text { Sachurteil erfolgt auf der Basis im Text } \\
\text { erkennbarer Kriterien. } \\
\text { Es ist eine klare Wertung erkennbar, das } \\
\text { Sachurteil basiert auf der vorausgegan- } \\
\text { genen Analyse, ist deshalb inhaltlich dif- } \\
\text { ferenziert und nicht pauschal. }\end{array}$ & $\begin{array}{l}\text { „Abschließend kann festgehalten wer- } \\
\text { den, dass insbesondere durch die Herr- } \\
\text { schaftsstrukturen die Industrialisie- } \\
\text { rung in Deutschland erst später begin- } \\
\text { nen konnte." }\end{array}$ \\
\hline $\begin{array}{l}\text { Sprachliche Mittel des Beurteilens: } \\
\text { Verknüpfung von Wertung und Begründestrukturen, } \\
\text { realisiert durch Verben, Adjektive, Nomen, die eine } \\
\text { Wertung einschließen, }\end{array}$ & & $\begin{array}{l}\text { Mögliche Erscheinungsformen: } \\
\text { - es gibt eine abschließende Beurteilung } \\
\text { der Gegenüberstellung }\end{array}$ & \\
\hline
\end{tabular}




\begin{tabular}{|c|c|c|c|}
\hline $\begin{array}{l}\text { z. B.: } \\
\text { macht deutlich, wird deutlich, ist einzuordnen, ist } \\
\text { festzustellen, man sieht..., insgesamt lässt sich sa- } \\
\text { gen... }\end{array}$ & & $\begin{array}{l}\text { oder } \\
\text {-es gibt Teilbeurteilungen der Ver- } \\
\text { gleichsobjekte (zu jedem Vergleichsob- } \\
\text { jekt mind. eine) }\end{array}$ & \\
\hline \multicolumn{4}{|l|}{ Wiss. Textkompetenz I: Umgang mit der Textgrundlage } \\
\hline \multirow{2}{*}{$\begin{array}{l}\text { Unterscheidung zwischen Vorlagentext und eigener } \\
\text { Position } \\
\text { Sind alle Übernahmen aus zugrundeliegenden Tex- } \\
\text { ten gekennzeichnet (durch Anführungsstriche, indi- } \\
\text { rekte Rede oder sprachliche Kennzeichnungen wie } \\
\text { "der Autor sagt...")? }\end{array}$} & $\begin{array}{l}0=\text { nicht er- } \\
\text { kennbar }\end{array}$ & $\begin{array}{l}\text { Es wird nicht unterschieden, Textpassa- } \\
\text { gen oder Teilsätze etc. aus dem Vorla- } \\
\text { gentext werden ohne korrekte Kenn- } \\
\text { zeichnung übernommen. }\end{array}$ & $\begin{array}{l}\text { Z. B.: Übernahme des gesamten ersten } \\
\text { Satzes aus dem zugrunde liegenden } \\
\text { Darstellungstextes ohne Kennzeich- } \\
\text { nung) }\end{array}$ \\
\hline & $\begin{array}{l}1=\text { teilweise } \\
\text { erkennbar }\end{array}$ & $\begin{array}{l}\text { Unterscheidung teilweise erkennbar: Es } \\
\text { wird teilweise, aber nicht vollständig un- } \\
\text { terschieden, Textpassagen oder Teil- } \\
\text { sätze aus dem Vorlagentext werden teil- } \\
\text { weise korrekt gekennzeichnet oder um- } \\
\text { formuliert. } \\
\text { Nicht alle Übernahmen sind gekenn- } \\
\text { zeichnet. }\end{array}$ & $\begin{array}{l}\text { Z. B.: Fehlende Kennzeichnung von } \\
\text { Wendungen aus dem zugrundeliegen- } \\
\text { den Darstellungstext. }\end{array}$ \\
\hline Wird fachgerecht zitiert? & $\begin{array}{l}2=\text { erkenn- } \\
\text { bar }\end{array}$ & $\begin{array}{l}\text { Es wird vollständig unterschieden, Text- } \\
\text { passagen oder Teilsätze aus dem Vorla- } \\
\text { gentext werden vollständig korrekt ge- } \\
\text { kennzeichnet oder umformuliert. }\end{array}$ & \\
\hline
\end{tabular}




\section{Modalisierungsmittel:}

Im Unterschied zu einer Tatsachenbehauptung kann mit Hilfe der Modalisierung der Grad einer Möglichkeit oder Wahrscheinlichkeit ausgedrückt werden.

Der Autor verdeutlicht über Modalisierungen, ob er eine Aussage für faktisch hält bzw. wie hoch er ihre Faktizität einschätzt (zur Darstellung verschiedener Grade „unsicherer Faktizität“ vgl. Diewald, 2013. S. 90). Modalisierungsmittel stellen ein wichtiges historisches Werkzeug dar.

Sprachliche Mittel zur Modalisierung, z. B.:

- Modalverben: müssen, können, sollen, dürfen, mögen, wollen, mögen/möchte

- Modalverben in Verbindung mit Part. + Hilfsverb, z. B.: ...soll gegeben haben, mag erreicht sein...

- Verbmodi: Konj. I und II, z. B. sei, würde, wäre, ginge...

- modale Satzadverbialia, z. B. wahrscheinlich, vielleicht, sicher, offenbar, offensichtlich, gewiss, bestimmt, angeblich anscheinend

- Modalpartikel, z. B. doch, eben, ja, möglicherweise, vielleicht, normalerweise

\begin{tabular}{|c|c|c|}
\hline $\begin{array}{l}0=\text { nicht er- } \\
\text { kennbar }\end{array}$ & $\begin{array}{l}\text { Es werden keine Modalisierungsmittel } \\
\text { eingesetzt }\end{array}$ & \\
\hline $\begin{array}{l}1=\text { teilweise } \\
\text { erkennbar }\end{array}$ & $\begin{array}{l}\text { Modalisierungsmittel werden z. T. ein- } \\
\text { gesetzt oder kennzeichnen nur zum Teil } \\
\text { eindeutig und zutreffend Grade von Fak- } \\
\text { tizität. }\end{array}$ & \\
\hline $\begin{array}{l}2=\text { erkenn- } \\
\text { bar }\end{array}$ & $\begin{array}{l}\text { Modalisierungsmittel werden durchgän- } \\
\text { gig passend eingesetzt und kennzeich- } \\
\text { nen die Faktizität von Aussagen }\end{array}$ & $\begin{array}{l}\text { „....könnte einen weiteren Hinweis auf } \\
\text { die Fortschrittlichkeit Englands darstel- } \\
\text { len" }\end{array}$ \\
\hline
\end{tabular}




\begin{tabular}{|c|c|c|c|}
\hline $\begin{array}{l}\text { - Modalpartikel in Kombination mit Satzadverb, z. B. } \\
\text { wahrscheinlich ja (vgl. Péteri, S. 272) } \\
\text { - formelhafte Verkürzungen, z. B. wie angenommen } \\
\text { - Anführungsstriche („,...") } \\
\text { (vgl. Zifonum, Gisela (1997); S. } 1252 \text { u.a.) } \\
\text { Weitere Modalisierungsformen: } \\
\text {-(nicht) brauchen, haben sein mit zu-Infinitiv } \\
\text {-bleiben, stehen, gehören mit Part. II } \\
\text {-pflegen, scheinen, drohen mit zu-Infinitiv } \\
\text {-...wie Historiker sagen... }\end{array}$ & & & \\
\hline $\begin{array}{l}\text { Personalisierung von Handelnden } \\
\text { Sichtbarmachung der Handelnden } \\
\text { (vgl. dazu Schneider (2012), Bergmann (1997): Prin- } \\
\text { zip der Personalisierung und Personifizierung) }\end{array}$ & $\begin{array}{l}0=\text { nicht er- } \\
\text { kennbar }\end{array}$ & Handelnde werden nicht personalisiert & $\begin{array}{l}\text { „Deutschland war der Vorreiter" } \\
\text { „ertragsschwache Kleinbetriebe muss- } \\
\text { ten das Geschäft aufgeben" } \\
\text { „Das Handwerk wollte das nicht" } \\
\text { „Der Staat..." }\end{array}$ \\
\hline Historische Akteure werden beim Namen genannt: & $\begin{array}{l}1=\text { teilweise } \\
\text { erkennbar }\end{array}$ & $\begin{array}{l}\text { Handelnde werden im Gesamttext nur z. } \\
\text { T. personalisiert }\end{array}$ & \\
\hline $\begin{array}{l}\text { statt: „England ging schnell voran“ werden die Han- } \\
\text { delnden genannt: „Die englischen Regierungsmit- } \\
\text { glieder sorgten dafür" oder "der Minister..." } \\
\text { Personalisierungen aus dem Text dürfen/sollen } \\
\text { übernommen werden. }\end{array}$ & $\begin{array}{l}2=\text { erkenn- } \\
\text { bar }\end{array}$ & $\begin{array}{l}\text { Handelnde werden im Gesamttext weit- } \\
\text { gehend (ggf. bis auf eine Ausnahme) } \\
\text { personalisiert } \\
\underline{\text { Historische Akteure werden genannt }}\end{array}$ & "Hargreaves..." \\
\hline
\end{tabular}




\begin{tabular}{|c|c|c|c|}
\hline \multicolumn{4}{|c|}{$\begin{array}{l}\text { Wiss. Textkompetenz II: Entwicklung eines vergleichsspezifischen „Roten Fadens“ } \\
\text { (Mittel zur Themenentwicklung) }\end{array}$} \\
\hline Themensplitting (Entwicklung durch Teilung) & $\begin{array}{l}0=\text { nicht er- } \\
\text { kennbar }\end{array}$ & $\begin{array}{l}\text { Es werden keine Gegensatzpaare ge- } \\
\text { nannt. }\end{array}$ & \\
\hline $\begin{array}{l}\text { genübergestellt wird (z. B. England und Deutschland) } \\
\text { (vgl. Hoffmann 2014, S. 202) } \\
\text { Hinweis: Das Gegensatzpaar muss sich im Text befin- }\end{array}$ & $\begin{array}{l}\text { 1= teilweise } \\
\text { erkennbar }\end{array}$ & $\begin{array}{l}\text { Es fehlen Gegensatzpaare oder sie wer- } \\
\text { den nur indirekt dargestellt und nicht } \\
\text { eindeutig benannt }\end{array}$ & $\begin{array}{l}\text { z. B. wird nur ein Gegensatzpaar ge- } \\
\text { nannt }\end{array}$ \\
\hline $\begin{array}{l}\text { den, es muss nicht unbedingt in einem Satz formu- } \\
\text { liert sein. } \\
\text { Versprachlichung über Ausdruckspaare: } \\
\text { die eine - die andere } \\
\text { er - sie } \\
\text { der - der } \\
\text { das erste - das zweite } \\
\text { einer - ein weiterer... } \\
\text { auch: Weiterführung über semantische Relationen: } \\
\text { die Industrieländer - England, Deutschland }\end{array}$ & $\begin{array}{l}2=\text { erkenn- } \\
\text { bar }\end{array}$ & $\begin{array}{l}\text { Die notwendigen Gegensatzpaare wer- } \\
\text { den eindeutig benannt }\end{array}$ & $\begin{array}{l}\text { Bsp.: „England und Deutschland" } \\
\text { oder } \\
\text { "das eine Land war schneller, das an- } \\
\text { dere langsamer" } \\
\text { In England begann die Industrialisie- } \\
\text { rung schon früh. [...weitere Textteile...] } \\
\text { In Deutschland dagegen... } \\
\text { etc. } \\
\text { oder: deutsch-englisch }\end{array}$ \\
\hline Gesamteindruck & \multicolumn{3}{|c|}{ (individueller Kommentar) } \\
\hline
\end{tabular}

Die gewählten Beispiele orientieren sich am Vergleich des Beginns der Industriellen Revolution in England und ,Deutschland’. 


\subsubsection{Hinweise zur Auswertung mit dem Vergleichs-Manual}

Das Kriterienraster ist für die Lehrerhand konzipiert. Als Strukturhilfe für Lernende ist es nicht gedacht, da es als Mittel zur Strukturierung oder Reflexion eigener Schreibprodukte zu komplex ist. Im nachfolgenden Ausblick werden deshalb Hinweise zur Didaktisierung von Vergleichsaufgaben im Geschichtsunterricht mit Hilfe einer Strukturskizze angefügt, die sich viel eher für die unterrichtliche Verwendung adaptieren lässt.

Das Kriterienraster sollte zur Einschätzung eines historischen Vergleichstextes in seiner Gesamtheit genutzt werden, es ist aber möglich, einzelne Items herauszulösen bzw. nur Ausschnitte des Manuals zu nutzen, wenn das Erkenntnisinteresse auf bestimmte Ausschnitte gerichtet ist.

Die Zuordnungen der einzelnen Items zu den Codes stellt eine Einordnung der Textqualität in Bezug auf einzelne Strukturen dar, so dass es möglich ist, die Punkte zu einer Gesamtpunktzahl aufzuaddieren und ins Verhältnis zu setzen (bspw. die Gesamtpunktzahl eines Einzeltextes im Vergleich zu den Gesamtpunktzahlen bzw. der mittleren Gesamtpunktzahl der Gesamtheit der erhobenen Texte). Dabei sollte aber auch im Blick gehalten werden, wie die Einordnung einzelner Items ausfällt. So fiel in der zuvor dargestellten Erhebung der Korpustexte in Bezug auf das Item „Versprachlichung des Beurteilens" (ST04.2) ein starker Bodeneffekt auf: Von 84 erfassten Texten wurde für 77 Texte (92\%) festgestellt, dass sprachliche Mittel, die das Beurteilen kennzeichnen, nicht erkennbar sind. Ein solcher Effekt weist darauf hin, dass entweder eine sprachliche Kennzeichnung für die Bearbeitung des Vergleichs nicht nötig ist ${ }^{75}$ oder zu schwierig war. Dies ist wiederum ein Hinweis, darauf im Unterricht zu reagieren.

Das Kriterienraster ist insgesamt reduziert auf solche Strukturen, die aufgrund der in der Arbeit dargestellten normativen Grundlage und der Korpusanalyse als grundlegend für den historischen Vergleich angenommen werden können. Es ist jedoch möglich, dass in weniger komplexen Zusammenhängen oder solchen, in denen bestimmte Wissensstrukturen bei den Lesern vorausgesetzt werden können und nicht erst transferiert werden müssen, Erklär-Strukturen nur eine untergeordnete Rolle spielen. In diesem Fall kann die Berücksichtigung des Items entfallen. Umgekehrt können bspw. zugrundeliegende Materialien dazu führen, dass für die Aufgabenbearbeitung weitere sprachlich-kognitive Teilhandlungen eine Rolle für die Aufgabenlösung spielen, wenn z. B. aus Materialien bestimmte historische Sachverhalte erst herausgefunden müssen, weil diese nicht explizit genannt werden, und Zusammenhänge zwischen ihnen herstellt werden müssen. ${ }^{746}$ In diesem Fall müsste das Herausarbeiten ${ }^{747}$ berücksichtigt werden.

\footnotetext{
745 Dem widerspricht im untersuchten Fall jedoch die Auswertung des Items ST04.1 „Beurteilen fachlich/pragmatisch“. Die Auswertung zeigt, dass es in der überwiegenden Zahl der Lernendentexte nur teilweise gelingt, das Beurteilen zu realisieren. Dies spricht für eine zu hohe Anforderung.

${ }^{746}$ Vgl. dazu die Bestimmung des Operators „Herausarbeiten“ in den EPA Geschichte, S. 8.

${ }^{747}$ Vgl. ebd.
} 
Abschließend bleibt festzustellen, dass sowohl die Einordung in den historischen Kontext als auch die der Vergleichsaufgabe zugrundeliegenden Materialien die Bearbeitung der Aufgabe wesentlich strukturieren. Dies macht es notwendig, auf der Grundlage einer Bedarfsanalyse über das Kriterienraster hinausgehende Elemente (wie im Beispiel das Herausarbeiten) aus der Fachperspektive heraus zu bestimmen und für die Einschätzung der Textqualität einer Vergleichsaufgabe im Geschichtsunterricht zu berücksichtigen.

\subsection{Entwurf für eine Strukturierung von Vergleichstexten für die Schülerhand}

In Kapitel 2.6 konnte dargelegt werden, dass das historische Vergleichen als Textmuster ${ }^{748}$ eingeordnet werden kann. Wenn in diesem Kapitel nun der Vorschlag für eine Strukturierung gemacht wird, dann ist dieser als eine Art Schreibplan zu verstehen. Ein solches Vorgehen nimmt allerdings an, dass das Vergleichen im Geschichtsunterricht nicht nur ein Textmuster, sondern vielmehr eine feste Textsorte darstellt. Grund für dieses Vorgehen ist im Sinne einer unterrichtlichen Progression eine didaktische Entscheidung: Die zunächst feste Struktur für das Vergleichen im Geschichtsunterricht soll Schreibnovizen dabei unterstützen, strukturelles Wissen zu antizipieren, das anschließend in ein Musterwissen überführt werden kann. ${ }^{749}$ Hinter diesem Ansatz steht die Idee des lernenden Schreibens. ${ }^{750}$ Der Schreibplan soll dazu dienen, wesentliche Elemente des Vergleichs im Geschichtsunterricht offenzulegen und ein Beispiel für die Anordnung dieser Elemente geben. Es reicht jedoch nicht aus, den Lernenden die Strukturierung bereitzustellen, vielmehr müssen die Elemente besprochen, inhaltlich ausgefüllt und vor allem auf der Metaebene hinsichtlich ihres Zwecks reflektiert werden, damit sie nicht auf der Ebene deklarativen Wissens verbleiben. Gerade in Verbindung mit der Klärung der Elemente im Hinblick auf den Zweck des Vergleichs kann nach einer solchen Textsortenorientierung durch den Schreibplan eine Musterklärung angebahnt werden, die den Vergleich als Textmuster kenntlich macht. Damit würde die Annahme des Vergleichs als Textsorte ein didaktisches Hilfsmittel darstellen, über welches das Muster des historischen Vergleichs transparent gemacht werden kann. Die Realisierung dieses Musters kann dann über das Kriterienraster erfasst werden, so dass sich eine enge Verzahnung zwischen dem didaktischen Strukturvorschlag und dem Manual für die Hand der Lehrkraft ergibt. Dem Strukturierungsvorschlag liegen Reduktionsentscheidungen zugrunde, um ihn für Schreibnovizen überschaubar und anwendbar zu machen. Gleichwohl werden darin die in dieser Arbeit herausgearbeiteten wesentlichen Strukturelemente des Vergleichs im Geschichtsunterricht berücksichtigt.

Nachfolgend wird nun die Grundstruktur dargestellt. Sprachliche Mittel zur Unterstützung oder Beispielformulierungen befinden sich im Kriterienraster, sie können für die Aufarbeitung des Strukturvorschlags im Unterrichtskontext herangezogen werden.

\footnotetext{
748 Zur Bedeutung von Textformen vgl. auch Haueis, Eduard (2003) Formen schriftlicher Texte. In: Bredel, Ursula et al. (Hrsg.) Didaktik der deutschen Sprache. Ein Handbuch. Band 1. Paderborn et al.: Schöningh, S. 224-236. S. 234.

${ }^{749}$ Vgl. dazu Steinhoff (Pohl, Thorsten; Steinhoff, Torsten; 2010), S. 17.

${ }^{750}$ Vgl. ebd. S. 20.
} 
9.2.1 Eine Strukturskizze als Hilfe für Schreibnovizen

\section{Einen Vergleich im Geschichtsunterricht schreiben}

- Einleitung:

Ziel: Transparenz für den Leser schaffen

- Vergleichselemente aufnehmen:

\section{Vergleichsobjekt}

Vergleichsgröße

Tertium Comparationis

-These aufstellen

- Hauptteil:

Ziel: Mit Hilfe des Gegenüberstellens Unterschiede, Gemeinsamkeiten, Ähnlichkeiten erkennen und formulieren

\section{Gegenüberstellen \\ (mit Hilfe des Tertium Comparationis)}

Vergleichsgröße

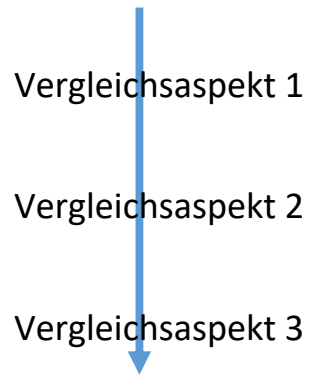

Vergleichsobjekt

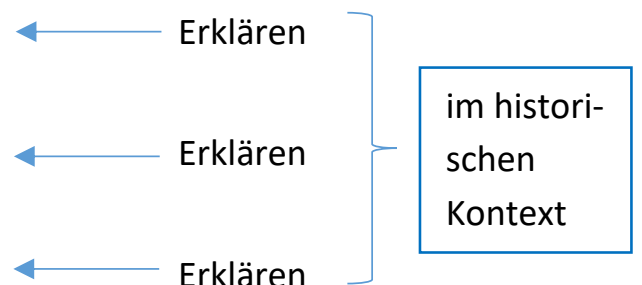

- Schluss:

Ziel: Beurteilen im historischen Kontext:

Einbettung in den historischen Kontext

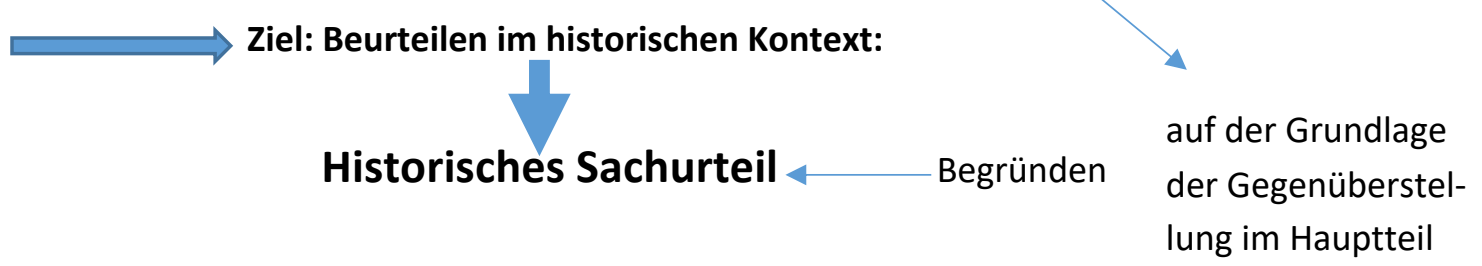

Abbildung 58: Strukturskizze für das Vergleichen im Geschichtsunterricht (eigene Darstellung) 


\subsubsection{Hinweise zur Strukturskizze}

Die Strukturskizze wurde wie das Kriterienraster aus den zuvor dargestellten Befunden abgeleitet und ist darüber fundiert. Sie ist untergliedert in Einleitung, Hauptteil und Schluss. Über die vorangestellte Zielformulierung kann die jeweilige Funktion der Textteile aufgegriffen und expliziert werden. Für die Lernenden soll zunächst deutlich werden, dass der Vergleich in der Einleitung angelegt wird, indem sowohI Vergleichsgröße, Vergleichsobjekt als auch das Tertium Comparationis explizit genannt und ins Verhältnis gesetzt werden. Hierüber kann auch die Ausgangsthese formuliert werden, die der Leserlenkung dient und am Ende überprüft werden muss. Da es sich um einen Vergleich im Geschichtsunterricht handelt, muss die Anlage des Vergleichs in der Einleitung in Bezug auf das historische Thema geleistet werden, der Vergleich also explizit in den historischen Kontext eingebettet werden.

Über die These kann in den Hauptteil übergeleitet werden, da es diese ja zu überprüfen gilt. Hier wird nun die Gegenüberstellung durchgeführt, um so Gemeinsamkeiten, Ähnlichkeiten und/oder Unterschiede herauszuarbeiten. ${ }^{751}$ Die Darstellung soll verdeutlichen, dass hier nun einzelne Vergleichsaspekte gegenübergestellt werden, die in Bezug auf beide Vergleichselemente (also Vergleichsobjekt und Vergleichsgröße) überprüft werden, um so zu einem (Teil-)Schluss zu kommen, ob hinsichtlich des überprüften Vergleichsaspekts Gemeinsamkeiten, Ähnlichkeiten oder Unterschiede für die Vergleichselemente festzustellen sind. Da hierüber das abschließende Sachurteil angebahnt wird, ist ein enger Bezug auf den historischen Kontext bedeutsam.

Es kann nicht vorausgesetzt werden, dass alle benötigten Wissenselemente dem Leser bekannt sind. Diese müssten dann unter Einbezug des historischen Kontextes erklärt werden. Insbesondere um Zusammenhänge nachvollziehbar zu machen, sind in Bezug auf die einzelnen Vergleichsaspekte Erklärungen anzufügen.

Aus der Gegenüberstellung leitet sich als Textschluss das abschließende historische Sachurteil ab. Den Lernenden muss dazu vorher bekannt sein, welches Ziel das Sachurteil hat und wodurch es gekennzeichnet ist. Diese Beurteilung wird abgesichert durch Begründungen, die durch den Hauptteil zuvor angelegt wurden.

Dieser Strukturvorschlag verzichtet auf eine eingangs zu formulierende Überschrift. Gleichwohl wird sie als wichtiges Element der Textgestaltung angesehen. Es handelt sich bei der Formulierung einer Überschrift um eine komplexe Anforderung. In der Analyse der Vergleichstexte konnte gezeigt werden, dass viele Lernende keine passende Überschrift für ihren Vergleichstext formuliert haben. Es wurde vermutet, dass die Anforderung zu hoch war, obwohl eine Überschrift ein wesentliches Element wissenschaftlichen Schreibens darstellt. Deshalb wird sie als Strukturelement hier nicht aufgeführt, da der Strukturvorschlag ihrer Komplexität nicht gerecht werden kann. Gleichwohl sollte sie im Unterricht

\footnotetext{
${ }^{751}$ Die einzelnen Aspekte, die gegenübergestellt werden sollen, müssen dazu bereits aus der Materialgrundlage herausgearbeitet worden sein. Auch hierzu benötigen Schreibnovizen in der Regel eine Anleitung, bevor sie dies selbständig leisten können. Dazu bietet sich Vorarbeit mit Hilfe einer Tabelle an, deren Funktion vorab geklärt werden muss.
} 
thematisiert und ihre Funktion geklärt werden. Für die Formulierung einer Überschrift für einen Vergleich im Geschichtsunterricht sollten dann insbesondere die folgenden Elemente berücksichtigt werden: der Hinweis auf das Thema (bezogen auf den historischen Kontext) und die Operation (Vergleich). ${ }^{752}$

Strukturvorschlag und Kriterienraster für die Lehrkraft stellen Werkzeuge für die Explizierung des Vergleichs im Geschichtsunterricht dar. Hieran muss sich nun eine Überführung dieser Ansätze in konkrete Unterrichtsplanungen oder Materialien anschließen, die im Rahmen dieser Arbeit nicht geleistet werden kann.

${ }^{752}$ Die besonderen Anforderungen, die der Formulierung von Überschriften innewohnen, arbeitet bspw. Feilke auf: Feilke, Helmuth (2010). 


\section{Zusammenfassung und weiterführende Überlegungen}

Am Beispiel der ausgewählten Vergleichsaufgabe konnte gezeigt werden, welche Herausforderung die Lösung einer solchen Aufgabe fachlich wie sprachlich an die Lernenden stellt. Insbesondere muss die Realisierung eines Vergleichs im Geschichtsunterricht vom Fach heraus gedacht werden. Nur darüber kann erklärt werden, warum in den EPA Geschichte das Beurteilen für das Vergleichen eine wesentliche Rolle spielt, in den EPA Politik hingegen nicht. Ein zentrales Ziel des Vergleichs ist die Bildung eines Sachurteils (oder je nach Aufgabe darüber hinaus auch eines Werturteils). Diese kognitive Operation des Beurteilens ist wiederum mit Sprache verknüpft, sie wird so zum Werkzeug des Denkens oder in diesem Fall des Beurteilens und letztlich des Vergleichens.

Dem historischen Vergleich kommt die Rolle einer Denkfigur zu: „Denkfiguren, so kann man festhalten, sind erkenntnisleitend, wissensorganisierend, integrativ, sie markieren epistemische Bedingungen des Wissens und sprachlicher Bedeutungskonstitution, sie verbinden wissenschaftliche und nicht-wissenschaftliche Diskurse. “753 Die genannten Bedingungen treffen auf den historischen Vergleich zu. Der Vergleich im Geschichtsunterricht nimmt die fachwissenschaftliche Ausformung des Vergleichs in didaktisierter Weise auf und macht den Vergleich so zum Lernwerkzeug. Damit dies im Sinne eines kompetenzorientierten Unterrichts gelingen kann, sind verschiedene Bedingungen notwendig, die im Folgenden auf der Grundlage der Ergebnisse der vorausgehenden Kapitel zusammengeführt werden sollen.

\subsection{Zum Aufgabenformat}

In den Kapiteln 2.2 und 5.3 wurde bereits analysiert, was kompetenzorientierte Aufgaben ausmacht und warum es sich bei der ausgewählten Vergleichsaufgabe nicht um ein kompetenzorientiertes Aufgabenformat handelt. Dies liegt, wie zuvor gezeigt, sowohl am Aufgabensetting als auch an der Formulierung der Aufgabe und spitzt sich zu in der für jede unterrichtliche Zielformulierung grundlegenden Frage: Was lernen Schüler*innen eigentlich bei der Bearbeitung dieser Aufgabe? Im besten Fall lernen sie auf der inhaltlichen Ebene etwas über den Beginn der Industrialisierung in England und ,Deutschland', so wie der Darstellungstext dies vermittelt. Sie lernen jedoch nicht, dass die dargestellten Wissenselemente und Zusammenhänge eine Konstruktion darstellen und damit kritisch zu prüfen sind z. B. im Hinblick auf die Konzepte, die sie vermitteln. Durch den weitgehenden Verzicht auf Modalisierungsformen im Darstellungstext eröffnet der Text diesen kritischen Blick gar nicht, sondern erweckt den Anschein, dass an den vermittelten Inhalten nicht zu rütteln sei. Dabei wird schon in der Aufgabenstellung von ,Deutschland' gesprochen, das Konzept dieses ,Deutschlands' ist jedoch mindestens fragwürdig, weil nicht erkennbar ist, dass es sich Ende des 18. bzw. zu Beginn des 19. Jhd. noch

\footnotetext{
753 Müller-Tamm, Jutta (2014): Die Denkfigur als wissensgeschichtliche Kategorie. In: Gess, Nicola; Janßen, Sandra: Wissensordnungen: Zu einer historischen Epistemologie der Literatur. Berlin/Boston: De Guyter. S. 100120. S. 104.
} 
längst nicht um einen Nationalstaat gehandelt hat, der inzwischen mit dem Begriff ,Deutschland' verbunden wird. Auch auf implizite Wertungen wie durch den Ausdruck „Entfesselung der Wirtschaftskräfte ${ }^{\prime 754}$ werden weder durch Text noch Aufgabe Hinweise gegeben.

Im Hinblick auf die Form des Vergleichens wird vorausgesetzt, dass den Lernenden das Textmuster geläufig ist und sie ein historisches Vergleichen bzw. ein Vergleichen im Geschichtsunterricht von einem alltäglichen Vergleichen differenzieren können. Dies ist jedoch fraglich, da das Vergleichen für den Geschichtsunterricht hinsichtlich seines Formats und seiner Anforderungen bisher nicht oder nur oberflächlich kleingearbeitet wurde. Ein wirkliches historisches Lernen, in dem Sinne, dass Schüler*innen bspw. die durch unterrichtliche Kontexte erworbenen Teilhandlungen auf diese Aufgabe anwenden, kann kaum angenommen werden, zumal auch die untersuchten Methodenseiten keine echte Lösungsstruktur für eine Vergleichsaufgabe im Geschichtsunterricht liefern. Entweder bringen Lernende eine Idee vom Vergleich im Geschichtsunterricht mit oder eben nicht. Dann (das zeigt die Analyse der Korpustexte) gelingt auch die Lösung der Aufgabe nur teilweise oder eben nicht und manche Lernende nutzen andere Strategien wie das Aneinanderreihen von Inhalten aus dem Darstellungstext. Ein offensichtliches Scheitern an der Aufgabe durch die Abgabe eines leeren Blattes (was auch in der Erhebung vorkam) kann durch solche ,Ausweichstrategien' zumindest zunächst verschleiert werden. Bleiben manche Lernende, sprachlos' und geben ein leeres Blatt ab, so schreiben andere ihre Schwierigkeiten mit der Aufgabe in kleinen Metatexten auf:

Ein Lernender/ eine Lernende aus der Erhebung 1 (unveränderter Text und Aufgabe) meldet zurück: „Ich fand die Aufgabenstellung doof, weil man genau den Text wiedergeben konnte. Also ich fand die Aufgabenstellung war nicht klar formuliert. “755 Damit fasst der oder die Lernende die Analyse von Text und Aufgabe in Kapitel 5 knapp zusammen: Er/sie zeigt mit dieser Aussage ein sehr deutliches Bewusstsein dafür, dass das Vergleichen zu einer Neustrukturierung von Wissenselementen herausfordert und eben nicht durch das Wiedergeben vorgegebener Vergleichsstrukturen erfüllt werden kann. Dieser Text hat eine Gesamtpunktzahl von 31 Punkten erhalten und liegt damit im oberen Drittel der Skala. Er ist somit als stärkerer Text einzuordnen. Dennoch bereiten dem/der Lernenden Text und Aufgabe Schwierigkeiten. Bei der Aufgabenstellung kann vermutet werden, dass sie so kompliziert erscheint, weil sie sehr komprimiert viele verschiedene Aspekte vereint, die den Fokus der Aufgabe darstellen.

Ein weiterer Lernender oder eine Lernende aus Erhebung 5 (veränderter Text, unveränderte Aufgabe als Bearbeitungsgrundlage) fügt einen längeren Kommentar als Postscriptum an:

„PS: Es ist nicht so wie du denkst,

wenn du denkst, was ich denke,

was du denkst.

\footnotetext{
${ }^{754}$ Laschewski-Müller, Karin; Rauh, Robert (Hrsg.) (2010), S. 257.

755 Metatext (auf dem Erhebungsbogen vermerkt) aus Text 1_03. Für Erhebung 1 wurden Einschätzungen zum Text im Nachgang systematisch erhoben. Es gibt also auch neutrale bis positive Rückmeldungen. Interessanter sind jedoch die kritischen Rückmeldungen, weil sie viel weniger allgemein bleiben. Eine Prüfung von Text und Art der Rückmeldung zeigt übrigens, dass es keinen eindeutigen Zusammenhang zwischen Gesamtpunktzahl und Rückmeldung gibt. Man kann deshalb nicht sagen: Je höher die Gesamtpunkzahl, desto besser die Einschätzung von Aufgabe und Text. Deshalb werden im Folgenden nur solche Aussagen betrachtet, die etwas zur Einschätzung der Anforderungen dieser beitragen können.
} 
Denn das Denken der Gedanken ist

ein gedankenloses Denken darum denke

nicht gedacht zu haben.

Wenn du versuchst daran zu denken,

nicht daran zu denken, dass du dumm bist,

obwohl du nicht dumm bist, was hast du

dann gedacht? HÄ? HÄÄ? “756

Der Kommentar zeigt, dass sich Lernende an mehr abarbeiten als an der Aufgabe, vor allem, wenn die Lösung dieser zu misslingen droht. Der zu dem Kommentar gehörende Text hat eine Gesamtpunktzahl von 16 Punkten erhalten. Damit ist er als deutlich schwächer als der zuvor genannte Text einzuordnen. Der oder die Lernende arbeitet sich an der Frage ab, was Denken eigentlich bedeutet und inwiefern davon Dummheit abzuleiten ist. Dazu nutzt er/sie einen bekannten Aphorismus als Grundlage, der hier verändert wird. Es geht in diesem Text nicht nur um die Sorge an der Aufgabe zu scheitern, sondern um die ganz grundlegende Frage nach Dummheit, die durch die Art des Textes und das darin angelegte Verwirrspiel wieder in Frage gestellt wird. Es muss deshalb vermutet werden, dass die Schwierigkeit bei der Lösung von Aufgaben ein negatives Selbstbild fördern kann. Dem muss Unterricht durch das Offenlegen von Fachprinzipien und (sprachlichen) Handlungen entgegenwirken. Für den Geschichtsunterricht bedeutet dies, Aufgabenformate zu finden, die historisches Lernen anregen und eben nicht auf der Ebene des Reproduzierens vorgegebener Wissenselemente verbleiben. Solche Aufgaben müssen darauf hin angelegt sein, mit zugrundeliegendem Material selbstständig umzugehen, es zu strukturieren, neu zusammenzusetzen, auszuwählen, um Geschichte als Konstruktion erfahrbar zu machen. Dazu gehört untrennbar die Auseinandersetzung mit den zugehörigen sprachlichen Mitteln, wie z. B. den Modalisierungsformen, über die angezeigt werden kann, welche Faktizität historischen Aussagen, Folgerungen, Schlüssen etc. zugrunde liegen.

Barricelli/Gautschi/Körber bieten 7 Prinzipien eines kompetenzorientierten Geschichtsunterrichts $\mathrm{an}^{757}$, bei denen Aufgaben eine zentrale Rolle spielen. Diese Prinzipien sollen im Hinblick auf die Vergleichsaufgabe in den Blick genommen werden. Die Autoren definieren Aufgaben im Rahmen dieser Prinzipien als ,[...] schriftlich abgefasste inhaltsbezogene Arbeitsanleitungen mit Materialien“758 und machen damit deutlich, dass es nicht ausreichend ist (wie bei der untersuchten Vergleichsaufgabe) in einer Aufgabenstellung die Aspekte zu komprimieren, die die Lernenden in den Fokus stellen sollen. Vielmehr sprechen sie von einer Arbeitsanleitung. Lernende müssen also angeleitet werden, wie sie bspw. einen Vergleich im Geschichtsunterricht anfertigen können. Dazu reicht eine Aufgabenstellung in einem Satz nicht aus. Auf das Fehlen einer konkreten Anleitung in der Vergleichsaufgabe weist der erste Schülerkommentar sehr deutlich hin.

\footnotetext{
756 Metatext aus Text 5_01.

${ }^{757}$ Vgl. Barriceli, Michele; Gautschi, Peter; Körber, Andreas (2017).

758 Ebd. 231.
} 
Weiterhin sollen kompetenzorientierte Aufgaben Lernende „[...] mit Problemstellungen [Hervorh. i. Orig.] konfrontieren, die zentrale Fragen der Gegenwart aufnehmen. ${ }^{1759}$ Auch diese ist aus der vorliegenden Aufgabe nicht zu entnehmen.

Die Autoren stellen zudem fest, dass kompetenzorientierter Unterricht individualisiert und differenziert sein müsse ${ }^{760}$, sie folgern: „Die Aufgaben sind also offen, erlauben verschiedene Lösungswege und möglicherweise sogar einander widersprechende Resultate. ${ }^{7761}$ Diesen Ansatz ermöglicht die vorliegende Aufgabe nicht, vielmehr gibt der Darstellungstext einen engen Fokus vor, wodurch das Konzept von ,Pionier' und ,Nachzügler' reproduziert werden muss.

Der Lerneffekt scheint im Verhältnis zum Umfang der Aufgabe hinsichtlich des historischen Lernens doch eher gering zu sein. Gibt es bessere Aufgabenfomate in Lehrwerken? Eine Antwort darauf kann nur eine Lehrwerksanalyse bieten.

\subsection{Zum Stellenwert einer kritischen Lehrwerkanalyse}

Die Frequenzanalyse in Kapitel 4 konnte zeigen, welche Operatoren in welchem Umfang in zugelassenen Geschichtslehrwerken der Sekundarstufe II in Nordrhein-Westfalen verwendet werden. Die Ergebnisse daraus verweisen auf die Bedeutsamkeit eines kritischen Umgangs mit Aufgabenformaten und eben auch mit Operatoren. Die Analyse konnte zeigen, inwieweit manche Operatoren überhaupt verwendet werden und damit als geübt im Hinblick auf eine Abiturprüfung gelten können. Es wurde weiterhin deutlich, dass zwischen den Lehrwerken der Einführungs- und Qualifikationsphase keine Progression erkennbar ist. Hier ist eine viel stärkere Abstimmung der Operatoren hinsichtlich ihrer Verwendung in den Lehrwerken notwendig. Es ist allerdings auch die Frage zu stellen, inwiefern dies zur Zeit überhaupt leistbar ist, da die Analyse auch eine fehlende Trennschärfe zwischen Operatoren zeigen konnte. Eine erste ausschnitthafte Untersuchung für Aufgaben in Lehrwerken der Sekundarstufe I zeigt ähnliche Ergebnisse. ${ }^{762}$

Die Frequenzanalyse konnte außerdem offenlegen, dass W-Fragen durch Operatoren fast vollständig abgelöst wurden. Dabei könnten sie im Sinne einer Arbeitsanleitung hilfreich zur Strukturierung eines übergeordneten Operators sein.

Über die Frequenzanalyse hinaus wird eine umfassende quantitative und qualitative Auswertung von Aufgaben benötigt, in der die gesamte Formulierung einer Aufgabe in den Blick genommen und hinsichtlich ihres Potentials als Lernaufgaben untersucht wird. Hieraus könnten dann auch Hinweise für Lehrkräfte generiert werden, wie sie selber Aufgaben kompetenzorientiert formulieren und gestalten können.

\footnotetext{
$759 \mathrm{Ebd}$.

760 Ebd.

${ }^{761} \mathrm{Ebd}$

${ }^{762}$ Altun, Tülay; Günther, Katrin (2015).
} 
Als wesentliche Ergebnisse der Frequenzanalyse ist festzuhalten, dass Aufgaben in den Geschichtslehrwerken viel strukturierter eingesetzt werden müssen. Dabei wäre es für die Operation des historischen Vergleichs sinnvoll, zunächst mit sprachlichen Teilhandlungen des Vergleichs zu arbeiten, wie etwa dem Gegenüberstellen, bevor das Vergleichen als Ganzes gefordert wird. Eine solche strukturierte Herangehensweise müsste in der Sekundarstufe I beginnen und in der Sekundarstufe II im Hinblick auf die Anforderungen dieser Schulstufe (etwa dem wissenschaftspropädeutischen Schreiben und Arbeiten) ausgebaut werden. Dazu müsste aber überhaupt erst einmal klar sein, welche Operatoren welche sprachlichen Teilhandlungen beinhalten und wie diese zu realisieren sind, so wie dies in dieser Arbeit für das Vergleichen vorgenommen wurde.

\subsection{Zum Umgang mit Operatoren im Geschichtsunterricht}

Die Überlegungen aus Kapitel 10.2 zeigen auf der Grundlage der Frequenzanalyse bereits deutlich die Bedeutsamkeit eines strukturierten Umgangs mit Operatoren auf. Die Untersuchung der ausgewählten Vergleichsaufgabe hat zudem gezeigt, dass es nicht genügt, Lernenden die entsprechende Operatorenbestimmung aus den EPA oder eine Umformulierung dieser (wie in einigen Lehrwerken abgedruckt) an die Hand zu geben, damit die entsprechenden Aufgaben gelöst werden können. Der Umgang mit Operatoren ist alles andere als einfach, vor allem wenn die Fachspezifik berücksichtigt wird. Die geschichtsdidaktische Diskussion der neueren Zeit bildet diese Schwierigkeit ab und legt ebenfalls einen kritischen Umgang mit den Operatoren nahe. ${ }^{763}$

Solche Schwierigkeiten können auch auf der Grundlage der Bestimmung des Begriffs ,Operator' in den EPA aufgezeigt werden:

„Operatoren sind handlungsinitiierende Verben, die signalisieren, welche Tätigkeiten beim Lösen von Prüfungsaufgaben erwartet werden. In der Regel sind sie den einzelnen Anforderungsbereichen zugeordnet. Einige Operatoren haben integrierenden Charakter, beinhalten in sich ohne weitere Differenzierung alle drei Anforderungsbereiche und formulieren übergeordnete Prüfungsaufgaben, die durch untergeordnete Teilaufgaben ergänzt werden können. ${ }^{4764}$

Diese Zuordnung ist in mehrfacher Hinsicht problematisch: Zunächst einmal gibt es hier keinen Bezug auf das Fach Geschichte. Sicherlich ist der Begriff ,Operator' zunächst dem Fach übergeordnet, dennoch fehlt der Ausblick auf die für das Fach Geschichte angenommene Fachspezifik, die grundlegend ist, wenn Operatoren für ein Fach festgelegt und bestimmt werden. Der eigentliche Kernpunkt der Kritik steckt aber im Nebensatz, dass die Operatoren signalisierten, welche Tätigkeiten von den Lernenden erwartet werden dürfen. Zwar geben die Bestimmungen einige Hinweise, eine Aufschlüsselung der erwarteten Tätigkeiten stellen sie jedoch nicht dar. Für das Vergleichen konnte gezeigt werden, dass die Teilhandlungen sehr vielfältig sind. Es stellt sich die Frage, ob die Aufschlüsselung dieser, die durch die Entwicklung des Kriterienrasters vorgenommen wurde, wirklich von vorneherein ,erwartete Tätigkeiten' darstellen. Wäre eindeutig geklärt, was die verschiedenen Operatoren ausmacht, was also

\footnotetext{
${ }^{763}$ Vgl. dazu z. B. Schönemann, Bernd et al. (2011) oder Hartung, Olaf (2013).

764 Vgl. Kultusministerkonferenz (2005): EPA Geschichte, S. 7.
} 
tatsächlich bei der Lösung einer operationalisierten Aufgabe erwartet werden darf, könnte man diese genauso aufschlüsseln wie in dieser Arbeit das Vergleichen. Dies ist bisher jedoch kaum geschehen. Eine weitere Kritik gilt der Zuordnung in die verschiedenen Anforderungsbereiche. Die EPA berücksichtigen nicht, in welchen Kontext die Operation gestellt wird. Diese kann jedoch ganz maßgeblich Auswirkungen auf den Anforderungsbereich haben: Sollen bspw. Zusammenhänge dargestellt, also erklärt werden oder ist durch den Aufgabenkontext eher ein Wiedergeben gemeint? Diese Bandbreite bilden auch Aufgaben in den Lehrwerken ab. Dabei könnte dieses Phänomen durchaus für eine unterrichtliche Progression genutzt werden, wenn Operatoren im Unterricht eingeführt und ausdifferenziert werden müssen.

Berücksichtigt man die Anzahl von 32 Operatoren nur für den Geschichtsunterricht, wird deutlich, dass die Anforderung an Lernende, diese hinsichtlich ihrer Fachspezifik zu erfassen, deutlich zu hoch ist. Auf der Grundlage der Frequenzanalyse, die gezeigt hat, dass einige Operatoren kaum, andere dagegen häufig Verwendung finden, muss diskutiert werden, welche Operatoren verzichtbar sind.

Schönemann et al. zeigen die Folgen eines indifferenten Umgangs mit den Operatoren für den Geschichtsunterricht auf, wenn sie bemängeln, dass in Abiturklausuren inhaltliche Korrekturen und Notenbegründungen häufig fehlen. ${ }^{765}$ Mit der methodischen Klärung der Operatoren (zu der unabdingbar die Klärung des Zwecks gehört) könnte hier Abhilfe geschaffen werden: Wenn Lehrkräften klar ist, welche Tätigkeiten eine Operation beinhaltet, können sie diese im Unterricht thematisieren. Solche Tätigkeiten (oder eben sprachliche Teilhandlungen) könnten außerdem in einen didaktischen Erwartungshorizont aufgenommen werden, der als Aufgabenlösung im Unterricht oder in Klausuren dienen kann. Dadurch werden Korrekturen und Bewertungen nachvollziehbarer und können so außerdem zur Lernhilfe werden. Das in Kapitel 9 vorgeschlagene Kriterienraster für die Lösung einer Vergleichsaufgabe im Geschichtsunterricht kann sowohl (etwa zusammen mit der dort vorgestellten Strukturierungshilfe) zur Klärung dienen, was einen Vergleich im Geschichtsunterricht ausmacht, es kann außerdem eine Grundlage für die Bewertung darstellen und wirkt dem von Lernenden zuweilen geäußerten Eindruck entgegen, dass die Note in gesellschaftswissenschaftlichen Fächern ,vom Lehrer' abhinge.

\subsection{Zu den Schreibprodukten}

Das Fach Geschichte als (zumindest in NRW) klassisches ,Nebenfach' bedeutet, dass in der Sekundarstufe I nur wenige Wochenstunden zur Vermittlung historischer Themen, aber auch Methoden und Arbeitstechniken zur Verfügung stehen. Als Teildisziplin des Konglomeratsfachs Gesellschaftslehre kann davon ausgegangen werden, dass sich die für Geschichte zur Verfügung stehende Zeit noch weiter verkürzt. Das bedeutet auch, dass nur wenig Zeit für das Schreiben bleibt, dem wiederum in der Sekundarstufe II eine wachsende Bedeutung zukommt, da spätestens zu diesem Zeitpunkt Klausuren geschrieben werden müssen.

${ }^{765}$ Ebd. S. 21. 
Es konnte anhand der Vergleichsaufgabe gezeigt werden, wie komplex die schriftliche Lösung einer Aufgabe sein kann. Lernende müssen dazu zunächst die Textgrundlage verstehen, sie müssen wissen, was Aufgabenformat und Operation in Bezug auf das Fach Geschichte von ihnen verlangen, und dieses Wissen schließlich in ein Textformat transferieren.

Gerade aufgrund dieser vielfältigen und hohen Anforderungen muss Schreiben auf der Grundlage von Lernaufgaben im Geschichtsunterricht systematisch und auf die Fachspezifika hin ausgerichtet angeleitet werden, auch wenn es wertvolle Unterrichtszeit kostet. Um Lernende in ihrem Schreibprozess zu unterstützen, brauchen kompetenzorientierte Schreibaufgaben die von Barricelli et al. geforderte Anleitung. ${ }^{766}$

Die vorliegenden Schreibprodukte der Lernenden sind ohne eine solche Anleitung entstanden. Zwar kann vorausgesetzt werden, dass Lernende der Sekundarstufe II im Laufe ihrer Schulzeit mit Vergleichsaufgaben in Berührung gekommen sind, es kann aber nicht davon ausgegangen werden, dass ein Vergleich im Geschichtsunterricht systematisch eingeführt wurde. Dies erklärt sicherlich einige der Schwierigkeiten, die sich in den Textprodukten zeigen und die die Lernenden manchmal in Metakommentaren thematisiert haben. Des Weiteren zeigt sich, dass auch in der gymnasialen Oberstufe bei Lernenden mit einer fortgeschrittenen Bildungsbiographie die Klärung des Zwecks einer Aufgabe (und des zugehörigen Operators) grundlegend ist. Weiterhin ist eine Profilierung von Teilhandlungen von Nöten, wie für das Vergleichen im Geschichtsunterricht vorgeschlagen, und zuletzt eine Modellierung dieser.

Aus seiner Untersuchung zum konzeptionellen Schreibhandeln im Geschichtsunterricht resümiert Hartung:

„Es konnte gezeigt werden, dass Schüler/innen beider Sekundarbereiche in einem an den Erkenntnissen der Schreibforschung ausgerichteten Geschichtsunterricht selbstständig schreibhandelnd historischen Sinn ausbilden können. Bei entsprechenden Aufgabenstellungen sind Schüler/innen in der Lage, konzeptionell über Geschichte zu schreiben, ihre Texthandlungen am kommunikativen Zweck des Textziels auszurichten und dabei auf Basis von Ausgangstexten und Vorwissen selbstständig Wissensstrukturen über historische Zusammenhänge zu generieren. ${ }^{1767}$

Damit betont Hartung zunächst, dass Lernende durchaus in der Lage sind, historischen Sinn auszubilden. Blendet man für die untersuchte Vergleichsaufgabe Setting und Aufgabenformulierung aus, könnte man den Eindruck gewinnen, dass Lernende mit einer solchen Sinnbildung überfordert seien. Solch ein Schluss ist jedoch immer erst nach der Prüfung dessen möglich, was den Lernenden überhaupt zur Verfügung gestellt wurde. In Kapitel 5 wurde bereits ausgeführt, dass es sich bei der Aufgabe zum Vergleich des Beginns der Industrialisierung in England und ,Deutschland' eben nicht um eine kompetenzorientierte Aufgabe handelt. Insofern darf es auch nicht verwundern, wenn die geforderte Urteilsbildung und damit historischen Sinnbildung kaum oder sogar gar nicht in den Texten gefunden werden konnte oder aus dem Darstellungstext übernommen wurde.

\footnotetext{
${ }^{766}$ Barricelli, Michele; Gautschi, Peter; Körber, Andreas (2017), S. 231.

767 Hartung, Olaf (2013), S. 401.
} 
Hartung gibt darüber hinaus auch Hinweise auf die Frage, wie ein schreibförderlicher Geschichtsunterricht aufgebaut werden kann: indem Ergebnisse aus dem Wissen um den Schreibprozess und der didaktischen Schreibforschung auf den Geschichtsunterricht übertragen werden. Dies kann in der konkreten Umsetzung bedeuten, dass der Schreibprozess in seine Teilprozesse aufgeschlüsselt wird und Lernende schon beim Planen, dann aber auch beim Formulieren und Überarbeiten ihres Textes unterstützt werden. Außerdem stellt auch Hartung die Ausrichtung am kommunikativen Zweck als bedeutsam für das Gelingen einer Schreibhandlung heraus. Das Ergebnis aus Erhebungsteil 3 der vorliegenden Korpusanalyse verweist gerade auf diesen Aspekt. Obwohl die Lernenden vor der eigentlichen Lösung der Schreibaufgabe eine Tabelle ausfüllen sollten, die das Gegenüberstellen im eigentlichen Vergleichstext vorbereiten sollte, sind die Textprodukte nicht besser als die derjenigen, die diese Unterstützung nicht hatten. Ein Schüler oder eine Schülerin formuliert sogar in seinem/ihrem Metakommentar: "Meinung Ich finde den Text zu „zäh" und verstehe nicht wirklich das Prinzip dieser Tabelle." "768 Weder für das Vergleichen noch für das durch die Tabelle angelegte Gegenüberstellen wurde eine Zweckklärung vorgenommen. Dies verhindert die Wahrnehmung der Tabelle als Unterstützungsmittel. Erst das Zusammenspiel aus Unterstützungsmitteln und Reflexion dieser auf der Metaebene kann die Aufgabenlösung unterstützen.

\subsection{Zum wissenschaftspropädeutischen Schreiben von Texten in der Sekundar- stufe II}

Ebenso bedeutsam wie die explizite Auseinandersetzung mit den Operatoren ist gerade für den Unterricht in der Sekundarstufe II die Thematisierung von Merkmalen und Werkzeugen des (vor-)wissenschaftlichen Schreibens im Geschichtsunterricht. Dies zeigt auch die Analyse der Lernendentexte, in denen nur wenig mit Distanzierungsmitteln gearbeitet wird oder in denen die Kennzeichnung von Übernahmen aus dem Text kaum erfolgt.

Die Auseinandersetzung mit solchen Mitteln des wissenschaftlichen Arbeitens und damit auch des wissenschaftlichen Schreibens kann über die der Aufgaben zugrundeliegenden Texte angeregt werden. Die Berücksichtigung einer fachlichen Perspektive für das Schreiben im Geschichtsunterricht der Sekundarstufe II ist gerade für diskursive Textsorten hoch bedeutsam. Dies hat bestimmte Entscheidungen für die Didaktisierung und Explizierung dieser im Unterricht zur Folge:

Mittel zum Verfassen (vor-)wissenschaftlicher Texte und sprachliche Teilhandlungen stehen nicht nebeneinander, sondern müssen als zusammengehörig gedacht werden ${ }^{769}$. Dies bedeutet für den Unterricht, dass Werkzeuge des wissenschaftlichen Arbeitens und sprachliche Teilhandlungen in einen Zusammenhang gebracht werden müssen, statt sie isoliert voneinander zu vermitteln. In der Analyse der vorliegenden Lernendentexte fällt bspw. auf, dass es den Lernenden, die die Vergleichsaufgabe gelöst haben, schwerfällt, Übernahmen aus dem Darstellungstext zu kennzeichnen, sich also von dem dort

\footnotetext{
${ }^{768}$ Korpustext 6_07.

${ }^{769}$ Vgl. dazu auch Hartung, Olaf (2013), S. 92.
} 
Gesagten zu distanzieren. In dem Moment, wo jedoch etwa durch den Vergleich ein Sachurteil herbeigeführt werden soll, also beurteilt und begründet werden muss, ist eine solche Distanzierung essentiell. Diese verbindende Herangehensweise stellt also wieder die Klärung des Zwecks an den Anfang. Es reicht für den Unterricht von Geschichte nicht, das wissenschaftspropädeutische Arbeiten in Verbindung mit bestimmten Erarbeitungsformen wie bspw. dem Referat zu bringen (vgl. dazu Kap. 2.4). Vielmehr wird ein Geschichtsunterricht benötigt, der etwa im Sinne Pandels wissenschaftspropädeutisches Arbeiten als „Erkenntnisverfahren “770 erfahrbar macht. Dazu müsste eine viel differenziertere Aufnahme und klare Füllung des Begriffs „Wissenschaftspropädeutisches Arbeiten“ in den Lehrplänen erfolgen, auf deren Grundlage die Arbeit an didaktischen Planungen für den konkreten Unterricht ausgestaltet werden kann.

770 Pandel, Hans-Jürgen (2012), S. 183. 


\section{Ableitungen: Welche Lehr- und Lernmaterialien werden für den Vergleich im Geschichtsunterricht benötigt, die fachliches und sprachliches Lernen fördern?}

Aus den vorliegenden Befunden lässt sich ableiten, welches Textmusterwissen von Lernenden gefordert wird, um eine Vergleichsaufgabe im Geschichtsunterricht zu lösen, und welche Strategien Lernende zur Lösung der ausgewählten Aufgabe nutzen. Aus diesen Ergebnissen konnte wiederum ein Kriterienraster für die Lehrkraft und ein Schreibplan zum Umgang mit dem Vergleichen im Geschichtsunterricht entwickelt werden. Beide sind sowohl quantitativ fundiert in den Ergebnissen der Korpusanalyse mit der anschließenden Faktorenanalyse sowie qualitativ abgeleitet aus der Analyse der drei Lernendentexte.

Welche Lehr- und Lernmaterialien werden neben einem solchen Kriterienraster und dem Schreibplan für einen Gesellschaftslehre- und Geschichtsunterricht benötigt, die das Vergleichen sprachlich und fachlich fördern? Folgende Bedingungen lassen sich auf der Grundlage der Untersuchung und unter Hinzuziehung der ,Checkliste Lernaufgaben ${ }^{1771}$ zur Beantwortung dieser Frage ausmachen:

- Lernende benötigen sowohl ein Verständnis für den Zweck einer historischen Vergleichsaufgabe als auch eine Anleitung, damit sie ein Musterwissen für die Operation des historischen Vergleichs aufbauen können. Beides sollte in der Konzeption von Material berücksichtigt werden.

- Für die vorliegende Aufgabe kann festgestellt werden, dass sie erst durch den Aufgabenkontext zu einer geschichtlichen Vergleichsaufgabe wird. Darüber hinaus sollten im Aufgabenformat die von Thünemann als genuin geschichtsspezifisch ausgemachten Kriterien wiederzufinden $\operatorname{sein}^{772}$. Sie wurden hier für das Format des historischen Vergleichens leicht abgewandelt: Dazu gehören eine historische Leitfrage, die den Lernprozess strukturiert und über eine Faktendarstellung hinausgeht, sowie die Möglichkeit einer echten historischen Urteilsbildung, die nicht durch das Material schon vorgegeben wird. Für das Vergleichen muss es sich zumindest um ein begründetes Sachurteil handeln, über das in einem weiteren Schritt das von Thünemann geforderte Werturteil angebahnt werden kann. Schließlich muss die Chance für eine historische Reflexion eröffnet werden, durch die Lernende ihren Weg reflektieren, der sie zum abschließenden Urteil geführt hat. Diese Kriterien führen unweigerlich zu dem Schluss, dass die Formulierung einer geschichtlichen Vergleichsaufgabe in ein oder zwei Sätzen nicht ausreichend ist, sondern eine kompetenzorientierte Vergleichsaufgabe eine Anleitung benötigt. Für das Verfertigen einer solchen Anleitung kann der Schreibplan eine Grundlage bieten.

- Der zugrundeliegende Darstellungstext sollte keine Vergleichsstrukturen oder Teilvergleiche vorwegnehmen, die die Lernenden nur wiedergeben müssen (vgl. dazu auch die Kritik durch den Metakommentar eines/einer Lernenden in Kapitel 10.1). Vielmehr muss das Material

\footnotetext{
${ }^{771}$ Vgl. Köster, Manuel; Bernhardt, Markus; Thünemann, Holger (2016), S. 7. / abgedruckt in Kap. 2.2.2, Abb. 4.

772 Thünemann, Holger (2013), S. 142.
} 
Lernenden die Möglichkeit bieten, selbstständig Wissenselemente, die sie etwa für die Gegenüberstellung nutzen können, auszuwählen. Dazu kann ein Setting hilfreich sein, das unterschiedliche Texte anbietet, aus denen die Lernenden wählen können.

Bei der Auswahl oder Gestaltung solcher Texte können neben genuin geschichtsdidaktischen Forschungsergebnissen auch die Ergebnisse aus der didaktischen Schreibforschung zum materialgestützten Schreiben Ansätze bieten, um so eine echte Weiterverarbeitung von Wissenselementen in einen historischen Vergleich anzuregen. An eine solche Arbeitsform müssen Lernende durch unterrichtliche Progression herangeführt werden.

- Bevor es um die Erarbeitung des eigentlichen Vergleichs geht, ist die Sicherung des historischinhaltlichen Wissens, das sehr häufig über Texte aufgebaut wird, nötig. Dies bedeutet zudem, die Klärung von Textsorte und Musterwissen zu leisten sowie Leseverständnis zu sichern, z. B. durch den Einsatz von Lesestrategien ${ }^{773}$. Der der ausgewählten Vergleichsaufgabe zugrundeliegende Darstellungstext beinhaltet bspw. bildhafte Sprache, deren Bedeutung eingeordnet werden müsste. Ebenso müssen für diesen Text implizite argumentative Strukturen herausgearbeitet werden, so dass sie für Lernende erkennbar werden. Um Leseverständnis sichern zu können, muss zunächst die Textgrundlage analysiert und didaktisch bspw. durch die Anwendung von Lesestrategien aufbereitet werden.

- Unterrichtsmaterial für den historischen Vergleich muss insbesondere die Operation aufschlüsseln und damit die sprachlichen Teilhandlungen klären: Warum bspw. sind für den historischen Vergleich Begründungen hilfreich und an welcher Stelle sind sie einzusetzen, warum ist eine Reihung von Wissenselementen kein strukturelles Element des Vergleichs? Dazu gehört auch immer die Berücksichtigung des konkreten geschichtlichen Kontextes. Fordert der Kontext die Berücksichtigung weiterer Teilhandlungen, können diese entsprechend ergänzt werden und so beim notwendigen Aufbau kontextgebundenen thematischen Wissens unterstützen.

- Sprachliche Hilfen wie Satzbausteine, Strukturhilfen, Begriffssammlungen etc. sollten erst nach der Klärung von Zweck und Textmuster eingesetzt werden, damit sie als wirkliche Hilfe wahrgenommen werden können (im Gegensatz zur in der Erhebung eingesetzten Tabelle, bei der auf eine Zweckklärung verzichtet wurde und die nicht zu besseren Vergleichstexten geführt hat).

Dies sind nicht wenige Anforderungen, die deutlich machen, dass Geschichtsunterricht auch Schreibunterricht ist. Diese Anforderungen gilt es nun umzusetzen in konkrete Settings für das Vergleichen im Geschichtsunterricht und neue echte kompetenzorientierte Aufgabenformate.

Dabei ist dieses Vorgehen zentral für die Förderung fachsprachlicher Kompetenzen für alle Schüler*innen und Aufgabe von Schule und Geschichtsunterricht auch in der Sekundarstufe II.

\footnotetext{
773 Zum Umgang mit Lesestrategien legt Handro Vorschläge vor, um diese geschichtsdidaktisch zu profilieren: Handro, Saskia (2018): Geschichte lesen, aber wie? Pladoyer für eine geschichtsdidaktische Profilierung von Lesestrategien. In: Sandkühler, Thomas; Bühl-Gramer, Charlotte; John, Anke; Schwabe, Astrid; Bernhardt, Markus (Hrsg.): Geschichtsunterricht im 21. Jahrhundert. Bonn: Bundeszentrale für politische Bildung. S. 275-293.
} 
Diese auf das Vergleichen im Geschichtsunterricht bezogenen Überlegungen sind übertragbar auf andere Aufgaben bzw. Operationen im Unterrichtsfach Geschichte. Sollen Operatoren die Ausbildung historischer Kompetenzen anregen, ist wie für das Vergleichen eine Aufarbeitung dieser nötig. Dazu bietet sich eine fächerübergreifende Perspektive an, die fachliches und sprachliches Lernen aus einem kommunikativen Ansatz heraus verbindet und im Hinblick auf den konkreten Geschichtsunterricht ausdifferenziert. 


\section{Anhang}

\section{Verzeichnis der Anhänge:}

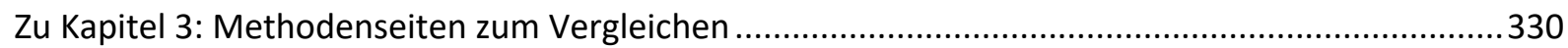

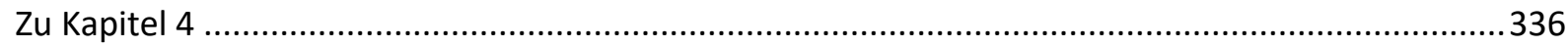

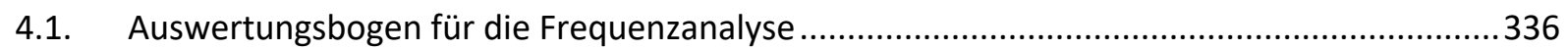

4.2 Liste der ausgezählten Schulbücher nach Erscheinungsjahr:..............................................340

4.3 Aufschlüsselung der ausgewerteten Lehrwerke für die Darstellungen in Kapitel 4.2.1: ........342

4.4 Aufschlüsselung der ausgewerteten Lehrwerke für die Darstellungen in Kapitel 4.2.2: ........343

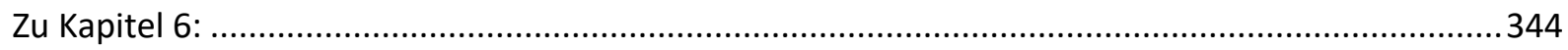

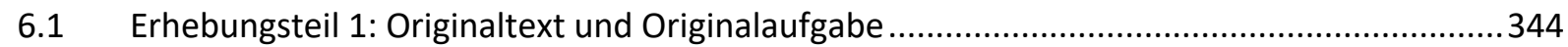

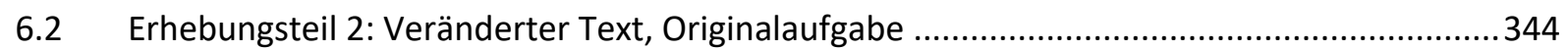

6.3 Erhebungsteil 3: Veränderter Text, veränderte Aufgabe (Scaffold) ......................................349

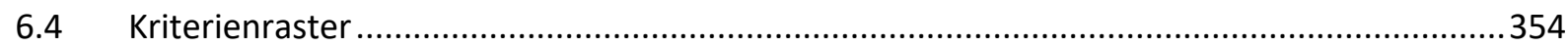

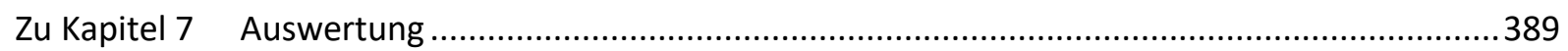

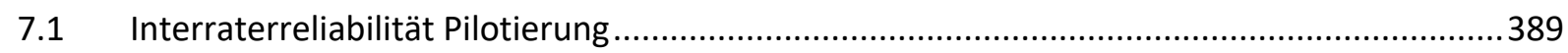

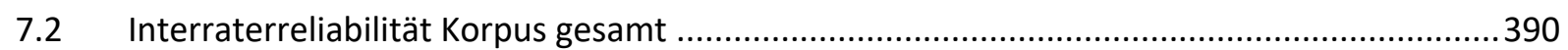

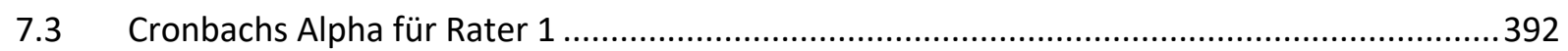

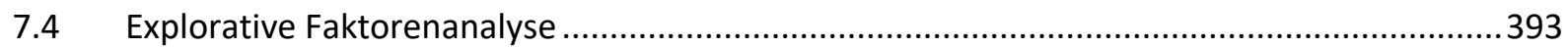

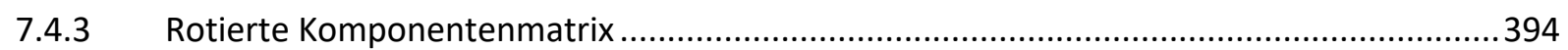

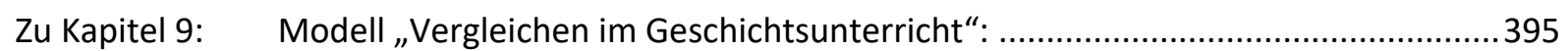

9.1 Zuordnung der Ratings zu den Kompetenzeniveaus ..............................................................395 


\section{Zu Kapitel 3: Methodenseiten zum Vergleichen}

\begin{tabular}{|c|c|}
\hline Lehrwerkstitel & Methodenhinweise zum Vergleichen \\
\hline $\begin{array}{l}\text { Bage, Claudia et.al. (2012): Anstöße - } \\
\text { Gesellschaftslehre mit Geschichte. Be- } \\
\text { rufliche Gymnasien. Stuttgart: Ernst } \\
\text { Klett Verlag. }\end{array}$ & $\begin{array}{l}\text { Bestimmung der Operatoren entspr. Lehrplan Politik im } \\
\text { Einband }\end{array}$ \\
\hline $\begin{array}{l}\text { Bahr, Frank (2006): Horizonte I. Ge- } \\
\text { schichte für die Oberstufe. Von der grie- } \\
\text { chischen Antike bis zur Frühen Neuzeit. } \\
\text { Braunschweig: Bildungshaus Schulbuch- } \\
\text { verlage (Westermann). }\end{array}$ & - \\
\hline $\begin{array}{l}\text { Bahr, Frank (2006): Horizonte II. Ge- } \\
\text { schichte für die Oberstufe. Von der grie- } \\
\text { chischen Antike bis zur Frühen Neuzeit. } \\
\text { Braunschweig: Bildungshaus Schulbuch- } \\
\text { verlage (Westermann). }\end{array}$ & - \\
\hline $\begin{array}{l}\text { Bahr, Frank (2006): Horizonte III. Ge- } \\
\text { schichte für die Oberstufe. Von der grie- } \\
\text { chischen Antike bis zur Frühen Neuzeit. } \\
\text { Braunschweig: Bildungshaus Schulbuch- } \\
\text { verlage (Westermann). }\end{array}$ & - \\
\hline $\begin{array}{l}\text { Bender, Daniela u.a. (2010): Geschichte } \\
\text { und Geschehen. Neuzeit. Oberstufe. } \\
\text { Leipzig: Ernst Klett Schulbuchverlag. }\end{array}$ & - \\
\hline $\begin{array}{l}\text { Brütting, Rolf u.a. (2002): Geschichte } \\
\text { Geschehen exempla. Geschichtliches } \\
\text { Unterrichtswerk für die Sekundarstufe } \\
\text { II. Leipzig: Ernst Klett Schulbuchverlag. }\end{array}$ & - \\
\hline $\begin{array}{l}\text { Edinger, Thomas u.a. (2011): Forum Ge- } \\
\text { schichte/Gemeinschaftskunde. Lern- } \\
\text { und Arbeitsbuch für berufliche Gymna- } \\
\text { sien, Jahrgangsstufe } 1 \text { und 2. 2. Auflage. } \\
\text { Köln: Bildungsverlag } 1 .\end{array}$ & - \\
\hline $\begin{array}{l}\text { Günther-Arndt, Hilke u.a. (2006): Ge- } \\
\text { schichtsbuch Oberstufe, Band 1: Von } \\
\text { der Antike bis zum Ende des 19. Jahr- } \\
\text { hunderts. Berlin: Cornelsen Verlag. }\end{array}$ & - \\
\hline
\end{tabular}




\begin{tabular}{|c|c|}
\hline $\begin{array}{l}\text { Günther-Arndt, Hilke u.a. (2006): Ge- } \\
\text { schichtsbuch Oberstufe, Band 2: Das } 20 . \\
\text { Jahrhundert. Berlin: Cornelsen Verlag. }\end{array}$ & - \\
\hline $\begin{array}{l}\text { Kochendörfer, Jürgen (Hrsg.): Ge- } \\
\text { schichte und Geschehen (2010). Berufs- } \\
\text { kolleg. Stuttgart: Ernst Klett Verlag. }\end{array}$ & - \\
\hline $\begin{array}{l}\text { Kochendörfer, Jürgen (Hrsg). (2003): } \\
\text { Geschichte Geschehen. Berufliche Ober- } \\
\text { stufe. Ernst Klett-Verlag. }\end{array}$ & - \\
\hline $\begin{array}{l}\text { Laschewski-Müller, Karin; Rauh, Robert } \\
\text { (Hrsg.) (2010). Kursbuch Geschichte. } \\
\text { Neue Ausgabe Nordrhein-Westfalen. } \\
\text { Von der Antike bis zur Gegenwart. Ber- } \\
\text { lin: Cornelsen-Verlag. }\end{array}$ & $\begin{array}{l}\text { S. 488: Methodenseiten zu „Interpretation und Vergleich } \\
\text { schriftlicher Quellen“, ähnlich wie in der Neuauflage }\end{array}$ \\
\hline $\begin{array}{l}\text { Lendzian, Hans-Jürgen (Hrsg.) (2007): } \\
\text { Zeiten und Menschen. Geschichte Ober- } \\
\text { stufe. Band 1. Braunschweig, Pader- } \\
\text { born, Darmstadt: Schöningh Verlag. }\end{array}$ & - \\
\hline $\begin{array}{l}\text { Lendzian, Hans-Jürgen (Hrsg.) (2006): } \\
\text { Zeiten und Menschen. Geschichte Ober- } \\
\text { stufe. Band 2. Braunschweig, Pader- } \\
\text { born, Darmstadt: Schöningh Verlag. }\end{array}$ & $\begin{array}{l}\text {-Methodenseiten S. 291f zu: „Historischer Vergleich“ } \\
\text { Verfassertext zur Unterscheidung synchroner und dia- } \\
\text { chroner Vergleiche, dann folgen „Schritte des histori- } \\
\text { schen Vergleichs“, Aufschlüsselung in } 5 \text { Schritte: } \\
\text { 1. Leitfragen und Vergleichstyp klären bzw. festlegen, } 2 . \\
\text { Einzelphänomene in ihrer besonderen historischen Be- } \\
\text { dingtheit klären und beschreiben, 3. Vergleichsaspekte } \\
\text { festlegen, 4. Vergleichen, 5. Vergleichsergebnisse beur- } \\
\text { teilen und bewerten } \\
\text {-keine Aufschlüsselung sprachlicher Mittel }\end{array}$ \\
\hline $\begin{array}{l}\text { Lendzian, Hans-Jürgen (Hrsg.) (2011): } \\
\text { Zeiten und Menschen. Geschichte Ein- } \\
\text { führungsphase Oberstufe. Nordrhein- } \\
\text { Westfalen. Braunschweig, Paderborn, } \\
\text { Darmstadt: Schöningh Verlag. }\end{array}$ & $\begin{array}{l}\text {-Methodenkasten zu „Lesekompetenz - Historische Texte } \\
\text { aspektorientiert lesen, systematisch auswerten und ver- } \\
\text { gleichen“ (S. 40f): Hauptaspekt liegt auf Lesen und aus- } \\
\text { werten; 5. Schritt: Vergleichen, dazu werden die Unter- } \\
\text { punkte } \\
\text { „-Prinzipielle Unterschiede und Gegensätze aufzeigen. } \\
\text {-Übereinstimmungen und Ähnlichkeiten aufzeigen. } \\
\text {-Positionen erklären und beurteilen“ genannt. (S. 40f) } \\
\text {-Methodenkasten zu „Historische Urteile analysieren und } \\
\text { vergleichen (S. 70) }\end{array}$ \\
\hline
\end{tabular}




\begin{tabular}{|c|c|}
\hline & $\begin{array}{l}\text {-Methodenkasten zu: „Historischer Vergleich“ (S. 263) } \\
\text { (wie in Auflage von 2006) } \\
\text { Verfassertext zur Unterscheidung synchroner und dia- } \\
\text { chroner Vergleiche, dann folgen „Schritte des histori- } \\
\text { schen Vergleichs“, Aufschlüsselung in } 5 \text { Schritte: } \\
\text { 1. Leitfragen und Vergleichstyp klären bzw. festlegen, } 2 . \\
\text { Einzelphänomene in ihrer besonderen historischen Be- } \\
\text { dingtheit klären und beschreiben, 3. Vergleichsaspekte } \\
\text { festlegen, 4. Vergleichen, 5. Vergleichsergebnisse beur- } \\
\text { teilen und bewerten } \\
\text {-keine Aufschlüsselung sprachlicher Mittel }\end{array}$ \\
\hline $\begin{array}{l}\text { Rauh, Robert (2011): Grundwissen Ge- } \\
\text { schichte Sekundarstufe II. Berlin: Cor- } \\
\text { nelsen Verlag. }\end{array}$ & $\begin{array}{l}\text { Teilkapitel zu „Vergleich von Sekundärtexten“ (S. 273): } \\
\text { Hinweise zur Bedeutung des Vergleichens, dann tabellari- } \\
\text { sche Übersicht: } \\
\text { 1. Leitfragen, 2. Analyse der Texte (inhaltlich und formal), } \\
\text { 3. Historischer Kontext, 4. Urteilen: Sachurteil (z. B. wel- } \\
\text { cher Text ist aus welchen Gründen überzeugender; Wert- } \\
\text { urteil: Bewertung aus heutiger Sicht. } \\
\text { Wenig zum Vergleichen an sich, nur in Urteilen z.T. abge- } \\
\text { bildet }\end{array}$ \\
\hline $\begin{array}{l}\text { Schade, Peter; Stark, Hans-Joachim; Ma- } \\
\text { yer, Ines (2010): Geschichte in der Ge- } \\
\text { genwart. Lehr- und Arbeitsbuch für Ge- } \\
\text { schichte und Gemeinschaftskunde/Sozi- } \\
\text { alkunde in der gymnasialen Oberstufe. } \\
\text { Troisdorf: Bildungsverlag Eins. }\end{array}$ & - \\
\hline $\begin{array}{l}\text { von Reeken, Dietmar (2012): Ge- } \\
\text { schichtskultur - Module für die Ober- } \\
\text { stufe. Berlin: Cornelsen Verlag. }\end{array}$ & $\begin{array}{l}\text { Im Anhang gibt es unter dem Kapitel „Arbeitsaufträge in } \\
\text { der Abiturklausur“ Operatorendef. (S. 134f), auf S. } 136 \\
\text { folgen Formulierungshilfen; zum Vergleich von Texten: } \\
\text { gibt es Satzbausteine zu „Übereinstimmung“ und „Ge- } \\
\text { gensatz“ }\end{array}$ \\
\hline $\begin{array}{l}\text { Arand, Tobias et al. (2011): Geschichte } \\
\text { und Geschehen Oberstufe NRW. Ge- } \\
\text { samtband. Stuttgart/Leipzig: Ernst Klett } \\
\text { Verlag. }\end{array}$ & $\begin{array}{l}\text { Operatorenliste: Hinweise zum Vergleichen (S. 640): Auf- } \\
\text { schlüsselung in „Erläuterung“ (EPA-Def.), Arbeitsschritte } \\
\text { (5 Schritte), Hinweise auf Aufgabenbeispiele, eine visuelle } \\
\text { Merkhilfe („Apfel und Birne“) sowie Hinweise auf Leistun- } \\
\text { gen im AFB III allgemein. } \\
\text { Zu den } 5 \text { Schritten: Aufschlüsselung des Vorgehens: } \\
\text {-Wiedergabe der zu vergleichenden Sachverhalte } \\
\text {-Nennen der Vergleichsaspekte }\end{array}$ \\
\hline
\end{tabular}




\begin{tabular}{|c|c|}
\hline & $\begin{array}{l}\text {-wesentliche von unwesentlichen Vergleichsaspekten } \\
\text { trennen } \\
\text {-gleiche, ähnliche und unterschiedliche Merkmale her- } \\
\text { ausstellen } \\
\text {-abschließende Einschätzung zur Vergleichbarkeit formu- } \\
\text { lieren } \\
\text { in der Operatorenliste wird unter „Aufgabenbeispiele“ } \\
\text { auf ein Operatorentraining zum Vergleichen hingewiesen } \\
\text { (S. 373): } \\
\text { Aufgabe: „Vergleichen Sie Mommsens und Wehlers Im- } \\
\text { perialismustheorien hinsichtlich ihrer Nähe oder Distanz } \\
\text { zu Lenins Imperialismustheorie“, Hinweise auf Material } \\
\text { zur Aufgabe sowie zum Operator: } \\
\text { Zunächst wird die EPA-Definition indirekt zitiert, dann } \\
\text { wird darauf hingewiesen, dass es zwei grundlegende } \\
\text { Dinge zu beachten gelte: } 1 \text {. die Bestandteile des Ver- } \\
\text { gleichs (Gemeinsamkeiten und Unterschiede nennen, Fa- } \\
\text { zit), } \\
\text { 2. Vergleich anhand eines klaren Kriteriums (Tertium } \\
\text { Comparationis) wird erläutert. } \\
\text { Es fehlt ein Bezug auf das Historische des Vergleichs }\end{array}$ \\
\hline \multicolumn{2}{|l|}{ nach neuem Kernlehrplan } \\
\hline $\begin{array}{l}\text { Baumgärtner, Ulrich et al. (Hrsg.) } \\
\text { (2014): Horizonte Geschichte Einfüh- } \\
\text { rungsphase. Sekundarstufe II Nord- } \\
\text { rhein-Westfalen, Braunschweig: West- } \\
\text { ermann. }\end{array}$ & - \\
\hline $\begin{array}{l}\text { Baumgärtner, Ulrich et al. (Hrsg.) } \\
\text { (2015): Horizonte Geschichte Qualifika- } \\
\text { tionsphase. Sekundarstufe II Nordrhein- } \\
\text { Westfalen, Braunschweig: Westermann. }\end{array}$ & $\begin{array}{l}\text { Methodentraining zu „Analyse und Vergleich von Darstel- } \\
\text { lungen - Ein Beispiel mit einem Lösungsvorschlag; } \\
\text { dabei werden Darstellungen, jedoch keine DT verglichen: } \\
\text { Ausschnitte aus Monographien zweier Historiker (S. } \\
\text { 206ff); } \\
\text { Aufschlüsselung in Bearbeitungsschritte: } \\
\text { „Schritt 1: Historische Darstellungen analysieren“ } \\
\text { „Schritt 2: Erläuterungen und historische Einordnung der } \\
\text { Sachverhalte, auf die die Texte Bezug nehmen.“ }\end{array}$ \\
\hline
\end{tabular}




\begin{tabular}{|c|c|}
\hline & $\begin{array}{l}\text { „Schritt 3: Vergleich beider Darstellungen“: Allgemeine } \\
\text { Hinweise zu Inhalten und Aufbau eines Vergleichs, An- } \\
\text { nahme, dass der Vergleich in ein Fazit mündet und in ei- } \\
\text { nem abschließenden Urteil die Beurteilungsmaßstäbe } \\
\text { und Kriterien offengelegt würden. Dies wird jedoch nicht } \\
\text { weiter konkretisiert. } \\
\text { Die Darstellung geht aus vom Vergleich mind. zweier } \\
\text { Texte zu einem gemeinsamen Thema. }\end{array}$ \\
\hline $\begin{array}{l}\text { Dzubiel, Christiane et al (Hrsg.) (2014): } \\
\text { Geschichte und Geschehen. Oberstufe } \\
\text { Einführungsphase Nordrhein-Westfalen. } \\
\text { Ernst Klett Verlag. }\end{array}$ & $\begin{array}{l}\text { Hinweise in Operatorenübersicht (S. 247), grobes Schema } \\
\text { zum Vorgehen in fünf Unterpunkten, keine gesonderte } \\
\text { Methodenseite }\end{array}$ \\
\hline $\begin{array}{l}\text { Droste, Peter Johannes u.a. (Hrsg.) } \\
\text { (2015): Geschichte und Geschehen. } \\
\text { Oberstufe Qualifikationsphase. Nord- } \\
\text { rhein-Westfalen. Ernst Klett Verlag. }\end{array}$ & $\begin{array}{l}\text { Innerhalb einer aufklappbaren Doppelseite + Einband fin- } \\
\text { den sich die wichtigsten Informationen zum Umgang mit } \\
\text { dem Lehrwerk „Geschichte und Geschehen“ } \\
\text { Im Anhand dann detaillierte Auflistung der Anforderungs- } \\
\text { bereiche und der dazugehörigen Operatoren mit Erläute- } \\
\text { rung und Arbeitsschritten } \\
\text { Vorangehend Einweisung in den Begriff des Operators } \\
\text { und seine Rolle und Auslegung speziell im Fach Ge- } \\
\text { schichte } \\
\text { Innerhalb der Einführung in das Lehrwerk allerdings } \\
\text { kein Verweis auf die hinteren Seiten } \\
\text { auch innerhalb der einzelnen Buchkapitel keine Metho- } \\
\text { denseiten oder Aufgabenstellungen, in denen auf Metho- } \\
\text { den und Methodenseiten verwiesen wird. } \\
\text { Am Ende des Lehrwerkes Aufzeigen verschiedener Ar- } \\
\text { beitsschritte, z. B. für die Interpretation von Karikaturen } \\
\text { oder Bildquellen, aber innerhalb des gesamten Buches } \\
\text { keinerlei Hinweise auf die Existenz dieser Seiten. } \\
\text { und: Seiten noch hinter dem Begriffs- und Personen- } \\
\text { glossar und dem Bildquellennachweis }\end{array}$ \\
\hline $\begin{array}{l}\text { Lanzinner, Maximilian (Hrsg.) (2014): } \\
\text { Buchners Geschichte Oberstufe. Aus- } \\
\text { gabe Nordrhein-Westfalen, Einfüh- } \\
\text { rungsphase, Bamberg: C.C. Buchner. }\end{array}$ & - \\
\hline
\end{tabular}




\begin{tabular}{|c|c|}
\hline $\begin{array}{l}\text { Laschewski-Müller, Karin/Rauh, Robert } \\
\text { (Hrsg.) (2014): Kursbuch Geschichte. } \\
\text { Nordrhein-Westfalen Einführungsphase, } \\
\text { Berlin: Cornelsen. }\end{array}$ & $\begin{array}{l}\text { Methodenseiten zu „Schriftliche Quellen vergleichen“ (S. } \\
\text { 146f), vergleichbar mit nächstem Titel }\end{array}$ \\
\hline $\begin{array}{l}\text { Laschewski-Müller, Karin/Rauh, Robert } \\
\text { (Hrsg.) (2014): Kursbuch Geschichte. } \\
\text { Nordrhein-Westfalen Qualifikations- } \\
\text { phase, Berlin: Cornelsen } 2015 .\end{array}$ & $\begin{array}{l}\text { Methodenseite zu „Darstellungen vergleichen“ (S. 90f). } \\
\text { Zur Methodenklärung: nur S. 90, auf S. } 91 \text { folgt Bsp. } \\
\text { Einführungstext mit Hinweisen zu Perspektivität und } \\
\text { Kontroversität und der Notwendigkeit der Diskussion. } \\
\text { Dann folgen Arbeitsschritte. Dabei stehen jedoch allge- } \\
\text { meine Analyseaspekte im Vordergrund (Leitfrage, Ana- } \\
\text { lyse von Darstellungsart, Texten, Zielgruppe etc., Histori- } \\
\text { scher Kontext). Hinweise auf den eigentlichen Vergleich: } \\
\text { „Unter welchen Gesichtspunkten lassen sich die Texte } \\
\text { vergleichen?“ (S.90). Dann Urteil: gefordert werden Sach- } \\
\text { und Werturteil }\end{array}$ \\
\hline $\begin{array}{l}\text { Lendzian, Hans-Jürgen (Hrsg.) (2014): } \\
\text { Zeiten und Menschen. Geschichte Ein- } \\
\text { führungsphase Oberstufe Nordrhein- } \\
\text { Westfalen, Braunschweig u.a.: Schö- } \\
\text { ningh Verlag. }\end{array}$ & $\begin{array}{l}\text { Methodenseite zu „Historische Darstellungen analysieren } \\
\text { und kritisch vergleichen“ (S. 101), Aufschlüsselung ver- } \\
\text { schiedener Arbeitsschritte mit Textbausteinen zu Leitfra- } \\
\text { gen, Analyse (Vorstellung des Textauszugs), strukturierte } \\
\text { Textwiedergabe. Der eigentliche Vergleich wird allerdings } \\
\text { nur sehr kurz thematisiert: } \\
\text { „- Zum Inhalt (Thesen, Kernaussagen, Argumente) } \\
\text { des/der Texte(s) kritisch Stellung nehmen, um zu Ansät- } \\
\text { zen für eine eigene Einschätzung oder ein eigenes Urteil } \\
\text { zu gelangen. } \\
\text { - Die Ausführungen der Autoren vergleichen.“ (ebd.) }\end{array}$ \\
\hline $\begin{array}{l}\text { Lendzian, Hans-Jürgen (Hrsg.) (2015): } \\
\text { Zeiten und Menschen. Geschichte Qua- } \\
\text { lifikationsphase Oberstufe Nordrhein- } \\
\text { Westfalen, Braunschweig u.a.: Schö- } \\
\text { ningh Verlag. }\end{array}$ & - \\
\hline
\end{tabular}




\section{Zu Kapitel 4}

\subsection{Auswertungsbogen für die Frequenzanalyse}

Operatoren und Aufgabenstellungen in Geschichtsbüchern der Sek. II/gymnasiale Oberstufe

Titel:

Anforderungsbereich I

\begin{tabular}{|l|l|l|l|l|}
\hline Operatoren & $\begin{array}{l}\text { Nennung allge- } \\
\text { mein }\end{array}$ & $\begin{array}{l}\text { Nennung in Be- } \\
\text { zug auf Darstel- } \\
\text { lungstexte in Ver- } \\
\text { bindung mit an- } \\
\text { deren Materia- } \\
\text { lien }\end{array}$ & $\begin{array}{l}\text { Nenng in Be- } \\
\text { zungstexte ohne } \\
\text { luarstel- } \\
\text { Baterialien } \\
\text { Menf andere }\end{array}$ & Bemerkungen \\
\hline $\begin{array}{l}\text { nennen } \\
\text { aufzählen }\end{array}$ & & & & \\
\hline $\begin{array}{l}\text { bezeichnen } \\
\text { schildern } \\
\text { skizzieren }\end{array}$ & & & & \\
\hline $\begin{array}{l}\text { aufzeigen } \\
\text { beschreiben } \\
\text { zusammenfassen } \\
\text { wiedergeben }\end{array}$ & & Gesamt: & Gesamt: & \\
\hline Statistik & Gesamt: & & & \\
\hline
\end{tabular}

Anforderungsbereich II

\begin{tabular}{|l|l|l|l|l|}
\hline Operatoren & $\begin{array}{l}\text { Nennung allge- } \\
\text { mein }\end{array}$ & $\begin{array}{l}\text { Nennung in Be- } \\
\text { zug auf Darstel- } \\
\text { lungstexte in Ver- } \\
\text { bindung mit an- } \\
\text { deren Materia- } \\
\text { lien }\end{array}$ & $\begin{array}{l}\text { zenng in Be- } \\
\text { lungstexte ohne } \\
\text { Bezug auf andere } \\
\text { Materialien }\end{array}$ & Bemerkungen \\
\hline $\begin{array}{l}\text { analysieren } \\
\text { untersuchen }\end{array}$ & & & & \\
\hline $\begin{array}{l}\text { begründen } \\
\text { nachweisen }\end{array}$ & & & & \\
\hline charakterisieren & & & & \\
\hline einordnen & & & & \\
\hline
\end{tabular}




\begin{tabular}{|l|l|l|l|l|}
\hline erklären & & & & \\
\hline erläutern & & & & \\
\hline herausarbeiten & & & & \\
\hline gegenüberstellen & & & & \\
\hline widerlegen & & & & \\
\hline Statistik & Gesamt: & Gesamt: & Gesamt: & \\
\hline & & & & \\
\hline
\end{tabular}

Anforderungsbereich III

\begin{tabular}{|l|l|l|l|l|}
\hline Operatoren & $\begin{array}{l}\text { Nennung allge- } \\
\text { mein }\end{array}$ & $\begin{array}{l}\text { Nennung in Be- } \\
\text { zug auf Darstel- } \\
\text { lungstexte in Ver- } \\
\text { bindung mit an- } \\
\text { deren Materia- } \\
\text { lien }\end{array}$ & $\begin{array}{l}\text { zug auf Darstel- } \\
\text { lungstexte ohne } \\
\text { Bezug auf andere } \\
\text { Materialien }\end{array}$ & Bemerkungen \\
\hline beurteilen & & & & \\
\hline $\begin{array}{l}\text { bewerten } \\
\text { Stellung nehmen }\end{array}$ & & & & \\
\hline entwickeln & & & & \\
\hline $\begin{array}{l}\text { sich auseinander- } \\
\text { setzen } \\
\text { diskutieren }\end{array}$ & & & & \\
\hline $\begin{array}{l}\text { prüfen } \\
\text { überprüfen }\end{array}$ & & & & \\
\hline vergleichen & & Gesamt: & Gesamt: & \\
\hline Statistik & Gesamt: & & & \\
\hline & & & & \\
\hline
\end{tabular}

\section{„Breitbandoperatoren“}

\begin{tabular}{|l|l|l|l|l|}
\hline Operatoren & $\begin{array}{l}\text { Nennung allge- } \\
\text { mein }\end{array}$ & $\begin{array}{l}\text { Nennung in Be- } \\
\text { zug auf Darstel- } \\
\text { lungstexte in Ver- } \\
\text { bindung mit an- } \\
\text { deren Materia- } \\
\text { lien }\end{array}$ & $\begin{array}{l}\text { Nenng in Be- } \\
\text { lungstexte ohne } \\
\text { Bezug auf andere } \\
\text { Materialien }\end{array}$ & Bemerkungen \\
\hline erörtern & & & & \\
\hline interpretieren & & & & \\
\hline darstellen & & & & \\
\hline
\end{tabular}




\begin{tabular}{|l|l|l|l|l|}
\hline Statistik & Gesamt: & Gesamt: & Gesamt: & \\
\hline & & & & \\
\hline
\end{tabular}

Weitere Operatoren, die im Schulbuch verwendet werden

\begin{tabular}{|c|c|c|c|c|}
\hline Operatoren & $\begin{array}{l}\text { Nennung allge- } \\
\text { mein }\end{array}$ & $\begin{array}{l}\text { Nennung in Be- } \\
\text { zug auf Darstel- } \\
\text { lungstexte in Ver- } \\
\text { bindung mit an- } \\
\text { deren Materia- } \\
\text { lien }\end{array}$ & $\begin{array}{l}\text { Nennung in Be- } \\
\text { zug auf Darstel- } \\
\text { lungstexte ohne } \\
\text { Bezug auf andere } \\
\text { Materialien }\end{array}$ & Bemerkungen \\
\hline & & & & \\
\hline \multicolumn{5}{|l|}{$\ldots$} \\
\hline \multirow[t]{2}{*}{ Statistik } & Gesamt: & Gesamt: & Gesamt: & \\
\hline & $\begin{array}{l}\text { Gesamtzahl der } \\
\text { Operatoren }\end{array}$ & $\begin{array}{l}\text { Gesamtzahl der } \\
\text { Darstellungstexte } \\
\text { in Verbindung } \\
\text { mit anderen Ma- } \\
\text { terialien }\end{array}$ & $\begin{array}{l}\text { Gesamtzahl der } \\
\text { Darstellungstexte } \\
\text { ohne Bezug auf } \\
\text { andere Materia- } \\
\text { lien }\end{array}$ & \\
\hline & & & & \\
\hline
\end{tabular}

Platz für Anmerkungen: 


\begin{tabular}{|c|c|c|c|c|}
\hline W-Frage & $\begin{array}{l}\text { Nennung allgemein } \\
\text { (Gesamtzahl der } \\
\text { Aufgaben mit W- } \\
\text { Fragen) }\end{array}$ & $\begin{array}{l}\text { Nennung in Bezug } \\
\text { auf Darstellungs- } \\
\text { texte in Verbin- } \\
\text { dung mit anderen } \\
\text { Materialien }\end{array}$ & $\begin{array}{l}\text { Nennung in Bezug } \\
\text { auf Darstellungs- } \\
\text { texte ohne Bezug } \\
\text { auf andere Materi- } \\
\text { alien }\end{array}$ & $\begin{array}{l}\text { Bemer- } \\
\text { kungen }\end{array}$ \\
\hline \multicolumn{5}{|l|}{ wer } \\
\hline \multicolumn{5}{|l|}{ was } \\
\hline \multicolumn{5}{|l|}{ wann } \\
\hline \multicolumn{5}{|l|}{ wo } \\
\hline \multicolumn{5}{|l|}{ wie } \\
\hline \multicolumn{5}{|l|}{ warum } \\
\hline \multicolumn{5}{|c|}{$\begin{array}{l}\text { welche/wel- } \\
\text { cher/welches }\end{array}$} \\
\hline \multicolumn{5}{|c|}{ inwiefern } \\
\hline \multicolumn{5}{|c|}{ inwieweit } \\
\hline \multicolumn{5}{|l|}{ worin } \\
\hline \multicolumn{5}{|l|}{ worauf } \\
\hline \multicolumn{5}{|l|}{ wodurch } \\
\hline \multicolumn{5}{|c|}{ in welcher Weise } \\
\hline Statistik & Gesamt: & Gesamt: & Gesamt: & \\
\hline
\end{tabular}

Quantitativer Vergleich Operatoren/W-Fragen:

Gesamtzahl: Operatoren und W-Fragen:

Anzahl Operatoren: __ (Anteil an Gesamtzahl der Fragen:__ \%)

Anzahl W-Fragen:___ (Anteil an Gesamtzahl der Fragen:__ \%)

Bei __ Seiten (Gesamtzahl einschl. Glossar etc.) entspricht dies __ Aufgaben pro Seite

Anteil der Aufgaben, die sich auf Darstellungstexte beziehen (Spalte 3): __ (entspricht __ \%) 


\subsection{Liste der ausgezählten Schulbücher nach Erscheinungsjahr:}

vor 2014

\begin{tabular}{|c|c|}
\hline Erscheinungsjahr & Titel \\
\hline 2002 & $\begin{array}{l}\text { Brütting, Rolf u.a. (2002): Geschichte Geschehen exempla. Geschichtliches Un- } \\
\text { terrichtswerk für die Sekundarstufe II. Leipzig: Ernst Klett Schulbuchverlag. }\end{array}$ \\
\hline 2003 & $\begin{array}{l}\text { Kochendörfer, Jürgen (Hrsg). (2003): Geschichte Geschehen. Berufliche Ober- } \\
\text { stufe. Ernst Klett-Verlag. }\end{array}$ \\
\hline 2006 & $\begin{array}{l}\text { Bahr, Frank (2006): Horizonte I. Geschichte für die Oberstufe. Von der griechi- } \\
\text { schen Antike bis zur Frühen Neuzeit. Braunschweig: Bildungshaus Schulbuch- } \\
\text { verlage (Westermann). }\end{array}$ \\
\hline 2006 & $\begin{array}{l}\text { Bahr, Frank (2006): Horizonte III. Geschichte für die Oberstufe. Von der Weima- } \\
\text { rer Republik bis zum Beginn des 21. Jahrhunderts. Braunschweig: Bildungshaus } \\
\text { Schulbuchverlage (Westermann). }\end{array}$ \\
\hline 2006 & $\begin{array}{l}\text { Günther-Arndt, Hilke u.a. (2006): Geschichtsbuch Oberstufe, Band 1: Von der } \\
\text { Antike bis zum Ende des 19. Jahrhunderts. Berlin: Cornelsen Verlag (unverän- } \\
\text { derte 1. Auflage von 1995). }\end{array}$ \\
\hline 2006 & $\begin{array}{l}\text { Günther-Arndt, Hilke u.a. (2006): Geschichtsbuch Oberstufe, Band 2: Das } 20 . \\
\text { Jahrhundert. Berlin: Cornelsen Verlag (unveränderte 1. Auflage von 1995). }\end{array}$ \\
\hline 2006 & $\begin{array}{l}\text { Lendzian, Hans-Jürgen (Hrsg.) (2006): Zeiten und Menschen. Geschichte Ober- } \\
\text { stufe. Geschichtswerk für das Gymnasium (G8) in Nordrhein-Westfalen. Band } 2 . \\
\text { Braunschweig, Paderborn, Darmstadt: Schöningh Verlag. }\end{array}$ \\
\hline 2007 & $\begin{array}{l}\text { Lendzian, Hans-Jürgen (Hrsg.) (2007): Zeiten und Menschen. Geschichte Ober- } \\
\text { stufe. Geschichtswerk für das Gymnasium (G8) in Nordrhein-Westfalen. Band } 1 . \\
\text { Braunschweig, Paderborn, Darmstadt: Schöningh Verlag. }\end{array}$ \\
\hline 2009 & $\begin{array}{l}\text { Baumgärtner, Ulrich; Fieberg, Klaus (Hrsg.) (2009): Horizonte III. Geschichte } \\
\text { Gymnasium Nordrhein-Westfalen. Braunschweig: Bildungshaus Schulbuchver- } \\
\text { lage (Westermann). }\end{array}$ \\
\hline 2010 & $\begin{array}{l}\text { Bender, Daniela u.a. (2010): Geschichte und Geschehen. Neuzeit. Oberstufe } \\
\text { Leipzig: Ernst Klett Schulbuchverlag. }\end{array}$ \\
\hline 2010 & $\begin{array}{l}\text { Kochendörfer, Jürgen (Hrsg.): Geschichte und Geschehen (2010). Berufskolleg. } \\
\text { Stuttgart: Ernst Klett Verlag. }\end{array}$ \\
\hline 2010 & $\begin{array}{l}\text { Laschewski-Müller, Karin; Rauh, Robert (Hrsg.) (2010). Kursbuch Geschichte. } \\
\text { Neue Ausgabe Nordrhein-Westfalen. Von der Antike bis zur Gegenwart. Berlin } \\
\text { Cornelsen-Verlag. }\end{array}$ \\
\hline 2010 & $\begin{array}{l}\text { Schade, Peter; Stark, Hans-Joachim; Mayer, Ines (2010): Geschichte in der Ge- } \\
\text { genwart. Lehr- und Arbeitsbuch für Geschichte und Gemeinschaftskunde/Sozi- } \\
\text { alkunde in der gymnasialen Oberstufe. Troisdorf: Bildungsverlag Eins. }\end{array}$ \\
\hline
\end{tabular}




\begin{tabular}{|l|l|}
\hline 2011 & $\begin{array}{l}\text { Droste, Peter-Johannes (Hrsg.) (2011): Geschichte und Geschehen. Oberstufe } \\
\text { Nordrhein-Westfalen. Stuttgart: Ernst Klett-Verlag. }\end{array}$ \\
\hline 2011 & $\begin{array}{l}\text { Edinger, Thomas u.a. (2011): Forum Geschichte/Gemeinschaftskunde. Lern- } \\
\text { und Arbeitsbuch für berufliche Gymnasien, Jahrgangsstufe 1 und 2. 2. Auflage. } \\
\text { Köln: Bildungsverlag 1. }\end{array}$ \\
\hline 2012 & $\begin{array}{l}\text { Bage, Claudia et.al. (2012): Anstöße - Gesellschaftslehre mit Geschichte. Beruf- } \\
\text { liche Gymnasien. Stuttgart: Ernst Klett Verlag. }\end{array}$ \\
\hline
\end{tabular}

nach 2014

\begin{tabular}{|l|l|}
\hline Erscheinungsjahr & Titel \\
\hline 2014 & $\begin{array}{l}\text { Baumgärtner, Ulrich (Hrsg.) (2014): Horizonte Geschichte Einführungsphase. } \\
\text { Sekundarstufe II Nordrhein-Westfalen, Braunschweig: Westermann. }\end{array}$ \\
\hline 2015 & $\begin{array}{l}\text { Baumgärtner, Ulrich (Hrsg.) (2015): Horizonte Geschichte. Oberstufe Qualifika- } \\
\text { tionsphase NRW. Braunschweig: Westermann. }\end{array}$ \\
\hline 2014 & $\begin{array}{l}\text { Dzubiel, Christiane u.a. (Hrsg.) (2014): Geschichte und Geschehen. Oberstufe } \\
\text { Einführungsphase Nordrhein-Westfalen. Stuttgart: Klett. }\end{array}$ \\
\hline 2015 & $\begin{array}{l}\text { Droste, Peter Johannes (Hrsg.) (2015): Geschichte und Geschehen. Qualifikati- } \\
\text { onsphase Oberstufe Nordrhein-Westfalen. Stuttgart: Klett. }\end{array}$ \\
\hline 2015 & $\begin{array}{l}\text { Lanzinner, Maximilian (Hrsg.) (2014): Buchners Geschichte Oberstufe. Ausgabe } \\
\text { Nordrhein-Westfalen Einführungsphase. Bamberg: C.C. Buchner. }\end{array}$ \\
\hline 2014 & $\begin{array}{l}\text { Langendorf, Elke (Hrsg.) (2015): Buchners Geschichte Oberstufe. Ausgabe } \\
\text { Nordrhein-Westfalen Qualifikationsphase. Bamberg: C.C. Buchner. }\end{array}$ \\
\hline 2015 & $\begin{array}{l}\text { Laschewski-Müller, Karin / Rauh, Robert (Hrsg.) (2014): Kursbuch Geschichte. } \\
\text { Nordrhein-Westfalen Einführungsphase. Berlin: Cornelsen. }\end{array}$ \\
\hline 2014 & $\begin{array}{l}\text { Laschewski-Müller, Karin; Rauh, Robert (Hrsg.) (2015): Kursbuch Geschichte. } \\
\text { Qualifikationsphase Nordrhein-Westfalen. Berlin: Cornelsen }\end{array}$ \\
\hline $\begin{array}{l}\text { Lendzian, Hans-Jürgen (Hrsg.) (2014): Zeiten und Menschen. Geschichte Einfüh- } \\
\text { rungsphase NRW. Braunschweig: Schöning. }\end{array}$ \\
$\begin{array}{l}\text { Lendzian, Hans-Jürgen (2015) (Hrsg.): Zeiten und Menschen. Qualifikations- } \\
\text { phase Oberstufe Nordrhein-Westfalen. Paderborn: Schöningh Verlag. }\end{array}$ \\
\hline
\end{tabular}




\subsection{Aufschlüsselung der ausgewerteten Lehrwerke für die Darstellungen in Ka-}

pitel 4.2.1:

vor 2014

\begin{tabular}{|c|c|}
\hline Nr. & Titel \\
\hline 1 & $\begin{array}{l}\text { Bage, Claudia et.al. (2012): Anstöße - Gesellschaftslehre mit Geschichte. Berufliche Gym- } \\
\text { nasien. Stuttgart: Ernst Klett Verlag. }\end{array}$ \\
\hline 2 & $\begin{array}{l}\text { Bahr, Frank (2006): Horizonte I. Geschichte für die Oberstufe. Von der griechischen An- } \\
\text { tike bis zur Frühen Neuzeit. Braunschweig: Bildungshaus Schulbuchverlage (Wester- } \\
\text { mann). }\end{array}$ \\
\hline 3 & $\begin{array}{l}\text { Bahr, Frank (Hrsg.) (2006): Horizonte II. Geschichte für die Oberstufe. Vom Absolutismus } \\
\text { bis zum Ersten Weltkrieg. Braunschweig: Westermann. }\end{array}$ \\
\hline 4 & $\begin{array}{l}\text { Bahr, Frank (2006): Horizonte III. Geschichte für die Oberstufe. Von der Weimarer Re- } \\
\text { publik bis zum Beginn des 21. Jahrhunderts. Braunschweig: Bildungshaus Schulbuchver- } \\
\text { lage (Westermann). }\end{array}$ \\
\hline 5 & $\begin{array}{l}\text { Bender, Daniela u.a. (2010): Geschichte und Geschehen. Neuzeit. Oberstufe. Leipzig: } \\
\text { Ernst Klett Schulbuchverlag. }\end{array}$ \\
\hline 6 & $\begin{array}{l}\text { Brütting, Rolf u.a. (2002): Geschichte Geschehen exempla. Geschichtliches Unterrichts- } \\
\text { werk für die Sekundarstufe II. Leipzig: Ernst Klett Schulbuchverlag. }\end{array}$ \\
\hline 7 & $\begin{array}{l}\text { Droste, Peter-Johannes (Hrsg.) (2011): Geschichte und Geschehen. Oberstufe Nordrhein- } \\
\text { Westfalen. Stuttgart: Ernst Klett-Verlag. }\end{array}$ \\
\hline 8 & $\begin{array}{l}\text { Edinger, Thomas u.a. (2011): Forum Geschichte/Gemeinschaftskunde. Lern- und Arbeits- } \\
\text { buch für berufliche Gymnasien, Jahrgangsstufe } 1 \text { und 2. 2. Auflage. Köln: Bildungsverlag } \\
\text { 1. }\end{array}$ \\
\hline 9 & $\begin{array}{l}\text { Günther-Arndt, Hilke u.a. (2006): Geschichtsbuch Oberstufe, Band 1: Von der Antike bis } \\
\text { zum Ende des 19. Jahrhunderts. Berlin: Cornelsen Verlag (unveränderte 1. Auflage von } \\
\text { 1995). }\end{array}$ \\
\hline 10 & $\begin{array}{l}\text { Günther-Arndt, Hilke u.a. (2006): Geschichtsbuch Oberstufe, Band 2: Das 20. Jahrhun- } \\
\text { dert. Berlin: Cornelsen Verlag (unveränderte 1. Auflage von 1995). }\end{array}$ \\
\hline 11 & $\begin{array}{l}\text { Kochendörfer, Jürgen (Hrsg). (2003): Geschichte Geschehen. Berufliche Oberstufe. Ernst } \\
\text { Klett-Verlag. }\end{array}$ \\
\hline 12 & $\begin{array}{l}\text { Kochendörfer, Jürgen (Hrsg.): Geschichte und Geschehen (2010). Berufskolleg. Stuttgart: } \\
\text { Ernst Klett Verlag. }\end{array}$ \\
\hline 13 & $\begin{array}{l}\text { Laschewski-Müller, Karin; Rauh, Robert (Hrsg.) (2010). Kursbuch Geschichte. Neue Aus- } \\
\text { gabe Nordrhein-Westfalen. Von der Antike bis zur Gegenwart. Berlin: Cornelsen-Verlag. }\end{array}$ \\
\hline 14 & $\begin{array}{l}\text { Lendzian, Hans-Jürgen (Hrsg.) (2007): Zeiten und Menschen. Geschichte Oberstufe. Ge- } \\
\text { schichtswerk für das Gymnasium (G8) in Nordrhein-Westfalen. Band 1. Braunschweig, } \\
\text { Paderborn, Darmstadt: Schöningh Verlag. }\end{array}$ \\
\hline
\end{tabular}




\begin{tabular}{|l|l|}
\hline 15 & $\begin{array}{l}\text { Lendzian, Hans-Jürgen (Hrsg.) (2006): Zeiten und Menschen. Geschichte Oberstufe. Ge- } \\
\text { schichtswerk für das Gymnasium (G8) in Nordrhein-Westfalen. Band 2. Braunschweig, } \\
\text { Paderborn, Darmstadt: Schöningh Verlag. }\end{array}$ \\
\hline 16 & $\begin{array}{l}\text { Schade, Peter; Stark, Hans-Joachim; Mayer, Ines (2010): Geschichte in der Gegenwart. } \\
\text { Lehr- und Arbeitsbuch für Geschichte und Gemeinschaftskunde/Sozialkunde in der gym- } \\
\text { nasialen Oberstufe. Troisdorf: Bildungsverlag Eins. }\end{array}$ \\
\hline
\end{tabular}

\subsection{Aufschlüsselung der ausgewerteten Lehrwerke für die Darstellungen in Ka-} pitel 4.2.2:

\section{ab 2014}

\begin{tabular}{|l|l|}
\hline Nr. & Titel \\
\hline 1 & $\begin{array}{l}\text { Baumgärtner, Ulrich (Hrsg.) (2014): Horizonte Geschichte Einführungsphase. Sekundar- } \\
\text { stufe Il Nordrhein-Westfalen, Braunschweig: Westermann. }\end{array}$ \\
\hline 2 & $\begin{array}{l}\text { Baumgärtner, Ulrich (Hrsg.) (2015): Horizonte Geschichte. Oberstufe Qualifikationsphase } \\
\text { NRW. Braunschweig: Westermann. }\end{array}$ \\
\hline 3 & $\begin{array}{l}\text { Droste, Peter Johannes (Hrsg.) (2015): Geschichte und Geschehen. Qualifikationsphase } \\
\text { Oberstufe Nordrhein-Westfalen. Stuttgart: Klett. }\end{array}$ \\
\hline 5 & $\begin{array}{l}\text { Dzubiel, Christiane u.a. (Hrsg.) (2014): Geschichte und Geschehen. Oberstufe Einfüh- } \\
\text { rungsphase Nordrhein-Westfalen. Stuttgart: Klett. }\end{array}$ \\
\hline 6 & $\begin{array}{l}\text { Langendorf, Elke (Hrsg.) (2015): Buchners Geschichte Oberstufe. Ausgabe Nordrhein- } \\
\text { Westfalen Qualifikationsphase. Bamberg: C.C. Buchner. }\end{array}$ \\
\hline 7 & $\begin{array}{l}\text { Lanzinner, Maximilian (Hrsg.) (2014): Buchners Geschichte Oberstufe. Ausgabe Nord- } \\
\text { rhein-Westfalen Einführungsphase. Bamberg: C.C. Buchner. }\end{array}$ \\
\hline 8 & $\begin{array}{l}\text { Waschewski-Müller, Karin; Rauh, Robert (Hrsg.) (2015): Kursbuch Geschichte. Qualifikati- } \\
\text { onsphase Nordrhein-Westfalen. Berlin: Cornelsen }\end{array}$ \\
\hline 9 & $\begin{array}{l}\text { Lendzian, Hans-Jürgen (Hrsg.) (2014): Zeiten und Menschen. Geschichte Einführungs- } \\
\text { phase NRW. Braunschweig: Schöning. }\end{array}$ \\
\hline 10 & $\begin{array}{l}\text { Lendzian, Hans-Jürgen (2015) (Hrsg.): Zeiten und Menschen. Qualifikationsphase Ober- } \\
\text { stufe Nordrhein-Westfalen. Paderborn: Schöningh Verlag. }\end{array}$ \\
\hline
\end{tabular}




\section{Zu Kapitel 6:}

\subsection{Erhebungsteil 1: Originaltext und Originalaufgabe}

Im Erhebungsteil 1 wurde der in Kapitel 5 vorgestellte originale Schulbuchtext mit der dort ebf. abgedruckten Aufgabe eingesetzt (s. S.178-188).

\subsection{Erhebungsteil 2: Veränderter Text, Originalaufgabe}

Ausgangsbedingungen für die Industrialisierung in England und Deutschland im ausgehenden 18. und beginnenden 19. Jahrhundert

-Die Anfänge der Industrialisierung-

\section{Text 1}

England

Die Industrialisierung begann im England des ausgehenden 18. Jahrhunderts und erfasste im beginnenden 19. Jahrhundert den westlichen Teil des europäischen Kontinents sowie Nordamerika. Während der zweiten Hälfte des 19. Jahrhunderts strahlte sie auf Osteuropa und Japan aus. Im 20. Jahrhundert entfaltete sie dann ihre Wirksamkeit in der gesamten Welt dieser Prozess dauert bis in die Gegenwart an.

England wird wegen des frühen Beginns der Industrialisierung oft eine „Pionierrolle“ zugesprochen.

Die Beantwortung der Frage, warum England eine Pionierrolle im Industrialisierungsprozess einnahm, ist also eng verknüpft mit den Ursachen der Industriellen Revolution. Historiker nennen mehrere begünstigende Umstände und Vorgänge, die unabhängig voneinander das englische Wirtschaftswachstum beschleunigt haben. Zu den wichtigsten gehören die schnellen Fortschritte in der Landwirtschaft, die die stark wachsende Bevölkerung ernähren und ihr einen gewissen Wohlstand garantieren konnte. Die Bevölkerungszunahme und die Tatsache, dass die Landbevölkerung nicht an die Scholle gebunden war, sondern in den Städten nach neuen Betätigungsfeldern suchte, sorgten sowohl für ein großes Angebot an Arbeitskräften als auch für eine steigende Güternachfrage auf dem englischen Binnenmarkt. England besaß außerdem große und leicht abzubauende Kohlevorkommen, kurze und kostengünstige Verkehrswege und ausreichend Kapital zum Investieren. Hinzu kam, dass der Staat den Unternehmern durch innere Reformen die für die Betätigung notwendigen Freiräume verschaffte. Zudem erlaubte die relativ offene Gesellschaftsstruktur des Königreichs flexible Reaktionen auf die unterschiedlichsten wirtschaftlichen Herausforderungen. 
Aufsehenerregende Erfindungen wie die Dampfmaschine von Watt (1765/69) oder die Spinnmaschinen von Hargreaves („Spinning Jenny“ 1764$)$ und Arkwright (1769) beschleunigten das englische Wirtschaftswachstum. Mit der Mechanisierung der Baumwollspinnerei, die zum ersten Führungssektor in der englischen Industriegeschichte aufstieg, begann das Zeitalter der Massenproduktion im Textilgewerbe. Für die Entfesselung der Wirtschaftskräfte noch bedeutsamer wurde jedoch das Vordringen des Energieträgers Kohle und der damit einhergehende Ausbau der Eisenindustrie. Die Verbilligung und Verbesserung des Eisens sowie die Modernisierung der Produktionsverfahren schufen die Voraussetzungen für die Entstehung einer leistungsfähigen Maschinenindustrie und später für den Eisenbahnbau. Damit wirkte die Eisenindustrie in viele andere Wirtschaftszweige hinein und veränderte vom Verkehrswesen bis zu individuellen Reisen alle Bereiche des gesellschaftlichen Lebens. 
Text 2

\section{Deutschland}

\section{Ausgangsbedingungen}

Deutschland wird oft als "Nachzügler“ in der Industrialisierungsgeschichte eingeordnet. Historiker gehen davon aus, dass dies mit der Ausgangssituation in Deutschland zu tun hat. Bis zum Ende des Heiligen Römischen Reiches Deutscher Nation im Jahre 1806 war Deutschland in 300 zum Teil ausgesprochen kleine Territorialstaaten zersplittert. Eine Vielzahl von Zollschranken, abweichende Maß-, Münz- und Gewichtssysteme, Handelsmonopole sowie schlecht erschlossene Verkehrsverbindungen hemmten die wirtschaftliche Expansion. Trotz mancher Fortschritte bei der Agrarproduktion gab es viele ertragsschwache Kleinbetriebe (ca. 70-80 \% aller Höfe), deren Betreiber oft einem Nebenerwerb nachgehen mussten, um ihre Existenz zu sichern. Die Abhängigkeiten der bäuerlichen Bevölkerung von ihren Gutsherren waren häufig noch so stark, dass dadurch die zur Bildung freier Arbeitsmärkte notwendige individuelle Mobilität eingeschränkt war. Feudale Abgaben, staatliche Steuern und große Unterschiede bei der Verteilung des Wohlstandes behinderten die Entstehung von Massenkaufkraft, die der gewerblichen Wirtschaft hätten zugute kommen können. Auch war die deutsche Gesellschaftsstruktur unflexibel. Starre Standesschranken und konservative Grundeinstellungen engten den Spielraum für innovatorisches Denken und Handeln ein. Und im Handwerk bildete das Festhalten an der überkommenen Zunftverfassung ein zentrales Hindernis für individuelles Erfolgsstreben und wirtschaftliche Neuerungen. Die absolutistischen deutschen Fürsten gängelten mit ihren merkantilistischen Konzepten die wirtschaftliche Entwicklung durch massive Eingriffe und Beschränkungen; staatsfreie Märkte für Kapital, Boden und Waren konnten sich daher nur schwer entfalten.

\section{Staatliche Reformen}

Erst im Verlauf des 19. Jahrhunderts wurden diese Hemmnisse für eine dynamische Industriegesellschaft und -wirtschaft allmählich beseitigt. Dabei nahm der Staat eine herausragende Rolle ein. Durch die Liberalisierung der Agrar- und Gewerbeverfassung, den Abbau von Zollschranken oder die Vereinheitlichung des Rechts- und Finanzwesens schuf er die Voraussetzungen zur Entfesselung einer modernen Wirtschafts- und für den Übergang zur modernen Marktgesellschaft.

Entscheidende Grundlagen dafür wurden in den preußischen Reformen während der ersten beiden Jahrzehnte des 19. Jahrhunderts gelegt, die das Überleben und den Wiederaufbau des von den napoleonischen Armeen besiegten Landes garantieren sollten. Die Reformbeamten, die sich seit 1807 an die Modernisierung des preußischen Staates begaben, kannten nicht nur die englische Entwicklung. Auch „die Bibel des Kapitalismus“, Adam Smiths Buch 
über den „Wohlstand der Nationen“ („The Wealth of Nations") hatte bei den Reformkräften in Deutschland begeisterte Aufnahme gefunden. Die Leitbegriffe dieses Werks, allen voran „Besitzindividualismus“, „Leistungsprinzip“, „Arbeitsteilung“, „freie Märkte“ und „Konkurrenz" prägten daher die Wirtsschafts- und Gesellschaftsreformen auf dem Kontinent. Das zeigte sich am preußischen Oktoberedikt von 1807, das eine Mischung aus politischem Manifest und national-ökonomischem Programm darstellte. An die Stelle einer gebundenen Ständegesellschaft, die jedem Menschen eine feste, durch Geburt und Recht erworbene soziale Position zuwies, sollte nun eine mobile Marktgesellschaft treten. Dazu passten auch die Ablösung sozialer Abhängigkeitsverhältnisse auf dem Land und der Erlass der Gewerbefreiheit im Jahre 1810. Die Reformbürokratie wollte vor allem durch die Einführung der allgemeinen Gewerbefreiheit die Wirtschaftskraft des Landes stärken und damit zugleich die Steuereinnahmen des Staates erhöhen. Am Ideal einer Gesellschaft freier Wirtschaftssubjekte (mit Subjekten sind hier die einzelnen, wirtschaftlich frei handelnden Menschen gemeint) waren zudem die Bemühungen ausgerichtet, die die Macht der Zünfte brechen und die traditionellen Begrenzungen gewerblicher Produktion aufheben sollten. Die Reformer wollten damit alle kreativen Energien des Landes mobilisieren und eine dynamische Konkurrenzwirtschaft begründen, die den Wohlstand der Einwohner und die Macht des Staates garantierte.

Der Staat beseitigte zum einen die rechtlichen Hindernisse, die den Wirtschaftsaufschwung behinderten. Zu diesem Zweck schuf er Bedingungen für die Entstehung freier Arbeits-, Kapital- und Bodenmärkte. Zudem wollten die Reformer infrastrukturelle Voraussetzungen schaffen, die eine Verbindung einzelner Wirtschaftsfaktoren ermöglichten. Mithilfe staatlicher Investitionen wurde das Verkehrsnetz erweitert und leistungsfähiger gemacht. Durch die Abschaffung von Zollschranken wurde der Binnenmarkt ausgebaut - ein Prozess, der mit dem 1834 gegründeten Zollverein seinen vorläufigen Höhepunkt erreichte. Staatliche Gewerbeschulen und-akademien wurden errichtet, mit denen Preußen seinen technologischen Rückstand aufzuholen hoffte. 


\section{Aufgabe:}

Vergleichen Sie die unterschiedlichen Ausgangsbedingungen für die Industrialisierung in England und Deutschland im ausgehenden 18. und beginnenden 19. Jahrhundert. Arbeiten Sie dabei besonders heraus, welche Strukturen und Prozesse fördernd oder hemmend auf die Industrialisierung wirkten

(entnommen aus: Laschewski-Müller, Karin; Rauh, Robert (Hrsg.) (2010). Kursbuch Geschichte. Neue Ausgabe Nordrhein-Westfalen. Von der Antike bis zur Gegenwart. Berlin: Cornelsen. S. 257f. Text geändert) 


\title{
6.3 Erhebungsteil 3: Veränderter Text, veränderte Aufgabe (Scaffold)
}

\author{
Ausgangsbedingungen für die Industrialisierung in England und Deutschland \\ im ausgehenden 18. und beginnenden 19. Jahrhundert
}

-Die Anfänge der Industrialisierung-

Text

\section{England}

Die Industrialisierung begann im England des ausgehenden 18. Jahrhunderts und erfasste im beginnenden 19. Jahrhundert den westlichen Teil des europäischen Kontinents sowie Nordamerika. Während der zweiten Hälfte des 19. Jahrhunderts strahlte sie auf Osteuropa und Japan aus. Im 20. Jahrhundert entfaltete sie dann ihre Wirksamkeit in der gesamten Welt dieser Prozess dauert bis in die Gegenwart an.

England wird wegen des frühen Beginns der Industrialisierung oft eine „Pionierrolle“ zugesprochen.

Die Beantwortung der Frage, warum England eine Pionierrolle im Industrialisierungsprozess einnahm, ist also eng verknüpft mit den Ursachen der Industriellen Revolution. Historiker nennen mehrere begünstigende Umstände und Vorgänge, die unabhängig voneinander das englische Wirtschaftswachstum beschleunigt haben. Zu den wichtigsten gehören die schnellen Fortschritte in der Landwirtschaft, die die stark wachsende Bevölkerung ernähren und ihr einen gewissen Wohlstand garantieren konnte. Die Bevölkerungszunahme und die Tatsache, dass die Landbevölkerung nicht an die Scholle gebunden war, sondern in den Städten nach neuen Betätigungsfeldern suchte, sorgten sowohl für ein großes Angebot an Arbeitskräften als auch für eine steigende Güternachfrage auf dem englischen Binnenmarkt. England besaß außerdem große und leicht abzubauende Kohlevorkommen, kurze und kostengünstige Verkehrswege und ausreichend Kapital zum Investieren. Hinzu kam, dass der Staat den Unternehmern durch innere Reformen die für die Betätigung notwendigen Freiräume verschaffte. Zudem erlaubte die relativ offene Gesellschaftsstruktur des Königreichs flexible Reaktionen auf die unterschiedlichsten wirtschaftlichen Herausforderungen.

Aufsehenerregende Erfindungen wie die Dampfmaschine von Watt (1765/69) oder die Spinnmaschinen von Hargreaves („Spinning Jenny“ , 1764) und Arkwright (1769)_beschleunigten das englische Wirtschaftswachstum. Mit der Mechanisierung der Baumwollspinnerei, die zum ersten Führungssektor in der englischen Industriegeschichte aufstieg, begann das Zeitalter der Massenproduktion im Textilgewerbe. Für die Entfesselung der Wirtschaftskräfte noch 
bedeutsamer wurde jedoch das Vordringen des Energieträgers Kohle und der damit einhergehende Ausbau der Eisenindustrie. Die Verbilligung und Verbesserung des Eisens sowie die Modernisierung der Produktionsverfahren schufen die Voraussetzungen für die Entstehung einer leistungsfähigen Maschinenindustrie und später für den Eisenbahnbau. Damit wirkte die Eisenindustrie in viele andere Wirtschaftszweige hinein und veränderte vom Verkehrswesen bis zu individuellen Reisen alle Bereiche des gesellschaftlichen Lebens. 
Text 2

\section{Deutschland}

\section{Ausgangsbedingungen}

Deutschland wird oft als „Nachzügler“ in der Industrialisierungsgeschichte eingeordnet. Historiker gehen davon aus, dass dies mit der Ausgangssituation in Deutschland zu tun hat. Bis zum Ende des Heiligen Römischen Reiches Deutscher Nation im Jahre 1806 war Deutschland in 300 zum Teil ausgesprochen kleine Territorialstaaten zersplittert. Eine Vielzahl von Zollschranken, abweichende Maß-, Münz- und Gewichtssysteme, Handelsmonopole sowie schlecht erschlossene Verkehrsverbindungen hemmten die wirtschaftliche Expansion. Trotz mancher Fortschritte bei der Agrarproduktion gab es viele ertragsschwache Kleinbetriebe (ca. 70-80 \% aller Höfe), deren Betreiber oft einem Nebenerwerb nachgehen mussten, um ihre Existenz zu sichern. Die Abhängigkeiten der bäuerlichen Bevölkerung von ihren Gutsherren waren häufig noch so stark, dass dadurch die zur Bildung freier Arbeitsmärkte notwendige individuelle Mobilität eingeschränkt war. Feudale Abgaben, staatliche Steuern und große Unterschiede bei der Verteilung des Wohlstandes behinderten die Entstehung von Massenkaufkraft, die der gewerblichen Wirtschaft hätten zugute kommen können. Auch war die deutsche Gesellschaftsstruktur unflexibel. Starre Standesschranken und konservative Grundeinstellungen engten den Spielraum für innovatorisches Denken und Handeln ein. Und im Handwerk bildete das Festhalten an der überkommenen Zunftverfassung ein zentrales Hindernis für individuelles Erfolgsstreben und wirtschaftliche Neuerungen. Die absolutistischen deutschen Fürsten gängelten mit ihren merkantilistischen Konzepten die wirtschaftliche Entwicklung durch massive Eingriffe und Beschränkungen; staatsfreie Märkte für Kapital, Boden und Waren konnten sich daher nur schwer entfalten.

\section{Staatliche Reformen}

Erst im Verlauf des 19. Jahrhunderts wurden diese Hemmnisse für eine dynamische Industriegesellschaft und -wirtschaft allmählich beseitigt. Dabei nahm der Staat eine herausragende Rolle ein. Durch die Liberalisierung der Agrar- und Gewerbeverfassung, den Abbau von Zollschranken oder die Vereinheitlichung des Rechts- und Finanzwesens schuf er die Voraussetzungen zur Entfesselung einer modernen Wirtschafts- und für den Übergang zur modernen Marktgesellschaft.

Entscheidende Grundlagen dafür wurden in den preußischen Reformen während der ersten beiden Jahrzehnte des 19. Jahrhunderts gelegt, die das Überleben und den Wiederaufbau des von den napoleonischen Armeen besiegten Landes garantieren sollten. Die Reformbeamten, die sich seit 1807 an die Modernisierung des preußischen Staates begaben, kannten 
nicht nur die englische Entwicklung. Auch „die Bibel des Kapitalismus“, Adam Smiths Buch über den „Wohlstand der Nationen“ („,The Wealth of Nations") hatte bei den Reformkräften in Deutschland begeisterte Aufnahme gefunden. Die Leitbegriffe dieses Werks, allen voran „Besitzindividualismus“, „Leistungsprinzip“, „Arbeitsteilung“, „, freie Märkte“ und „Konkurrenz" prägten daher die Wirtsschafts- und Gesellschaftsreformen auf dem Kontinent. Das zeigte sich am preußischen Oktoberedikt von 1807, das eine Mischung aus politischem Manifest und national-ökonomischem Programm darstellte. An die Stelle einer gebundenen Ständegesellschaft, die jedem Menschen eine feste, durch Geburt und Recht erworbene soziale Position zuwies, sollte nun eine mobile Marktgesellschaft treten. Dazu passten auch die Ablösung sozialer Abhängigkeitsverhältnisse auf dem Land und der Erlass der Gewerbefreiheit im Jahre 1810. Die Reformbürokratie wollte vor allem durch die Einführung der allgemeinen Gewerbefreiheit die Wirtschaftskraft des Landes stärken und damit zugleich die Steuereinnahmen des Staates erhöhen. Am Ideal einer Gesellschaft freier Wirtschaftssubjekte (mit Subjekten sind hier die einzelnen, wirtschaftlich frei handelnden Menschen gemeint) waren zudem die Bemühungen ausgerichtet, die die Macht der Zünfte brechen und die traditionellen Begrenzungen gewerblicher Produktion aufheben sollten. Die Reformer wollten damit alle kreativen Energien des Landes mobilisieren und eine dynamische Konkurrenzwirtschaft begründen, die den Wohlstand der Einwohner und die Macht des Staates garantierte.

55 Der Staat beseitigte zum einen die rechtlichen Hindernisse, die den Wirtschaftsaufschwung behinderten. Zu diesem Zweck schuf er Bedingungen für die Entstehung freier Arbeits-, Kapital- und Bodenmärkte. Zudem wollten die Reformer infrastrukturelle Voraussetzungen schaffen, die eine Verbindung einzelner Wirtschaftsfaktoren ermöglichten. Mithilfe staatlicher Investitionen wurde das Verkehrsnetz erweitert und leistungsfähiger gemacht. Durch die Abschaffung von Zollschranken wurde der Binnenmarkt ausgebaut - ein Prozess, der mit dem 1834 gegründeten Zollverein seinen vorläufigen Höhepunkt erreichte. Staatliche Gewerbeschulen und-akademien wurden errichtet, mit denen Preußen seinen technologischen Rückstand aufzuholen hoffte.

(Texte und folgende Aufgabe entnommen aus: Laschewski-Müller, Karin; Rauh, Robert (Hrsg.) (2010). Kursbuch Geschichte. Neue Ausgabe Nordrhein-Westfalen. Von der Antike bis zur Gegenwart. Berlin: Cornelsen. S. $257 f$. Text geändert) 


\section{Aufgabe:}

Vergleichen Sie die unterschiedlichen Ausgangsbedingungen für die Industrialisierung in England und Deutschland im ausgehenden 18. und beginnenden 19. Jahrhundert. Arbeiten Sie dabei besonders heraus, welche Strukturen und Prozesse fördernd oder hemmend auf die Industrialisierung wirkten.

Vorgehen bei der Aufgabenlösung:

1. Bevor Sie losschreiben: Füllen Sie die nachstehende Tabelle aus, um die Ausgangsbedingungen in England und Deutschland gegenüberzustellen.

Ausgangsbedingungen der Industrialisierung

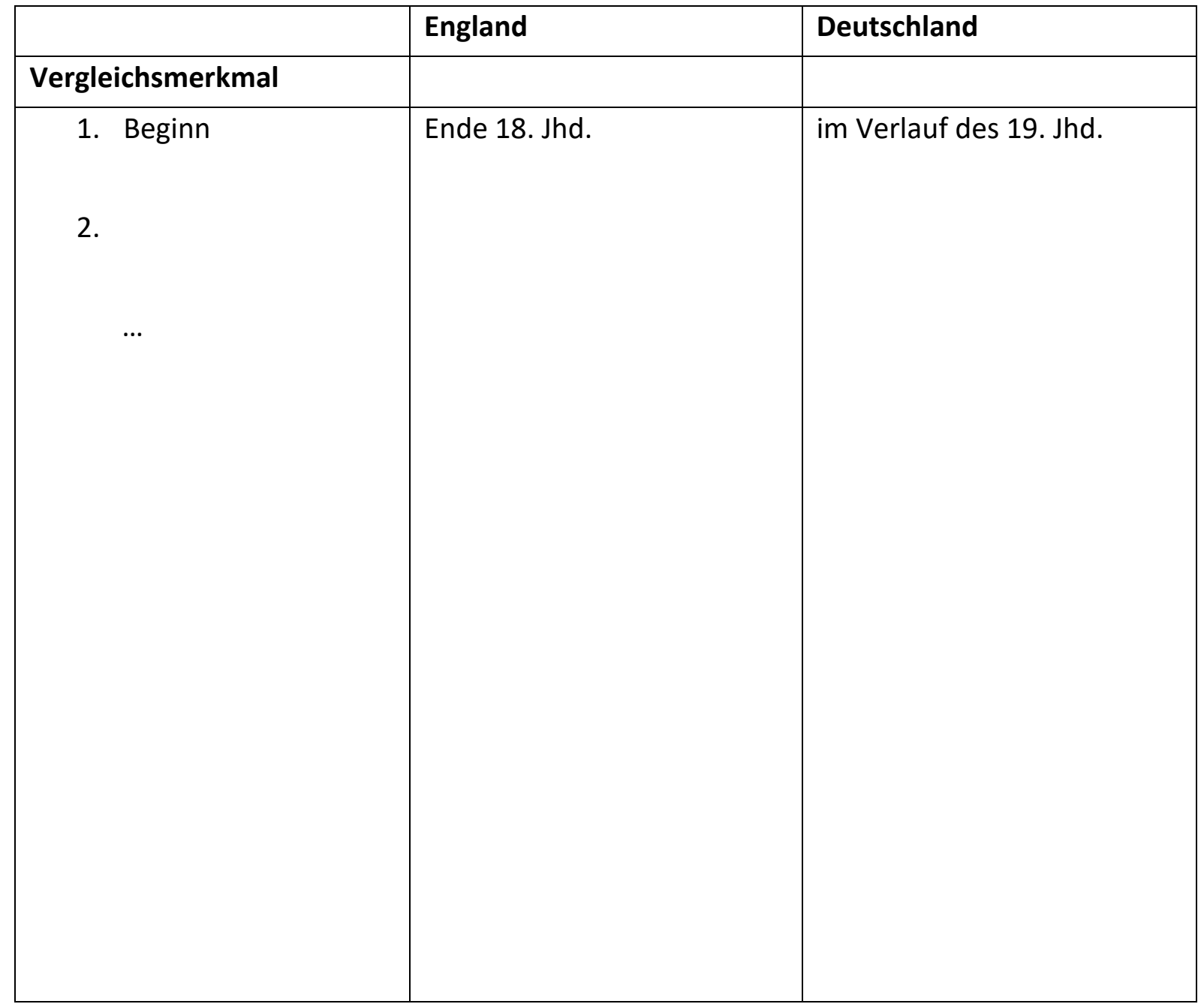

Hinweis: Falls Ihnen die Spalten zu klein sind, dürfen Sie die Tabelle auch auf ein leeres Blatt übertragen und darin ausfüllen. Bitte legen Sie diese Tabelle dann der Lösung bei.

2. Lösen Sie nun die oben gestellte Aufgabe. 


\subsection{Kriterienraster}

\section{Auswertungsmanual mit Anmerkungen}

\section{Überblick und Inhalt}

\begin{tabular}{|c|c|}
\hline Inhalt & \\
\hline $\mathrm{BA}$ & Bearbeitete Aufgabenvariante \\
\hline VS & $\begin{array}{l}\text { Nutzung von Mitteln zur Vorstrukturierung, die nicht in der Aufgabe gefordert werden } \\
\text { (z. B. Gliederung oder Tabelle) }\end{array}$ \\
\hline \multicolumn{2}{|c|}{ Formale Kriterien, die vergleichsunabhängig auf Textqualität hinweisen } \\
\hline \multicolumn{2}{|c|}{ Textlänge } \\
\hline \multicolumn{2}{|c|}{ Mittlere Satzlänge } \\
\hline \multicolumn{2}{|c|}{ Bearbeitung der Aufgabe } \\
\hline \multicolumn{2}{|c|}{ Formale Kriterien, die vergleichsabhängig zu bewerten sind: Textaufbau } \\
\hline \multicolumn{2}{|l|}{ TA } \\
\hline \multicolumn{2}{|c|}{$\underline{\text { Inhaltliche Gestaltung }}$} \\
\hline IG 01 & Eigenständige Überschrift \\
\hline IG 02 & Einleitung \\
\hline IG 03 & Hauptteil \\
\hline IG 04 & Schluss \\
\hline \multicolumn{2}{|c|}{ Inhaltliche Fokussierung } \\
\hline \multicolumn{2}{|c|}{ Thematische Fokussierung } \\
\hline TF 01 & Nennung einer zentralen These \\
\hline TF 02 & Explizite Fokussierung auf den Vergleich durch Aufnahme direkter Vergleichselemente \\
\hline TF 03 & $\begin{array}{l}\text { Nennung von wesentlichen historisch-inhaltlichen Vergleichsaspekten (Orientierung an den } \\
\text { Vorgaben des Darstellungstextes) }\end{array}$ \\
\hline TF 03.1 & England \\
\hline
\end{tabular}




\begin{tabular}{|c|c|}
\hline TF 03.2 & Deutschland \\
\hline TF 03.3 & Darstellung entsprechend Vorlage \\
\hline TF 04 & Vergleichsabschluss \\
\hline \multicolumn{2}{|c|}{$\begin{array}{l}\text { Fokussierung auf den Vergleich durch verwendete sprachliche Teilhand- } \\
\text { lungen }\end{array}$} \\
\hline ST 01 & Gegenüberstellen \\
\hline ST 01.1 & Gegenüberstellen fachlich/pragmatisch \\
\hline ST 01.2 & $\begin{array}{l}\text { Versprachlichung des Gegenüberstellens: Verwendung expliziter sprachlicher Mittel für das Ge- } \\
\text { genüberstellen }\end{array}$ \\
\hline ST 02 & Begründen \\
\hline ST 02.1 & Begründen fachlich/pragmatisch \\
\hline ST 02.2 & $\begin{array}{l}\text { Versprachlichung des Begründens } \\
\text { Begründen von Sachverhaltsbeziehungen }\end{array}$ \\
\hline ST 03 & Erklären \\
\hline ST 03.1 & $\begin{array}{l}\text { Erklären fachlich/pragmatisch (über propositionalen Gehalt) } \\
\text { (Erklären-warum) }\end{array}$ \\
\hline ST 03.2 & Versprachlichung des Erklärens (Erklären-warum) \\
\hline ST 04 & Beurteilen \\
\hline ST 04.1 & Beurteilen fachlich/pragmatisch \\
\hline ST 04.2 & Versprachlichung des Beurteilens \\
\hline ST 05 & Andere sprachliche Teilhandlungen \\
\hline ST 05.1 & Die sprachliche Handlung Folge \\
\hline ST 05.2 & Die sprachliche Handlung Reihung \\
\hline \multicolumn{2}{|c|}{ Wiss. Textkompetenz I: Umgang mit der Textgrundlage } \\
\hline UT & Unterscheidung zwischen Vorlagentext und eigener Position \\
\hline DM 01 & Distanzierungsmittel: Modalisierung \\
\hline DM 02 & Personalisierung Handelnder \\
\hline
\end{tabular}


Wiss. Textkompetenz II: Entwicklung eines „Roten Fadens“

Mittel zur Themenentwicklung

MT 01

Themensplitting (Entwicklung durch Teilung)

MT 02

Themenfortführung 


\section{Erläuterungen zur Auswertung}

\begin{tabular}{|c|c|c|c|}
\hline Item & Code & Erläuterungen & Beispiele und Bemerkungen \\
\hline BA & 1 & $\begin{array}{l}\text { unveränderter Ausgangstext, unverän- } \\
\text { derte Aufgabe }\end{array}$ & Texte der Erhebungen 1-3 \\
\hline \multirow[t]{2}{*}{ Bearbeitete Aufgabenvariante } & 2 & $\begin{array}{l}\text { Ausgangstext zu } 2 \text { Texten verändert, un- } \\
\text { veränderte Aufgabe }\end{array}$ & Texte der Erhebungen 4-5 \\
\hline & 3 & $\begin{array}{l}\text { Ausgangstext zu } 2 \text { Texten verändert, ver- } \\
\text { änderte Aufgabe: Tabelle vorab }\end{array}$ & Texte der Erhebung 6 \\
\hline VS & 0 & keine Nutzung & \\
\hline $\begin{array}{l}\text { Nutzung von Mitteln zur Vorstrukturierung, } \\
\text { (z. B. Gliederung oder Tabelle) }\end{array}$ & 1 & $\begin{array}{l}\text { Nutzung eines Mittels zur Vorstrukturie- } \\
\text { rung }\end{array}$ & $\begin{array}{l}\text { z. B.: Tabelle, dann folgt der eigentli- } \\
\text { che Text zur Aufgabe. Die Inhalte der } \\
\text { Tabelle finden sich zumindest zum } \\
\text { Teil im Text wieder }\end{array}$ \\
\hline $\begin{array}{l}\text { die in Erhebung } 6 \text { geforderte Tabelle wird als } \\
\text { ein Mittel berücksichtigt }\end{array}$ & 2 & $\begin{array}{l}\text { Nutzung mehrerer Mittel zur Vorstruktu- } \\
\text { rierung }\end{array}$ & Tabelle, Gliederung, Schaubild \\
\hline \multicolumn{4}{|l|}{$\begin{array}{l}\text { Formale Kriterien, die vergleichsunabhängig } \\
\text { auf Textqualität hinweisen }\end{array}$} \\
\hline Textlänge & -kein Code- & Eintragung des numerischen Ergebnisses & $\begin{array}{l}\text { Anzahl der Wörter eintragen, } \\
\text { Zahlen werden als Wörter gezählt, da } \\
\text { sie wichtige Bedeutungsträger sind. } \\
\text { Auch Überschriften und Tabellen- } \\
\text { überschriften werden als Wörter ge- } \\
\text { zählt und für die mittlere Satzlänge } \\
\text { berücksichtigt. } \\
\text { Auch einzelne Wörter zählen. }\end{array}$ \\
\hline
\end{tabular}




\begin{tabular}{|c|c|c|c|}
\hline & & & $\begin{array}{l}\text { Wenn Lernende erst ihre Sorgen we- } \\
\text { gen der Aufgabenlösung o.ä. nieder- } \\
\text { schreiben, gehört dieser Teil mit in } \\
\text { den Text, diese Wörter werden mitge- } \\
\text { zählt, das gilt auch für ähnliche Hin- } \\
\text { weise am Schluss oder ein P.S. }\end{array}$ \\
\hline Mittlere Satzlänge & -kein Code- & Eintragung des numerischen Ergebnisses & $\begin{array}{l}\text { Anzahl der Wörter durch Anzahl der } \\
\text { Sätze teilen, auf eine Nachkommas- } \\
\text { telle runden } \\
\text { Zählung der Sätze: Gesamtzahl aller } \\
\text { Sätze (entspr. Zählvorgaben Fast } \\
\text { Catch Bumerang: Reich/Roth/Döll, } \\
\text { 2009, S. 12f). } \\
\text { Wenn nur Stichpunkte, aber keine } \\
\text { Ganzsätze vorhanden sind (z. B. bei } \\
\text { Tabellen), dann ist das Ergebnis der } \\
\text { Satzlänge 0. } \\
\text { Bsp. } \\
\text { „[...] mehr. (z.B. Dampfmaschine, } \\
\text { Spinnenmaschine).“: Der unterstri- } \\
\text { chene Bereich stellt keinen eigenstän- } \\
\text { digen Satz dar. }\end{array}$ \\
\hline
\end{tabular}




\begin{tabular}{|c|c|c|c|}
\hline Bearbeitung der Aufgabe & $0=$ nicht erkennbar & eine Bearbeitung der Aufgabe fehlt ganz & leeres Blatt oder „bekritzeltes Blatt“ \\
\hline \multirow{2}{*}{$\begin{array}{l}\text { Hinweis: } \\
\text { Es handelt sich um einen holistischen Zugang: } \\
\text { Es soll nur geklärt werden, ob der Text grund- } \\
\text { sätzlich Bezug auf die Aufgabe nimmt; es ist } \\
\text { hier nicht zu entscheiden, ob er fachlich kor- } \\
\text { rekt ist, ein Vergleich wirklich geleistet wird } \\
\text { etc. }\end{array}$} & $1=$ Spaßantwort & es handelt sich um eine Spaßantwort & $\begin{array}{l}\text { die Antwort hat mit der Aufgabe } \\
\text { nichts zu tun } \\
\text { z. B. "Spruch“ }\end{array}$ \\
\hline & $2=$ erkennbar & $\begin{array}{l}\text { eine Beantwortung der Aufgabe liegt vor } \\
\text { oder liegt teilweise vor }\end{array}$ & $\begin{array}{l}\text { Leere Tabelle (nur in Erhebungsteil } 1 \\
\text { und 2): teilweise Bearbeitung der Auf- } \\
\text { gabe, da der Schüler Kategorien in } \\
\text { eine Tabelle überführt hat (in Erhe- } \\
\text { bungsteil } 3 \text { sind Kriterien vorgegeben, } \\
\text { deshalb keine teilweise Bearbeitung), } \\
\text { Stichwörter, Text etc.: es wird zumin- } \\
\text { dest inhaltlich knapp ein Bezug zur } \\
\text { Frage hergestellt }\end{array}$ \\
\hline \multicolumn{4}{|l|}{$\begin{array}{l}\text { Formale Kriterien, die vergleichsabhängig zu } \\
\text { bewerten sind: Textaufbau }\end{array}$} \\
\hline \multirow[t]{2}{*}{ TA } & $0=$ Stichwörter & $\begin{array}{l}\text { Es sind durchgängig Stichwörter vorhan- } \\
\text { den, dabei gibt es keine Struktur einer Ge- } \\
\text { genüberstellung }\end{array}$ & \\
\hline & 1= Tabelle/Kombination & $\begin{array}{l}\text { Es gibt eine Tabelle oder eine Art Gegen- } \\
\text { überstellung, die zum Beispiel dadurch er- } \\
\text { kennbar wird, dass erst stichwortartig As- } \\
\text { pekte zum einen und dann zum anderen } \\
\text { Vergleichsobjekt aufgelistet sind } \\
\text { oder }\end{array}$ & $\begin{array}{l}\text { Bsp.: Struktur von Stichwörtern, } \\
\text { durch die Zuweisung Pro und Contra } \\
\text { Gegenüberstellung (als Kombination } \\
\text { einzuordnen): } \\
\text { „England } \\
\text { - Wro } \\
\text { - Wirtschaftswachstum }\end{array}$ \\
\hline
\end{tabular}




\begin{tabular}{|c|c|c|c|}
\hline & & $\begin{array}{l}\text { zwei oder mehrere unterschiedliche } \\
\text { Textaufbau-Strukturen sind miteinander } \\
\text { kombiniert. } \\
\text { Wenn eine Tabelle vorangestellt wird und } \\
\text { dann der Fließtext folgt, handelt es sich } \\
\text { ebf. um eine Kombination. }\end{array}$ & $\begin{array}{l}\text { - Landwirtschaftliche Fortschritte } \\
\text { - wachsende Bevölkerung } \\
\text {-enormer Arbeitsmarkt } \\
\text { - steigende Güternachfrage } \\
\text { - offene Gesellschaftsstruktur } \\
\text { [...] Contra } \\
\text { - 18. Jahrhunder } \\
\text { - war nachzügler } \\
\text { - verschiedene Währungen } \\
{[\ldots . .]^{\prime \prime}}\end{array}$ \\
\hline & $2=$ Fließtext & $\begin{array}{l}\text { Ein Fließtext ist weitgehend erkennbar. } \\
\text { Die meisten Sätze sind ausformuliert und } \\
\text { aufeinander bezogen. }\end{array}$ & $\begin{array}{l}\text { nur Tabelle als Überschrift, dann aber } \\
\text { vollständiger Fließtext }\end{array}$ \\
\hline \multicolumn{4}{|l|}{ Inhaltliche Gestaltung } \\
\hline IG 01 & $0=$ nicht erkennbar & Es gibt keine Überschrift & \\
\hline Eigenständige Überschrift & $1=$ teilweise erkennbar & $\begin{array}{l}\text { Die Überschrift ist eine Übernahme der } \\
\text { vorgegebenen Überschrift im Darstel- } \\
\text { lungstext oder } \\
\text { es wird die Aufgabenstellung wiedergege- } \\
\text { ben } \\
\text { oder }\end{array}$ & $\begin{array}{l}\text { "Ausgangsbedingungen für } \\
\text { die Industrialisierung von } \\
\text { England und Deutschland“ } \\
\text { (hierunter folgt Tabelle, da es etwas } \\
\text { über die reinen Tabellenkategorien } \\
\text { hinausgeht: teilweise erkennbar) }\end{array}$ \\
\hline
\end{tabular}




\begin{tabular}{|c|c|c|c|}
\hline & & $\begin{array}{l}\text { die Überschrift ist nicht eindeutig als } \\
\text { Überschrift zu identifizieren }\end{array}$ & $\begin{array}{l}\text { „Die Anfänge der Industrialisierung“ } \\
\text { „England“ } \\
\text { „Aufgabe 1“ }\end{array}$ \\
\hline & $2=$ erkennbar & $\begin{array}{l}\text { Die Überschrift ist kaum vom Darstel- } \\
\text { lungstext übernommen (Bezugnahmen } \\
\text { auf die Teilüberschriften oder der Hin- } \\
\text { weis, dass es sich um die Anfänge der In- } \\
\text { dustrialisierung handelt, sind zulässig, da- } \\
\text { mit das Thema getroffen wird) }\end{array}$ & $\begin{array}{l}\text { „Ausgangsbedingungen der Industri- } \\
\text { ellen Revolution In England und } \\
\text { Deutschland“ }\end{array}$ \\
\hline \multirow[t]{2}{*}{$\begin{array}{l}\text { IG } 02 \\
\text { Einleitung } \\
\end{array}$} & $0=$ nicht erkennbar & $\begin{array}{l}\text { Es ist keine Einleitung im Sinne einer text- } \\
\text { eröffnenden Sprachhandlung vorhanden } \\
\text { (es kann sich um einen Satz handeln, der } \\
\text { kausal-, lokal- und temporalstrukturie- } \\
\text { rend den Hauptteil vorbereitet ggf. auch } \\
\text { schon Vergleichsobjekte und Tertium } \\
\text { Comparationis nennt). }\end{array}$ & $\begin{array}{l}\text { z. B. Start mit Tabelle ohne einleiten- } \\
\text { den Satz }\end{array}$ \\
\hline & $1=$ teilweise erkennbar & $\begin{array}{l}\text { Eine Einleitung ist teilweise vorhanden, } \\
\text { das heißt, es werden Teilaspekte genannt, } \\
\text { um die es im Vergleich/Hauptteil gehen } \\
\text { soll. } \\
\text { Sie ist sachlich ggf. nicht oder nur zum Teil } \\
\text { korrekt. } \\
\text { Sie nimmt nur einen Teil der Aufgaben- } \\
\text { stellung auf (z. B. nur ein Vergleichsobjekt }\end{array}$ & $\begin{array}{l}\text { "Die Industrialisierung in England im } \\
\text { ausgehenden 18. Jahrhundert wurde } \\
\text { begünstigt durch Faktoren wie Land- } \\
\text { wirtschaftliches Wachstum, welches } \\
\text { der wachsenden Bevölkerung Wohl- } \\
\text { stand gebracht hatte." } \\
\text { oder }\end{array}$ \\
\hline
\end{tabular}




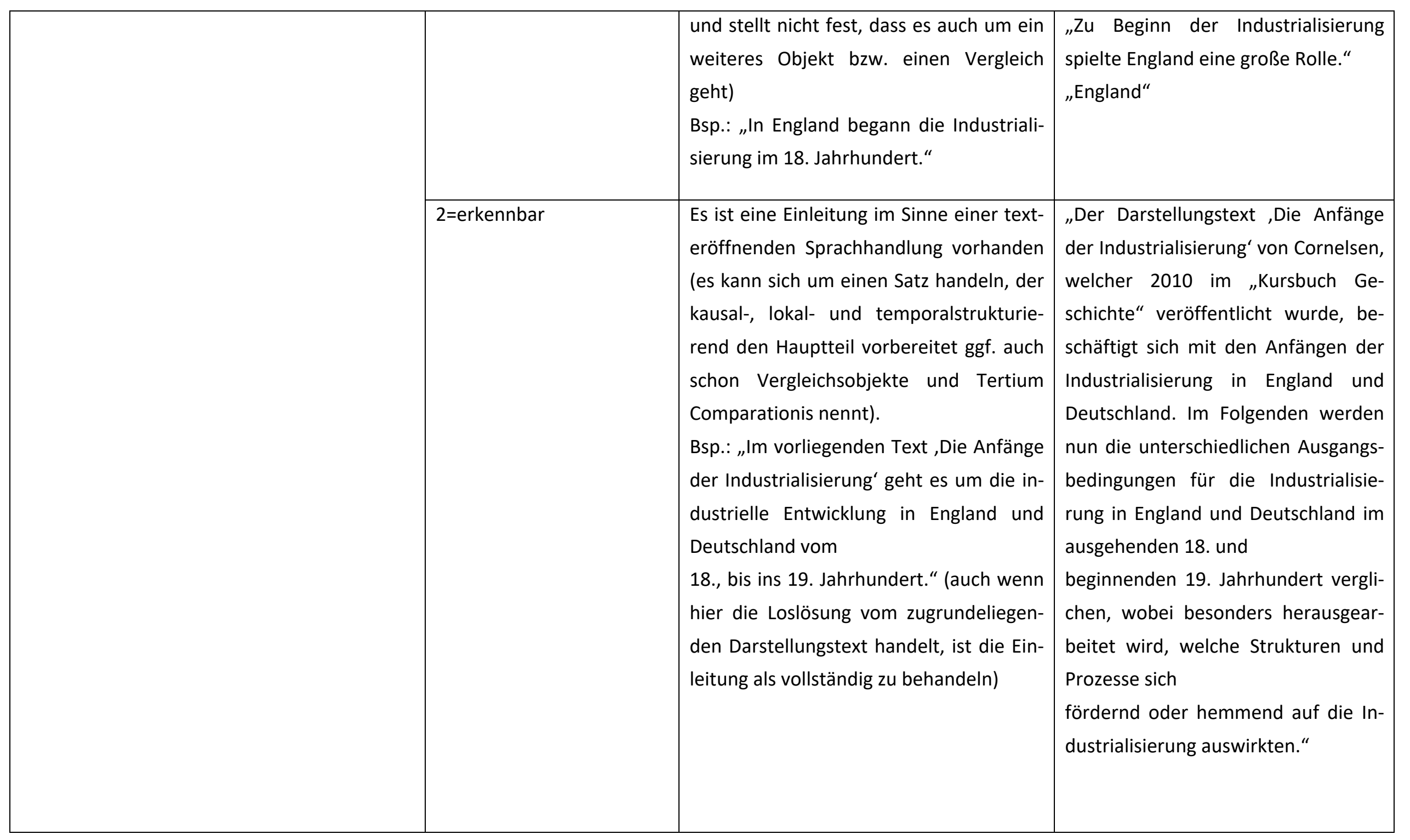




\begin{tabular}{|c|c|c|c|}
\hline \multirow[t]{3}{*}{$\begin{array}{l}\text { IG } 03 \\
\text { Hauptteil } \\
\end{array}$} & $0=$ nicht erkennbar & $\begin{array}{l}\text { Der Vergleich wird nicht entfaltet, das } \\
\text { heißt: } \\
\text { es ist kein Hauptteil vorhanden oder } \\
\text { der Hauptteil geht nicht auf mind. ein Ver- } \\
\text { gleichsobjekt ein }\end{array}$ & \\
\hline & $1=$ teilweise erkennbar & $\begin{array}{l}\text { Der Hauptteil geht nur auf ein Vergleichs- } \\
\text { objekt ein oder es werden nur ein oder } \\
\text { zwei Vergleichsaspekte benannt oder er } \\
\text { geht nicht auf Gemeinsamkeiten oder Un- } \\
\text { terschiede ein, die nicht direkt in Bezug } \\
\text { gesetzt werden oder nur aufgelistet wer- } \\
\text { den. Insgesamt ist ein Hauptteil vorhan- } \\
\text { den, für den Vergleich erscheint er aber } \\
\text { unvollständig. }\end{array}$ & \\
\hline & $2=$ erkennbar & $\begin{array}{l}\text { Der Vergleich wird entfaltet, das heißt } \\
\text { der Hauptteil geht auf die Vergleichsob- } \\
\text { jekte England und Deutschland ein, } \\
\text { er nimmt Bezug auf mind. } 3 \text { Vergleichsas- } \\
\text { pekte aus dem Darstellungstext (z. B. Ent- } \\
\text { wicklung in der Landwirtschaft etc.), die in } \\
\text { Bezug gesetzt werden; } \\
\text { er setzt England und Deutschland in Be- } \\
\text { zug, } \\
\text { es werden Gemeinsamkeiten und Unter- } \\
\text { schiede genannt (möglich ist auch die }\end{array}$ & $\begin{array}{l}\text { (auch tabellarisch oder stichwortartig } \\
\text { möglich) } \\
\text { Bezug muss deutlich herausgestellt } \\
\text { sein: } \\
\text { „England war Vorreiter, weil die Fort- } \\
\text { schritte in der Landwirtschaft groß } \\
\text { waren, die Bevölkerung konnte wach- } \\
\text { sen, die Infrastruktur war gut und } \\
\text { wurde gestärkt. } \\
\text {... }\end{array}$ \\
\hline
\end{tabular}




\begin{tabular}{|c|c|c|c|}
\hline & & $\begin{array}{l}\text { Nennung von Ähnlichkeiten, die Aufga- } \\
\text { benstellung fordert allerdings zur Darstel- } \\
\text { lung dieser nicht direkt heraus), } \\
\text { er zeigt Strukturen und Prozesse, die hem- } \\
\text { mend oder fördernd waren (z. B. unter- } \\
\text { schiedliche Gesellschaftsstrukturen in } \\
\text { England und Deutschland, die für England } \\
\text { Fortschritt und für Deutschland ein } \\
\text { Hemmnis bedeuteten) }\end{array}$ & $\begin{array}{l}\text { In Deutschland waren die Fortschritte } \\
\text { in der Landwirtschaft geringer, weil...; } \\
\text { das Bevölkerungswachstum war we- } \\
\text { niger stark; die Verkehrswege wurden } \\
\text { nicht genügend ausgebaut." } \\
\text { (kann auch untereinander abgearbei- } \\
\text { tet werden) }\end{array}$ \\
\hline \multirow[t]{2}{*}{$\begin{array}{l}\text { IG } 04 \\
\text { Schluss } \\
\end{array}$} & $0=$ nicht erkennbar & $\begin{array}{l}\text { Es fehlt eine textschließende Sprachhand- } \\
\text { lung ganz, das heißt: } \\
\text { es fehlt ein Abschluss des Vergleichstex- } \\
\text { tes, } \\
\text { es gibt kein Fazit oder eine Zusammenfas- } \\
\text { sung; } \\
\text { ggf. gibt es eine Markierung, dass der Text } \\
\text { nicht fertiggestellt wurde („....", etc., usw. } \\
\text { o.ä.) }\end{array}$ & $\begin{array}{l}\text { z. B. wenn es nur eine Tabelle gibt, } \\
\text { aber kein Fazit o.ä. daraus } \\
\text { oder } \\
\text { wenn der Fließtext mit der Aufzäh- } \\
\text { lung weiterer Einzelaspekte schließt, } \\
\text { die vorher noch nicht genannt und /o- } \\
\text { der nur aufgezählt werden. } \\
\text { z. B. „Die Reformbürokratie stärke die } \\
\text { Wirtschaftskraft. Der Binnenmark } \\
\text { wird ausgebaut und das Verkehrs- } \\
\text { netz.“ Aufzählung der Fortentwick- } \\
\text { lung Deutschlands ohne Einordnung }\end{array}$ \\
\hline & $1=$ teilweise erkennbar & $\begin{array}{l}\text { Der Schluss ist nicht vollständig: } \\
\text { Der Text nimmt nur einen Aspekt oder ein }\end{array}$ & $\begin{array}{l}\text { „Das englische Wirtschaftswachstum } \\
\text { wurde durch aufsehenerregende } \\
\text { Erfindungen (z.B. Dampfmaschine }\end{array}$ \\
\hline
\end{tabular}




\begin{tabular}{|c|c|c|c|}
\hline & & $\begin{array}{l}\text { Vergleichsobjekt auf, ist aber grundsätz- } \\
\text { lich als gedanklicher Abschluss zu erken- } \\
\text { nen (z. B. „Der technologische Rückstand } \\
\text { im Vergleich zu England sollte durch staat- } \\
\text { liche Gewerbeschulen aufgeholt wer- } \\
\text { den."). Ein Schluss ist auch teilweise er- } \\
\text { kennbar, wenn der 3. Teile des Darstel- } \\
\text { lungstextes aufgegriffen wird (Weiterent- } \\
\text { wicklung Deutschlands) und zum gedank- } \\
\text { lichen Abschluss führt. }\end{array}$ & $\begin{array}{l}\text { oder Spinnmaschinen) beschleunigt } \\
\text { und durch das Vordringen des Ener- } \\
\text { gieträgers Kohle konnte die Eisenin- } \\
\text { dustrie ausgebaut werden.“ } \\
\text { Durch „..." wird angedeutet, dass der } \\
\text { Text eigentlich noch nicht abgeschlos- } \\
\text { sen ist. }\end{array}$ \\
\hline & $2=$ erkennbar & $\begin{array}{l}\text { Textschließende Sprachhandlung (An- } \\
\text { knüpfung an den Hauptteil, Abschluss des } \\
\text { Vergleichstextes), verweist durch Fazit } \\
\text { oder Zusammenfassung auf den Zweck } \\
\text { des Vergleichs }\end{array}$ & $\begin{array}{l}\text { z. B. „Deutschland hatte viele hem- } \\
\text { mende Probleme, weshalb es etwas } \\
\text { schwierig war. England hatte gute Ab- } \\
\text { sichten und Voraussetzungen.“: Zwar } \\
\text { wird auf der inhaltlichen Seite nicht } \\
\text { erklärt, warum England „gute Absich- } \\
\text { ten“ hatte, aber ein Textschluss im } \\
\text { Sinne einer abschließenden Einord- } \\
\text { nung ist erkennbar. }\end{array}$ \\
\hline \multicolumn{4}{|l|}{ Inhaltliche Fokussierung } \\
\hline \multicolumn{4}{|l|}{ Thematische Fokussierung } \\
\hline $\begin{array}{l}\text { TF } 01 \\
\text { Nennung einer zentralen These }\end{array}$ & $0=$ nicht erkennbar & $\begin{array}{l}\text { Es wird keine These formuliert, an der die } \\
\text { Vergleichsaspekte abgearbeitet werden. }\end{array}$ & \\
\hline
\end{tabular}




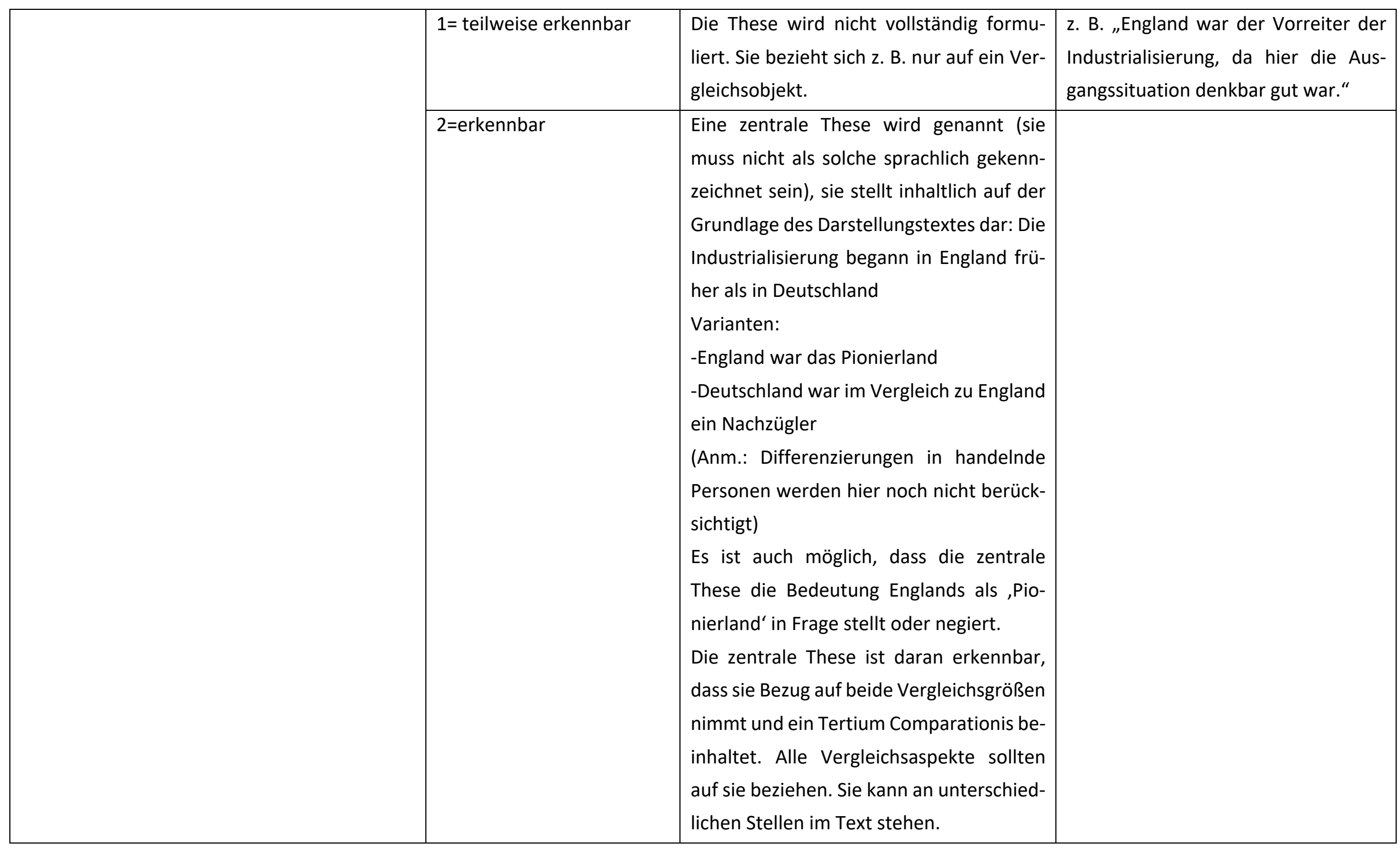




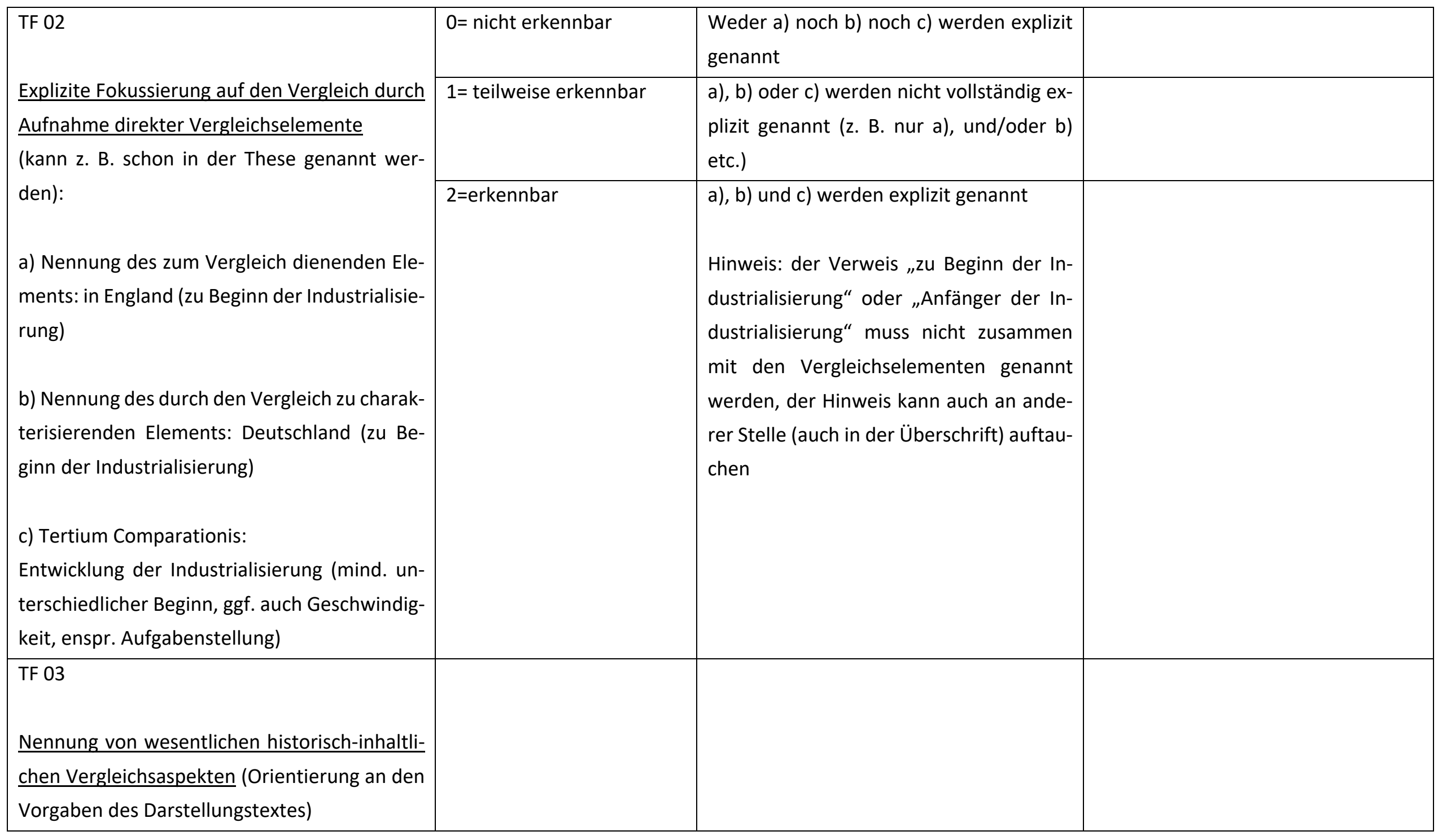




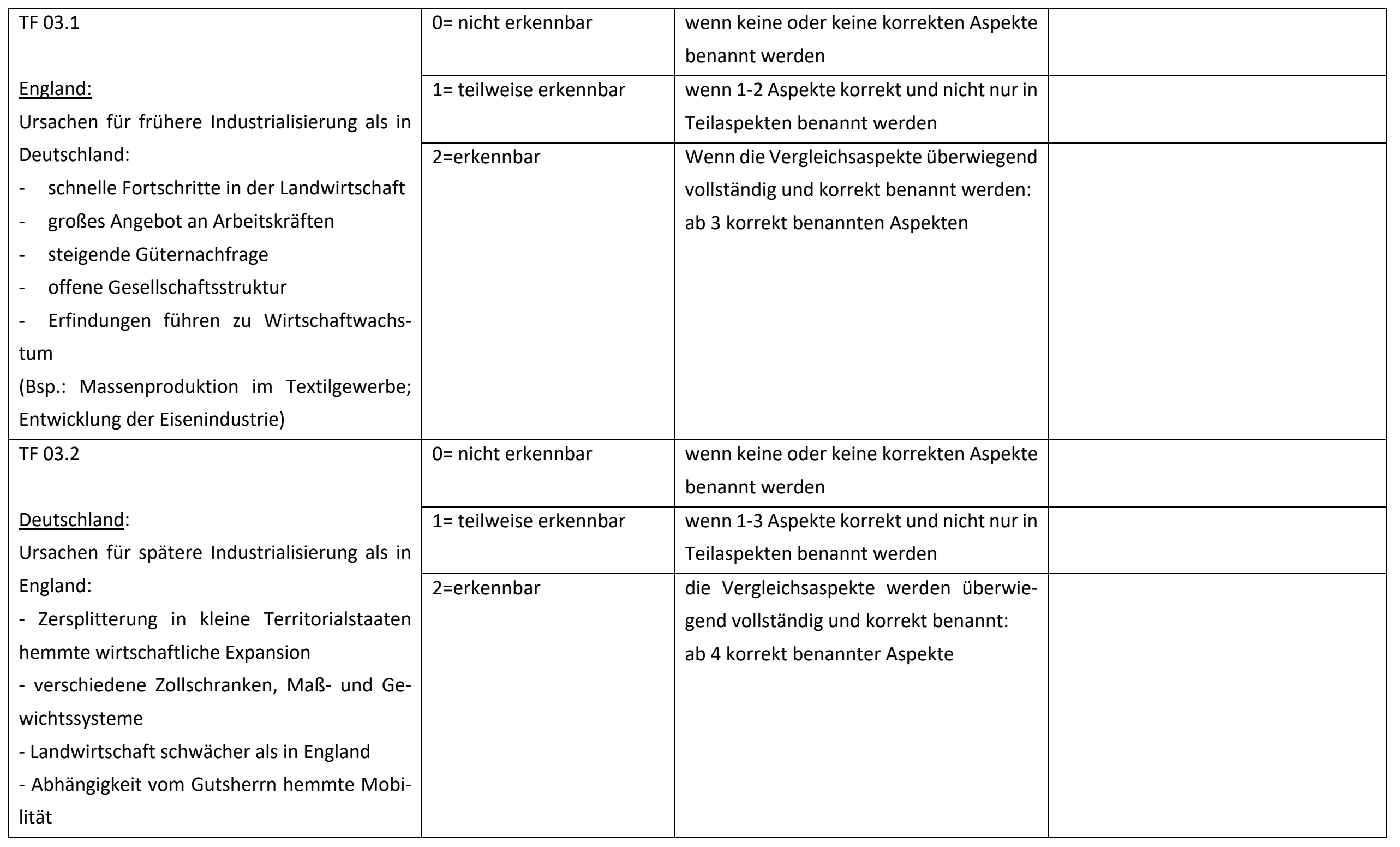




\begin{tabular}{|c|c|c|c|}
\hline $\begin{array}{l}\text { - Entwicklung Massenkaufkraft gehemmt } \\
\text { - vergleichsweise geschlossenere Gesell- } \\
\text { schaftsstruktur hemmte Innovationen } \\
\text { - Zunftverfassung hinderte wirtschaftliche } \\
\text { Neuerungen } \\
\text { - Merkantilismus hinderte wirtschaftliche Ent- } \\
\text { wicklung }\end{array}$ & & & \\
\hline \multirow{3}{*}{$\begin{array}{l}\text { TF } 03.3 \\
\text { Darstellung entspr. Vorlage, aber über die Mi- } \\
\text { nimalanforderungen hinaus: } \\
\text { Ergänzend wird gezeigt, wie die Veränderun- } \\
\text { gen der Strukturen in Deutschland die Indus- } \\
\text { trialisierung förderten } \\
\text { (Liberalisierung der Agrar- und Gewerbever- } \\
\text { fassung, Abbau von Zollschranken, Vereinheit- } \\
\text { lichung des Rechts- und Finanzwesens, preußi- } \\
\text { sche Reformen: Wirtschafts- und Gesell- } \\
\text { schaftsreformen; Oktoberedikt von } 1807 \text { zur } \\
\text { Aufhebung der Ständegesellschaft; Gewerbe- } \\
\text { freiheit; Entstehung freier Arbeits-, Kapital- } \\
\text { und Bodenmärkte; Erweiterung des Verkehrs- } \\
\text { netzes, Zollverein, Errichtung staatlicher Ge- } \\
\text { werbeschulen und-akademien, um technolog. } \\
\text { Rückstand aufzuholen) }\end{array}$} & $0=$ nicht erkennbar & $\begin{array}{l}\text { wenn keine oder keine korrekten Aspekte } \\
\text { benannt werden }\end{array}$ & \\
\hline & 1= teilweise erkennbar & $\begin{array}{l}\text { beispielhafte Aufzählung von (einer oder } \\
\text { mehrerer) Strukturen, die zur Verände- } \\
\text { rung in D. führten, jedoch ohne Einord- } \\
\text { nung der Bedeutung bzw. Bezug auf die } \\
\text { Vergleichshypothese (reihend) oder fal- } \\
\text { sche Einordnung }\end{array}$ & $\begin{array}{l}\text { "Es begannen fördernde Prozesse [...]. } \\
\text { Der Staat liberalisierte die Agrar- und } \\
\text { Gewerbeverfassung, [...]. Es wurde } \\
\text { nicht nur nach englischem Vorbild ge- } \\
\text { handelt..." } \\
\rightarrow \text { der Zusammenhang zwischen Libe- } \\
\text { ralisierung und englischem Vorbild ist } \\
\text { nur implizit, jedoch nicht eindeutig } \\
\text { hergestellt. }\end{array}$ \\
\hline & $2=e r k e n n b a r$ & $\begin{array}{l}\text { beispielhafte Aufzählung von (einer oder } \\
\text { mehrerer) Strukturen, die zur Verände- } \\
\text { rung in D. führten, mit zutreffender Ein- } \\
\text { ordnung der Bedeutung bzw. Bezug auf } \\
\text { die Vergleichshypothese }\end{array}$ & $\begin{array}{l}\text { Es wurden für die Entwicklung der In- } \\
\text { dustrialisierung fördernde Prozesse } \\
\text { eingeleitet [...]. Der Staat liberalisierte } \\
\text { bspw. die Agrar- und Gewerbeverfas- } \\
\text { sung, [...]. Dafür diente England als }\end{array}$ \\
\hline
\end{tabular}




\begin{tabular}{|c|c|c|c|}
\hline & & & $\begin{array}{l}\text { Vorbild, wo es diese Strukturen be- } \\
\text { reits zu Beginn der Industrialisierung } \\
\text { gab.“ } \\
\text { Bezug zu England wird hergestellt! }\end{array}$ \\
\hline \multirow{3}{*}{$\begin{array}{l}\text { TF } 04 \\
\text { Vergleichsabschluss } \\
\text { Bildung eines abschließenden Sachurteils } \\
\text { Beurteilung im historischen Kontext (entspr. } \\
\text { Jeismann): Sinnbildung/Deutung }\end{array}$} & $0=$ nicht erkennbar & kein abschließendes Sachurteil & $\begin{array}{l}\text { z. B. Tabelle ohne Fazit, auch nicht in } \\
\text { einzelnen Spalten klar erkennbar }\end{array}$ \\
\hline & $1=$ teilweise erkennbar & $\begin{array}{l}\text { Das Sachurteil wird angedeutet bzw. teil- } \\
\text { weise realisiert } \\
\text { z. B. ist das Urteil nur auf ein Vergleichs- } \\
\text { objekt bezogen oder nimmt nur Teilas- } \\
\text { pekte auf oder es ist pauschal und kaum } \\
\text { fundiert. }\end{array}$ & \\
\hline & $2=$ erkennbar & $\begin{array}{l}\text { Das Sachurteil wird ausführlich realisiert, } \\
\text { es ist auf beide Vergleichsobjekte bezo- } \\
\text { gen. }\end{array}$ & $\begin{array}{l}\text { „Insgesamt lässt sich also sagen, dass } \\
\text { in England die Industrialisierung } \\
\text { schneller stattfinden konnte, da die } \\
\text { Gegebenheiten besser waren. } \\
\text { Durch die Hindernisse in Deutschland } \\
\text { hat alles etwas länger gedauert, } \\
\text { doch letzten Endes hat auch dort die } \\
\text { Industrialisierung erfolgreich } \\
\text { stattgefunden, so dass England und } \\
\text { Deutschland auf einem ähnlich } \\
\text { modernen Stand waren." }\end{array}$ \\
\hline
\end{tabular}




\begin{tabular}{|c|c|c|c|c|}
\hline \multicolumn{5}{|l|}{$\begin{array}{l}\text { Fokussierung auf den Vergleich durch ver- } \\
\text { wendete sprachliche Teilhandlungen }\end{array}$} \\
\hline \multicolumn{5}{|l|}{$\begin{array}{l}\text { ST } 01 \\
\text { Gegenüberstellen }\end{array}$} \\
\hline \multirow{3}{*}{$\begin{array}{l}\text { ST } 01.1 \\
\text { Gegenüberstellen fachlich/pragmatisch } \\
\text { "wie skizzieren, aber zusätzlich argumentie- } \\
\text { rend gewichten“ (Kultuministerkonferenz, } \\
\text { 2005: EPA Geschichte, S. 8) }\end{array}$} & $0=$ nicht erkennbar & $\begin{array}{l}\text { Der Text geht nur auf ein Vergleichsobjekt } \\
\text { (Deutschland oder England) ein. }\end{array}$ & & \\
\hline & \multirow[t]{3}{*}{$1=$ teilweise erkennbar } & \multirow{2}{*}{$\begin{array}{l}\text { sprachliche Handlung wird angedeutet } \\
\text { bzw. teilweise realisiert und nur implizit } \\
\text { realisiert }\end{array}$} & \multicolumn{2}{|c|}{$\begin{array}{l}\text {-Reihung/Aufzählung } \\
\text {-Tabelle }\end{array}$} \\
\hline & & & England & $\begin{array}{l}\text { Deutsch- } \\
\text { land }\end{array}$ \\
\hline \multirow[t]{2}{*}{$\begin{array}{l}\text { Herstellung eines Zusammenhanges zwischen } \\
\text { mind. zwei Vergleichselementen }\end{array}$} & & $\begin{array}{l}\text { z. B. gibt es nur eine stichwortartige Auf- } \\
\text { zählung } \\
\text { oder } \\
\text { es gibt „nur“ eine Tabelle, die einzelne As- } \\
\text { pekte untereinander aufreiht, wie eine } \\
\text { Aufzählung, die Aspekte werden aber } \\
\text { nicht gegenüber angeordnet }\end{array}$ & $\begin{array}{l}\text {-schneller Fort- } \\
\text { schritt der Land- } \\
\text { wirtschaft } \\
\text {-Landbevölkerung } \\
\text { nicht an Scholle } \\
\text { gebunden } \\
\ldots\end{array}$ & $\begin{array}{l}\text {-in Territorialstaa- } \\
\text { ten gegliedert } \\
\text {-Vielzahl von Zoll- } \\
\text { schranken } \\
\text {-abweichende } \\
\text { Maß-, Münz- und } \\
\text { Gewichtssysteme } \\
\text {... }\end{array}$ \\
\hline & $2=$ erkennbar & $\begin{array}{l}\text { sprachliche Handlung wird ausführlich } \\
\text { und explizit realisiert: Es gibt einen geglie- } \\
\text { derten und strukturierten Text, der weit- } \\
\text { gehend einen Zusammenhang zwischen } \\
\text { England und Deutschland herstellt und } \\
\text { die Aspekte nicht nur untereinander auf- } \\
\text { reiht (sie inhaltlich gewichtet). }\end{array}$ & \multicolumn{2}{|c|}{$\begin{array}{l}\text { z. B.: } \\
\text { „Als Pionier der Industrialisierung } \\
\text { können anhand des Beispiels ,Eng- } \\
\text { land' mehrere Faktoren dargestellt } \\
\text { werden, die den Beginn dieses Pro- } \\
\text { zesses begünstigen und fördern, so- } \\
\text { gar regelrecht bedingen. [...] }\end{array}$} \\
\hline
\end{tabular}




\begin{tabular}{|c|c|c|c|}
\hline & & $\begin{array}{l}\text { oder } \\
\text { eine Tabelle markiert eine Gegenüberstel- } \\
\text { lung erkennbar, wenn die einzelnen As- } \\
\text { pekte weitgehend ausdrücklich, gegen- } \\
\text { über' angeordnet sind }\end{array}$ & $\begin{array}{l}\text { Demgegenüber konnte aufgrund von } \\
\text { rückständigen Gesetzen und Struktu- } \\
\text { ren die Industrialisierung erst verspä- } \\
\text { tet, nämlich nach Abschaffung dieser, } \\
\text { in Deutschland einsetzen. [...]“ }\end{array}$ \\
\hline $\begin{array}{l}\text { ST } 01.2 \\
\text { Versprachlichung des Gegenüberstellens: Ver- } \\
\text { wendung expliziter sprachlicher Mittel für das }\end{array}$ & $0=$ nicht erkennbar & $\begin{array}{l}\text { Es kommen keine sprachlichen Mittel des } \\
\text { Gegenüberstellens vor. }\end{array}$ & \\
\hline $\begin{array}{l}\text { Gegenüberstellen } \\
\text { Gegenüberstellung über Antonyme oder ge- } \\
\text { gensätzlich wirkende Begriffe (insbesondere } \\
\text { Verben; Adjektive) } \\
\text { Mischung von Qualität und Quantität: Viele } \\
\text { sprachliche Mittel des Gegenüberstellens sind } \\
\text { nicht unbedingt ein Qualitätskriterium, wenn } \\
\text { der Leser nach der Vergleichsgröße suchen } \\
\text { muss: „Pseudo-Gegenüberstellung“. } \\
\text { Abweichung von der Realverteilung: da } \\
\text { sprachliche Mittel des Gegenüberstellens häu- }\end{array}$ & $\begin{array}{l}\text { 1= teilweise erkennbar: } \\
\text { implizites Gegenüberstel- } \\
\text { len }\end{array}$ & $\begin{array}{l}\text { Es sind nur wenige Mittel des Gegenüber- } \\
\text { stellens vorhanden (max. 2) } \\
\text { oder } \\
\text { die ausgewählten sprachlichen Mittel ver- } \\
\text { balisieren überwiegend eine Gegenüber- } \\
\text { stellung, ohne auf beide Vergleichsob- } \\
\text { jekte direkt Bezug zu nehmen; sie markie- } \\
\text { ren den Vergleich, jedoch muss der Leser } \\
\text { die zusammenpassenden Vergleichsele- } \\
\text { mente im Text weitgehend selbst suchen. }\end{array}$ & $\begin{array}{l}\text { Bsp.: „Deutschland befand sich in ei- } \\
\text { ner } \\
\text { anderen Ausgangsposition“ } \\
\text { „Deutschland war (noch) nicht so } \\
\text { weit“ (vergleichend gebraucht) } \\
\text { „In England ging die Industriealisie- } \\
\text { rung schneller und besser voran, weil } \\
\text { das Land nicht so aufgeteilt war wie in } \\
\text { Deutschland. England hatte bessere } \\
\text { Voraussetzung und eine größere Be- } \\
\text { völkerung.“ }\end{array}$ \\
\hline fig gebraucht werden können, aber dennoch & $\begin{array}{l}2=\text { erkennbar: explizites } \\
\text { Gegenüberstellen }\end{array}$ & $\begin{array}{l}\text { sprachliche Mittel des Gegenüberstellens } \\
\text { werden überwiegend differenziert ge- } \\
\text { nutzt: sie machen explizit deutlich, welche }\end{array}$ & $\begin{array}{l}\text { Bsp.: „Im Gegensatz zu England verlief } \\
\text { die Industrialisierung in Deutschland } \\
\text { langsamer“ }\end{array}$ \\
\hline
\end{tabular}




\begin{tabular}{|c|c|c|}
\hline 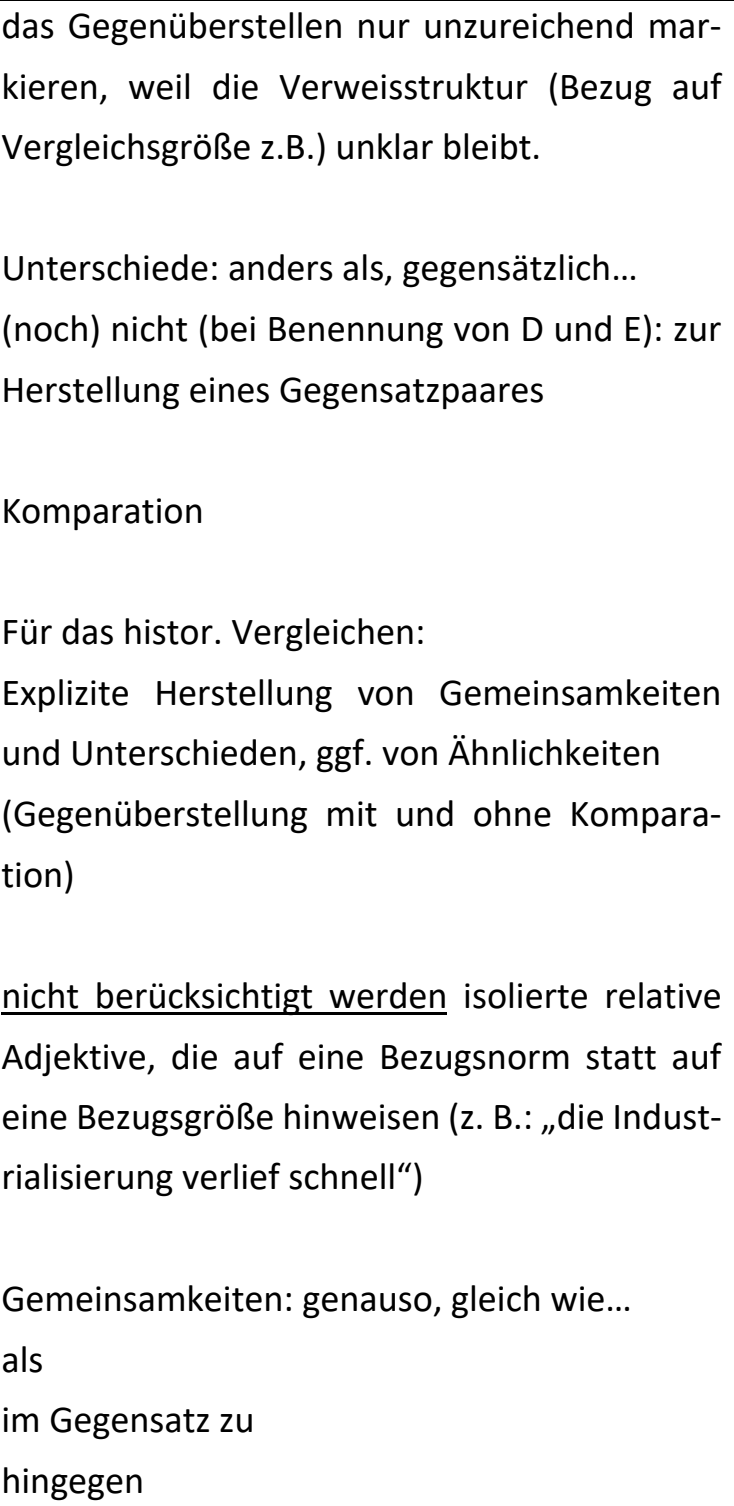 & $\begin{array}{l}\text { Kriterien gegenübergestellt werden, in- } \\
\text { dem sie auf beide Vergleichselemente di- } \\
\text { rekt Bezug nehmen. }\end{array}$ & $\begin{array}{l}\text { „... blieb hinter den Leistungen Eng- } \\
\text { lands zurück..." } \\
\text { „..., die nicht der Englands entsprach." } \\
\text { „Zum Ausgangspunkt der Jahre um } \\
\text { 1770, wo das britische Königreich sich } \\
\text { bereits schon im „großen Aufbruch“ } \\
\text { befand, steckte das noch nicht einmal } \\
\text { vereinte Deutschland, welches in (noch) } \\
\text { über } 300 \text { Territorialteile zerstückelt } \\
\text { war“ } \\
\text { „England war von vorneherein wohl- } \\
\text { habend [...]. Deutschland dagegen } \\
\text { [...].“ } \\
\text { Hier stehen die Vergleichselemente } \\
\text { direkt hintereinander und werden } \\
\text { durch das „hingegen“ sprachlich ge- } \\
\text { kennzeichnet und verbunden. }\end{array}$ \\
\hline
\end{tabular}




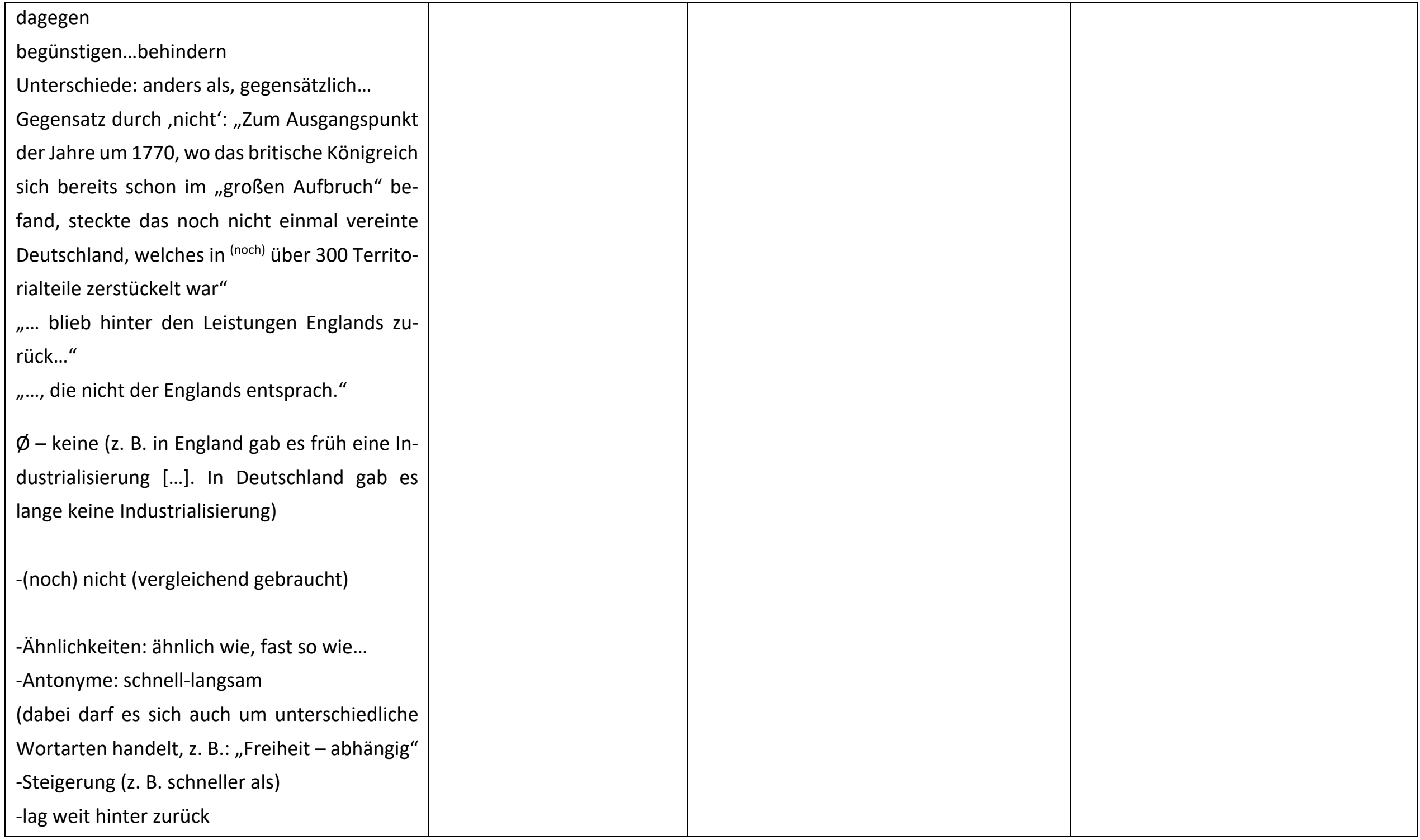




\begin{tabular}{|c|c|c|c|}
\hline $\begin{array}{l}\text {-Gegensatzpaare: begünstigte - hemmte, of- } \\
\text { fen - gebunden ( } 1 x \text { zählen) } \\
\text { (muss sich auf ein Vergleichspaar beziehen) }\end{array}$ & & & \\
\hline $\begin{array}{l}\text { ST } 02 \\
\text { Begründen }\end{array}$ & & & \\
\hline ST 02.1 & $0=$ nicht erkennbar & $\begin{array}{l}\text { Es gibt keine Begründungen, es wird kein } \\
\text { Einwand vorweggenommen. }\end{array}$ & \\
\hline $\begin{array}{l}\text { Begründen fachlich/pragmatisch } \\
\text { Nach Ehlich/Rehbein (1986): Begründen IV: } \\
\text { kognitives Begründen } \\
\text { Vorwegnahme von Einwänden (argumentativ: } \\
\text { eine Handlung/Aussage dem Leser verständ- } \\
\text { lich machen: aus Nicht-Akzeptierbarem Akzep- } \\
\text { tierbares machen) } \\
\text { Begründen: }\end{array}$ & $1=$ teilweise erkennbar & $\begin{array}{l}\text { Es gibt nur Begründungen, die sich auf je- } \\
\text { weils ein Vergleichsobjekt beziehen, aber } \\
\text { nicht in direktem Zusammenhang stehen. } \\
\text { Es gibt implizite Begründungen, die (funk- } \\
\text { tional-pragm.) Abgrenzung von Er- } \\
\text { klärstrukturen ist nicht eindeutig zu er- } \\
\text { kennen. }\end{array}$ & $\begin{array}{l}\text { z. B.: Aussage, dass England schneller } \\
\text { in der Entwicklung war, wird begrün- } \\
\text { det, aber nicht vergleichend mit } \\
\text { Deutschland: } \\
\text { Bsp.: „Die Ursachen der Industriellen } \\
\text { Revolution spielen } \\
\text { eine große Rolle, wieso England einer } \\
\text { der ersten Länder der Industrialisie- } \\
\text { rung war“ (nachfolgend werden die } \\
\text { Ursachen erklärt) }\end{array}$ \\
\hline $\begin{array}{l}\text { „Aussagen (z. B. Urteil, These, Wertung) durch } \\
\text { Argumente stützen, die auf historischen Bei- } \\
\text { spielen und anderen Belegen gründen“ (Kul- } \\
\text { tusministerkonferenz, 2005: EPA Geschichte, } \\
\text { S. 8) } \\
\text { Logikorientierung (Graefen/Moll, 2007) }\end{array}$ & $2=$ erkennbar & $\begin{array}{l}\text { Es gibt mind. eine explizite Begründung, } \\
\text { die sich auf beide Vergleichsobjekte be- } \\
\text { zieht. }\end{array}$ & $\begin{array}{l}\text { Es wird z. B. dargelegt, warum von der } \\
\text { Annahme ausgegangen wird, dass ein } \\
\text { Pionierland der Industrialisierung } \\
\text { war. } \\
\text { z. B.: Ein Grund für die Annahme, dass } \\
\text { England ein Pionierland war, ist... }\end{array}$ \\
\hline
\end{tabular}




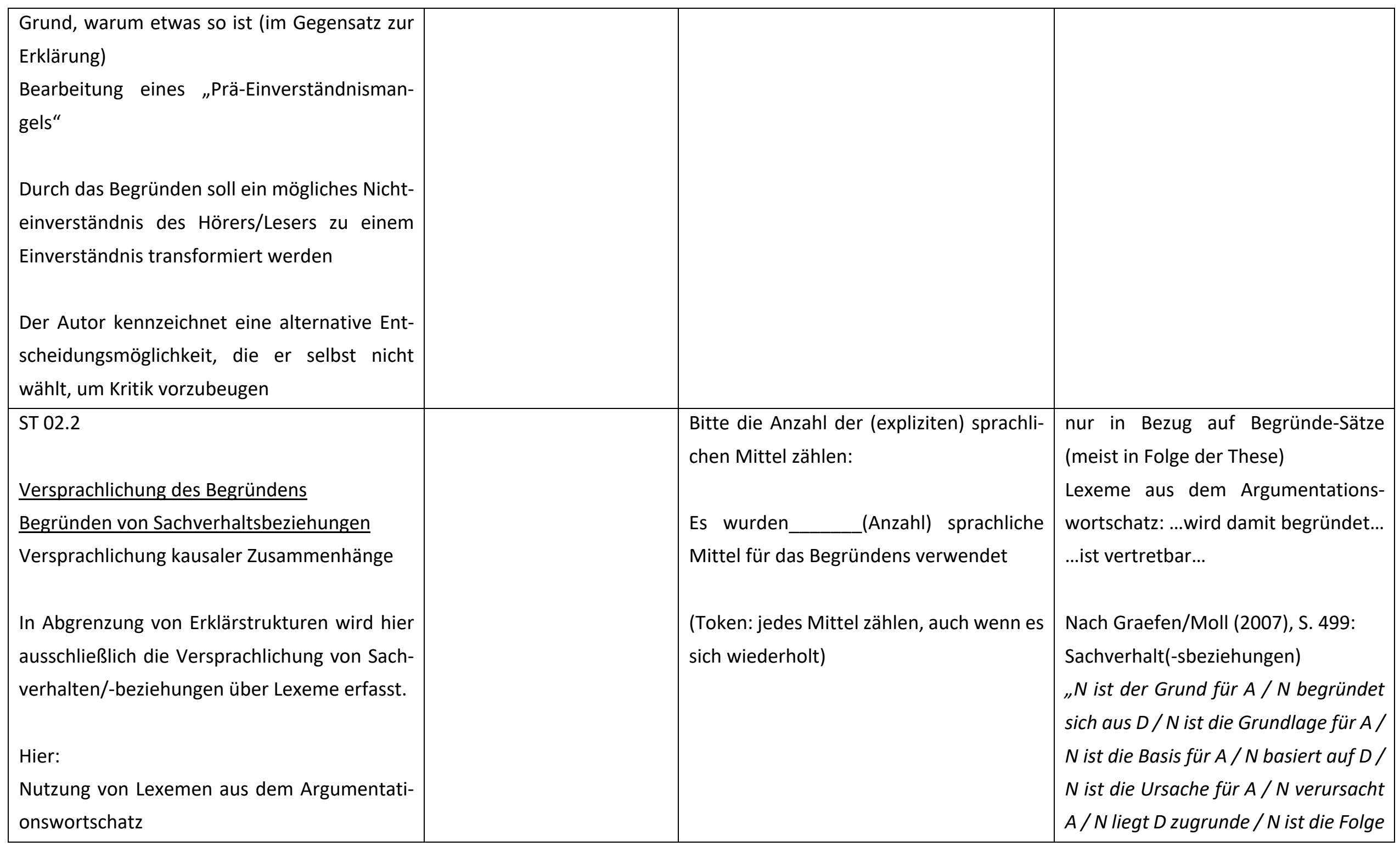




\begin{tabular}{|c|c|c|c|}
\hline $\begin{array}{l}\text { Es geht hier darum, bewusstes Begründen zu } \\
\text { erfassen. Deshalb Beschränkung auf die ge- } \\
\text { nannten sprachlichen Strukturen. }\end{array}$ & & & $\begin{array}{l}\text { von } D / N \text { folgt aus } D / N \text { ruft } A \text { hervor } \\
/ N \text { führt zu } D / N \text { ist die Wirkung von } \\
D^{\prime \prime}\end{array}$ \\
\hline $\begin{array}{l}\text { ST } 03 \\
\text { Erklären }\end{array}$ & & & \\
\hline $\begin{array}{l}\text { ST } 03.1 \\
\text { Erklären fachlich/pragmatisch (über propositi- } \\
\text { onalen Gehalt) }\end{array}$ & $0=$ nicht erkennbar & $\begin{array}{l}\text { sprachliche Handlung wird nicht realisiert, } \\
\text { es kommen keine Erklärungen vor }\end{array}$ & \\
\hline $\begin{array}{l}\text { (Erklären-warum) } \\
\text { „historische Sachverhalte durch Wissen und } \\
\text { Einsichten in einen Zusammenhang (Theorie, } \\
\text { Modell, Regel, Gesetz, Funktionszusammen- } \\
\text { hang) einordnen und begründen“ (Kultusmi- } \\
\text { nisterkonferenz, 2005, S. 8: EPA Geschichte) } \\
\text {-gewährt Einsicht in funktionale Zusammen- } \\
\text { hänge } \\
\text {-Erklären zur Herstellung von Wissen beim Hö- } \\
\text { rer/Leser } \\
\text {-Zusammenhänge darstellen } \\
\text {-Ziel: Wissensvermittlung }\end{array}$ & $1=$ teilweise erkennbar & $\begin{array}{l}\text { teilweise erkennbar: sprachliche Hand- } \\
\text { lung wird angedeutet bzw. teilweise oder } \\
\text { nur implizit realisiert }\end{array}$ & $\begin{array}{l}\text { Bsp.: anstatt einer ausgeführten Er- } \\
\text { klärung werden mehrere Aspekte } \\
\text { ohne weitere Ausführung aneinan- } \\
\text { dergereiht, um so einen zuvor ge- } \\
\text { nannten (meist übergeordneten As- } \\
\text { pekt zu erklären): } \\
\text { „Weitere hemmende Faktoren sind } \\
\text { schlechte Verkehrswege und die er- } \\
\text { tragsschwache Wirtschaft“ (dadurch } \\
\text { soll erklärt werden, warum in } \\
\text { Deutschland die Industrialisierung so } \\
\text { langsam und spät startete, statt einer } \\
\text { richtigen Erklärung wird hier aber e- } \\
\text { her aufgezählt) }\end{array}$ \\
\hline
\end{tabular}




\begin{tabular}{|c|c|c|c|}
\hline $\begin{array}{l}\text { „Mit einer Erklärung macht eine Person ande- } \\
\text { ren oder sich selbst einen Zusammenhang von } \\
\text { Sachverhalten oder Sachverhaltselementen so } \\
\text { klar, dass er ins Wissen integriert und als allge- } \\
\text { meine Orientierung des Handelns genommen } \\
\text { werden kann.“ (Hofmann, 2014; S. 527) } \\
\text { "Aufzuklären ist stets eine Warum-Frage“ } \\
\text { (Hoffmann, 2014; S. 528) }\end{array}$ & $2=$ erkennbar & $\begin{array}{l}\text { sprachliche Handlung wird mind. dreimal } \\
\text { ausführlich und explizit realisiert, dabei ist } \\
\text { der fachliche Zusammenhang korrekt: es } \\
\text { gibt } 3 x \text { fachlich richtige Antworten auf die } \\
\text { Frage "Warum" }\end{array}$ & \\
\hline ST 03.2 & & $\begin{array}{l}\text { Bitte die Anzahl der sprachlichen Mittel } \\
\text { zählen: }\end{array}$ & $\begin{array}{l}\text { Nur Antworten auf Warum-Fragen } \\
\text { zählen }\end{array}$ \\
\hline $\begin{array}{l}\text { Versprachlichung des Erklärens (Erklären-wa- } \\
\text { rum): } \\
\text { sprachliche Mittel für kausale Zusammen- } \\
\text { hänge in Bezug auf das Ziel der Wissensver- } \\
\text { mittlung }\end{array}$ & & $\begin{array}{l}\text { Es wurden___ (Anzahl) sprachliche } \\
\text { Mittel für das Erklären verwendet }\end{array}$ & $\begin{array}{l}\text { Konjunktionen: } \\
\text { weil, da, daher, wegen, um, aufgrund, } \\
\text { denn }\end{array}$ \\
\hline $\begin{array}{l}\text { (hier kann die fachliche Richtigkeit nicht be- } \\
\text { rücksichtigt werden) }\end{array}$ & & & $\begin{array}{l}\text { Adverbien: } \\
\text { darum, daher, deswegen, deshalb } \\
\text { warum (im Fragemodus), weshalb }\end{array}$ \\
\hline $\begin{array}{l}\text { Berücksichtigt werden kausale Konjunktionen } \\
\text { und Adverbien (Adverbien: mit Zeigewortkom- } \\
\text { ponente, reorientierend, (vgl. Hoffmann, } \\
\text { 2014; S. 208) sowie feststehende Wendungen) }\end{array}$ & & & $\begin{array}{l}\text { feststehende Wendungen: ...liegt da- } \\
\text { ran, dass..., der Grund ist..., aus die- } \\
\text { sem Grund... }\end{array}$ \\
\hline
\end{tabular}




\begin{tabular}{|c|c|c|c|}
\hline $\begin{array}{l}\text { ST } 04 \\
\text { Beurteilen }\end{array}$ & & & \\
\hline ST 04.1 & $0=$ nicht erkennbar & $\begin{array}{l}\text { historisches Sachurteil wird nicht reali- } \\
\text { siert }\end{array}$ & \\
\hline $\begin{array}{l}\text { Beurteilen fachlich/pragmatisch } \\
\text { Wertung } \\
\text { Beurteilung im Sinne eines Sach-, ggf. auch ei- } \\
\text { nes Werturteils: histor. Vergleich fordert zum } \\
\text { Sachurteil heraus } \\
\text { „den Stellenwert historischer Sachverhalte in } \\
\text { einem Zusammenhang bestimmen, um ohne } \\
\text { persönlichen Wertebezug zu einem begründe- } \\
\text { ten Sachurteil zu gelangen“ (Kultusminister- } \\
\text { konferenz, 2005, S. 8: EPA Geschichte) } \\
\text { Sachurteil } \\
\text { Interpretation aus der Sachanalyse, Einord- } \\
\text { nung in einen Zusammenhang von Ursache } \\
\text { und Wirkung im Universum des Historischen } \\
\text { (vgl. Gautschi, 2011)) }\end{array}$ & 1= teilweise erkennbar & $\begin{array}{l}\text { Sachurteil wird angedeutet bzw. teilweise } \\
\text { (z. B. nur in Teilbeurteilung) oder nur im- } \\
\text { plizit realisiert } \\
\text { Das Sachurteil ist pauschal, es kann nur } \\
\text { oberflächlich auf die Vergleichsaspekte } \\
\text { zurückgeführt werden } \\
\text { z. B.: } \\
\text {-es gibt Teilurteile über Positiv-/Negativ- } \\
\text { zuschreibungen } \\
\text {-das Urteil steckt in der zentralen These in } \\
\text { der Textmitte, wird jedoch nicht im } \\
\text { Schluss zu Ende geführt, es gibt außerdem } \\
\text { keine Teilbeurteilungen } \\
\text {-die Teilurteile sind pauschal und undiffe- } \\
\text { renziert }\end{array}$ & $\begin{array}{l}\text { "Die Industrialisierung begann wegen } \\
\text { der ganzen Faktoren erst später." }\end{array}$ \\
\hline $\begin{array}{l}\text { analytisch-sachliche histor. Beurteilung auf der } \\
\text { Grundlage des Darstellungstextes, möglichst } \\
\text { differenziert }\end{array}$ & $2=$ erkennbar & $\begin{array}{l}\text { Sachurteil ist klar erkennbar (in Teil- } \\
\text { und/oder Gesamtbeurteilung), das Sach- } \\
\text { urteil erfolgt auf der Basis im Text erkenn- } \\
\text { barer Kriterien }\end{array}$ & $\begin{array}{l}\text { „Abschließend kann festgehalten } \\
\text { werden, dass insbesondere durch die }\end{array}$ \\
\hline
\end{tabular}




\begin{tabular}{|c|c|c|}
\hline $\begin{array}{l}\text { Im Unterschied zum abschließenden Sachurteil } \\
\text { (vgl. TF04) werden hier auch „Teilurteile“ be- } \\
\text { rücksichtigt } \\
\text {-sachliche Wertungen: Bewerten } \\
\text {-kann im Vergleich stecken/erwächst u.a. aus } \\
\text { dem Vergleich } \\
\text { (werten, wie etwas eingeordnet wird) } \\
\text { kriteriengeleitete Conclusio } \\
\text { histor. Beurteilung verlangt fundiertes Sachur- } \\
\text { teil; Unterschied zu allgemeiner Begründung: } \\
\text { Es entsteht auf der Basis der vorangegangenen } \\
\text { Analyse. }\end{array}$ & $\begin{array}{l}\text { klare Wertung erkennbar, es basiert auf } \\
\text { der vorausgegangenen Analyse, ist des- } \\
\text { halb inhaltlich differenziert und nicht pau- } \\
\text { schal } \\
\text { z. B.: } \\
\text { - es gibt eine abschließende Beurteilung } \\
\text { des Verlaufs der Industrialisierung in Eng- } \\
\text { land und Deutschland } \\
\text { oder } \\
\text { - es gibt Teilbeurteilungen der Entwick- } \\
\text { lung von England und Deutschland (zu je- } \\
\text { dem Vergleichsobjekt mind. eine) }\end{array}$ & $\begin{array}{l}\text { Herrschaftsstrukturen die Industriali- } \\
\text { sierung in Deutschland erst später be- } \\
\text { ginnen konnte." }\end{array}$ \\
\hline $\begin{array}{l}\text { ST } 04.2 \\
\text { Versprachlichung des Beurteilens } \\
\text { Verknüpfung von Wertung- und Begrün- } \\
\text { destrukturen } \\
\text {-realisiert durch Verben, Adjektive, Nomen, die } \\
\text { eine Wertung einschließen }\end{array}$ & $\begin{array}{l}\text { Bitte die Anzahl der sprachlichen Mittel } \\
\text { zählen: } \\
\text { Es wurden__(Anzahl) sprachli- } \\
\text { che Mittel für das Beurteilen verwendet }\end{array}$ & $\begin{array}{l}\text { z. B.: } \\
\text {...macht deutlich..., ... wird deutlich..., } \\
\text {...ist einzuordnen..., ...ist festzustel- } \\
\text { len..., ...man sieht..., ...insgesamt lässt } \\
\text { sich sagen... }\end{array}$ \\
\hline $\begin{array}{l}\text { ST } 05 \\
\text { Andere sprachliche Teilhandlungen oder Ele- } \\
\underline{\text { mente solcher (holistisch) }}\end{array}$ & $\begin{array}{l}\text { Bei der Zuordnung der einzelnen sprachli- } \\
\text { chen Handlung zum Code zählt der Ge- } \\
\text { samteindruck. }\end{array}$ & \\
\hline
\end{tabular}




\begin{tabular}{|c|c|c|c|}
\hline \multirow[t]{3}{*}{ ST 05.1 Die sprachliche Handlung Folge } & $\begin{array}{l}0=\text { trägt nicht zum Ver- } \\
\text { gleich bei }\end{array}$ & $\begin{array}{l}\text { trägt nicht dazu bei oder ist nicht vorhan- } \\
\text { den }\end{array}$ & \multirow{3}{*}{$\begin{array}{l}\text { z. B. „England hatte eine schnell stei- } \\
\text { gende Landwirtschaft die die Bevölke- } \\
\text { rung ernähren konnte. Somit enstand } \\
\text { eine Bevölkerung die mehr Wohl- } \\
\text { stand besaß als in Deutschland diese } \\
\text { konnte sich dementsprechen mehr } \\
\text { leisten. Dadurch enstand auch eine } \\
\text { höhere nachfrage die die Wirtschaft } \\
\text { ankurbelte." } \\
\text { (kenntlich gemacht durch Folgepfeile) }\end{array}$} \\
\hline & $\begin{array}{l}\text { 1=trägt teilweise zum Ver- } \\
\text { gleich bei }\end{array}$ & & \\
\hline & $\begin{array}{l}2=\text { trägt in hohem Maße } \\
\text { zum Vergleich bei }\end{array}$ & & \\
\hline \multirow{3}{*}{$\begin{array}{l}\text { ST } 05.2 \text { E Die Reihung } \\
\text { Vergleichsaspekte werden aneinandergereiht, } \\
\text { aber nicht kommentiert }\end{array}$} & $\begin{array}{l}0=\text { trägt nicht zum Ver- } \\
\text { gleich bei }\end{array}$ & $\begin{array}{l}\text { trägt nicht dazu bei oder ist nicht vorhan- } \\
\text { den }\end{array}$ & \multirow{3}{*}{$\begin{array}{l}\text { z. B. } \\
\text { „Abbau von Zoll- } \\
\text { schranken } \\
\text { - Vereinheitl. d. Rechts- } \\
\text { \& Finanzwesens } \\
\text { - an England orientiert } \\
\text { - Besitzindividualismus } \\
\text { - Leistungsprinzip } \\
\text { - Arbeitsteilung } \\
\text { - Konkurrenz“ } \\
\text { (Reihung erkennbar durch Spiegelstri- } \\
\text { che) }\end{array}$} \\
\hline & $\begin{array}{l}\text { 1=trägt teilweise zum Ver- } \\
\text { gleich bei }\end{array}$ & & \\
\hline & $\begin{array}{l}2=\text { trägt in hohem Maße } \\
\text { zum Vergleich bei }\end{array}$ & & \\
\hline
\end{tabular}




\begin{tabular}{|c|c|c|c|}
\hline $\begin{array}{l}\text { Wiss. Textkompetenz I: Umgang mit der Text- } \\
\text { grundlage }\end{array}$ & & & \\
\hline \multirow{3}{*}{$\begin{array}{l}\text { UT } \\
\text { Unterscheidung zwischen Vorlagentext und ei- } \\
\text { gener Position } \\
\text { Globaleindruck: Sind alle Übernahmen ge- } \\
\text { kennzeichnet (durch Anführungsstriche, indi- } \\
\text { rekte Rede oder sprachliche Kennzeichnungen } \\
\text { wie „der Autor sagt..."): } \\
\text { wenn nicht: } 0 \\
\text { wenn nicht alle: } 1 \\
\text { wenn alle: } 2\end{array}$} & $0=$ nicht erkennbar & $\begin{array}{l}\text { Es wird nicht unterschieden, Textpassa- } \\
\text { gen oder Teilsätze oder Schlüsselbegriffe } \\
\text { aus dem Vorlagentext werden ohne kor- } \\
\text { rekte Kennzeichnung übernommen } \\
\text { oder ist nicht vorhanden }\end{array}$ & $\begin{array}{l}\text { Die Industrialisierung begann im Eng- } \\
\text { land des ausgehenden } 18 \text {. Jahrhun- } \\
\text { derts und erfasste im beginnenden } \\
\text { 19. Jahrhundert den westlichen Teil } \\
\text { des europäischen Kontinents sowie } \\
\text { Nordamerika (Übernahme des ge- } \\
\text { samten ersten Satzes ohne Kenn- } \\
\text { zeichnung) }\end{array}$ \\
\hline & $1=$ teilweise erkennbar & $\begin{array}{l}\text { Unterscheidung teilweise erkennbar: Es } \\
\text { wird teilweise, aber nicht vollständig un- } \\
\text { terschieden, Textpassagen oder Teilsätze } \\
\text { aus dem Vorlagentext werden teilweise } \\
\text { korrekt gekennzeichnet oder umformu- } \\
\text { liert. } \\
\text { Nicht alle Übernahmen sind gekennzeich- } \\
\text { net }\end{array}$ & $\begin{array}{l}\text { Bsp.: England hatte die „Pionierrolle" } \\
\text { und Deutschland die der Nachzügler } \\
\text { (eigenes Bsp.). Der Begriff "Nachzüg- } \\
\text { ler“ ist nicht gekennzeichnet, obwohl } \\
\text { er aus dem Text stammt } \\
\text { oder: fehlende Kennzeichnung von } \\
\text { „an die Scholle gebunden“ }\end{array}$ \\
\hline & $2=$ erkennbar & $\begin{array}{l}\text { Es wird vollständig unterschieden, Text- } \\
\text { passagen oder Teilsätze aus dem Vorla- } \\
\text { gentext werden vollständig korrekt ge- } \\
\text { kennzeichnet oder umformuliert. }\end{array}$ & $\begin{array}{l}\text { Bsp. „Aus diesem Grund wurde Eng- } \\
\text { land auch als „Pionierrolle“ in der zu } \\
\text { Beginn der Industrialisierung gese- } \\
\text { hen." Die Übernahme des Begriffs Pi- } \\
\text { onierrolle aus dem Darstellungstext } \\
\text { wird gekennzeichnet. }\end{array}$ \\
\hline
\end{tabular}




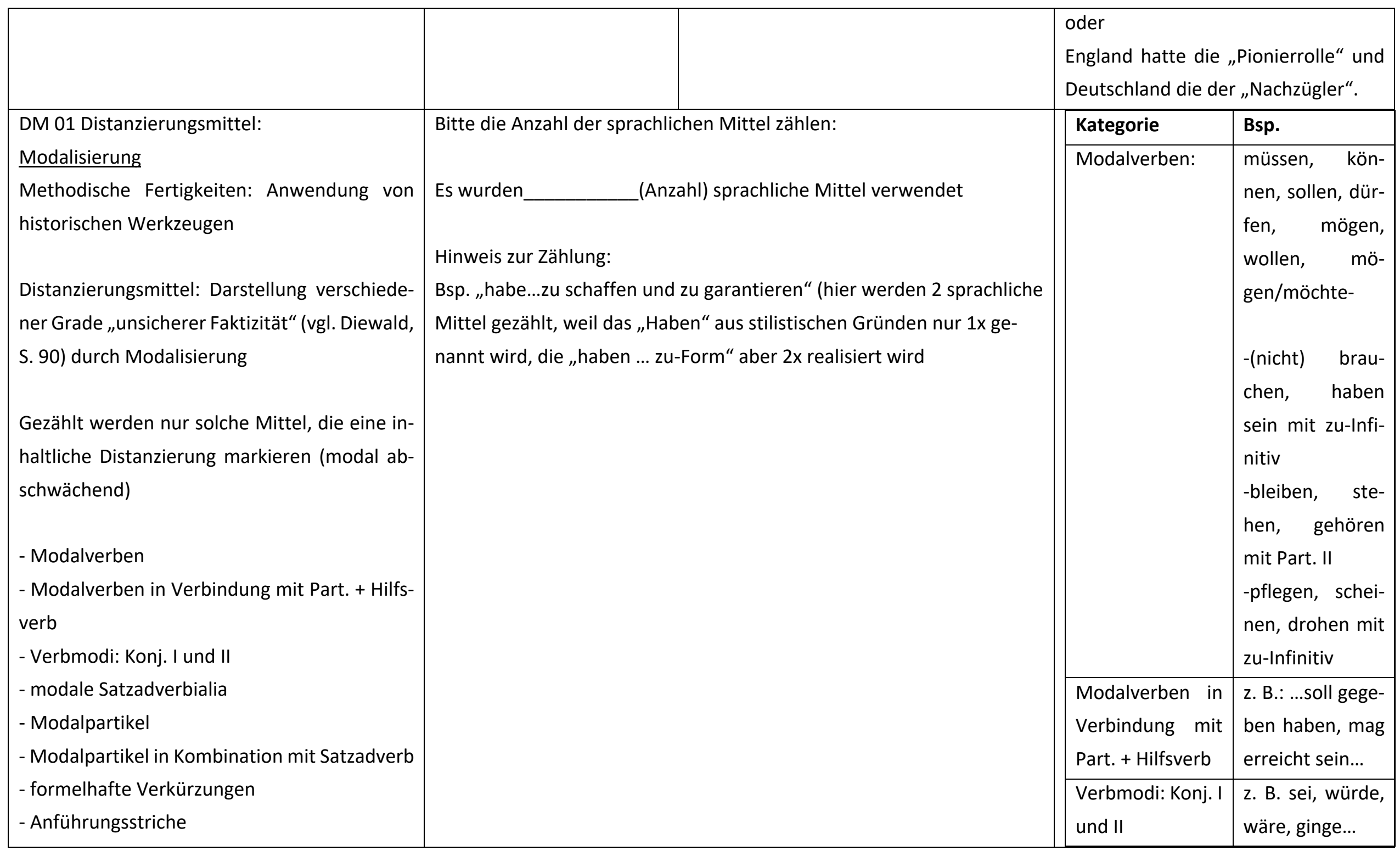




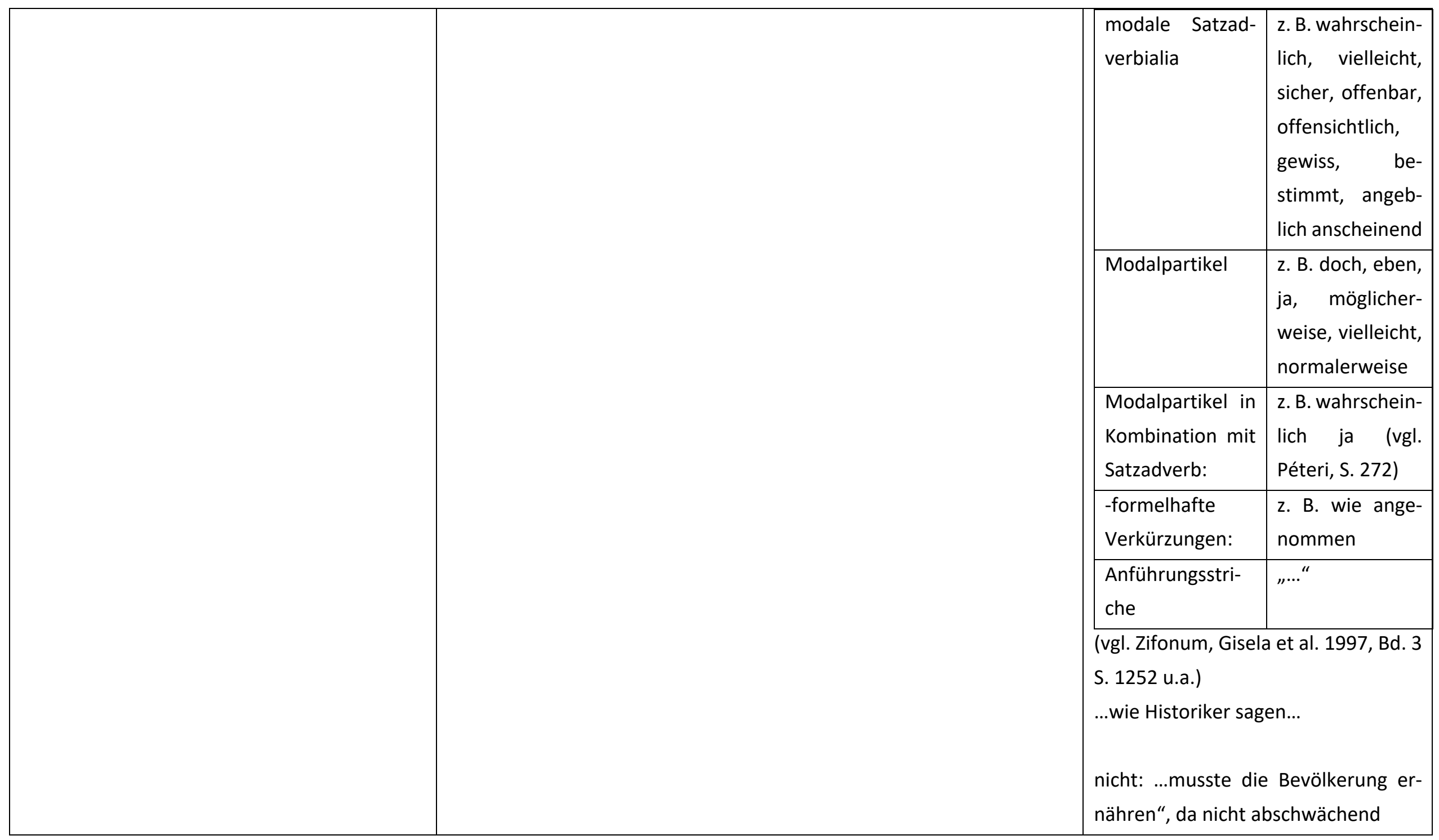




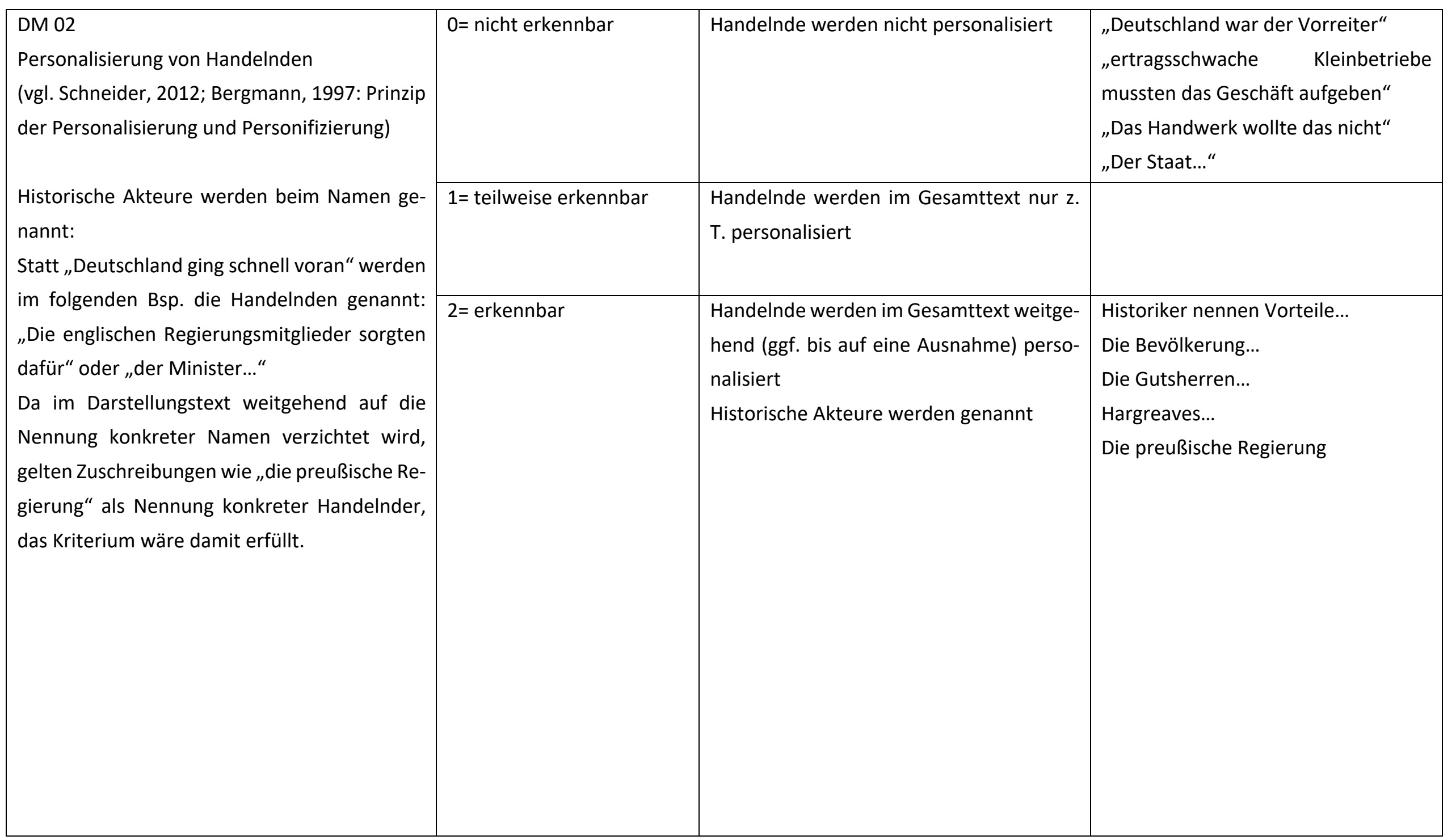




\begin{tabular}{|c|c|c|}
\hline $\begin{array}{l}\text { Wiss. Textkompetenz II: Entwicklung eines } \\
\text { „Roten Fadens“ }\end{array}$ & & \\
\hline Mittel zur Themenentwicklung & & \\
\hline $\begin{array}{l}\text { MT } 01 \\
\text { Themensplitting (Entwicklung durch Teilung) } \\
\text { Ein Redegegenstand enthält mind. ein Paar } \\
\text { (hier: Deutschland und England), daraus wer- } \\
\text { den einzelne Elemente gewählt und thema- } \\
\text { tisch weiterentwickelt (vgl. Hoffmann 2014, S. } \\
\text { 202) } \\
\text { Versprachlichung über Ausdruckspaare: } \\
\text { die eine - die andere } \\
\text { er - sie (Phorik) } \\
\text { der - der (Objektdeixis) } \\
\text { das erste - das zweite } \\
\text { einer - ein weiterer... } \\
\text { auch: Weiterführung über semantische Relati- } \\
\text { onen: } \\
\text { die Industrieländer - England, Deutschland }\end{array}$ & $\begin{array}{l}\text { Bitte die Anzahl der sprachlichen Mittel zählen: } \\
\text { Es wurden__(Anzahl) sprachliche Mittel für das Gegenüber- } \\
\text { stellen verwendet } \\
\text { Anmerkung: das jew. Paar (z. B. Deutschland - England) wird nur 1x als } \\
\text { Mittel gezählt (Type-Zählung), Wechsel in den Wortarten wird als neuer } \\
\text { Type gezählt (England - Deutschland; englisch - deutsch sind } 2 \text { Paare) }\end{array}$ & $\begin{array}{l}\text { Bsp.: „England und Deutschland“ } \\
\text { oder } \\
\text { „das eine Land war schneller, das an- } \\
\text { dere langsamer“ } \\
\text { etc. } \\
\text { oder: deutsch-englisch } \\
\text { die eine-die andere } \\
\text { er-sie (Phorik) } \\
\text { der - der (Objektdeixis) } \\
\text { das erste-das zweite } \\
\text { einer - ein weiterer... } \\
\text { auch: Weiterführung über semanti- } \\
\text { sche Relationen: } \\
\text { die Industrieländer - England, } \\
\text { Deutschland }\end{array}$ \\
\hline MT 02 & $\begin{array}{l}\text { Bitte die Anzahl der sprachlichen Mittel zählen: } \\
\text { Es wurden_ sprachliche Mittel für das Gegenüberstellen } \\
\text { verwendet }\end{array}$ & $\begin{array}{l}\text { Bsp.: „England war Pionierland in der } \\
\text { Industrialisierung. Es war früher als } \\
\text { die anderen Länder.“ } \rightarrow \text { „Es“ (Ana- } \\
\text { pher): Zählung als ein Mittel }\end{array}$ \\
\hline
\end{tabular}




\begin{tabular}{|c|c|}
\hline $\begin{array}{l}\text { Themenfortführung (Auswahl entspr. Relevanz } \\
\text { für wiss.propäd. Arbeiten) (Hoffmann, 2014, S. } \\
\underline{196 f f)} \\
\text { über Anapher: } \\
\text { er, sie es (angepasst an Numerus und Kasus) } \\
\text { über Zeigwörter (Objektdeixis): } \\
\text { der, die, das, dieser, diese, dieses (angepasst } \\
\text { an Numerus und Kasus) } \\
\text { dieses, welches, jenes } \\
\text { da, darauf, hier, dort (nicht das kausale da = } \\
\text { weil) } \\
\text { oder in zusammengesetzten Ausdrücken: da- } \\
\text { mit, hiermit, dadurch, dafür. hierdurch } \\
\text { zug) } \\
\text { das expletive „es“ wird nicht gezählt, wie in: } \\
\text { (Bsp.: Deutschland, das später mit der Indust- } \\
\text { rialisierung begann) }\end{array}$ & $\begin{array}{l}\text { oder } \\
\text { „Deutschland, das/welches später } \\
\text { mit der Industrialisierung begann...“ } \\
\rightarrow \text { „das“/“welches“ als Wiederauf- } \\
\text { nahme von Deutschland in der Einlei- } \\
\text { tung des Relativsatzes: Zählung als ein } \\
\text { Mittel } \\
\text { oder: } \\
\text { "Das heißt“ } \rightarrow \text { deiktisches Mittel, Zäh- } \\
\text { lung als ein Mittel } \\
\text { etc. } \\
\text { was, wenn als Relativum gebraucht } \\
\text { kein wodurch! }\end{array}$ \\
\hline
\end{tabular}


-Hanser, Cornelia/Nussbaumer, Markus/Sieber, Peter (1992): Zürcher Textanalyseraster.

-Bushati, Bora/Ebner, Christoph/Schmölzer-Eibinger, Sabine (Universität Graz) (2015): Vom Lesen zum Schreiben wissenschaftlicher Texte - Untersuchungsdesign und erste Auswertungen einer Interventionsstudie in der 11. Schulstufe. Vorstellung im Rahmen eines Workshops der dieS-Sommerschule „Lesen, um zu schreiben - Reading-to-Write“ vom 18. bis 20. Juni 2015 in Kassel.

-Reich, Hans H. / Roth, Hans-Joachim / Döll, Marion (2009): Fast Catch Bumerang - Auswertungshinweise, Schreibimpuls und Auswertungsbogen. In: Lengyel, Drorit / Reich, Hans H. / Roth, Hans-Joachim / Döll, Marion (Hrsg.): Von der Sprachdiagnose zur Sprachförderung. FörMig Edition Band 5. Münster, S. $209-241$.

Die mit Anführungsstrichen gekennzeichneten Beispiele in Spalte 3 entstammen aus den Erhebungstexten. 
Zu Kapitel 7 Auswertung

\subsection{Interraterreliabilität Pilotierung}

\begin{tabular}{|c|c|c|c|c|c|c|}
\hline & $\begin{array}{l}\text { Cohens } \\
\text { Kappa }\end{array}$ & & & & $\begin{array}{l}\text { Krippendorfs } \\
\text { Alpha }\end{array}$ & \\
\hline $\begin{array}{l}\text { Rater- } \\
\text { paar }\end{array}$ & & $1 \_2$ & 1_3 & 2_3 & & 1_2_3 \\
\hline BA & & 1 & 1 & 0,185 & & 0.513 \\
\hline VS & & 1 & 0,593 & 0,506 & & 0.623 \\
\hline $\begin{array}{l}\text { Text- } \\
\text { länge }\end{array}$ & & 0,952 & 0,672 & 0,718 & & 0.982 \\
\hline $\begin{array}{l}\text { Mittlere } \\
\text { Satz- } \\
\text { länge }\end{array}$ & & 0,708 & 0,199 & 0,19 & & 0.76 \\
\hline $\begin{array}{l}\text { Bearbei- } \\
\text { tung der } \\
\text { Aufgabe }\end{array}$ & & 1 & 0,651 & 0,651 & & 0.741 \\
\hline TA & & 0,817 & 0,941 & 0,731 & & 0.824 \\
\hline IG 01 & & 0,839 & 1 & 0,838 & & $\begin{array}{l}\text { no varia- } \\
\text { tion }\end{array}$ \\
\hline IG 02 & & 0,77 & 1 & 0,77 & & 0.871 \\
\hline IG 03 & & 1 & 0,916 & 0,916 & & 0.947 \\
\hline IG 04 & & 0,888 & 1 & 0,888 & & 0.928 \\
\hline TF 01 & & 0,917 & 1 & 0,917 & & 0.964 \\
\hline TF 02 & & 0,916 & 1 & 0,916 & & 0.948 \\
\hline TF 03.1 & & 0,782 & 1 & 0,782 & & 0.873 \\
\hline TF 03.2 & & 1 & 0,917 & 0,917 & & 0.965 \\
\hline TF 03.3 & & 0,913 & 1 & 0,913 & & 0.982 \\
\hline TF 04 & & 1 & 1 & 1 & & 1.0 \\
\hline ST 01.1 & & 0,859 & 0,703 & 0,561 & & 0.843 \\
\hline ST 01.2 & & 0,899 & 0,899 & 1 & & 0.959 \\
\hline ST 02.1 & & 0,81 & 1 & 0,81 & & 0.843 \\
\hline ST 02.2 & & 0,754 & 0,921 & 0,667 & & 0.887 \\
\hline ST 03.1 & & 0,776 & 1 & 0,776 & & 0.773 \\
\hline ST 03.2 & & 0,846 & 1 & 0,846 & & 0.945 \\
\hline ST 04.1 & & 0,784 & 1 & 0,784 & & 0.86 \\
\hline ST 04.2 & & 0,654 & 1 & 0,645 & & 0.787 \\
\hline
\end{tabular}




\begin{tabular}{|l|l|l|l|l|l|l|l|}
\hline ST 05.1 & & 0,239 & 1 & 0,293 & & & 0.544 \\
\hline ST 05.2 & & 0,786 & 1 & 0,786 & & & 0.889 \\
\hline UT & & 0,771 & 0,488 & 0,744 & & & 0.675 \\
\hline MT 01 & & 0,941 & 0,763 & 0,836 & & & 0.951 \\
\hline MT 02 & & 0,692 & 0,44 & 0,54 & & & 0.801 \\
\hline DM 01 & & 0,763 & 0,709 & 0,942 & & & 0.81 \\
\hline DM 02 & & 0,891 & 0,879 & 0,733 & & & 0.85 \\
\hline
\end{tabular}

\subsection{Interraterreliabilität Korpus gesamt}

\begin{tabular}{|c|c|c|c|c|c|c|}
\hline $\begin{array}{l}\text { Krippen- } \\
\text { dorffs alpha }\end{array}$ & $\begin{array}{l}\mathrm{N} \\
\text { coders }\end{array}$ & $\begin{array}{l}\mathrm{N} \\
\text { cases: }\end{array}$ & $\begin{array}{l}\text { N decisi- } \\
\text { ons: }\end{array}$ & & $\begin{array}{l}\text { Nomi- } \\
\text { nal }\end{array}$ & Name \\
\hline & 3 & 22 & 66 & Ordinal & & BA \\
\hline 0.946 & 3 & 22 & 66 & Ratio & 0.946 & VS \\
\hline 0.985 & 3 & 22 & 66 & $R$ & 0.828 & Textlänge \\
\hline 0.954 & 3 & 22 & 66 & $R$ & 0.603 & $\begin{array}{l}\text { Mittlere Satz- } \\
\text { länge }\end{array}$ \\
\hline 0 & 3 & 22 & 66 & 0 & 0 & $\begin{array}{l}\text { Bearbeitung der } \\
\text { Aufgabe }\end{array}$ \\
\hline 0.917 & 3 & 22 & 66 & 0 & 0.842 & TA \\
\hline 0.906 & 3 & 22 & 66 & 0 & 0.888 & IG 01 \\
\hline 0.933 & 3 & 22 & 66 & 0 & 0.897 & IG 02 \\
\hline 0.785 & 3 & 22 & 66 & 0 & 0.764 & IG 03 \\
\hline 0.799 & 3 & 22 & 66 & 0 & 0.787 & IG 04 \\
\hline 0.767 & 3 & 22 & 66 & 0 & 0.744 & TF 01 \\
\hline 0.748 & 3 & 22 & 66 & 0 & 0.729 & TF 02 \\
\hline 0.816 & 3 & 22 & 66 & 0 & 0.786 & TF 03.1 \\
\hline 0.864 & 3 & 22 & 66 & 0 & 0.8 & TF 03.2 \\
\hline 0.808 & 3 & 22 & 66 & 0 & 0.75 & TF 03.3 \\
\hline 0.914 & 3 & 22 & 66 & 0 & 0.865 & TF 04 \\
\hline 0.802 & 3 & 22 & 66 & 0 & 0.73 & ST 01.1 \\
\hline 0.765 & 3 & 22 & 66 & 0 & 0.652 & ST 01.2 \\
\hline 0.77 & 3 & 22 & 66 & $R$ & 0.742 & ST 02.1 \\
\hline 0.699 & 3 & 22 & 66 & 0 & 0.64 & ST 02.2 \\
\hline
\end{tabular}




\begin{tabular}{|l|l|l|l|l|l|l|l|l|}
\hline 0.835 & 3 & 22 & 66 & $R$ & 0.833 & & & ST 03.1 \\
\hline 0.92 & 3 & 22 & 66 & 0 & 0.817 & & & ST 03.2 \\
\hline 0.849 & 3 & 22 & 66 & 0 & 0.833 & & & ST 04.1 \\
\hline 0.623 & 3 & 22 & 66 & $R$ & 0.623 & & & ST 04.2 \\
\hline 0.734 & 3 & 22 & 66 & 0 & 0.684 & & & ST 05.1 \\
\hline 0.736 & 3 & 22 & 66 & 0 & 0.708 & & & ST 05.2 \\
\hline 0.745 & 3 & 22 & 66 & 0 & 0.718 & & & UT \\
\hline 0.769 & 3 & 22 & 66 & $R$ & 0.677 & & & MT 01 \\
\hline 0.865 & 3 & 22 & 66 & $R$ & 0.646 & & & MT 02 \\
\hline 0.852 & 3 & 22 & 66 & $R$ & 0.787 & & & DM 01 \\
\hline 0.893 & 3 & 22 & 66 & 0 & 0.893 & & & DM 02 \\
\hline
\end{tabular}




\subsection{Cronbachs Alpha für Rater 1}

\section{Item-Skala-Statistiken}

\begin{tabular}{|c|c|c|c|c|}
\hline & $\begin{array}{l}\text { Skalenmittel- } \\
\text { wert, wenn Item } \\
\text { weggelassen }\end{array}$ & $\begin{array}{l}\text { Skalenvarianz, } \\
\text { wenn Item weg- } \\
\text { gelassen }\end{array}$ & $\begin{array}{l}\text { Korrigierte Item- } \\
\text { Skala-Korrela- } \\
\text { tion }\end{array}$ & $\begin{array}{l}\text { Cronbachs Alpha, } \\
\text { wenn Item wegge- } \\
\text { lassen }\end{array}$ \\
\hline TA & 15,99 & 42,132 & ,422 & ,895 \\
\hline IG 01 & 16,95 & 42,986 & ,229 & ,902 \\
\hline IG 02 & 16,71 & 41,026 & ,443 & ,895 \\
\hline IG 03 & 16,38 & 41,130 & ,654 & ,889 \\
\hline IG 04 & 17,01 & 40,663 &, 525 & ,892 \\
\hline TF 01 & 16,65 & 39,409 & ,683 & ,887 \\
\hline TF 02 & 16,12 & 40,540 & ,613 & ,889 \\
\hline TF 03.1 & 15,80 & 40,067 & ,634 & ,888 \\
\hline TF 03.2 & 16,17 & 40,743 & ,435 & ,896 \\
\hline TF 03.3 & 16,92 & 42,511 & ,357 & ,897 \\
\hline TF 04 & 17,26 & 41,834 &, 533 & ,892 \\
\hline ST 01.1 & 16,44 & 40,370 & ,651 & ,888 \\
\hline ST 01.2 & 16,54 & 41,408 & ,549 & ,891 \\
\hline ST 02.1 & 16,87 & 40,669 & ,681 & ,888 \\
\hline ST 03.1 & 16,55 & 40,805 & ,656 & ,888 \\
\hline ST 04.1 & 16,83 & 40,574 & ,678 & ,888 \\
\hline ST 05.1 & 16,62 & 41,468 & ,674 & ,889 \\
\hline ST 05.2 & 16,70 & 42,428 &, 567 & ,892 \\
\hline UT & 17,15 & 43,891 & ,230 & ,899 \\
\hline DM 02 & 16,83 & 42,502 & ,461 & ,894 \\
\hline
\end{tabular}

Betrachtet man zunächst die "Korrigierte Item-Skala-Korrelation" (Trennschärfekoeffizient), ist festzustellen, dass zwei Werte unter 0,3 liegen. Für gute Werte wird eine Grenze von 0,3 angenommen. Diese Grenze erreichen die Items IG 01 („Eigenständige Überschrift“) und UT („Unterscheidung zwischen Vorlagentext und eigener Position") nicht. Da in beiden Fällen Cronbachs Alpha nur marginal über dem Gesamtwert liegt, wenn diese Items weggelassen würden, kann aufgrund dieser geringen Differenz der Wert erhalten bleiben. Zudem sind beide Items für die Erfassung des Vergleichs bedeutsam, so dass sie nicht weggelassen werden können. 


\subsection{Explorative Faktorenanalyse}

7.4.1 Kommunalitäten

\begin{tabular}{ll|l} 
& Anfänglich & Extraktion \\
\hline TA & 1,000 &, 550 \\
\hline IG 01 & 1,000 &, 760 \\
\hline IG 02 & 1,000 &, 789 \\
\hline IG 03 & 1,000 &, 596 \\
\hline IG 04 & 1,000 &, 821 \\
\hline TF 01 & 1,000 &, 823 \\
\hline TF 02 & 1,000 &, 628 \\
\hline TF 03.1 & 1,000 &, 745 \\
\hline TF 03.2 & 1,000 &, 708 \\
\hline TF 03.3 & 1,000 &, 597 \\
\hline TF 04 & 1,000 &, 821 \\
\hline ST 01.1 & 1,000 &, 742 \\
\hline ST 02.1 & 1,000 &, 734 \\
\hline ST 03.1 & 1,000 &, 652 \\
\hline ST 01.2 & 1,000 &, 589 \\
\hline ST 04.1 & 1,000 &, 660 \\
\hline ST 05.1 & 1,000 &, 648 \\
\hline UT & 1,000 &, 474 \\
\hline DM 02 & 1,000 &, 570 \\
\hline ST 05.2 & 1,000 &, 657 \\
\hline Ex 0 (1, 000 & \\
\hline
\end{tabular}

Extraktionsmethode: Hauptkomponentenanalyse.

\subsubsection{KMO- und Bartlett-Test}

Maß der Stichprobeneignung nach Kaiser-Meyer-Olkin. 838

Bartlett-Test auf Sphärizität Ungefähres Chi-Quadrat 907,782

\begin{tabular}{ll}
\hline df & 190 \\
\hline Signifikanz nach Bartlett &, 000
\end{tabular}




\subsubsection{Rotierte Komponentenmatrix}

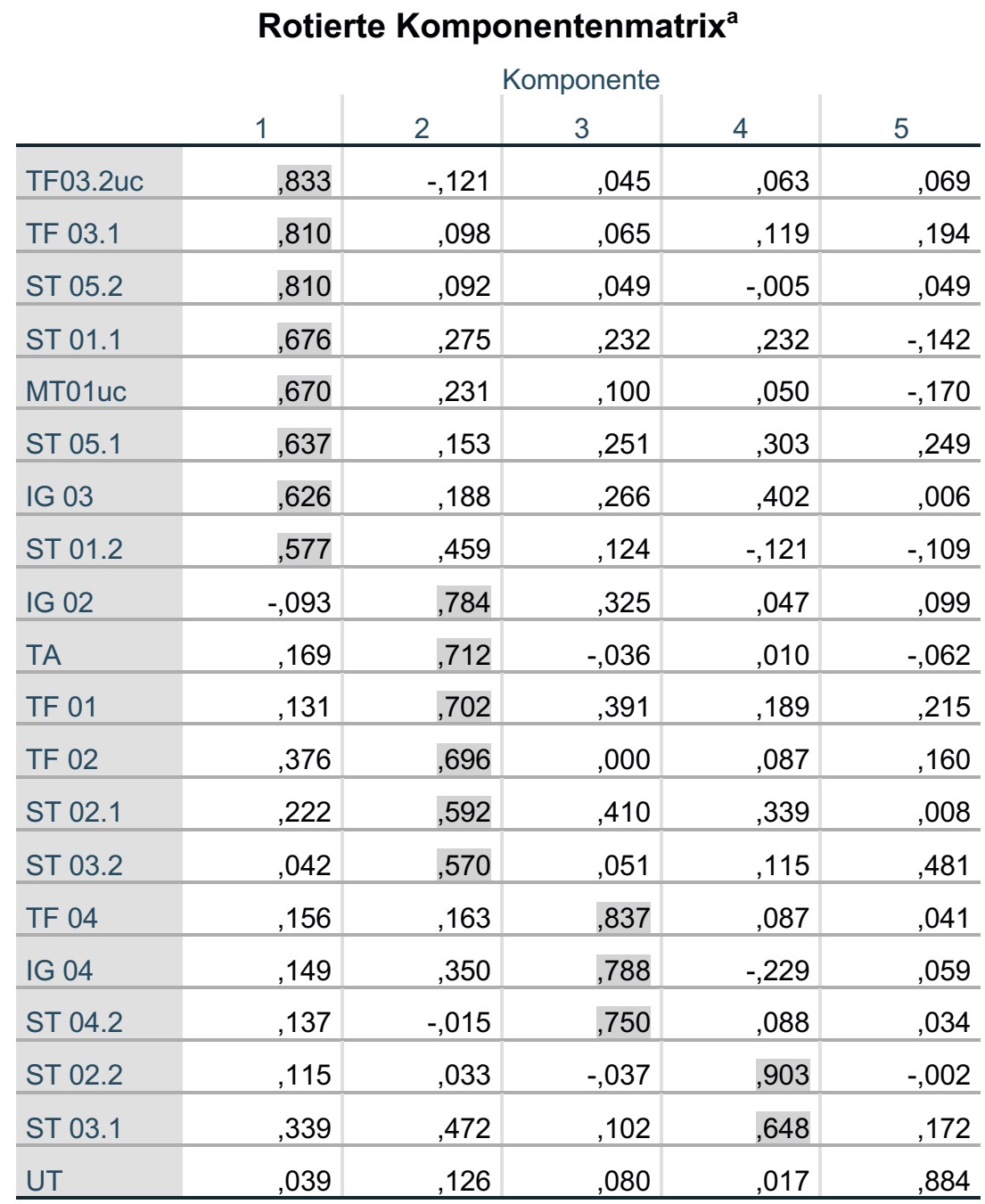

Extraktionsmethode: Hauptkomponentenanalyse.

Rotationsmethode: Varimax mit Kaiser-Normalisierung.

a. Die Rotation ist in 5 Iterationen konvergiert. 
Zu Kapitel 9: Modell „Vergleichen im Geschichtsunterricht“:

\subsection{Zuordnung der Ratings zu den Kompetenzeniveaus ${ }^{774}$}

\begin{tabular}{|l|l|l|l|l|}
\hline Kompetenzniveau I & Items & $\mathbf{0}$ & $\mathbf{1}$ & $\mathbf{2}$ \\
\hline & TF03.2 & $15(17,9 \%)$ & $26(31,0 \%)$ & $43(51,2 \%)$ \\
\hline & TF03.1 & $8(9,5 \%)$ & $9(10,7 \%)$ & $67(79,8 \%)$ \\
\hline & ST05.2 & $17(20,2 \%)$ & $67(79,8 \%)$ & $0(0 \%)$ \\
\hline & ST01.1 & $12(14,3 \%)$ & $55(65,5 \%)$ & $17(20,2 \%)$ \\
\hline & MT01 (uc) & $9(10,7 \%)$ & $59(70,2 \%)$ & $16(19,0 \%)$ \\
\hline & ST05.1 & $14(16,7 \%)$ & $66(78,6 \%)$ & $4(4,8 \%)$ \\
\hline & IG03 & $6(7,1 \%)$ & $62(73,8 \%)$ & $16(19,0 \%)$ \\
\hline & ST01.2 & $14(16,7 \%)$ & $59(70,2 \%)$ & $11(13,1 \%)$ \\
\hline
\end{tabular}

\begin{tabular}{|l|l|l|l|l|}
\hline Kompetenzniveau II & Items & $\mathbf{0}$ & $\mathbf{1}$ & $\mathbf{2}$ \\
\hline & IG02 & $32(38,1 \%)$ & $38(45,2 \%)$ & $14(16,7 \%)$ \\
\hline & TA & $3(3,6 \%)$ & $35(41,7 \%)$ & $46(54,8 \%)$ \\
\hline & TF01 & $26(31,0 \%)$ & $45(53,6 \%)$ & $13(15,5 \%)$ \\
\hline & TF02 & $5(6,0 \%)$ & $42(50 \%)$ & $37(44 \%)$ \\
\hline & ST02.1 & $33(39,3 \%)$ & $49(58,3 \%)$ & $2(2,4 \%)$ \\
\hline & ST03.2 & $43(51,2 \%)$ & $24(28,6 \%)$ & $17(20,2 \%)$ \\
\hline
\end{tabular}

\begin{tabular}{|l|l|l|l|l|}
\hline Kompetenzniveau III & Items & $\mathbf{0}$ & $\mathbf{1}$ & $\mathbf{2}$ \\
\hline & TF04 & $67(79,8 \%)$ & $14(16,7 \%)$ & $3(3,6)$ \\
\hline & IG04 & $51(60,7 \%)$ & $25(29,8 \%)$ & $8(9,5)$ \\
\hline & ST04.2 & $77(91,7)$ & $7(8,3 \%)$ & $0(0 \%)$ \\
\hline
\end{tabular}

Kein Text wird in allen drei Kategorien mit einer "2" geratet, nur drei Texte werden in zwei Kategorien mit einer „2“ geratet (4_24, 5_05, 5_08).

\begin{tabular}{|l|l|l|l|l|}
\hline $\begin{array}{l}\text { Sprachliche Hand- } \\
\text { lungskompetenz }\end{array}$ & Items & $\mathbf{0}$ & $\mathbf{1}$ & $\mathbf{2}$ \\
\hline & ST02.2 & $73(86,9 \%)$ & $8(9,5 \%)$ & $3(3,6 \%)$ \\
\hline & ST03.1 & $14(16,7 \%)$ & $60(71,4 \%)$ & $10(11,9 \%)$ \\
\hline
\end{tabular}

Nur drei Texte (1_02, 1_09, 1_10) werden für beide Items mit einer „2“ geratet.

${ }^{774}$ Die Prozentzahlen lesen sich zeilenweise, die Angabe dieser erfolgen gerundet, so dass Ungenauigkeiten auf Rundungen zurückzuführen sind. 


\section{Literaturverzeichnis}

Abraham, Ulf; Baurmann, Jürgen; Feilke, Helmuth (2015): Materialgestütztes Schreiben. In: Praxis Deutsch 251. S. 4-11.

Altun, Tülay; Günther, Katrin (2018): Begründen als Arbeitsauftrag im Geschichtsunterricht. In: Grannemann, Katharina; Kuchler, Christian; Oleschko, Sven (Hrsg.): Sprachbildung im Geschichtsunterricht. Münster: Waxmann Verlag.

Altun, Tülay; Günther, Katrin (2015): Operatoren am Übergang von der Sekundarstufe I zur Sekundarstufe II als Vorbereitung auf wissenschaftspropädeutisches Arbeiten in der Sekundarstufe II? Eine Auszählung von Aufgabenstellungen in 10 Schulbüchern der Sekundarstufen I und II. Verfügbar unter: https://www.uni-due.de/imperia/md/content/prodaz/altun_g\%C3\%BCnther_operatoren.pdf (zuletzt eingesehen am 22.05.2020).

Arndt, Agnes; Häberlen, Joachim; Reinecke, Christiane (Hrsg.) (2011): Vergleichen, verflechten, verwirren? Europäische Geschichtsschreibung zwischen Theorie und Praxis. Göttingen: Vandenhoeck \& Ruprecht.

Bachmann, Thomas; Becker-Mrotzek, Michael (2017): Schreibkompetenz und Textproduktion modellieren. In: Becker-Mrotzek, Michael; Grabowski, Joachim; Steinhoff, Torsten (Hrsg.): Forschungshandbuch empirische Schreibdidaktik. Münster: Waxmann. S.25-53.

Bäuerle, Rainer (1997): Relativierung, Perspektivierung, Beschränkung: als und wie. In: Umbach, Carla; Grabski, Michael; Hörnig, Robin (Hrsg.): Perspektive in Sprache und Raum. Wiesbaden: Deutscher Universitätsverlag. S. 243-259.

Barriceli, Michele; Gautschi, Peter; Körber, Andreas (2017): Historische Kompetenzen und Kompetenzmodelle. In: Barricelli, Michele; Lücke, Martin (Hrsg.): Handbuch Praxis des Geschichtsunterrichts. Bd. 1. 2. Aufl. Schwalbach/Ts.: Wochenschau Verlag. S. 207-235.

Barricelli, Michele (2017): Narrativität. In: Barricelli, Michele; Lücke, Martin (Hrsg.): Handbuch Praxis des Geschichtsunterrichts. Bd. 1. 2. Aufl. Schwalbach/Ts.: Wochenschau Verlag. S. 255-280.

Barricelli, Michele (2005): Schüler erzählen Geschichte. Narrative Kompetenz im Geschichtsunterricht. Schwalbach/Ts.: Wochenschau-Verlag.

Barricelli, Michele (2002): Narrative Kompetenz als Ziel des Geschichtsunterrichts - Eine empirische Studie zu Erfahrungen von Schülern, Geschichte zu erzählen, in: Handro, Saskia; Schönemann; Bernd (Hrsg.): Methoden geschichtsdidaktischer Forschung (Zeitgeschichte - Zeitverständnis 10), Münster. S. 73-84. 
Becker, Axel (2017): Historische Urteilsbildung. In: Barricelli, Michele; Lücke, Martin (Hrsg.): Handbuch Praxis des Geschichtsunterrichts. Bd. 1. 2. Aufl. Schwalbach/Ts.: Wochenschau Verlag. S. 316-325.

Becker-Mrotzek, Michael; Böttcher, Ingrid (2012): Schreibkompetenz entwickeln und beurteilen. 4. Aufl. Berlin: Cornelsen.

Becker-Mrotzek, Michael; Schindler, Kirsten (2007): Schreibkompetenz modellieren. In: Becker-Mrotzek, Michael; Schindler, Kirsten: Texte schreiben. Duisburg: Gilles \& Francke. S. 7-26.

Beese, Melanie; Roll, Heike (2015): Textsorten im Fach - zur Förderung von Literalität im Sachfach in Schule und Lehrerbildung. In: Benholz, Claudia; Frank, Magnus; Gürsoy, Erkan (Hrsg.). (2015). Deutsch als Zweitsprache in allen Fächern. Konzepte für Lehrerbildung und Unterricht. Beiträge zu Sprachbildung und Mehrsprachigkeit aus dem Modellprojekt ProDaZ. Stuttgart: Fillibach bei Klett. S. 51-72.

Bereiter, Carl (1980): Development in Writing. In: Gregg, Lee W.; Steinberg, Erwin R. (Hrsg.): Cognitive Processes in Writing. Hillsdale: Erlbaum. S. 73-93.

Bergerová, Hana (1997): Vergleichssätze in der deutschen Gegenwartssprache. Frankfurt a. M. u.a.: Peter Lang Verlag.

Bergmann, Klaus (1997): Personalisierung, Personifizierung in: Bergmann, Klaus; Fröhlich, Klaus; Kuhn, Annette; Rüsen, Jörn; Schneider, Gerhard (Hrsg.): Handbuch der Geschichtsdidaktik. Seelze-Velber: Kallmeyer Verlag. S. 298-300.

Bernhardt, Markus; Conrad, Franziska (2018): Sprachsensibler Geschichtsunterricht. Sprachliche Bildung als Aufgabe des Fachs Geschichte. In: Geschichte lernen 31 (2018). H. 182, S. 2-9.

Bernhardt, Markus; Wickner, Mareike-Cathrine (2015): Die narrative Kompetenz vom Kopf auf die Füße stellen - Sprachliche Bildung als Konzept der universitären Geschichtslehrerausbildung. In Benholz, Claudia; Frank, Magnus; Gürsoy, Erkan (Hrsg.): Deutsch als Zweitsprache in allen Fächern. Konzepte für Lehrerbildung und Unterricht. Beiträge zu Sprachbildung und Mehrsprachigkeit aus dem Modellprojekt ProDaZ. Stuttgart: Fillibach bei Klett. S. 281-296.

Bernhardt, Markus (2013): Erarbeitung. In: Mayer, Ulrich; Pandel, Hans-Jürgen; Schneider, Gerhard (Hrsg.): Handbuch Methoden im Geschichtsunterricht. Klaus Bergmann zum Gedächtnis, 4. Aufl. Schwalbach/Ts.: Wochenschau Verlag. S. $619-633$.

Bernhardt, Markus, Gautschi, Peter; Meyer, Ulrich (2011): Historisches Lernen angesichts neuer Kerncurricula. Von Bildungsstandards und Inhaltsfeldern zur Themenbestimmung und Unterrichtsplanung im Geschichtsunterricht. Wiesbaden: Institut für Qualitätsentwicklung. S. 14. Online einsehbar unter: https://www.google.com/url?sa=t\&rct=j\&q=\&esrc=s\&source=web\&cd=1\&ved=2ahUKEwiP17Ljx7vpAhUS3hoKHVTnBBIQFjAAegQIAhAB\&url=https\%3A\%2F\%2Fdieter-vaupel.jimdo- 
free.com\%2Fapp\%2Fdownload\%2F7230429781\%2FBernhardt\%2BGautschi\%2BMayer\%2BHistorisches\%2BLernen\%2B-Kerncurricula.pdf\%3Ft\%3D1586423116\&usg=AOvVawOVGaNTSmVz4IvSTjiNp8c- (zuletzt eingesehen am 17.05.2020).

Bildungsportal des Landes Nordrhein-Westfalen: Zentralabitur in der gymnasialen Oberstufe. Geschichte. Online einsehbar: https://www.standardsicherung.schulministerium.nrw.de/cms/zentralabitur-gost/faecher/fach.php?fach=12 (zuletzt eingesehen am 22.05.2020).

Bloch, Marc (1994): Für eine vergleichende Geschichtsbetrachtung der europäischen Gesellschaften. In: Middel, Matthias; Sammler, Steffen (Hrsg.): Alles Gewordene hat Geschichte. Die Schule der Annales in ihren Texten. Leipzig: Reclam Verlag. S. 121-167.

Bourdieu, Pierre (1982): Die feinen Unterschiede. Kritik der gesellschaftlichen Urteilskraft. 1. Aufl. Frankfurt a. M.: Suhrkamp Verlag.

Bortz, Jürgen; Döring, Nicola (2009): Forschungsmethoden und Evaluation in den Sozial- und Humanwissenschaften. 4. Aufl. Heidelberg: Springer Verlag.

Brinker, Klaus (2010): Linguistische Textanalyse. Eine Einführung in Grundbegriffe und Methoden. 7. Aufl. Berlin: Erich Schmidt Verlag. S. 135.

Brinker, Klaus (1983): Textfunktionen. Ansätze zu ihrer Beschreibung. In: Zeitschrift für germanistische Linguistik. Bd. 11 H. 2, S. 127-148. Online verfügbar unter:

https://www.degruyter.com/downloadpdf/j/zfgl.1983.11.issue-

2/zfgl.1983.11.2.127/zfgl.1983.11.2.127.pdf (zuletzt eingesehen am 17.05.2020).

Bubenhofer, Noah; Lange, Willi; Okamura, Saburo; Scharloth, Joachim (2015): Wortschätze in Lehrbüchern für Deutsch als Fremdsprache - Möglichkeiten und Grenzen frequenzorientierter Ansätze. In: Ott, Christine; Kiesendahl, Jana (Hrsg.): Linguistik und Schulbuchforschung: Gegenstände - Methoden - Perspektiven. Göttingen: V\&R unipress. S. 85-107.

Bubenhofer, Noah (2009): Sprachgebrauchsmuster. Korpuslinguistik als Methode der Diskurs- und Kulturanalyse. Berlin/New York.

Demandt, Alexander (1987): Metaphern für Geschichte. Sprachbilder und Gleichnisse im historischpolitischen Denken. München: Beck Verlag.

Diewald, Gabriele (2013): Modus und Modalverben - Kategorisierungsoptionen im grammatischen Kernbereich der Modalität. In: Abraham, Werner; Leiss, Elisabeth (Hrsg.): Funktionen von Modalität. Berlin/Boston: de Gruyter. S. 77-112.

Doherty, Monika (1970): Zur Komparation antonymer Adjektive. In: ASG-Bericht 6, Berlin: Deutsche Akademie der Wissenschaft. 
Drüding, Markus; Schlutow, Martin (2019): Vergleich(en) im Geschichtsunterricht. Frankfurt a. M.: Wochenschau Verlag.

Eggs, Frederike (2007): Adjunktor. In: Hoffmann, Ludger (Hrsg.): Handbuch der deutschen Wortarten. 2. Aufl. Berlin u.a.: de Gruyter. S. 189-221.

Eggs, Frederike (2006a): die Grammatik von als und wie. Tübingen: Gunter Narr Verlag.

Eggs, Frederike (2006b): Vergleichen und Vergleiche - Implikationen der Sprachwissenschaft für die Sprachdidaktik In: Becker, Tabea und Peschel, Corinna (Hrsg.): Gesteuerter und ungesteuerter Grammatikerwerb. Hohengehren: Schneider. S. 45-62.

Eggers, Michael (2011): Vom Wissen zur Wissenschaft. Vergleich, Analogie, Klassifikation als wissenschaftliche Ordnungsmethoden im 18. und 19. Jahrhundert-zur Einleitung. In: Eggers, Michael (Hrsg.): Von Ähnlichkeiten und Unterschieden. Vergleich, Analogie und Klassifikation in Wissenschaft und Literatur (18./19. Jahrhundert). Heidelberg: Universitätsverlag Winter. S. 7-31.

Ehlich, Konrad (2009): Erklären verstehen - Erklären und Verstehen. In: Vogt, Rüdiger (Hrsg.): Erklären Gesprächsanalytische und fachdidaktische Perspektiven. Tübingen: Stauffenburg Verlag. S. 11-24.

Ehlich, Konrad (2007): Sprache und sprachliches Handeln. Bd. 3 (Diskurs - Narration - Text - Schrift). New York: de Gruyter.

Ehlich, Konrad (2005): dabar und logos. Kursorische Überlegungen zum Verhältnis von Sprache und Geschichte. In: Trabant, Jürgen (Hrsg.): Sprache der Geschichte. München: Oldenbourg. S. 27-39.

Ehlich, Konrad (1999): Alltägliche Wissenschaftssprache. In: Info DaF 1/1999. S. 3-24.

Ehlich, Konrad (1993): Deutsch als fremde Wissenschaftssprache. In: Wierlacher, Alois et al. (Hrsg.) Jahrbuch Deutsch als Fremdsprache 19, 13-42.

Ehlich, Konrad; Rehbein, Jochen (1986): Muster und Institution. Untersuchungen zur schulischen Kommunikation. Tübingen: Narr.

Ehlich, Konrad (1984): Zum Textbegriff. In: Rothkegel, Annely; Sandig, Barbara (Hrsg.) Text - Textsorten - Semantik. Hamburg: Buske. S. 9-25.

Ehlich, Konrad (1981): Schulischer Diskurs als Dialog? In: Schröder, Peter; Steger, Hugo (Hrsg.): Dialogforschung. Düsseldorf: Schwann, S. 334-369.

Ehlich, Konrad; Rehbein, Jochen (1979) Sprachliche Handlungsmuster. In: Soeffner, Hans-Georg (Hrsg.): Interpretative Verfahren in den Sozial- und Textwissenschaften. Stuttgart: Metzler, 243-274. 
Erben, Johannes (1988): Vergleichsurteile und Vergleichsstrukturen im Deutschen. In: Sprachwissenschaft 13, 1988. S. 309-329.

Feilke, Helmuth; Köster, Juliane; Steinmetz, Michael (2012): Zur Einführung - Textkompetenzen in der Sekundarstufe II. In: Feilke, Helmuth; Köster, Juliane; Steinmetz, Michael (Hrsg.): Textkompetenzen in der Sekundarstufe II. Stuttgart: Fillibach bei Klett. S. 7-18.

Feilke, Helmuth (2011): Was sind Textroutinen? In: Feilke, Helmuth; Lehnen, Katrin (Hrsg.): Schreibund Textroutinen. Frankfurt a. M. Lang. S. 1-31.

Feilke, Helmuth; Lehnen, Katrin (Hrsg.) (2011): Schreib- und Textroutinen. Theorie, Erwerb und didaktisch-mediale Modellierung. Frankfurt a. M.: Peter Lang Verlag. S. 1- 31.

Feilke, Helmuth (2010): „Aller guten Dinge sind drei“ - Überlegungen zu Textroutinen \& literalen Prozeduren. In: Fest-Platte für Gerd Fritz. Hrsg. und betreut von Iris Bons, Thomas Gloning und Dennis Kaltwasser. Gießen 17.05.2010. Online-Zugriff unter: http://www.festschrift-gerd-fritz.de/files/feilke_2010_literale-prozeduren-und-textroutinen.pdf (zuletzt eingesehen am 22.05.2020).

Fix, Martin (2008): Texte schreiben. Schreibprozesse im Deutschunterricht. Paderborn: Schöningh UTB. Fix, Ulla (2008): Texte und Textsorten - sprachliche, kommunikative und kulturelle Phänomene. Berlin: Frank \& Timme.

Gautschi, Peter (2016): Plausibilität der Theorie, Spuren der Empirie, Weisheit der Praxis. Zum Stand der geschichtsdidaktischen Kompetenzdiskussion. In: Geschichte für heute. Zeitschrift für historischpolitische Bildung, Heft 3 (2016), S. 5-19.

Gautschi, Peter (2011): Guter Geschichtsunterricht. Grundlagen, Erkenntnisse, Hinweise. 2. Aufl. Schwalbach/Ts.: Wochenschau-Verlag.

Goertz, Hans-Jürgen (2002): Wirklichkeit. In: Jordan, Stefan (Hrsg.): Lexikon Geschichtswissenschaft. Hundert Grundbegriffe. Stuttgart: Reclam. S. 328-330.

Graefen, Gabriele (2008) Versteckte Metaphorik - ein Problem im Umgang mit der fremden deutschen Wissenschaftssprache. In: Dalmas, Martine; Foschi-Albert, Marina; Neuland, Eva (Hrg.) Wissenschaftliche Textsorten im Germanistikstudium deutsch-italienisch-französisch kontrastiv. Trilaterales Forschungsprojekt in der Villa Vigoni (2007-2008). 2. Auflage (2013), Teil 2, 151-168. Online einsehbar unter:

www.villavigoni.it/contents/editions/ VV_Gesamtmanuskript_nuova_edizione_04.03.13.pdf (zuletzt eingesehen am 02.06.18). 
Graefen, Gabriele; Moll, Melanie (2007): Das Handlungsmuster Begründen: Wege zum Unterricht „Deutsch als fremde Wissenschaftssprache“. In: Redder, Angelika (Hrsg.): Diskurse und Texte. Festschrift für Konrad Ehlich zum 65. Geburtstag. Tübingen: Stauffenburg Verlag. S. 419-515.

Gabriele Graefen, München (2002): Probleme mit der Alläglichen Wissenschaftssprache in Hausarbeiten ausländischer StudentInnen. In: Rehbein, Jochen; Riedel, Sabine (Hrsg.): Lernen in der zweiten Sprache. Online-Zugriff: www.wissenschaftssprache.de/Texte/GG_Probleme_mit_AWS_2002.pdf (zuletzt eingesehen am 09.02.17).

Graefen, Gabriele (1997): Der wissenschaftliche Artikel - Textart und Textorganisation. Frankfurt a. M. (Arbeiten zur Sprachanalyse Bd. 27).

Günther-Arndt, Hilke (2010): Hinwendung zur Sprache in der Geschichtsdidaktik - Alte Fragen und neue Antworten. In: Handro, Saskia; Schönemann, Bernd (Hrsg.): Geschichte und Sprache (Zeitgeschichte Zeitverständnis 21). Berlin: LiT-Verlag, S. 17-46.

Hahn, Hans Werner (2011): Die industrielle Revolution in Deutschland (Enzyklopädie deutscher Geschichte 49), 3. Auflage München

Hahnemann, Suzan (1999): Vergleiche im Vergleich. Zur Syntax und Semantik ausgewählter Vergleichsstrukturen mit >als< und >wie< im Deutschen. Tübingen: Niemeyer (Linguistische Arbeiten; 397).

Hallet, Wolfgang (2013): Generisches Lernen im Fach. In: Becker-Mrotzek, Michael et al. (Hrsg.) (2013): Sprache im Fach. Münster/New York: Waxmann. S. 59-76.

Hammann, Marcus (2002): Kriteriengeleitetes Vergleichen im Biologieunterricht. Innsbruck, München: Studienverlag.

Handro, Saskia (2018): Geschichte lesen, aber wie? Plädoyer für eine geschichtsdidaktische Profilierung von Lesestrategien. In: Sandkühler, Thomas; Bühl-Gramer, Charlotte; John, Anke; Schwabe, Astrid; Bernhardt, Markus (Hrsg.): Geschichtsunterricht im 21. Jahrhundert. Bonn: Bundeszentrale für politische Bildung. S. 275-293.

Handro, Saskia (2014): Sprachlos im Geschichtsunterricht. Blogbeitrag vom 9.1.14. In: Public History Weekly. The International Blogjournal. Online-Ressource: https://public-history-weekly.degruyter.com/2-2014-1/sprachlos-im-geschichtsunterricht/ (zuletzt eingesehen am 07.03.17).

Handro, Saskia (2013): Sprache und historisches Lernen. In: Becker-Mrotzek, Michael; Schramm, Karen; Thürmann, Eike; Vollmer; Helmut Johannes (Hrsg.): Sprache im Fach. Sprachlichkeit und fachliches Lernen. Münster: Waxmann S. 318-333.

Handro, Saskia; Schönemann, Bernd (2010): Geschichte und Sprache - Eine Einführung. In: Dies. (Hrsg.): Geschichte und Sprache (Zeitgeschichte - Zeitverständnis 21). Berlin: LiT-Verlag, S. 3-15. 
Hanser, Cornelia; Nussbaumer, Markus; Sieber, Peter (1996): Zürcher Textanalyseraster. In: Nussbaumer, Markus: Lernerorientierte Textanalyse - Eine Hilfe zum Textverfassen? In: Feilke, Helmuth / Portmann, Paul R. (Hrsg.): Schreiben im Umbruch. Stuttgart: Klett.

Hartung, Olaf; Memminger, Josef (2017): Schreibend Geschichte lernen. Mehr als ein „mündliches Fach“ - die Vielfalt des Schreibens im Geschichtsunterricht entdecken. In: Geschichte lernen, 176. S. 2-11.

Hartung, Olaf (2016): Generische Lernaufgaben im Geschichtsunterricht. In: Handro, Saskia; Schönemann, Bernd (Hrsg.): Aus der Geschichte lernen? Weiße Flecken der Kompetenzdebatte. Münster, Hamburg, Berlin, London: LIT. S. 187-198.

Hartung, Olaf (2014): Die Fragen, die Saskia Handro in ihrem Beitrag zum Forschungsfeld Sprache und Geschichtslernen stellt, sind für die Gestaltung historischer Lernprozesse essentiell [Blog-Kommentar], veröffentlicht am 27.01.2014 um 14:25 auf Public History Weekly. The International Blogjournal. Online-Ressource: https://public-history-weekly.degruyter.com/2-2014-1/sprachlos-im-geschichtsunterricht/ (zuletzt eingesehen am 07.03.17).

Hartung, Olaf (2013a): Sprache und konzeptionelles Schreibhandeln im Fach Geschichte. Ergebnisse der empirischen Fallstudie "Geschichte-Schreiben-Lernen". In: Becker Mrotzek, Michael; Schramm, Karen; Thürmann, Eike; Vollmer, Helmut Johannes (Hrsg.): Sprache im Fach. Sprachlichkeit und fachliches Lernen. Münster u.a.: Waxmann, S. 335-353.

Hartung, Olaf (2013b): Geschichte Schreiben Lernen. Empirische Erkundungen zum konzeptionellen Schreibhandeln im Geschichtsunterricht. Berlin: LIT Verlag.

Hartung, Olaf (2010a): Die ,sich ewig wiederholende Arbeit' des Geschichtsbewusstseins - Sprache als Medium des historischen Lernens, in: Zeitschrift für Geschichtsdidaktik 9 (2010), S. 181-191.

Hartung, Olaf (2010b): Historisches Lernen und (Schreib-)Kultur. Zur Bedeutung einer „Kulturtechnik" für das Geschichtslernen. In: Hartung, Olaf u.a. (Hrsg.): Lernen und Kultur. Kulturwissenschaftliche Perspektiven in den Bildungswissenschaften (Schule und Gesellschaft 46). Wiesbaden: Verlag für Sozialwissenschaften, S. 67-79.

Hartung; Olaf (2010c): Geschichte - Schreiben - Lernen. Eine empirische Studie. In: Handro, Saskia; Schönemann, Bernd (Hrsg.): Sprache und Geschichte (Zeitgeschichte - Zeitverständnis, Bd. 21). Berlin: LIT-Verlag. S. 61-77.

Haueis, Eduard (2003) Formen schriftlicher Texte. In: Bredel, Ursula; Günther, Hartmut; Klotz, Peter; Ossner, Jakob; Siebert-Ott, Gesa (Hrsg.): Didaktik der deutschen Sprache. Ein Handbuch. Band 1. Paderborn et al.: Schöningh, S. 224-236. 
Haupt, Heinz-Gerhard; Kocka, Jürgen (1996): Historischer Vergleich: Methode, Aufgaben, Probleme. Eine Einleitung. In: Haupt, Heinz-Gerhard; Kocka, Jürgen: Geschichte und Vergleich. Ansätze und Ergebnisse international vergleichender Geschichtsschreibung. New York: Campus Verlag. S. 9-45.

Hayes, John R.; Flower, Linda S.: Identifying the Organization of Writing Processes. In: Gregg, Lee W.; Steinberg, Erwin R.: Cognitive processes in writing. Hillsdale: Lawrence Erlbaum Associates, 1980, S.330.

Hayes, Andrew F.; Krippendorff, Klaus (2007): Answering the Call for a Standard reliability Measure for Coding data. In: Communication Methods and Measures 1 Jg., Nr. 1, 77-89. Online verfügbar unter: http://www.afhayes.com/public/kalpha.pdf (zuletzt eingesehen am 25.06.2018).

Hodel, Jan (2013): Verkürzen und Verknüpfen. Geschichte als Netz narrativer Fragmente: Wie Jugendliche digitale Netzmedien für die Erstellung von Referaten im Geschichtsunterricht verwenden. Bern: hep Verlag.

Hodel, Jan; Waldis, Monika (2007): Sichtstrukturen im Geschichtsunterricht - die Ergebnisse der Videoanalyse. In: Gautschi, Peter; Moser, Daniel; Reusser, Kurt; Wiher, Pit (Hrsg.): Geschichtsunterricht heute. Eine empirische Analyse ausgewählter Aspekte. Bern: h.e.p. Verlag. S. 91-142.

Hoffmann, Ludger (2014): Deutsche Grammatik. Grundlagen für Lehrerausbildung, Schule, Deutsch als Zweitsprache und Deutsch als Fremdsprache. Berlin: Erich Schmidt Verlag.

Hoffmann, Lothar (1998): Syntaktische und morphologische Eigenschaften von Fachsprachen. In: Hoffmann, Lothar; Kalverkämper, Hartwig; Wiegand, Herbert Ernst (Hrsg.): Fachsprachen / Languages for Special Purposes. Ein internationales Handbuch zur Fachsprachenforschung und Terminologiewissenschaft / An International Handbook of Special-Language and Terminology Research. Berlin: de Gruyter. S. 416-427.

Hofmannsthal, Hugo von (1927): Wert und Ehre deutscher Sprache. München: Verlag der Bremer Presse.

Humboldt, Wilhelm v. (1973): Über die Verschiedenheit des menschlichen Sprachbaues und ihren Einfluß auf die geistige Entwicklung des Menschengeschlechts. In: Humboldt, Wilhelm v. (Hrsg.): Schriften zur Sprache Stuttgart: Reclam. [Orig. v. 1836].

Humboldt, Wilhelm v. (1908): Fragmente der Monographie über die Basken. Hrsg.: Leitzmann, Albert: Gesammelte Schriften Bd. VII. 2. Hälfte. Berlin. S. 602ff. Zit. n.: Hofmannsthal, Hugo von (1927): Wert und Ehre deutscher Sprache. München: Verlag der Bremer Presse. S. 146.

Jeismann, Karl-Ernst (2000): "Geschichtsbewusstsein" als zentrale Kategorie der Didaktik des Geschichtsunterrichts, in: Jeismann, Karl-Ernst (Hrsg.): Geschichte und Bildung. Beiträge zur Geschichtsdidaktik und zur Historischen Bildungsforschung, Paderborn. S. 46-72. 
Kaelble, Hartmut (2012): Historischer Vergleich, Version: 1.0, in: Docupedia-Zeitgeschichte, 14.8.2012, URL: http://docupedia.de/zg/Historischer_Vergleich (zuletzt eingesehen am 01.08.2018).

Kaelble, Hartmut (2005): Die Debatte über Vergleich und Transfer und was jetzt? In: H-Soz-u-Kult. Nr. 08. Online einsehbar unter:

http://hsozkult.geschichte.hu-berlin.de/index.asp?id=574\&view=pdf\&pn=forum\&type=artikel (zuletzt eingesehen am 12.04.2018).

Kaelble, Hartmut (2002): Vergleich, historischer. In: Jordan, Stefan (Hrsg.): Lexikon Geschichtswissenschaft. Hundert Grundbegriffe. Stuttgart: Reclam. S. 303-306.

Kaelble, Hartmut (1999): Der historische Vergleich. Eine Einführung zum 19. und 20. Jahrhundert. Frankfurt a. M./ New York: Campus Verlag.

Kameyama, Sinichi (1999): Wiederholen. In: Bührig, Kristin; Matras, Yaron (Hrsg.): Sprachtheorie und sprachliches Handeln. Festschrift für Jochen Rehbein zum 60. Geburtstag. Tübingen: Stauffenburg Verlag. S. 187-202.

Kast, Bernd (2003): Fertigkeit Schreiben. Das Fernstudienangebot Deutsch als Fremdsprache. Fernstudieneinheit 12. München: Langenscheidt-Verlag.

Kilian, Jörg; Brouër, Birgit; Lüttenberg, Dina (2016): Handbuch Sprache in der Bildung. Berlin: de Gruyter.

Knopp, Matthias; Jost, Jörg; Linnemann, Markus; Becker-Mrotzek, Michael (2014): Textprozeduren als Indikatoren von Schreibkompetenz - ein empirischer Zugriff. In: Bachmann, Thomas; Feilke, Helmuth (2014): Werkzeuge des Schreibens. Stuttgart: Fillibach bei Klett. S. 111-128.

Koch, Peter; Oesterreicher, Wulf (1985): Sprache der Nähe - Sprache der Distanz. Mündlichkeit und Schriftlichkeit im Spannungsfeld von Sprachtheorie und Sprachgeschichte. In: Romanistisches Jahrbuch 36/85. S. $15-43$.

Körber, Andreas; Schreiber, Waltraud; Schöner, Alexander (Hrsg.) (2007): Kompetenzen historischen Denkens. Ein Strukturmodell als Beitrag zur Kompetenzorientierung in der Geschichtsdidaktik. (Kompetenzen: Grundlagen - Entwicklung - Förderung. Bd. 1). Neuried: Ars Una. S. 155.-193.

Köster, Manuel; Bernhardt, Markus; Thünemann, Holger (2016): Aufgaben im Geschichtsunterricht. Typen, Gütekriterien und Konstruktionsprinzipien. In: Geschichte Lernen 29 (2016), H. 174, S. 2-11.

Köster, Manuel (2013): Historisches Textverstehen. Rezeption und Identifikation in der multiethnischen Gesellschaft, Berlin: LIT Verlag.

Kühberger, Christoph (Hrsg.) (2012): Historisches Wissen. Geschichtsdidaktische Erkundung zu Art, Tiefe und Umfang für das historische Lernen. Schwalbach/Ts.: Wochenschau Verlag. 
Kultusministerkonferenz (2016): Vereinbarung zur Gestaltung der gymnasialen Oberstufe in der Sekundarstufe II, Beschluss der Kultusministerkonferenz vom 07.07.1972 i. d. F. vom 08.12.2016. S. 5. Online einsehbar unter: http://www.kmk.org/fileadmin/Dateien/veroeffentlichungen_beschluesse/1972/1972_07_07-Vereinbarung-Gestaltung-Sek2.pdf (zuletzt eingesehen am 07.02.2017).

Kultusministerkonferenz (2005): Einheitliche Prüfungsanforderungen der KMK. Geschichte. Beschluss der Kultusministerkonferenz vom 01.12.1989 i. d. F. vom 10.02.2005. Online einsehbar unter: https://www.kmk.org/fileadmin/veroeffentlichungen_beschluesse/1989/1989_12_01-EPA-Geschichte.pdf (zuletzt eingesehen am 01.08.2018).

Kultusministerkonferenz (2005): Einheitliche Prüfungsanforderungen in der Abiturprüfung Sozialkunde/Politik. Beschluss der Kultusministerkonferenz vom 01.12.1989 i. d. F. vom 17.11.2005. Online verfügbar unter:

http://www.kmk.org/fileadmin/veroeffentlichungen_beschluesse/1989/1989_12_01-EPA-Sozialk-Politik.pdf (zuletzt eingesehen am 01.08.2018).

Kultusministerkonferenz (2005): Einheitliche Prüfungsanforderungen in der Abiturprüfung Geographie (Beschluss der Kultusministerkonferenz vom 01.12.1989 i. d. F. vom 10.02.2005). Online verfügbar unter: http://www.kmk.org/fileadmin/veroeffentlichungen_beschluesse/1989/1989_12_01-EPA-Geographie.pdf (zuletzt eingesehen am 01.08.2018).

Lehrerfortbildungsserver Baden-Württemberg: Operatorenliste. Online einsehbar: http://lehrerfortbildung-bw.de/faecher/gwg/fb1/modul1/geo/operator/ (zuletzt eingesehen am 01.08.2018).

Lucas, Friedrich J. (1975): Zur Funktion der Sprache im Geschichtsunterricht. In: Die Funktion der Geschichte in unserer Zeit. Hrsg.: Jäckel, Eberhard; Weymar, Ernst. Stuttgart. S. 326-342.

Lurija, Alexander R. (1992): Das Gehirn in Aktion. Rowohlt: Reinbek. S. 90. Zitiert nach: Dörner, D. (2006). Sprache und Denken. In J. Funke (Ed.), Denken und Problem-lösen (=Enzyklopädie der Psychologie, Themenbereich C: Theorie und Forschung, Serie II: Kognition, Band 8). Göttingen: Hogrefe. S. 29.

Mayer, Ulrich (2014): Keine Angst vor Kompetenzen. Kompetenzorientierung - eine typologische, historische und systematische Einordnung. In: Geschichte für heute, Jg. 7/2014, Heft 3, S. 6-19.

Mayring, Philip (2003): Qualitative Inhaltsanalyse. Grundlagen und Techniken. Weinheim: Beltz.

Memminger, Josef (2007): Schüler schreiben Geschichte. Kreatives Schreiben zwischen Fiktionalität und Faktizität. Schwalbach/Ts.: Wochenschau-Verlag.

Mierwald, Marcel, Brauch, Nicola (2015): Historisches Argumentieren als Ausdruck historischen Denkens. Theoretische Fundierung und empirische Annäherungen. Zeitschrift für Geschichtsdidaktik 14/2015. S. 104-120. 
Middel, Matthias (2005): Konjunkturen vergleichender Geschichtswissenschaft. In: Schreiber, Waltraud (Hrsg.): Der Vergleich - Eine Methode zur Förderung historischer Kompetenzen. Ausgewählte Beispiele. Neuried: ars una. S. 11-30.

Mieles, Bernhard (1999): Wissenschaftspropädeutisches Arbeiten in der Sekundarstufe II. In: Geschichte lernen, 68/1999. S. 50-53.

Ministerium für Schule und Weiterbildung des Landes Nordrhein-Westfalen (jew. In der akt. Fassung), z. B. für 2018): Verzeichnis der zugelassenen Lehrmittel.

https://www.schulministerium.nrw.de/docs/Schulsystem/Unterricht/Lernmittel/index.html (zuletzt eingesehen am 01.08.2018).

Ministerium für Schule und Weiterbildung des Landes Nordrhein-Westfalen (2015): Kernlehrplan für das Abendgymnasium und Kolleg in Nordrhein-Westfalen Geschichte / Sozialwissenschaft https://www.schulentwicklung.nrw.de/lehrplaene/lehrplan/191/KLP_WbK_GESO.pdf

Ministerium für Schule und Weiterbildung des Landes Nordrhein-Westfalen (Hrsg.) (2014): Kernlehrplan für die Sekundarstufe II Gymnasium/Gesamtschule in Nordrhein-Westfalen. Geschichte. Online verfügbar unter:

https://www.schulentwicklung.nrw.de/lehrplaene/lehrplan/191/KLP_WbK_GESO.pdf (zuletzt eingesehen am 01.08.2018).

Ministerium für Schule und Weiterbildung des Landes Nordrhein-Westfalen (Hrsg.) (2012): Bildungspläne zur Erprobung für die Bildungsgänge, die zu einem Berufsabschluss nach Landesrecht und zur allgemeinen Hochschulreife oder zu beruflichen Kenntnissen und zur allgemeinen Hochschulreife führen. Fachlehrplan Gesellschaftslehre mit Geschichte Fachbereich Wirtschaft und Verwaltung. Grundkurs. Online einsehbar unter:

http://www.berufsbildung.schulministerium.nrw.de/cms/upload/_lehrplaene/d/wirtschaft_und_verwaltung/teil3/teil3_glg_wuv_gk.pdf (zuletzt eingesehen am 01.08.2018).

Ministerium für Schule und Weiterbildung des Landes Nordrhein-Westfalen (Hrsg.) (2007): Kernlehrplan für das Gymnasium - Sekundarstufe I (G8) in Nordrhein-Westfalen. Geschichte. Online einsehbar unter: http://www.schulentwicklung.nrw.de/lehrplaene/upload/lehrplaene_download/gymnasium_ g8/gym8_geschichte.pdf (zuletzt eingesehen am 01.08.2018).

Ministerium für Schule und Weiterbildung des Landes Nordrhein-Westfalen (Hrsg.) (2007): Lehrplan für das Berufskolleg in Nordrhein-Westfalen. Politik/Gesellschaftslehre bzw. Gesellschaftslehre mit Geschichte. Bildungsgänge der Fachoberschule (Anlage C9 bis C11 und D29). Online verfügbar unter: https://www.berufsbildung.nrw.de/cms/upload/_lehrplaene/d/politikgesellschaftslehrefos_40012.pdf (zuletzt eingesehen am 01.08.2018). 
Müller-Tamm, Jutta (2014): Die Denkfigur als wissensgeschichtliche Kategorie. In: Gess, Nicola; Janßen, Sandra: Wissensordnungen: Zu einer historischen Epistemologie der Literatur. Berlin/Boston: De Gruyter. S. 100-120.

Müller, Bernhard (2013): Wissenschaftspropädeutisches Arbeiten. in: Mayer, Ulrich; Pandel, Hans-Jürgen; Schneider, Gerhard (Hrsg.), Handbuch Methoden im Geschichtsunterricht. Klaus Bergmann zum Gedächtnis, Schwalbach/Ts. 4. Aufl. S. 308-324.

Nussbaumer, Markus; Sieber, Peter (1994): Texte analysieren mit dem Zürcher Textanalyseraster. In: Sieber, Peter (Hrsg.) Sprachfähigkeiten - Besser als ihr Ruf und nötiger denn je! Aarau u.a. S. 141-186.

Nussbaumer, Markus (1991): Was Texte sind und wie sie sein sollen. Ansätze zu einer sprachwissenschaftlichen Begründung eines Kriterienrasters zur Beurteilung von schriftlichen Schülertexten. Tübingen: Max Niemeyer Verlag.

OECD (2016): PISA 2015. Ergebnisse im Fokus. S. 4. Online verfügbar unter: https://www.oecd.org/berlin/themen/pisa-studie/PISA_2015_Zusammenfassung.pdf (zuletzt eingesehen am 01.08.2018).

Objartel, Georg (1970): Zur Semantik einfacherer Vergleichssätze im Deutschen. In: Moser, Hugo (Hrsg.): Linguistische Studien II. Düsseldorf: Schwann. S. 31-49.

Onken, Björn (2016): Theorie und Praxis im Konflikt. Überlegungen zur Narrativität von Verfassertexten in Geschichtsschulbüchern. In: Buchsteiner, Martin; Nitsche, Martin (Hrsg.): Historisches Erzählen und Lernen: Historische, theoretische, empirische und pragmatische Erkundungen. Wiesbaden: Springer VS. S. $69-84$.

Oppenrieder, Wilhelm (1991): Aussagesätze im Deutschen. In: Meibauer, Jörg (Hrsg.): Satzmodus zwischen Grammatik und Pragmatik. Tübingen: Niemeyer (=LA 180), S. 161-189.

Pandel, Hans-Jürgen (2010): Historisches Erzählen. Narrativität im Geschichtsunterricht. Schwalbach/Ts.: Wochenschau Verlag.

Pandel, Hans-Jürgen (2013): Geschichtsdidaktik. Eine Theorie für die Praxis. Schwalbach/Ts.: Wochenschau Verlag.

Pandel, Hans-Jürgen (2012): Geschichtsunterricht in der Sekundarstufe II. In: Barricelli, Michele; Lücke, Martin (Hrsg.): Handbuch Praxis des Geschichtsunterrichts. Bd. 1. Schwalbach/Ts.: Wochenschau Verlag. S. 176-188.

Pandel, Hans-Jürgen (2007): Geschichtsunterricht nach PISA. Kompetenzen, Bildungsstandards und Kerncurricula. Schwalbach/Ts.: Wochenschau Verlag.

Petersen, Inger (2014): Schreibfähigkeit und Mehrsprachigkeit. Berlin/Boston: de Gruyter. 
Petersen, Inger (2013): Entwicklung schriftlicher Argumentationskompetenz bei ein- und mehrsprachigen Oberstufenschüler/-innen und Studierenden. In: Brandl, Heike; Arslan, Emre; Langelahn, Elke; Riemer, Claudia (Hrsg.): Mehrsprachig in Wissenschaft und Gesellschaft. Mehrsprachigkeit, Bildungsbeteiligung und Potenziale von Studierenden mit Migrationshintergrund. Bielefeld, 69-79. Online verfügbar unter:

http://biecoll.ub.uni-bielefeld.de/volltexte/2013/5284/pdf/08_petersen_entwicklung\%20schriftlicher\%20argumentationskompetenz_korrigiert.pdf (zuletzt eingesehen am 01.08.2018).

Peuschel, Kristina, Burkhard, Anne (2019): Sprachliche Bildung und Deutsch als Zweitsprache in den geistes- und gesellschaftswissenschaftlichen Fächern. Tübingen: Narr Studienbücher.

Pingel, Falk (2010): UNESCO Guidebook on Textbook Research and Textbook Revision. 2. überarb. Auflage. Paris/Braunschweig.

Pohl, Karl Heinrich (2010): Wie evaluiert man Schulbücher? In: Fuchs, Eckhardt; Kahlert, Joachim; Sandfuchs, Uwe (Hrsg.): Schulbuch konkret. Kontexte - Produktion - Unterricht. Bad Heilbrunn: Julius Klinkhardt Verlag. S. 18-133.

Pohl, Torsten (2010): Das epistemische Relief wissenschaftlicher Texte - systematisch und ontogenetisch. In: Pohl, Thorsten; Steinhoff, Torsten (Hrsg.): Textformen als Lernformen. Duisburg: Gilles \& Francke. S. 97-116.

Pohl, Thorsten; Steinhoff, Torsten (2010): Textformen als Lernformen. In: Pohl, Thorsten; Steinhoff, Torsten (Hrsg.): Textformen als Lernformen. Duisburg: Gilles \& Francke. S. 5-26.

Prediger, Susanne (2013): Sprachmittel für mathematische Verstehensprozesse - Einblicke in Probleme, Vorgehensweisen und Ergebnisse von Entwicklungsforschungsstudien. In: Pallack, Andres (Hrsg.): Impulse für eine zeitgemäße Mathematiklehrer-Ausbildung. MNU-Dokumentation der 16. Fachleitertagung Mathematik. Neuss: Seeberger. Online verfügbar unter: http://www.mathematik.uni-dortmund.de/ prediger/veroeff/13-Prediger-MNU-FL-MuM_Sprachmittel.pdf (zuletzt eingesehen am 28.11.17).

Qualitäts- und Unterstützungsagentur - Landesinstitut für Schule (2017) Konstruktionsvorgaben für Abiturprüfungsaufgaben Geschichte (NRW). https://www.standardsicherung.schulministerium.nrw.de/abitur-gost/fach.php?fach=12 (zuletzt eingesehen am 03.02.18).

Raupp, Juliana; Vogelsang, Jens (2009): Medienresonanzanalyse. Eine Einführung in Theorie und Praxis. Wiesbaden: VS Verlag für Sozialwissenschaften Onlinezusatzmaterial. Springer. Online verfügbar unter: http://www.springer.com/cda/content/document/cda_downloaddocument/Berechnung-vonReliabilita\%CC\%88tskoeffizienten-Anhang.pdf?SGWID=0-0-45-1508109-p174296378 (zuletzt eingesehen am 08.08.17). 
Redder, Angelika; Guckelsberger, Susanne; Graßer, Barbara (2013): Mündliche Wissensprozessierung und Konnektierung: Sprachliche Handlungsfähigkeiten in der Primarstufe (Sprachvermittlungen 13). Münster: Waxmann Verlag.

Redder, Angelika (1986): Modalverben im Unterrichtsdiskurs. Pragmatik der Modalverben am Beispiel eines institutionellen Diskurses. Tübingen: Niemeyer Verlag.

Rehbein, Jochen (1978) Ankündigungen. In: Germanistische Linguistik (Varia V) 2-5, 340-387.

Rehbein, Jochen (1977): Komplexes Handeln. Elemente zur Handlungstheorie der Sprache. Stuttgart: Metzler.

Reich, Hans H.; Roth, Hans-Joachim; Döll, Marion (2009): Fast Catch Bumerang - Auswertungshinweise, Schreibimpuls und Auswertungsbogen. In: Lengyel, Drorit.; Reich, Hans H.; Roth, Hans-Joachim; Döll, Marion (Hrsg.): Von der Sprachdiagnose zur Sprachförderung. FörMig Edition Band 5. Münster, S. 209-241.

Riekenberg, Michael (2008): Der Vergleich. In: Mayer, Ulrich; Pandel, Hans-Jürgen, Schneider, Gerhard (Hrsg.): Handbuch Methoden im Geschichtsunterricht. Schwalbach/Ts. S. 269-285.

Roggenbruck, Simone (2011): Analogie als Ausgangspunkt für Vergleich und Klassifikation. Mit Beispielen aus der Sprachwissenschaft des 19. Jahrhunderts. In: Eggers, Michael (Hrsg.): Von Ähnlichkeiten und Unterschieden. Vergleich, Analogie und Klassifikation in Wissenschaft und Literatur (18./19. Jahrhundert). Heidelberg: Universitätsverlag Winter. S. 80-90.

Rüsen, Jörn (2013): Historik. Theorie der Geschichtswissenschaft. Köln, Weimar, Wien: Böhlau Verlag. Rüsen, Jörn (1997): Gesetze, Erklärungen. In: Bergmann, Klaus; Fröhlich, Klaus; Kuhn, Annette, Rüsen, Jörn; Schneider, Gerhard: Handbuch der Geschichtsdidaktik. 5. überarb. Aufl. Seelze-Velber: Kallmeyer. S. 164-169.

Rüsen, Jörn (1992): Das ideale Schulbuch. Überlegungen zum Leitmedium des Geschichtsunterrichts. In: Internationale Schulbuchforschung, 14. S. 237-250.

Rüsen, Jörn (1983): Historische Vernunft. Göttingen: Vandenhoeck und Ruprecht.

Rüth, Axel (2005): Erzählte Geschichte: narrative Strukturen in der französischen Annales-Geschichtsschreibung. Berlin, New York: de Gruyter. S. 22

Sauer, Michael (2012): Geschichte unterrichten. Eine Einführung in die Didaktik und Methodik. 10. Aufl. Seelze: Friedrich Verlag. 
Sauer, Michael (2002): Methodenkompetenz als Schlüsselqualifikation. Eine neue Grundlegung des Geschichtsunterrichts? In: Geschichte, Politik und ihre Didaktik: Zeitschrift für historisch-politische Bildung. Bd. 30, Heft 3/4. S. 183-192.

Schendera, Christian (2010): Clusteranalyse mit SPSS, Mit Faktorenanalyse. München: Oldenburg Wissenschaftsverlag.

Schindler, Kirsten; Siebert-Ott, Gesa (2012): Textkompetenzen im Übergang Oberstufe - Universität. In: Feilke, Helmuth; Köster, Juliane; Steinmetz, Michael (Hrsg.): Textkompetenzen in der Sekundarstufe II. Stuttgart: Fillibach bei Klett. S.151-175.

Schmölzer-Eibinger, Sabine (2013): Sprache als Medium des Lernens im Fach. In: Becker Mrotzek, Michael; Schramm, Karen; Thürmann, Eike; Vollmer, Helmut Johannes (Hrsg.): Sprache im Fach. Sprachlichkeit und fachliches Lernen. Münster u.a.: Waxmann, S. 25-40.

Schneider, Gerhard (2016): Transfer. In: Mayer, Ulrich; Pandel, Hans-Jürgen; Schneider, Gerhard (Hrsg.): Handbuch Methoden im Geschichtsunterricht. 5. Aufl. Schwalbach/Ts.: Wochenschau Verlag. S. 649-674.

Schneider, Gerhard (2012): Personalisierung/Personifizierung, in: Barricelli, Michele; Lücke, Martin (Hrsg.): Handbuch Praxis des Geschichtsunterrichts, Bd. 1, Schwalbach/Ts. S. 302-315.

Schönemann, Bernd; Thünemann, Holger; Zülsdorf-Kersting, Maik (2010): Was können Abiturienten? Zugleich ein Beitrag zur Debatte über Kompetenzen und Standards im Fach Geschichte. LIT Verlag, Münster.

Scholle, Dietrich (1997): Schulbuchanalyse. In: Bergmann, Klaus; Fröhlich, Klaus; Kuhn, Annette, Rüsen, Jörn; Schneider, Gerhard: Handbuch der Geschichtsdidaktik. 5. überarb. Aufl. Seelze-Velber: Kallmeyersche Verlagsbuchhandlung. S. 369-375.

Scholten-Akoun, Dirk; Kuhnen, Angela; Mashkovskaya, Anna (2012): Sprachkompetenzen Studierender. Design und erste Ergebnisse einer empirischen Studie. In: Feilke, Helmuth; Köster, Juliane; Steinmetz, Michael (Hrsg.): Textkompetenzen in der Sekundarstufe II. Stuttgart: Fillibach bei Klett, S. 207228.

Schrader, Viola, 2013: Geschichte als narrative Konstruktion. Eine funktional-linguistische Analyse von Darstellungstexten in Geschichtsschulbüchern. Berlin: LIT Verlag.

Schramm, Karen; Hardy, Ilonca; Saalbach, Henry; Gadow, Anne (2013): Wissenschaftliches Begründen im Sachunterricht. S. 299. In: Becker-Mrotzek, Michael; Schramm, Karen; Thürmann, Eike; Vollmer, Helmut Johannes (Hrsg.): Sprache im Fach. Sprachlichkeit und fachliches Lernen. Münster: Waxmann. S. 295-316. 
Schreiber, Waltraud (2007): Kompetenzbereich historische Methodenkompetenzen. In: Körber, Andreas et al. (Hrsg.): Kompetenzen historischen Denkens. Ein Strukturmodell als Beitrag zur Kompetenzorientierung in der Geschichtsdidaktik. (Kompetenzen: Grundlagen - Entwicklung - Förderung. Bd. 1). Neuried: Ars Una. S. 194-235.

Schreiber, Waltraud (2005a): Durch Vergleiche lernen - vergleichen lernen. In: Schreiber, Waltraud (Hrsg.): Der Vergleich - Eine Methode zur Förderung historischer Kompetenzen. Ausgewählte Beispiele. Neuried: ars una. S. 31-59.

Schreiber Waltraud (2005): Geschichte denken statt pauken. Theoretische Grundlegung für ein praktisches Konzept. Basisbeitrag. In: Mebus, Sylvia; Schreiber, Waltraud (Hrsg.): Geschichte denken statt pauken. Didaktisch-methodische Hinweise zur Förderung historischer Kompetenzen (Siebeneichener Diskurse 3). Meißen. S. 17-23.

Schriewer, Jürgen (2003): Problemdimensionen sozialwissenschaftlicher Komparatistik. In: Vergleich und Transfer. Kaelble, Hartmut; Schriewer, Jürgen (Hrsg.): Vergleich und Transfer. Komparatistik in den Sozial-, Geschichts- und Kulturwissenschaften. Frankfurt a. M.: Campus Verlag. S. 9-52.

Siegrist, Hannes (2003): Perspektiven der vergleichenden Geschichtswissenschaft. Gesellschaft, Kultur und Raum. In: Kaelble, Hartmut; Schriewer, Jürgen (Hrsg.): Vergleich und Transfer. Komparatistik in den Sozial-, Geschichts- und Kulturwissenschaften. Frankfurt a. M.: Campus Verlag. S. 303-339.

Spanhel, Dieter (1973): Die Sprache des Lehrers. Grundformen des didaktischen Sprechens (Sprache und Lernen. Internationale Studien zur pädagogischen Anthropologie, Band 12). 2. Aufl. Düsseldorf: Pädagogischer Verlag Schwann.

Ständige Konferenz der Kultusminister (2012): Bildungsstandards im Fach Deutsch für die Allgemeine Hochschulreife. S31. Online einsehbar unter:

http://www.kmk.org/fileadmin/Dateien/veroeffentlichungen_beschluesse/2012/2012_10_18-Bildungsstandards-Deutsch-Abi.pdf (zuletzt eingesehen am 05.04.17).

Steinhoff, Torsten (2017): Untersuchung von Textkorpora. In: Becker-Mrotzek, Michael; Grabowski, Joachim; Steinhoff, Torsten (Hrsg.): Forschungshandbuch empirische Schreibdidaktik. Münster: Waxmann. S. 353-368.

Steinhoff, Torsten; Grabowski, Joachim; Becker-Mrotzek, Michael (2017): Herausforderungen der empirischen Schreibdidaktik. In: Becker-Mrotzek, Michael; Grabowski, Joachim; Steinhoff, Torsten (Hrsg.): Forschungshandbuch empirische Schreibdidaktik. Münster: Waxmann. S. 9-24.

Steinhoff, Thorsten (2014): Lernen durch Schreiben. In: Feilke, Helmuth; Pohl, Thorsten (Hrsg.): Schriftlicher Sprachgebrauch - Texte verfassen. Baltmannsweiler: Schneider. S. 316-330. 
Steinhoff, Thorsten (2007): Wissenschaftliche Textkompetenz. Sprachgebrauch und Schreibentwicklung in wissenschaftlichen Texten von Studenten und Experten. Tübingen: Niemeyer.

Süssmann, Johannes (2000): Für eine Textsortenlehre der Geschichtsliteratur. In: ders.: Geschichtsschreibung oder Roman? Zur Konsitutionslogik von Geschichtserzählungen zwischen Schiller und Ranke (1780-1824). Stuttgart. S. 11-32.

Tilly, Charles (1984): Big Structures, Large Prozess, Huge Comparisons. New York: Russel Sage Foundation.

Trabant, Jürgen (2005): Zur Einführung: Vom linguistic turn der Geschichte zum historical turn der Linguistik. In: Trabant, Jürgen (Hrsg.): Sprache der Geschichte (Schriften des Historischen Kollegs 62). München: Oldenbourg Verlag. S. VII-XXII.

Thünemann, Holger (2013): Historische Lernaufgaben. Theoretische Überlegungen, empirische Befunde und forschungspragmatische Perspektiven. In: Zeitschrift für Geschichtsdidaktik 12, S. 141-155.

Thürmann, Eike (2012): Lernen durch Schreiben? Thesen zur Unterstützung sprachlicher Risikogruppen im Sachfachunterricht. Dies online. Online verfügbar unter: http://geb.uni-giessen.de/geb/volltexte/2012/8668/pdf/DieS_online-2012-1.pdf (zuletzt eingesehen am 01.08.2018).

Thurmair, Maria (2001): Vergleiche und Vergleichen. Eine Studie zu Form und Funktion der Vergleichsstrukturen im Deutschen. Linguistische Arbeiten, 43. Tübingen: Niemeyer.

Valentin, Paul (1988): Zu Natur, Funktion und Bedeutung der W-Wörter im heutigen Deutsch. In: Askedal, John Ole et al. (Hrsg.): Gedenkschrift für Ingerid Dal. Tübingen: Niemeyer. S. 202-213.

Van Norden, Jörg (2011): Was machst du für Geschichten? Didaktik eines narrativen Konstruktivismus. Freiburg: Centaurus Verlag.

Verband der Geschichtslehrer Deutschlands (2011): Bildungsstandards Geschichte (Sekundarstufe I) Kompetenzmodell und synoptische Darstellung der Kompetenzen und verbindlichen Inhalte des Geschichtsunterrichts - Entwurf. Online verfügbar unter:

http://cms.geschichtslehrerverband.de/typo/fileadmin/images/Bildungsstandards/Druckfassung/Standards_Druckformat_10.5.2011_.pdf (zuletzt eingesehen am 01.08.18).

Verband der Geschichtslehrer Deutschlands (Hrsg.) (2007): Bildungsstandards Geschichte. Rahmenmodell Gymnasium 5.-10. Jahrgangsstufe. Schwalbach/Ts.: Wochenschau-Verlag.

Welskopp, Thomas (2010): Vergleichende Geschichte. In: Europäische Geschichte Online (EGO), hrsg. vom Institut für Europäische Geschichte (IEG), Mainz 2010-12-03. Online einsehbar unter: http://iegego.eu/de/threads/theorien-und-methoden/vergleichende-geschichte (zuletzt eingesehen am 22.05.2020). 
Welskopp, Thomas (2007): Erklären, begründen, theoretisch begreifen. In: Goertz, Hans-Jürgen (Hrsg.): Geschichte. Ein Grundkurs. 3. rev. u. erw. Auflage. Reinbek bei Hamburg: Rowohlt. S. 137-177.

Welskopp, Thomas (2002): Erklären. In: Jordan, Stefan (Hrsg.): Lexikon Geschichtswissenschaft. Hundert Grundbegriffe. Stuttgart: Reclam. S. 81-84.

Wenzel, Birgit, (2007, hier in der Neuauflage von 2015): Aufgaben im Geschichtsunterricht. In: Günther-Arndt, Hilke; Handro, Saskia (Hrsg.): Geschichtsmethodik. Handbuch für die Sekundarstufe I und II. 5. Aufl. Berlin: Cornelsen. S. 84.

Wickner, Mareike-Cathrine (2018): So schließt sich der Kreis. Textsortenspezifische Schreibförderung im Geschichtsunterricht mit dem ,Genre Cycle'. In: Geschichte lernen 31 (2018). H. 182, S. 38-45.

Wirtz, Markus; Caspar, Franz, 2002: Beurteilerübereinstimmung und Beurteilerreliabilität. Methoden zur Bestimmung und Verbesserung der Zuverlässigkeit von Einschätzungen mittels Kategoriensystemen und Ratingskalen. Göttingen, Bern, Toronto, Seattle: Hogrefe.

Wohlrapp, Harald (2009): Der Begriff des Arguments. Über die Beziehungen zwischen Wissen, Forschen, Glauben, Subjektivität und Vernunft. 2. Aufl. Würzburg: Verlag Königshausen \& Neumann.

Wunderlich, Dieter (1973): Vergleichssätze. In: Kiefer, Ferenc; Ruwet, Nicolas (Hrsg.): Generative Grammar in Europe. Dordrecht: Reidel. S. 629-672.

Wunderer, Hartmann (2000): Geschichtsunterricht in der Sekundarstufe II. Schwalbach/Ts.: Wochenschau Verlag.

Ziegler, Dieter (2009): Die Industrielle Revolution in Deutschland, 2. bibliographisch aktualisierte Auflage. Darmstadt: WBG.

Zifonum, Gisela; Hoffmann, Ludger; Strecker, Bruno (1997): Grammatik der deutschen Sprache. Bd. 3. Berlin, New York: de Gruyter.

Zülsdorf-Kersting, Meik (2012): Was ist guter Geschichtsunterricht? Annäherung an eine verschüttete und wieder aktuelle Frage. In: Meyer-Hamme, Johannes; Thünemann, Holger; Zülsdorf-Kersting, Meik (Hrsg.): Was heißt guter Geschichtsunterricht? Perspektiven im Vergleich. Schwalbach/Ts.: Wochenschau Verlag. S. 7-19. 


\section{Verwendete Lehrwerke und Materialien}

Arand, Tobias et al. (2011): Geschichte und Geschehen Oberstufe NRW. Gesamtband. Stuttgart/Leipzig: Ernst Klett Verlag.

Asch, Bettina/Gehrke, Hans-Joachim/Geske-Wolf, Barbara u.a. (2010): Kursbuch Geschichte. Neue Ausgabe. Handreichungen für den Unterricht mit Kopiervorlagen, Cornelsen Verlag Berlin. S. 135.

Bage, Claudia et.al. (2012): Anstöße - Gesellschaftslehre mit Geschichte. Berufliche Gymnasien. Stuttgart: Ernst Klett Verlag.

Bahr, Frank (2006): Horizonte I. Geschichte für die Oberstufe. Von der griechischen Antike bis zur Frühen Neuzeit. Braunschweig: Bildungshaus Schulbuchverlage (Westermann).

Bahr, Frank (Hrsg.) (2006): Horizonte II. Geschichte für die Oberstufe. Vom Absolutismus bis zum Ersten Weltkrieg. Braunschweig: Westermann.

Bahr, Frank (2006): Horizonte III. Geschichte für die Oberstufe. Von der Weimarer Republik bis zum Beginn des 21. Jahrhunderts. Braunschweig: Bildungshaus Schulbuchverlage (Westermann).

Baumgärtner, Ulrich et. al (Hrsg.) (2015): Horizonte - Geschichte Qualifikationsphase S II NordrheinWestfalen. Braunschweig: Westermann.

Baumgärtner, Ulrich (Hrsg.) (2014): Horizonte Geschichte Einführungsphase. Sekundarstufe II Nordrhein-Westfalen, Braunschweig: Westermann.

Bender, Daniela u.a. (2010): Geschichte und Geschehen. Neuzeit. Oberstufe. Leipzig: Ernst Klett Schulbuchverlag.

Brütting, Rolf u.a. (2002): Geschichte Geschehen exempla. Geschichtliches Unterrichtswerk für die Sekundarstufe II. Leipzig: Ernst Klett Schulbuchverlag.

Droste, Peter Johannes (Hrsg.) (2015): Geschichte und Geschehen. Qualifikationsphase Oberstufe Nordrhein-Westfalen. Stuttgart: Klett.

Droste, Peter-Johannes (Hrsg.) (2011): Geschichte und Geschehen. Oberstufe Nordrhein-Westfalen. Stuttgart: Ernst Klett-Verlag.

Dzubiel, Christiane u.a. (Hrsg.) (2014): Geschichte und Geschehen. Oberstufe Einführungsphase Nordrhein-Westfalen. Ernst Klett Verlag.

Edinger, Thomas u.a. (2011): Forum Geschichte/Gemeinschaftskunde. Lern- und Arbeitsbuch für berufliche Gymnasien, Jahrgangsstufe 1 und 2. 2. Auflage. Köln: Bildungsverlag 1. 
Günther-Arndt, Hilke u.a. (2006): Geschichtsbuch Oberstufe, Band 1: Von der Antike bis zum Ende des 19. Jahrhunderts. Berlin: Cornelsen Verlag (unveränderte 1. Auflage von 1995).

Günther-Arndt, Hilke u.a. (2006): Geschichtsbuch Oberstufe, Band 2: Das 20. Jahrhundert. Berlin: Cornelsen Verlag (unveränderte 1. Auflage von 1995).

Kochendörfer, Jürgen (Hrsg). (2003): Geschichte Geschehen. Berufliche Oberstufe. Ernst Klett-Verlag.

Kochendörfer, Jürgen (Hrsg.) (2010): Geschichte und Geschehen. Berufskolleg. Stuttgart: Ernst Klett Verlag.

Langendorf, Elke (Hrsg.) (2015): Buchners Geschichte Oberstufe. Ausgabe Nordrhein-Westfalen Qualifikationsphase. Bamberg: C.C. Buchner.

Lanzinner, Maximilian (Hrsg.) (2014): Buchners Geschichte Oberstufe. Ausgabe Nordrhein-Westfalen Einführungsphase. Bamberg: C.C. Buchner.

Laschewski-Müller, Karin/Rauh, Robert (Hrsg.) (2015): Kursbuch Geschichte. Nordrhein-Westfalen Qualifikationsphase, Berlin: Cornelsen.

Laschewski-Müller, Karin/Rauh, Robert (Hrsg.) (2014): Kursbuch Geschichte. Nordrhein-Westfalen Einführungsphase, Berlin: Cornelsen.

Laschewski-Müller, Karin; Rauh, Robert (Hrsg.) (2010). Kursbuch Geschichte. Neue Ausgabe NordrheinWestfalen. Von der Antike bis zur Gegenwart. Berlin: Cornelsen-Verlag.

Lendzian, Hans-Jürgen (Hrsg.) (2015): Zeiten und Menschen. Geschichte Qualifikationsphase Oberstufe Nordrhein-Westfalen. Braunschweig u.a.: Schöningh-Verlag.

Lendzian, Hans-Jürgen (Hrsg.) (2014): Zeiten und Menschen. Geschichte Einführungsphase Oberstufe Nordrhein-Westfalen, Braunschweig u.a.: Schöningh Verlag.

Lendzian, Hans-Jürgen (Hrsg.) (2007): Zeiten und Menschen. Geschichte Oberstufe. Geschichtswerk für das Gymnasium (G8) in Nordrhein-Westfalen. Band 1. Braunschweig, Paderborn, Darmstadt: Schöningh Verlag.

Lendzian, Hans-Jürgen (Hrsg.) (2006): Zeiten und Menschen. Geschichte Oberstufe. Band 2. Braunschweig, Paderborn, Darmstadt: Schöningh Verlag.

Rauh, Robert (2011): Grundwissen Geschichte Sekundarstufe II. Berlin: Cornelsen Verlag.

von Reeken, Dietmar (2012): Geschichtskultur - Module für die Oberstufe. Berlin: Cornelsen Verlag. 
Schade, Peter; Stark, Hans-Joachim; Mayer, Ines (2010): Geschichte in der Gegenwart. Lehr- und Arbeitsbuch für Geschichte und Gemeinschaftskunde/Sozialkunde in der gymnasialen Oberstufe. Troisdorf: Bildungsverlag Eins.

\section{Benutztes Online-Forum}

http://www.schueler-talk.de/geschichte-f25/industrielle-revolution-vergleich-deutschland-englt1737.html (zu-letzt eingesehen am 09.10.15).

Hinweis: Die Seite ist inzwischen nicht mehr aktiv, kann aber über folgende Internet-Archiv-Adresse aufgerufen werden: https://web.archive.org/web/20130420021414/http://www.schueler-talk.de/geschichte-f25/industrielle-revolution-vergleich-deutschland-engl-t1737.html (zuletzt eingesehen am 18.07.18).

\section{Weitere Webseiten:}

Bildungsprotal NRW: Zulassung von Lernmitteln in NRW: https://www.schulministerium.nrw.de/docs/Schulsystem/Unterricht/Lernmittel/index.html

„Neue Statistik“: Kurs der Fernuniversität Hagen: http://www.fernuni-hagen.de/ksw/neuestatistik/content/MOD_23196/html/comp_23414.html (zuletzt eingesehen am 20.06.2018).

\section{Benutzte Online-Tools}

http://dfreelon.org/utils/recalfront/ 
Bisher erschienene Bände der Reihe

\section{Geschichtsdidaktische Studien}

ISSN 2363-670X

$\begin{array}{ll}1 \text { Marco Zerwas } & \text { Lernort "Deutsches Eck". Zur Variabilität } \\ & \text { geschichtskultureller Deutungsmuster } \\ & \text { ISBN 978-3-8325-3856-9 47.00 EUR }\end{array}$

Das auf dem Höhepunkt des Kaiser-Wilhelm-Kults am Deutschen Eck geschaffene Monumentaldenkmal unterlag wechselnden Konjunkturen; radikale Umnutzungen und bauliche Umgestaltungen prägen seine Geschichte. Es stellt sich die Frage, welche geschichtskulturellen Faktoren dabei maßgebend waren. Wie kam es immer wieder zu der Bezugnahme auf die Einheit Deutschlands, die als politischer Mythos an dem Geschichtsort wirkt? Einer Analyse der historischen Dynamiken geschichtskultureller Leitmuster widmet sich die vorliegende Studie, indem sie immer wieder nach stabilen Faktoren und wechselnden Motiven fragt.

Selten blieb der Diskurs um den Bestand des Denkmals auf das lokale Umfeld beschränkt und weckte häufig nationales Interesse. Der Skandal um die Reinszenierung des preußischen Denkmals um den Zeitpunkt der Wiedervereinigung macht bis heute fast sprachlos und ist ein Indiz für postmoderne Geschichtsinstrumentalisierung, die mehr an der Eventisierung öffentlicher Objekte als an Inhalten interessiert ist. Über die kurze Perspektive einer geschichtskulturellen Inszenierung an Festtagen hinaus wird der Nutzungsalltag langfristig transparent und ermöglicht eine epochenübergreifende Analyse. Mit genuin geschichtswissenschaftlichen und kunsthistorischen Methoden wird so ein für die Geschichtsdidaktik gangbarer Weg eingeschlagen, der das geschichtsbezogene Potenzial des Lernorts Deutsches Eck auf den Punkt bringt und in der Entwicklung eines didaktisch begründeten Lernszenarios mündet. 
2 Christoph Pallaske (Hrsg.)
Medien machen Geschichte. Neue Anforderungen an den geschichtsdidaktischen Medienbegriff im digitalen Wandel ISBN 978-3-8325-3956-6

Medien nehmen in der Praxis des Geschichtsunterrichts und in der Geschichtsdidaktik eine Schlüsselrolle ein. Quellen der Vergangenheit und Darstellungen von Geschichte sind immer nur über Medien vermittelbar. Über den Begriff Medien herrscht allerdings Unklarheit. Während in der Geschichtsdidaktik unter Medien meist Lernobjekte - also Quellen und Darstellungen - verstanden werden, weisen Praktiker des Geschichtslernens oft auch alle unterrichtlichen Hilfsmittel als Medien aus. Im Alltagssprachgebrauch oder anderen Wissenschaftsdisziplinen kommen Medien wiederum andere Bedeutungen zu.

Der digitale Wandel hat die Diskussion über Medienbegriffe des Geschichtslernens neu entfacht. Dabei rücken stärker der funktionale und kommunikative Aspekt von Medien und ihre kulturelle und gesellschaftliche Bedeutung - beispielsweise durch neue Kommunikationsformen und geschichtskulturelle Ausprägungen im Web 2.0 - in den Mittelpunkt. Auch in der Geschichtswissenschaft werden Medien nicht mehr nur als Abbild, sondern auch als ein Motor historischer Entwicklungen begriffen: Medien "machen" Geschichte. Der Band dokumentiert die Beiträge der Tagung "Geschichtsdidaktische Medienverständnisse" 2014 in Köln, auf der Entwicklungen des Medienbegriffs in der geschichtsdidaktischen und interdisziplinären Diskussion, neve Anforderungen an Medienbegriffe des Geschichtslernens im digitalen Wandel sowie Forschungsprojekte vorgestellt und diskutiert wurden. 
3 Christian Grieshaber (Hrsg.)
Sklaverei und Zwangsarbeit als Themen eines global orientierten Geschichtsunterrichts. Ein zentraler Beitrag zur Bildung eines globalen Geschichtsbewusstseins ISBN 978-3-8325-4284-9

Sklaverei besteht seit Anbeginn der Menschheitsgeschichte und ist in allen Völkerschaften bekannt. Der hohe Stellenwert der Sklaverei in der globalen geschichtskulturellen Auseinandersetzung zeigt sich zum einen an der Fülle großer Hollywoodproduktionen der letzten Zeit, die das Thema in den Mittelpunkt stellten (12 Years a Slave, Dido Elizabeth Belle, Django Unchained, Amazing Grace), sowie zum anderen an einer Vielzahl von wissenschaftlichen Publikationen, wie Michael Zeuskes Handbuch "Geschichte der Sklaverei". Nimmt man die Nachrichten in den Medien bewusst zur Kenntnis, so weiß man, dass bis auf den heutigen Tag die Unfreiheit immer noch ein trauriges Kapitel von Ungerechtigkeit und Unmenschlichkeit in unserer globalisierten Welt darstellt. Der Sammelband, der das Ergebnis der geschichtsdidaktischen Tagung "Die Sklaverei als Thema eines global orientierten Geschichtsunterrichts" an der Universität Rostock dokumentiert, zeigt, dass eine Auseinandersetzung mit der Sklaverei sehr große Chancen bietet, Schülern und jungen Erwachsenen historisches Denken in der longue durée zu vermitteln. Dazu werden exemplarisch neve, für den Geschichtsunterricht bisher unbeachtete Perspektiven zur Geschichte des Abolitionismus, zur Kolonialgeschichte, sowie zur Geschichte des anderen deutschen Staates vorgestellt und daraus konkrete Fragestellungen und Unterrichtsvorschläge entwickelt. 
Historische Romane waren und sind in der Öffentlichkeit erstaunlich konstant präsent - und das trotz konkurrierender, populärer Medien wie Kinofilmen, Serien oder Spielen. Durch historische Romane werden Geschichten erzählt und inszeniert und (sehr wahrscheinlich) historische Vorstellungen geprägt. Aber wie geschieht das genau? Welche Geschichtsbilder werden vermittelt? Und wer sind engagierte Akteure auf dem wirtschaftlich orientierten Buchmarkt? Mithilfe empirischer Verfahren nimmt diese Studie das geschichtskulturelle, kommerzielle Medium "Historischer Roman" in den Blick, lässt sich nicht von den gängigen Kanonlisten leiten, sondern blickt auf jene ,Schmöker', deren Anspruch auf Ästhetik schwerer wiegt als auf wissenschaftlich korrekte Historiographie. Diese Studie ist eine Produkt- und keine Rezeptionsanalyse. Diese Produktanalyse kann aber historisch kontextualisiert werden. Der diachrone Vergleich der in den Romanen vorwaltenden Geschichtsbilder zeigt nicht nur in einem wichtigen Segment die Dynamiken und Konjunkturen deutschsprachiger Geschichtskultur auf, sondern weist auch auf Korrespondenzen mit gesellschaftspolitischen Entwicklungen der Jahre 1913 bis 1933 hin. Der potentielle Dialog zwischen Roman und Publikum, oder genauer gesagt: die Marktantizipation von Publikumsverlagen und ihrer literarischen Produzenten, lässt für den untersuchten Zeitraum erkennen, dass Medien der Geschichtskultur auf eine seismographische Weise Verschiebungen im kollektiven Bewusstsein ihrer Nutzerinnen und Nutzer dokumentieren. 
Das übergeordnete Ziel von Geschichtsunterricht besteht darin, Schülerinnen und Schüler in die Lage zu versetzen, eigene historische Narrationen anzufertigen und mit vorliegenden historischen Narrationen umgehen zu können. Geschichtliche Spielfilme könnten eine Möglichkeit darstellen, an beiden Zielsetzungen zu arbeiten, da sie eine (geschichtskulturelle) Variante der historischen Narration darstellen und eine Auseinandersetzung mit den narrativen Strukturen dazu beitragen könnte, eigene Narrationskompetenzen anzubahnen und zu vertiefen. Die vorliegende Studie zeigt daher am Beispiel des Fernsehzweiteiles "Schicksalsjahre"(2011) auf, wie eine mögliche Unterrichtseinheit zur Analyse eines geschichtlichen Spielfilms im 10. Jahrgang einer Realschule bzw. 11. Jahrgang eines Gymnasiums gestaltet werden kann. Weiterhin legt die Studie erste Ergebnisse zur Frage vor, wie sich solch eine Unterrichtseinheit auf die narrativen Strukturen von Schülerinnen und Schülern auswirkt. Hierzu wird auf ein eigens entwickeltes Kriterienraster zurückgegriffen, das sowohl zur Tiefenbeschreibung schriftlicher Schülerprodukte herangezogen werden kann wie auch für die Analyse der filmischen Narrationsstrukturen. Die Studie bietet daher sowohl Anregungen für die Unterrichtspraxis (Materialien und Arbeitsblätter sind beigefügt) als auch forschungsbasierte Erkenntnisse zu möglichen Auswirkungen einer Spielfilmanalyse. Diese weisen darauf hin, dass es zu graduellen Verbesserungen in den Schülernarrationen kommt, so dass Geschichtsspielfilme offenbar tatsächlich als eine Möglichkeit (unter vielen) genutz† werden können, um Narrationskompetenzen aufzubauen und zu vertiefen. 
Die Kompetenz, andere Kulturen zu verstehen und mit Alterität umzugehen, scheint in einer interkulturellen Einwanderungsgesellschaft und einer globalisierten kulturellen und ökonomischen Wirklichkeit unerlässlich zu sein. Im Rahmen schulischer Bildung nimmt der Geschichtsunterricht hier eine Schlüsselrolle bei der Förderung von (interkultureller) Perspektivübernahme ein.

Der vorliegende Band befasst sich mit der theoretischen Modellierung von Perspektivübernahme als Partialkompetenz historischen Denkens. Das konzipierte Niveaustufenmodell wurde im Rahmen einer explorativen Studie an zwei Gesamtschulen einer ersten empirischen Prüfung unterzogen. Die Intervention geschah im Rahmen einer Unterrichtsreihe zur japanischen Mentalitätsgeschichte mit Fokus auf die Gesellschaft der Tokugawa- und Meiji-Periode zwischen 1600 und 1912. Dabei wurde der Schwerpunkt auf die gesellschaftlichen Strukturen und die Mentalität der Samurai als bestimmender Elite an den Wendepunkten der Epochen gelegt.

Die erfassten Datensätze wurden mittels einer qualitativen Inhaltsanalyse ausgewertet, um das Niveaustufenmodell zur Perspektivübernahme zu prüfen. Aus den Antworten auf offene Aufgabenformate zu unterschiedlichen Problemstellungen wurden jene Kernelemente destilliert, die als Performanzleistungen der Perspektivübernahme erfasst und in das Niveaustufenmodell eingeordnet werden konnten. 
Dem Vergleichen wird in der Geschichtswissenschaft die Funktion einer historischen Methode zugesprochen, für den Geschichtsunterricht dient es als Methode zur Förderung historischer Kompetenzen. In den Geschichtslehrwerken der Sekundarstufe II taucht es in der Regel in Form von Aufgaben auf. Doch wie relevant sind Vergleichsaufgaben in Lehrwerken überhaupt und welche Fertigkeiten und Kompetenzen benötigen Lernende in der Sekundarstufe II zur (schriftlichen) Lösung einer historischen Vergleichsaufgabe?

Diesen Fragen wird mit Hilfe einer zweiteiligen Untersuchung auf der Grundlage einer umfangreichen Frequenzanalyse von Aufgaben in Geschichtslehrwerken der Sekundarstufe II in NRW sowie einer Korpusanalyse von Lernendentexten zu einer authentischen Vergleichsaufgabe aus einem Geschichtslehrwerk nachgegangen.

Die Arbeit verbindet den geschichtsdidaktischen Zugang mit dem linguistischen Ansatz der Funktionalen Pragmatik, um das Vergleichen als Handlungsmuster des Geschichtsunterrichts zu bestimmen und es im Hinblick auf seinen kommunikativen Zweck in Teilhandlungen aufzuschlüsseln. Der Zugang wird ergänzł durch den Bezug zur Schreibdidaktik, der gerade im Hinblick auf wissenschaftspropädeutisches Arbeiten in der Sekundarstufe II eine wichtige Rolle spielt.

Aus den Ergebnissen der Untersuchungen wird auf der Grundlage eines Indikatorenmodells ein praxisorientierter Weg zum Umgang mit Vergleichsaufgaben im Geschichtsunterricht vorgeschlagen.

Alle erschienenen Bücher können unter der angegebenen ISBN-Nummer direkt online (http://www.logos-verlag.de) oder per Fax (030 - 428510 92) beim Logos Verlag Berlin bestellt werden. 

Dem Vergleichen wird in der Geschichtswissenschaft die Funktion einer historischen Methode zugesprochen, für den Geschichtsunterricht dient es als Methode zur Förderung historischer Kompetenzen. In den Geschichtslehrwerken der Sekundarstufe II taucht es in der Regel in Form von Aufgaben auf. Doch wie relevant sind Vergleichsaufgaben in Lehrwerken überhaupt und welche Fertigkeiten und Kompetenzen benötigen Lernende in der Sekundarstufe II zur (schriftlichen) Lösung einer historischen Vergleichsaufgabe?

Diesen Fragen wird mit Hilfe einer zweiteiligen Untersuchung auf der Grundlage einer umfangreichen Frequenzanalyse von Aufgaben in Geschichtslehrwerken der Sekundarstufe II in NRW sowie einer Korpusanalyse von Lernendentexten zu einer authentischen Vergleichsaufgabe aus einem Geschichtslehrwerk nachgegangen.

Die Arbeit verbindet den geschichtsdidaktischen Zugang mit dem linguistischen Ansatz der Funktionalen Pragmatik, um das Vergleichen als Handlungsmuster des Geschichtsunterrichts zu bestimmen und es im Hinblick auf seinen kommunikativen Zweck in Teilhandlungen aufzuschlüsseln. Der Zugang wird ergänzt durch den Bezug zur Schreibdidaktik, der gerade im Hinblick auf wissenschaftspropädeutisches Arbeiten in der Sekundarstufe II eine wichtige Rolle spielt.

Aus den Ergebnissen der Untersuchungen wird auf der Grundlage eines Indikatorenmodells ein praxisorientierter Weg zum Umgang mit Vergleichsaufgaben im Geschichtsunterricht vorgeschlagen.

\section{Logos Verlag Berlin}

ISBN 978-3-8325-5140-7

ISSN 2363-670X 